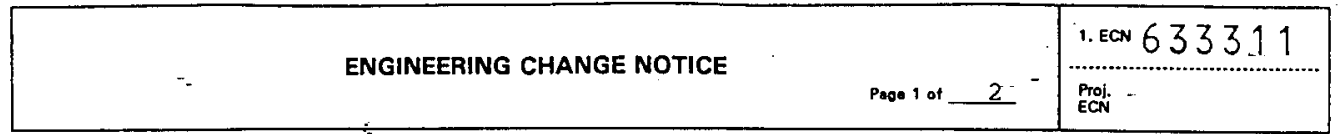

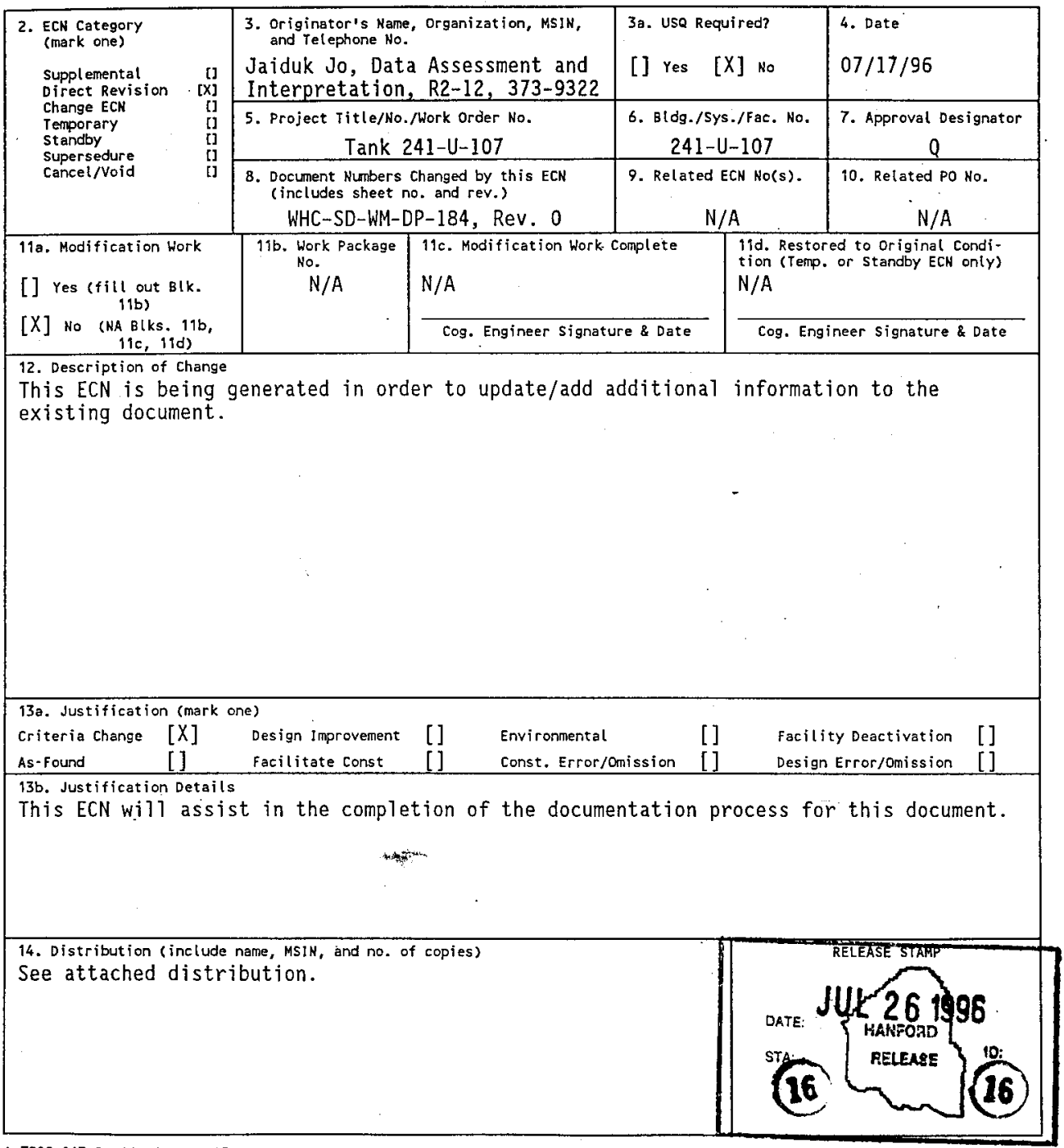




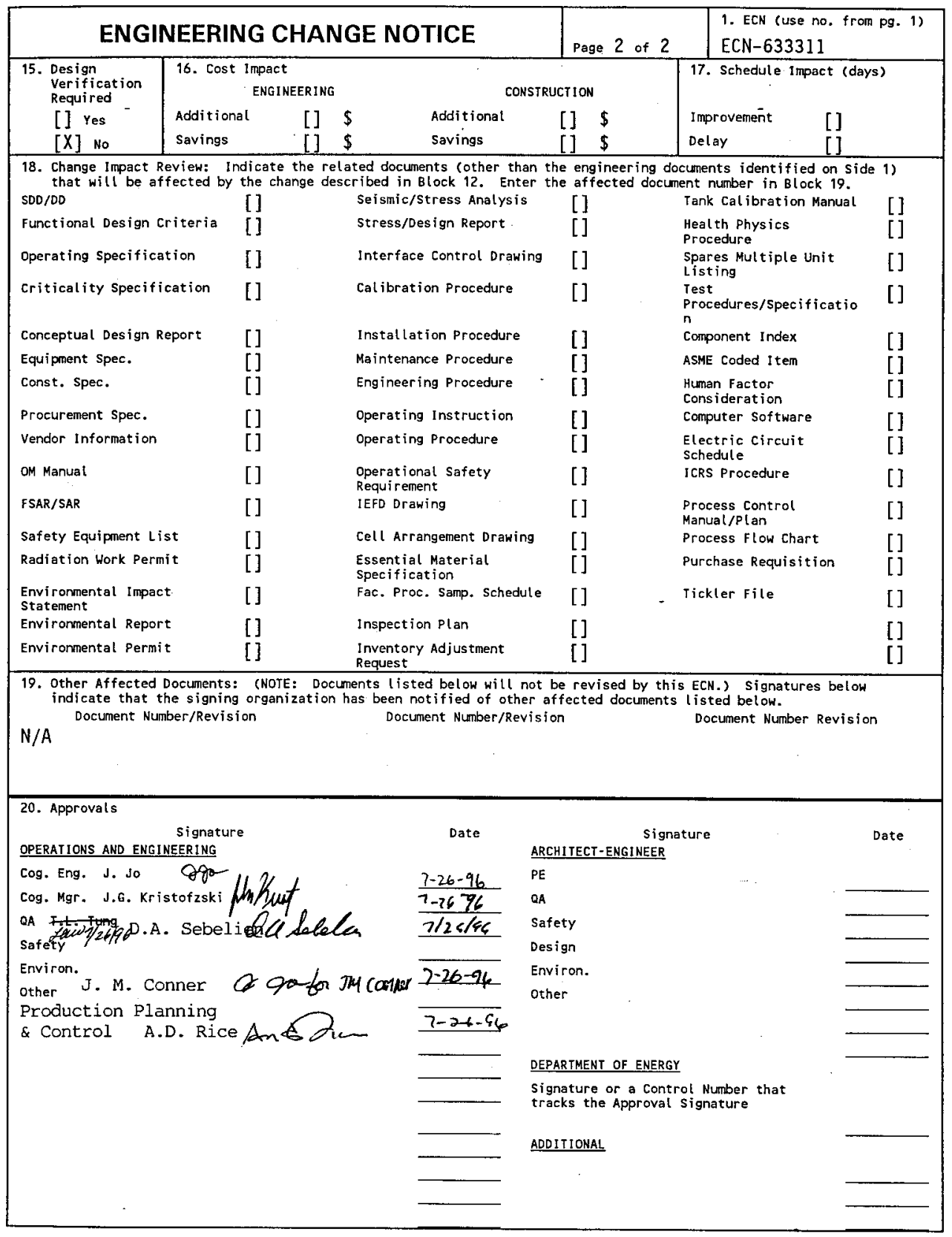




\section{Final Report for Tank 241-U-107, Push Mode Cores 129, 134 and 135}

Jaiduk Jo

Westinghouse Hanford Company, Richland, WA 99352

U.S. Department of Energy Contract DE-AC06-87RL10930

$\begin{array}{lll}\text { EDT/ECN: } & \text { ECN-633311 } & \text { UC: } 2070 \\ \text { Org Code: } & 79400 & \text { Charge Code: } N 4 G 4 C \\ \text { B\&R Code: } & \text { EW 3120074 } & \text { Tota7 Pages: } 1680\end{array}$

Key Words: Final Report, Tank 241-U-107, Tank U-107, U-107, U Farm, Push Mode, Push, Core 129, Core 134, Core 135

Abstract: N/A

TRADEMARK DISCLAIMER. Reference herein to any specific comercial product, process, or service by trade name, trademark, manufacturer, or otherwise, does not necessarily constitute or imply its endorsement, recommendation, or favoring by the United States Government or any agency thereof or its contractors or subcontractors.

Printed in the United States of America. To obtain copies of this document, contact: WhC/BCS Document Control Services, P.O. Box 1970, Mailstop H6-08, Richland WA 99352, Phone (509) 372-2420; Fax (509) 376-4989.
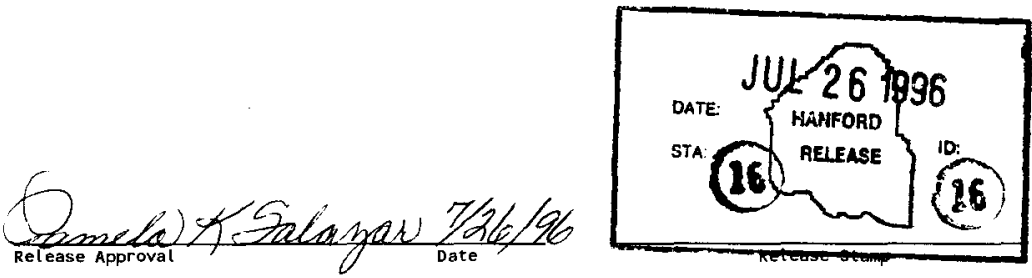

\section{Approved for Public Release}


(2) Title

Final Report for Tank 241-U-107, Push Mode Cores 129, 134 and 135

CHANGE CONTROL RECORD

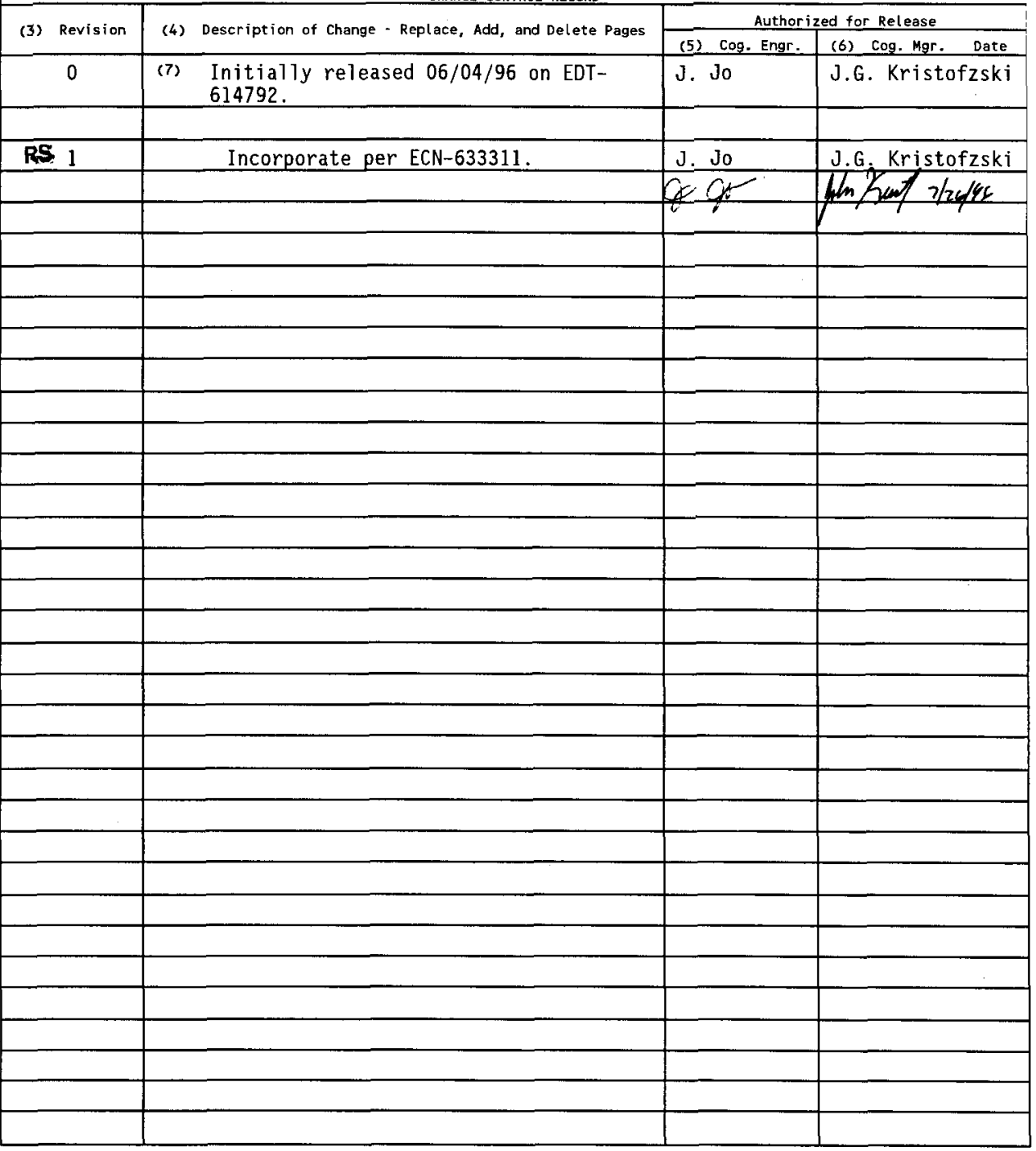


WHC-SD-WM-DP-184, REV. 1

\section{ANALYTICAL SERVICES}

\section{FINAL REPORT FOR TANK 241-U-107, PUSH MODE CORES 129, 134 AND 135}

PROJECT COORDINATOR: JAIDUK JO

Prepared for the U.S. Department of Energy Office of Environmental Restoration and Waste Management

by

Westinghouse Hanford Company 
WHC-SD-WM-DP-184, REV. I

THIS PAGE WAS INTENTIONALLY LEFT BLANK 
WHC-SD-WM-DP-184, REV, 1

TABLE OF CONTENTS

Narrative .. . . . . . . . . . . . . . . . . . . . 1

Sample Data Summary . . . . . . . . . . . . . . . . . . 6

U-107 Statistical Analysis . . . . . . . . . . . . . . . . . 54

Chain of Custody Forms ....................... . 57

Photographs . . . . . . . . . . . . . . . . . . . 78

Sample Handling ............................ . . 101

Extrusion Worklist \#5652 (610) . . . . . . . . . . . . . 103

Extrusion Worklist \#5653(612) ................ . 104

Extrusion Workl ist \#5654 (614) .................... 105

Extrusion Workl ist \#5655 (611) ................. . . . 106

Extrusion Worklist \#5656 (613) ................ 107

Extrusion Workl ist \#5800 (694) ................... . 108

Extrusion Worklist \#5854 (775) ................ 109

Extrusion Worklist $\$ 5857$ (778) ................. . . 110

Extrusion Worklist \# 5861 (781) ................... . 111

Extrusion Workl ist \#5863 (782) ................... 112

Extrusion Workl ist \# 5865 (783) ................... 113

Extrusion Workl ist \#5866 (784) .................. 114

Extrusion Workl ist \# 5867 (785) . . . . . . . . . . . . . . . 115

Extrusion Workl ist \#6049 (924) ..................... 116

Extrusion Worklist \#7293 (1667) . . . . . . . . . . . . . . 117

Extrusion Worklist \# 7296 (1668) . . . . . . . . . . . . . . . . . 118

Extrusion Work list \#7297 (1669) . . . . . . . . . . . . . . . . 119

Extrusion Worklist \# $7299(1670)$. . . . . . . . . . . . 120

Extrusion Workl ist \#7308 (1692) . . . . . . . . . . . . 121

Extrusion Worklist 6276 Core $129(665,682)$. . . . . . . . . . 122

Extrusion Workl ist $\# 6277$ Core $134(1110,1119,1120,1121)$. . . . 123

Extrusion Workl ist \# 8846 Core 135 (1866) . . . . . . . . . . . 124

Sample Preparations . . . . . . . . . . . . . . . . . . 125

Fusion Digestion Worklist \#5882 $(684,686,687)$. . . . . . . . . 127

Fusion Digestion Work1 ist \# $5883(1688,1689) . . . . . . . . . . . .128$

Fusion Digestion Workl ist \#6417 (1089, 1090, 1091)......... . 129

Fusion Digestion Workl ist \#6418 $(1092,1093,1094) . . . . . . . . ~ 130$

Fusion Digestion Workl ist \# $6419(1095,1096,1097,1132) . . . . .131$

Fusion Digestion Worklist * $6535(1153,1162) . . . . . . . . . . .133$

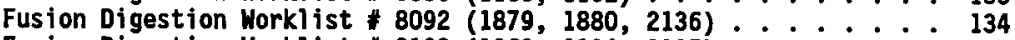

Fusion Digestion Worklist \#8102 $(1868,2134,2135)$. . . . . . . 135 
WHC-SD-WM-DP-184, REV. 1

TABLE OF CONTENTS (Continued)

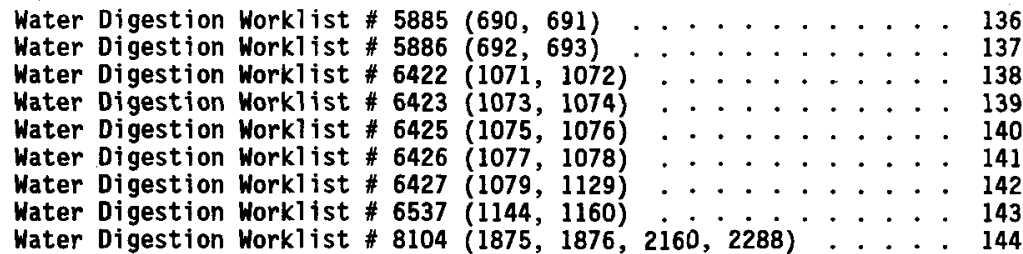

Acid Digestion Worklist \# $5884(788,789,790,791)$. . . . . . . . 145

Acid Digestion Workl ist \# $6420(1080,1081,1082,1083,1084)$. . . 147

Acid Digestion Workl ist \#6421 (1085, 1086, 1087, 1088, 1130) . . . 149

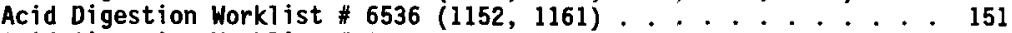

Acid Digestion Worklist \#8103 $(1877,1878,2161) \ldots . . . . . . .152$

Acid Digestion Worklist \# $8209(2284,2285,2286,2287)$. . . . . 154

Inorganic Analyses . . . . . . . . . . . . . . I56

Bulk Density Worksheet . . . . . . . . . . . . . . . . . . 158

Specific Gravity Analysis (SpG)

SpG Workl ist \#6053 (637) . . . . . . . . . . . . . . . . 159

SpG Work list \#6523 (1107) . . . . . . . . . . . . ..... 166

SpG Worklist \# $7984(1865)$. . . . . . . . . . . . . . . 170

SpG Work l ist \# $8440(2140,2141,2142)$. . . . . . . . . . . . . . 178

SpG Worklist \#8729 (1121) . . . . . . . . . . . . . . 187

OH Analys is Worklist \#6687 $(665,682)$. . . . . . . . . . . . . 207

OH Analysis Work 1 ist \# $7581(1110,1119,1120,1121)$. . . . . . . 214

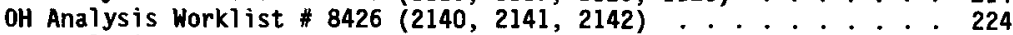

OH Analysis Work1 ist \# 9008 (1866) . . . . . . . . . . . . . . 233

pH Analysis Worklist \#6770 $(665,682)$. . . . . . . . . . . 241

pH Analys is Worklist \# $7582(1110,1119,1120,1121) . . . . . . .242$

pH Analys is Workl ist \#8428 $(2140,2141,2142)$. . . . . . . . . 243

pH Analysis Workl ist $\$ 8913(1866) \ldots . . . . . . . . . . . . . .244$

Ion Chromatograhic Analysis (IC)

IC Workl ist \# $6322(690,691)$. . . . . . . . . . . . . . . 245

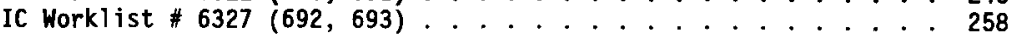

IC Workl ist \# $6622(682,665)$. . . . . . . . . . . . . . . . . . . 274

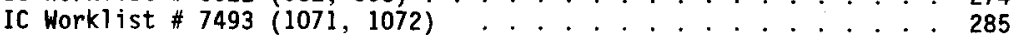

IC Worklist \#7494 (1073, 1074) .................... 300

IC Worklist $\$ 7495(1075,1076)$............... . . 313

IC Workl ist \# $7496(1077,1078)$. . . . . . . . . . . . . . . . 326 
WHC-SD-WM-DP-184, REV. 1

TABLE OF CONTENTS (Continued)

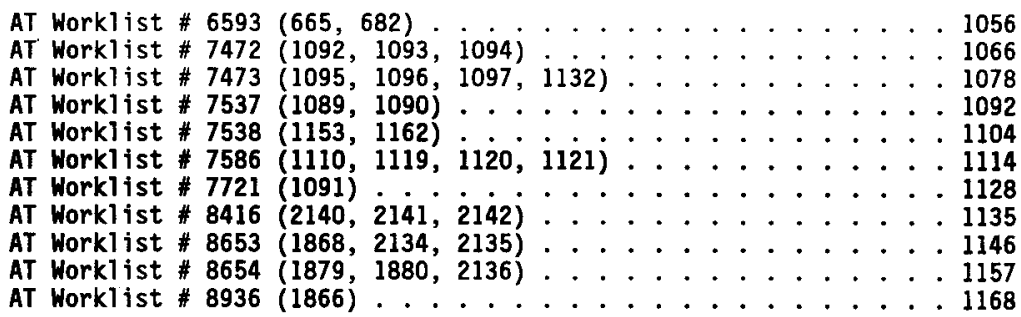

Gamma Energy Analysis (GEA)

GEA Worklist \#8418 $(2140,2141,2142) \ldots \ldots \ldots \ldots$

GEA Worklist \#8657 $(2134,2135,2136) \ldots \ldots . \ldots . \ldots 1211$

Strontium 90 Analysis $(\mathrm{Sr}-90)$

Sr-90 Workl ist \# 6901 $(665,682)$. . . . . . . . . . . . . . . . 1247

Sr-90 Worklist \# $7795(1119,1120,1121) \ldots \ldots . . \ldots . . . .1255$

Sr-90 Workl ist \#8417 (2141, 2142) . . . . . . . . . . . . 1265

Sr-90 Workl ist \# 8611 (2034) . . . . . . . . . . . . . . . . 1275

Sr-90 Workl ist \#8712 (2140) . . . . . . . . . . . . . . . . . 1281

Sr-90 Work1 ist \# $9300-8941(1866) \ldots \ldots . . . . . . . . .1287$

Plutonium 239 Analysis (Pu-239)

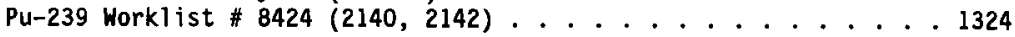

Part II 45-Day Safety Screening Results for Tank

241-U-107, Push Mode Cores 129, 134 and 135 ....... 2-1

Narrative .................. 2- . . . . .

Sample Data Summary . . . . . . . . . . . . . 2-16

Dome Space Vapor Flammability Data Sheets . . . . . . . . . . 2-34

Statistical Analysis .................. 2- . . . . . . .

Inorganic Analyses . . . . . . . . . . . . . . 2-48

Differential Scanning Calorimetry (DSC)

DSC Workl ist \# $5892(644,647) \ldots . . . . . . . . .2-50$

DSC Workl ist \#5893 $(650,653) \ldots \ldots \ldots \ldots . . \ldots . . . . .2-56$

DSC Work1 ist \#6035 (683) . . . . . . . . . . . . . 2-62

DSC Work list \#6511 $(1055,1056) \ldots \ldots . . \ldots 2-66$ 
WHC-SD-WM-DP-184, REV. I

TABLE OF CONTENTS (Cont inued)

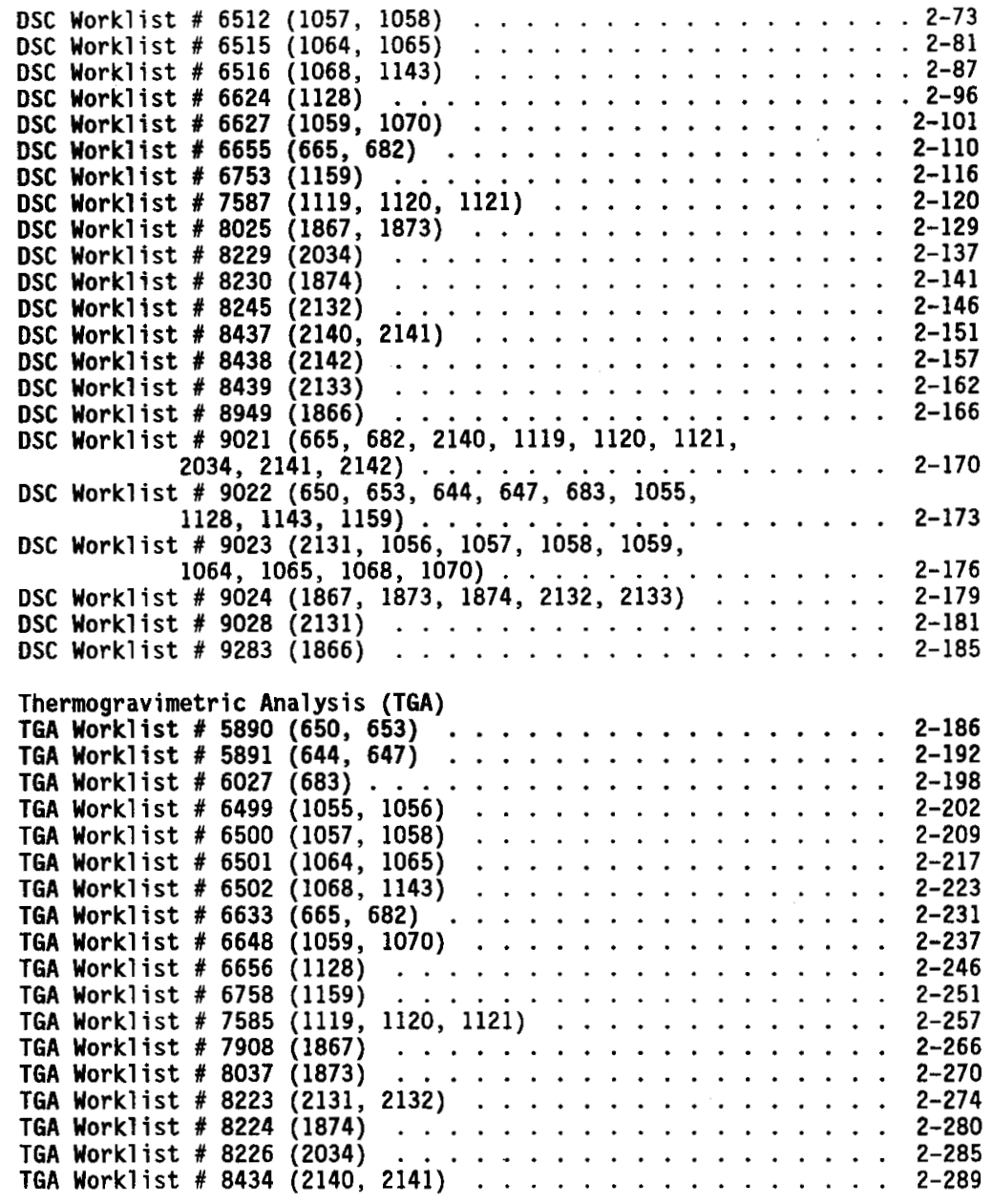


WHC-SD-WM-DP-184, REV. 1

TABLE OF CONTENTS (Continued)

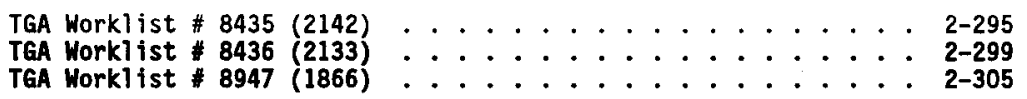

This document consists of two sections

Part I consists of pages 1 through 1357, plus pages 5.1, 77.1, 757.1 and pages $i j, 2,7,11,55,58,79,102,126,157,1038$ and 1055 were intentionally left blank.

Part II consists of pages 2-1 through 2-308, and pages 2-2, 2-6, 2-17, 2-35, 2-44, and 2-49 were intentionally left blank. 
WHC-SD-WM-DP-184, REV. 1

NARRATIVE 
WHC-SD-WH-DP-184, REV. 1

THIS PAGE WAS INTENTIONALLY LEFT BLANK 


\section{WHC-SD-WM-DP-184, REV. 1 \\ FINAL REPORT FOR TANK 241-U-107, PUSH MODE CORES 129,134 AND 135}

\section{SUMMARY}

This is the final laboratory report for tank 241-U-107 (U-107), cores 129, 134 and 135. A11 three core samples underwent safety screening analyses in accordance with the sampling and analysis plan (Raphael 1996)-differential scanning calorimetry (DSC), thermogravimetric analysis (TGA), total alpha analysis, and bulk density measurements. Bromide analysis by ion chromatography (IC) and lithium analys is by inductively coupled plasma atomic emission spectroscopy (ICP) were performed to determine if the samples were contaminated with any lithium bromide solution that was used during sampling. In addition to the core sample analysis, the tank headspace flammability was measured prior to core sampling as required by the SAP and Safety Screening DQ0. This final report contains the analysis results requested by the organic data quality objective (DQO), namely the total organic carbon (TOC) results. The analysis results requested by the compatibility DQO is contained in a letter report (Esch, 1995).

None of the data indicate that the tank is "unsafe" when compared to the criteria in the Safety Screening Data Quality Objective (Dukelow et al, 1995). with the exception of the energetic results for two samples (liquid samples). However, there is a high moisture content of greater than $45 \%$ in the liquid portion. Also, TOC analysis on these samples indicated negligible fuel content. The one-sided 95-percent confidence intervals for total alpha results are well below the notification 1 imit. Furthermore, the vapor in the tank U-107 dome space is far below the $25 \%$ lower flammability 1 imit (LFL) stated in the SAP. Therefore, the results show that this tank may be considered "conditionally" safe.

Water with a lithium bromide tracer, was used to soften the waste when pushing with $1900 \mathrm{lb}$ down force could not penetrate the layer. However, no lithium bromide solution blank was taken. The lithium bromide solution blank data from tank B-204 sampling event is used for comparison. The results indicates very small amounts of contamination in some samples and large amounts of contamination in other samples by the lithium bromide solution. Most of the samples with large amounts of contamination had lithium bromide solution added to soften the waste.

\section{SCOPE}

This document serves as the final report deliverable for the tank 241-U-107 core samples collected between February 6 and March 18, 1996. Each sample was received, extruded, and analyzed by the 222-5 Laboratory in accordance with the SAP (Jo 1996b).

This report includes the safety screening results (tank headspace flammability, DSC, TGA, total alpha, and bulk density or specific gravity) and the IC, ICP, and TOC results. As the final report, the following are also included: chain of custody forms; photographs of extruded segments; bulk 
WHC-SD-WM-DP-184, REV. 1

density worksheets; and worklists for extrusion, sample preparation, total alpha, specific gravity, IC, ICP, and TOC. Worklists for DSC and TGA were provided in the 45-day report (Jo 1996a). A copy of the 45-day report is provided as Part II of this final report.

\section{SAMPLE RECEIPT AND EXTRUSION}

A description of the sample receipt and extrusion is contained in the 45-day report (Jo 1996a). The chain of custody forms, photographs of the extruded segments, and extrusion worklists are provided in this report. Details of the extrusion and subsampling are provided in the hot cell logbook for tank U-107 (Fuller 1996).

\section{TANK HEADSPACE FLAMMABILITY}

A description of the tank headspace flammability and other headspace measurements taken in tank U-107 prior to core sampling is provided in the 45day report (Jo 1996a). The combustible gas meter readings were between 0 to 4 percent of the LFL, indicating no flammability concerns with this tank. Other measurements made were oxygen (20.8 to 21.1 percent), total organic vapors (14 to 75 parts per million $(\mathrm{ppm}))$, and ammonia (400 to $600 \mathrm{ppm}$ ).

The vapor in the tank headspace above the waste is safe from a selfpropagating chemical reaction (safe from flame or explosion) but is dangerous to breathe. Ammonia concentration is at the threshold (300 ppm) considered by the National Institute of Safety and Health to be "immediately dangerous to life or health." However, the ammonia concentration is 0 ppm at the breather filter and it is not dangerous for the workers to breathe.

\section{ANALYTICAL RESULTS}

The results of all analyses performed on the tank U-107 push samples are provided in the Sample Data Summary tables. Analytical results for DSC, TGA, and total alpha were discussed in the 45-day report (Jo 1996a). TOC, IC, and ICP analyses results are also discussed below.

\section{Total Organic Carbon (TOC)}

TOC analyses were performed by using procedure LA-342-100 Rev. C-0. The results for sludge samples ranged from $516 \mu \mathrm{g} / \mathrm{g}$ to $16,085 \mu \mathrm{g} / \mathrm{g}$ (dry weight basis). For the drainable liquid samples, the TOC values ranged from 6022 $\mu \mathrm{g} / \mathrm{ml}$ to $13,682 \mu \mathrm{g} / \mathrm{ml}$. The results show that the TOC concentration is we 11 below the action 1 imit of $30,000 \mu \mathrm{g} / \mathrm{g}$ (dry weight basis). All RPDs, spike recoveries, and standard recoveries for the TOC were within the limits required by the SAP.

The upper limit of a one-sided 95\% Confidence Interval were calculated (see attachment). The upper 1 imit of the $95 \%$ Confidence Intervals were 555 to $20,395 \mu \mathrm{g} / \mathrm{g}$ (dry weight basis) for drainable liquid and sludge samples. 
WHC-SD-WM-DP-184, REV. 1

Standards run with the samples had recoveries which fell within the required range of 80 to 120 percent for all of the samples. RPDs between the sample and duplicate sample analyses were below 20 percent for all but two of the samples. The RPDs for these two samples were 32.2 and 33.4 percent.

Triplicate analyses were run and the resulting RPDs between duplicate and triplicate were less than $10 \%$.

TIC analysis was also done for the samples. This additional analysis was not required by the Safety Screening DQ0. However, since these data are generated when the TOC analysis is performed, the SAP requested that the results be reported.

\section{Bromide by IC}

Bromide analyses were performed by IC using procedure LA-533-105, Rev. D-1. Solid samples were prepared by a water digestion prior to the IC analysis; the water digestion was performed using procedure LA-504-101, Rev. D-0. The lithium bromide solution sample from B-204 sampling event (1 ithium bromide solution blank was not collected for U-107 sampling event) had an average bromide concentration of $2.72 \mathrm{E}+04 \mu \mathrm{g} / \mathrm{ml}$. Results indicate that the samples for core 134, segment 6A (sample numbers S96T001079, S96T001120, and S96T001121) consist almost entirely of lithium bromide solution. Liner liquid from all three samples was contaminated with lithium bromide solution. Other results showed less than $10 \%$ of bromide, indicating very little contamination of the samples by the lithium bromide solution. All RPDs, spike recoveries, and standard recoveries for the bromide analyses were within the limits required by the SAP.

Nitrite, nitrate, sulfate, phosphate, fluoride, chloride, and oxalate results are also reported for the samples. These additional analyses were not required by the Safety Screening DQO. However, since these data are generated when the IC analysis is performed, the SAP requested that the results be reported.

\section{Lithium by ICP}

Lithium analyses were performed by ICP using procedure LA-505-151, Rev. D-3 or LA-505-161, Rev. B-0. Solid samples were prepared by acid digestion prior to the ICP analysis; the acid digestion was performed using procedure LA-505-159, Rev. $\mathrm{C}-0$. The lithium bromide solution sample (from B-204 sampling event) had an average 1ithium concentration of $1.98 \mathrm{E}+03 \mu \mathrm{g} / \mathrm{ml}$. Like the bromide results, the samples for core 134, segment 6A (sample numbers S96T001079, S96T001120, and S96T001121) consist almost entirely of lithium bromide solution. Liner liquid from all three samples were contaminated with lithium bromide solution. All the solid sample results were less than $100 \mu \mathrm{g} / \mathrm{g}$ value with an exception of core 134, segment $6 \mathrm{~A}$ as mentioned above. Therefore, no adjustments were made for the TGA results. All standard recoveries were with in the limits required by the SAP with one exception of $84 \%$. Spike recoveries for three of the samples ( 67.9 to $77.0 \%)$ were outside the range specified by the SAP. RPDs for all of the samples were below 15 percent as required by the SAP with one exception $(69.4 \%)$ 
WHC-SD-WM-DP-184, REV. 1

Results for other ICP elements are also reported for the samples. These additional analyses were not required by the Safety Screening DQO. However, since these data are generated when the ICP analys is is performed, the SAP requested that the results be reported.

Project Coordinator: Jaiduk Jo

\section{REFERENCES}

Dukelow, G. T., J. W. Hunt, H. Babad, and J. E. Meacham, 1995, Tank Safety Screening Data Quality Objective, WHC-SD-WM-SP-004, Rev. 2, Westinghouse Hanford Company, Richland, Washington.

Esch, R. A., 1995, Waste Compatibility Results for 241-U-107 Grab Samples", Internal Memo, 8E480-95-027, Westinghouse Hanford Company, Richland, Washington.

Fuller, R. K., 1996, Tank 241-U-107, WHC-N-1007, Westinghouse Hanford Company, Richland, Washington.

Jo, J., 1996a, 45-Day Safety Screening Resu7ts for Tank 241-U-107, Push Mode Cores 129, 134 and 135, WHC-SD-WM-DP-184, Rev. 0, Westinghouse Hanford Company, Richland, Washington.

Jo, J., 1996b, Tank 241-U-107 Push Mode Core Sampling and Analysis Plan, WHC-SD-WM-TSAP-076, Rev. OB, Westinghouse Hanford Company, Richland, Washington. 
WHC-SD-WM-DP-184, REV. 1

SAMPLE DATA SUMMARY

6 
WHC-SD-WM-DP-184, REV. 1

THIS PAGE WAS INTENTIONALLY LEFT BLANK 
WHC-SD-WWi-SF -184, REV.

$\underset{\Xi}{\ddot{\Xi}}$
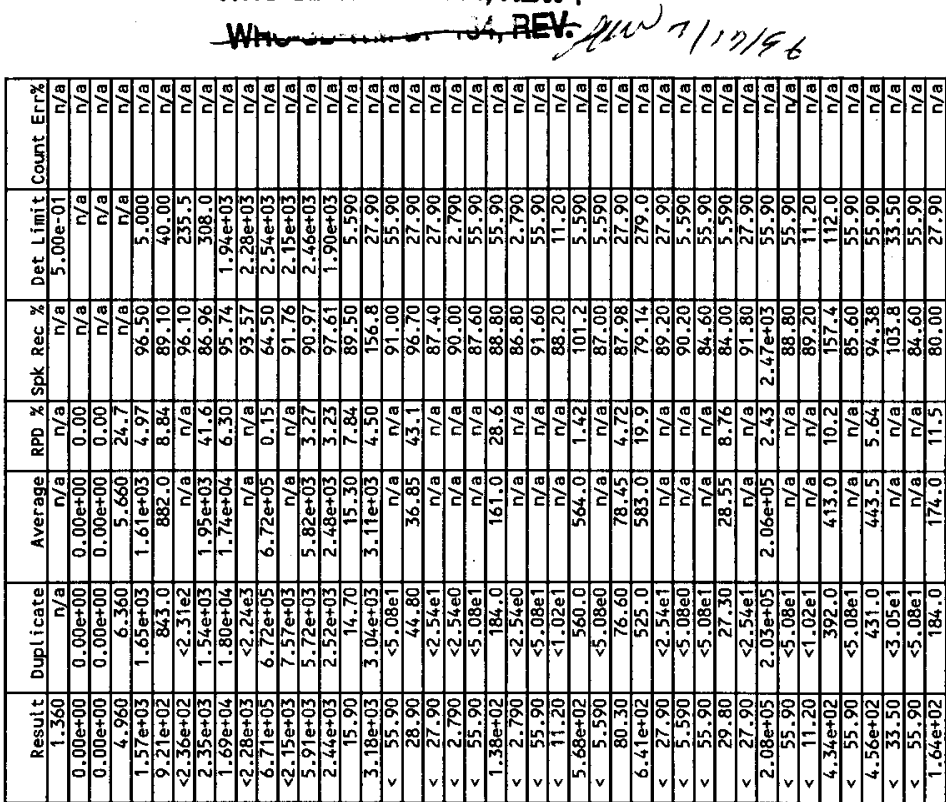

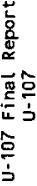

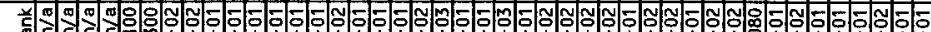

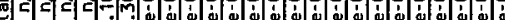

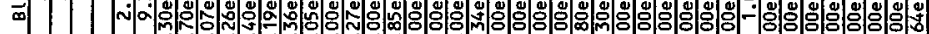

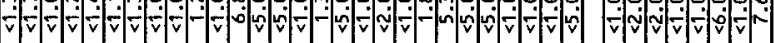
*0.0 च 苟

깅

(1)

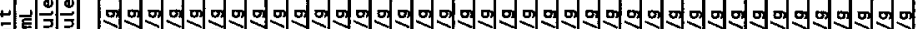

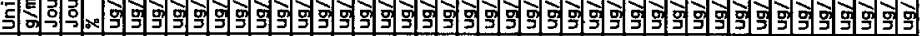

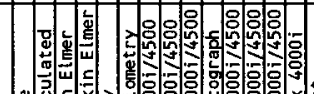

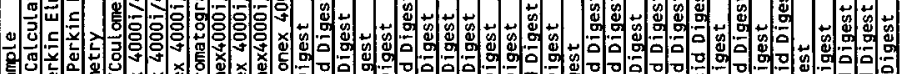
(0)

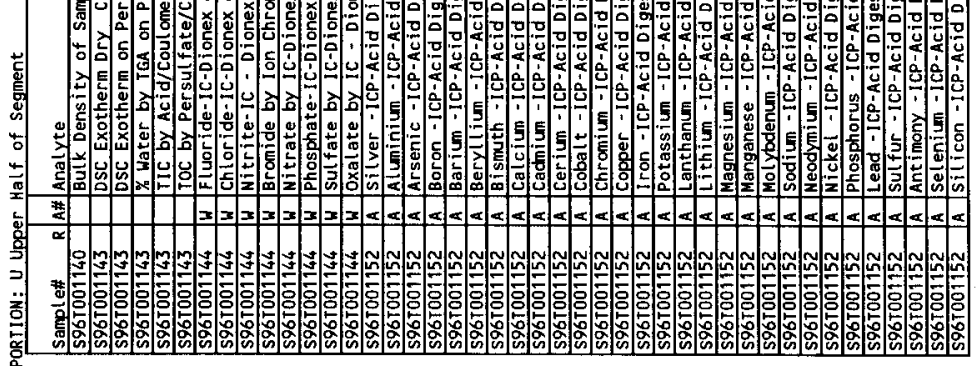

$\stackrel{2}{2}$

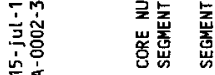

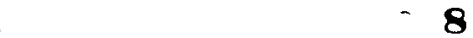



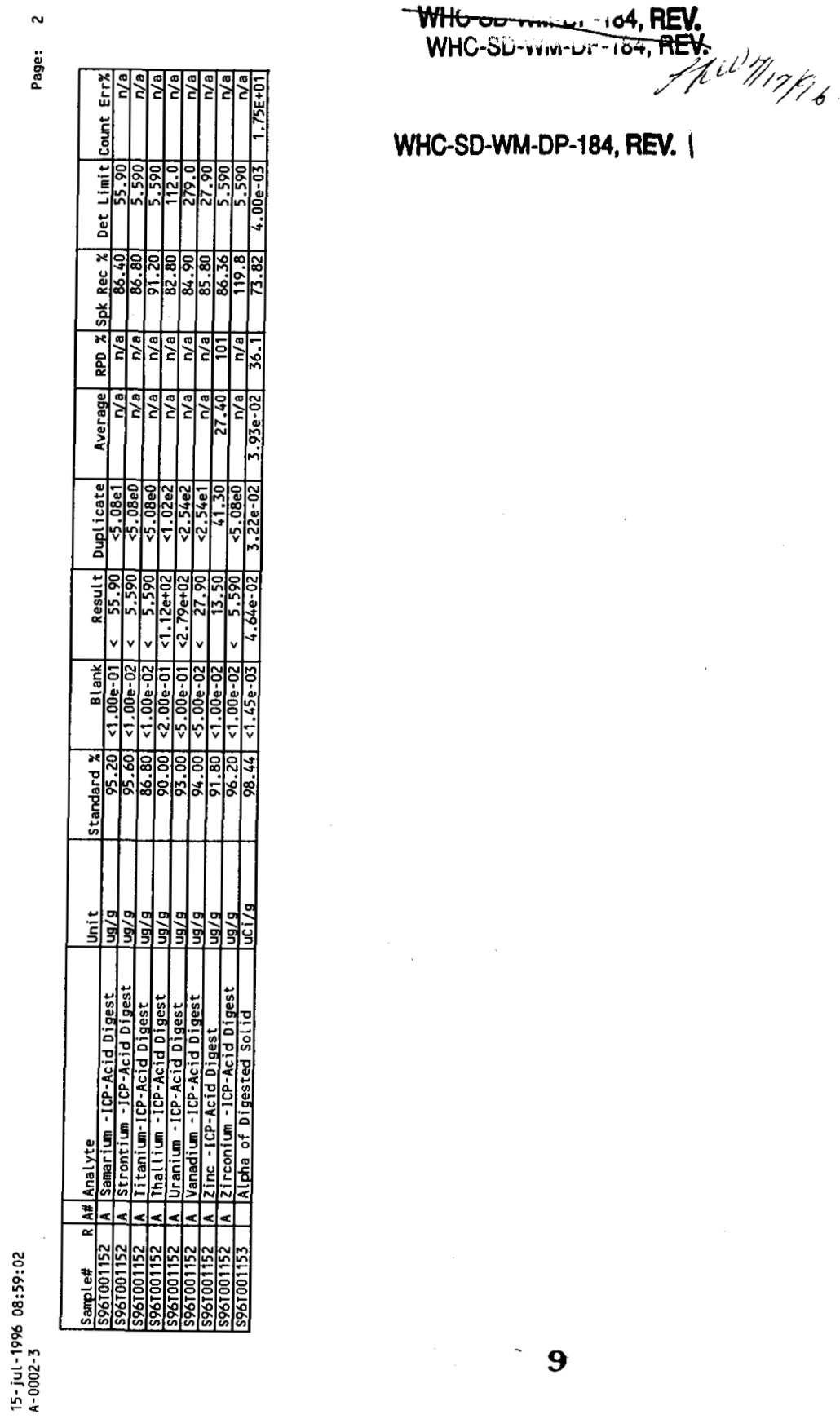

WHC-SD-WM-DP-184, REV. I 
WHC-SD-WM-DP-184, REV.

$\underset{\dot{8}}{\ddot{0}}$

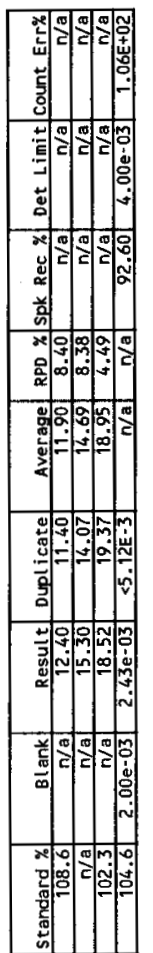

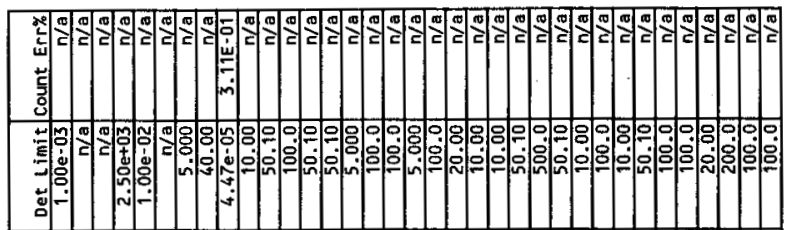

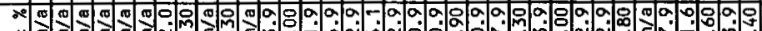

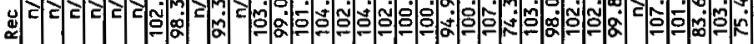
总

音

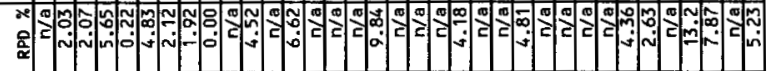

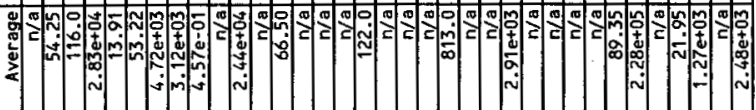

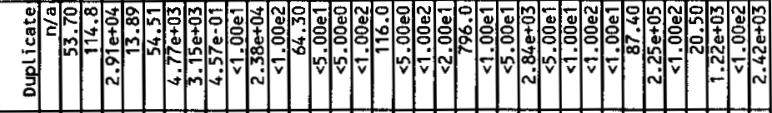

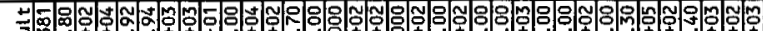
m.

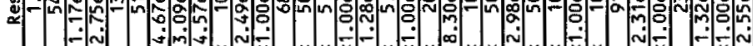

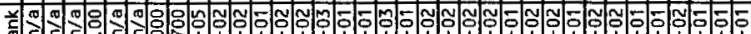

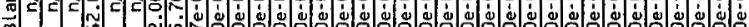
- $v$ n

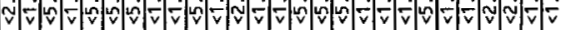

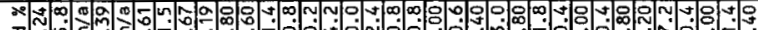

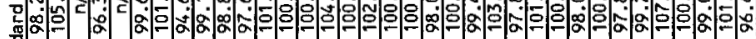
in

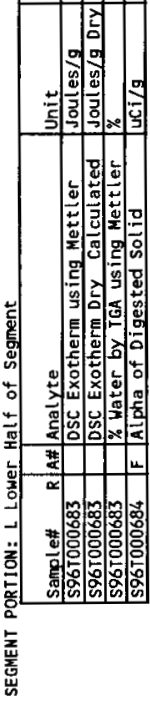

各

영

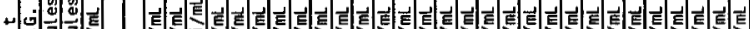

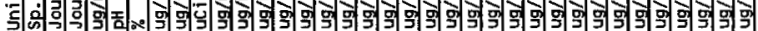

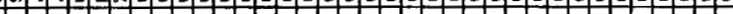

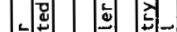

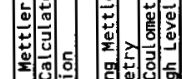

$>$ 每

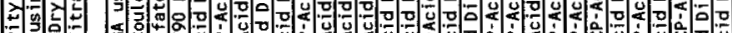

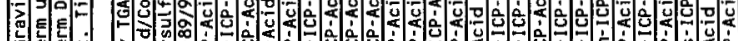

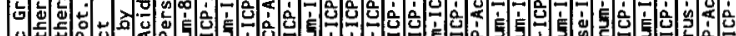

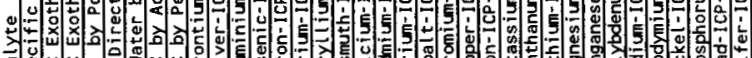

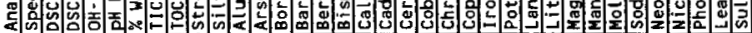
*

烈

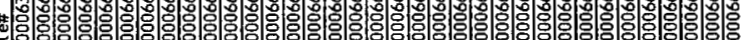


WHC-SD-WM-DP-184, REV. |

THIS PAGE WAS INTENTIONALLY LEFT BLANK 
$\underset{\substack{0 \\ 0 \\ 0}}{\ddot{0}}$

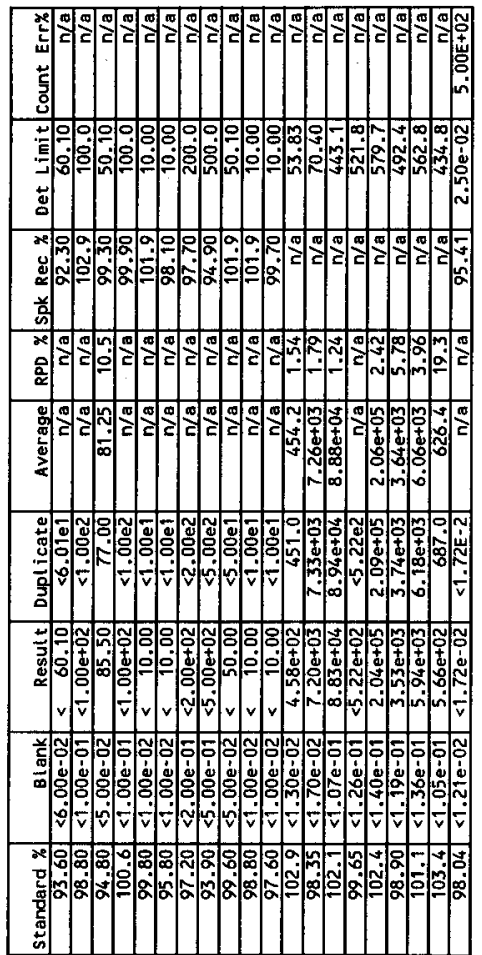

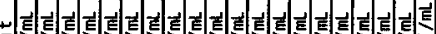

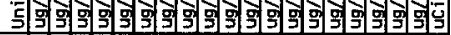


융ㅁำ

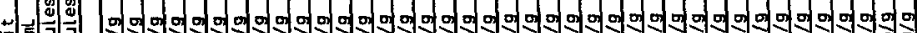

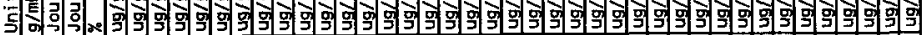
迹育

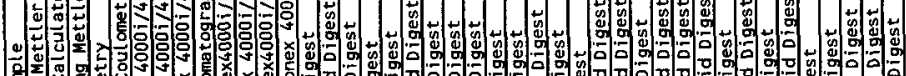

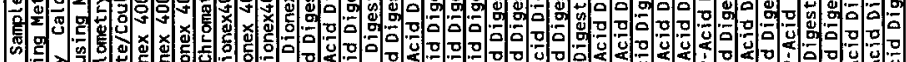

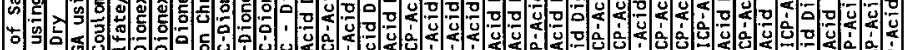

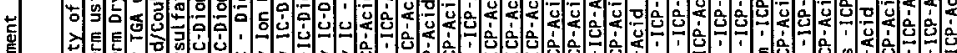

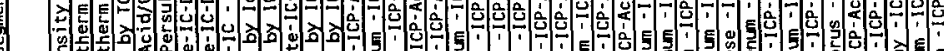

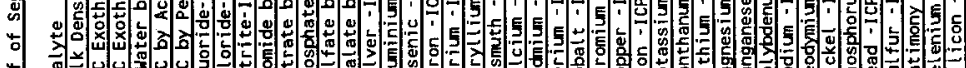

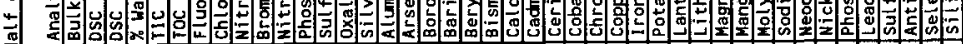

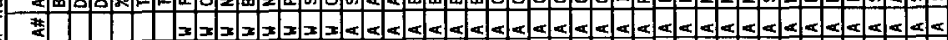




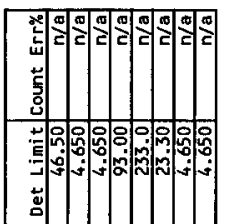

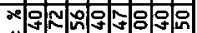

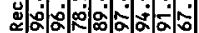
현

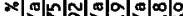

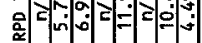

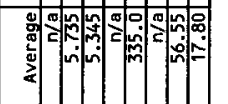

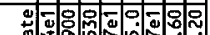

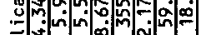
氙

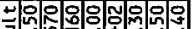

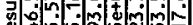

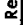

등융ㅇㅇㅇㅇㅇㅇㅇㅇㅇㅇㅇ

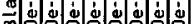

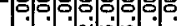

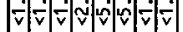
*응용ㅇㅇㅇㅇㅇㅇㅇㅇ 둥 密

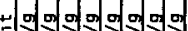

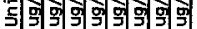

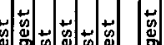

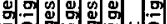

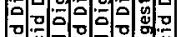

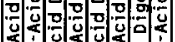
î

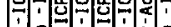

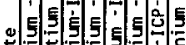

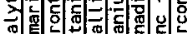

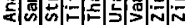
* < < < \& \& \& \&

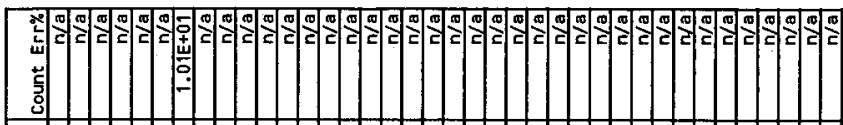

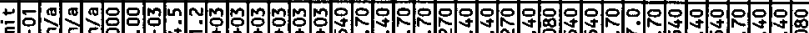
国 $=2=0=0$

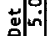

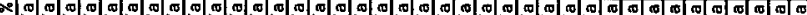

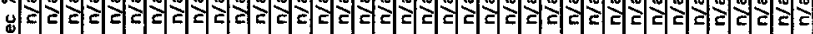
弟 일

"

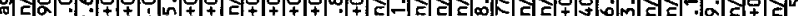

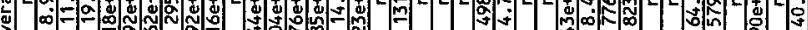

4 8 -

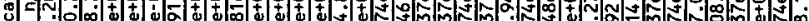

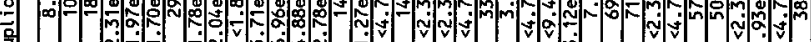
옴

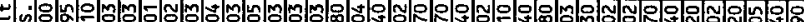

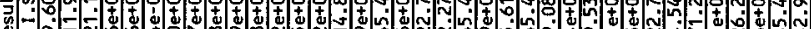

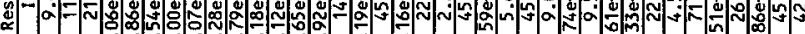

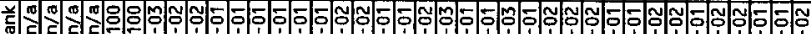

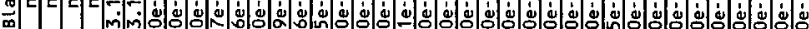

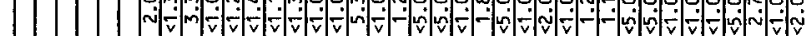

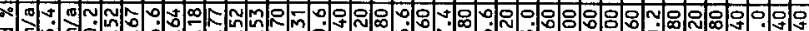
의 드 空

요요

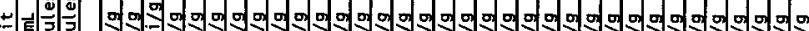

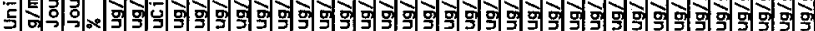

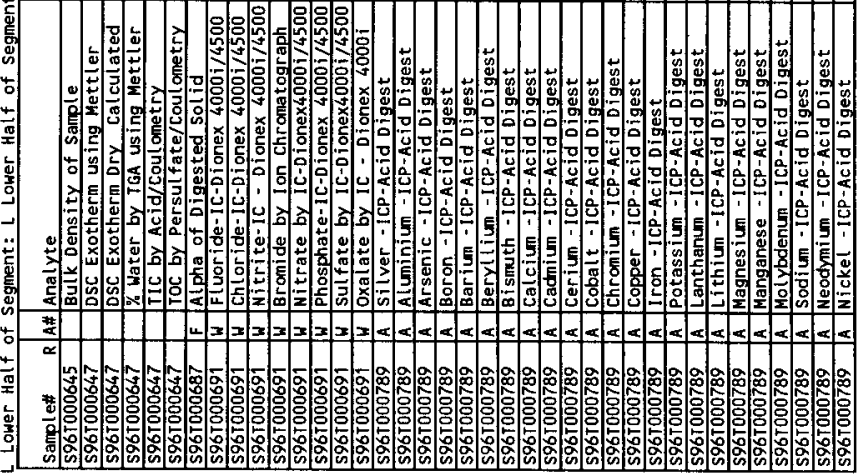




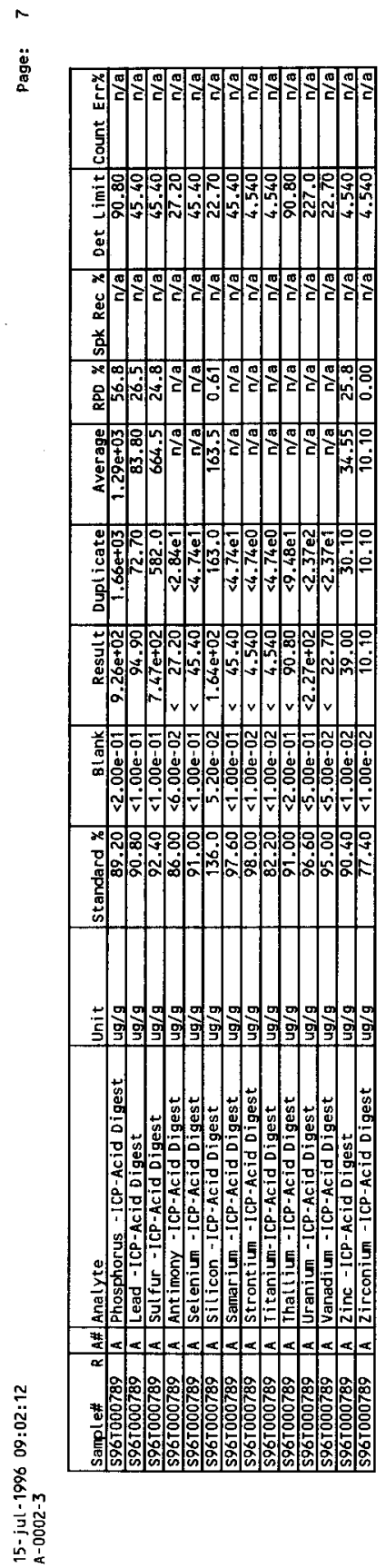

WHC-SD-WM-DP-184, REV. I

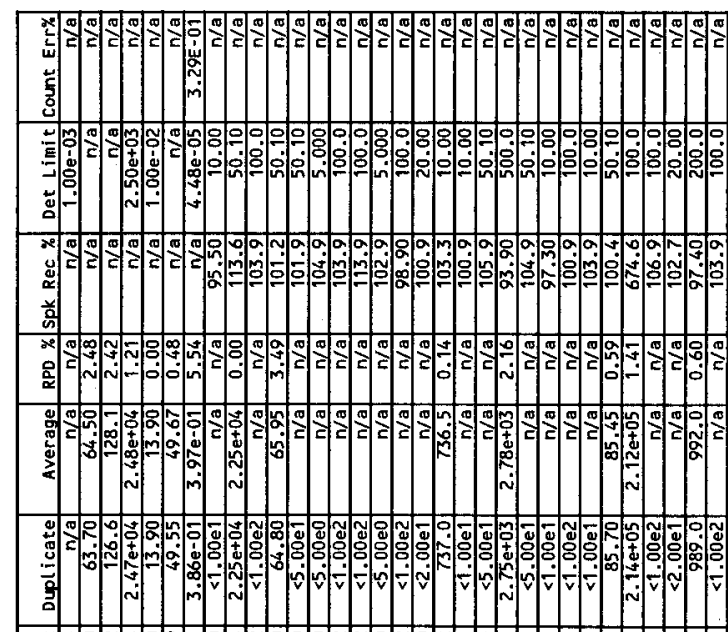

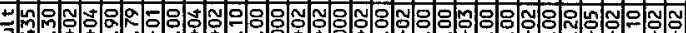

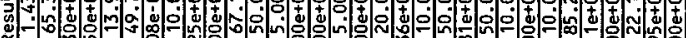

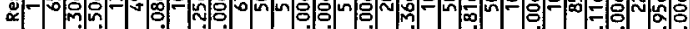

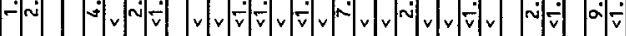

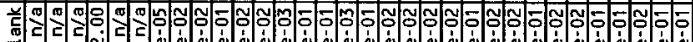

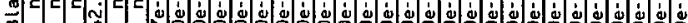
\%

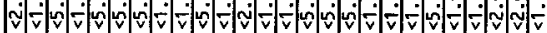

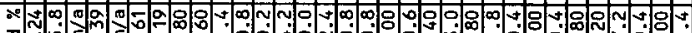
T

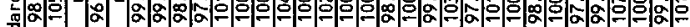
空

\section{중}

10.

영영

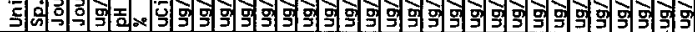

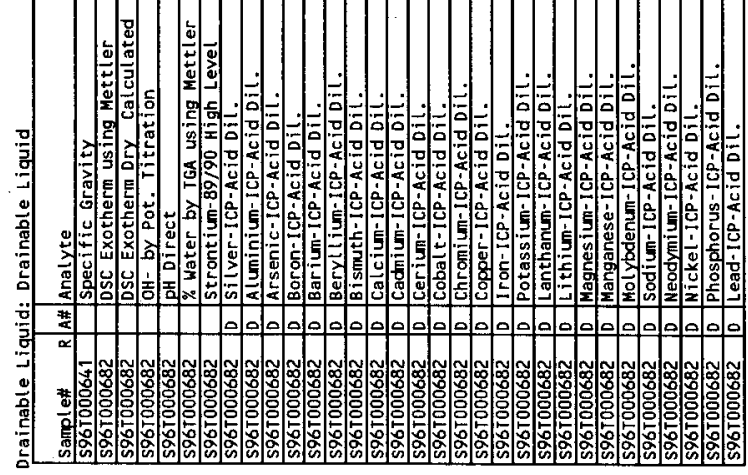




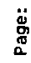

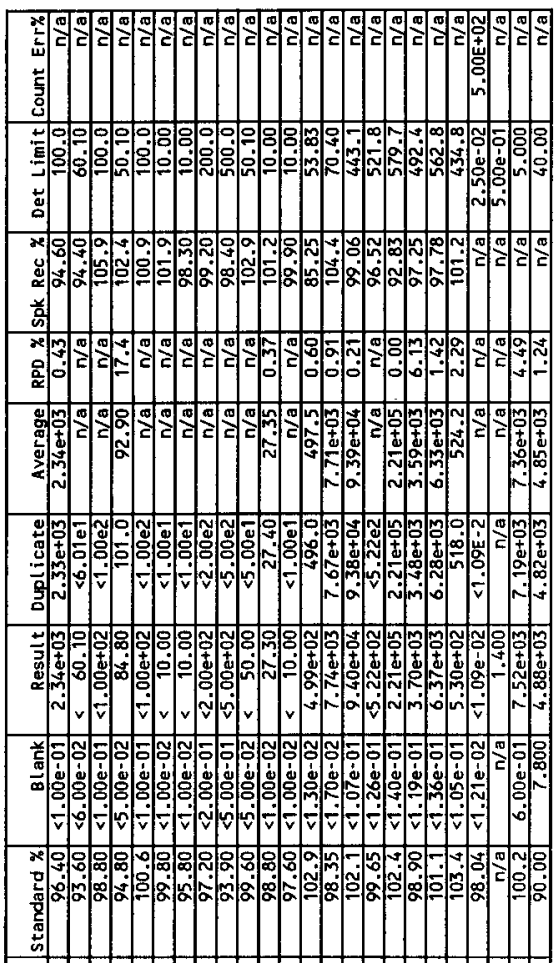

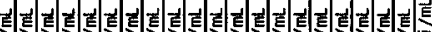

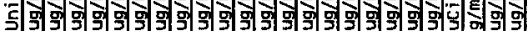

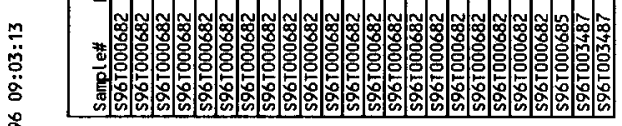

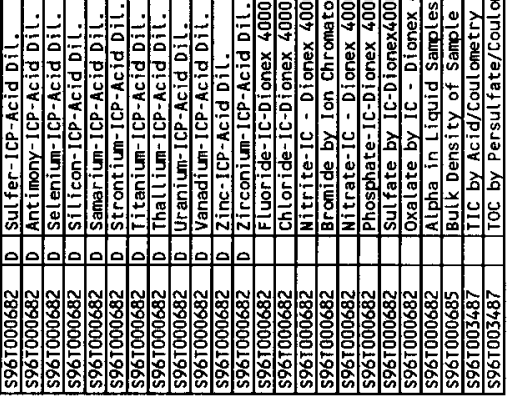




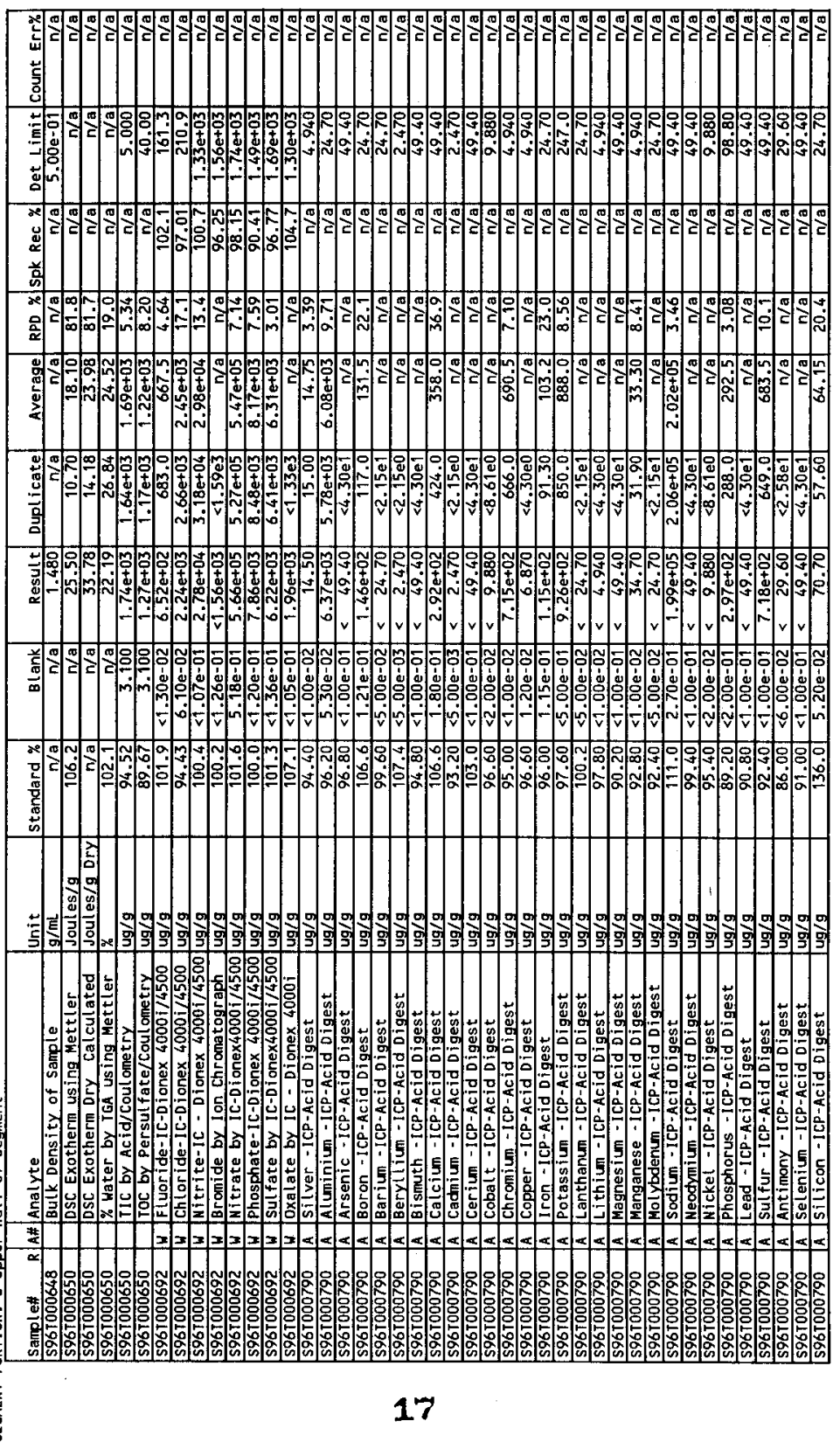




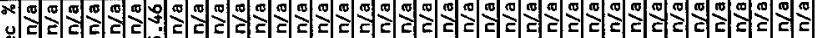

힘

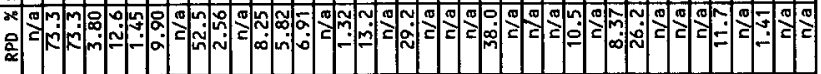

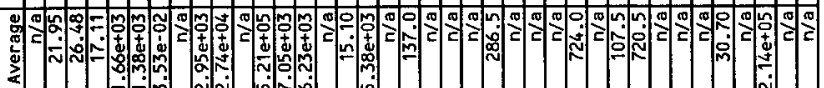

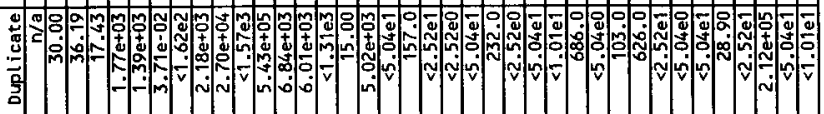

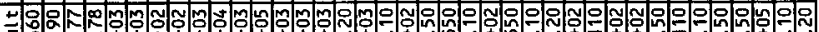
焉

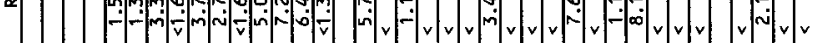

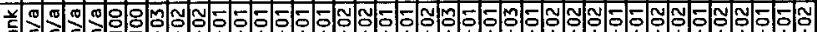

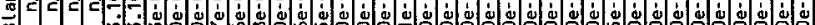

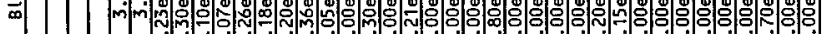

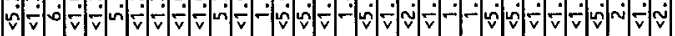

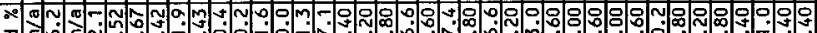
궁 다

है is

को

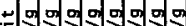

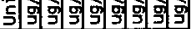

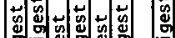
의 의의 음

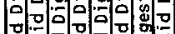
这通

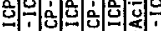

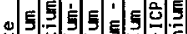

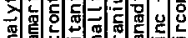

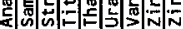
婪 $x<x<x<x$

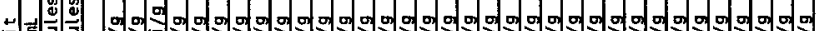

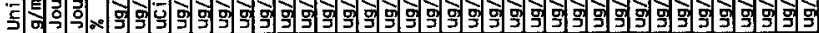

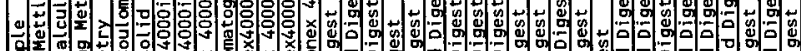

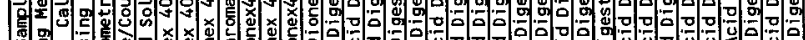

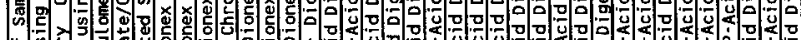

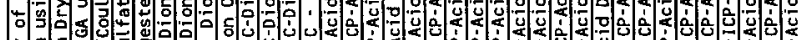

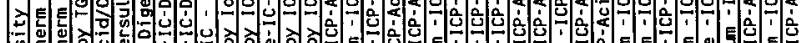

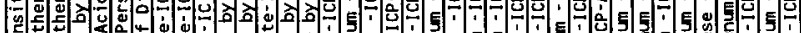

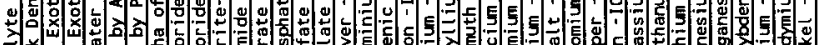
궁

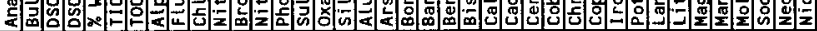

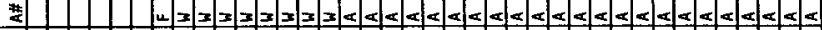
$\propto$

*

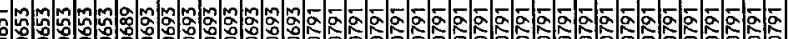




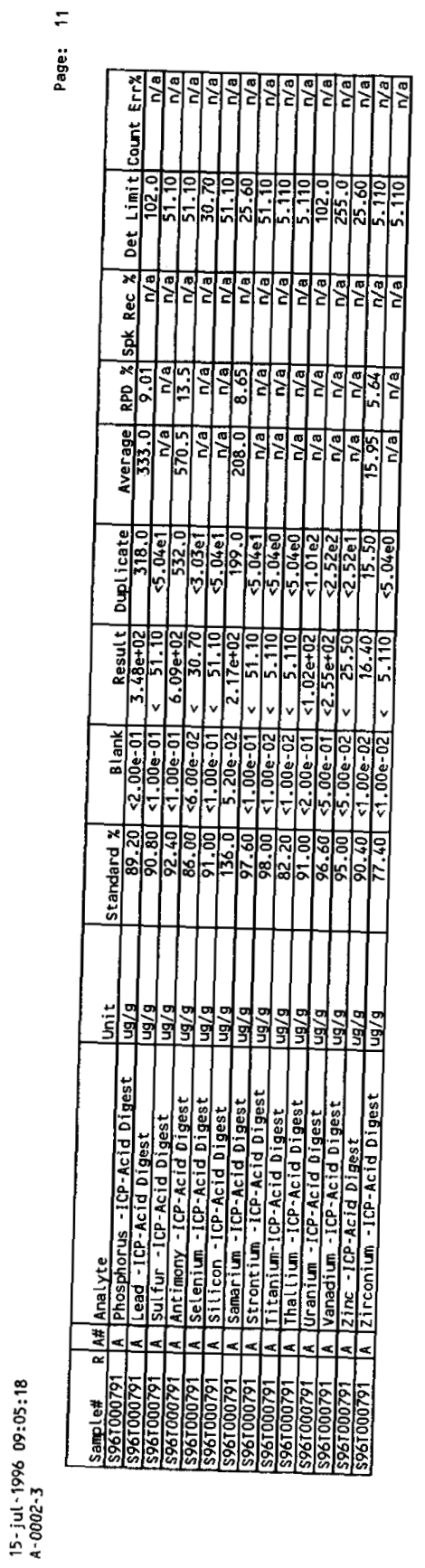

WHC-SD-WM-DP-184, REV. I 
$F \quad$ a $=0$ : $=0$ F

t

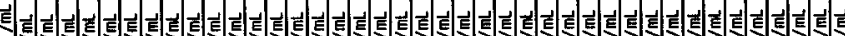

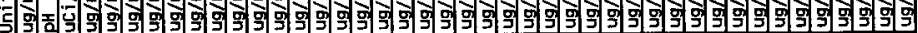

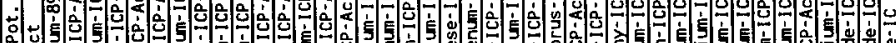

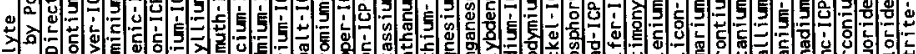

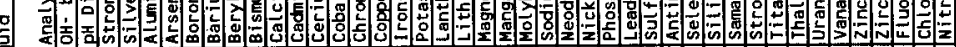

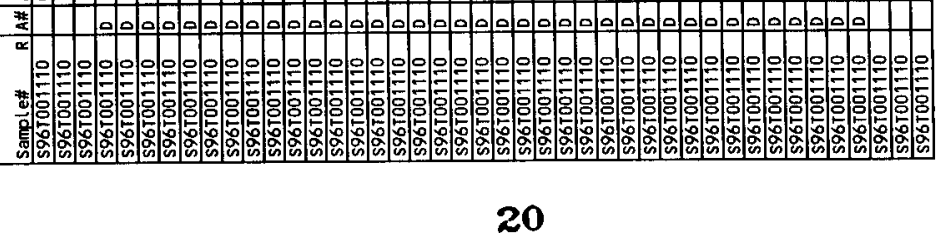


远

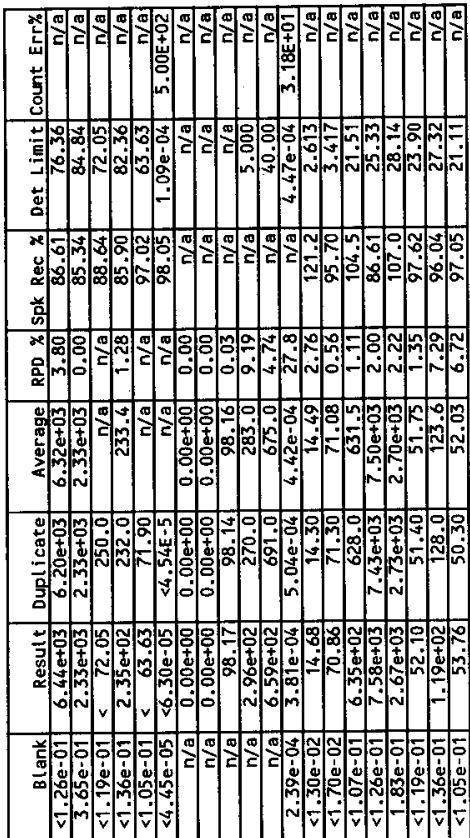

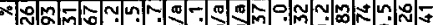

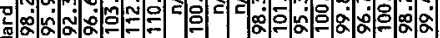
窵

잉

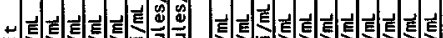

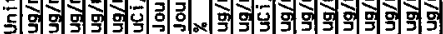

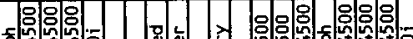

담

a. $\rightarrow$

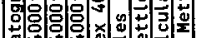

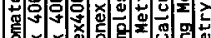

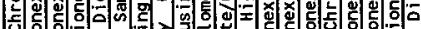

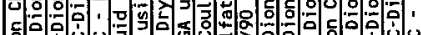

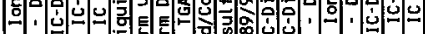

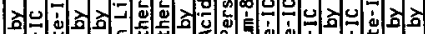

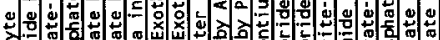

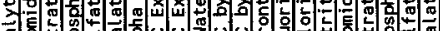

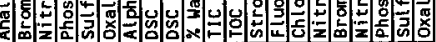
춘

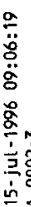

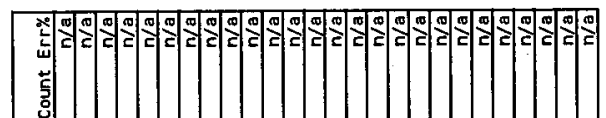

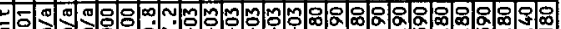

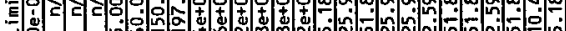
$\sim$

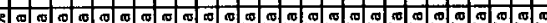

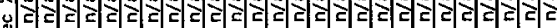

के

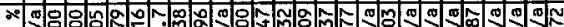
a a 이 두

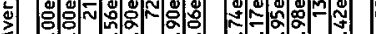
\&

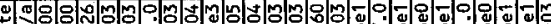
心 年 3

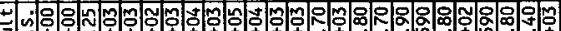

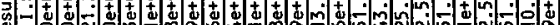
$\stackrel{2}{\alpha}$ ó.

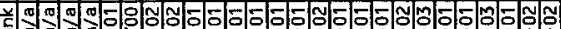

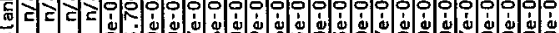
-

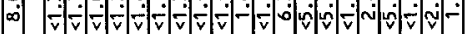

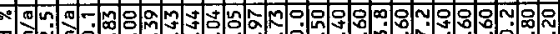
다 더영

in

.

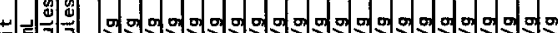

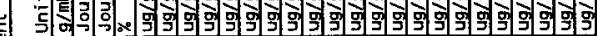

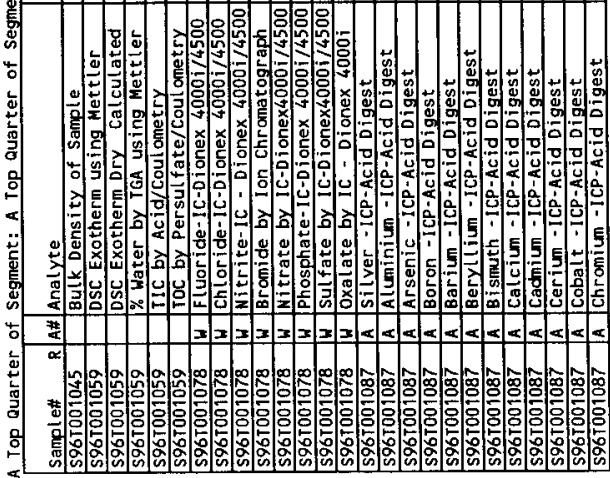




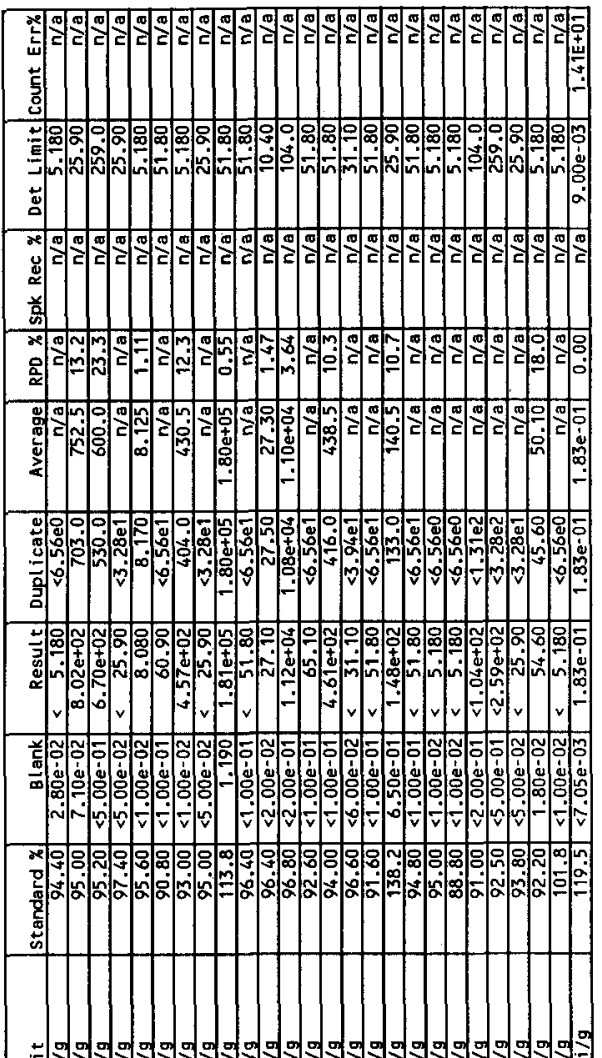

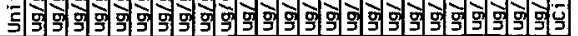

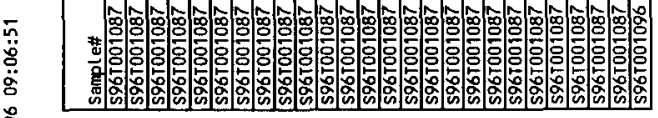

$\stackrel{\circ}{\circ}$

官苍

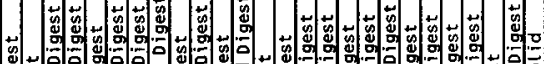

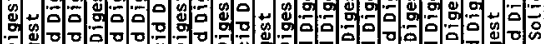
a

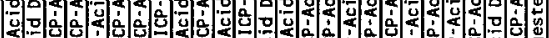
定

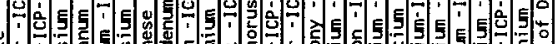
空产

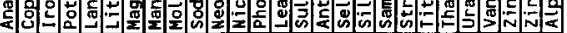

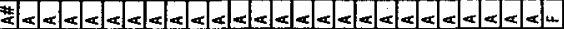

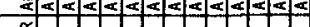
*

ñ 


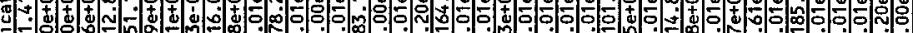

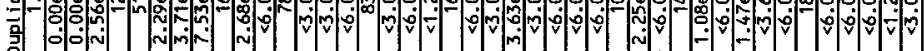

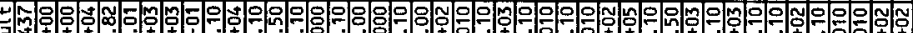

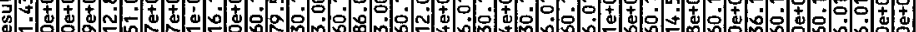
-

0 -

imin

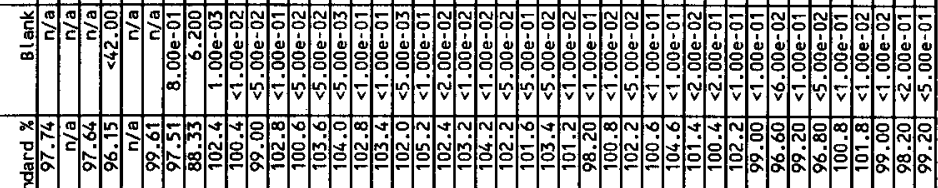

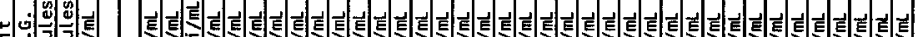

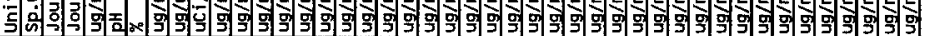
1
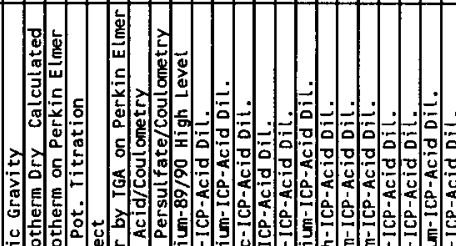

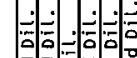

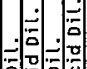




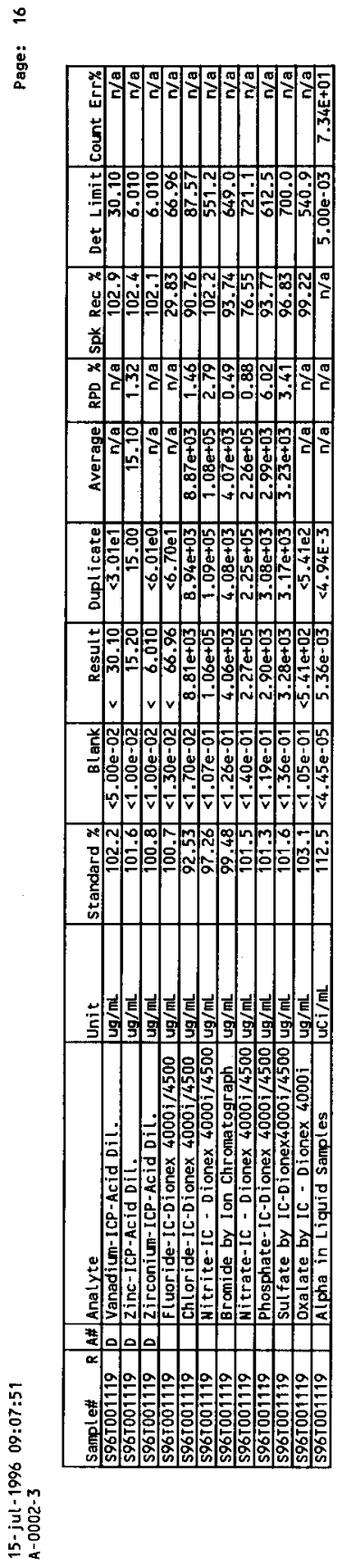




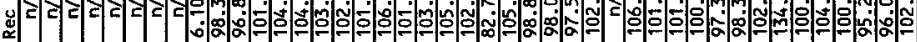
\%ั

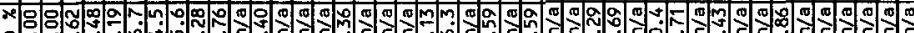
a

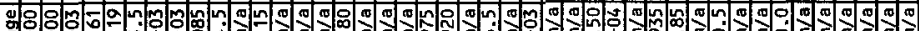
of to

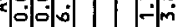

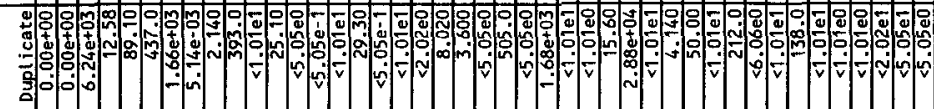

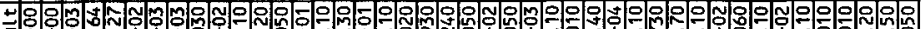

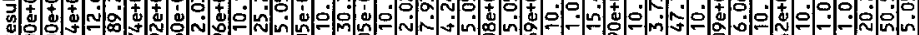

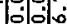
.

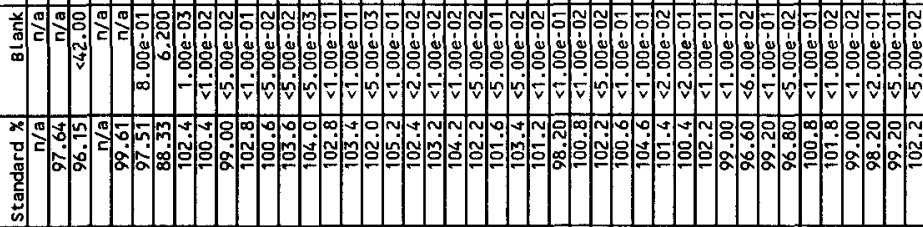

\section{군}

ㅇ. 영.

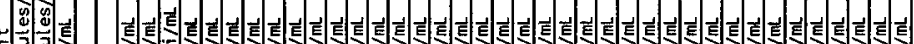

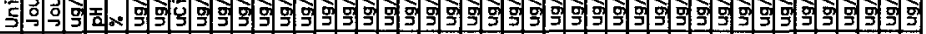

वृ

索

ज्ञ

递至

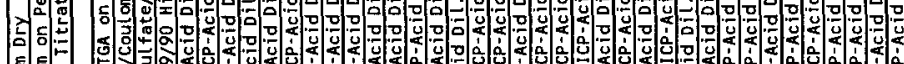

E气

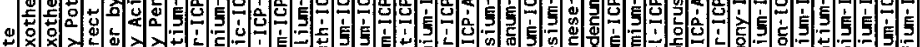

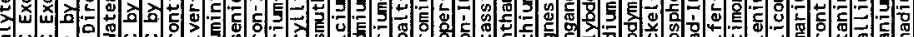

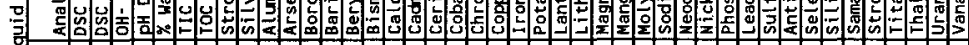

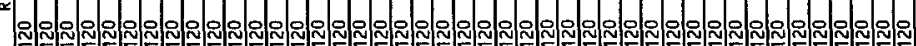




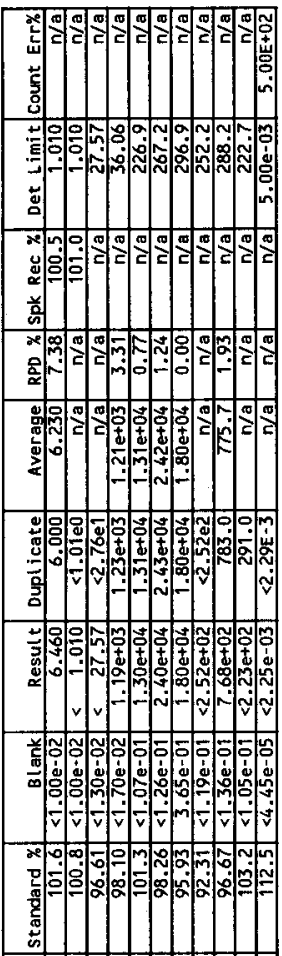

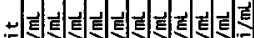

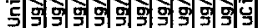

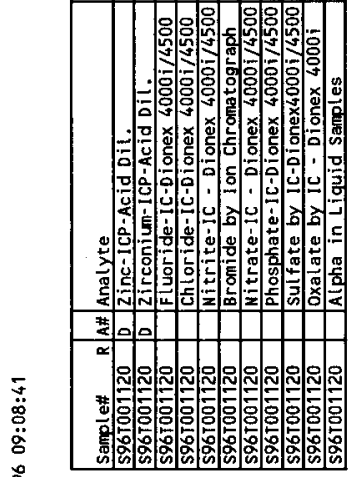

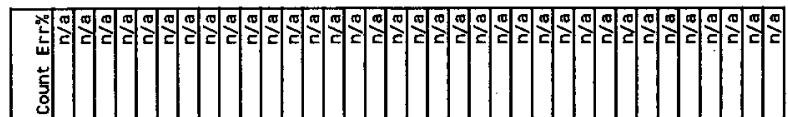

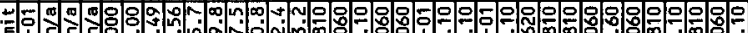

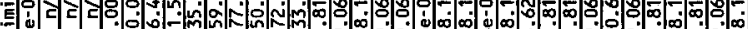
$=0$ 我 :

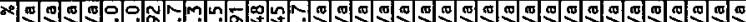

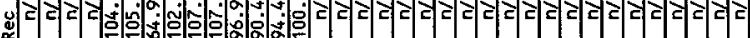
के

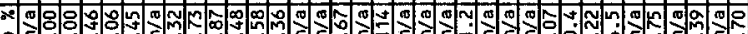
a

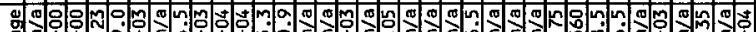

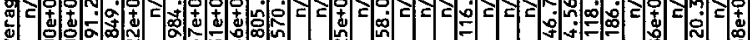

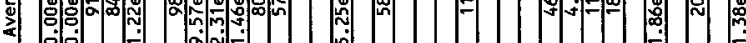

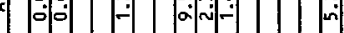

尝

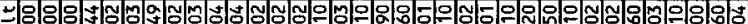

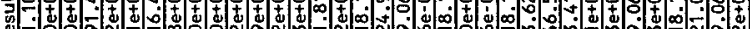
๕

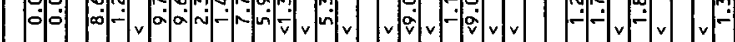

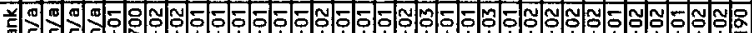

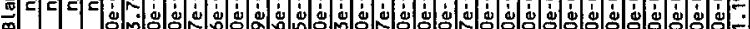
๓

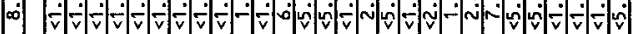

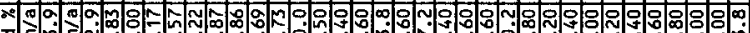

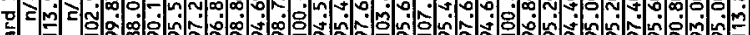

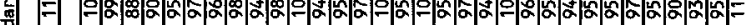
空

o. on

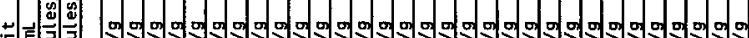

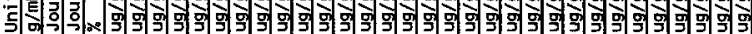

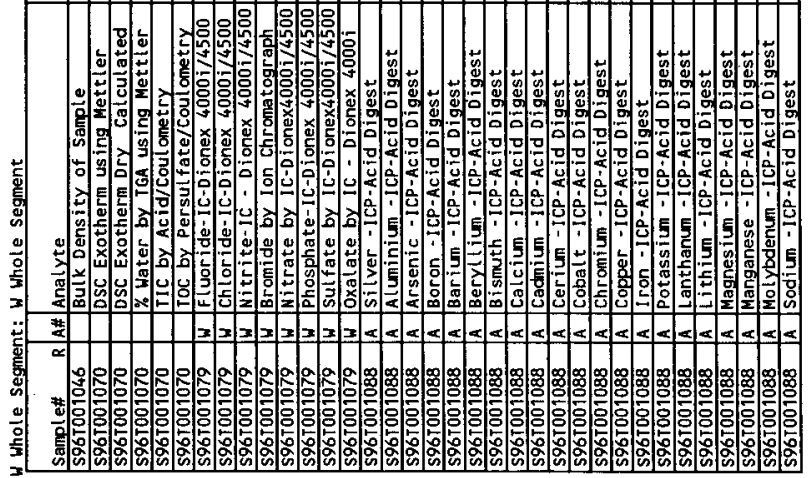




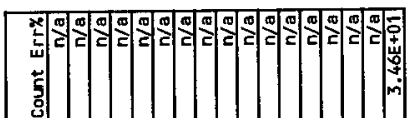

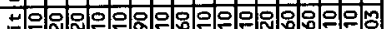
당

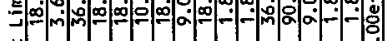
岁

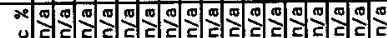

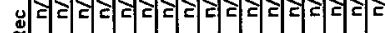
前

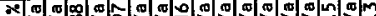

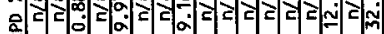
(2)

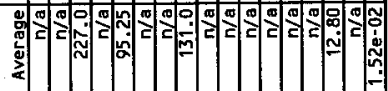

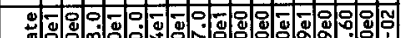

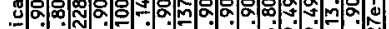

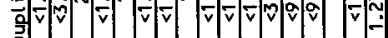

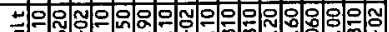
马े

$\checkmark \vee \sim \sim v$

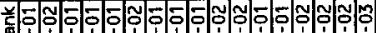
a d d d d

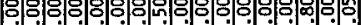

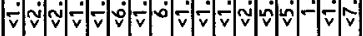

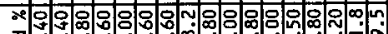
7

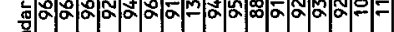
$\stackrel{5}{\infty}$

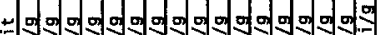

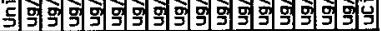

苟 苞

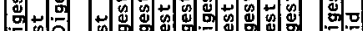

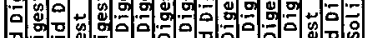

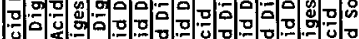
产 D

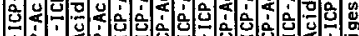

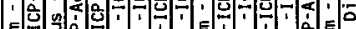

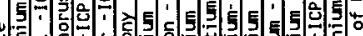

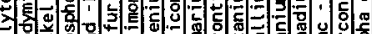

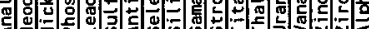

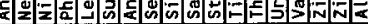

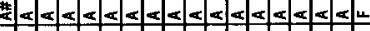

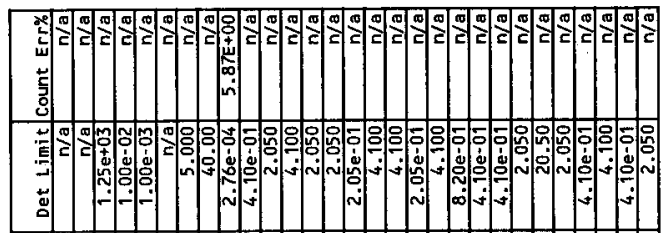

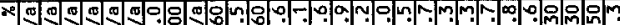

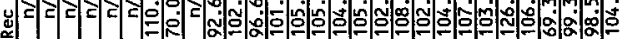
总

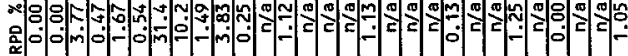

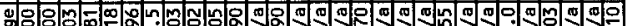 a d

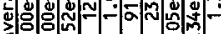 $\therefore 0.0$

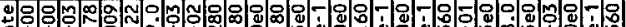
t t t

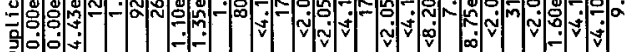
a. 0.0

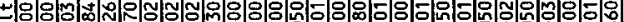

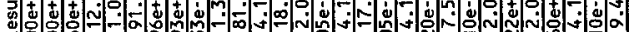
$\propto$ 잉잉

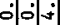

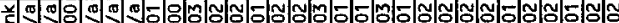

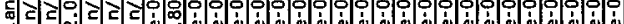

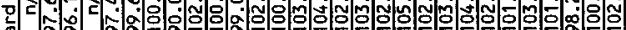
롱

요

包论

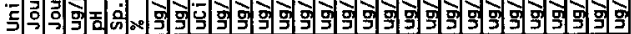

민

苟至

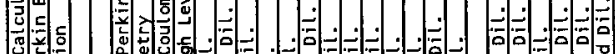

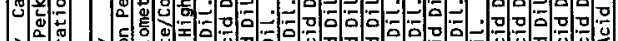

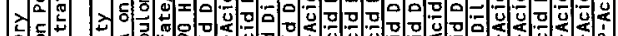

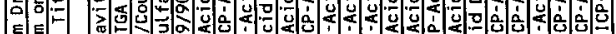
틀

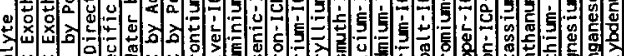

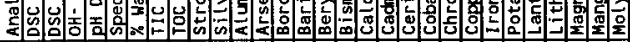

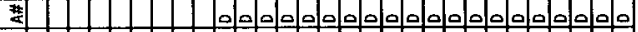

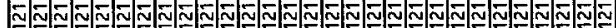

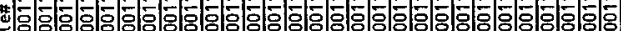

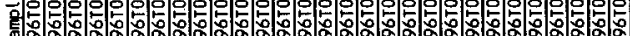

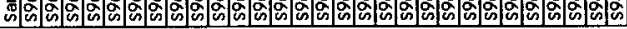




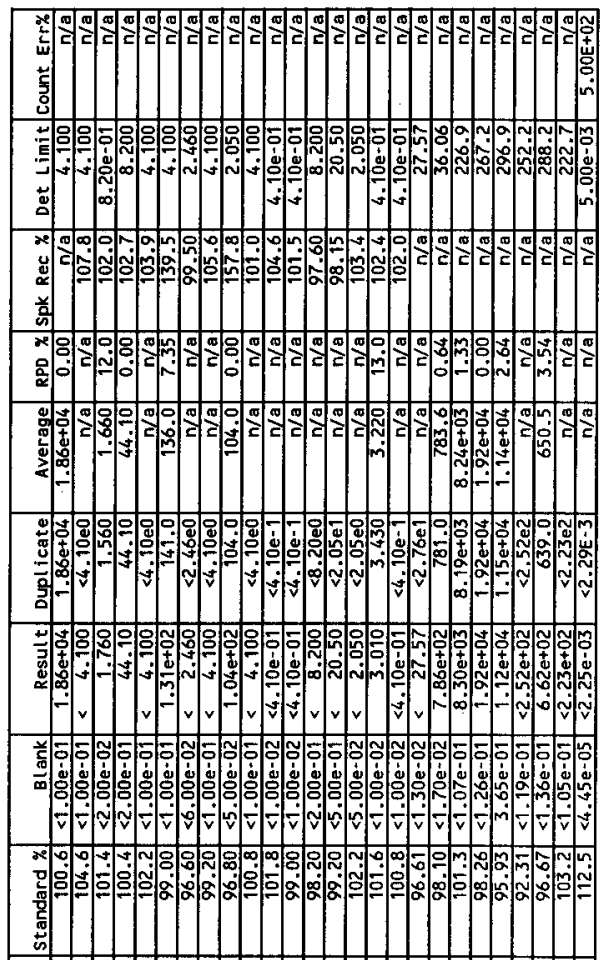

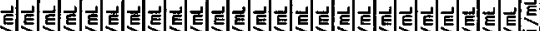

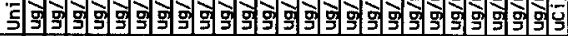

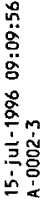

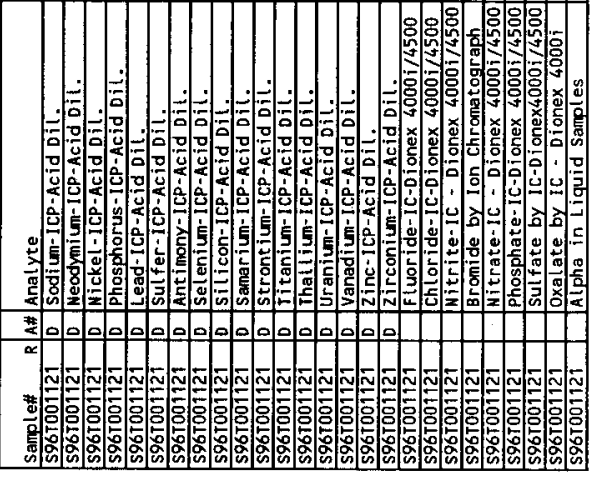




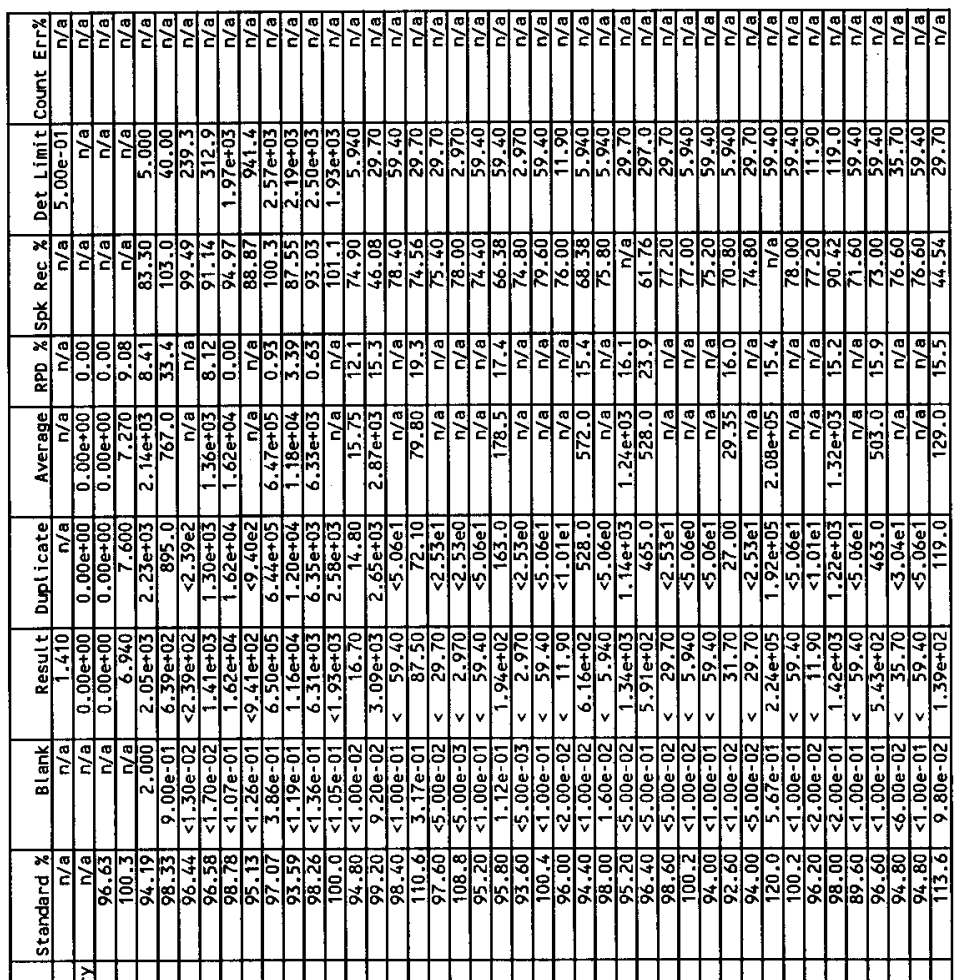

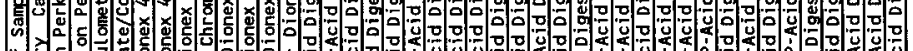
苟 基 士

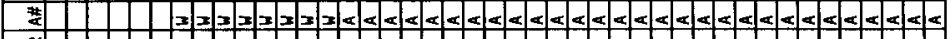

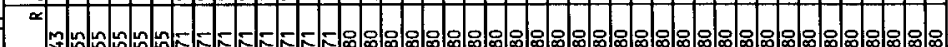

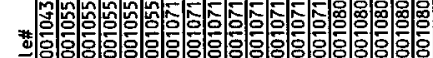

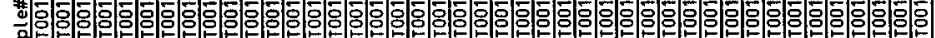

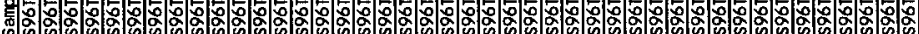




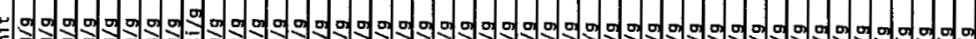

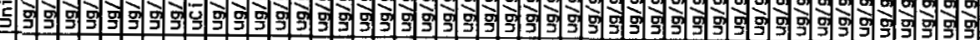

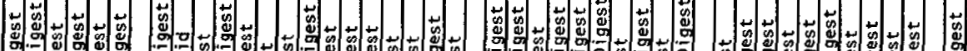

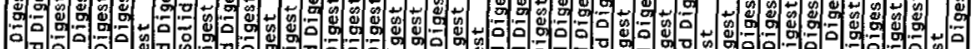
可

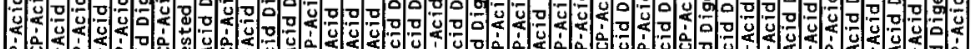

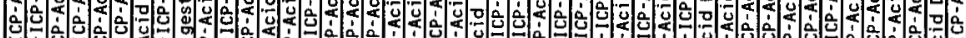

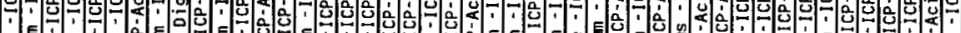

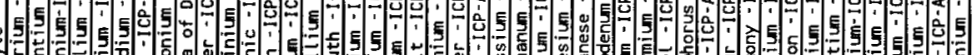

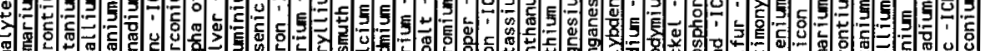

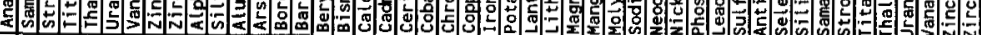

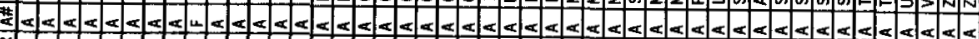




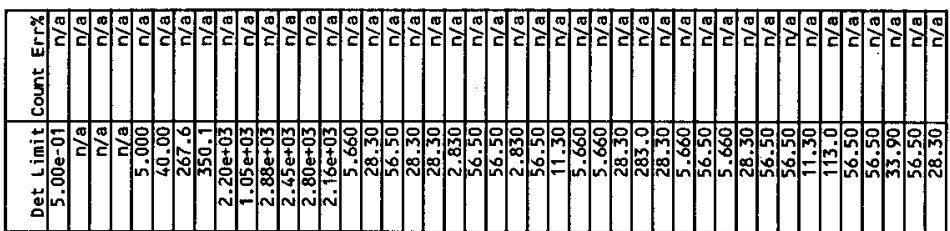

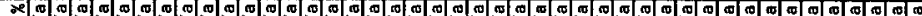

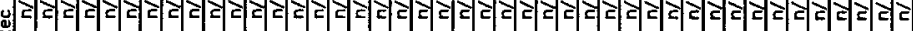
总

a

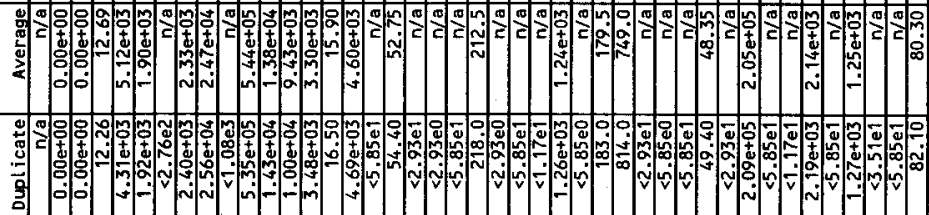

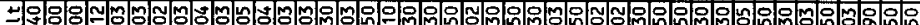
Ju -

- 0 vi- $\sim \sim \sim m-\infty$

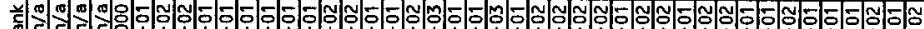
3 C C C

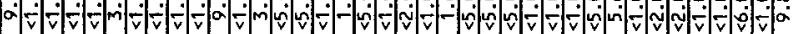

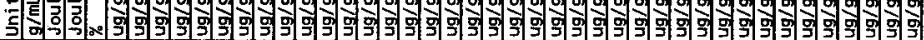

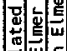

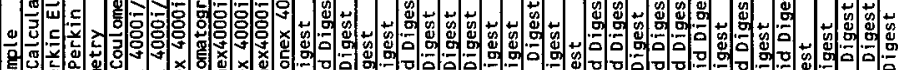

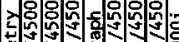

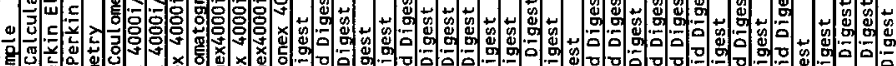
心

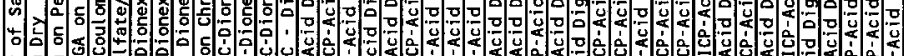

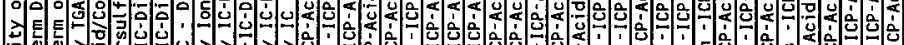

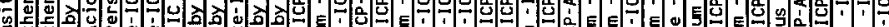

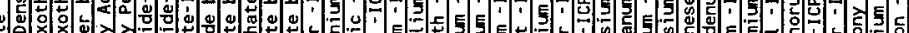

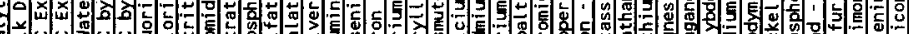

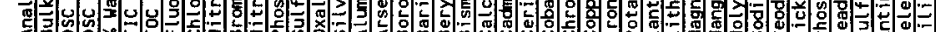

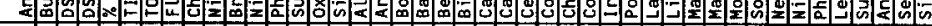

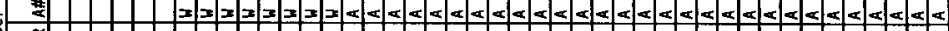


濉

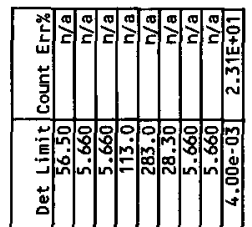

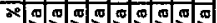

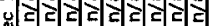
일

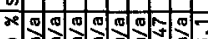

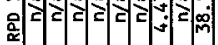
:

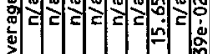
₹

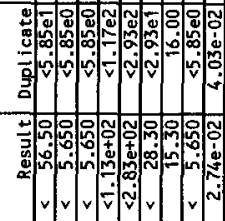

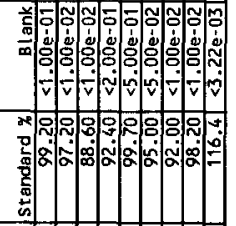

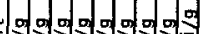

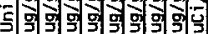

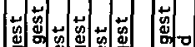

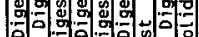

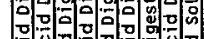
प) 은일 $\rightarrow=0.0$ - 5 5 5 S 50

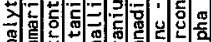

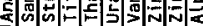
$<<<<<<<<<$

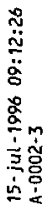
-

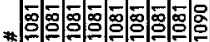

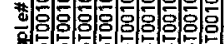

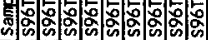

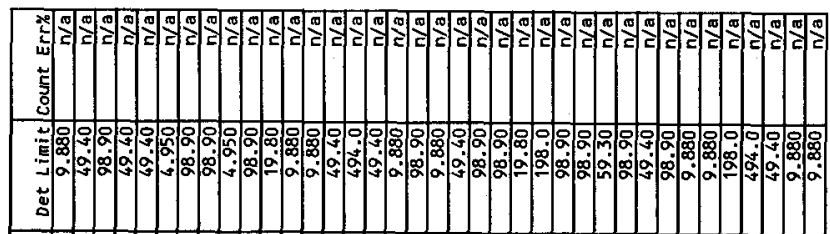

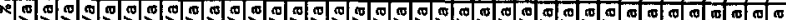

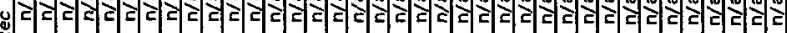
荺

r 0 ब

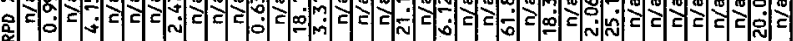

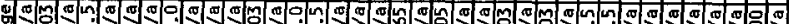

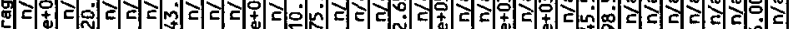

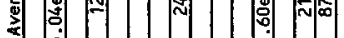

-

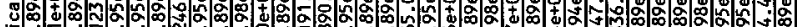

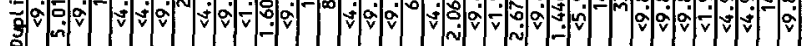

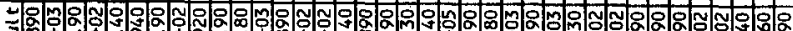

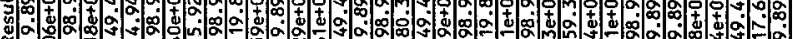

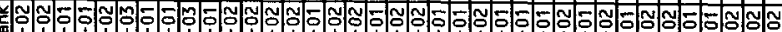

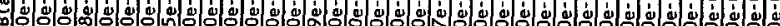
\%

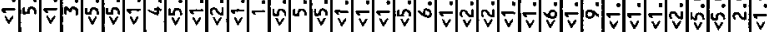

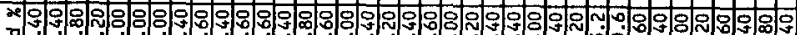

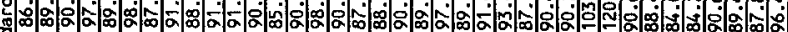

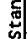

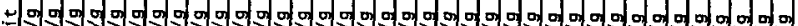

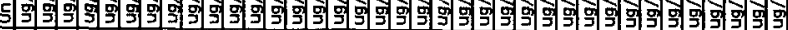

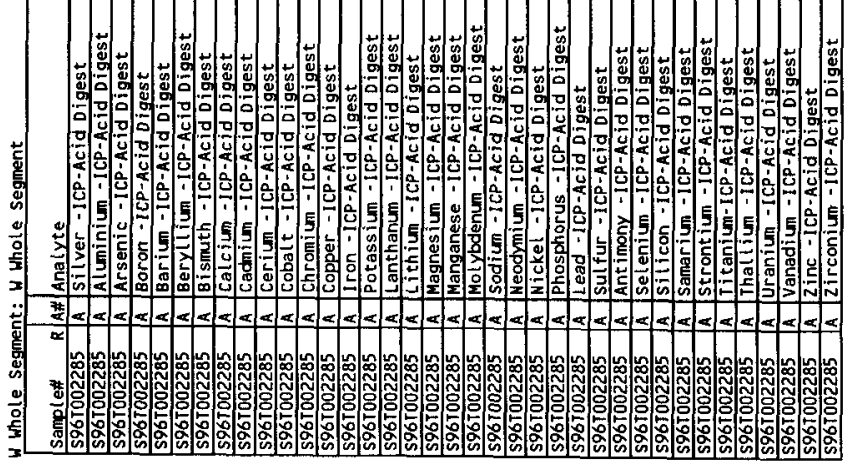


WHC-SD-WM-DP-184, REV. $\mid$

约

兽

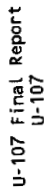

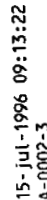

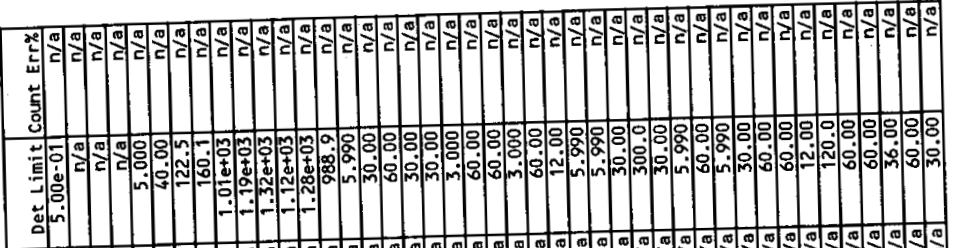

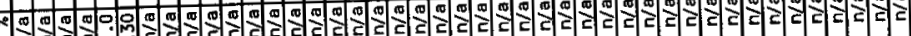

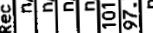

前

(

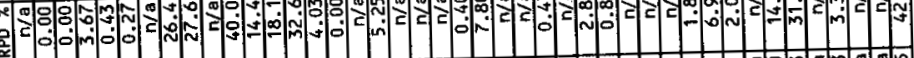

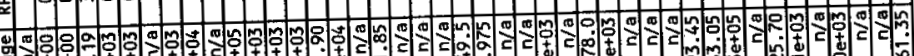
象

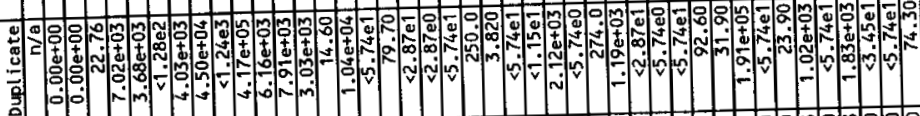

*1

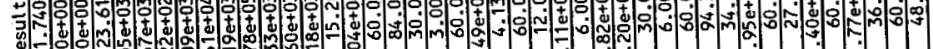
$\therefore$ : 0 :

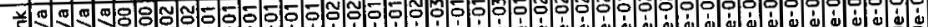

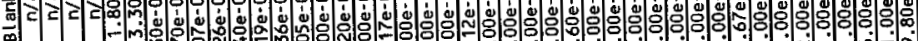

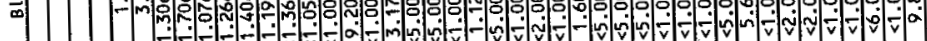

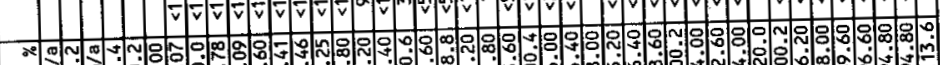
가의

ह (s)

0

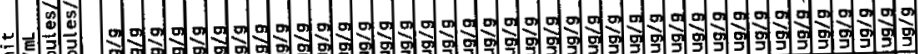

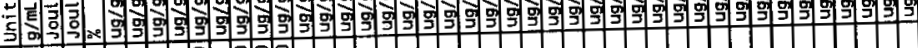
눈

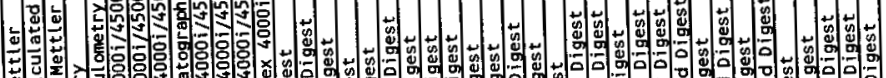

영 伺

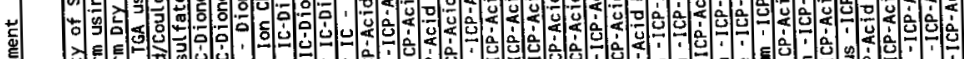

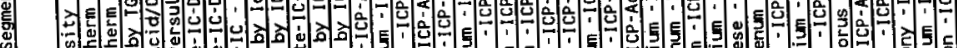
4 4

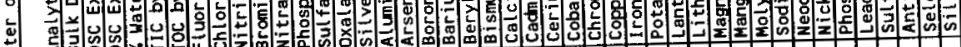
of

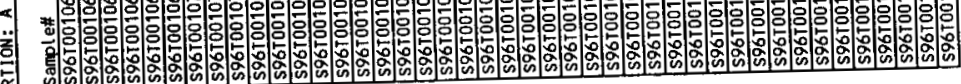

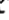


 \\ (1)}

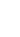

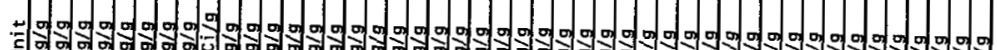

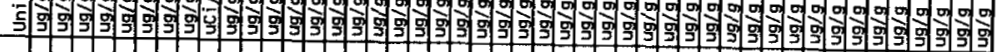

4

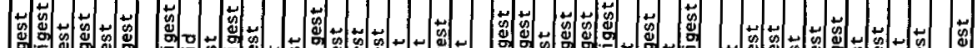

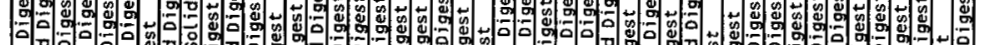
volo 0

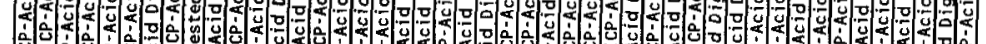
1

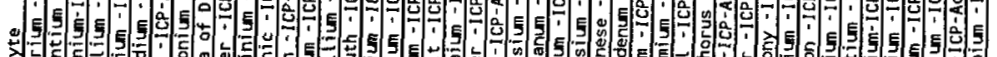

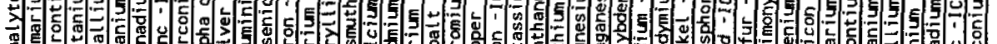

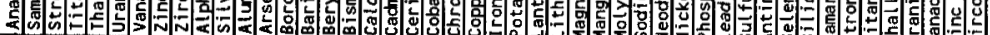

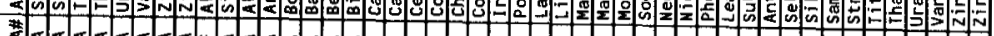

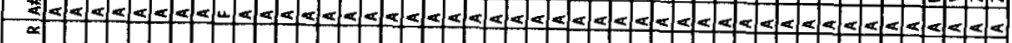




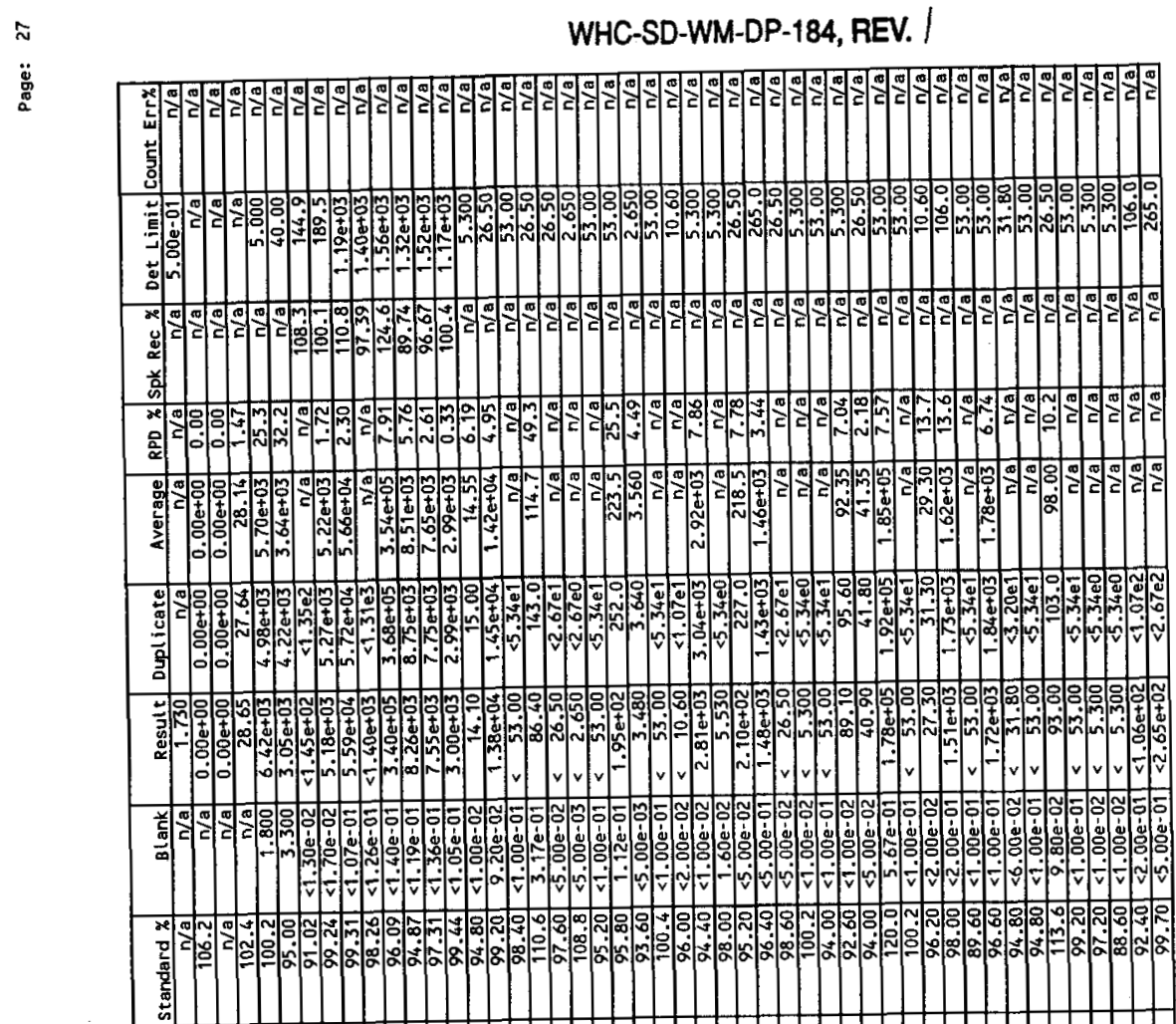

\section{|}

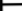

(2)

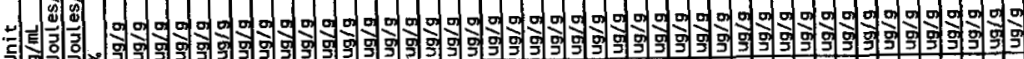

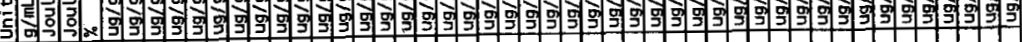

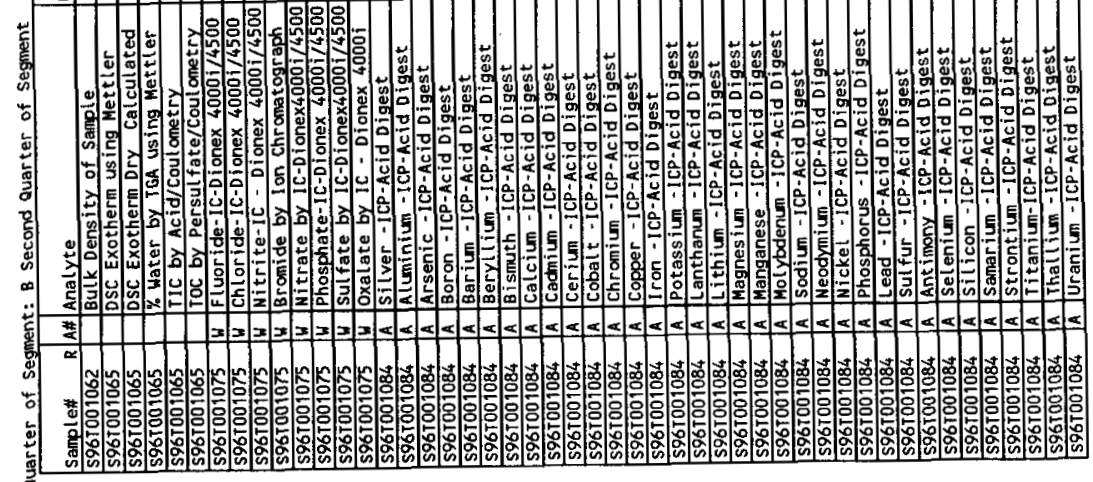



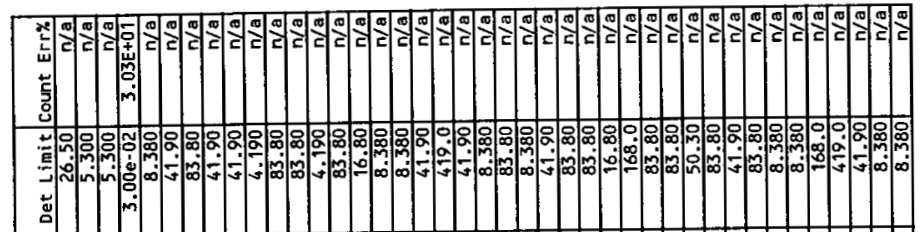

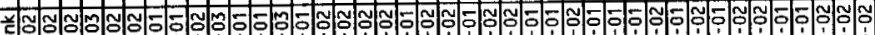
๓) o m.

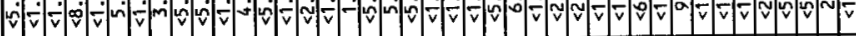

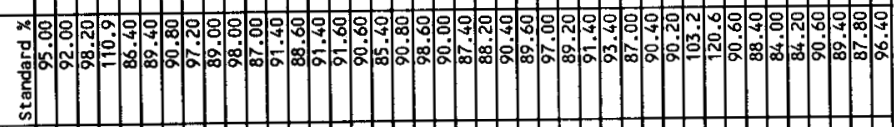
10 D D D D

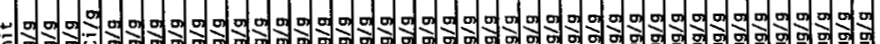

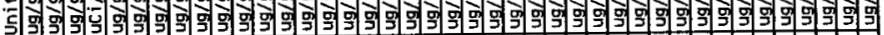

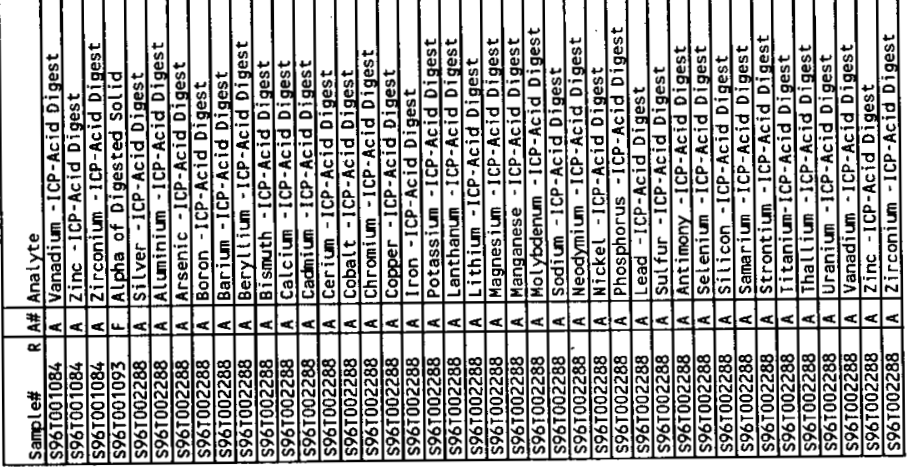

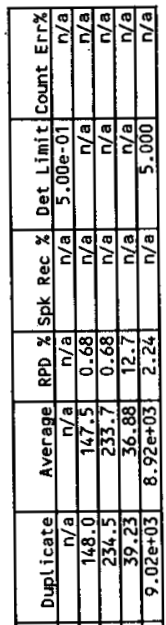

म०त्amm

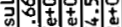
a.

羊可可

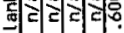
$\infty$

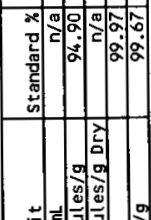

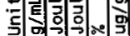
等 m 
范

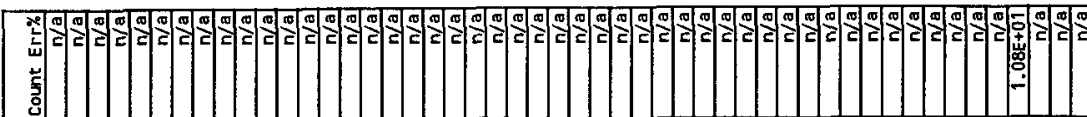

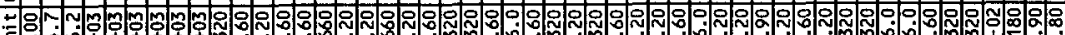

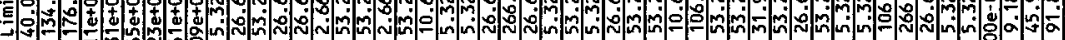

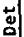

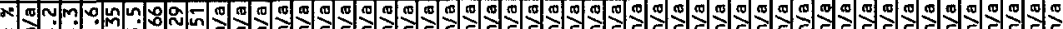
4 다

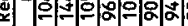

응

م-5

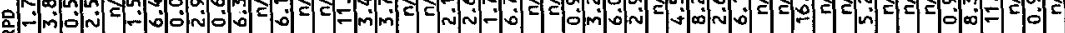

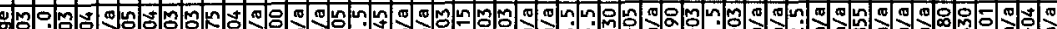

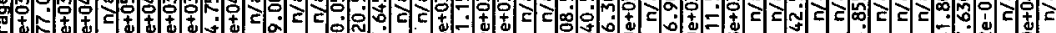
a

$\geqslant ?$

mo - m n t

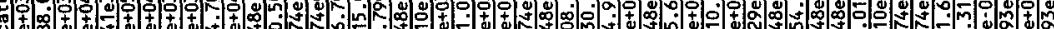

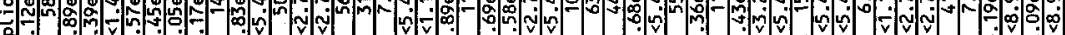

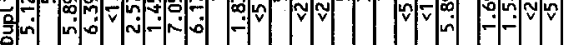

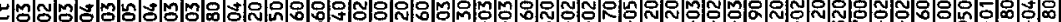

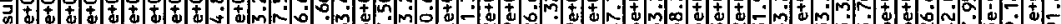

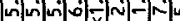

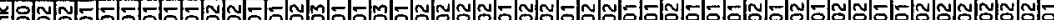

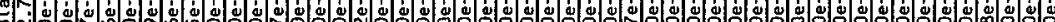

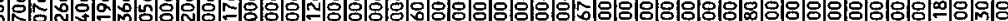

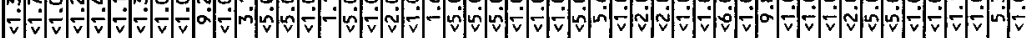

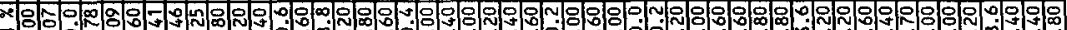

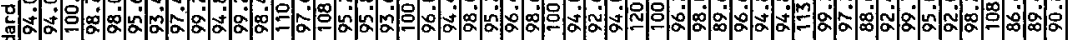

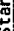

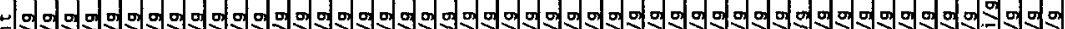
도의 응 8 응

等

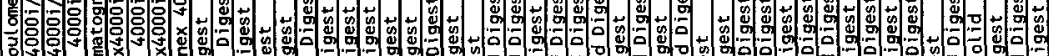

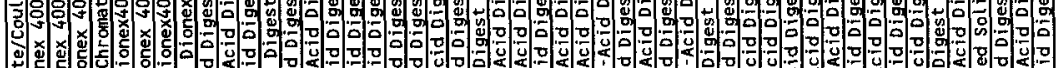
急

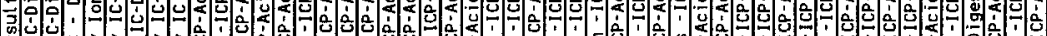

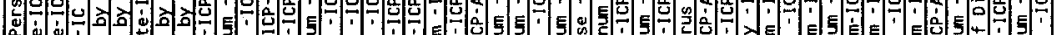

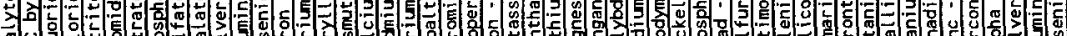

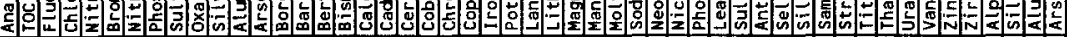

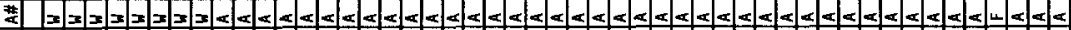


$\underset{\substack{\mathrm{D} \\ \mathrm{a}}}{\ddot{a}}$

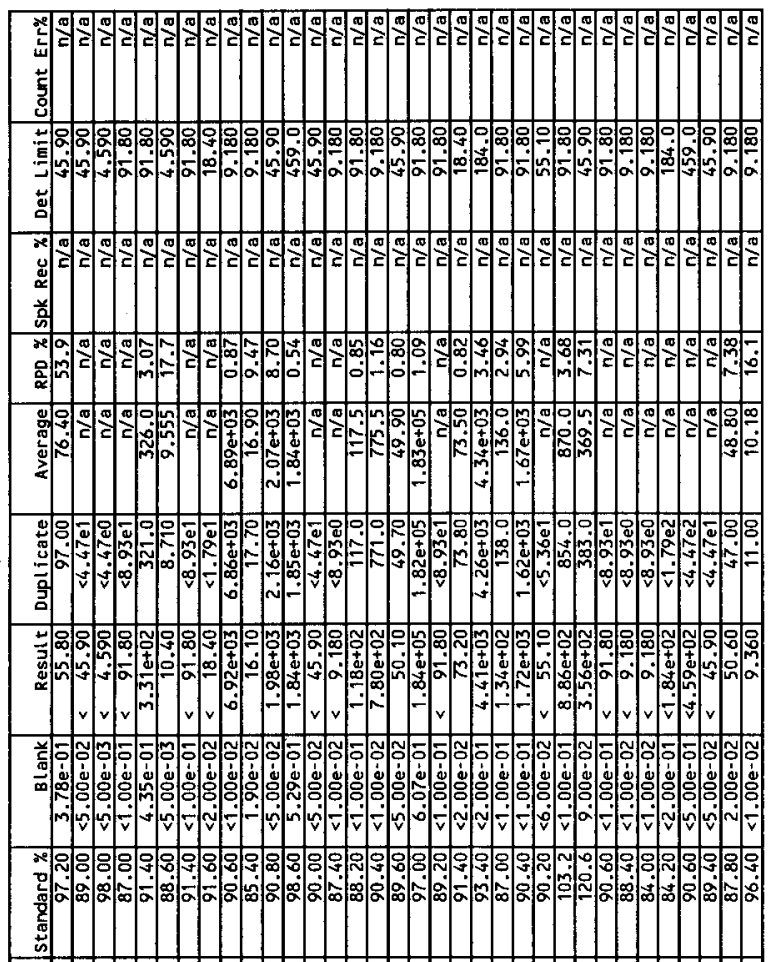

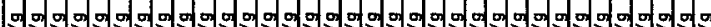

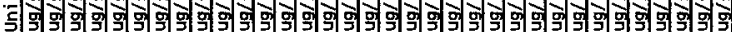

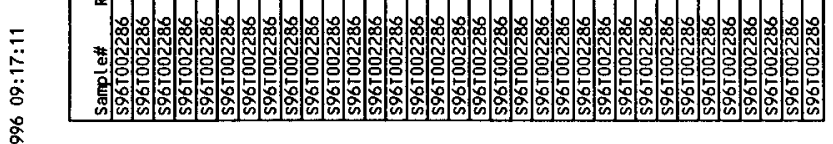

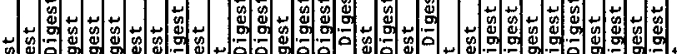

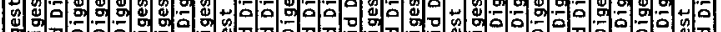

-

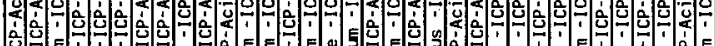

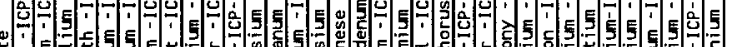

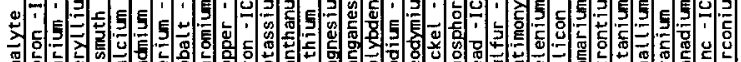

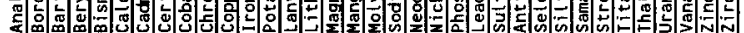

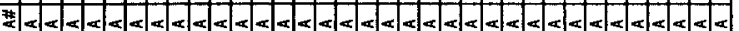
$\stackrel{m}{m}$ 


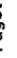

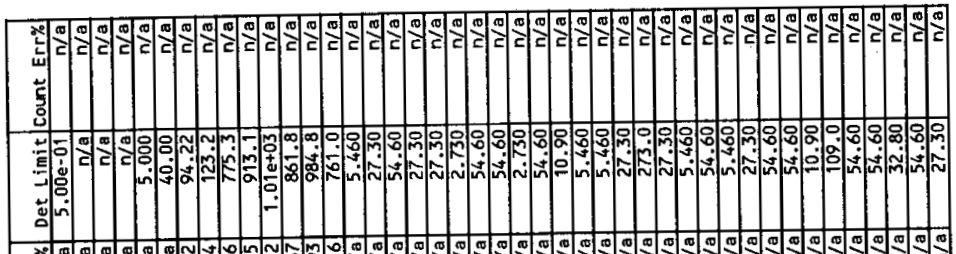

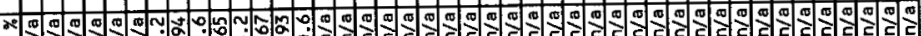

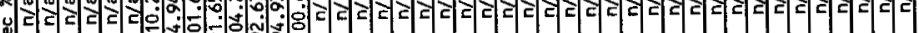

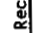

के

苾

졷으

흥 a

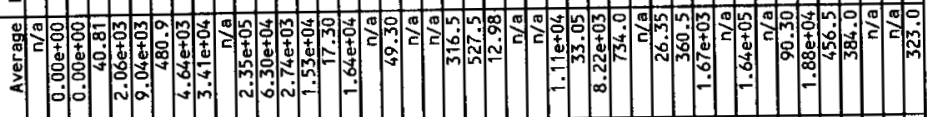

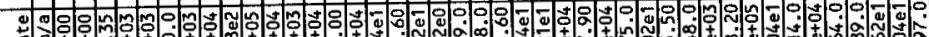

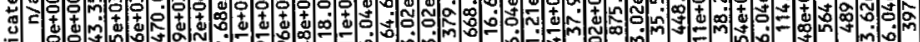
马े

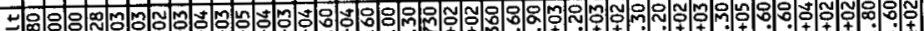

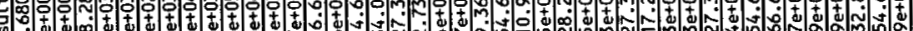

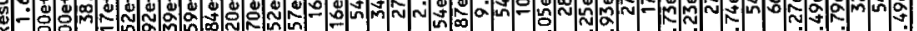

a.

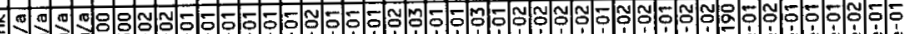
:

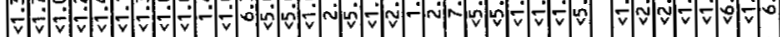

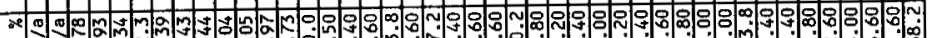

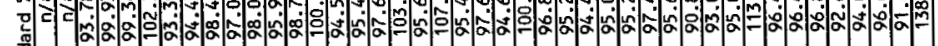
क्ष is

\section{츤.}

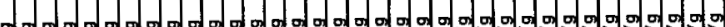

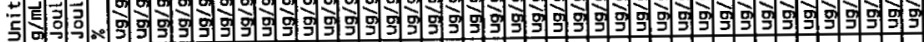

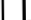

过

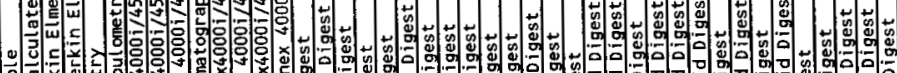

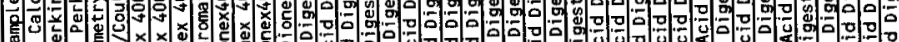
क "

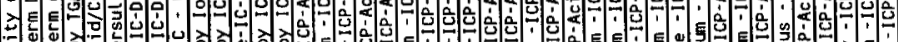

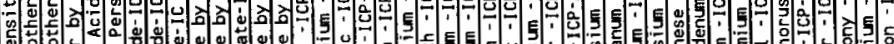

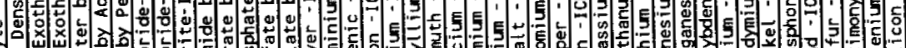

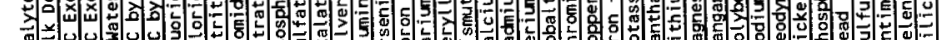

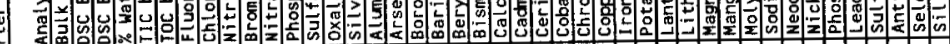

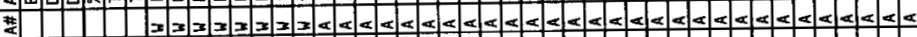

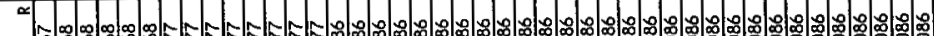
田:

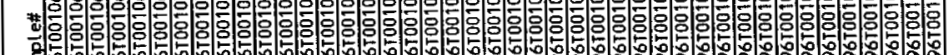

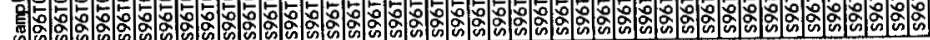




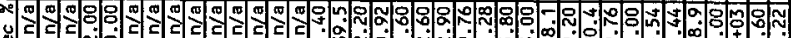

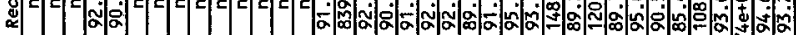
苚

国 这

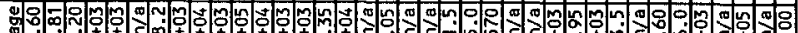

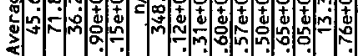

minn mim ninmai

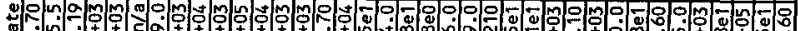

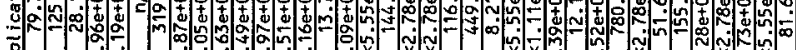
勿

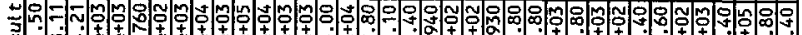

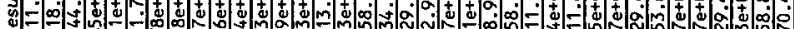

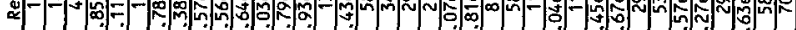

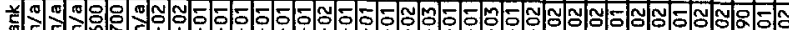

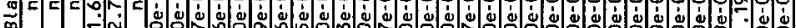

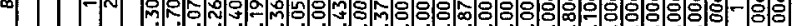

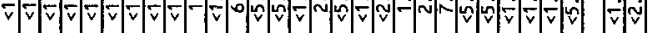

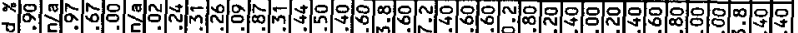

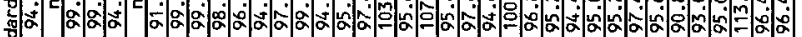
䒺

100.0100100 s

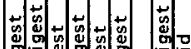
o. $\Rightarrow$ al a

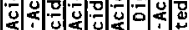
(1)

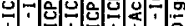

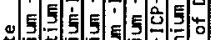

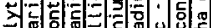

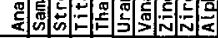

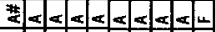
क

여용

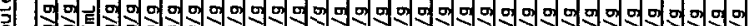

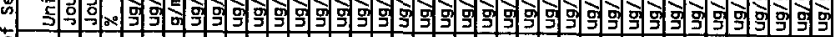

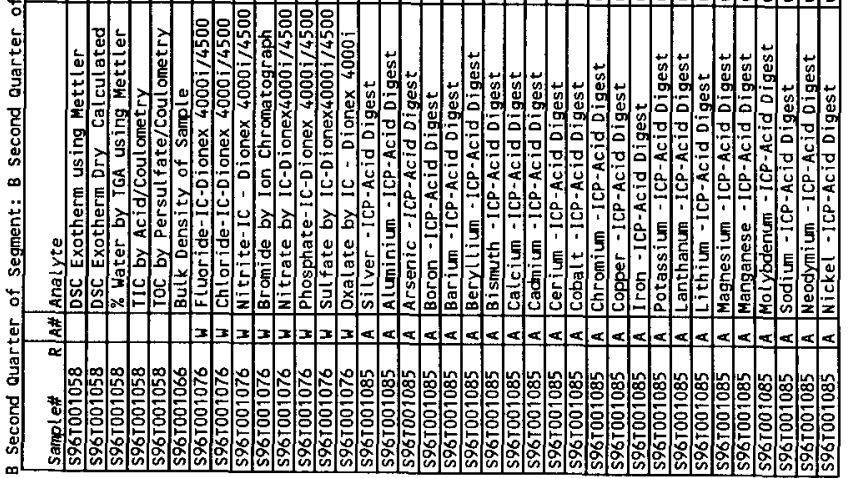
$\stackrel{2}{2}$ 


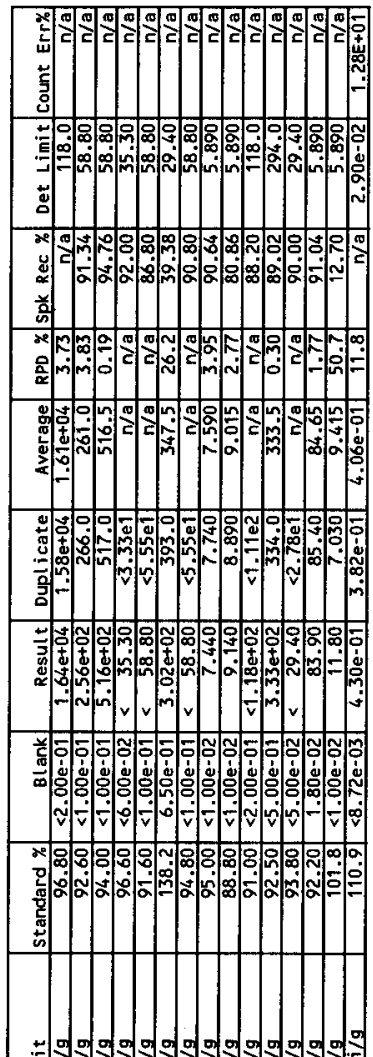

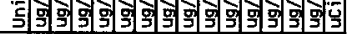

哭

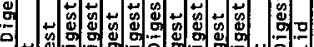

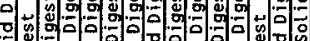
의 0 a 0 a $x-0 y$ o

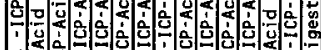

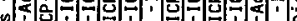

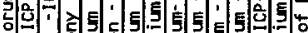

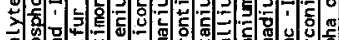

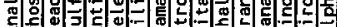
andiñ

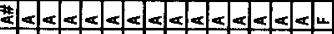

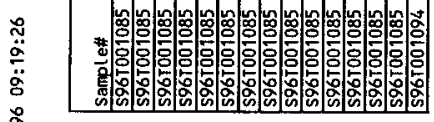

$\% m$ 


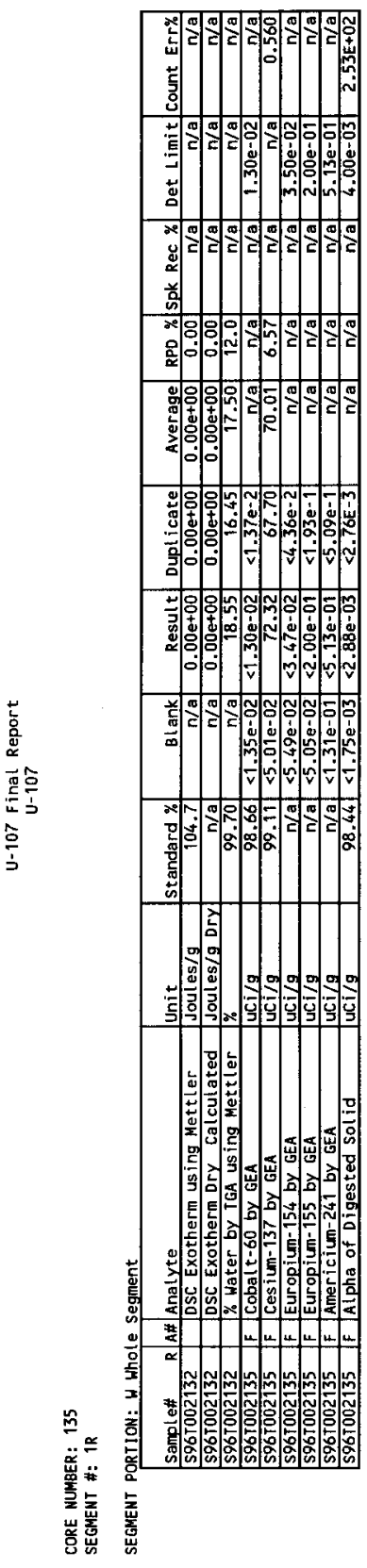

\begin{tabular}{|}
\hline \\
\hline \\
\hline
\end{tabular}

节

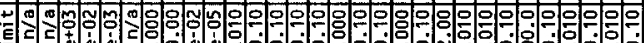
=1 = 范

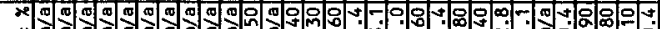

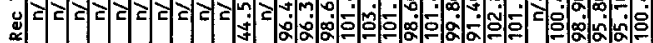
商

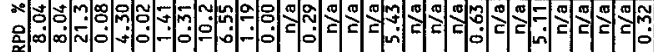

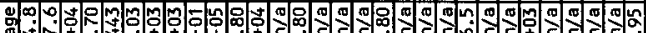

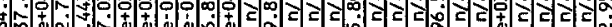

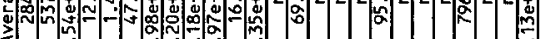
2

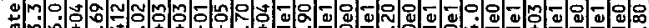

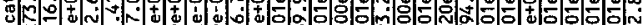

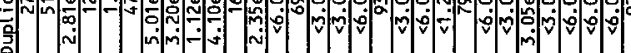

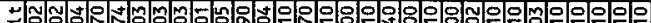

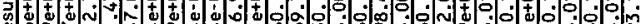
$\stackrel{\leftrightarrow}{\alpha}$ $m$

$x$ क

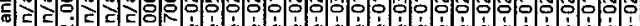

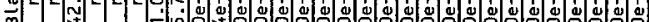

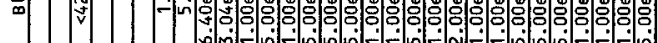

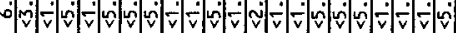

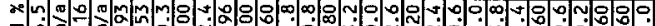

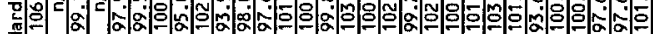
离

\section{1}

$\rightarrow$ $+$

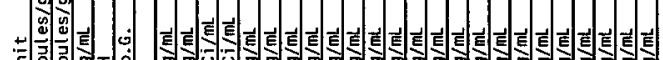

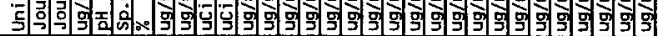

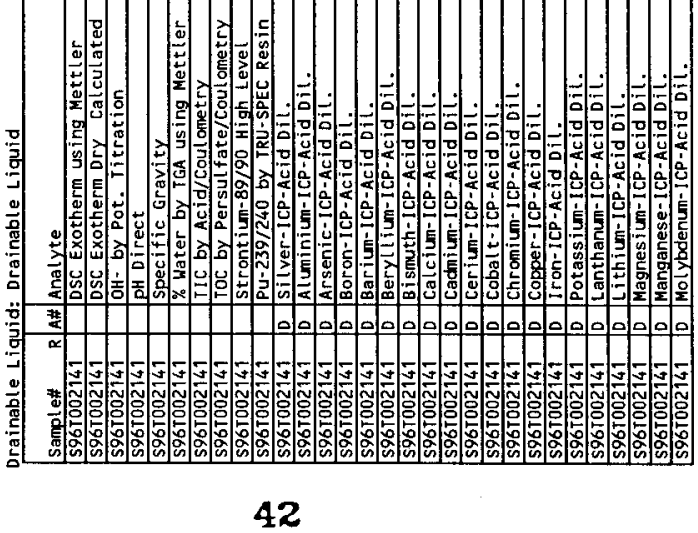




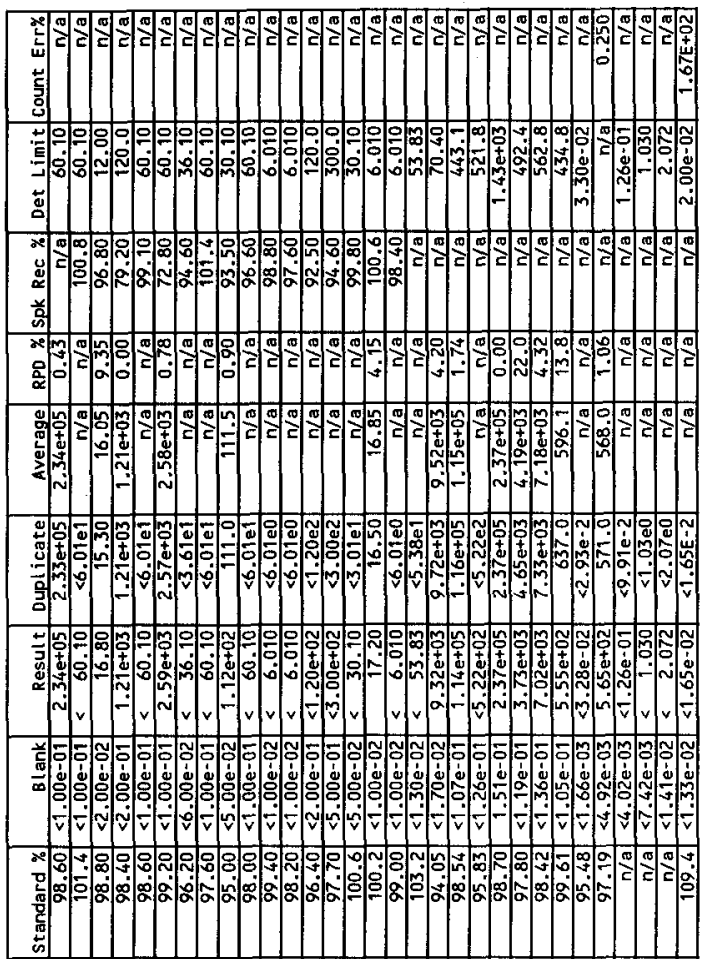

늘

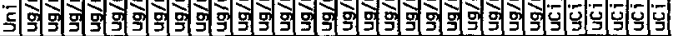

.

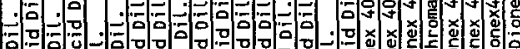

70

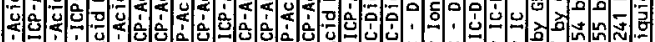

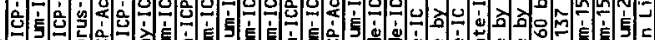
4 定

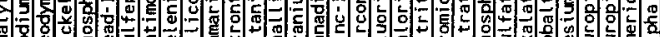

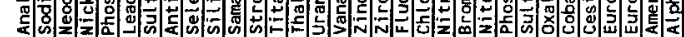
*0100010000100000

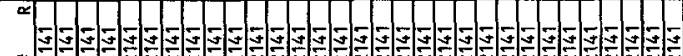


$\underset{\substack{i \\ ٌ}}{\ddot{m}}$

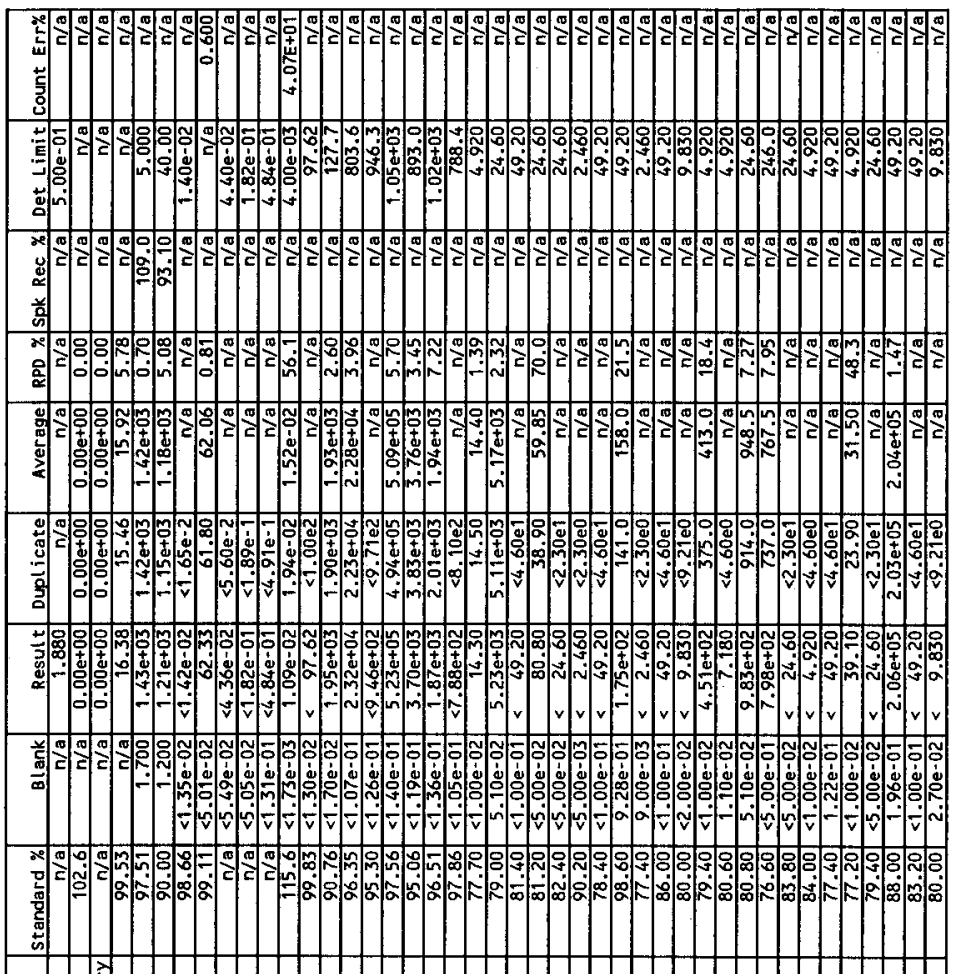

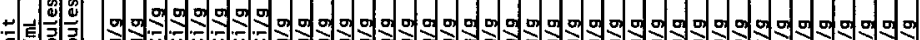

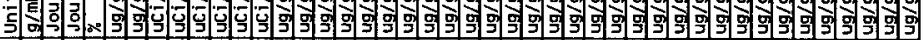

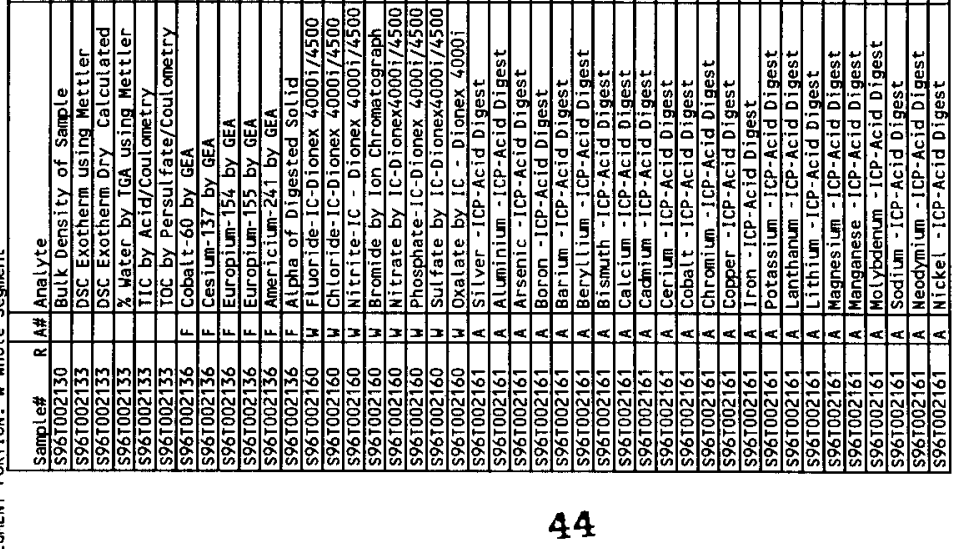




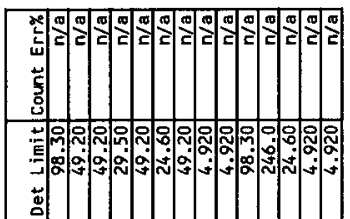

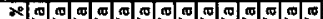

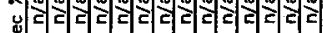
盖

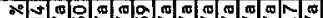

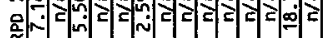

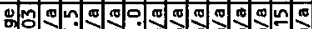

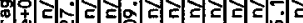
ํㅣㄴ व.

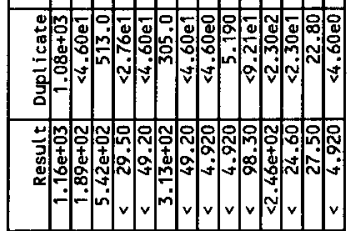

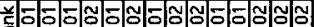

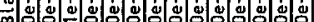

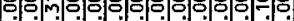

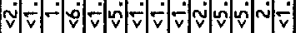

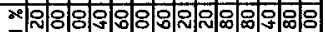
눈 产

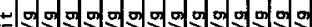

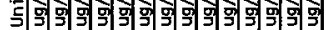

或

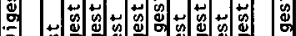
-

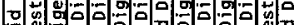

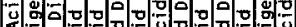

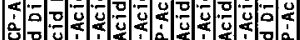

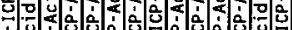
중의 $\rightarrow$ 은

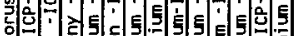

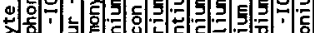

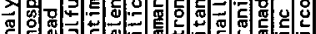

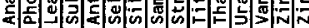

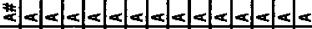

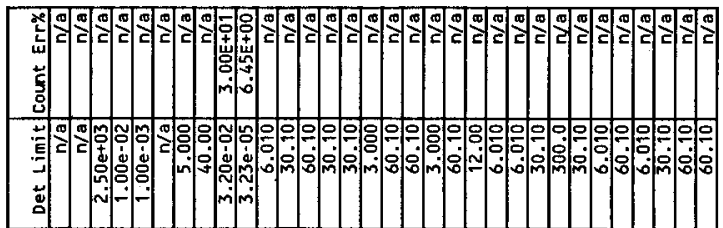

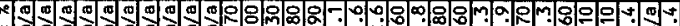

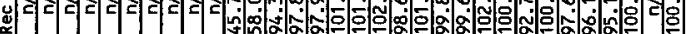
总

有

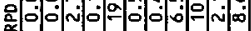

:

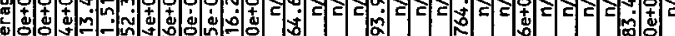
of 0 - in \&.ं $\therefore \rightarrow-\min$ i

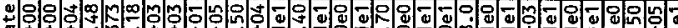

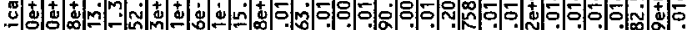

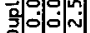

응ㅇㅇㅎㅇㅇㅛ

马े

$\approx 0$

óंn : i

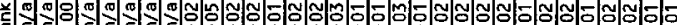

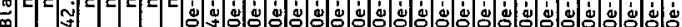

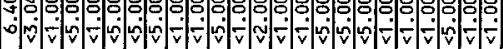

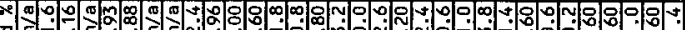

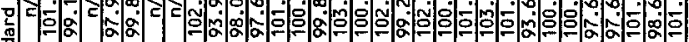
产 节

정

요응

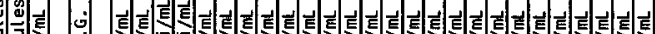

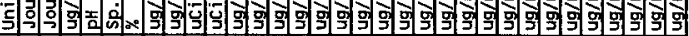

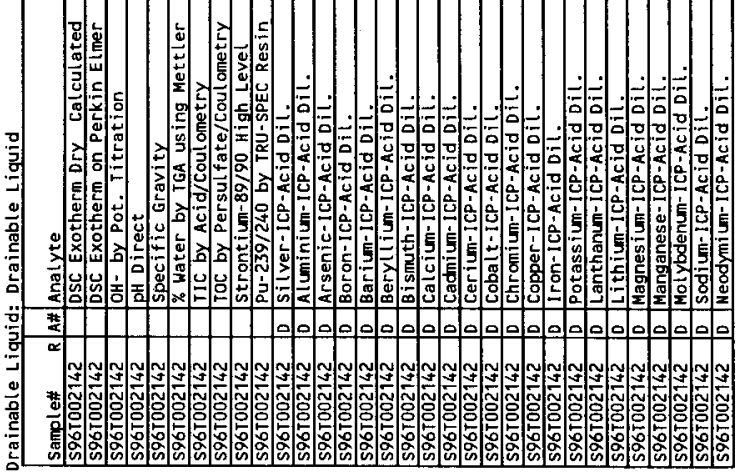




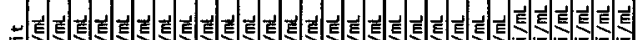

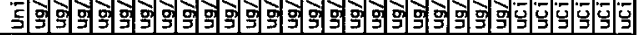

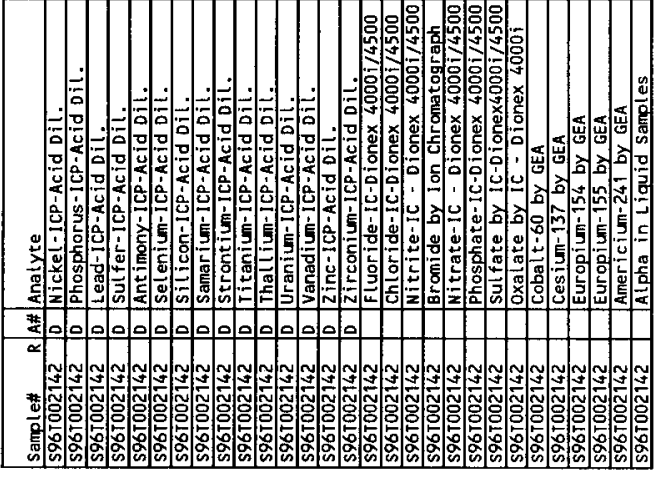




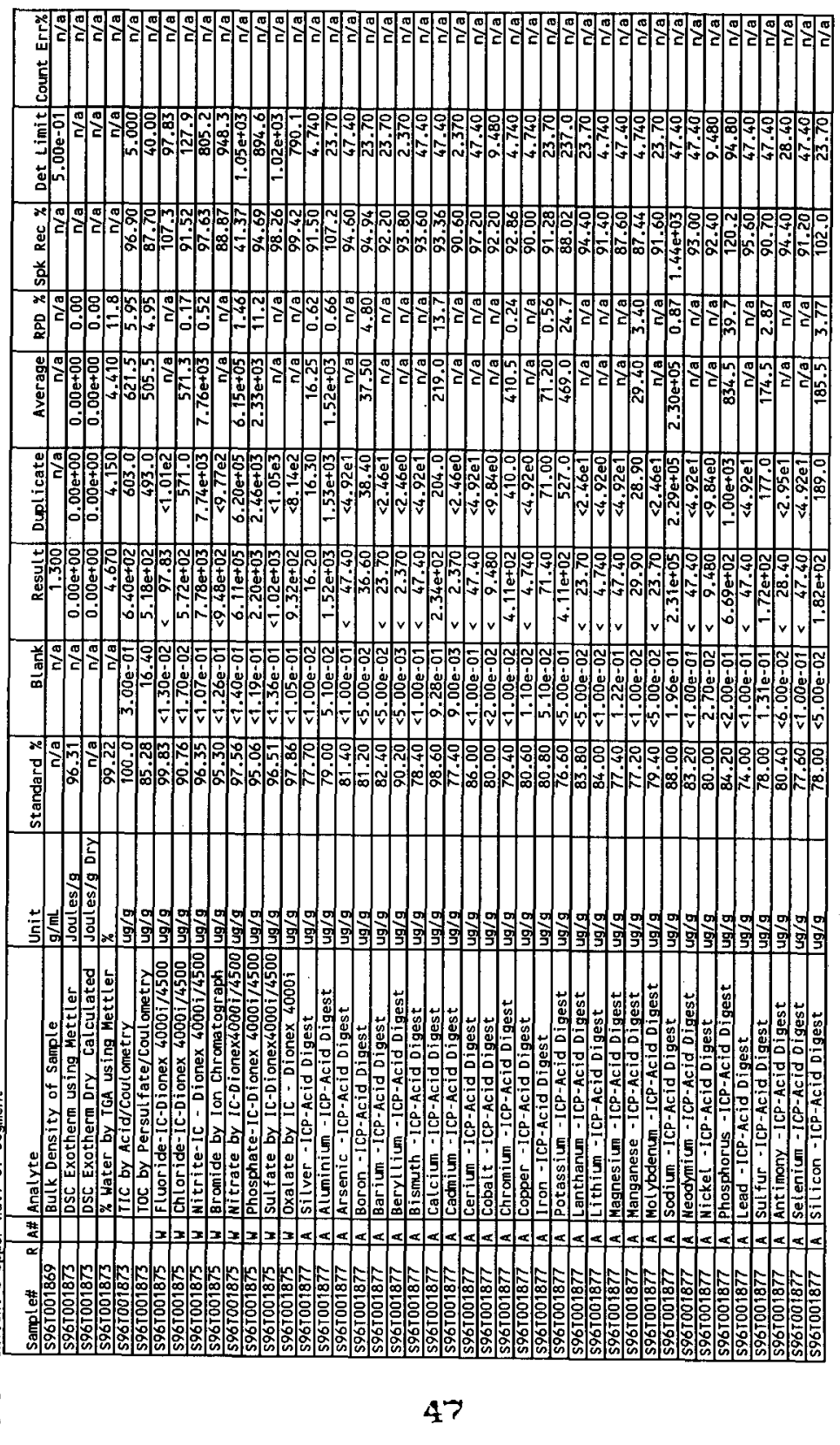




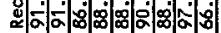
前

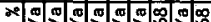
의드리드르드다눈 㠃可 $\sum$

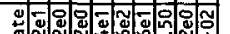
焉

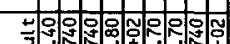

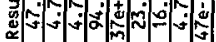
$\checkmark v \vee v \vee v \vee v$

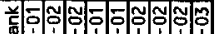

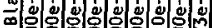

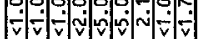

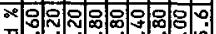
무요 क्ष i. :

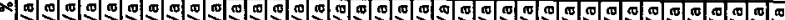

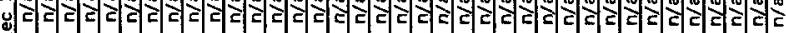
总

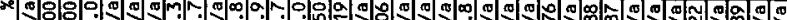

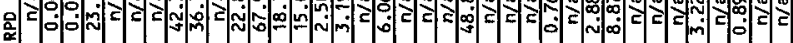
:

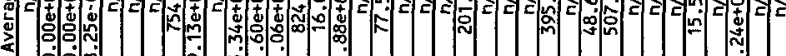

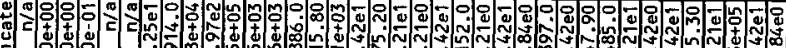
言

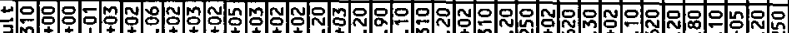

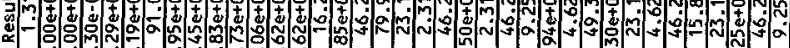
$0.0 \mathrm{~m} \cdot \mathrm{a}^{\circ}$

前

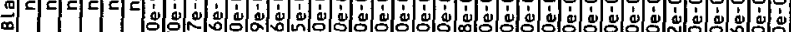

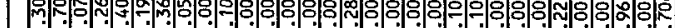

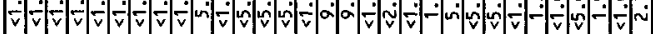
*0. 䒺 部

\section{능}

(1)

$\Rightarrow$ 의 0 on 의 0 영

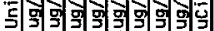

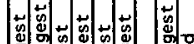
要

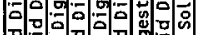
의 a $4: 00$ i

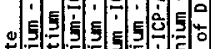

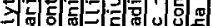

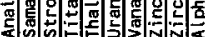
$x$ 的计 $\Rightarrow$ N

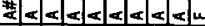

FENNNNO *

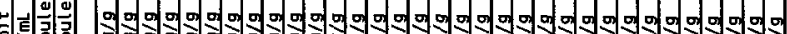

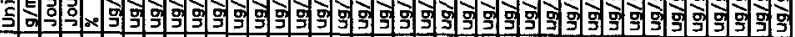

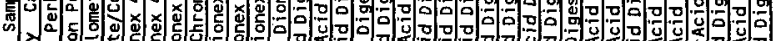

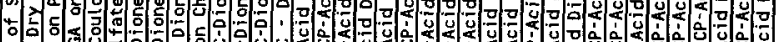

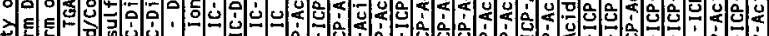

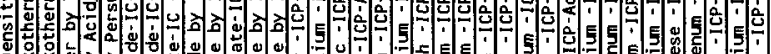

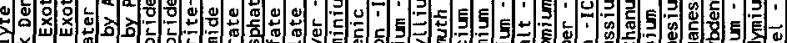
要

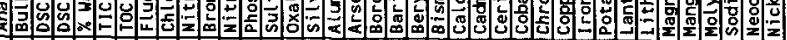
Z S r s

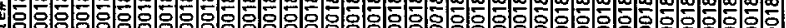

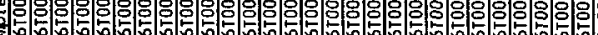




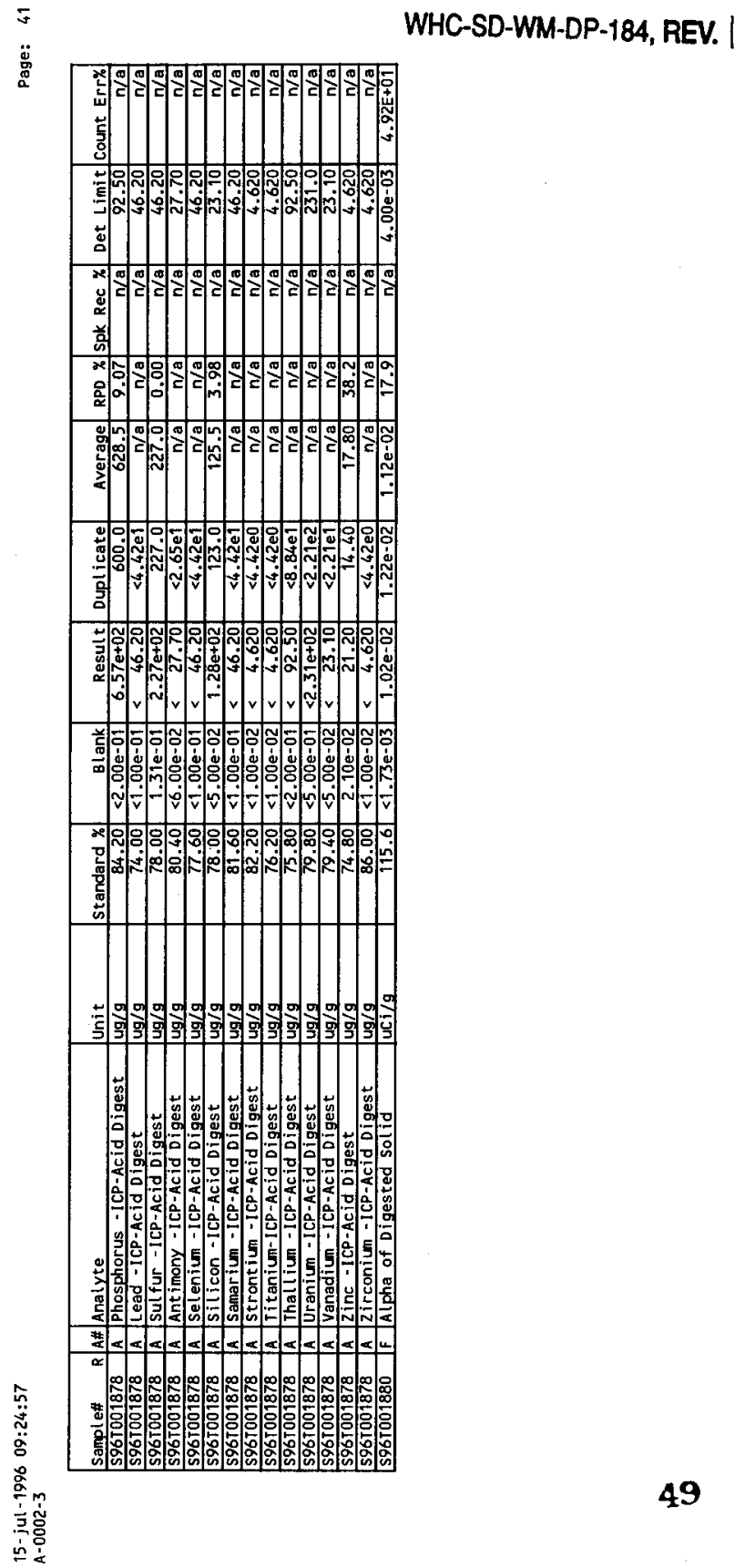


$\ddot{g}$
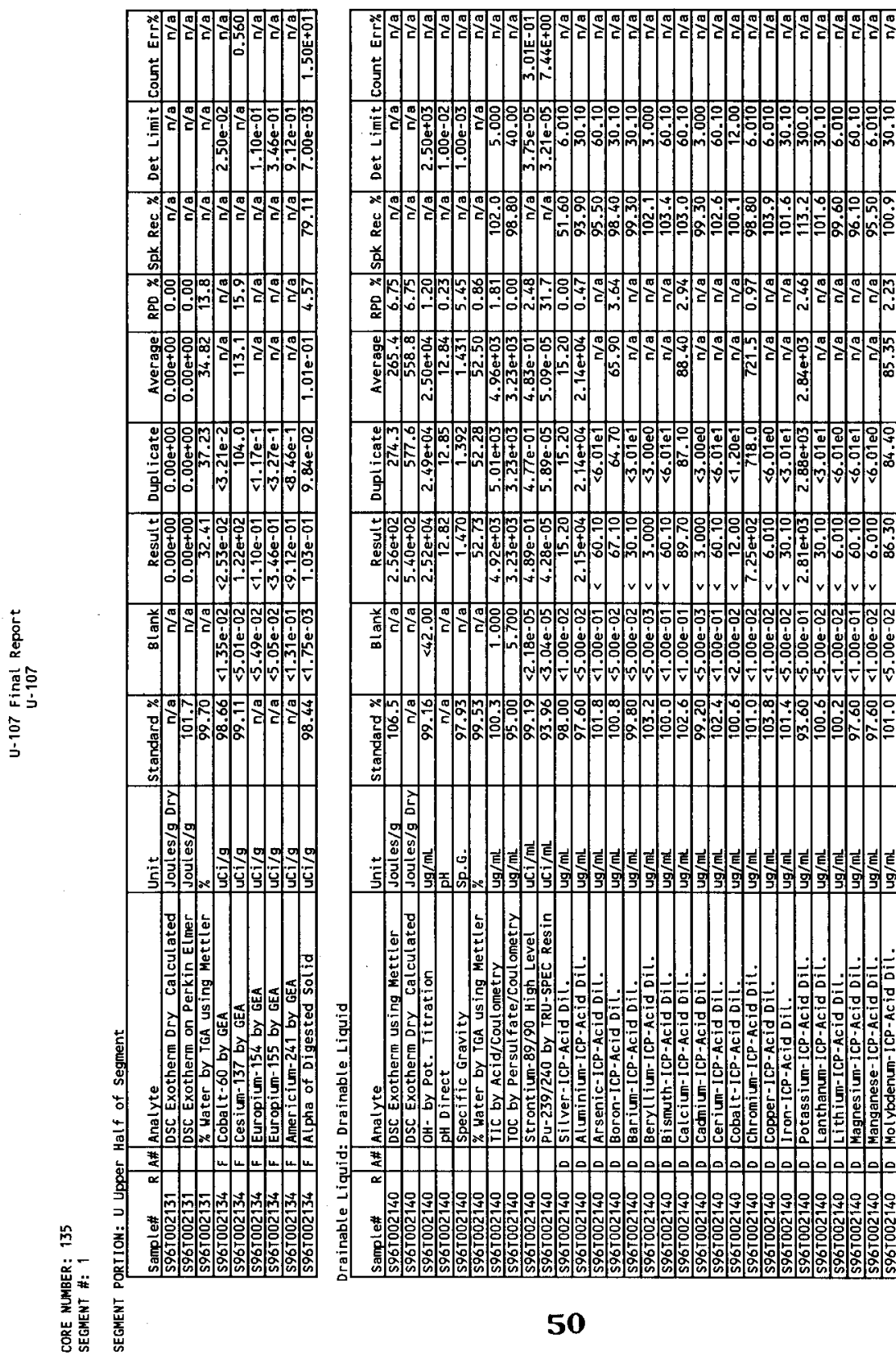

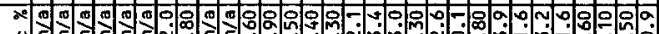

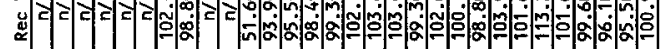
㚇

a

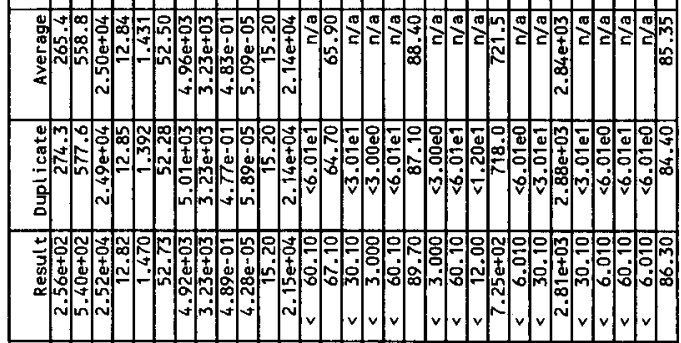

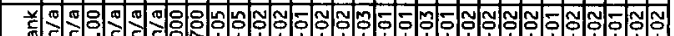

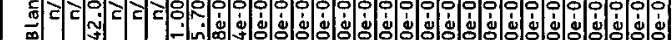

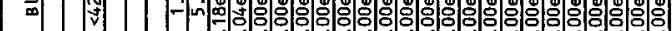

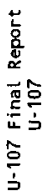

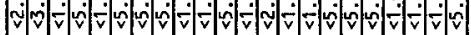

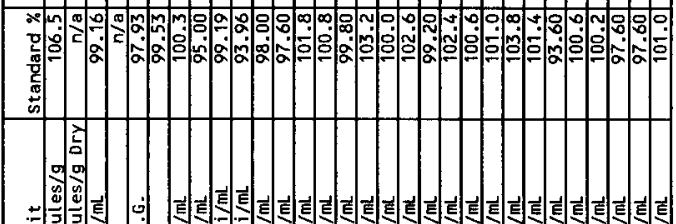

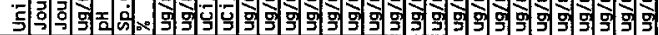

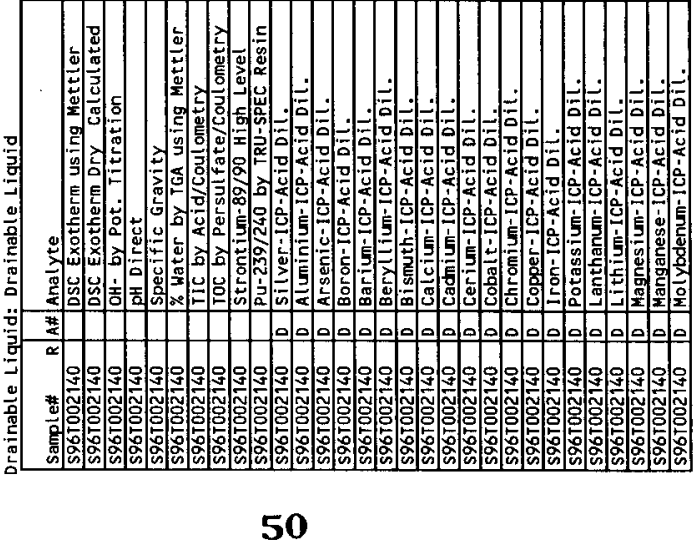


离

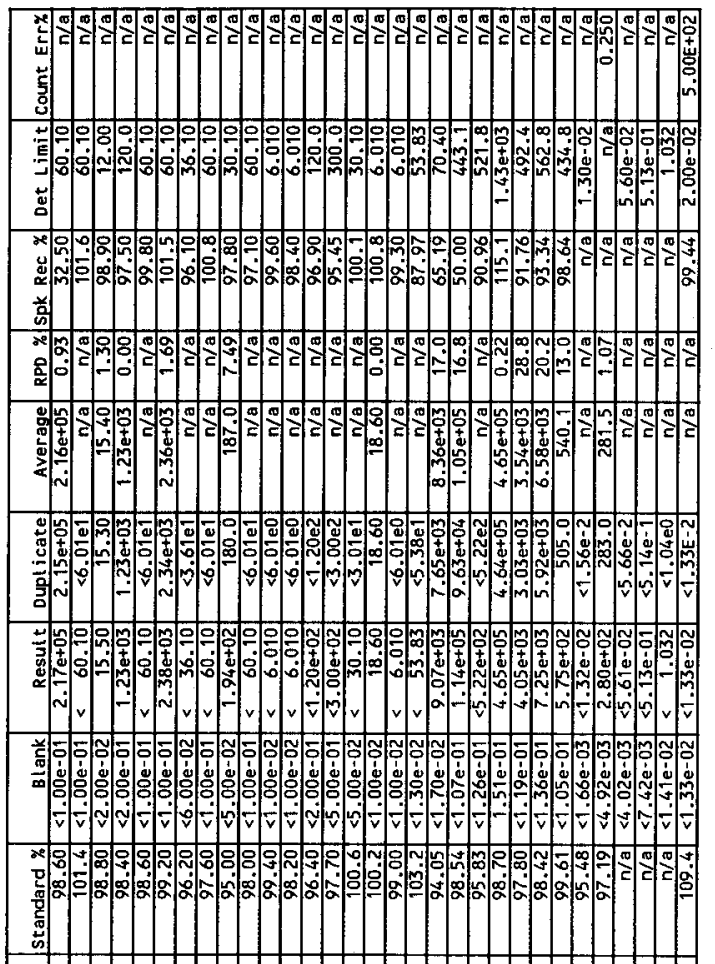

늘 흘

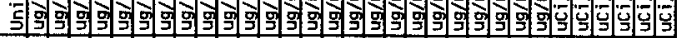

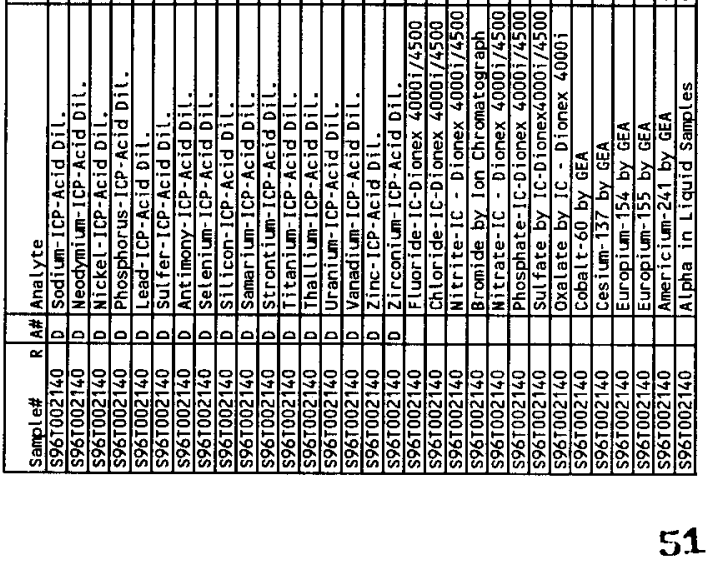




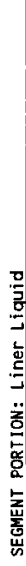


棁

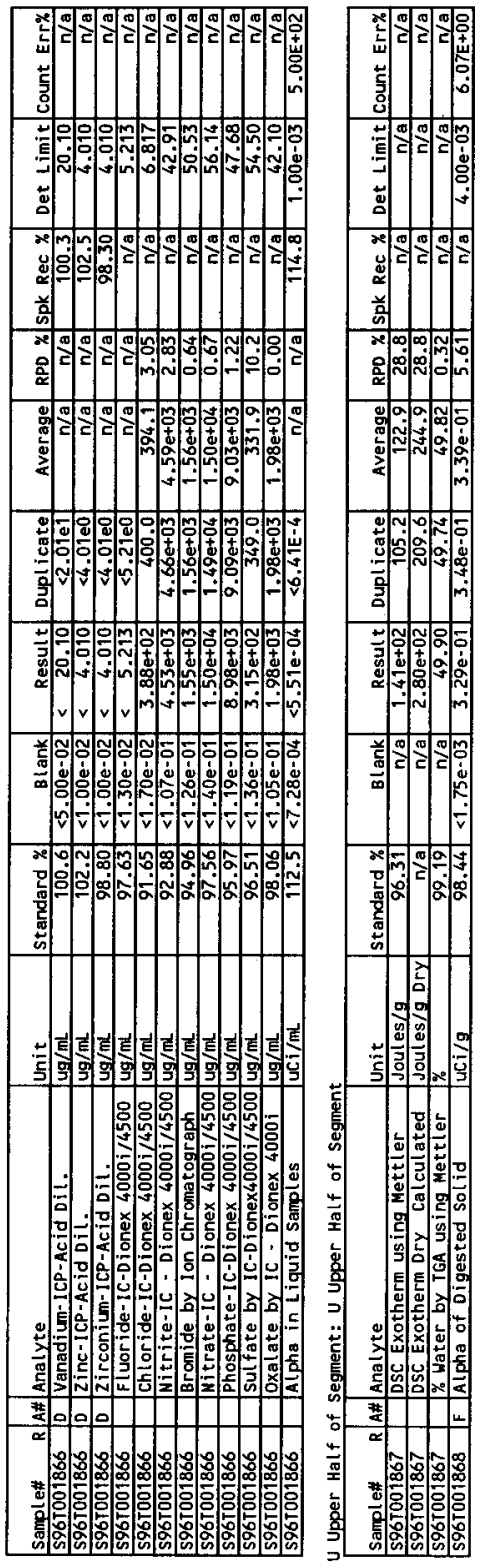

$\stackrel{\circ}{\circ}$

苟容 
WHC-SD-WM-DP-184, REV. 1

U-107 STATISTICAL ANALYSIS

54 
WHC-SD-WM-DP-184, REV. 1

THIS PAGE WAS INTENTIONALLY LEFT BLANK

55 
WHC-SD-WM-DP-184, REV. $\mid$

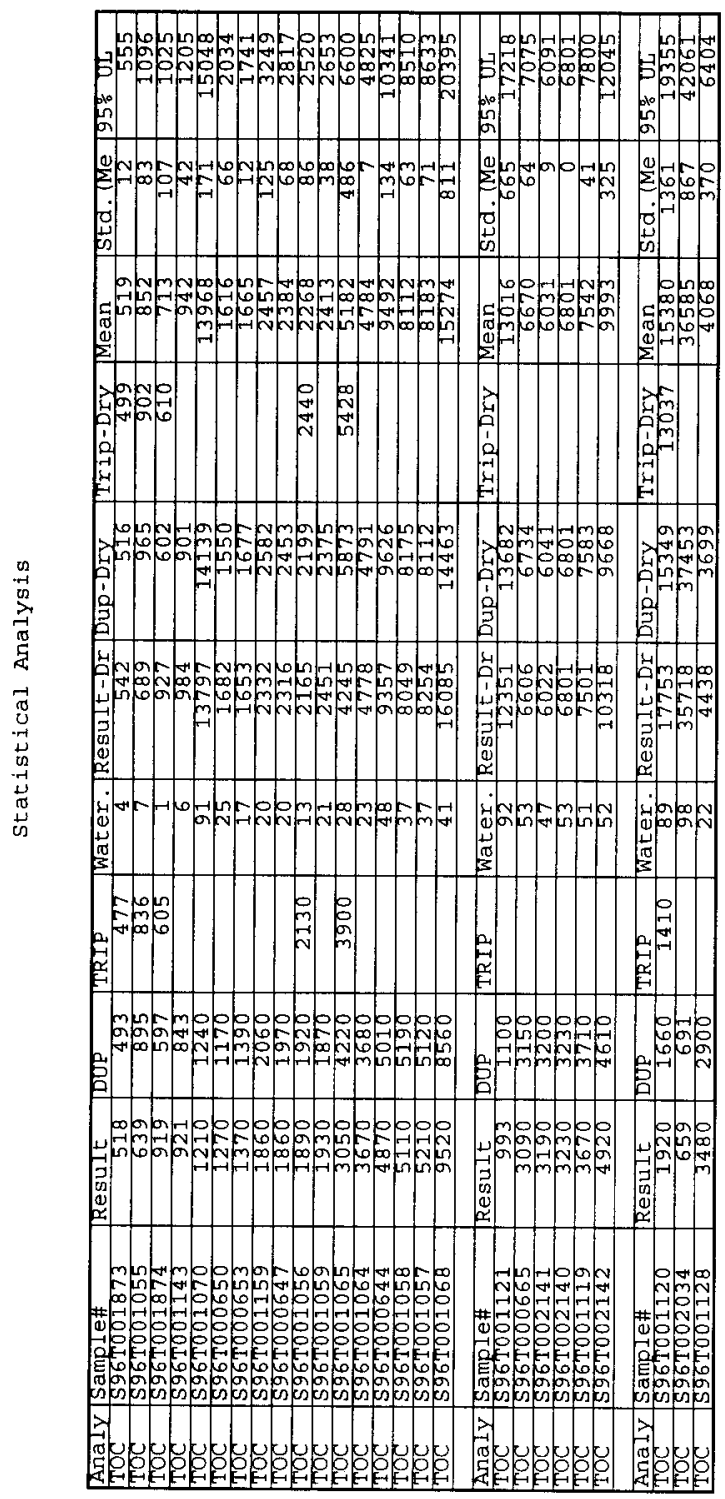


WHC-SD-WM-DP-184, REV. 1

CHAIN OF CUSTODY FORMS 
WHC-SD-WM-DP-184, REV. 1

THIS PAGE WAS INTENTIONALLY LEFT BLANK 


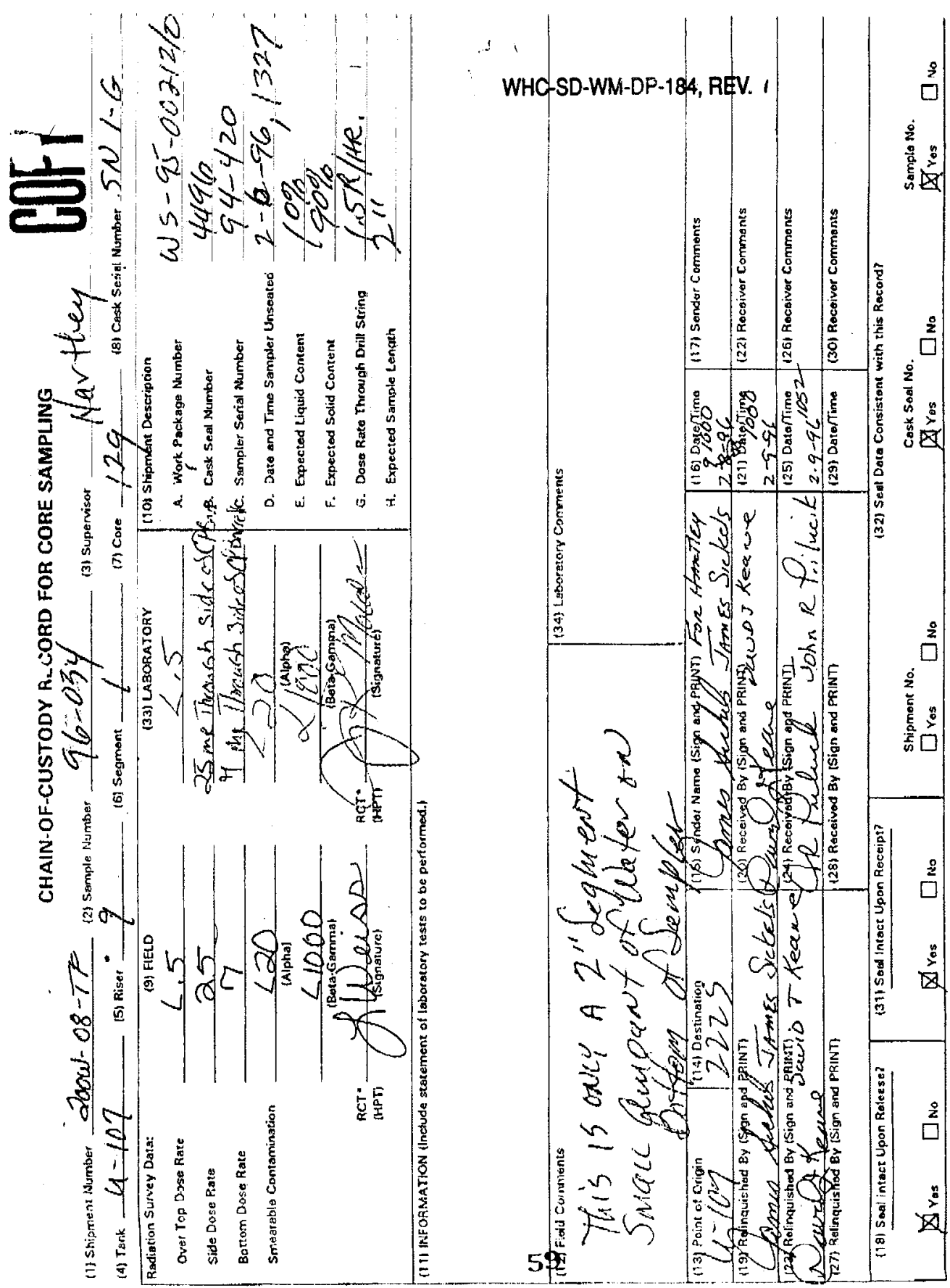




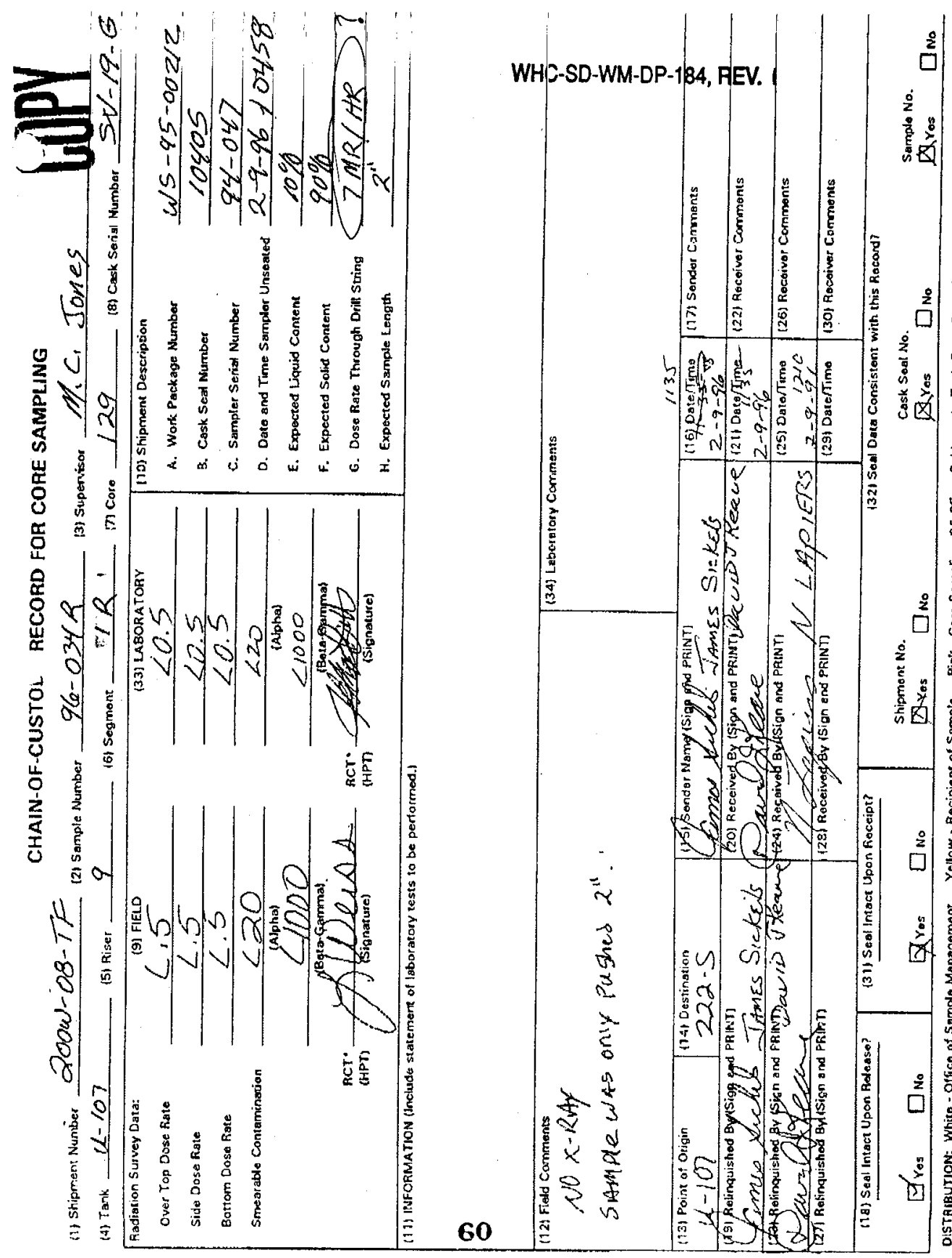




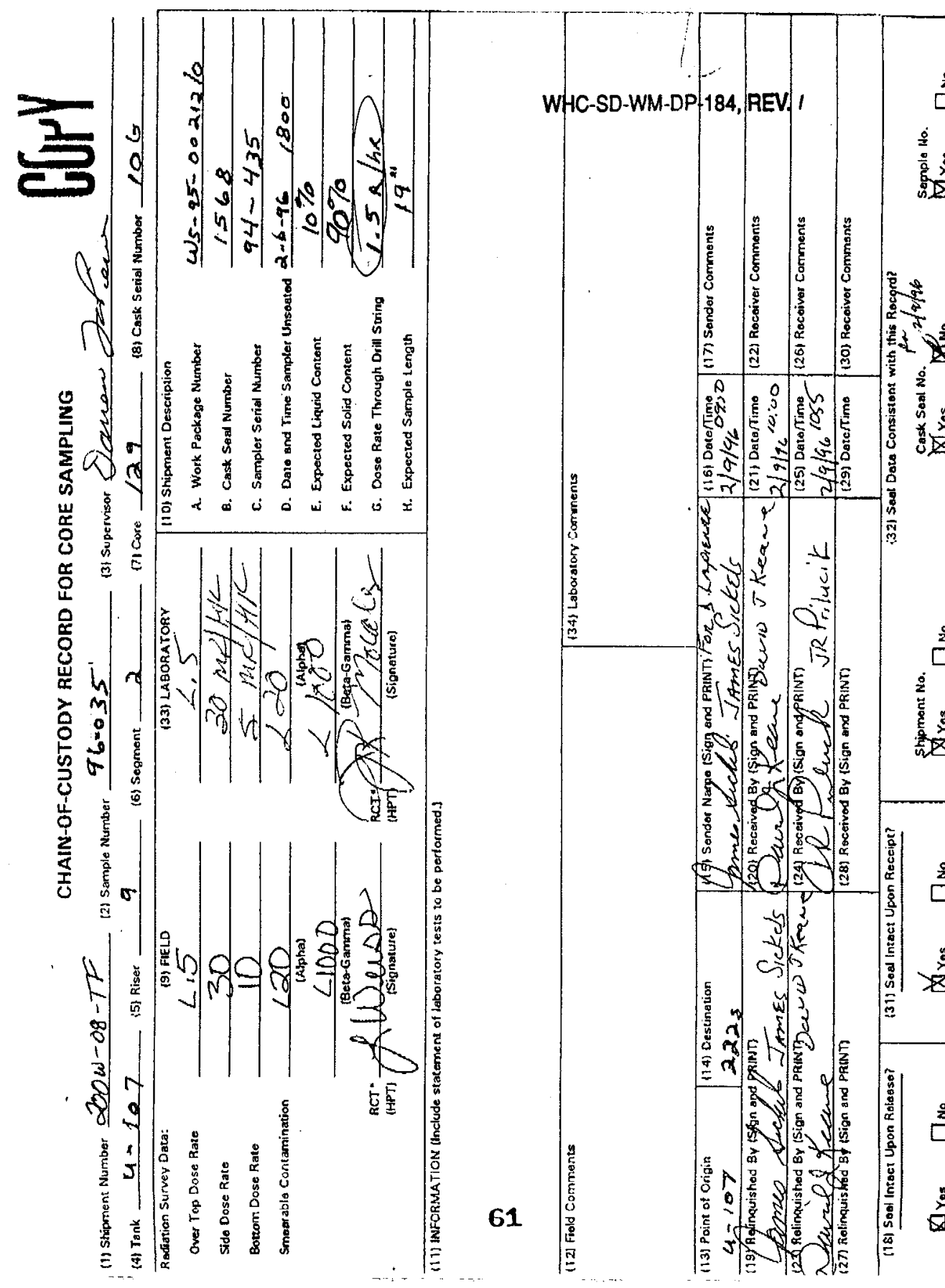




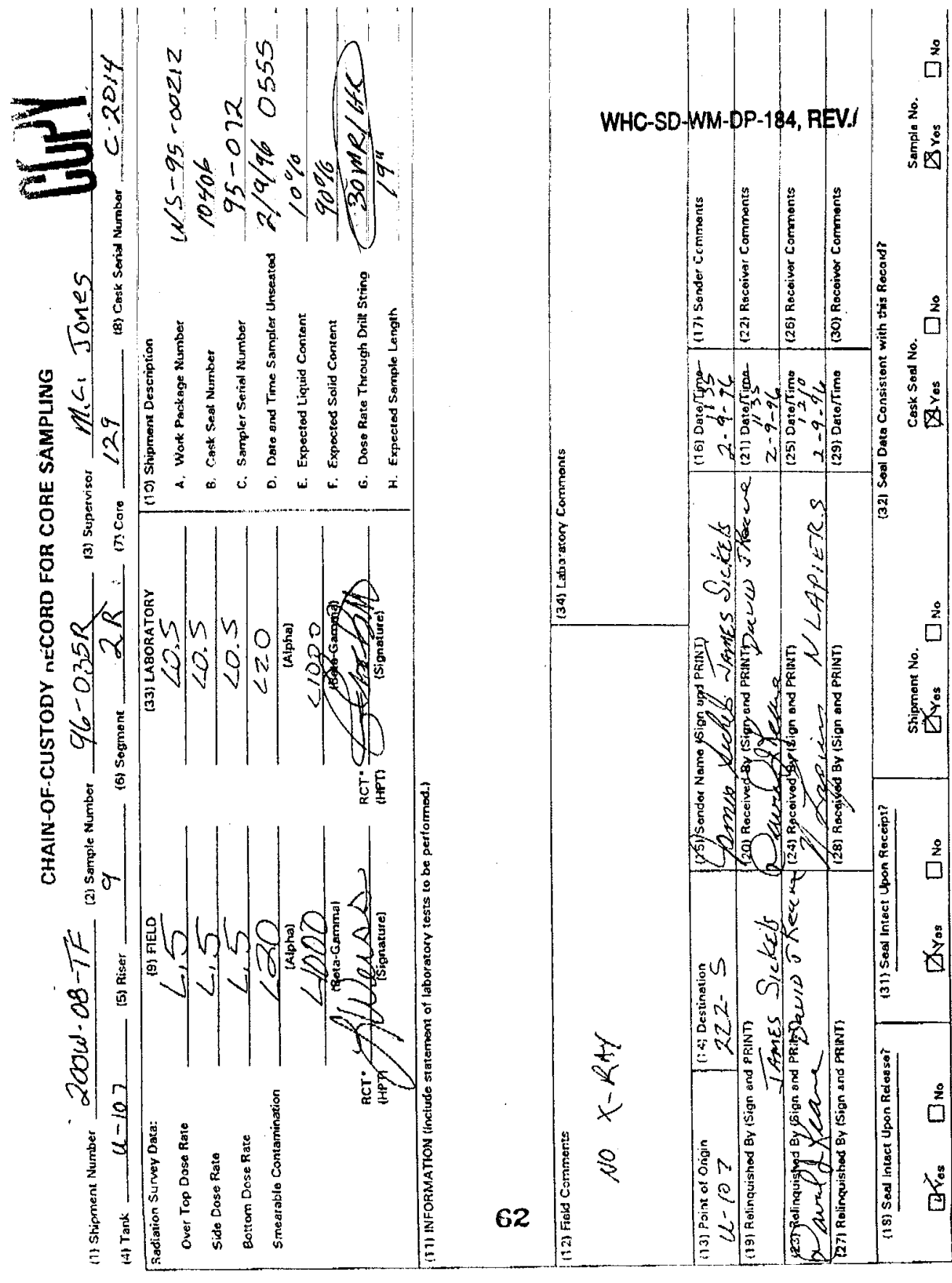




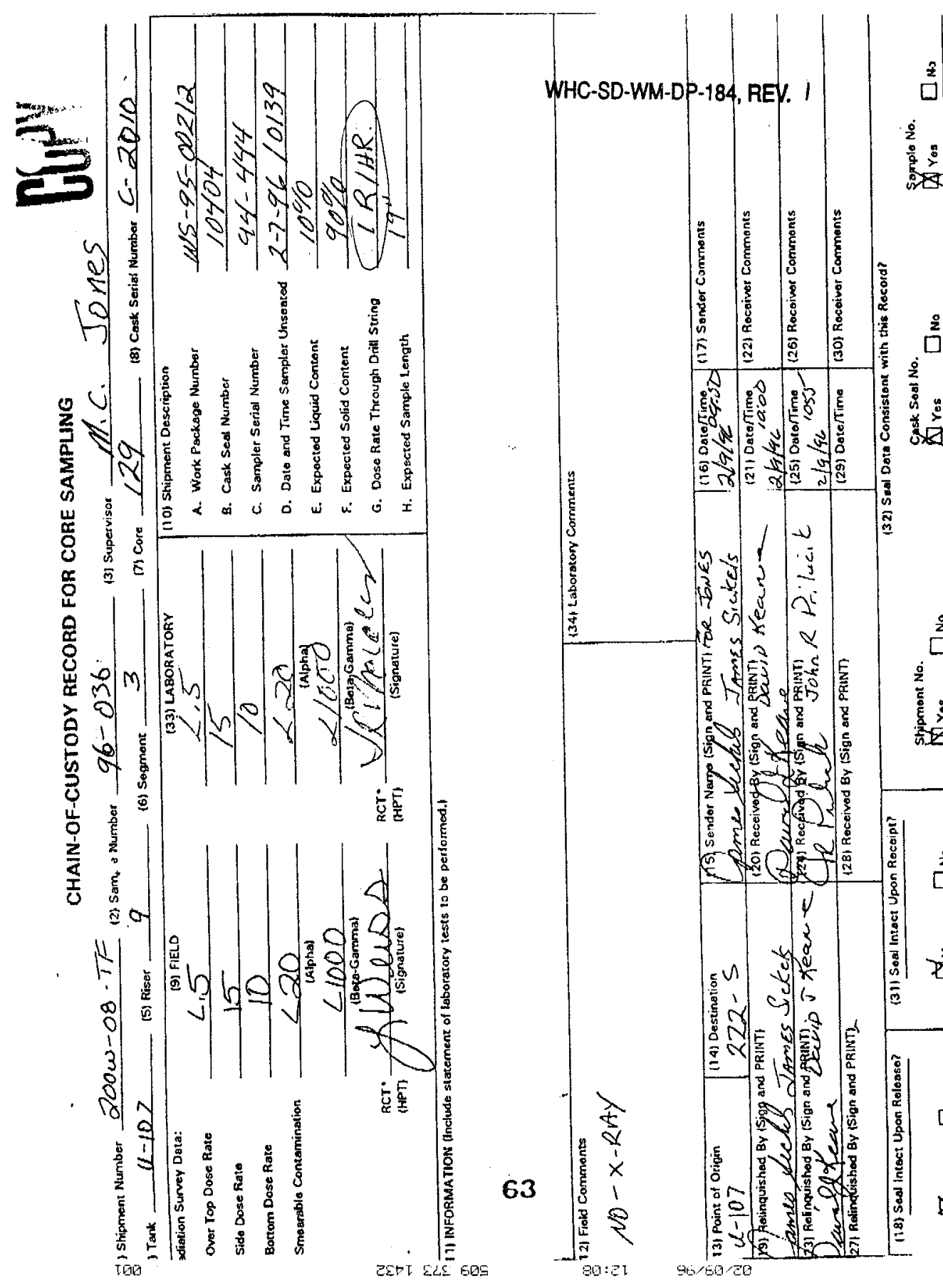




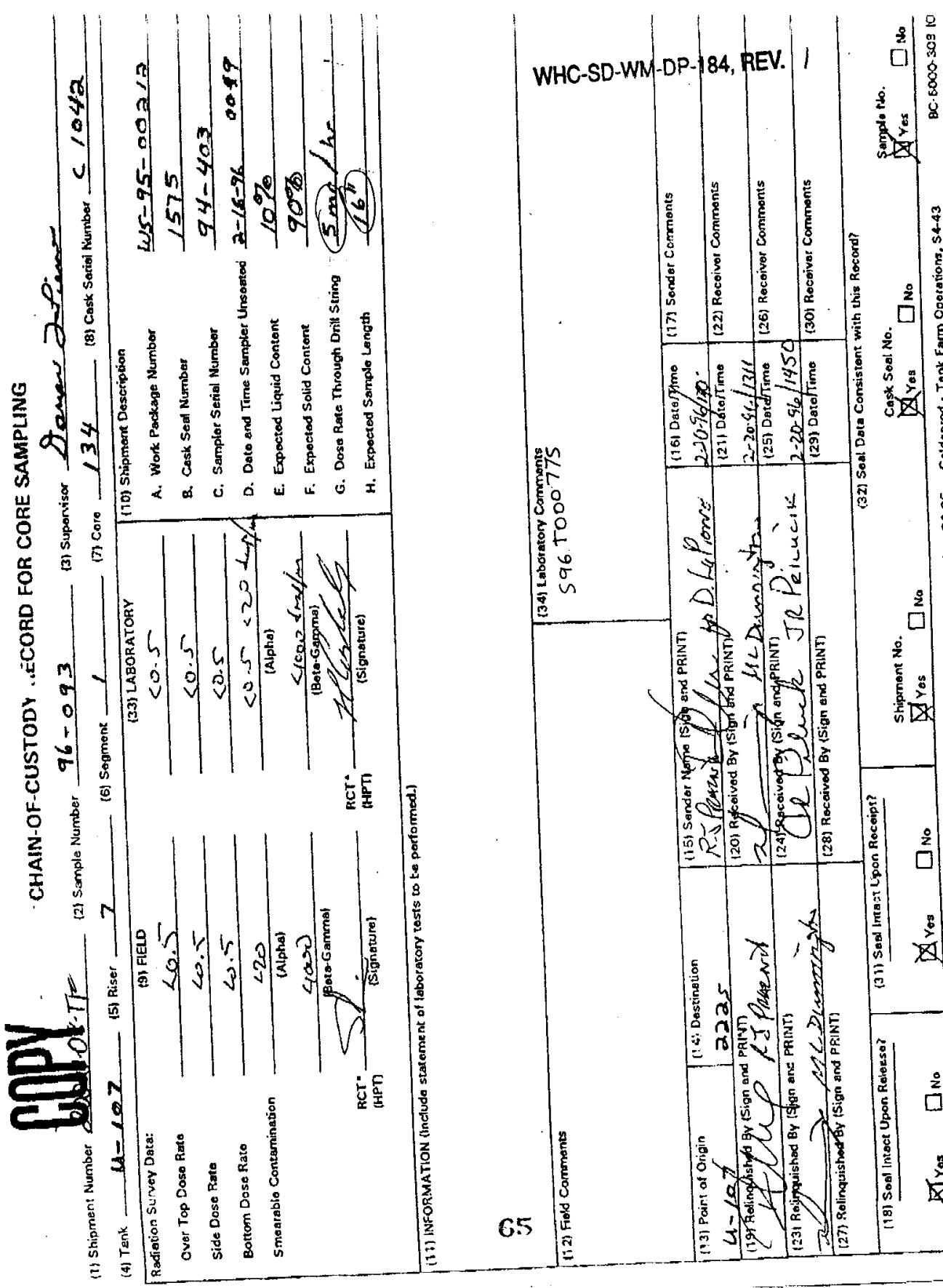




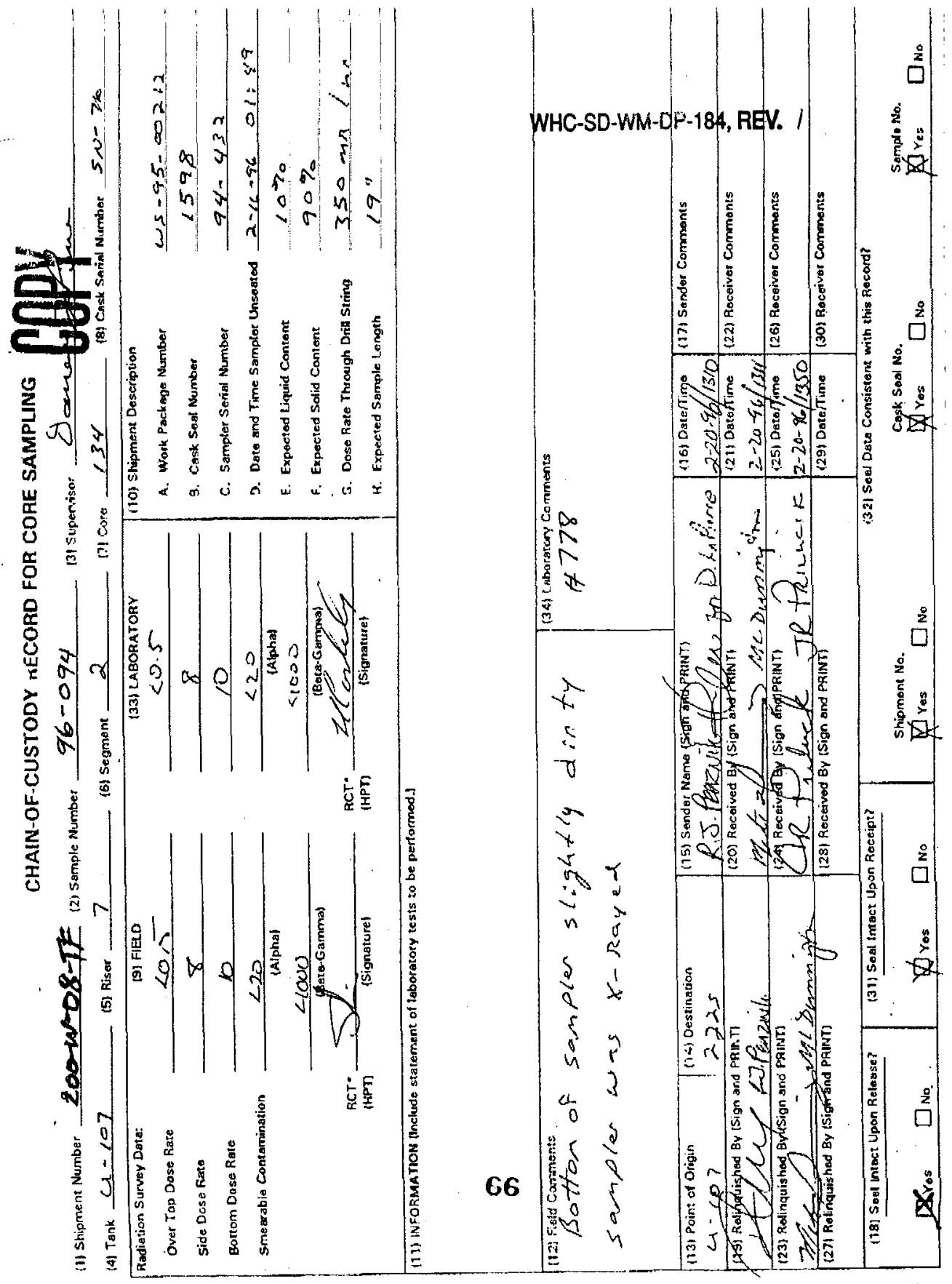




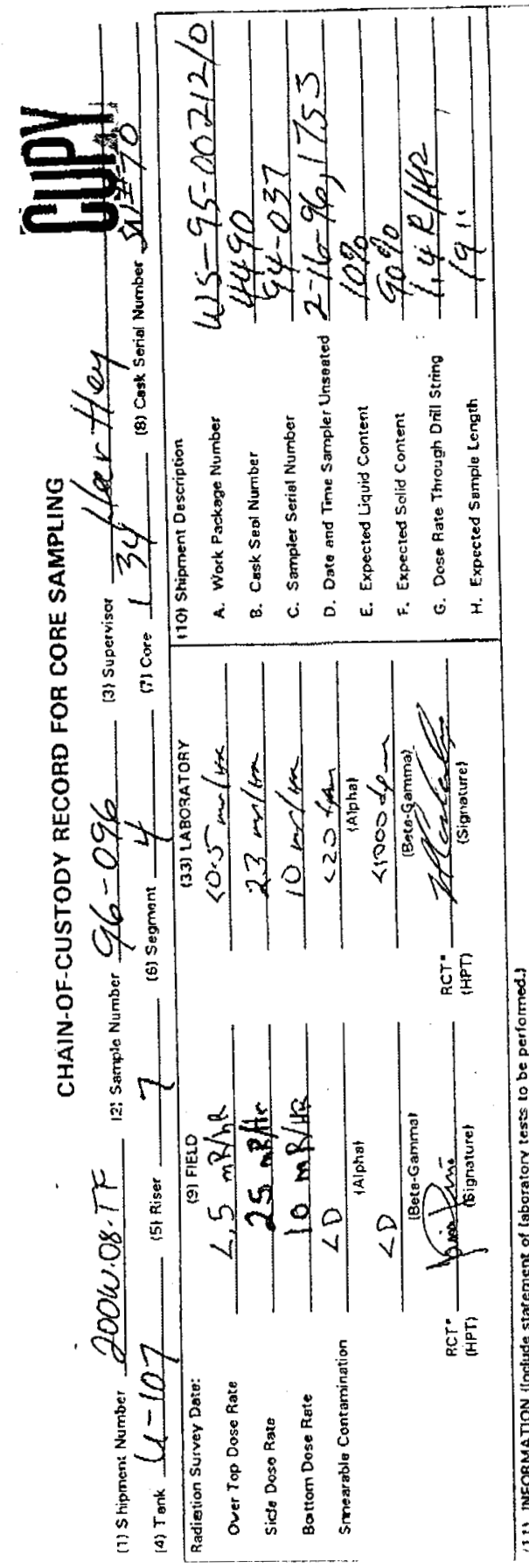

WHC-SD-WMM DP-184, REV.

要

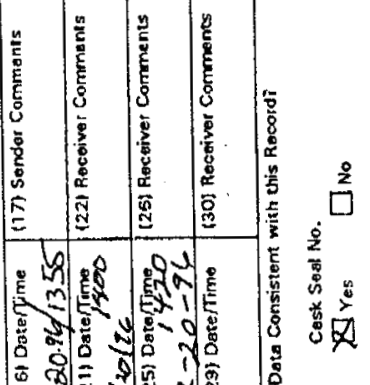

8

-

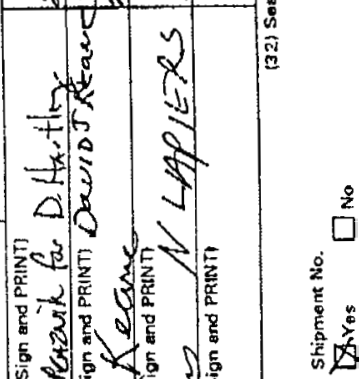

in का

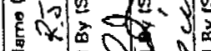

票 $\mathrm{P}$

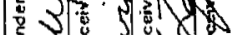

蒿

5)

(6)

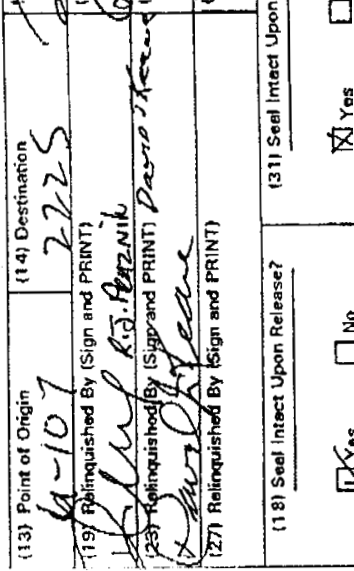




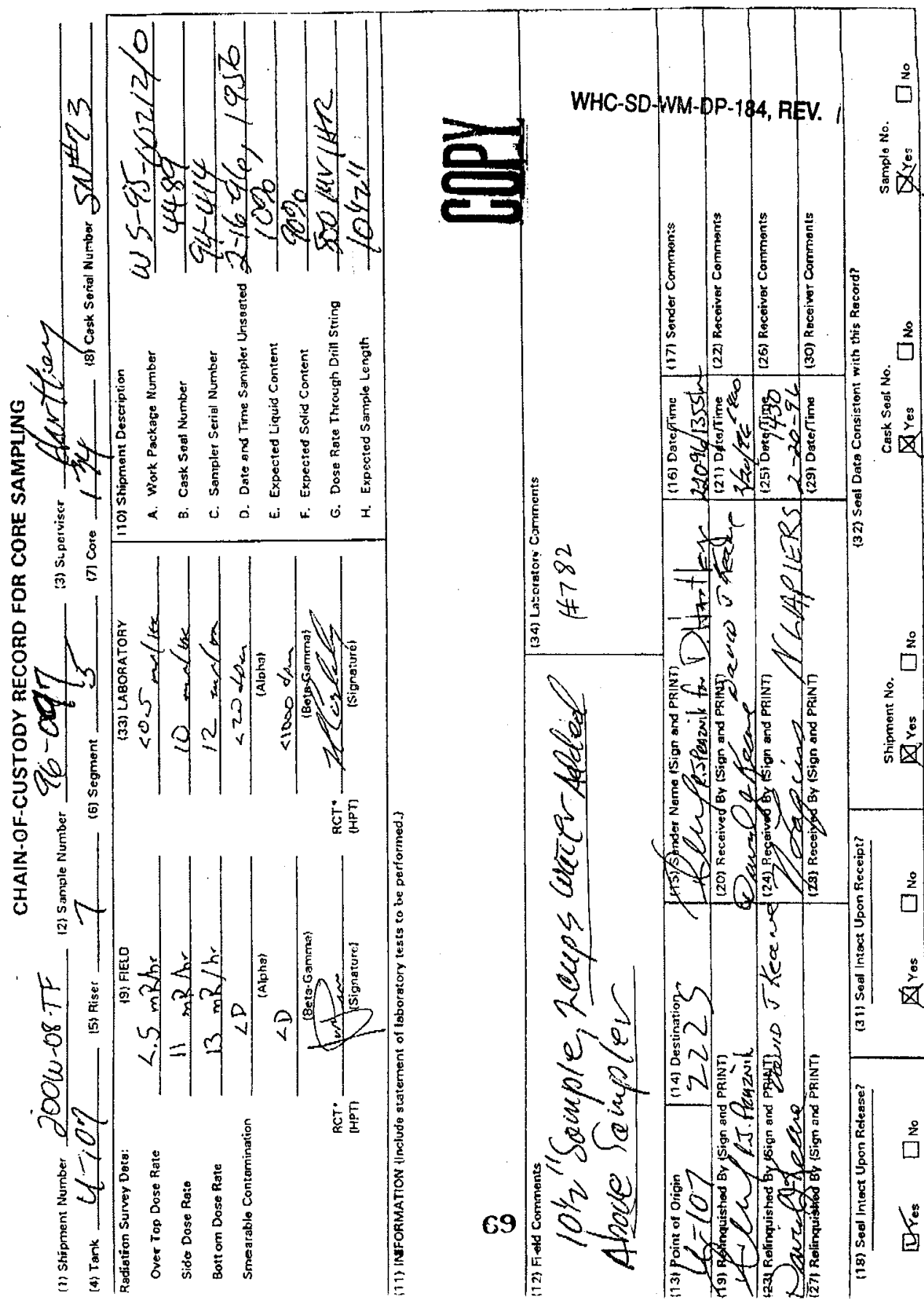




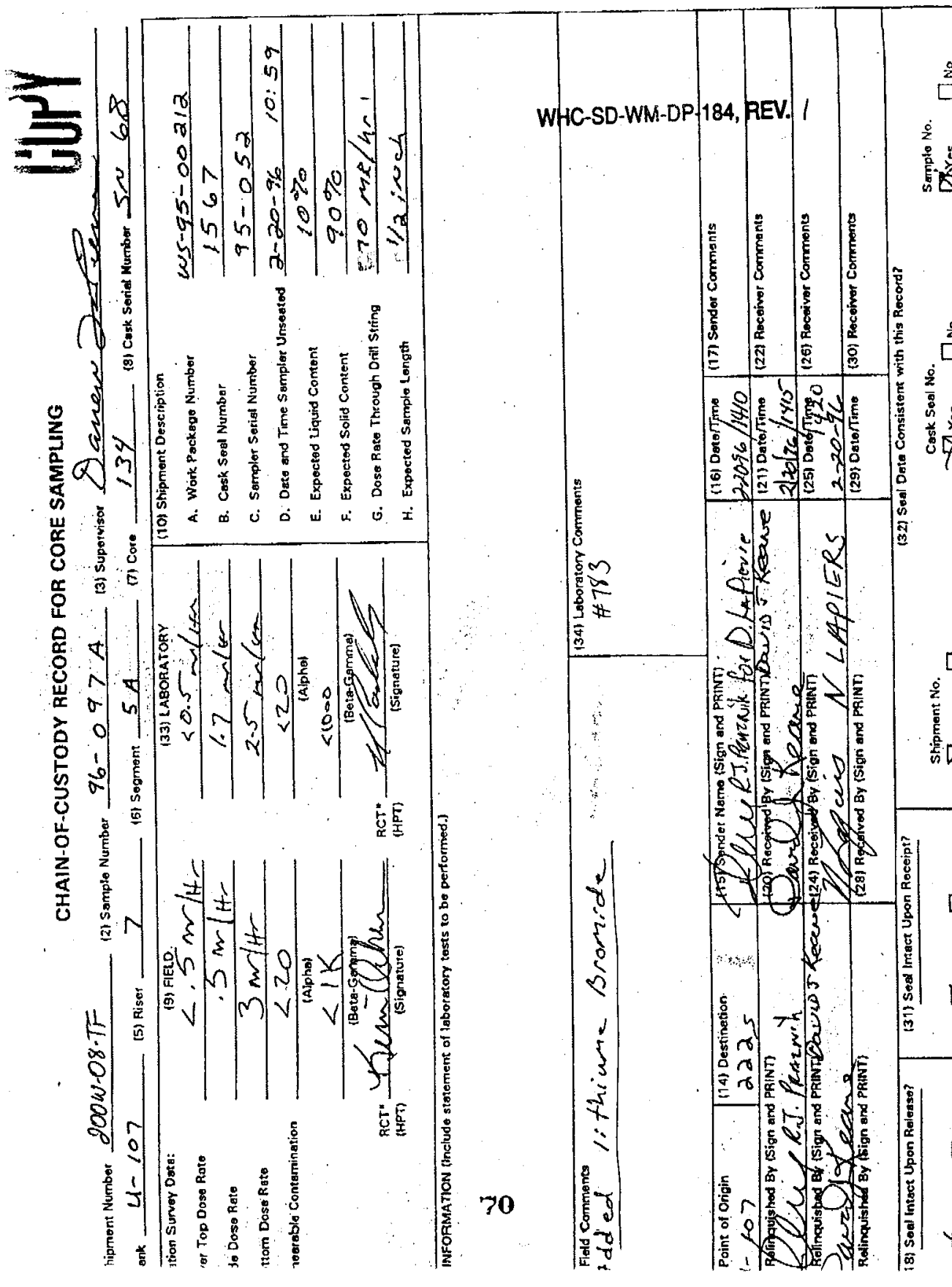




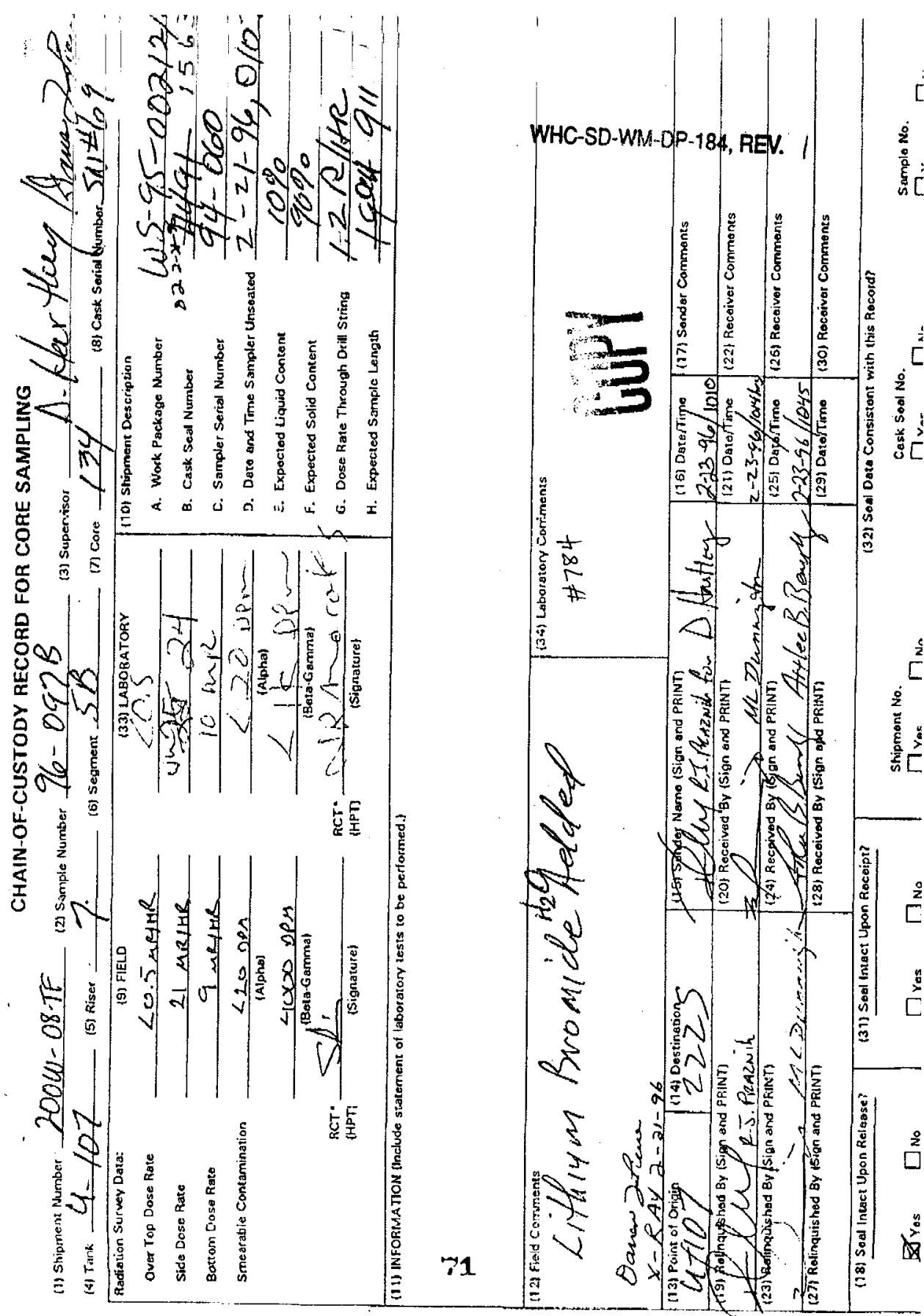




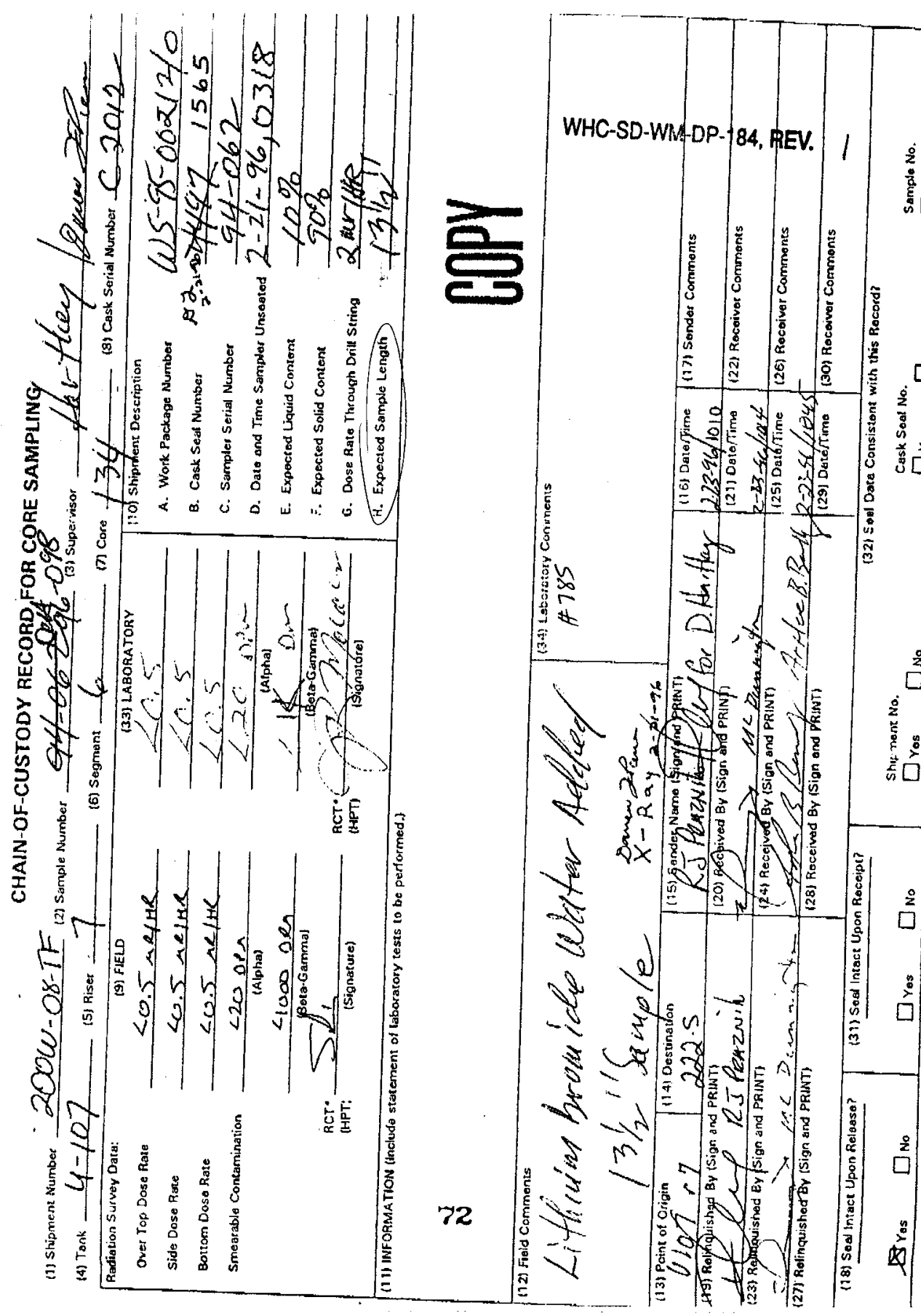




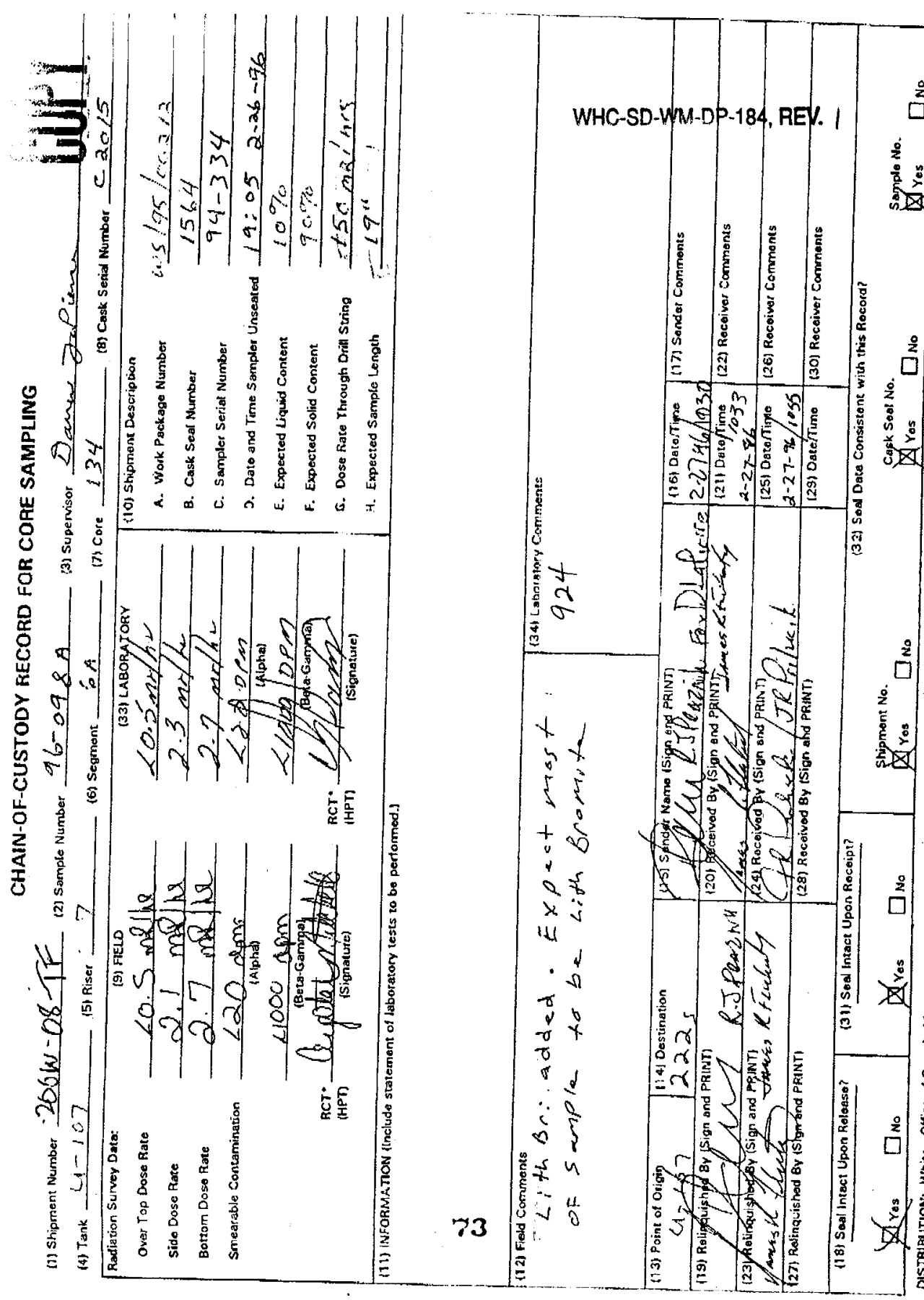




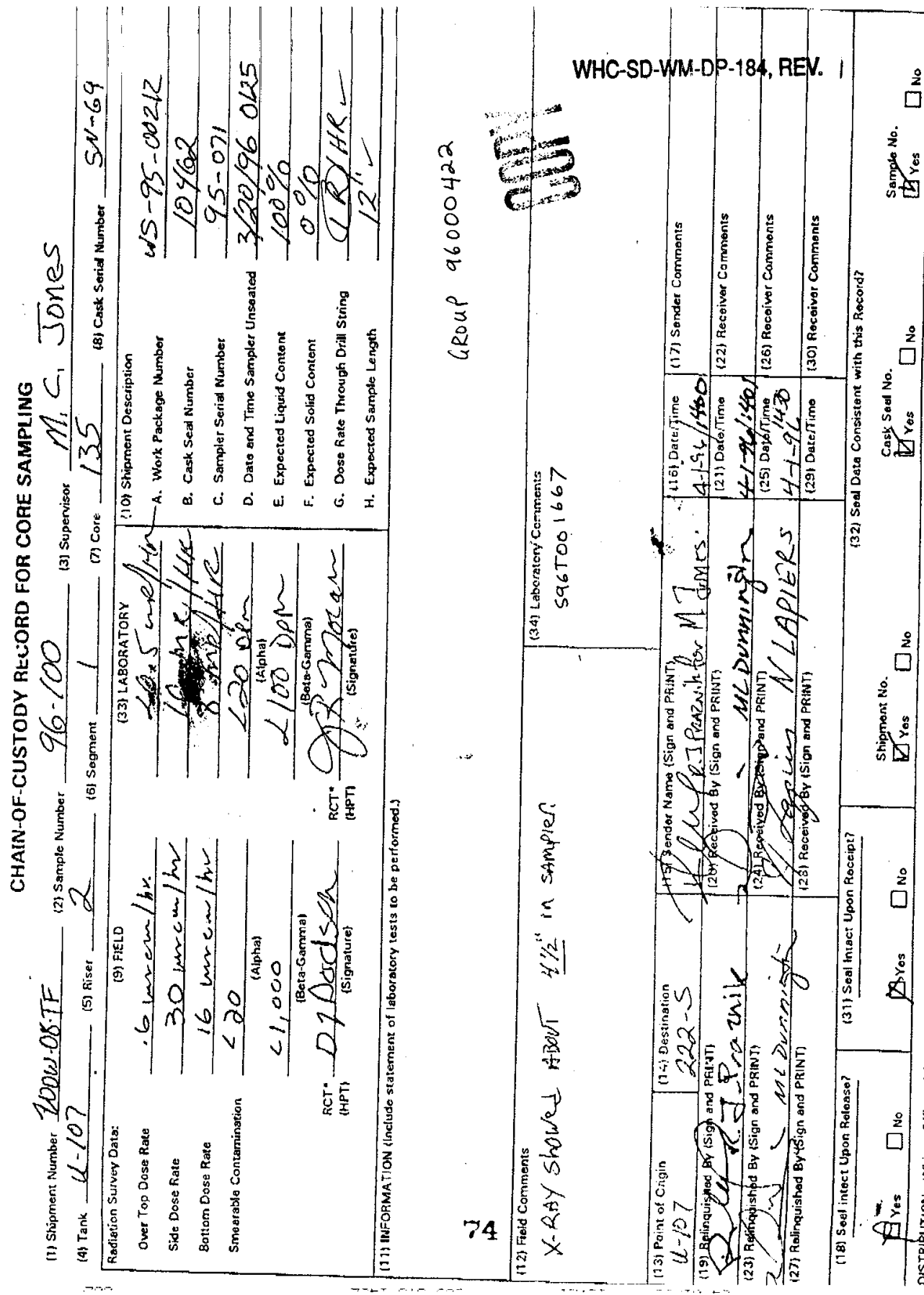




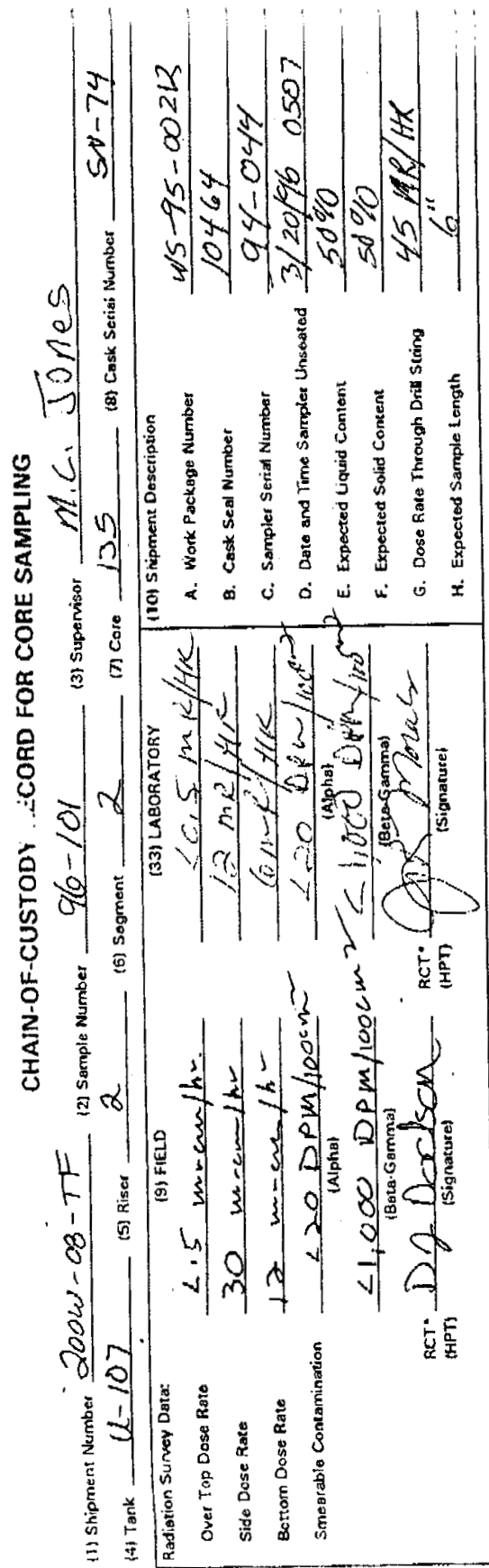




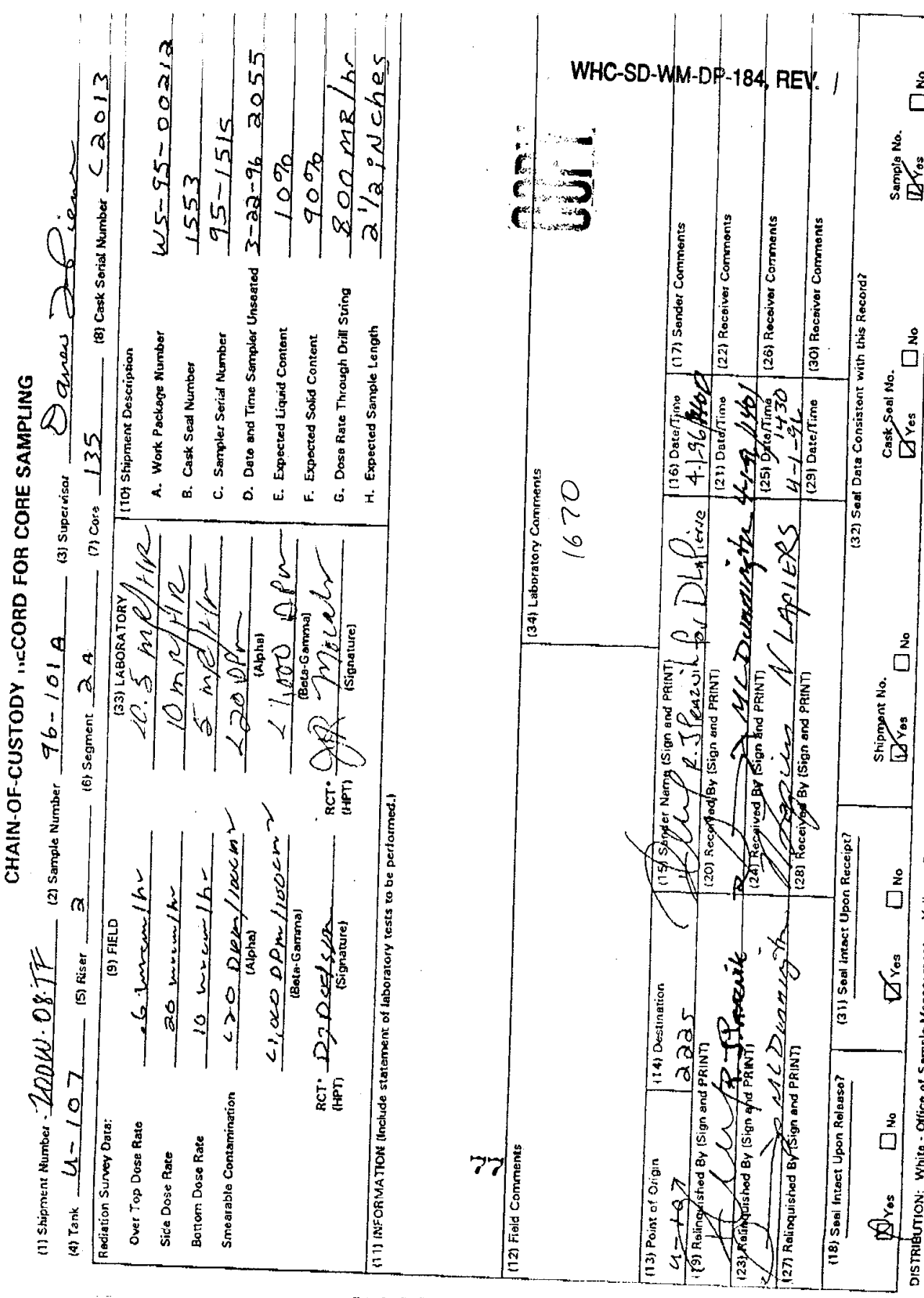




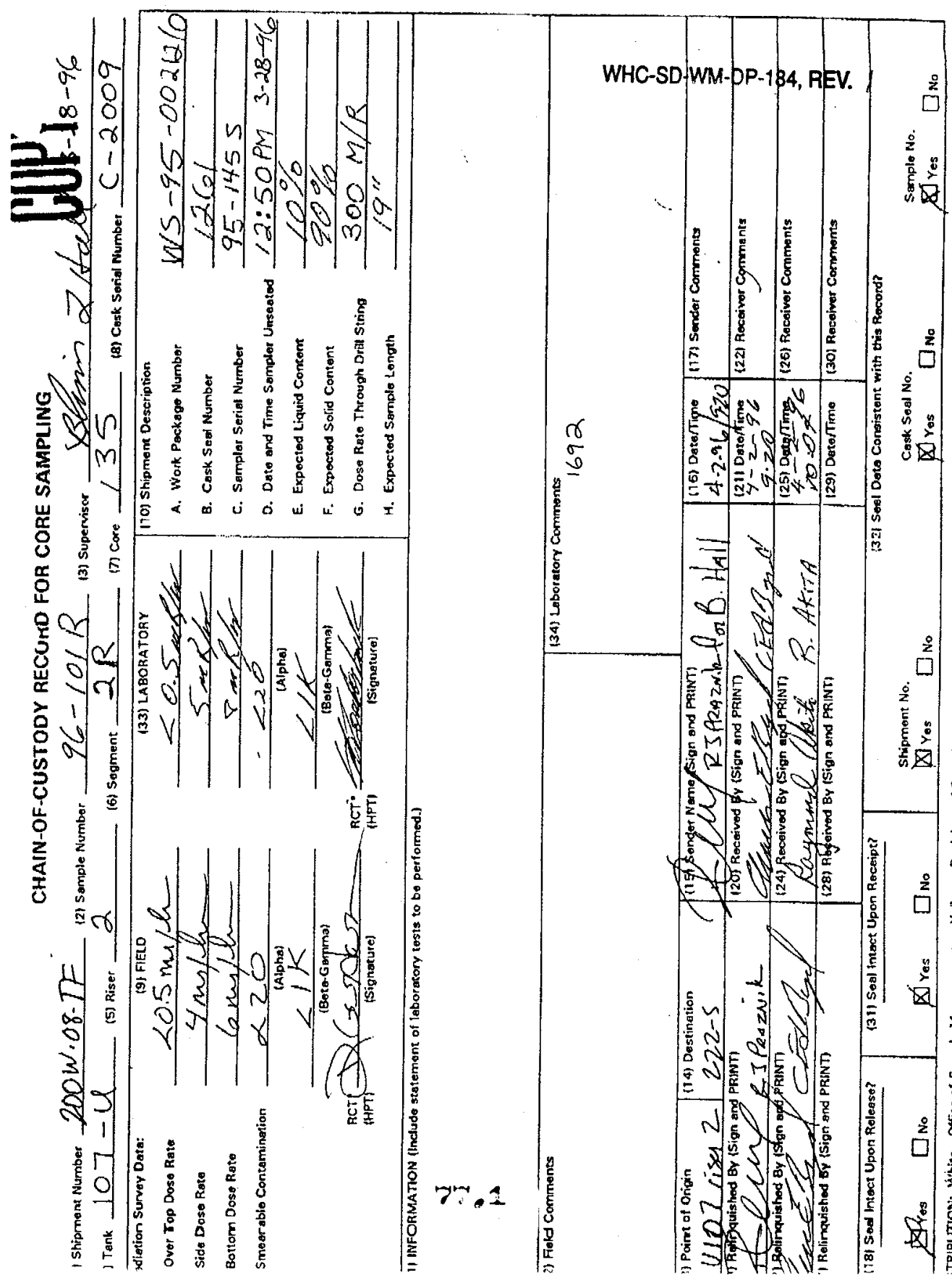


WHC-SD-WM-DP-184, REV. 1

PHOTOGRAPHS 
WHC-SD-WW-DP-184, REV. 1

THIS PAGE WAS INTENTIONALLY LEFT BLANK

79 
WHC-SD-WM-DP-184, REV.।

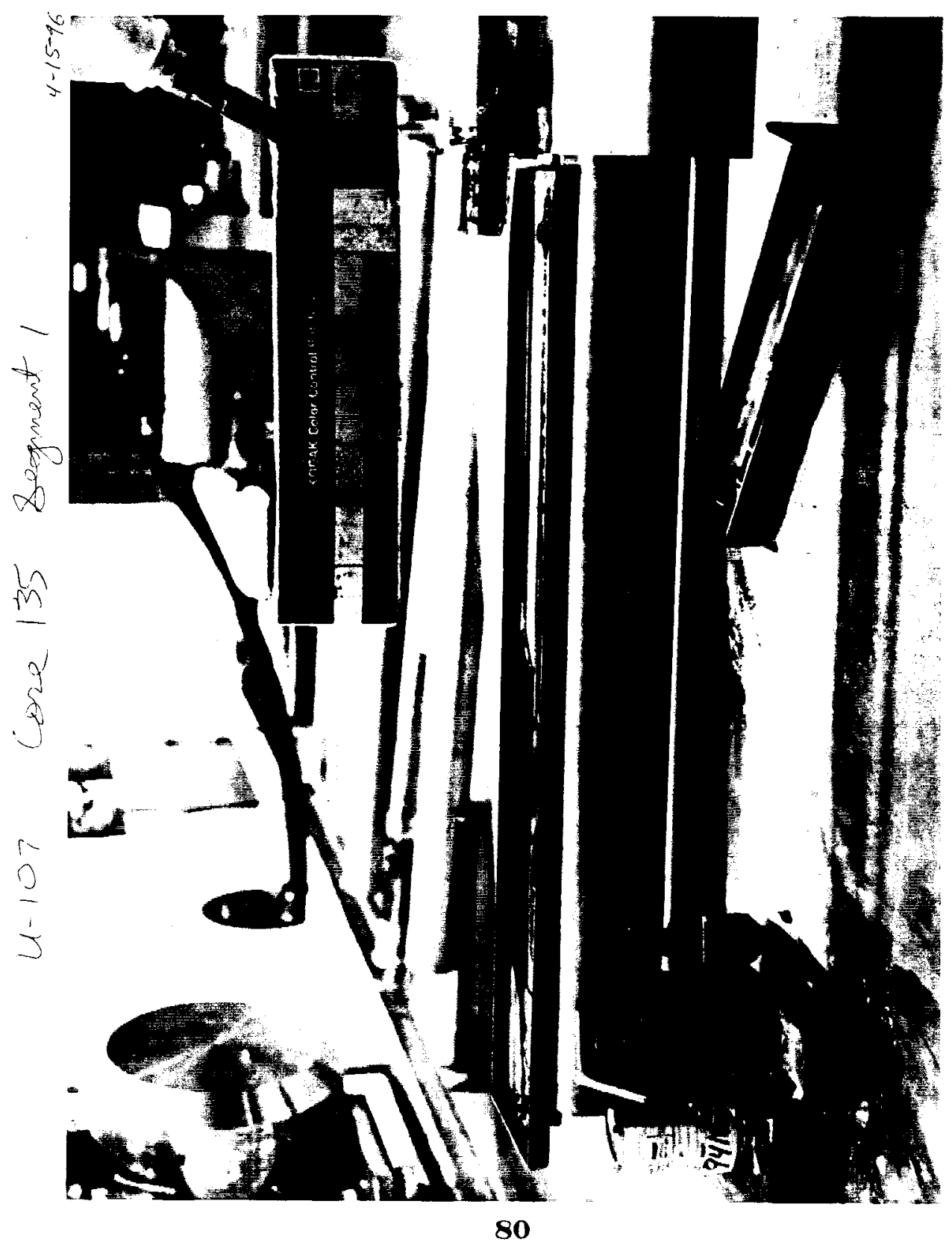


WHC-SD-WM-DP-184, REV.

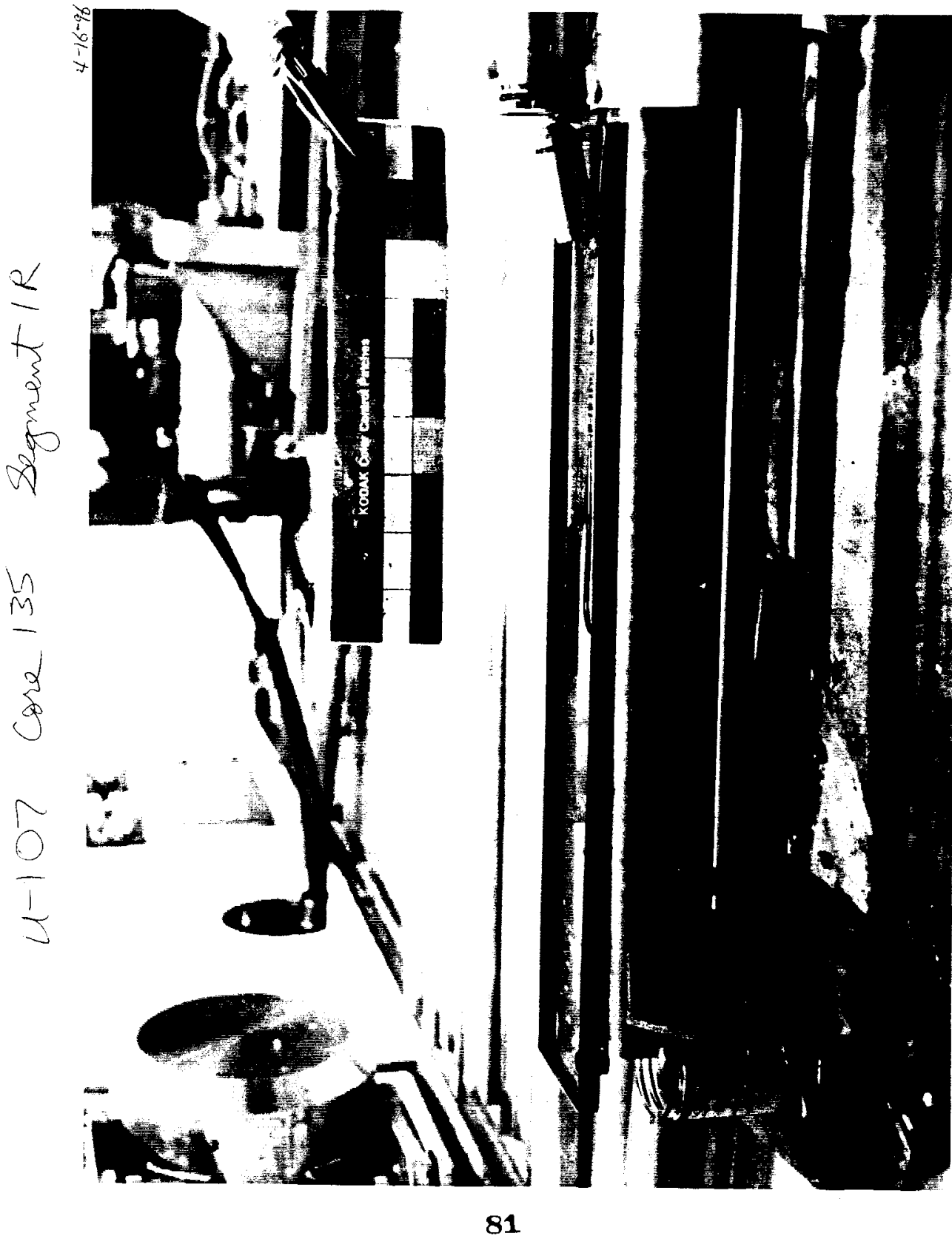


WHC-SD-WM-DP-184, REV.

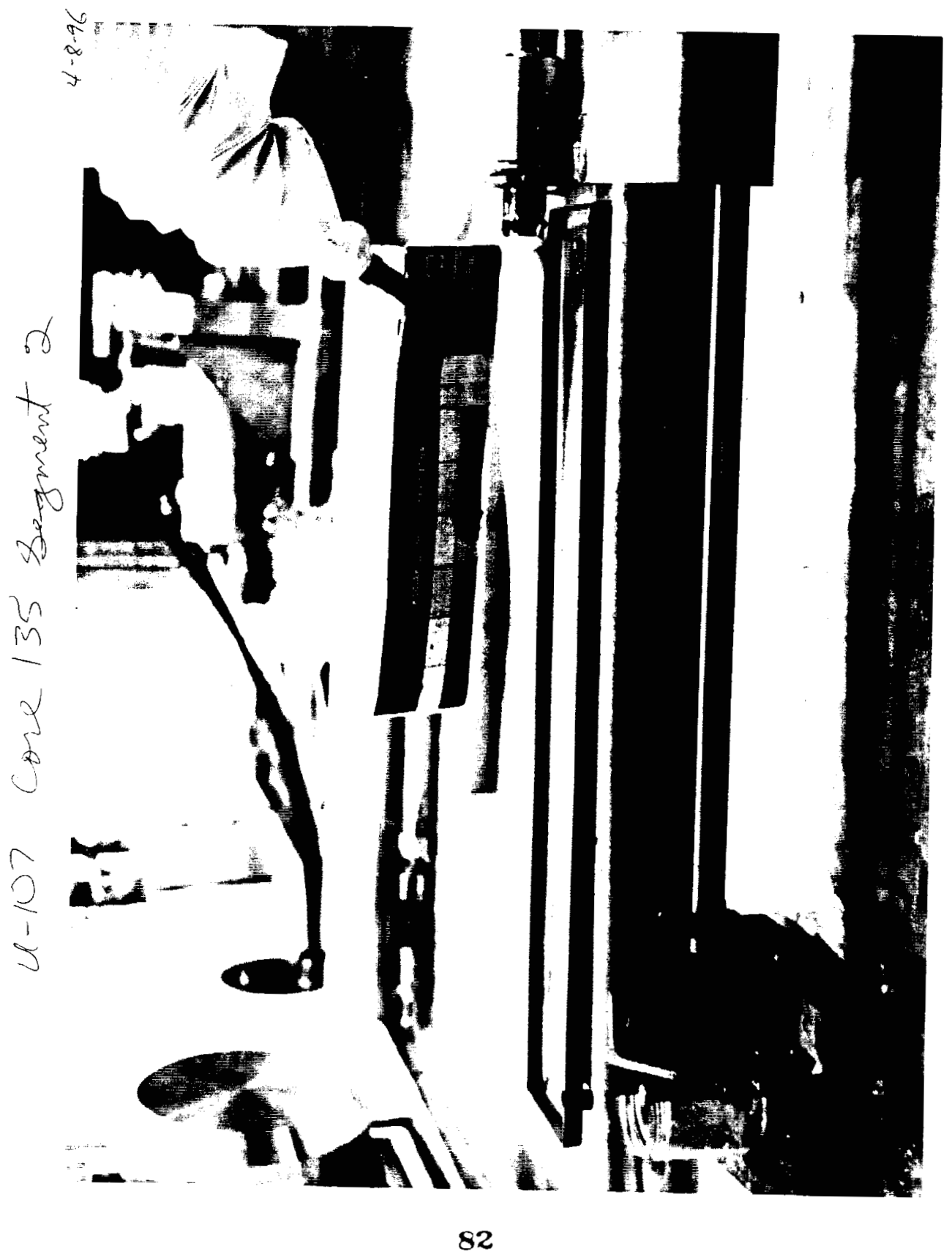


WHC-SD-WM-DP-184, REV.

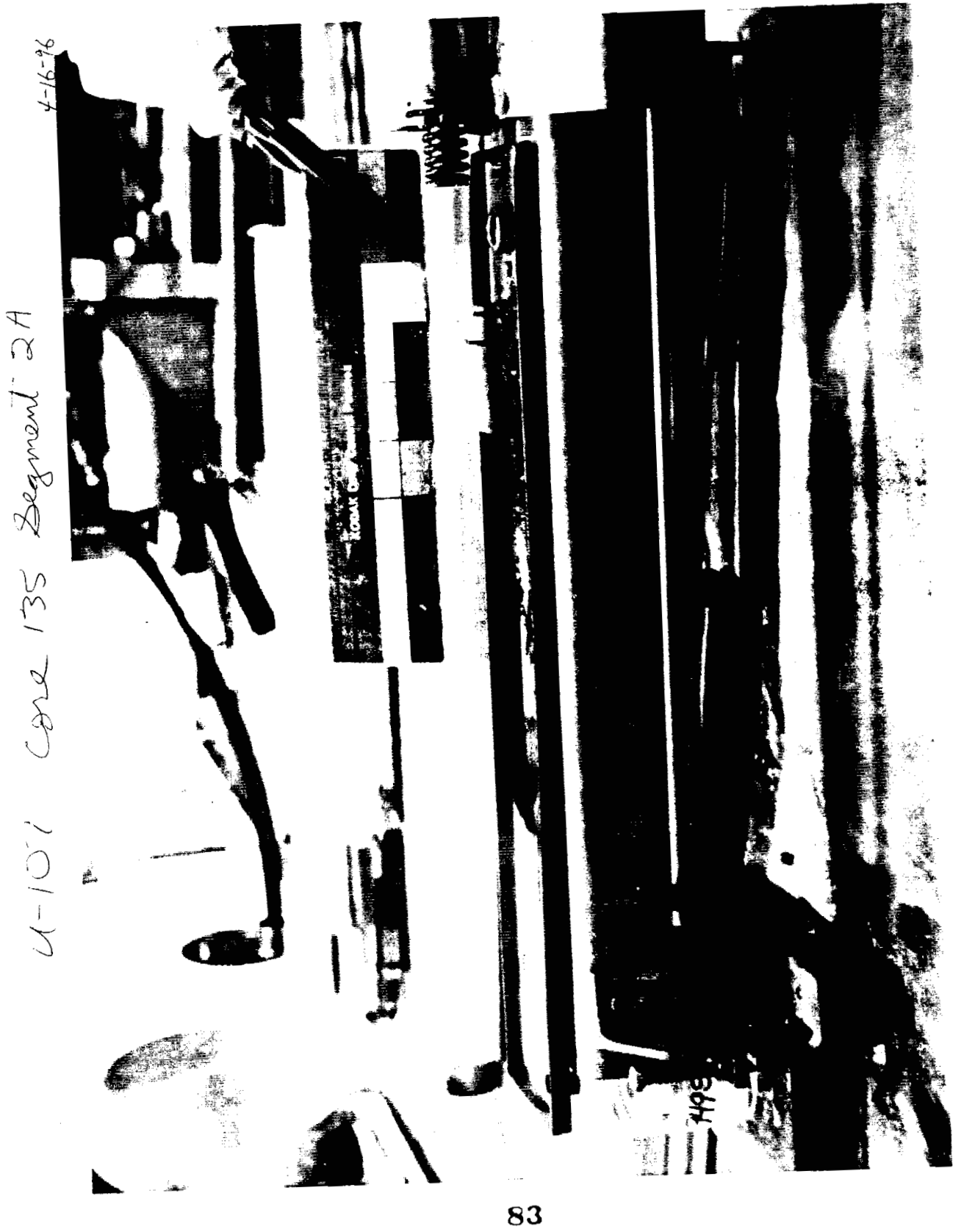


WHC-SD-WM-DP-184, REV.

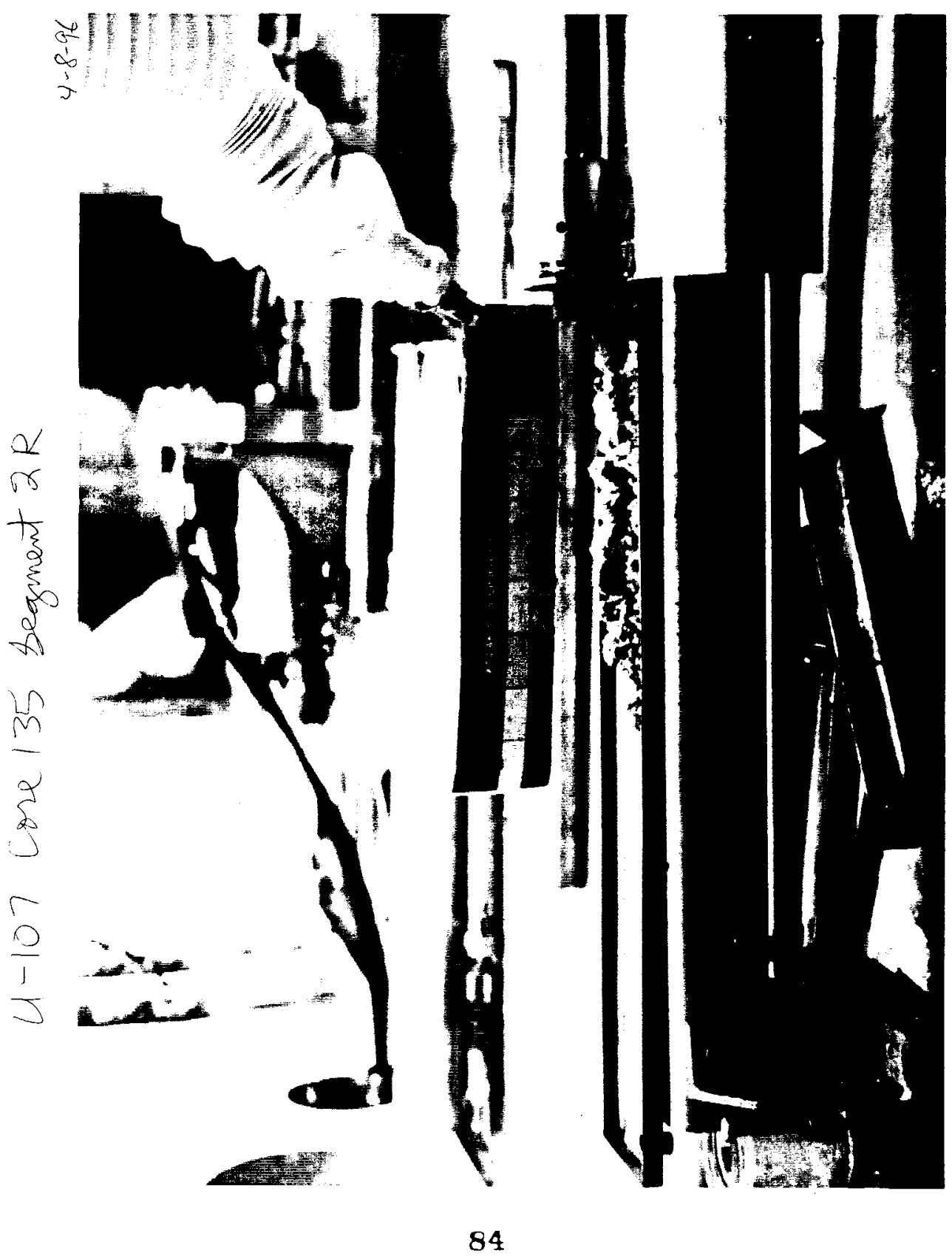


WHC-SD-WM-DP-184, REV.

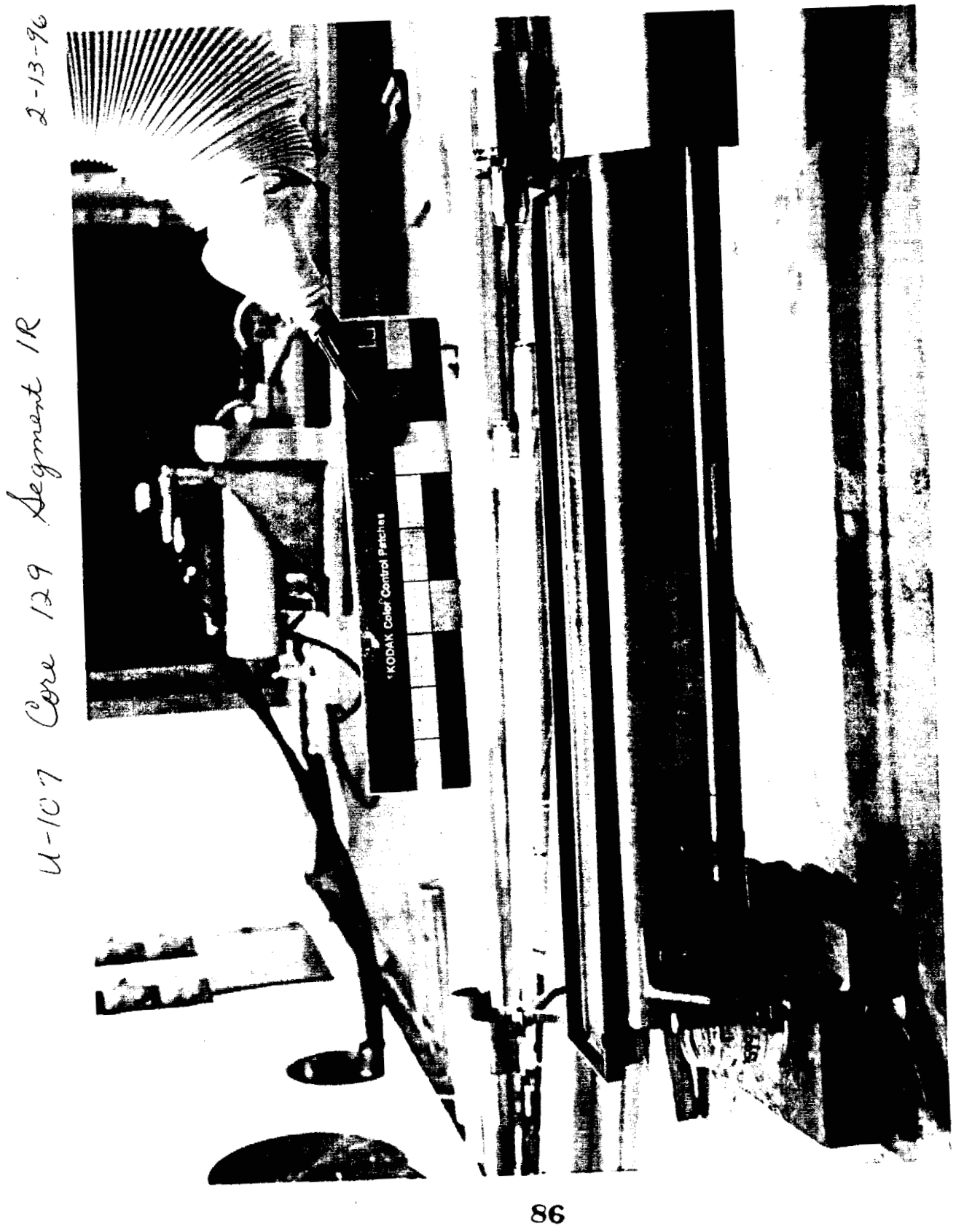


WHC-SD-WM-DP-184, REV. I

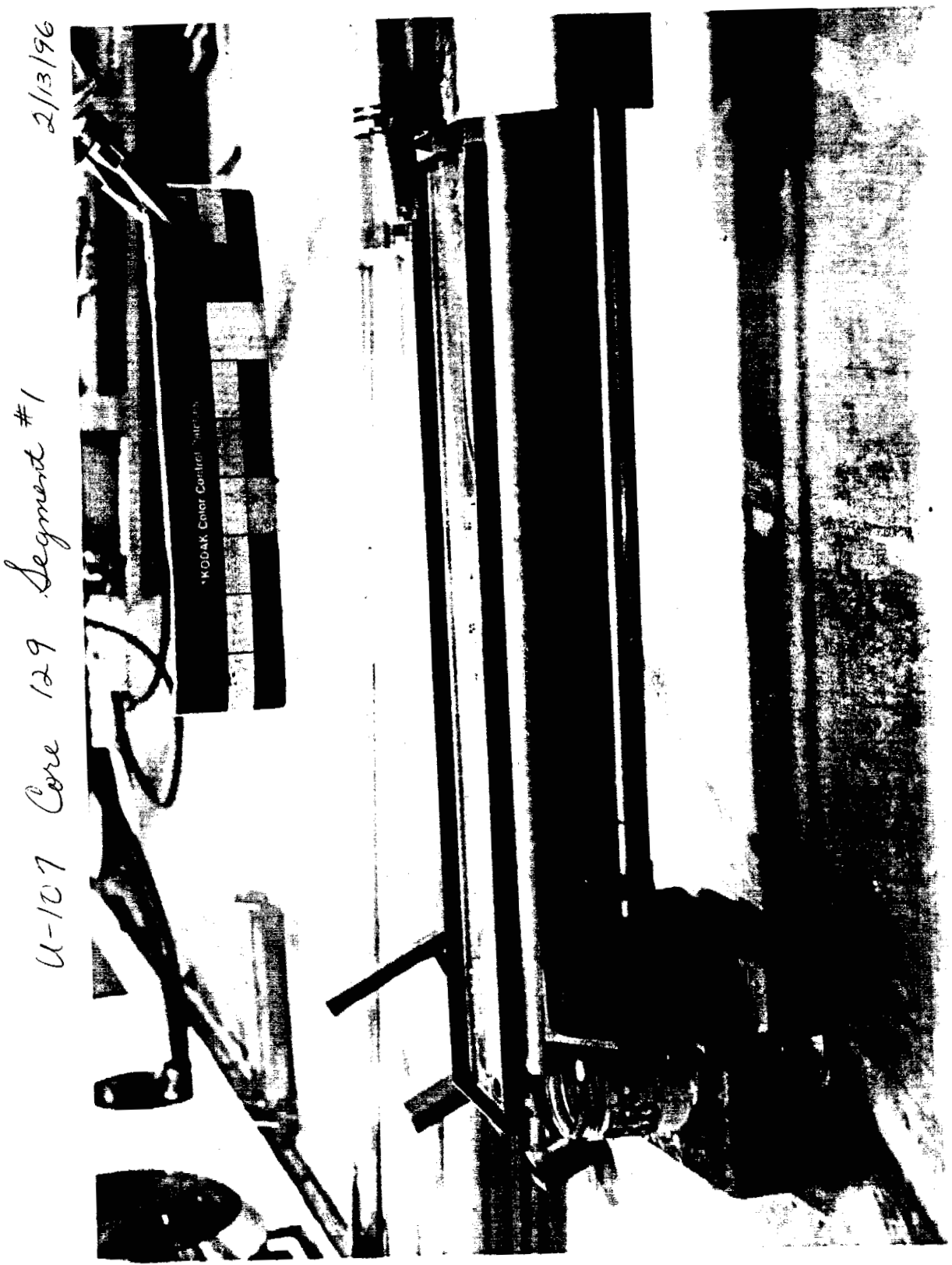




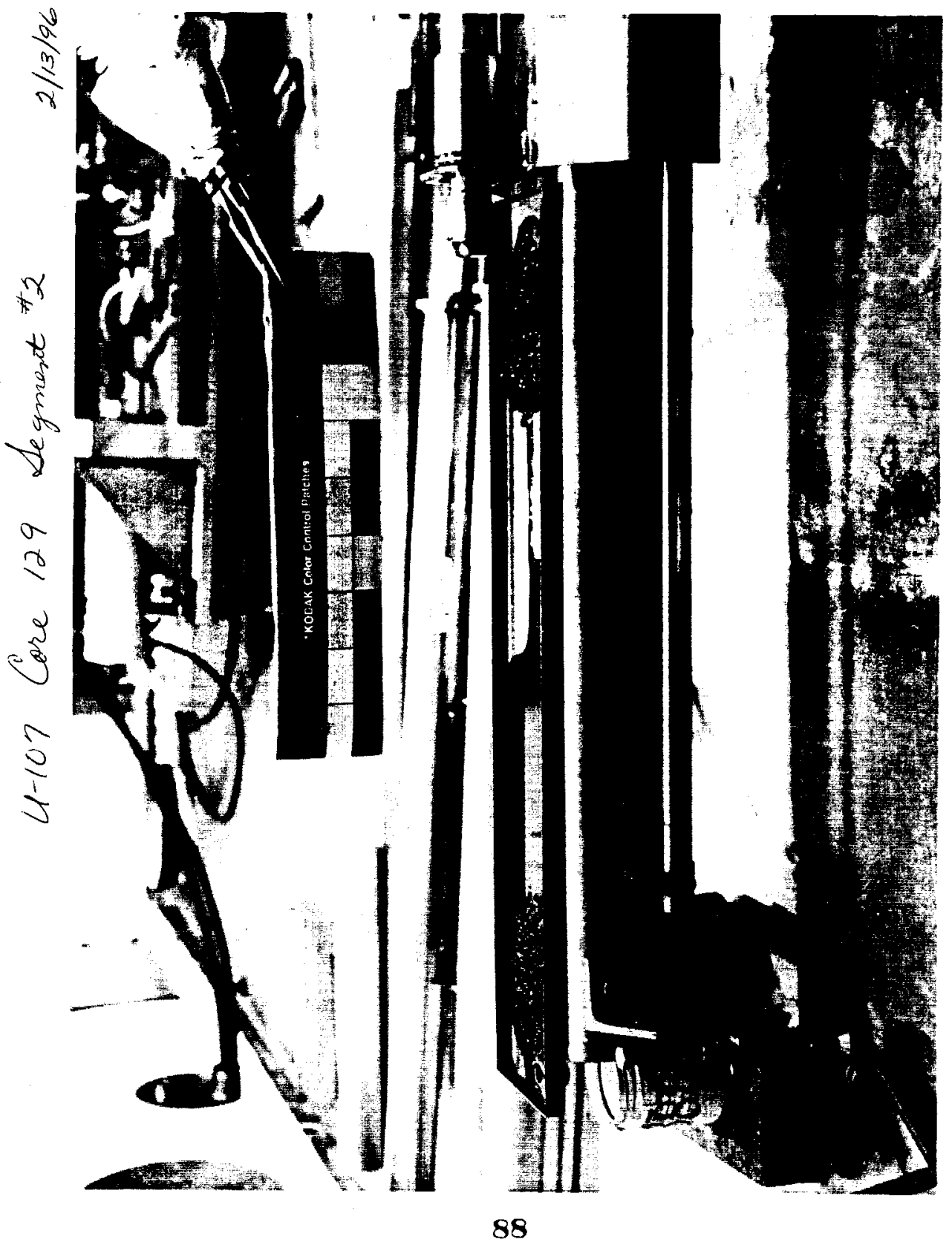


WHC-SD-WM-DP-184, REV.

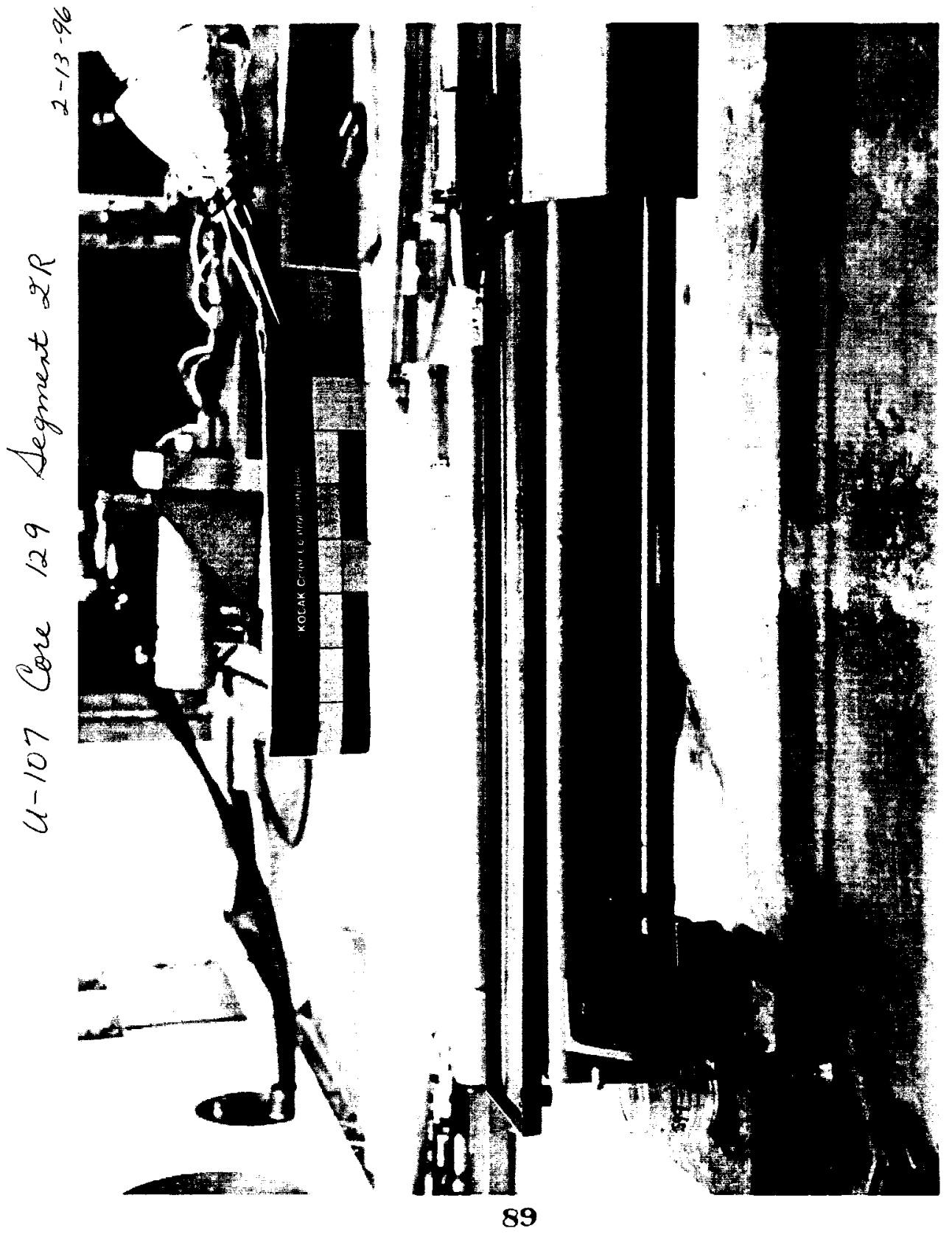


WHC-SD-WM-DP-184, REV.

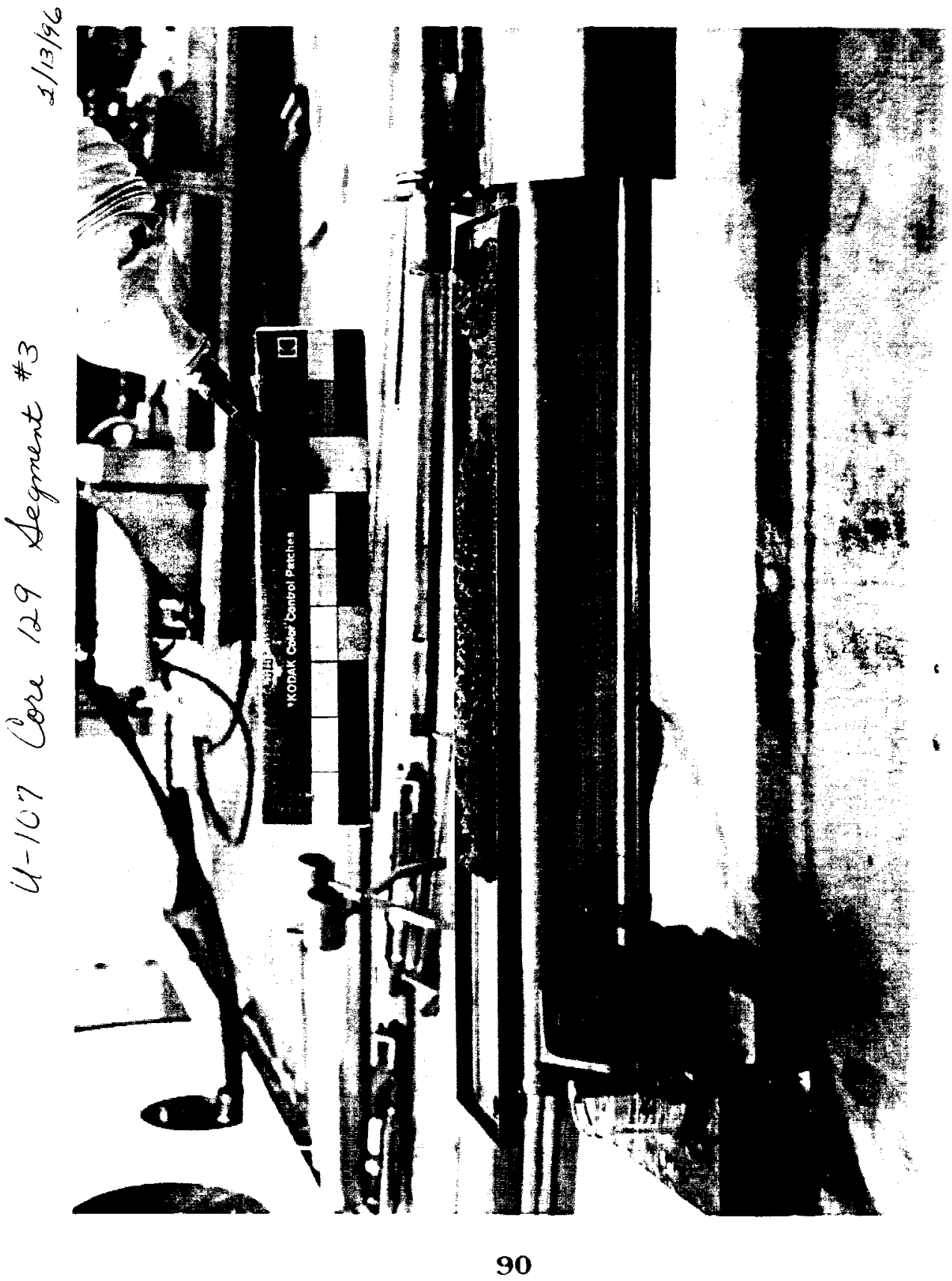


WHC-SD-WM-DP-184, REV.

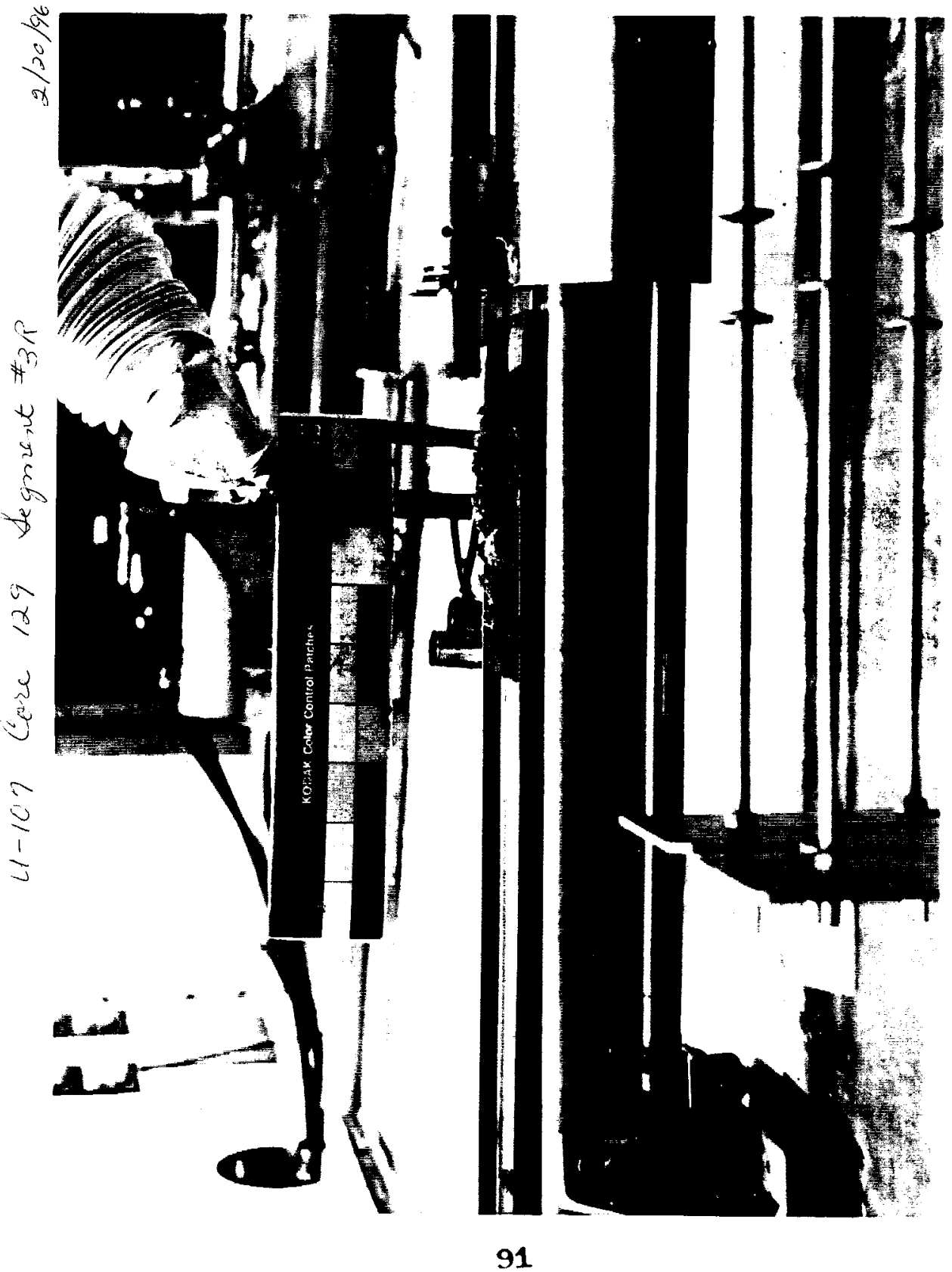


WHC-SD-WM-DP-184, REV.

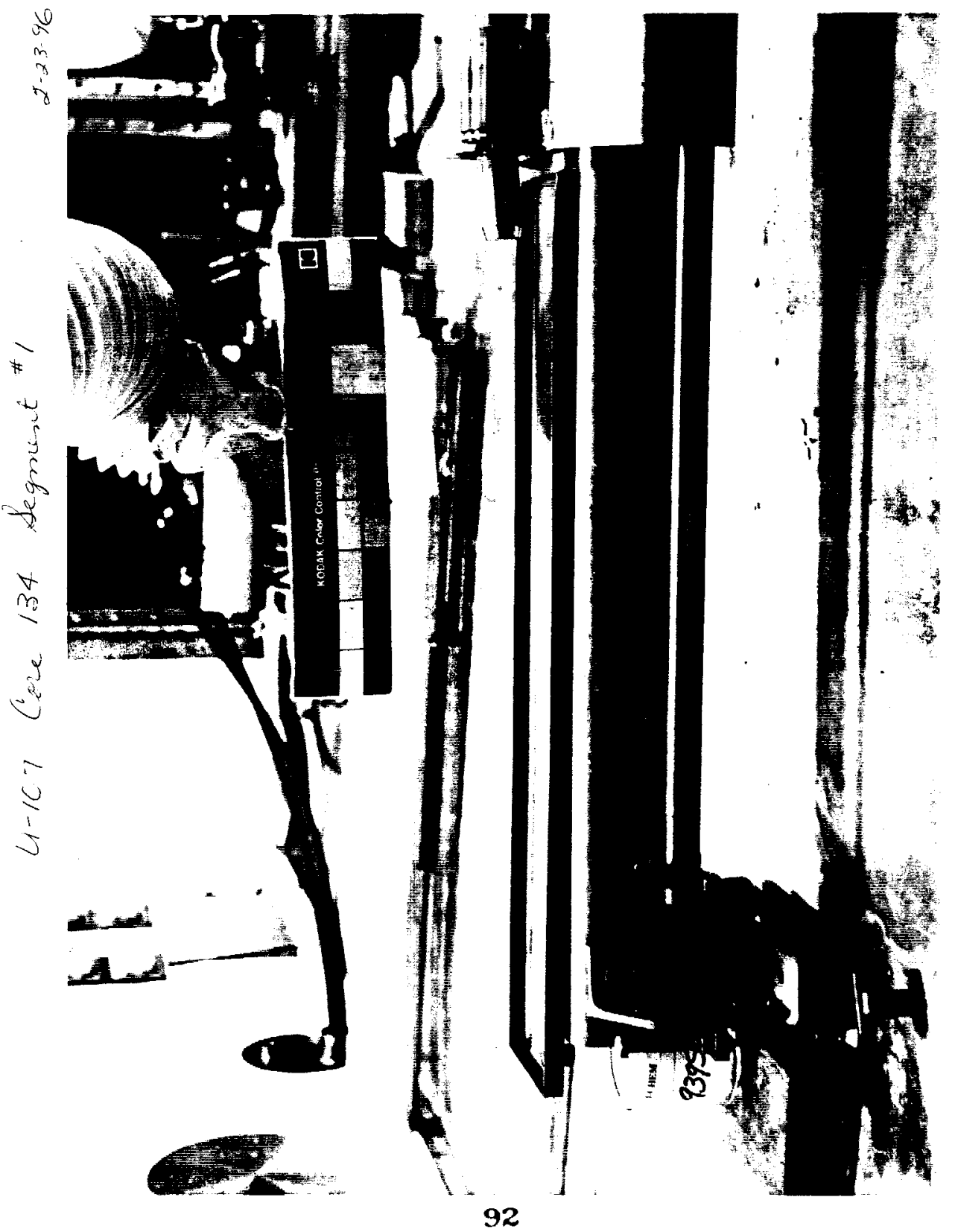




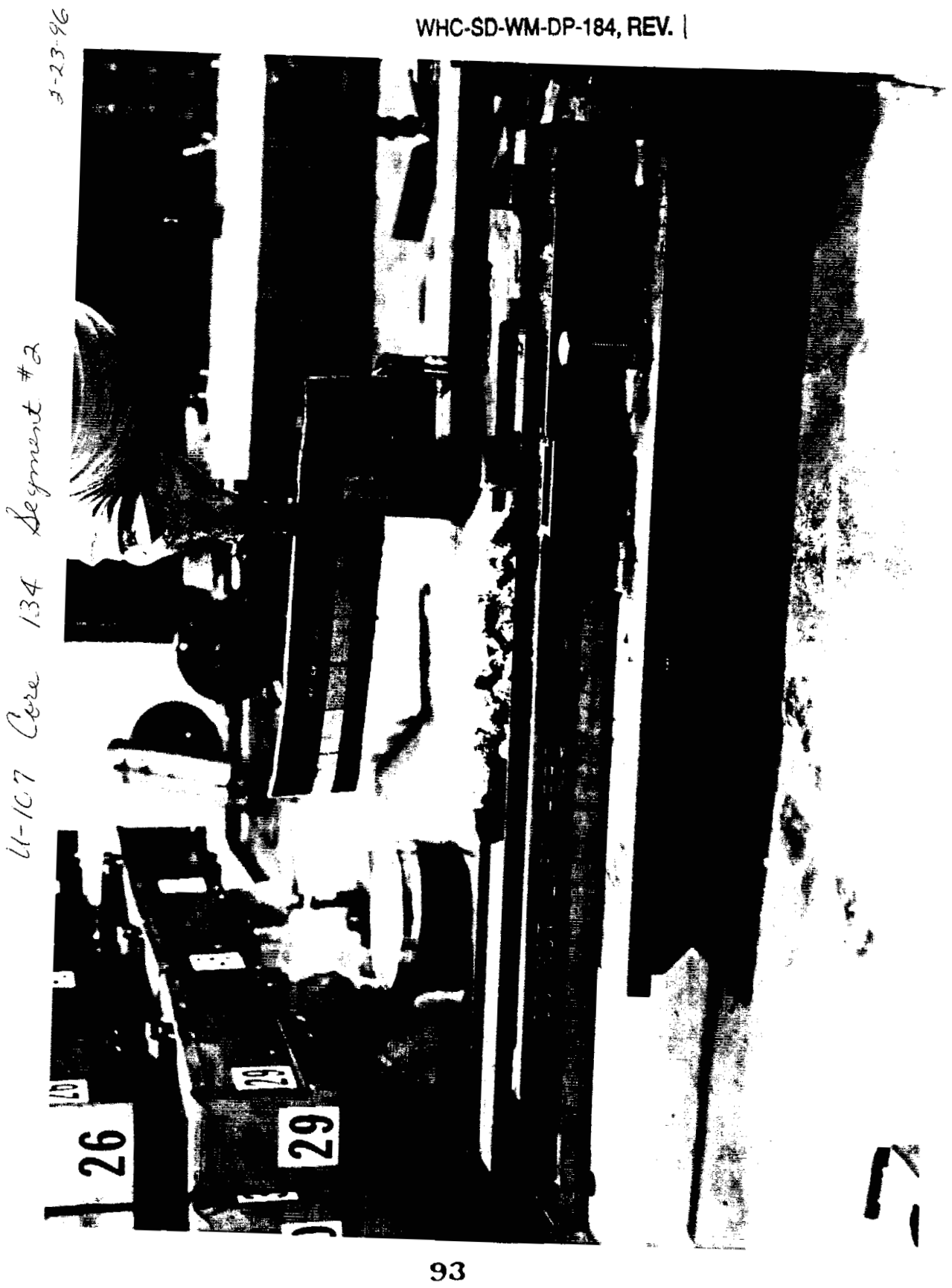




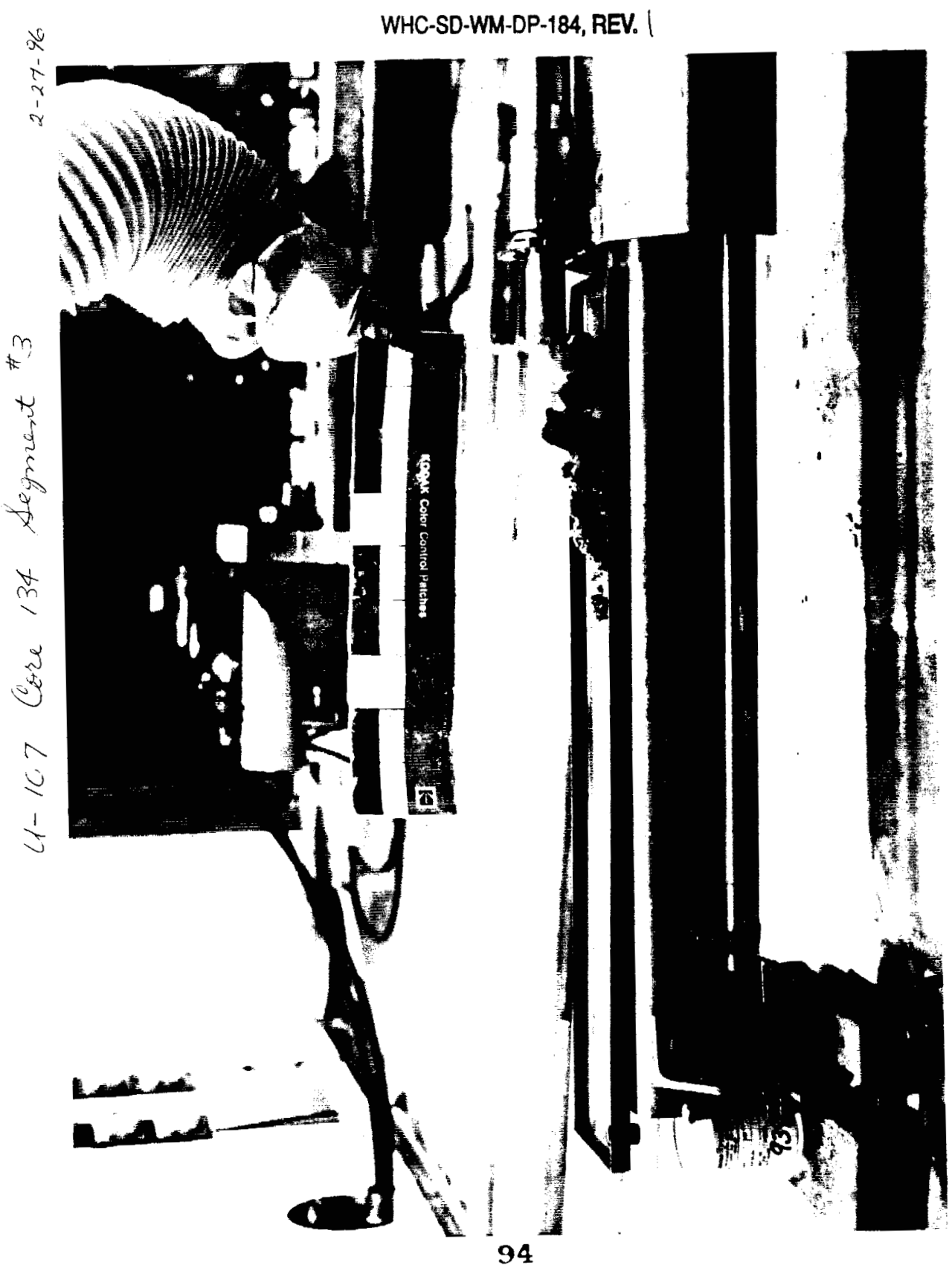


WHC-SD-WM-DP-184, REV.

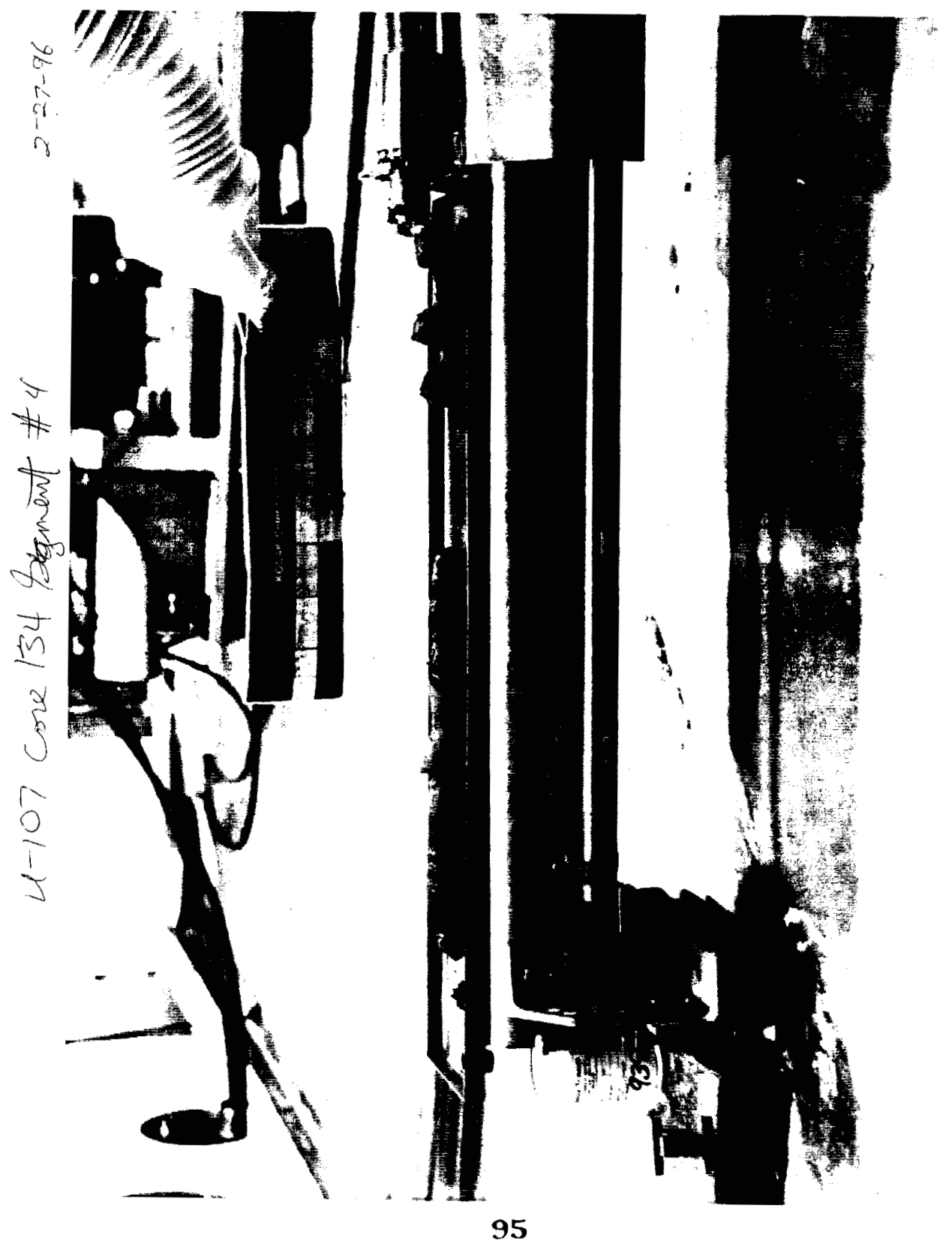


WHG-SD-WM-DP-184, REV.

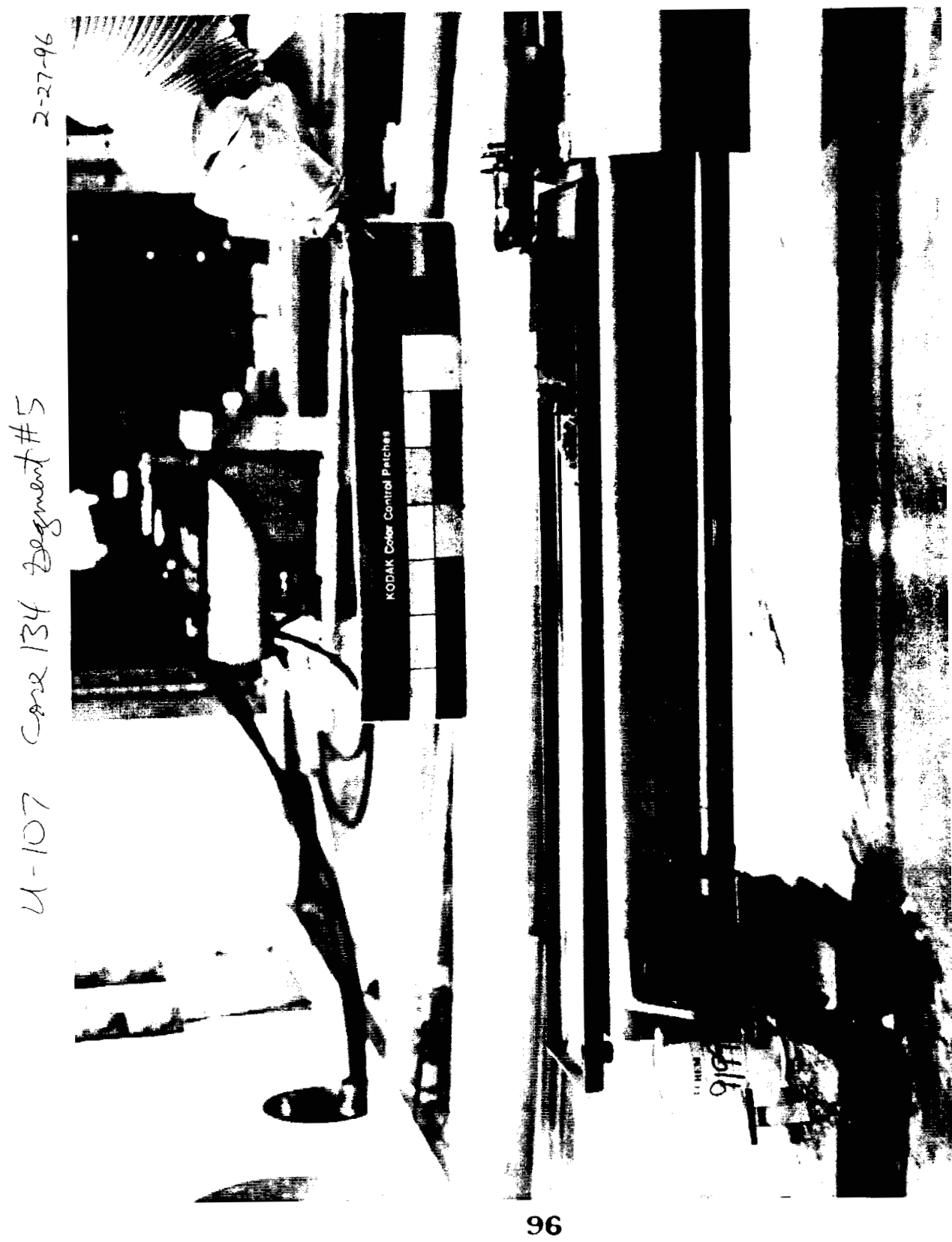


WHC-SD-WM-DP-184, REV. !

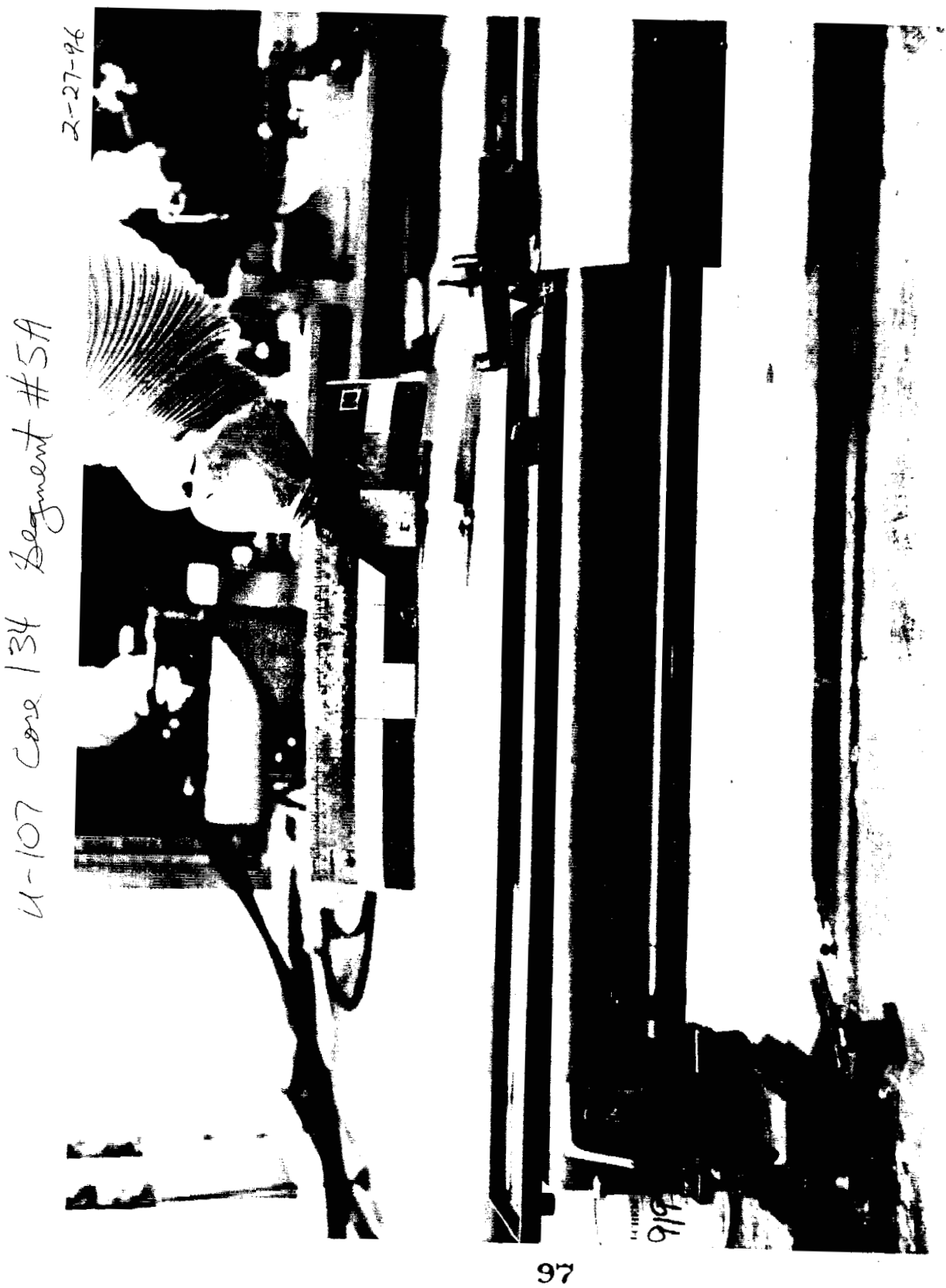


WHC-SD-WM-DP-184, REV. !

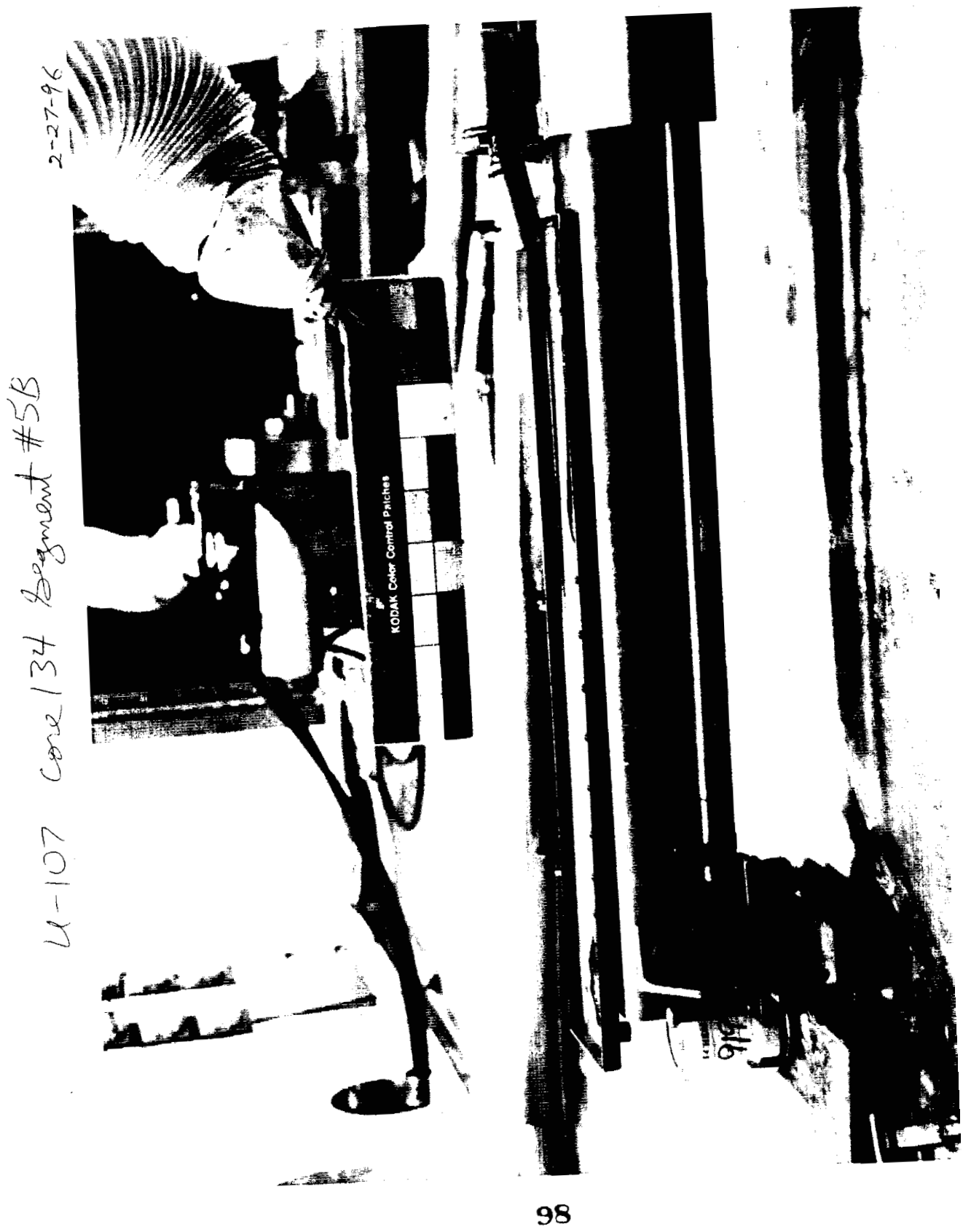


WHC-SD-WM-QP-184, REV. I

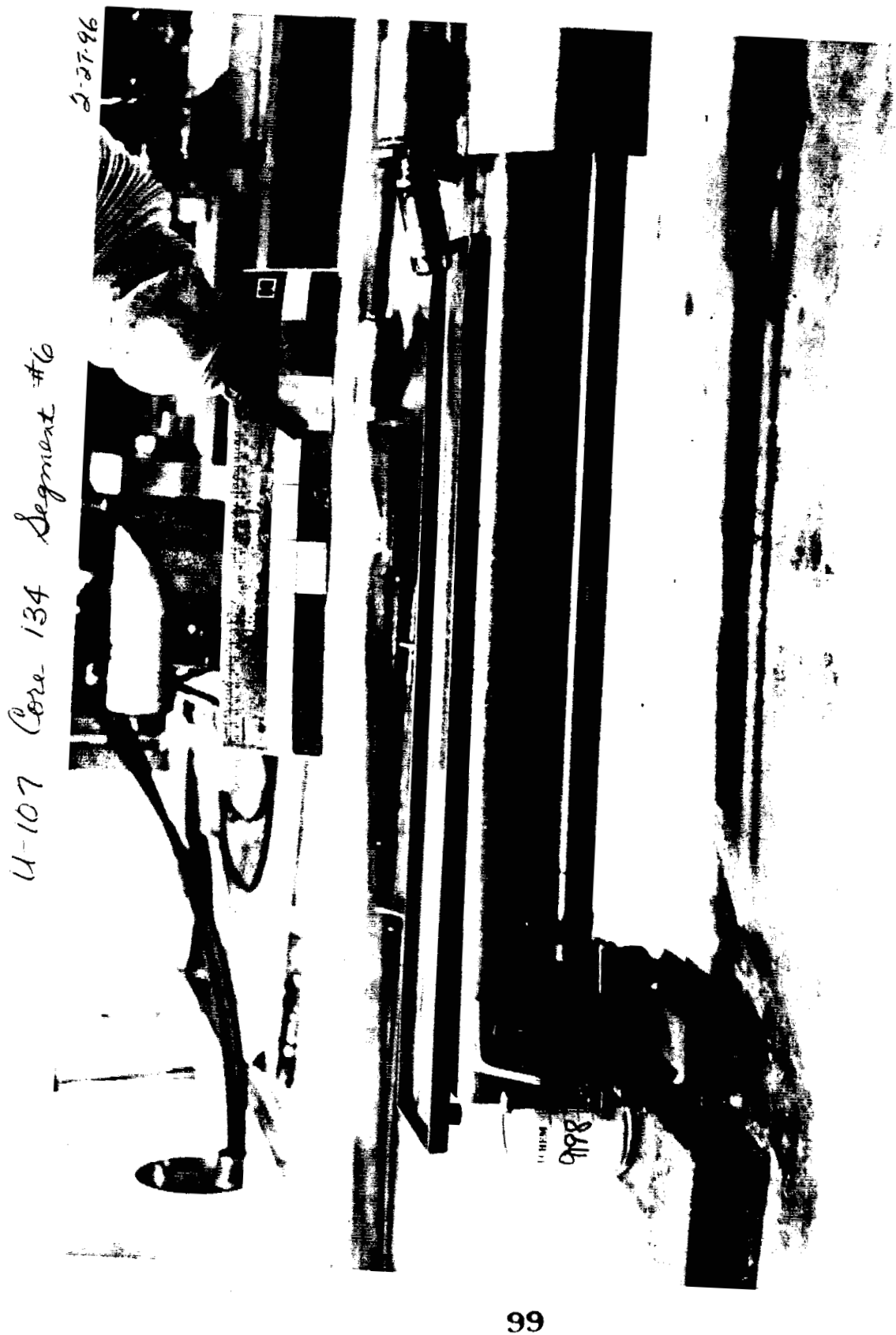


WHC-SD-WM-DP-184, REV. I
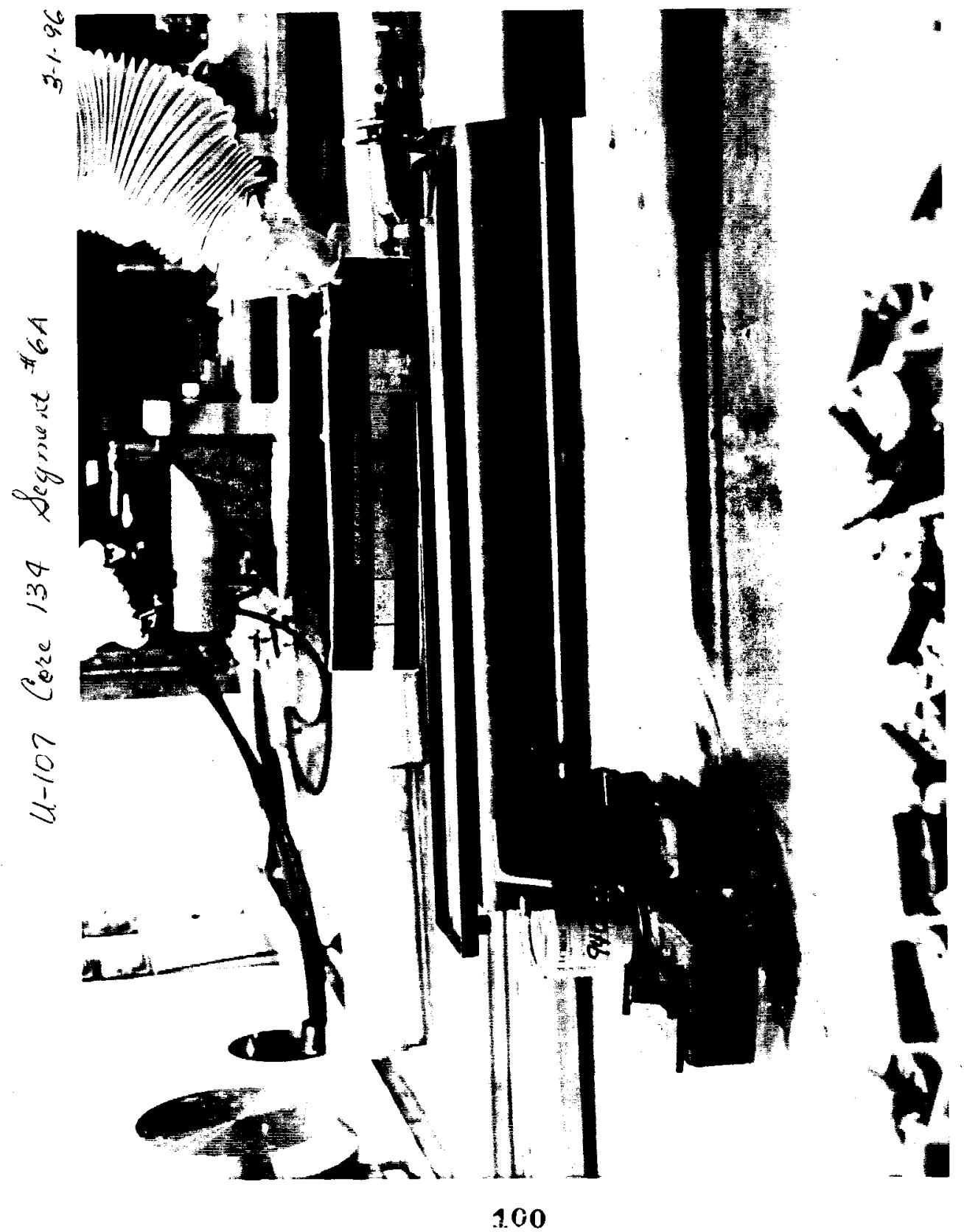
WHC-SD-WM-DP-184, REV. 1

\section{SAMPLE HANDLING}


WHC-SD-WM-DP-184, REV. 1

THIS PAGE WAS INTENTIONALLY LEFT BLANK 


\section{LABCORE Data Entry Template for Worklist\#}

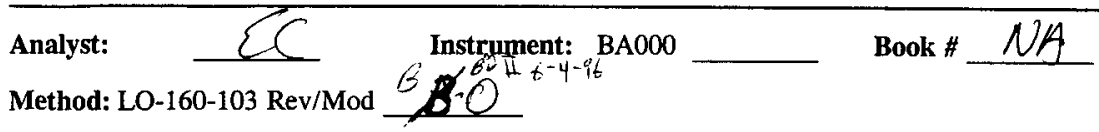

\section{Worklist Comment: U-107 C129 SEG \#1 RISER 9 EXTRUSION}

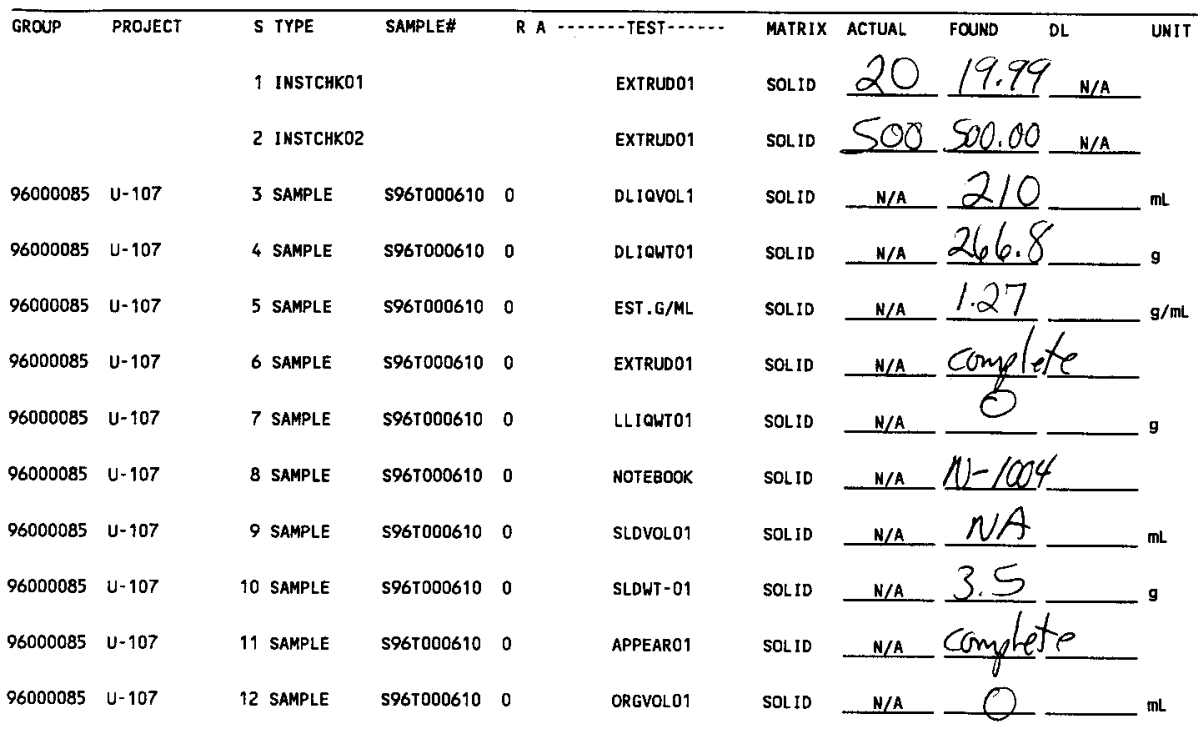

\section{Final page for worklist \# $\quad \mathbf{5 6 5 2}$}

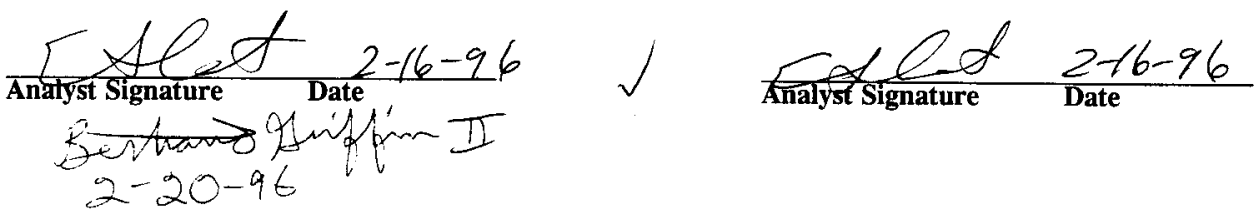

Data Entry Comments:

Units shown for $Q C$ (SPK \& STD) may not reflect the actual units. $D L=$ Detection Limit, $S=$ Worklist Slot Number, $R=$ Replicate Number, $A=$ Aliquor Code. 
worklistrpt Version 2.1 05/15/95 WHC-SD-WM-DP-184, REV. I

\section{LABCORE Data Entry Template for Worklist\#}

\section{Analyst: \\ Instrument: BA000 \\ Book \# NA}

Method: LO-160-103 Rev/Mod

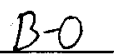

\section{Worklist Comment: U-107 C129 SEG \#2 RISER 9 EXTRUSION}

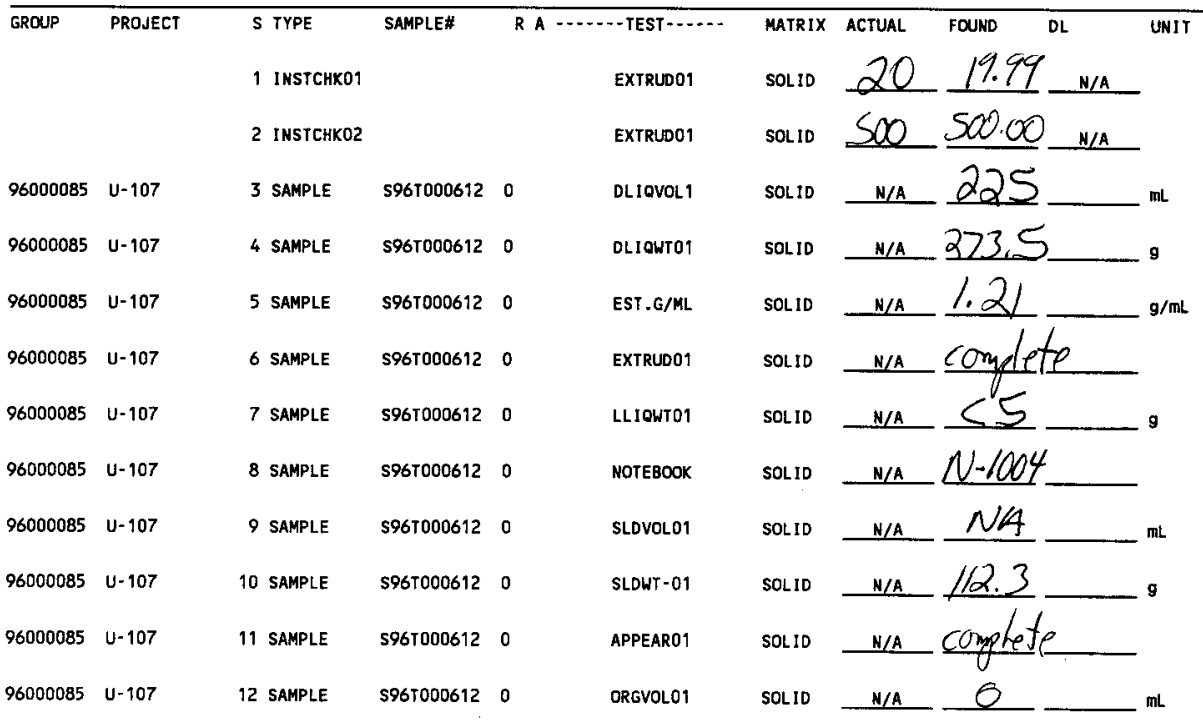

\section{Final page for worklist \# $\quad 5653$}

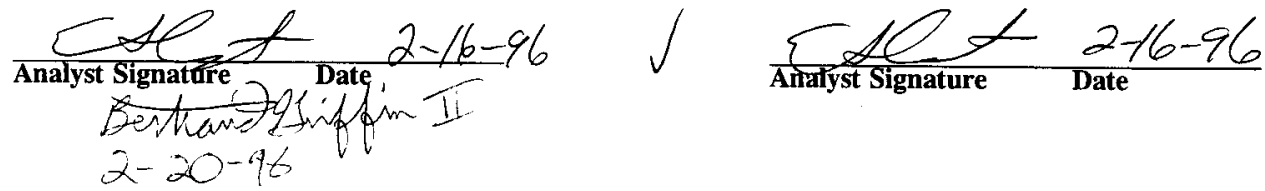

Data Entry Comments:

Units shown for $Q C$ (SPK \& STD) may not reflect the actual units. $D L=$ Detection Limit, $S=$ Worklist Slot Number, $R=$ Replicate Number, $A=$ Aliquot Code. 


\section{LABCORE Data Entry Template for Worklist\#}

Analyst:

Instrument: BA000

Book \# 1

Method: LO-160-103 Rev/Mod B-O

Worklist Comment: U-107 C129 SEG \#3 RISER 9 EXTRUSION

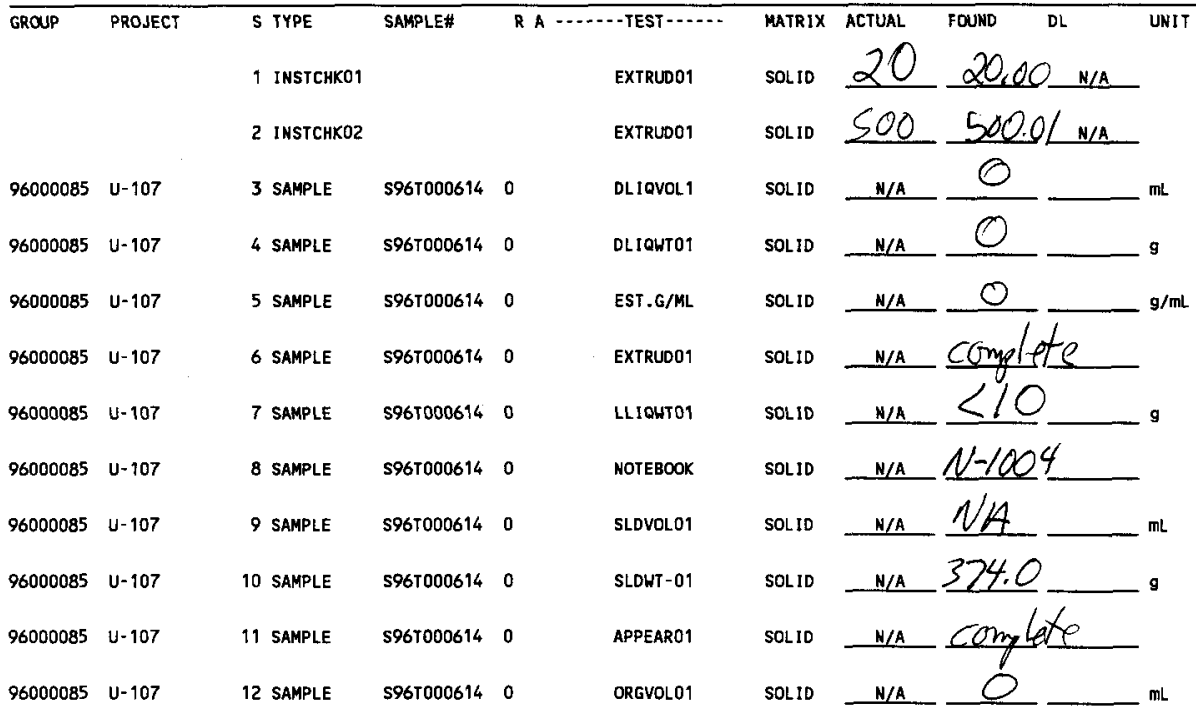

Final page for worklist \#

5654

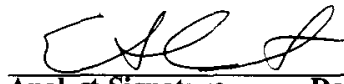

$2-16-46$

Analyst Signature

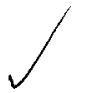
Signature

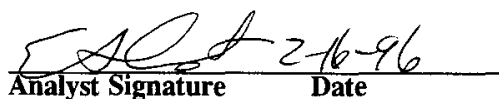

Data Entry Comments:

Units shown for $Q C$ (SPK \& STD) may not reflect the actual units. $D L=$ Detection Limit, $S=$ Worklist Slot Number, $R=$ Replicate Number, $A=$ Aliquot Code. 


\section{LABCORE Data Entry Template for Worklist\#}

\section{Analyst:}

Method: LO-160-103 Rev/Mod $Q-O$

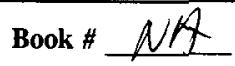

\section{Worklist Comment: U-107 C129 SEG \# 1R RISER 9 EXTRUSION}

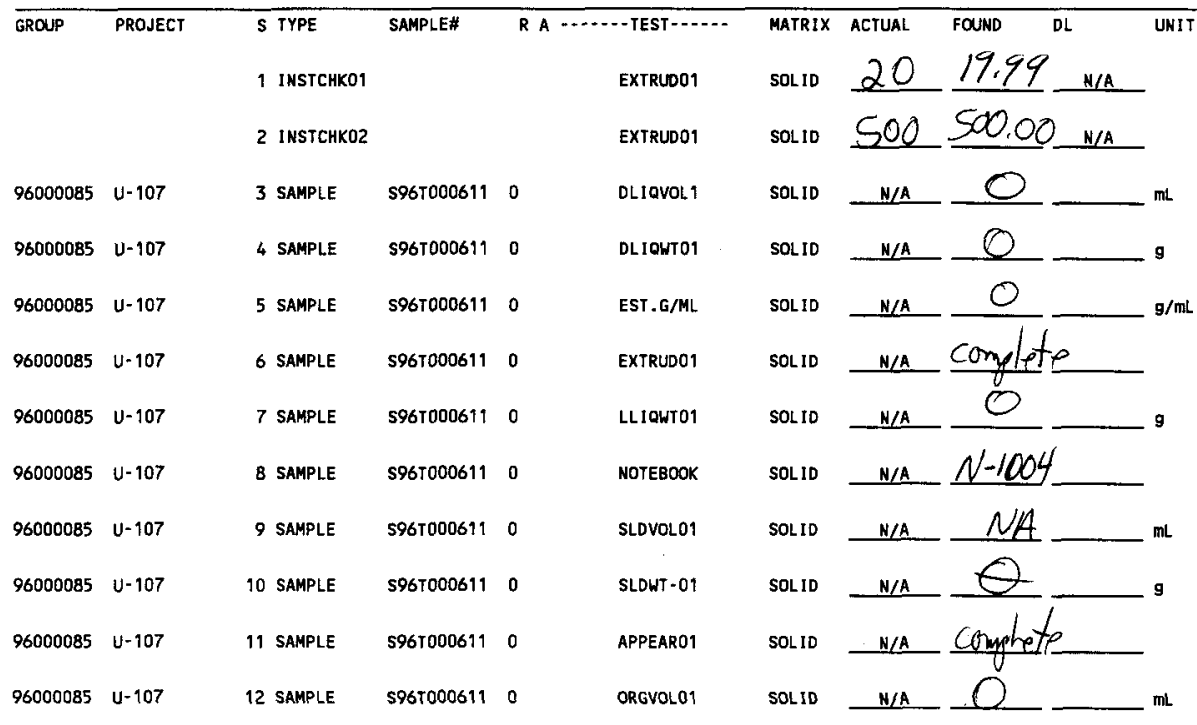

\section{Final page for worklist \# 5655}

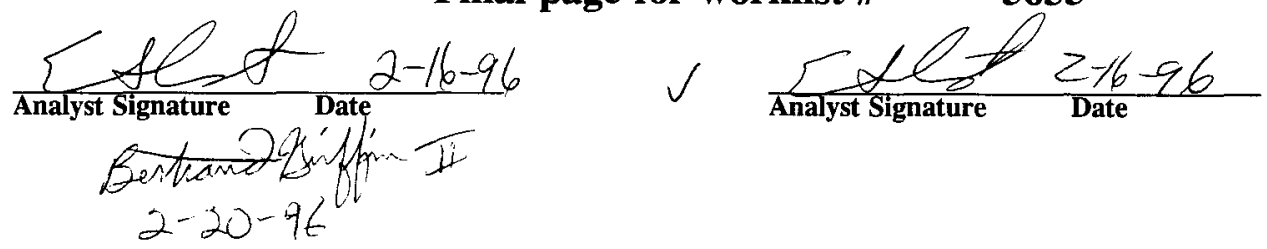

Data Entry Comments:

Units shown for $Q C$ (SPK \& STD) may not reflect the actual units. $D L=$ Detection Limit, $S=$ Worklist Slot Number, $R=$ Replicate Number, $A=$ Aliquot Code. 


\section{LABCORE Data Entry Template for Worklist\#}

Analyst: $\quad$ Instrument: BA000 _ Book \#

Method: LO-160-103 Rev/Mod

Q 0

Worklist Comment: U-107 C129 SEG \# 2R RISER 9 EXTRUSION

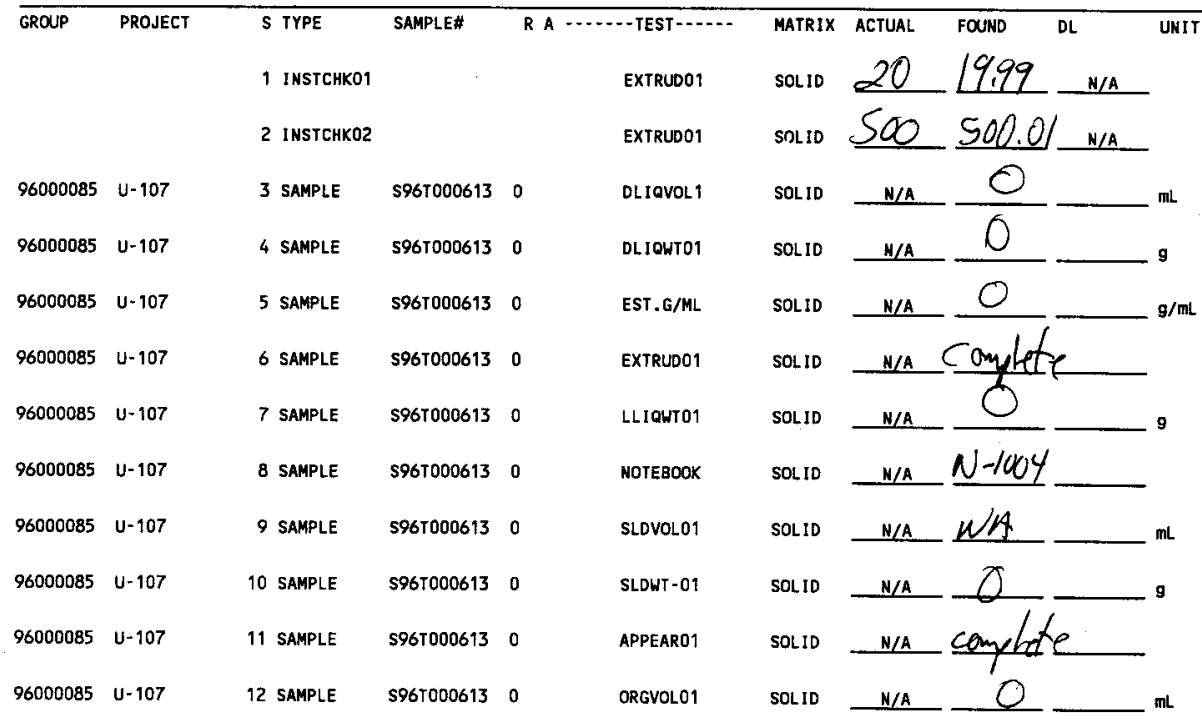

Final page for worklist \# $\quad 5656$

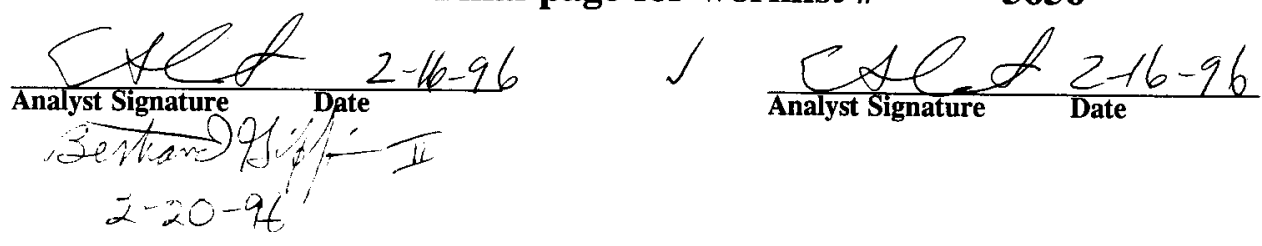

Data Entry Comments:

Units shown for $Q C$ (SPK \& STD) may not reflect the actual units. DL = Detection Limit, $S=$ Worklist Slot Number, $R=$ Replicate Number, $A=$ Aliquot Code. 


\section{LABCORE Data Entry Template for Worklist\#}

Analyst: $\quad$ Instrumeat: BA000_ Book \# NA

Method: LO-160-103 Rev/Mod $B-O$

\section{Worklist Comment: U-107 C129 SEG \# 3R RISER 9 EXTRUSION}

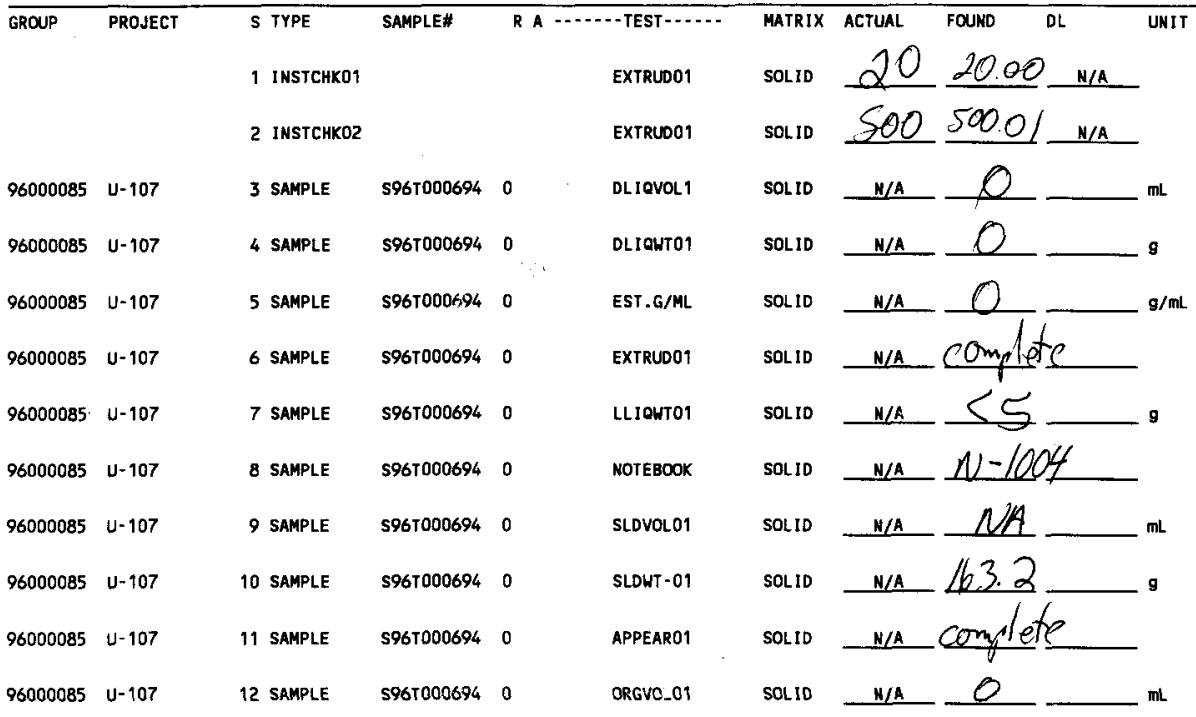

Final page for worklist \# $\quad \mathbf{5 8 0 0}$

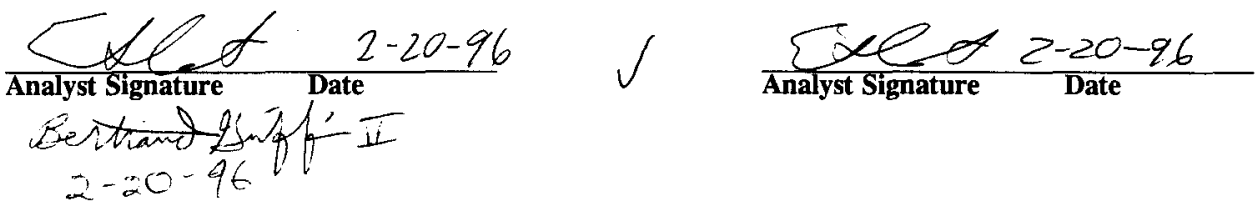

Data Entry Comments:

Units shown for $Q C$ (SPK \& STD) may not reflect the actical units. $D L=$ Detection Limit, $S=$ Worklist Slot Number,

$R=$ Replicate Number, $A=$ Aliquot Code. 
Analyst:

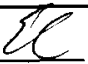

Instrument: BA000

Book $\#$

Method: LO-160-103 Rev/Mod

BO

\section{Worklist Comment: U-107 C134 SEG \# 1 RISER 7 EXTRUSION}

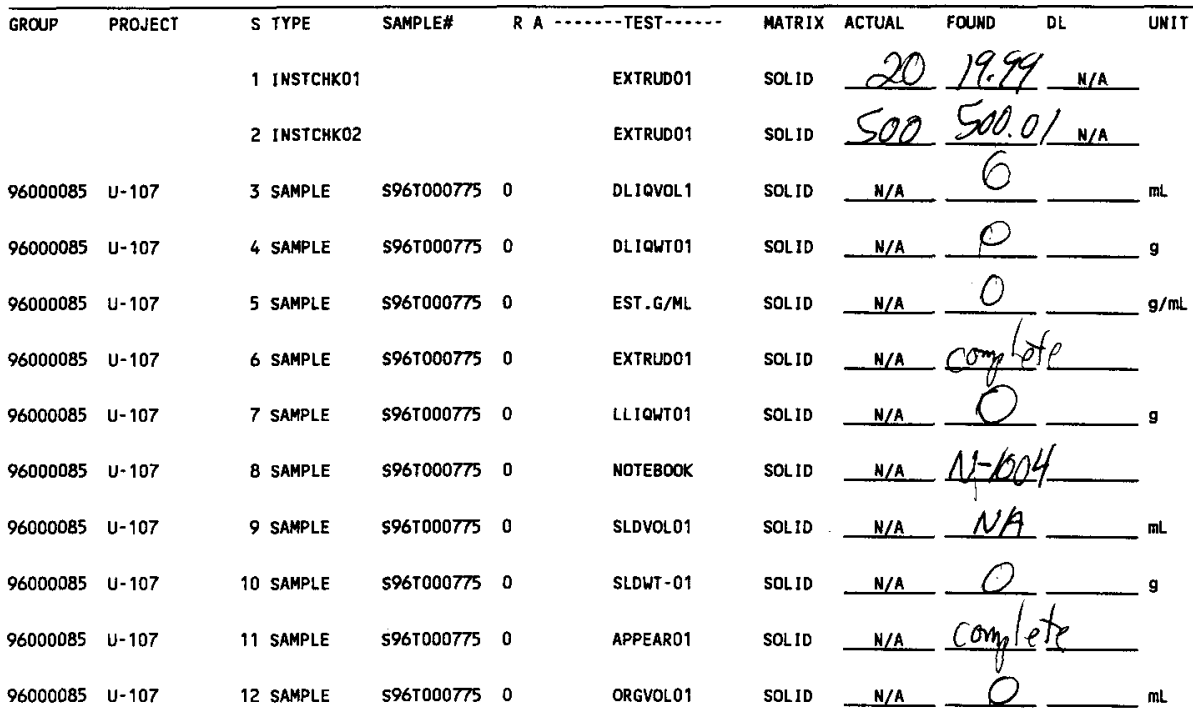

\section{Final page for worklist \#}

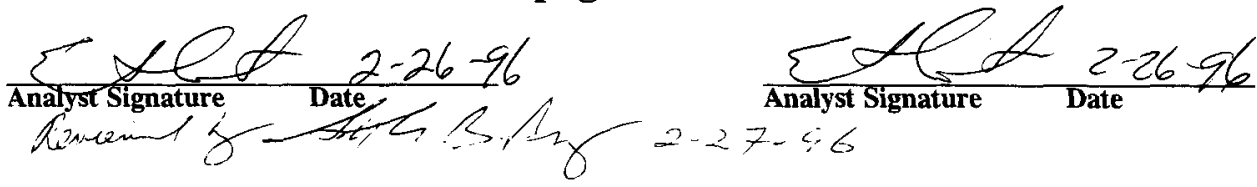

Data Entry Comments:

Units shown for $Q C$ (SPK \& STD) may not reflect the actual units. $D L=$ Detection Limit, $S=$ Worklist Slot Number, $R=$ Replicate Number, $A=$ Aliquot Code. 


\section{LABCORE Data Entry Template for Worklist\#}

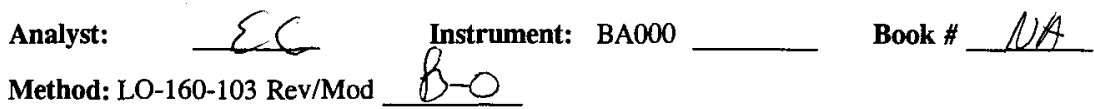

Worklist Comment: U-107 C134 SEG \# 2 RISER 7 EXTRUSION

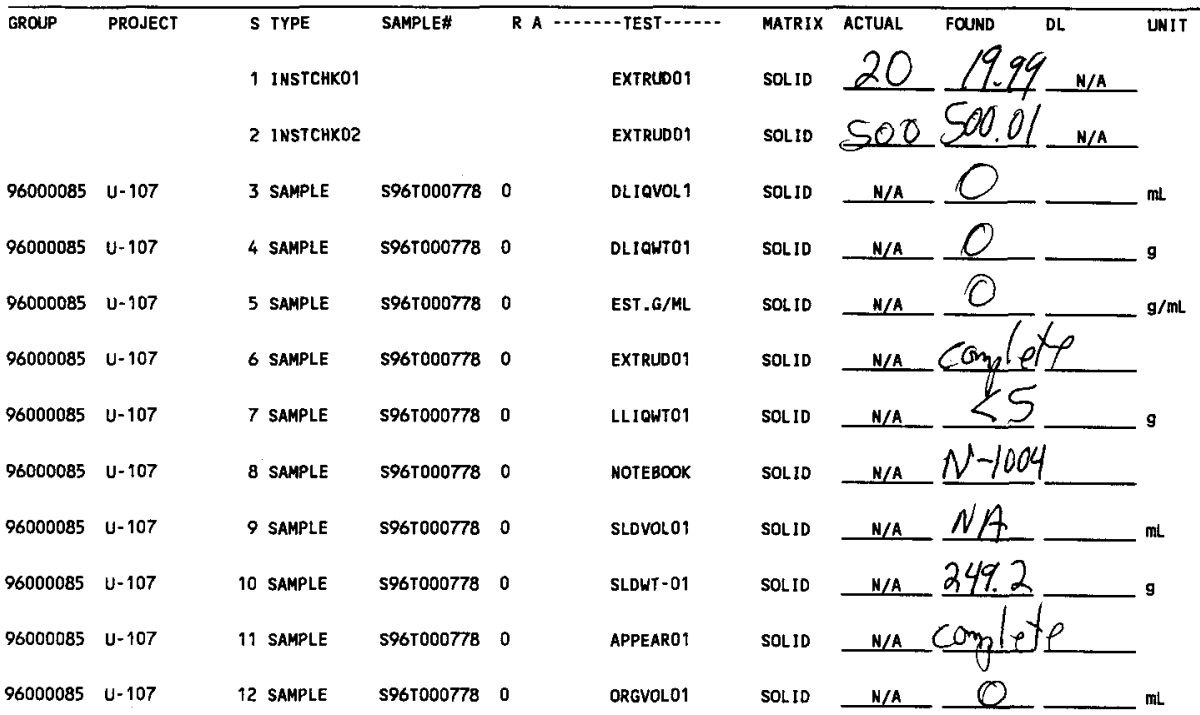

\section{Final page for worklist \#}

\section{7}

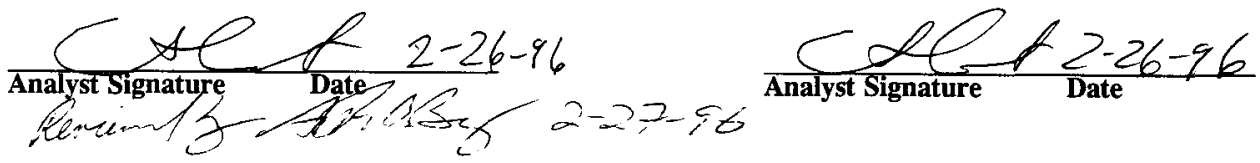

Data Entry Comments:

Units shown for $Q C$ (SPK \& STD) may not reflect the actual units. $D L=$ Detection Limit, $S=$ Worklist Slot Number, $R=$ Replicate Number, $A=$ Aliquot Code. 
Analyst:

Method: LO-160-103 Rev/Mod

Instrument: BA000

Book \# UA

\section{Worklist Comment: U-107 C134 SEG \# 4 RISER 7 EXTRUSION}

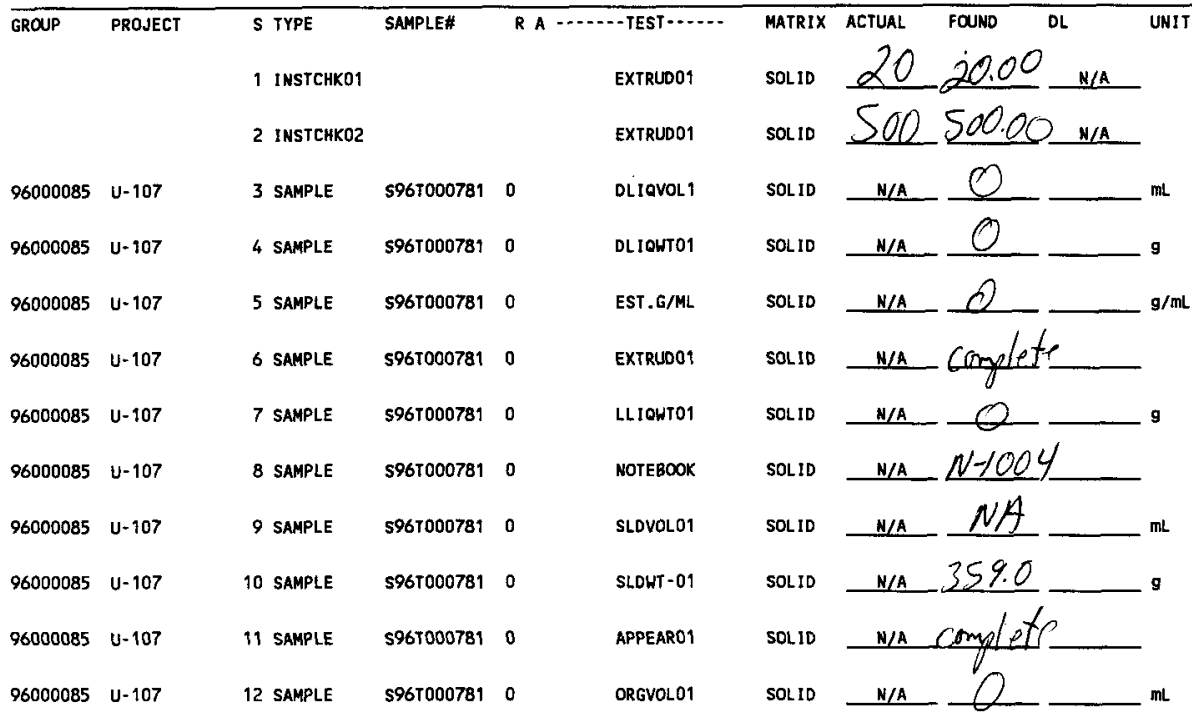

\section{Final page for worklist \# $\quad 5861$}
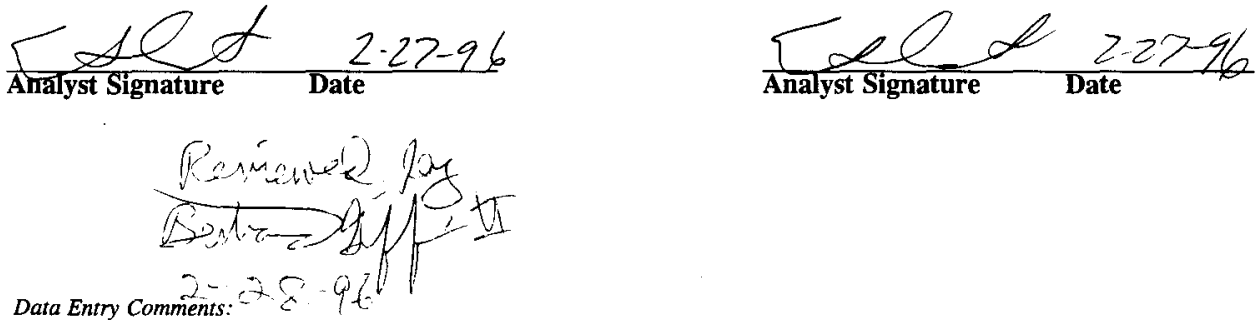

Units shown for $Q C$ (SPK \& STD) may not reflect the actual units. DL = Detection Limit, $S=$ Worklist Slot Number, $R=$ Replicate Number, $A=$ Aliquot Code. 


Analyst: $\quad[C \quad$ Instrument: BA000_ Book \# N/2

Method: LO-160-103 Rev/Mod B-O

Worklist Comment: U-107 C134 SEG \# 5 RISER 7 EXTRUSION

\begin{tabular}{|c|c|c|c|c|c|c|c|c|c|}
\hline \multirow[t]{2}{*}{ GROUP } & \multirow[t]{2}{*}{ PROJECT } & $\begin{array}{l}\text { S TYPE } \\
1 \text { INSTCHK01 }\end{array}$ & SAMPLE\# & RA & - TEST - & $\begin{array}{l}\text { MATRIX } \\
\text { SOL ID }\end{array}$ & $\begin{array}{l}\text { ACTUAL } \\
20\end{array}$ & 20.01 & $N / A$ \\
\hline & & 2 INSTCHKOZ & & & EXTRUDO9 & SOLID & 500 & & N/A \\
\hline 96000085 & $U \cdot 107$ & 3 SAMPLE & S96T000782 & 0 & DLIOVOLI & SOLID & $\mathrm{N} / \mathrm{A}$ & & \\
\hline 96000085 & $u-107$ & 4 SAMPLE & S96T000782 & 0 & DLIOLTOO1 & SOL ID & $\mathrm{N} / \mathrm{A}$ & & \\
\hline 96000085 & $u-107$ & 5 SAMPLE & s96T000782 & 0 & EST.G/ML & SOLID & N/A & & \\
\hline 96000085 & $u-107$ & 6 SAMPLE & S96T000782 & 0 & EXTRUD01 & SOL ID & N/A & & \\
\hline 96000085 & $U-107$ & 7 SAMPLE & S96T000782 & 0 & LLIDWTO1 & SOLID & N/A & & \\
\hline 96000085 & $U-107$ & 8 SAMPLE & S96T000782 & 0 & NOTEBOOK & SOLID & N/A & & \\
\hline 96000085 & $U-107$ & 9 SAMPLE & S96T000782 & 0 & SLOVOLOI & SOLID & N/A & $N A$ & \\
\hline 96000085 & $U-107$ & 10 SAMPLE & S96T000782 & 0 & SLDWT -01 & SOLID & N/A & & \\
\hline 96000085 & U-107 & 11 SAMPLE & 596T000782 & 0 & APPEAR01 & SOLID & N/A & & \\
\hline 96000085 & U-107 & 12 SAMPLE & S96T000782 & 0 & ORGVOL01 & SOL ID & N/A & & \\
\hline
\end{tabular}

\section{Final page for worklist \# $\quad \mathbf{5 8 6 3}$}
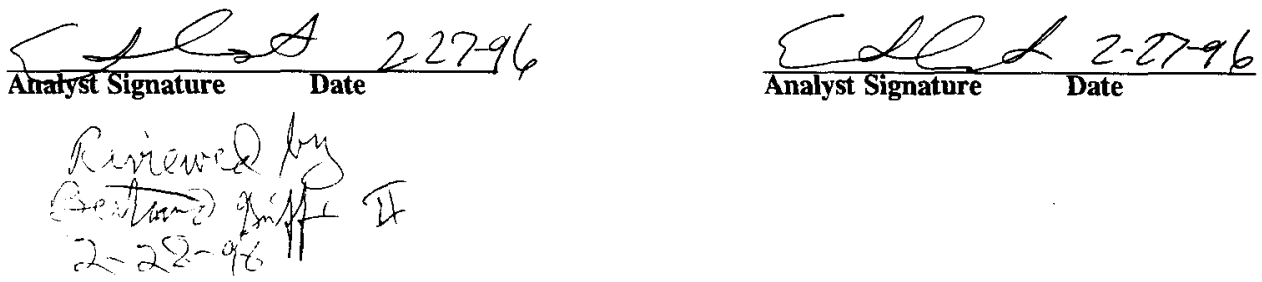

Data Entry Comments:

Units shown for $Q C$ (SPK \& STD) may not reflect the actual units. $D L=$ Detection Limit, $S=$ Worklist Slot Number, $R=$ Replicate Number, $A=$ Aliquot Code. 


\section{LABCORE Data Entry Template for Worklist\#}

Analyst: $\quad \sum f$ Instrument: $\mathrm{BA000}$

Book \#

Method: LO-160-103 Rev/Mod Q - O

Worklist Comment: U-107 C134 SEG \# 5A RISER 7 EXTRUSION

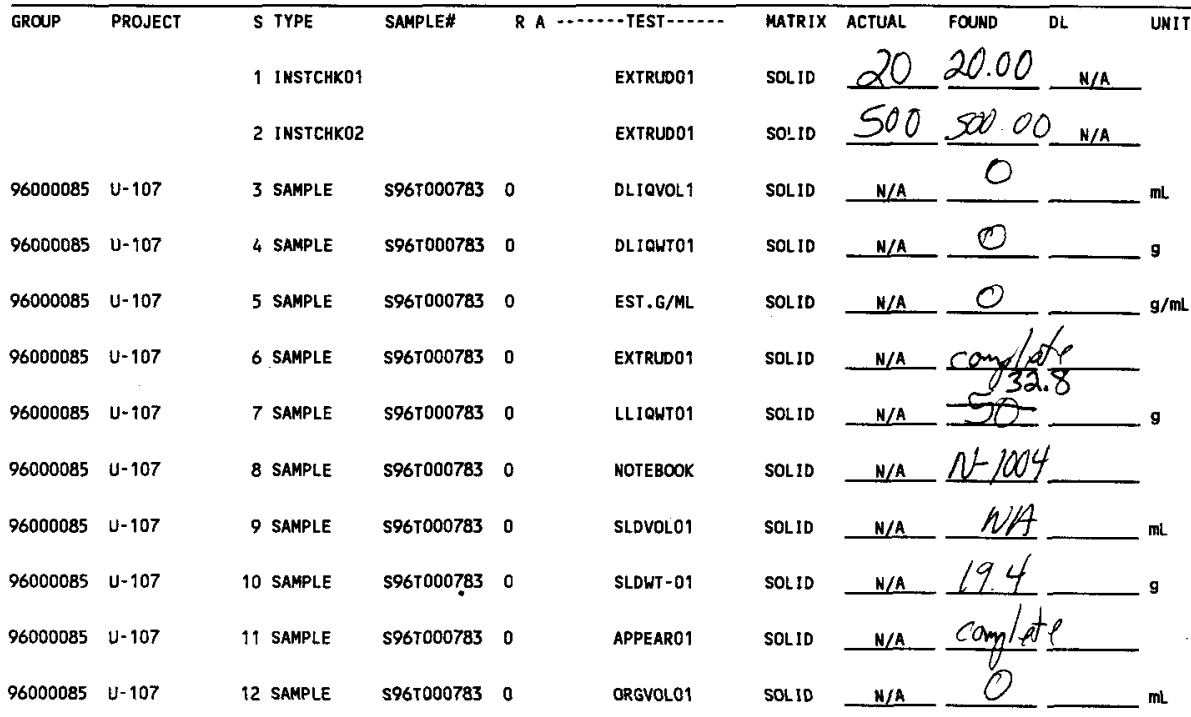

\section{Final page for worklist \# $\quad 5865$}
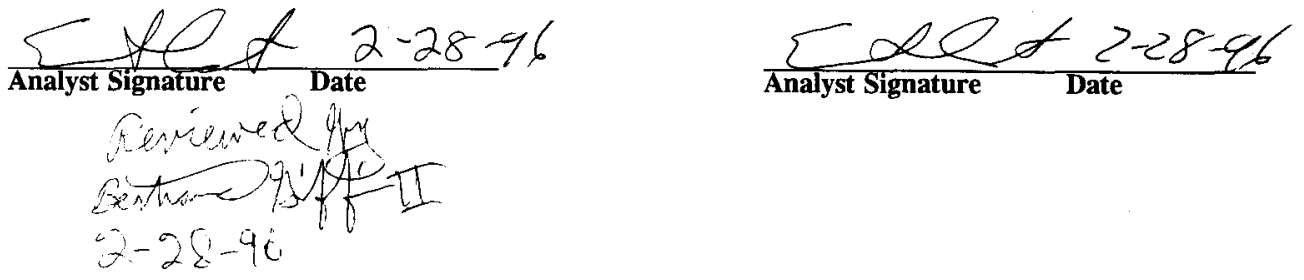

Data Entry Comments:

Units shown for $Q C$ (SPK \& STD) may not reflect the actual units. $D L=$ Detection Limit, $S=$ Worklist Slot Number, $R=$ Replicate Number, $A=$ Aliquot Code. 
Analyst: $\quad C C$ Instrument: BA000

Method: LO-160-103 Rev/Mod

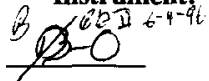

Book \# $\Perp A$

Worklist Comment: U-107 C134 SEG \# 5B RISER 7 EXTRUSION

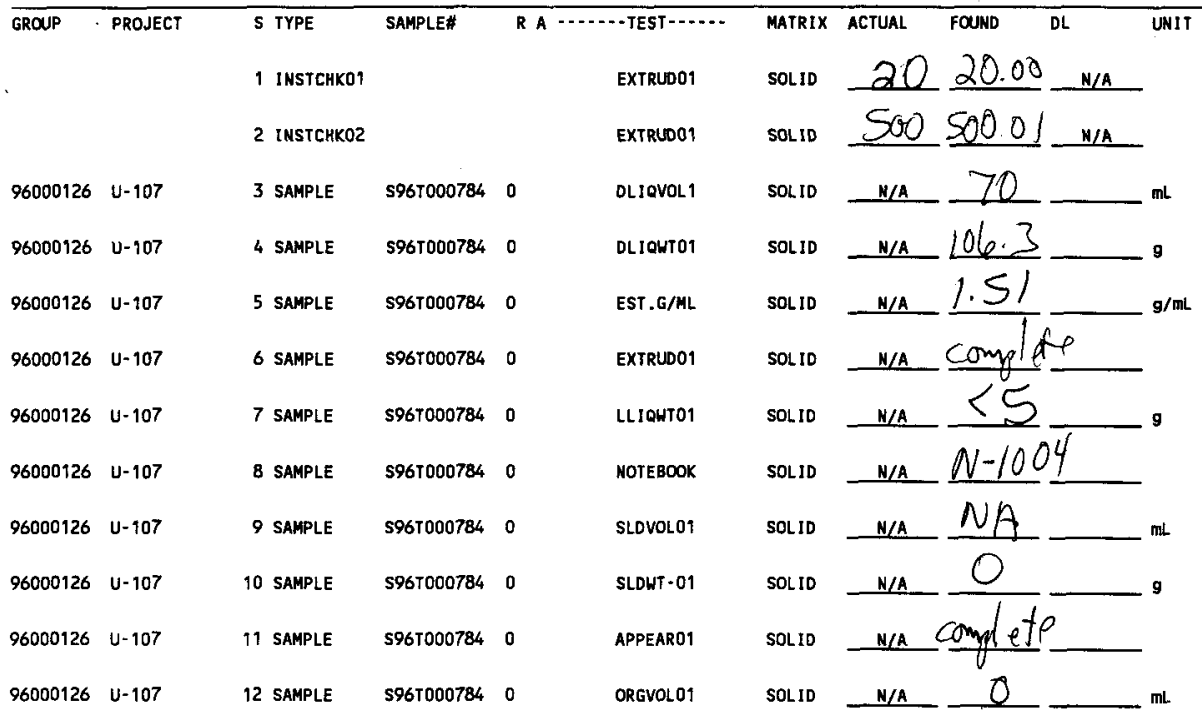

\section{Final page for worklist \# $\quad \mathbf{5 8 6 6}$}

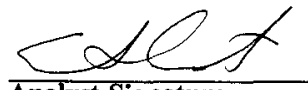

Analyst Signature

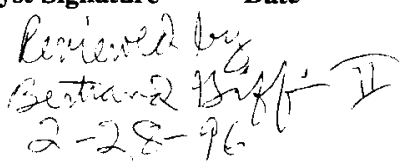

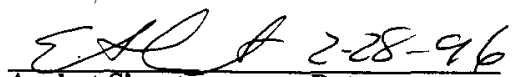

Analyst Signature Date

Data Entry Comments:

Units shown for $Q C$ (SPK \& STD) may not reflect the actual units. $D L=$ Detection Limit, $S=$ Worklist Slot Number, $R=$ Replicate Number, $A=$ Aliquot Code. 


\section{LABCORE Data Entry Template for Worklist\#}

Analyst: $\quad \sum S \quad$ Instrument: BA000_ Book \# $N A$

Method: LO-160-103 Rev/Mod B-O

Worklist Comment: U-107 C134 SEG \# 6 RISER 7 EXTRUSION

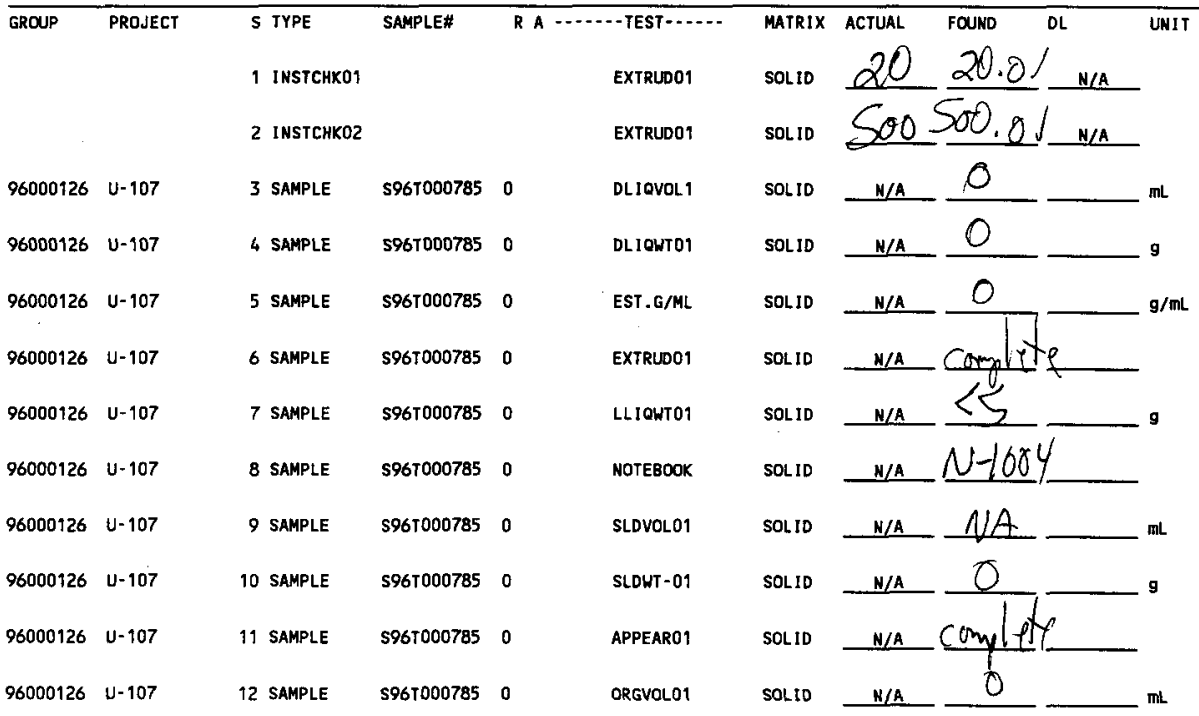

\section{Final page for worklist \# $\quad \mathbf{5 8 6 7}$}

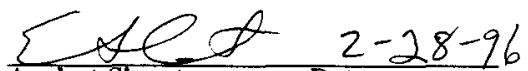

Analyst Signature

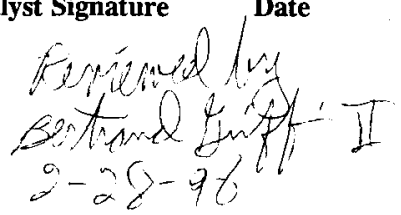

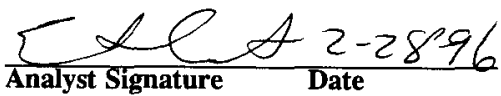

Data Entry Comments:

Units shown for $Q C$ (SPK \& STD) may not reflect the actual units. $D L=$ Detection Limit, $S=$ Worklist Slot Number, $R=$ Replicate Number, $A=$ Aliquot Code. 


\section{LABCORE Data Entry Template for Worklist\#}

\section{Analyst: \\ Instrument: BA000 \\ Book \#}

Method: LO-160-103 Rev/Mod B-O

Worklist Comment: U-107 C134 SEG \# 6A RISER 7 EXTRUSION

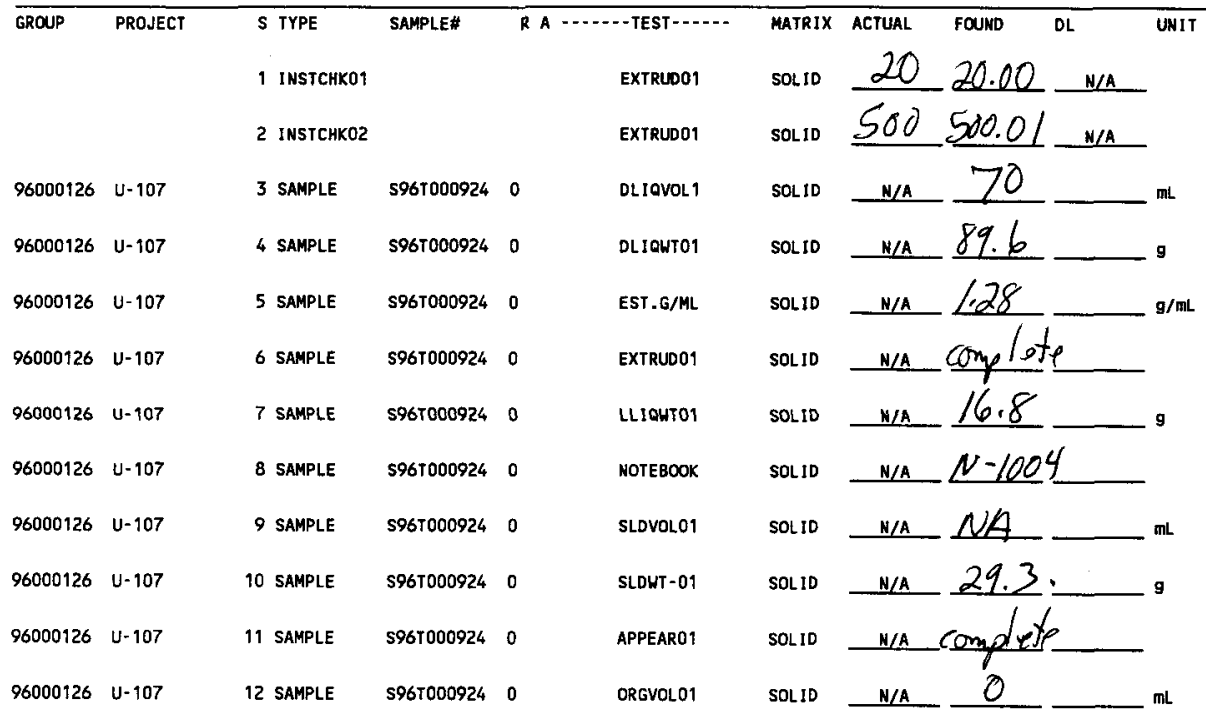

Final page for worklist \# 6049 .

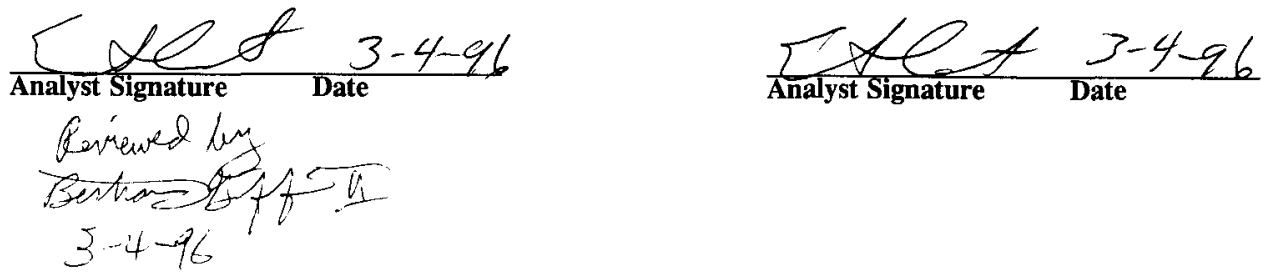

Data Entry Comments:

Units shown for $Q C$ (SPK \& STD) may not reflect the actual units. $D L=$ Detection Limit, $S=$ Worklist Slot Number, $R=$ Replicate Number, $A=$ Aliquot Code. 


Analyst: $\quad \sum E \quad$ Instrument: BA000 $\quad$ Book \# $1 / A$

Method: LO-160-103 Rev/Mod b)

Worklist Comment: U-107 C135 SEG \# 1 RISER 2 EXTRUSION

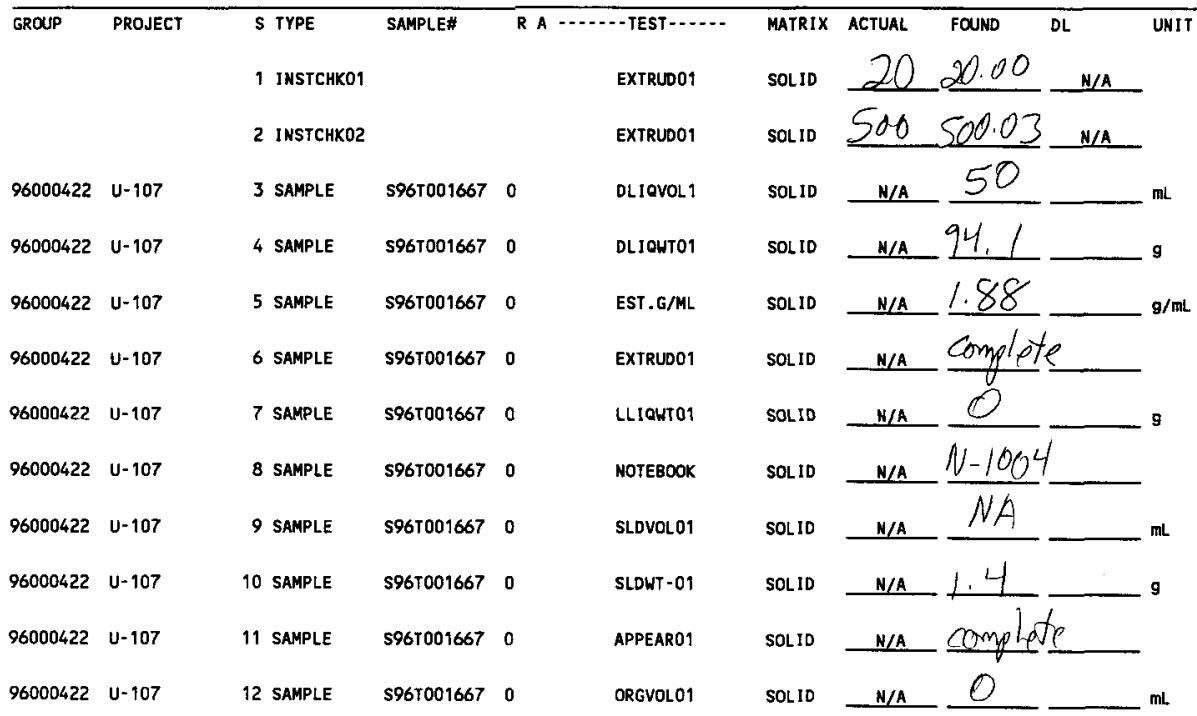

\section{Final page for worklist \# 7293}
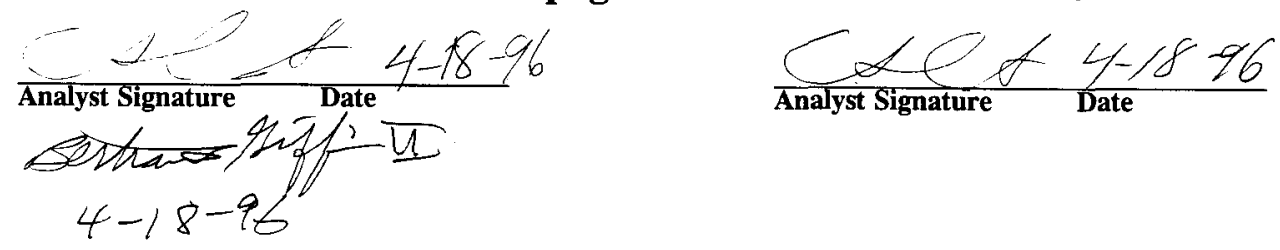

Data Entry Comments:

Units shown for $Q C$ (SPK \& STD) may not reflect the actual units. $D L=$ Detection Limit, $S=$ Worklist Slot Number, $R=$ Replicate Number, $A=$ Aliquot Code. 


\section{LABCORE Data Entry Template for Worklist\#}

Analyst: $\quad$ Instrument: BA000

Book \#

Method: LO-160-103 Rev/Mod

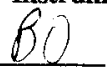

Worklist Comment: U-107 C135 SEG \# 1R RISER 2 EXTRUSION

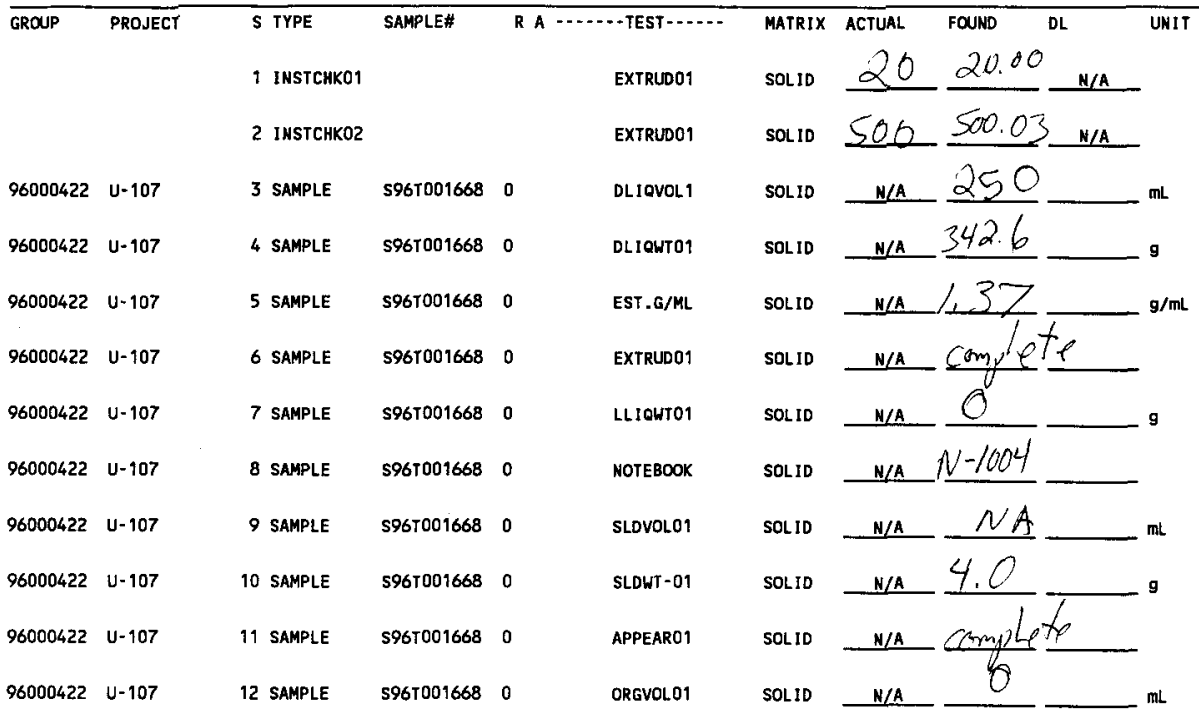

\section{Final page for worklist \#}

7296
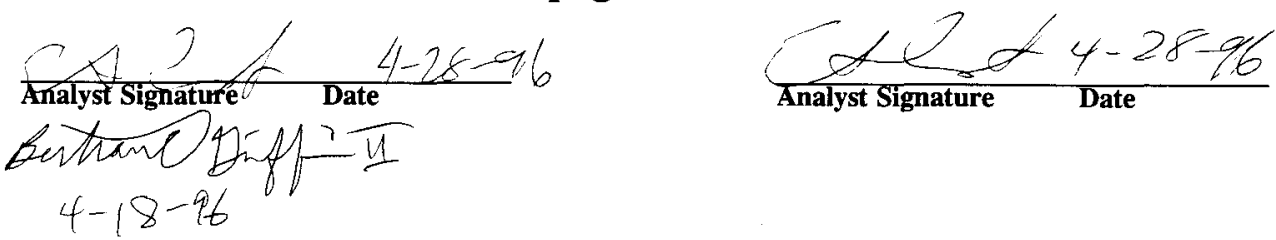

Data Entry Comments:

Units shown for $Q C$ (SPK \& STD) may not reflect the actual units. $D L=$ Detection Limit, $S=$ Worklist Slot Number, $R=$ Replicate Number, $A=$ Aliquot Code. 
Analyst:

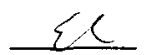

Instrument: BA000

Book \# NA

Method: LO-160-103 Rev/Mod _ 180

Worklist Comment: U-107 C135 SEG \# 2 RISER 2 EXTRUSION

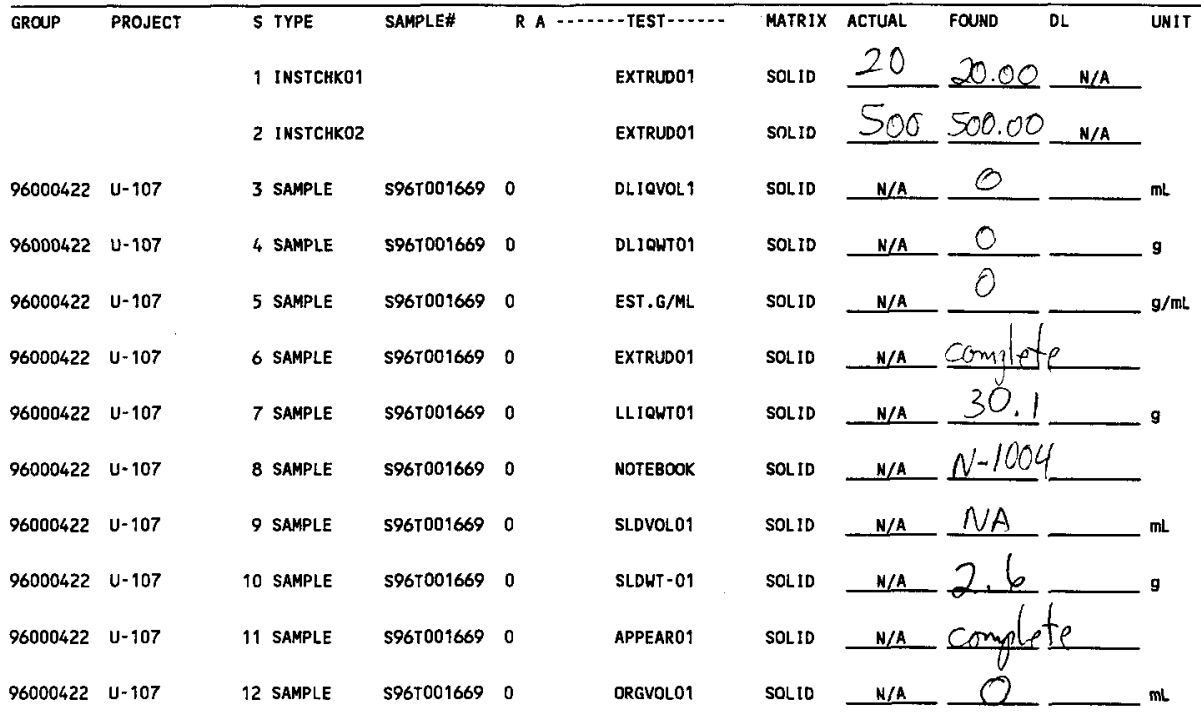

\section{Final page for worklist \# 7297}
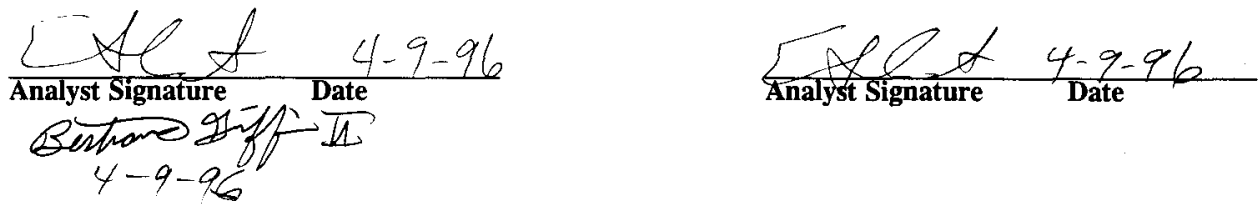

Data Entry Comments:

Units shown for $Q C$ (SPK \& STD) may not reflect the actual units. $D L=$ Detection Limit, $S=$ Worklist Slot Number, $R=$ Replicate Number, $A=$ Aliquot Code. 


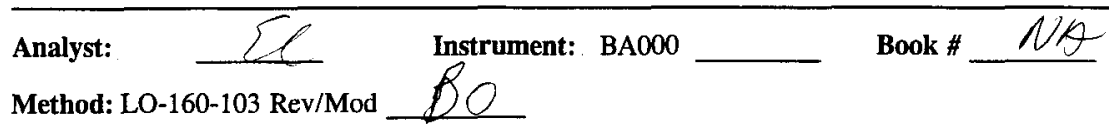

Worklist Comment: U-107 C135 SEG \# 2A RISER 2 EXTRUSION

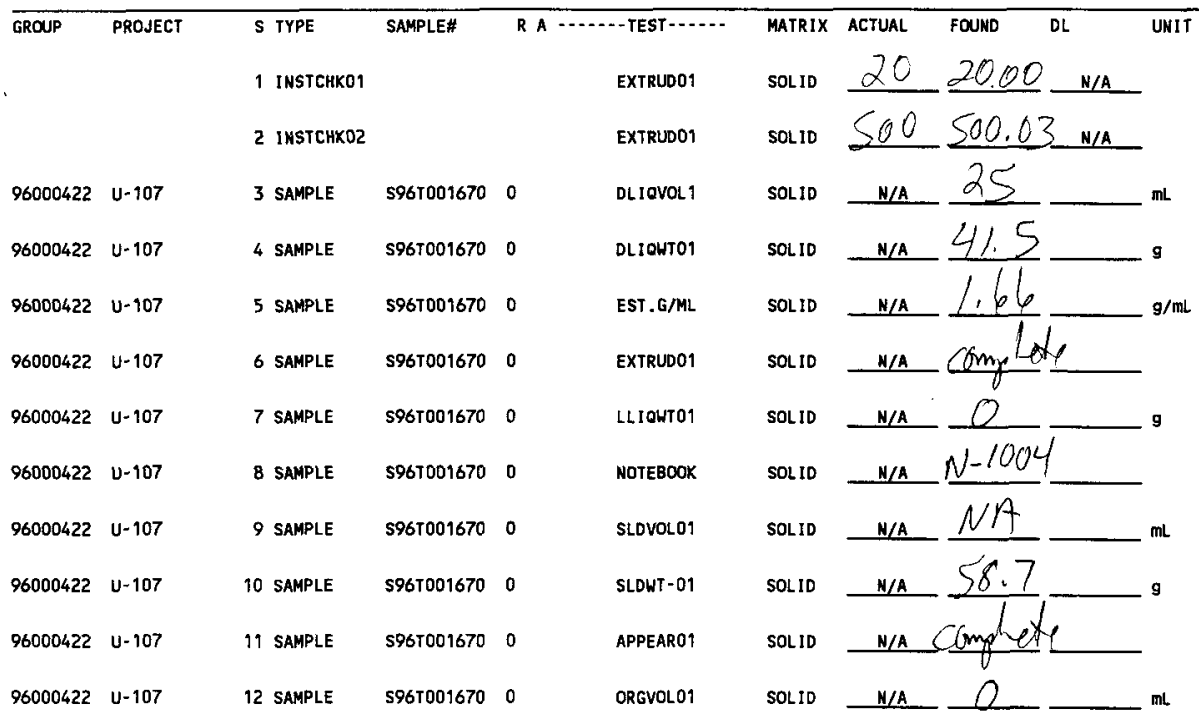

\section{Final page for worklist \# 7299}

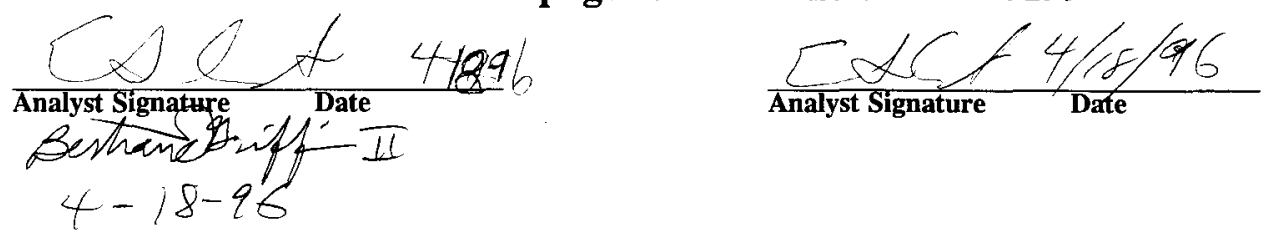

Data Entry Comments:

Units shown for QC (SPK \& STD) may not reflect the actual units. $D L=$ Detection Limit, $S=$ Worklist Slot Number. $R=$ Replicate Number, $A=$ Aliquot Code. 
Analyst:

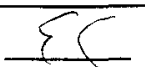

Instrument: BA000

Book \#

Method: LO-160-103 Rev/Mod

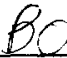

\section{Worklist Comment: U-107 C135 SEG \# 2R RISER 2 EXTRUSION}

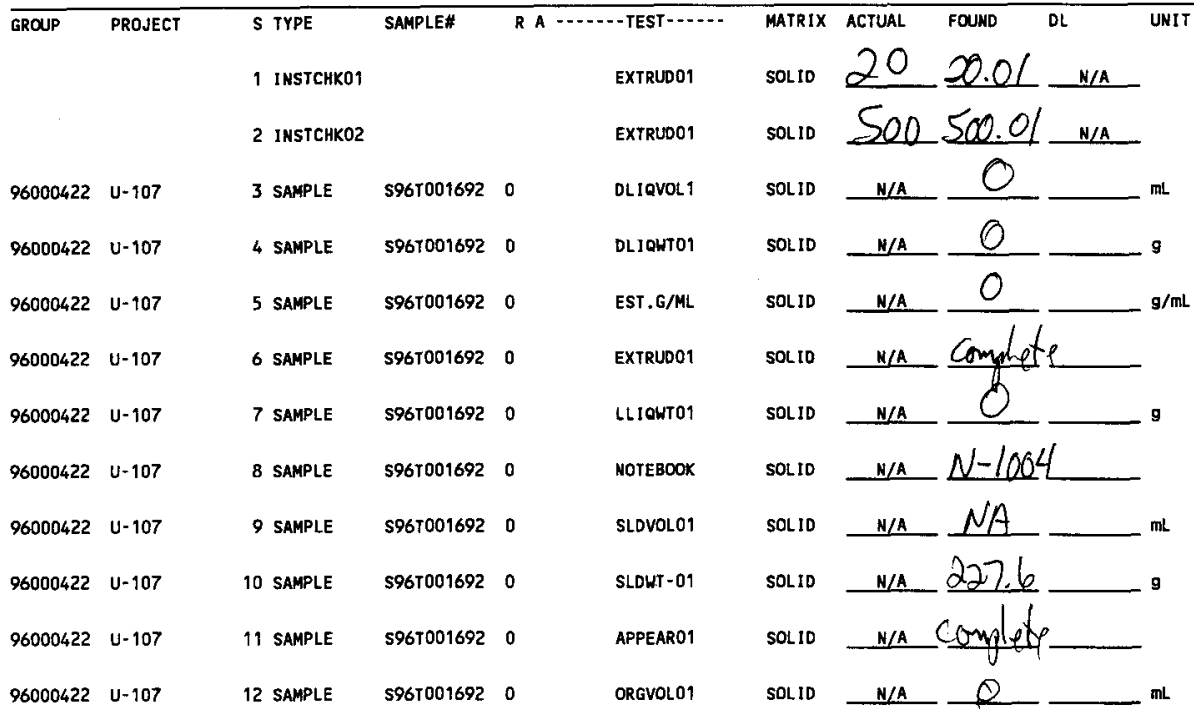

\section{Final page for worklist \# $\mathbf{7 3 0 8}$}
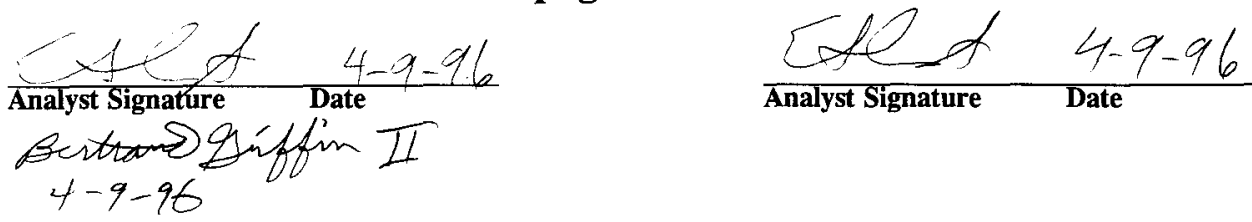

Analyst Signature Date

Data Entry Comments:

Units shown for $Q C$ (SPK \& STD) may not reflect the actual units. $D L=$ Detection Limit, $S=$ Worklist Slot Number, 


\section{LABCORE Data Entry Template for Worklist\#}

\section{Analyst: 4 Instrument: NONE — Book \#}

Method: LA-504-101 Rev/Mod $\varepsilon-0$

Worklist Comment: TK\#U-107,CORE 129, SEG 1DL,2DL,(DRAINABLE LIQUID)

\begin{tabular}{|c|c|c|c|c|c|c|c|c|c|c|}
\hline GROUP & PROJECT & & IYPE & SAMPLE\# & RA & - & MATRIX & ACTUAL & FOUND & UN!T \\
\hline 96000085 & $U-107$ & 1 & SAMPLE & S96T000665 & 0 & FILTER02 & LIQUID & N/A & & \\
\hline 96000085 & $u-107$ & 2 & SAMPLE & 5961000665 & 0 & DOSE-02 & LIQUID & N/A & & mrad/hour \\
\hline 96000085 & $u-107$ & 3 & SAMPLE & S96T000682 & 0 & FILTERO2 & LIQUID & $N / A$ & हा & \\
\hline 96000085 & U- 107 & 4 & SAMPLE & S96T000682 & 0 & DOSE-02 & LIQUID & $N / A$ & & mrad/hour \\
\hline
\end{tabular}

\section{Final page for worklist \# 6276}

\section{Analyst Signature Date}
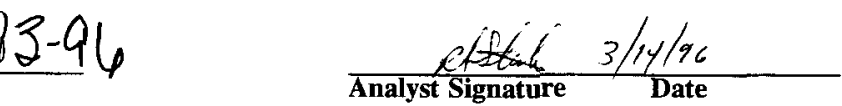

$5967000637 \rightarrow 5967000665$

$641 \rightarrow 682$

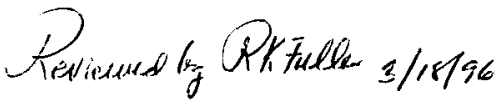

Data Entry Comments:

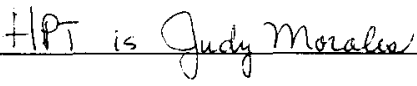

Units shown for QC (SPK \& STD) may not reflect the actual units. $D L=$ Detection Limit, $S=$ Worklist Slot Number, $R=$ Replicate Number, $A=$ Aliquot Code. 


\section{Analyst: GAM Instrument: NONE Book\#}

Method: LA-504-101 Rev/Mod $\mathcal{\varepsilon}-\mathcal{O}$

Worklist Comment: TK\#U-107,CORE C134,SEG 5A LL,5B DL,6A LL,6A DL, FILTERO2 RTS

\begin{tabular}{|c|c|c|c|c|c|c|c|c|c|}
\hline GROUP & PROJECT & $S$ TYPE & SAMPLE\# & & - TEST $-\cdots . .$. & MATRIX & ACTUAL & $D L$ & UNIT \\
\hline 96000126 & $U-107$ & i SAMPLE & S96T001110 & 0 & FILTER02 & LIQUiD & N/A & & \\
\hline 96000126 & $U-107$ & 2 SAMPLE & S96T001110 & 0 & DOSE-02 & LIQUID & $\mathbf{N} / \mathbf{A}$ & & mrad/hour \\
\hline 96000126 & $U-107$ & 3 SAMPLE & s96T001119 & 0 & FILTER02 & LIQUID & N/A & & \\
\hline 96000126 & $U-107$ & 4 SAMPLE & 596r001119 & 0 & DOSE-02 & LIQUID & N/A & & mrad/hour \\
\hline 96000126 & $u-107$ & 5 SAMPLE & S96T001120 & 0 & FILTER02 & LIQUID & N/A & & \\
\hline 96000126 & $u-107$ & 6 SAMPLE & S96T001120 & 0 & DOSE- 02 & LIOUID & N/A & & $\mathrm{mrad} / \mathrm{hour}$ \\
\hline 96000126 & $\mathrm{u}-107$ & 7 SAMPLE & S96T001121 & 0 & FILTER02 & LIQUID & DCNL & & \\
\hline 96000126 & U- 107 & 8 SAMPLE & S96r001121 & 0 & DOSE-02 & LIQUID & 115 & & $\mathrm{mrad} / \mathrm{hour}$ \\
\hline
\end{tabular}

\section{Final page for worklist \# 6277}

$\frac{\text { MAlloweng } 4-10-96}{\text { Amalyst Signature }}$

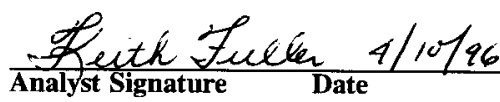

$$
\begin{aligned}
5965000 & \rightarrow 59670001110(4) \\
1107 & \rightarrow 1119(14) \\
1108 & \rightarrow 1120(4) \\
1109 & \rightarrow 1121(4) \text { ettrof }
\end{aligned}
$$

Peviound by PKFule

Data Entry Comments: 
worklistrpt Version 2.1 05/15/95 WHC-SD-WM-DP-184, REV. I
05/17/96 10:39

\section{LABCORE Data Entry Template for Worklist\#}

Analyst:

Method: LA-504-101 Rev/Mod \&-D

Worklist Comment: U-107 C135 SEG.2 FILTER02 SKB

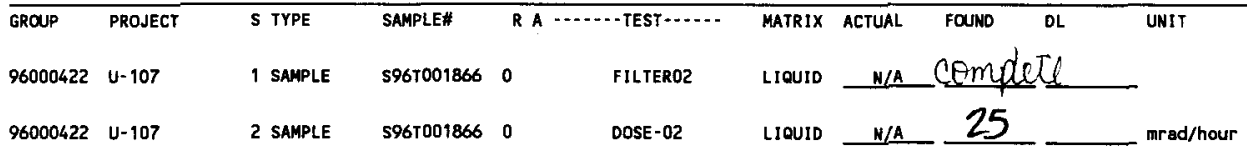

\section{Final page for worklist \# $\quad \mathbf{8 8 4 6}$}
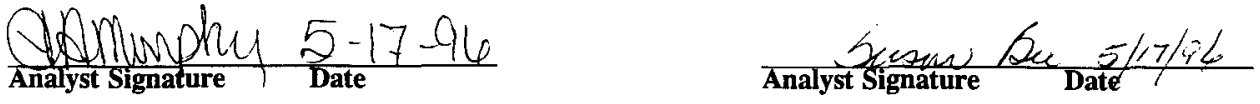

$$
\text { S967001865 } \rightarrow 5967001866
$$

Data Entry Comments:

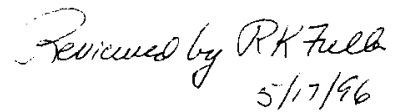

Units shown for $Q C$ (SPK \& STD) may not reflect the actual units. $D L=$ Detection Limit, $S=$ Worklist Slot Number, $R=$ Replicate Number, $A=$ Aliquot Code. 
WHC-SD-WM-DP-184, REV. 1

\section{SAMPLE PREPARATIONS}


WHC-SD-WM-DP-184, REV. 1

THIS PAGE WAS INTENTIONALLY LEFT BLANK 
WHC-SD-WM-DP-184, REV. 1

Page: $\quad 1$

worklistrpt Version $2.105 / 15 / 95$

LABCORE Data Entry Template for Worklist\#

5882

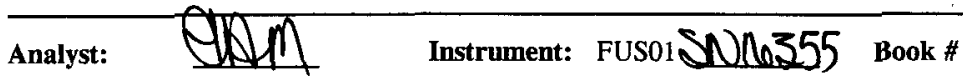

Method: LA-549-141 Rev/Mod $工-1$

Worklist Comment: U-107 Core 129 Stg 1sc,2U,2L Fusion Digestion raf

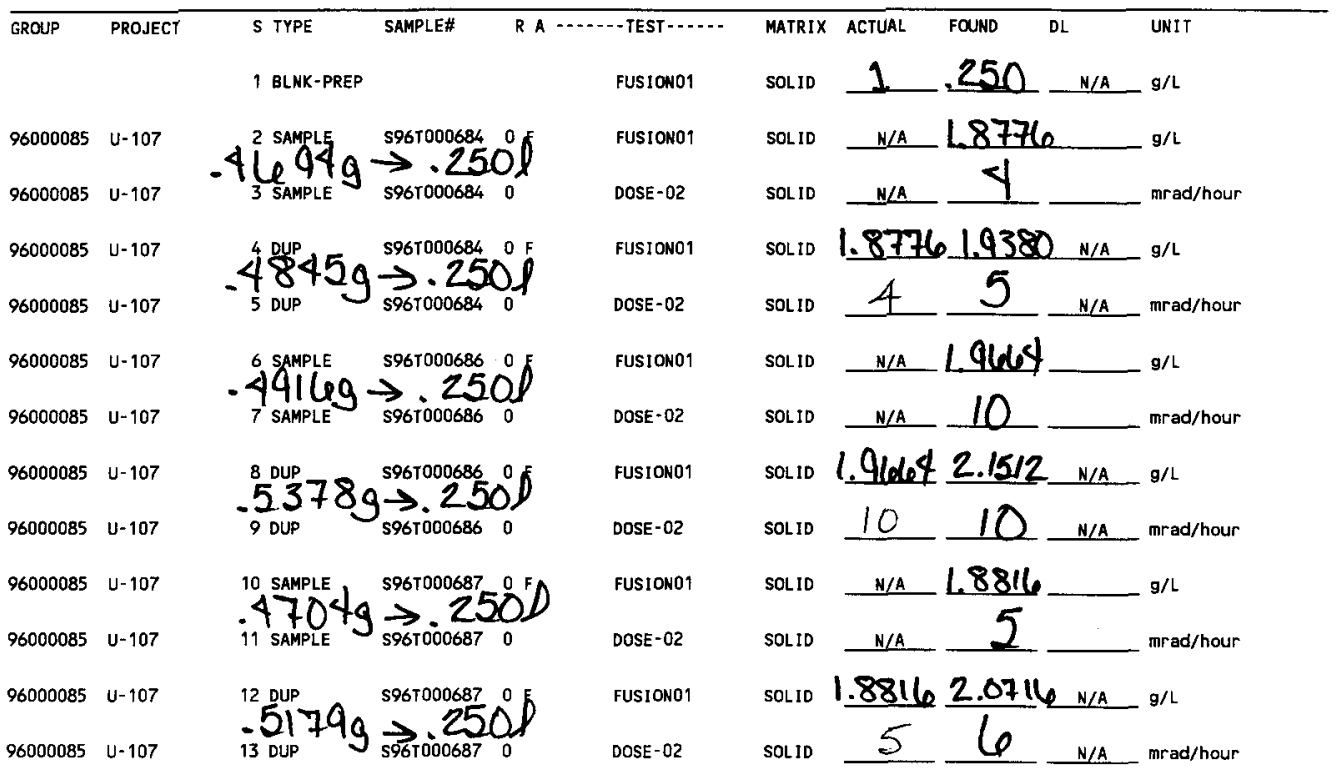

Final page for worklist \# $\mathbf{5 8 8 2}$

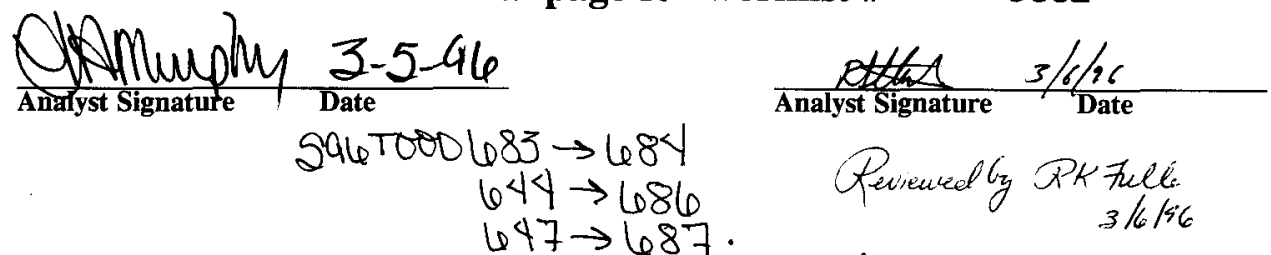

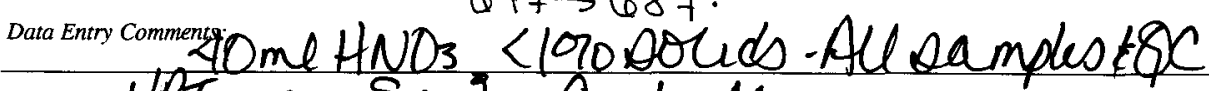
HPT was Scot Corbally

Units shown for $Q C$ (SPK \& STD) may not reflect the actual units. $D L=$ Detection Limit, $S=$ Worklist Slot Number, $R=$ Replicate Number, $A=$ Aliquot Code.

127 
WHC-SD-WM-DP-184, REV. |

worklistrpt Version $2.105 / 15 / 95$

Page: $\quad 1$ 02/25/96 15:56

LABCORE Data Entry Template for Worklist\#

5883

Analyst: Hind Instrument: Fus01 SN /6 555 Book \#

Method: LA-549-141 Rev/Mod \&-O

Worklist Comment: U-107 Core 129 Beg 3U,3L Fusion Digestion ref

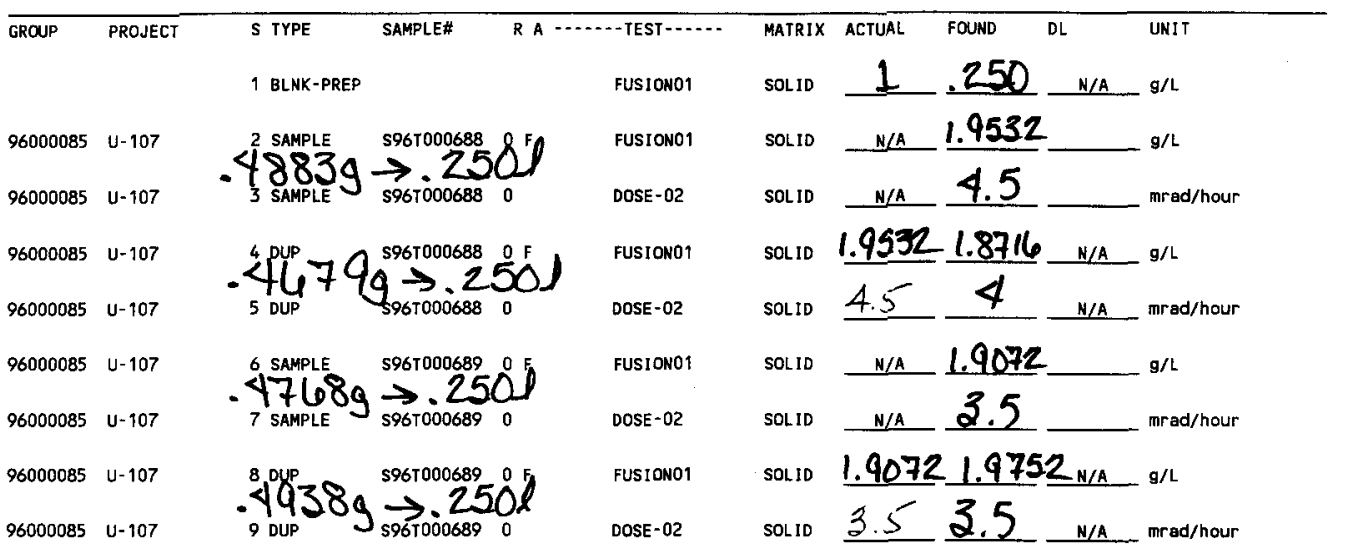

Final page for worklist \#

5883

AlAmumphy

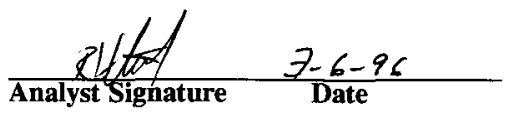

Reviewed by PK aneles

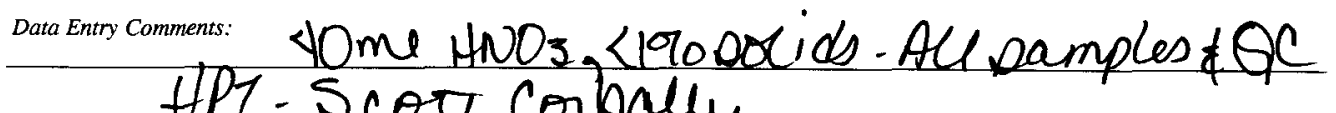
HPT - Scot Corbally

Units shown for QC (SPK \& STD) may not reflect the actual units. $D L=$ Detection Limit, $S=$ Worklist Slot Number, $R=$ Replicate Number, $A=$ Aliquot Code.

128 


\section{LABCORE Data Entry Template for Worklist\#}

Analyst:

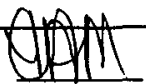
Instrument: FUS01 Shy $(0555)$

Book \#

Method: LA-549-141 Rev/Mod E 0

Worklist Comment: TK\#U-107,CORE 134,GRP\# 126,SEG 2,3,4L, FUSION RTS!

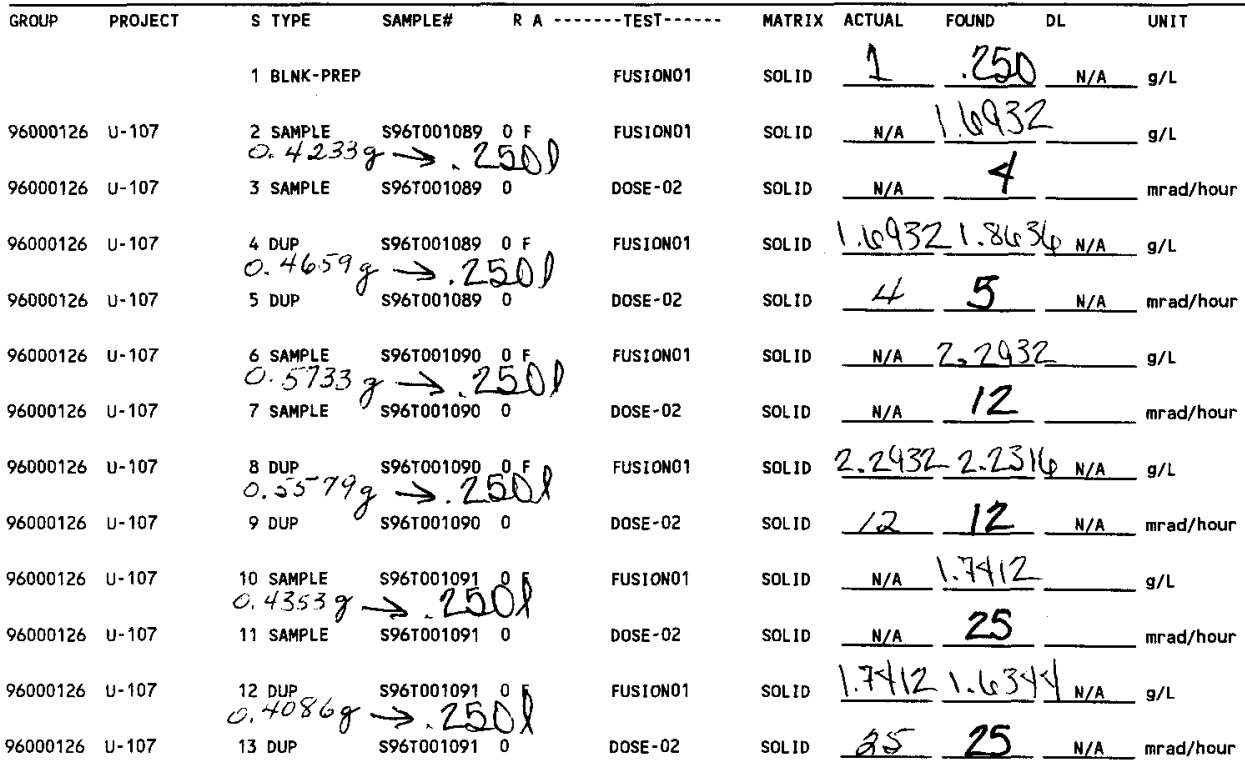

\section{Final page for worklist \# 6417}

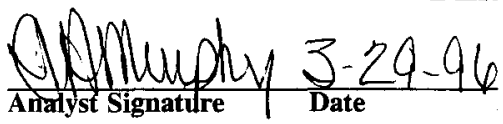

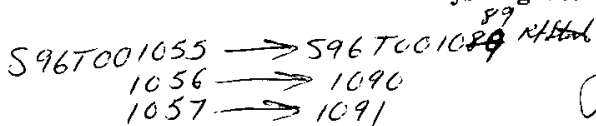

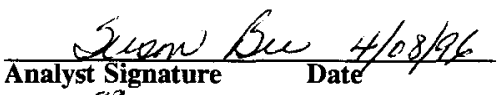

Analyst Signature

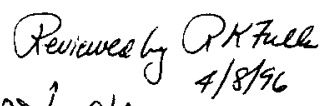

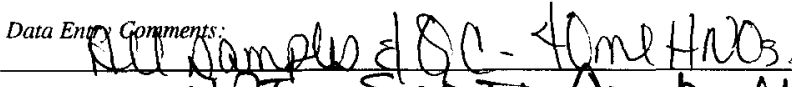

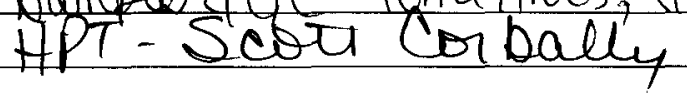

Units shown for QC (SPK \& STD) may not reflect the actual units. DL = Detection Limit, $S=$ Worklist Slot Number, $R=$ Replicate Number $A=$ Aliquot Code. 


\section{LABCORE Data Entry Template for Worklist\#}

Analyst: Instrument: FUS01 SN(6)

Worklist Comment: TK\#U-107,CORE 134,GRP\# 126,SEG 4A,4B,5B,FUSION RTS!

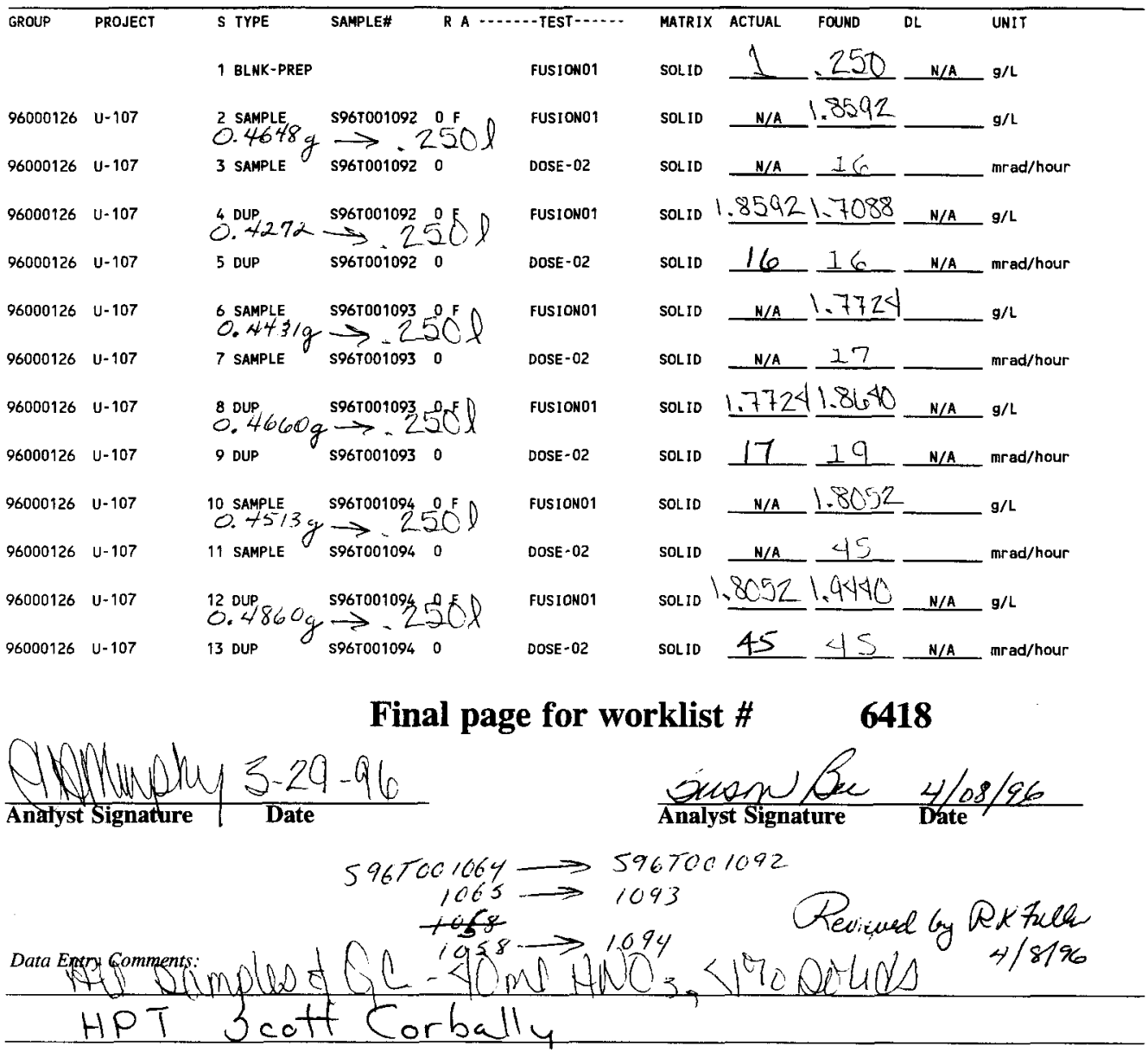

Units shown for $Q C$ (SPK \& STD) may not reflect the actual units. $D L=$ Detection Limit, $S=$ Worklist Slot Number, $R=$ Replicate Number, $A=$ Aliquot Code. 


\section{LABCORE Data Entry Template for Worklist\#}

Analyst:

Worklist Comment: TK\#U-107,CORE 134,GRP\# 126,SEG 5U,5A,6A,COMP FUSION RTS!

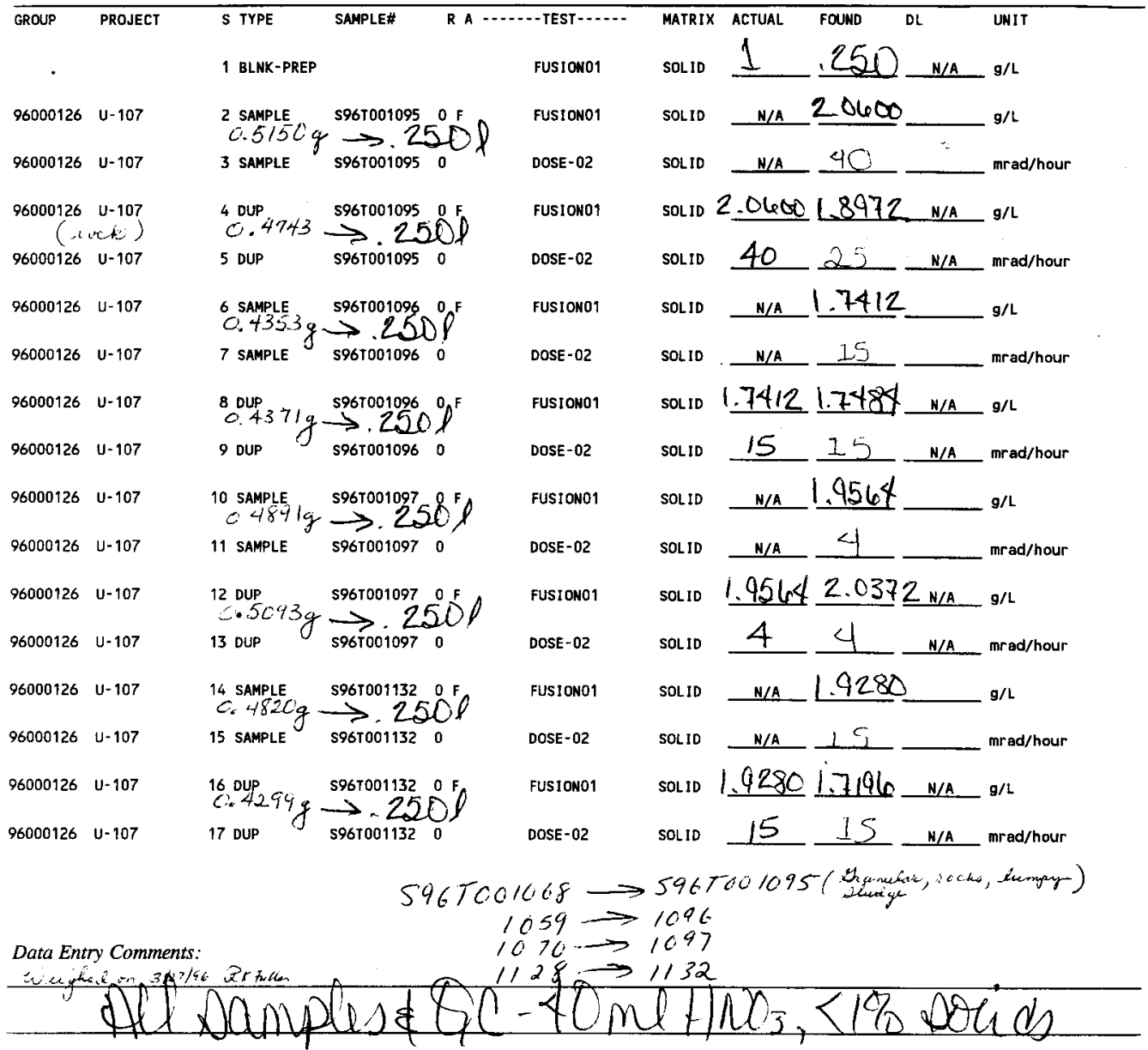

Units shown for QC (SPK \& STD) may not reflect the actual units. $D L=$ Detection Limit, $S=$ Worklist Slot Number, $R=$ Replicate Number, $A=$ Aliquot Code. 


\section{LABCORE Data Entry Template for Worklist\#}

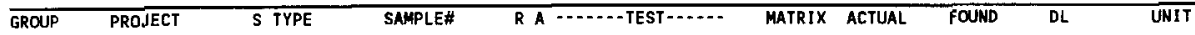

\section{Final page for worklist \# 6419}
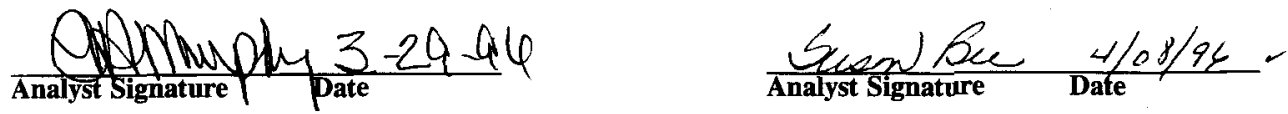

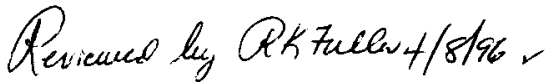

Data Entry Comments:

Units shown for $Q C$ (SPK \& STD) may not reflect the actual units. $D L=$ Detection Limit, $S=$ Worklist Slot Number, $R=$ Replicate Number, $A=$ Aliquot Code. 


\section{LABCORE Data Entry Template for Worklist\#}

Analyst: HNM Instrument: FUS01 SNilo555 Book \#

Method: LA-549-141 Rev/Mod $\mathcal{E}-\mathrm{O}$

Worklist Comment: TK\#U-107,CORE 129, SEG 3R,COMP FUSION01

RTS!

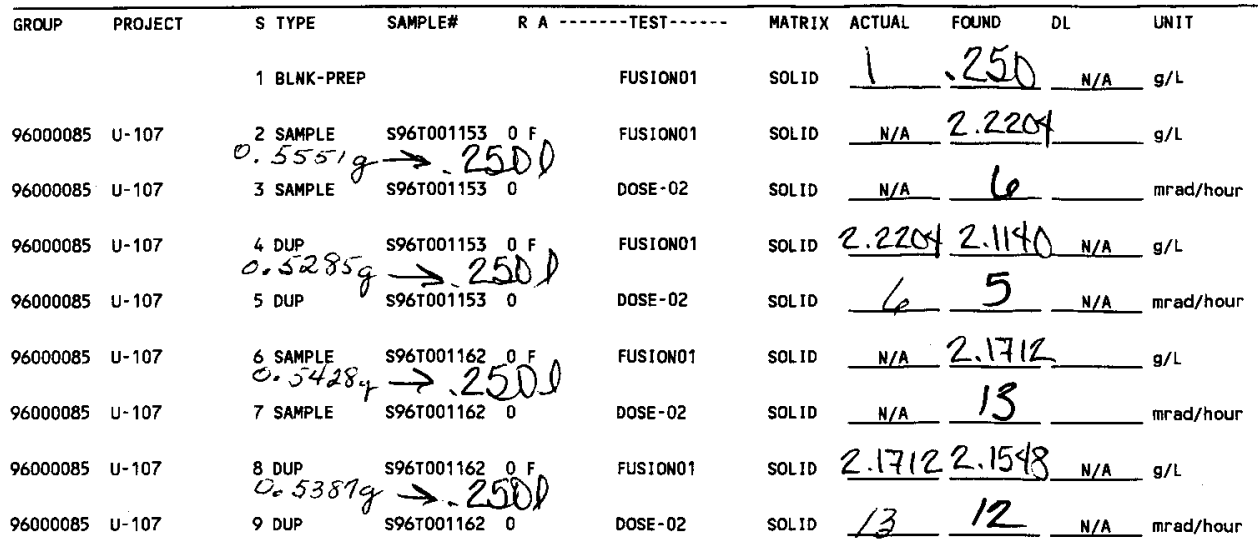

Units shown for $Q C$ (SPK \& STD) may not reflect the actual units. $D L=$ Detection Limit, $S=$ Worklist Slot Number, $R=$ Replicate Number, $A=$ Aliquot Code. 


\section{LABCORE Data Entry Template for Worklist\#}

Analyst: $\quad$ Instrument: FUS01___ Book \#

Method: LA-549-141 Rev/Mod F-O

Worklist Comment: U-107 C135 2RU QSA,2RU QSB,2A PUSH V FUSION01 SKB

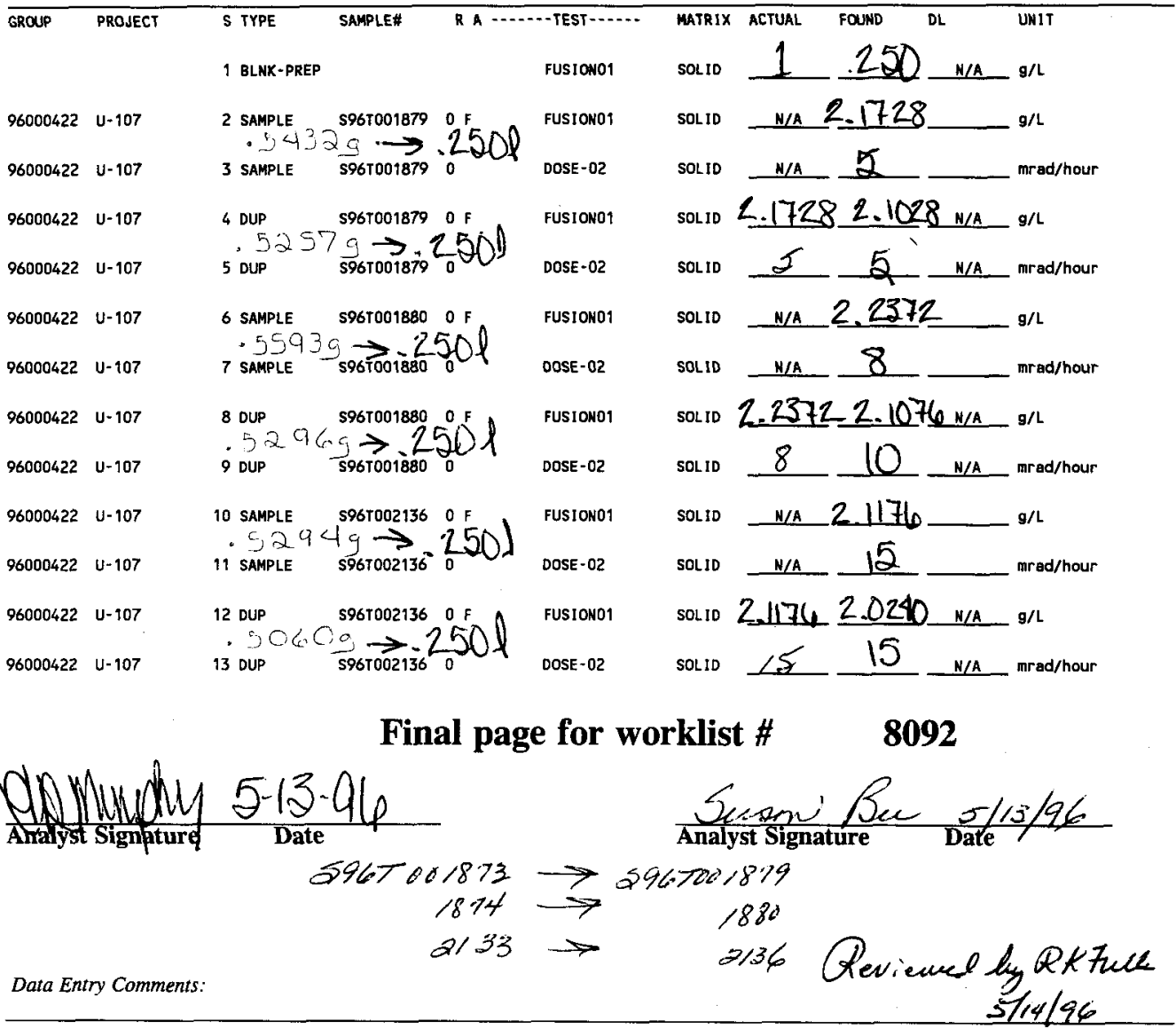

Units shown for $Q C$ (SPK \& STD) may not reflect the actual units. $D L=$ Detection Limit, $S=$ Worklist Slot Number, $R=$ Replicate Number, $A=$ Aliquot Code. 


\section{LABCORE Data Entry Template for Worklist\#}

Analyst: $\quad$ Instrument: FUS01 ines: Book \#

Method: LA-549-141 Rev/Mod F. P

Worklist Comment: U-107 C135 2S,1U,1R FUSION01 SKB

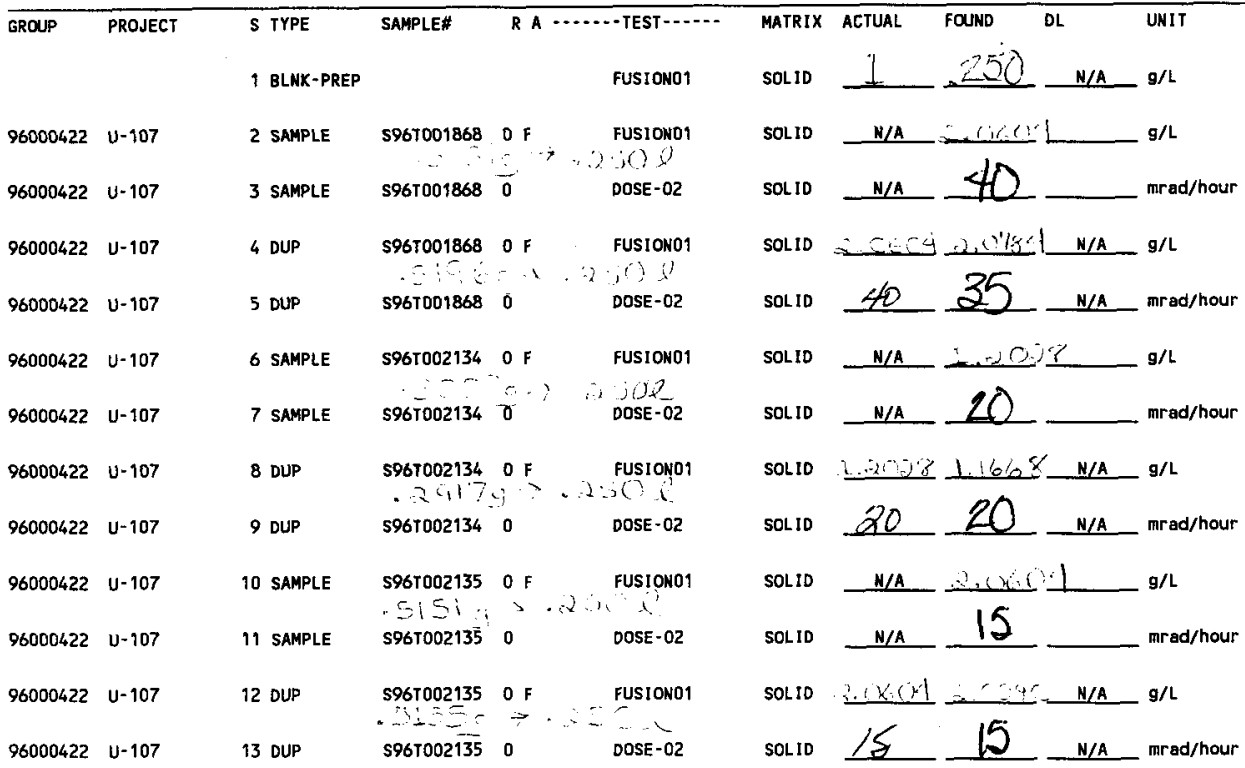

\section{Final page for worklist \# $\mathbf{8 1 0 2}$}

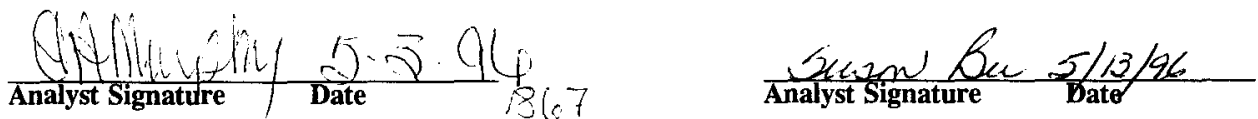

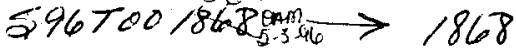

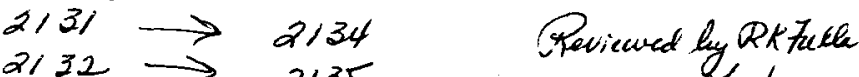

$$
\begin{aligned}
& 2 / 35
\end{aligned}
$$

Data Entry Comments:

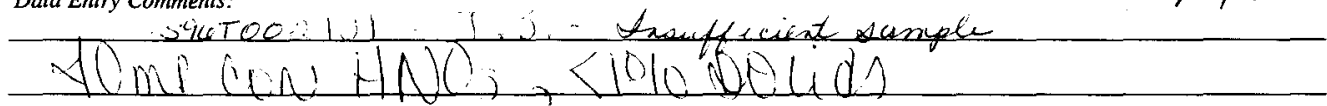

Units shown for $Q C$ (SPK \& STD) may not reflect the actual units. $D L=$ Detection Limit, $S=$ Worklist Slot Number,

$R=$ Replicate Number, $A=$ Aliquot Code. 


\section{LABCORE Data Entry Template for Worklist\#}

Analyst: VNMT Instrument: FUS01 SNIL355 Book \#

Method: LA-504-101 Rev/Mod $\varepsilon-0$

Worklist Comment: U-107 Core 129 Seg 2U,2L Water Digestion rkf

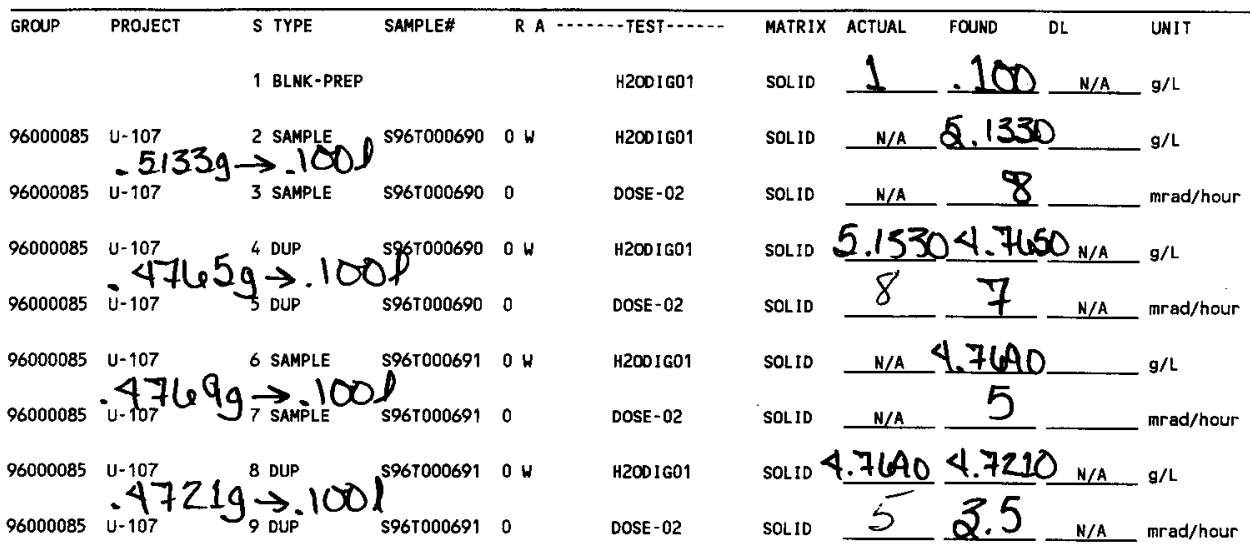

Final page for worklist \# $\mathbf{5 8 8 5}$ Andmenghy $3-5-96$

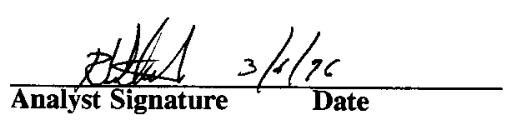

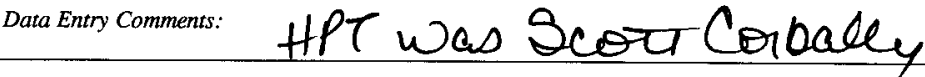

Units shown for $Q C$ (SPK \& STD) may not reflect the actual units. $D L=$ Detection Limit, $S=$ Worklist Slot Number, $R=$ Replicate Number, $A=$ Aliquot Code. 


\section{LABCORE Data Entry Template for Worklist\#}

\section{Analyst: UAm Instrument: FUS01 SN/6555 Book \#}

Method: LA-504-101 Rev/Mod $\&-0$

Worklist Comment: U-107 Core 129 Seg 3U,3L Water Digestion rkf

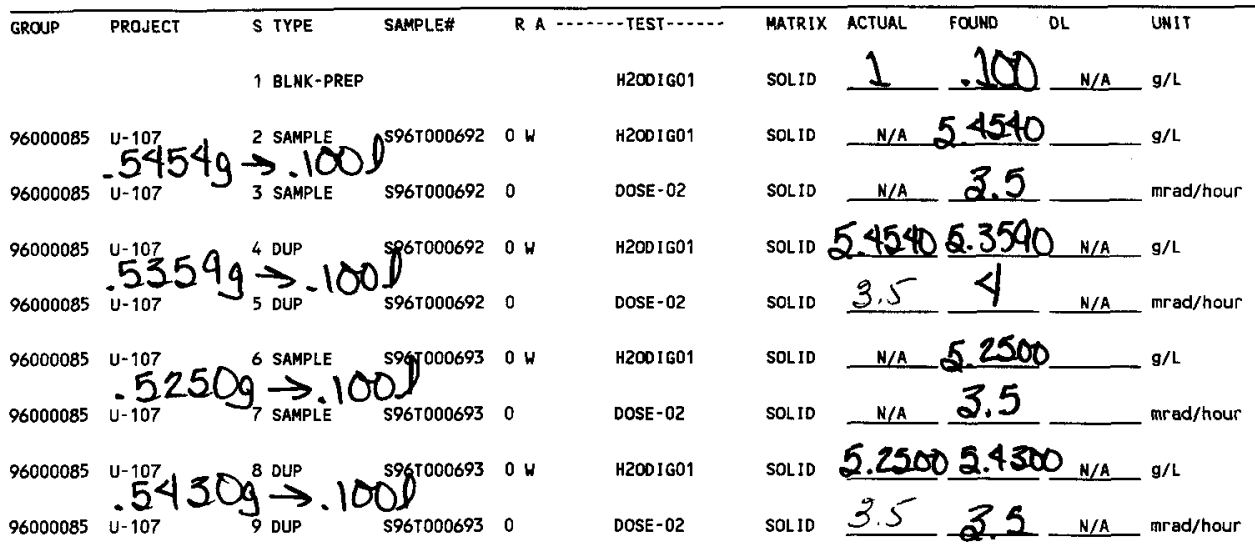

\section{Final page for worklist \# 5886}

SNmwotyy $3-5-96$

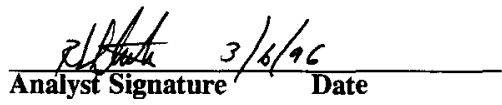

$$
\begin{aligned}
& 650 \rightarrow 692 \\
& 653 \rightarrow 693
\end{aligned} \quad \begin{array}{r}
\text { Pevieued by Xk full } \\
3 / 6 / 96
\end{array}
$$

\section{Data Entry Comments: HPT was Scou Combally}

Units shown for $Q C$ (SPK \& STD) may not reflect the actual units. $D L=$ Detection Limit, $S=$ Worklist Slot Number,

$R=$ Replicate Number, $A=$ Aliquot Code. 


\section{LABCORE Data Entry Template for Worklist\#}

Analyst: Un Instrument: H2001 Wula359 Book \#

Method: LA-504-101 Rev/Mod $\mathbf{E}-\mathrm{D}$

Worklist Comment: TK\#U-107,CORE 134,GRP\# 126,SEG 2,3, H2ODIG RTS!

\begin{tabular}{|c|c|c|c|c|c|c|c|c|c|c|}
\hline \multirow[t]{2}{*}{ GROUP } & PROJECT & S TYPE & SAMPLE\# & R A & - & MATRIX & ACTUAL & FOUND & $\mathrm{DL}$ & UNIT \\
\hline & & 1 BLNK-PREP & & & H2001G01 & SOL ID & & & & $\mathbf{g} / \mathbf{1}$ \\
\hline 96000126 & $U-107$ & 2 SAMPLE & $596 \mathrm{~T}$ & & H2ODI G01 & SOLID & $N / A$ & & & $g / L$ \\
\hline 96000126 & $U-107$ & 3 SAMPLE & S96T001071 & 0 & DOSE-02 & SOLID & $N / A$ & & & mrad/hour \\
\hline 96000126 & $U-107$ & 4 DUP & S96T00107 & & H2ODIG01 & SOLIDE & & & & $g / L$ \\
\hline 96000126 & $U-107$ & 5 DUP & s96T001071 & 0 & DOSE-02 & SOLID & & & & $\mathrm{mrad} / \mathrm{hour}$ \\
\hline 96000126 & $u-107$ & 6 SAMPLE & s96T001072 & & H2ODIG01 & SOLID & H/A & & & $\mathrm{g} / \mathrm{L}$ \\
\hline 96000126 & $u-107$ & 7 SAMPLE & S96T001072 & 0 & DOSE-02 & SOLID & $N / A$ & & & $\mathrm{mrad} /$ hour \\
\hline 96000126 & $u-107$ & 8 DUP & \$96T001072 & & H2ODIG01 & SOL ID & & & & $9 / 1$ \\
\hline 96000126 & $U-107$ & 9 DUP & S96T001072 & 0 & DoSE- 02 & SOL ID & & & & $\mathrm{mrad} /$ hour \\
\hline
\end{tabular}

\section{Final page for worklist \# 6422}
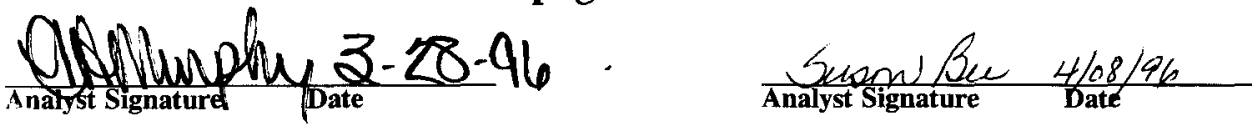

$$
\begin{aligned}
& 5967001055 \rightarrow 5967001071 \\
& 1056 \rightarrow 1072
\end{aligned}
$$

\section{Data Entry Comments: HPT-Scre Corkaley}

Units shown for $Q C$ (SPK \& STD) may not reflect the actual units. $D L=$ Detection Limit, $S=$ Worklist Slot Number, $R=$ Replicate Number, $A=$ Aliquot Code. 


\section{LABCORE Data Entry Template for Worklist\#}

\section{Analyst: Instrument: ACD01SWle 55 Book}

Method: LA-504-101 Rev/Mod $\mathcal{-} \mathrm{O}$

Worklist Comment: TK\#U-107,CORE 134,GRP\# 126,SEG 4L,4A, H2ODIG RTS!

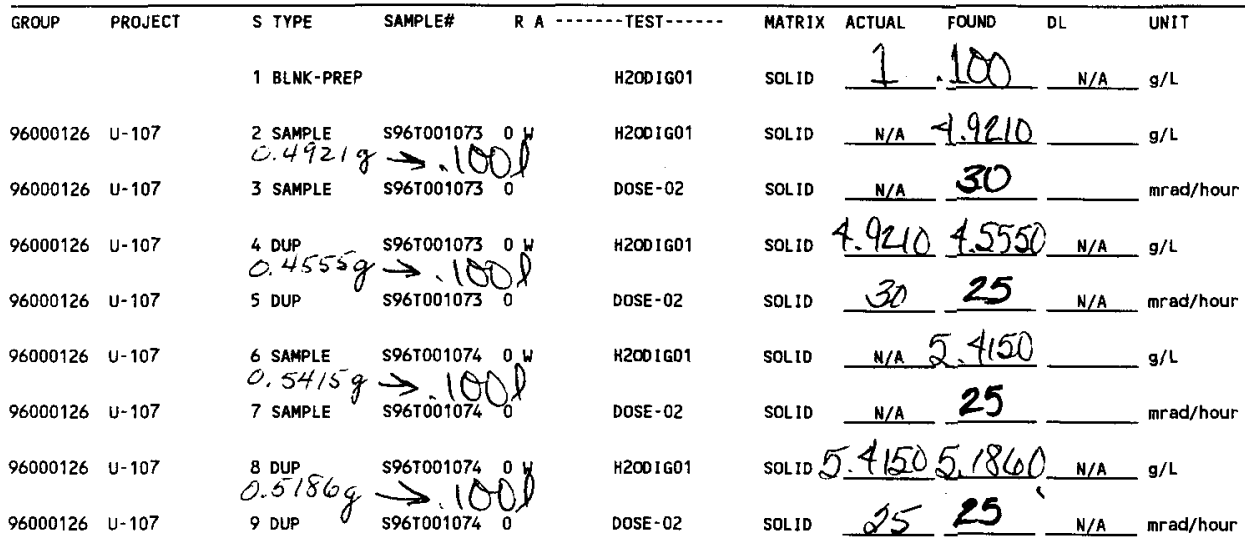

\section{Final page for worklist \#}

6423

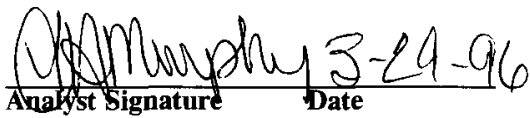

Mxpmoshy $\underset{\text { Date }}{3}-29-96$

$$
\begin{aligned}
& 596700105 \rightarrow 59670010 \\
& 1064 \Rightarrow 1074 \\
& \text { pittat }
\end{aligned}
$$

Data Entry Comments:

\section{HPT-Scou Corbally}

Units shown for $Q C$ (SPK \& STD) may not reflect the actual units. $D L=$ Detection Limit, $S=$ Worklist Slot Number,

$R=$ Replicate Number, $A=$ Aliquot Code. 
worklistrpt Version 2.1 05/15/95

WHC-SD-WM-DP-184, REV. I

$03 / 13 / 9615: 15$

LABCORE Data Entry Template for Worklist\#

Page: $\quad$ I

6425

Analyst: $\quad$ Instrument: $\mathrm{H} 2001 \mathrm{MN} / \mathrm{W} 5 \mathrm{~S}$ Book \#

Method: LA-504-101 Rev/Mod C-O

Worklist Comment: TK\#U-107, CORE 134,GRP\# 126,SEG 4B,5B, H2ODIG RTS!

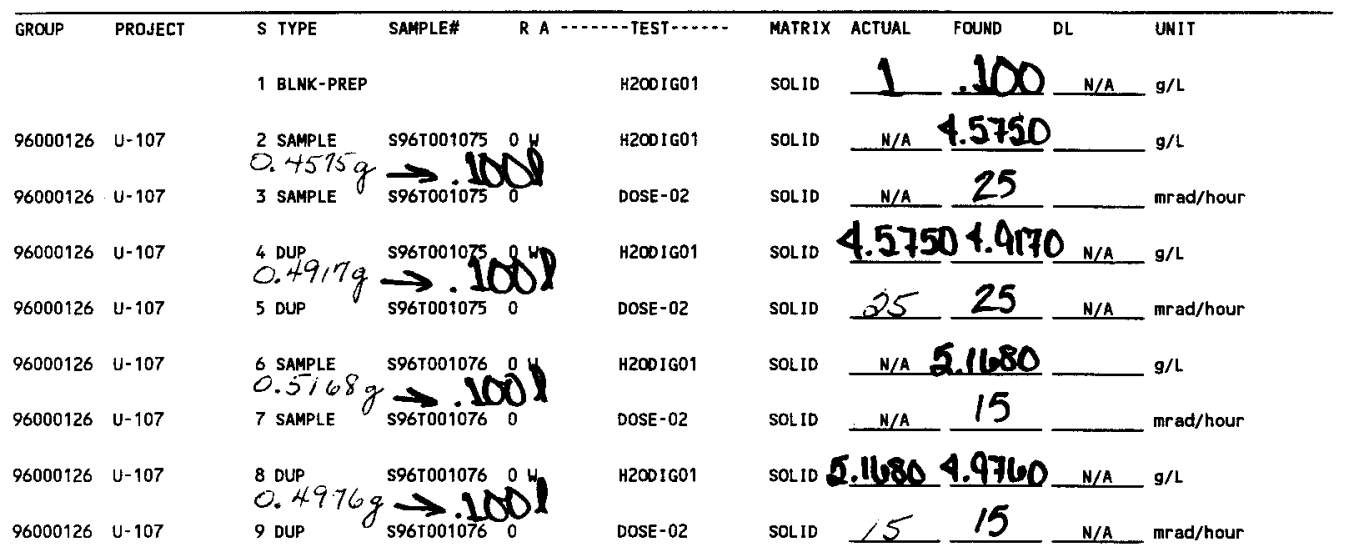

Final page for worklist \# 6425

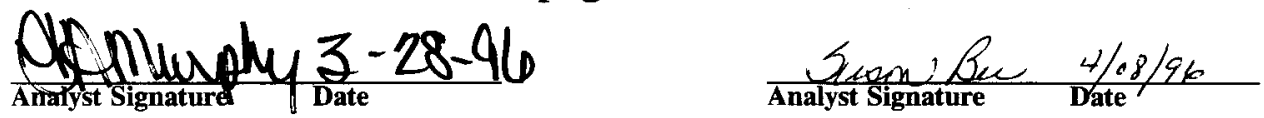

$$
\begin{aligned}
& 5 \% 7001065 \rightarrow 5467001075 \\
& 1058 \rightarrow 1076 \text { Pevieundly Przalle } \\
& \text { 4/816 }
\end{aligned}
$$

Data Entry Compute - Scout Corbally

Units shown for QC (SPK \& STD) may not reflect the actual units. DL = Detection Limit, $S=$ Worklist Slot Number, $R=$ Replicate Number, $A=$ Aliquot Code.

140 


\section{LABCORE Data Entry Template for Worklist\#}

Analyst:

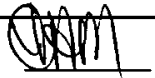

Instrument: ACD01SQ (16355

Book \#

Method: LA-504-101 Rev/Mod $\mathcal{E}-0$

Worklist Comment: TK\#U-107,CORE 134,GRP\# 126,SEG 5U,5A, H2ODIG RTS!

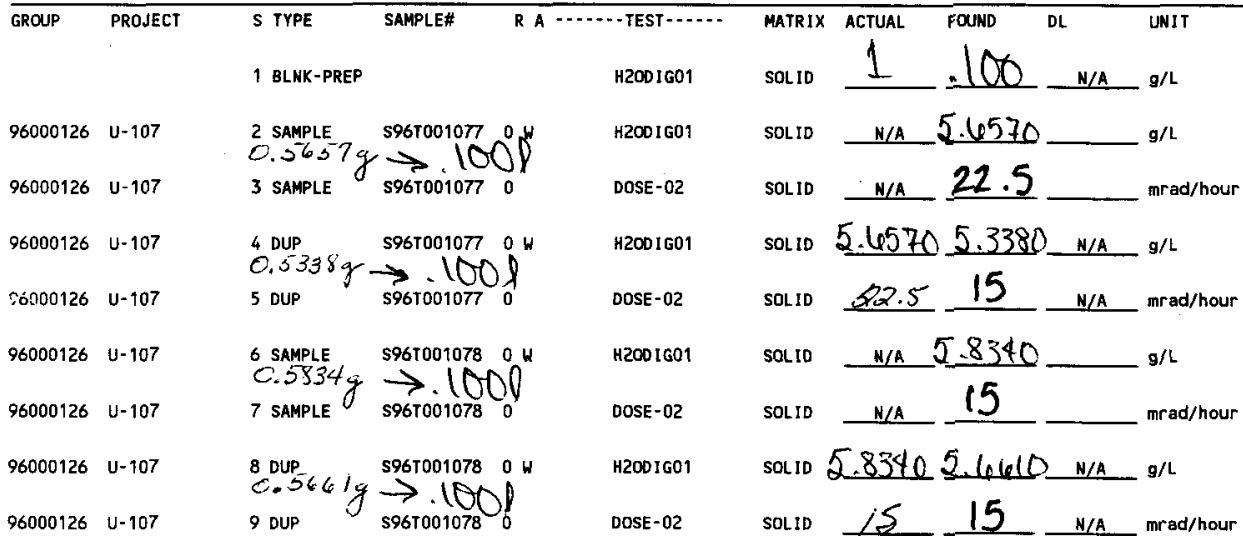

\section{Final page for worklist \#}

6426

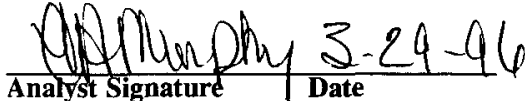

Analyst Signature 1 Date

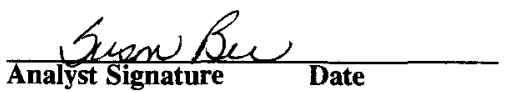

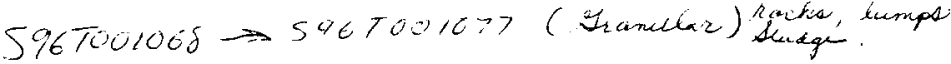

$$
\begin{aligned}
& 105 y \rightarrow 1078 \\
& \text { PHets } \\
& \text { Revieured loy Przule } \\
& 4 / 8196
\end{aligned}
$$

Data Entry Comments:

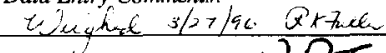

\section{HPT-Scou Corkally}

Units shown for QC (SPK \& STD) may not reflect the actual units. $D L=$ Detection Limit, $S=$ Worklist Slot Number,

$R=$ Replicate Number, $A=$ Aliquot Code. 


\section{LABCORE Data Entry Template for Worklist\#}

Analyst: Unf Instrument: H2001 SNllo355 Book \#

Method: LA-504-101 Rev/Mod $\mathcal{L}$

Worklist Comment: TK\#U-107,CORE 134,GRP\# 126,SEG 6A,COMP H2ODIG RTS!

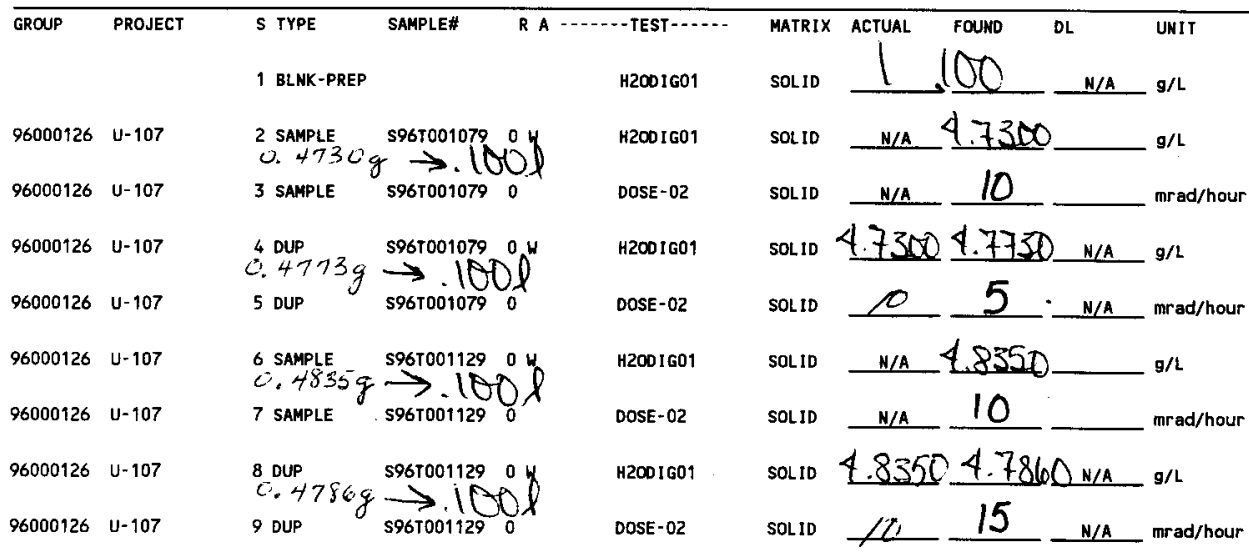

Final page for worklist \#
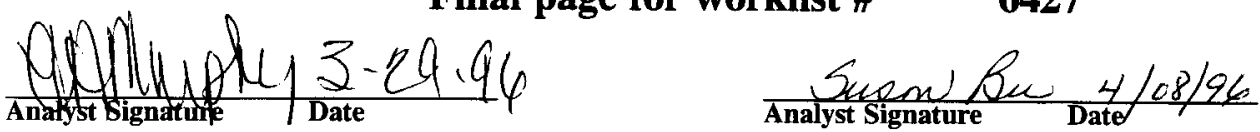

$\begin{aligned} 5967001070 & \rightarrow 5967001079 \text { (Dequace-sadge) } \\ 1128 & \rightarrow 1129\end{aligned}$

Rtbat

Peviruved log PK zulk
$4 / 8196$

Data Entry Comments: HPT - Scout Colbally

Units shown for $Q C$ (SPK \& STD) may not reflect the actual units. $D L=$ Detection Limit, $S=$ Worklist Slot Number,

$R=$ Replicate Number, $A=$ Aliquot Code. 


\section{LABCORE Data Entry Template for Worklist\#}

Analyst: Nifll Instrument: H2001 SWl6359 Book \#-_

Method: LA-504-101 Rev/Mod $\&-\mathrm{C}$

Worklist Comment: TK\#U-107,CORE 129,SEG 3R,COMP H2ODIG01 RTS!

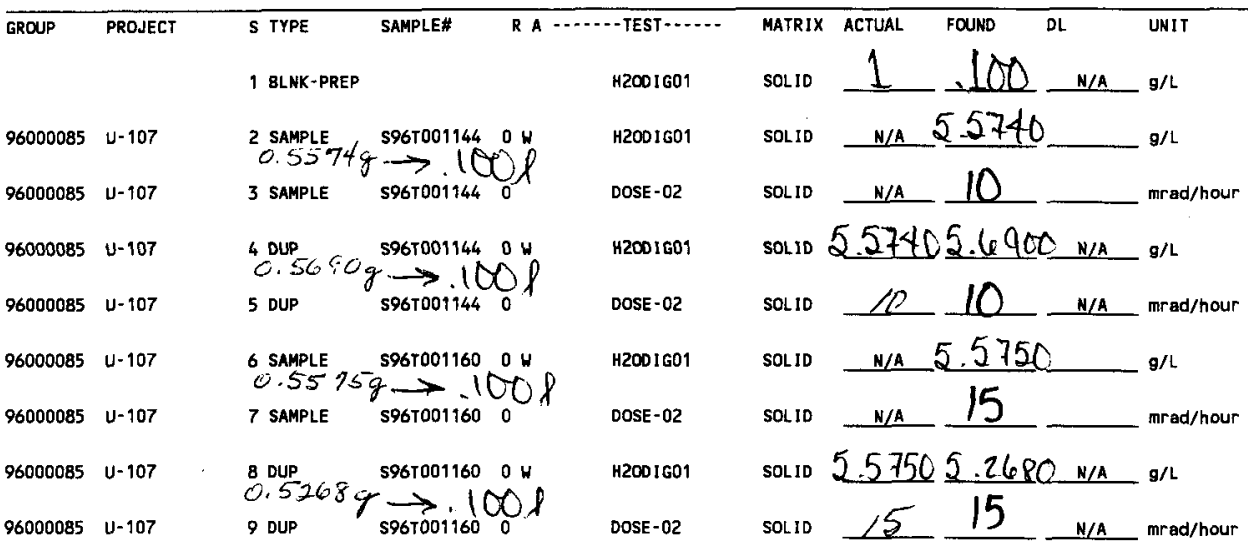

\section{Final page for worklist \#}

6537
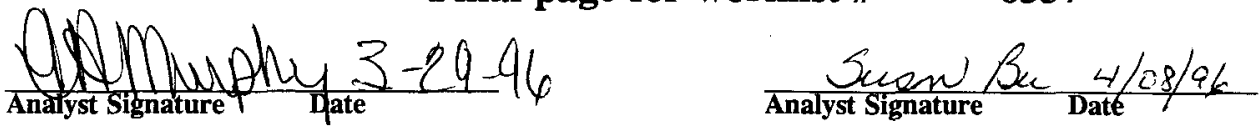

$$
\begin{aligned}
& S 467001143 \longrightarrow S 46 T 00114 \\
& S 967001154 \longrightarrow S 96 T 001160
\end{aligned}
$$

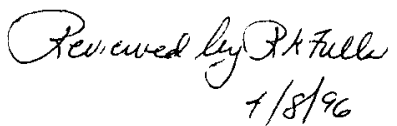

\section{Dat Entry Commantip T-Scour Corbally}

Units shown for $Q C$ (SPK \& STD) may not reflect the actual units. $D L=$ Detection Limit, $S=$ Worklist Slot Number,

$R=$ Replicate Number, $A=$ Aliquot Code. 


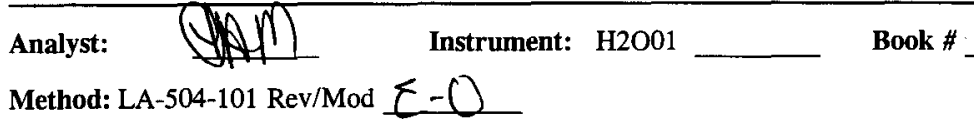

Worklist Comment: U-107 C135 2RA,2RB,2A H2ODIG01 SKB

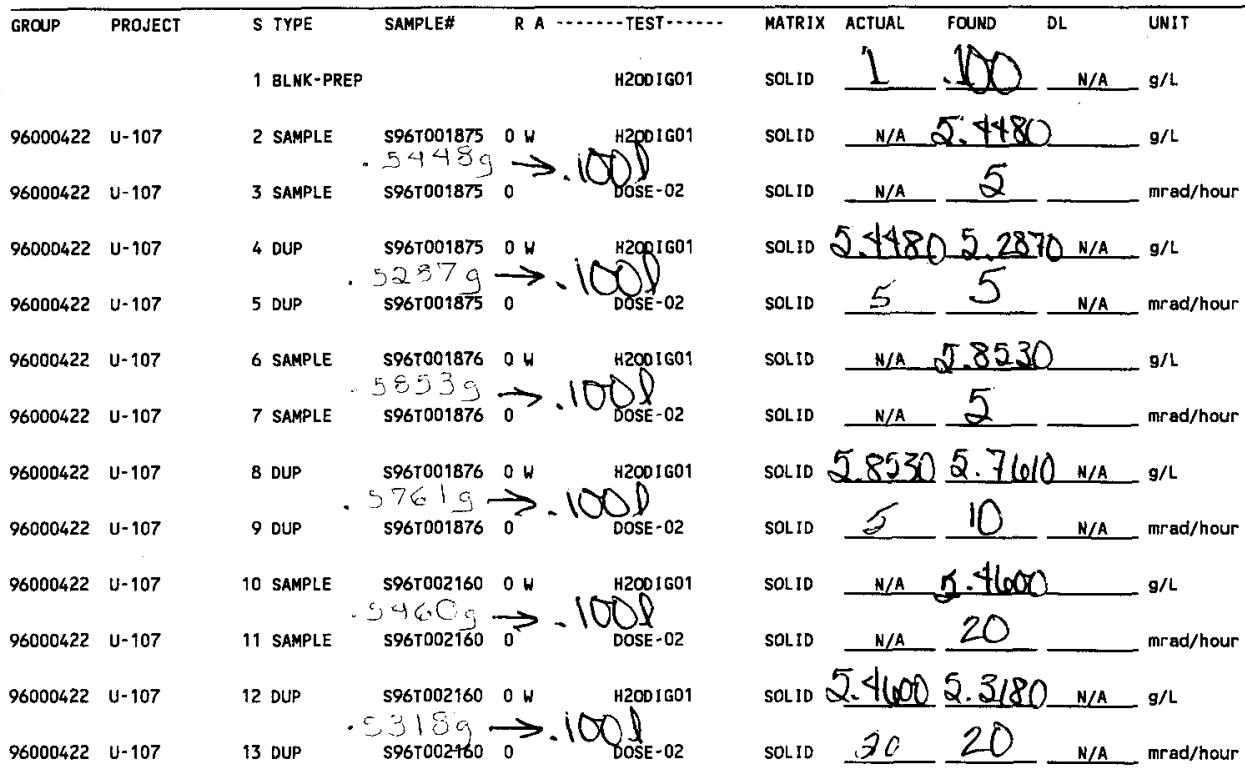

\section{Final page for worklist \# $\quad 8104$}

\section{AAmushy $5-13-96$ Antilyst Signature}

Data Entry Comments:

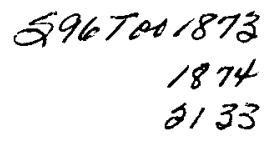

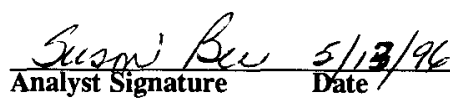

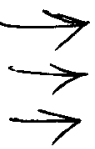

$596700 / 875$

Units shown for QC (SPK \& STD) may not reflect the actual units. $D L=$ Detection Limit, $S=$ Worklist Slot Number,

$R=$ Replicate Number, $A=$ Aliquot Code. 


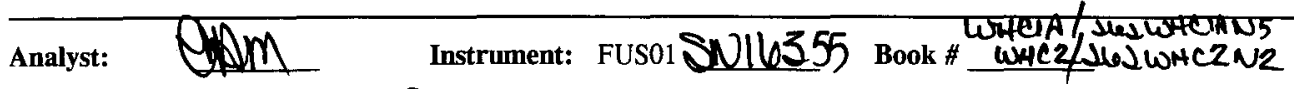

Method: LA-505-159 Rev/Mod $D-O$

Worklist Comment: U-107 Core 129 Seg 2U,2L,3U,3L Acid Digestion rkf

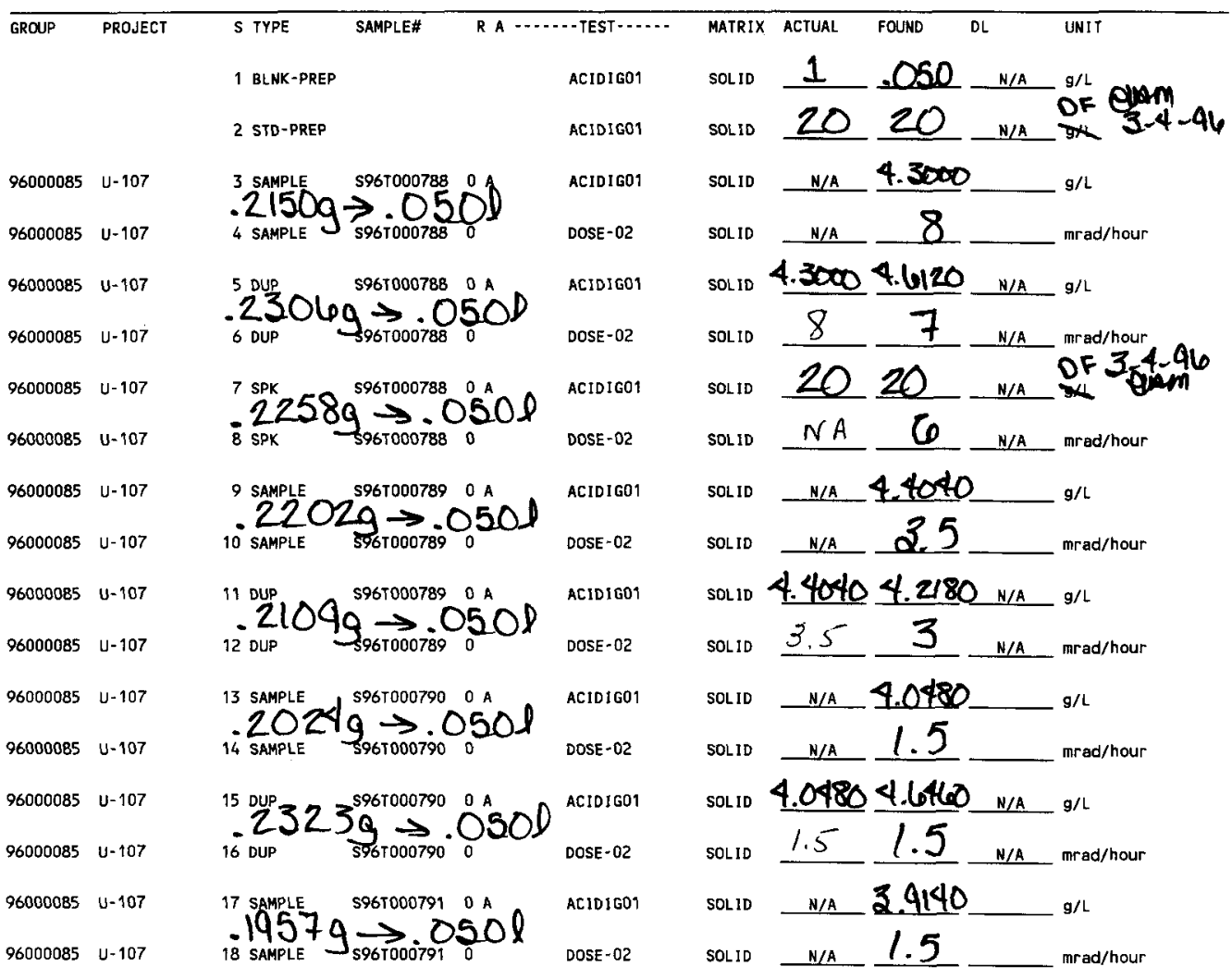

Data Entry Comments:

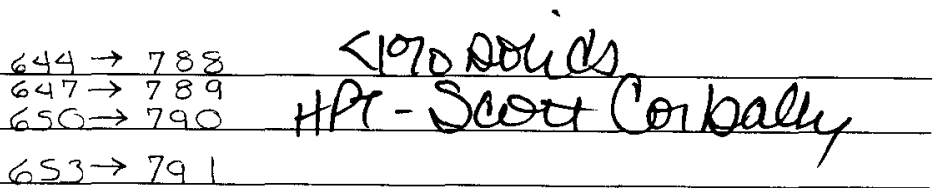

Units shown for QC (SPK \& STD) may not reflect the actual units. DL = Detection Limit, $S=$ Worklist Slot Number,

$R=$ Replicate Number, $A=$ Aliquot Code. 
worklistrpt Version $2.105 / 15 / 95$

WHC-SD-WM-DP-184, REV. 1

Page: 2

02/25/96 16:02

LABCORE Data Entry Template for Worklist\#

5884

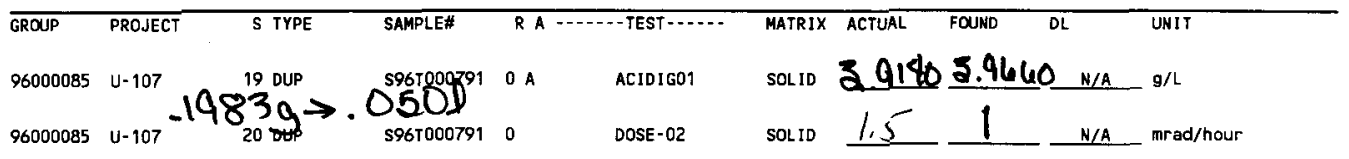

Final page for worklist \# $\quad \mathbf{5 8 8 4}$

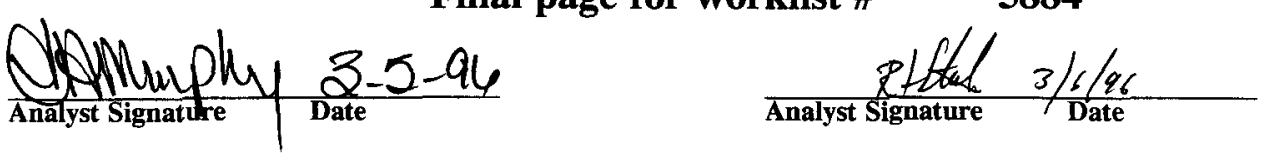

$$
\begin{aligned}
& 644 \rightarrow 788 \\
& 647 \rightarrow 789 \\
& 650 \rightarrow 790 \\
& 650 \rightarrow 791
\end{aligned}
$$

Rescued by RKFulla

$$
3 / 4 / 46
$$

Data Entry Comments:

Units shown for $Q C$ (SPK \& STD) may not reflect the actual units. $D L=$ Detection Limit, $S=$ Worklist Slot Number, $R=$ Replicate Number, $A=$ Aliquot Code.

146 


\section{LABCORE Data Entry Template for Worklist\#}

\section{Analyst:

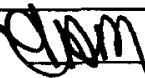 \\ Instrument: ACD0SNU1355

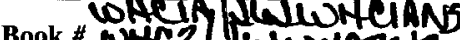

Method: LA-505-159 RevhMod $2-0$

Worklist Comment: TK\#U-107,CORE 134,GRP\# 126,SEG 2,3,4L,4A,4B ACIDIG RTS!

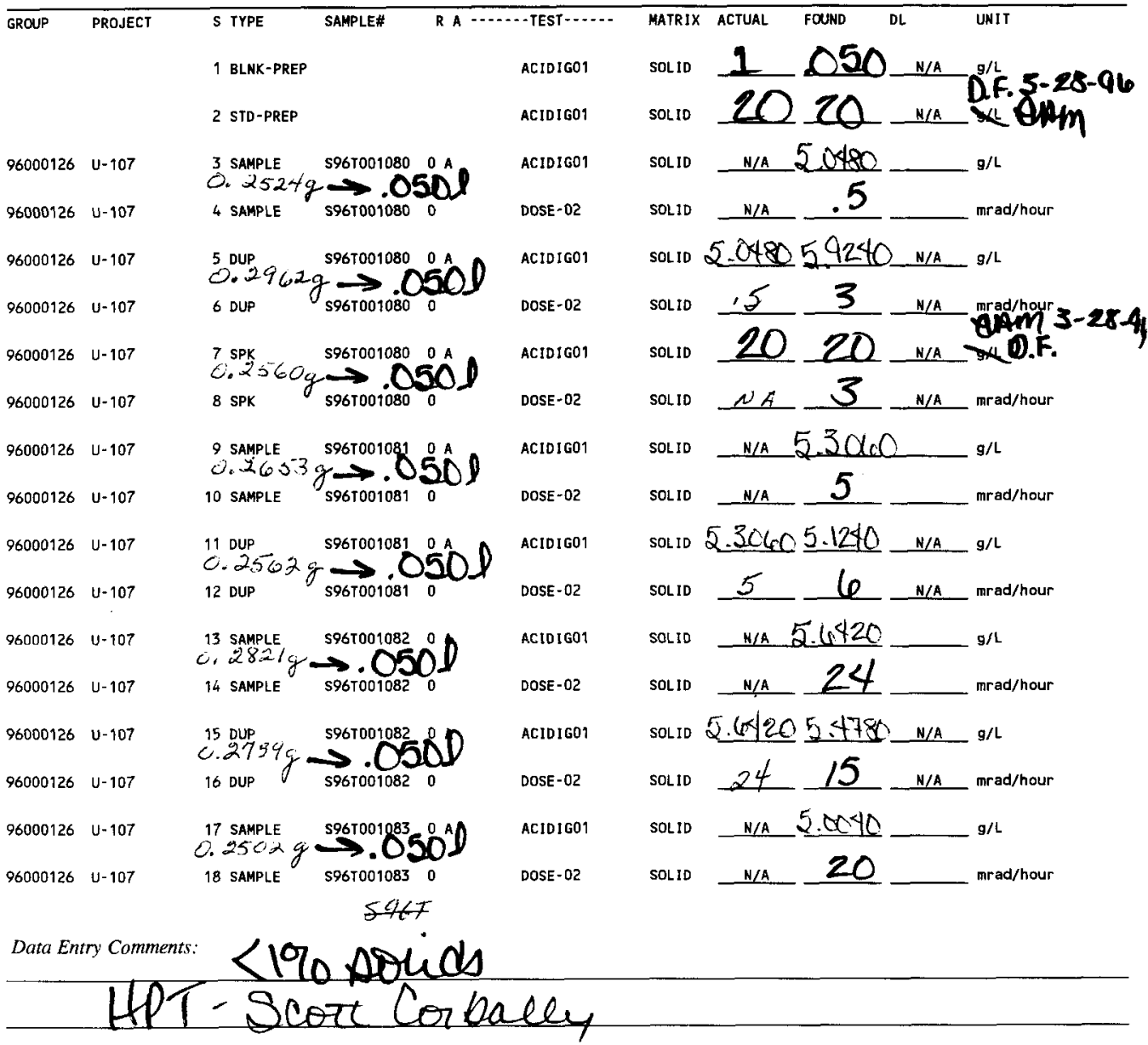

Units shown for $Q C$ (SPK \& STD) may not reflect the actual units. $D L=$ Detection Limit, $S=$ Worklist Slot Number,

$R=$ Replicate Number, $A=$ Aliquot Code. 


\section{LABCORE Data Entry Template for Worklist\#}

\begin{tabular}{|c|c|c|c|c|c|c|c|c|c|c|}
\hline$\overline{\text { GROUP }}$ & PROJECT & $\overline{S \text { TYPE }}$ & SAMPLE\# & $\overline{R A}$ & - -TEST $\cdots . . . .$. & MATRIX & ACTUAL & FOUND & & UNIT \\
\hline 96000126 & U-107 & 19 DUP & & & ACIDIGOI & SOLID & 2.0040 & 5.2220 & N/A & $g / L$ \\
\hline 96000126 & U-107 & 20 DUP & s96T00108 & 0 & DOSE-02 & SOLID & 15 & 2 & N/A & $\mathrm{mrad} /$ hour \\
\hline 96000126 & $U-107$ & $\begin{array}{l}21 \text { SAMPLE } \\
0.2828\end{array}$ & S96T001C & & ACIDIG01 & SOLID & N/A & & & $g / L$ \\
\hline 96000126 & $U-107$ & 22 SAMPLE & 596700108 & 0 & DOSE-02 & SOLID & N/A & & & $\mathrm{mrad} /$ hour \\
\hline 96000126 & U-107 & 23 oup 809 & S96T0010 & & ACIDIG01 & SOLID & 2.1056 & 10180 & N/A & $g / L$ \\
\hline 96000126 & $U \cdot 107$ & 24 DUP & 596700108 & & DOSE-02 & SOLID & 12 & 2 & N/A & $\mathrm{mrad} /$ hour \\
\hline
\end{tabular}

\section{Final page for worklist \# $\quad 6420$}
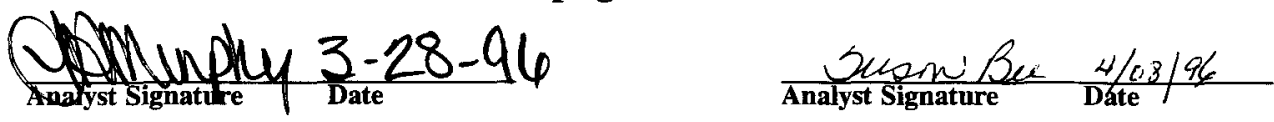

$$
\begin{aligned}
5967001055 \rightarrow 5967001080 \\
1056 \rightarrow 1081 \\
1057 \rightarrow 1082 \\
1064 \rightarrow 1053 \\
1065 \rightarrow 1054 \quad \text { Rlftort }
\end{aligned}
$$

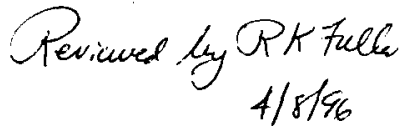


Analyst: Method: LA-505-159 Rev/Mod D-O

Worklist Comment: TK\#U-107,CORE 134,GRP\# 126,SEG 5B,5U,5A,6A,COMP ACIDIG RTS!

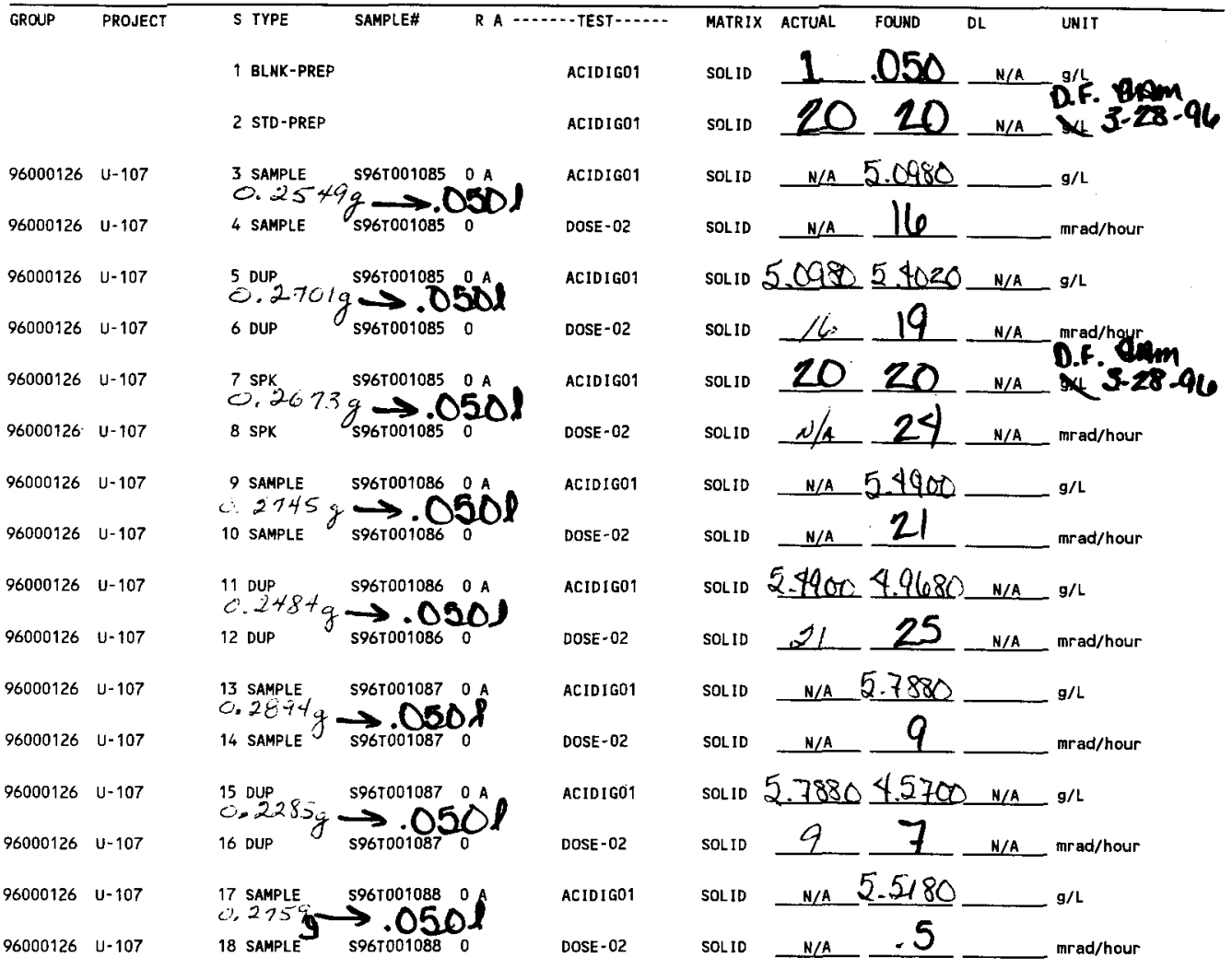

Data Entry Comments:

\section{Pro Dolids} HPT-Scot Corbally

Units shown for $Q C$ (SPK \& STD) may not reflect the actual units. $D L=$ Detection Limit, $S=$ Worklist Slot Number, $R=$ Replicate Number, $A=$ Aliquot Code. 


\section{LABCORE Data Entry Template for Worklist\#}

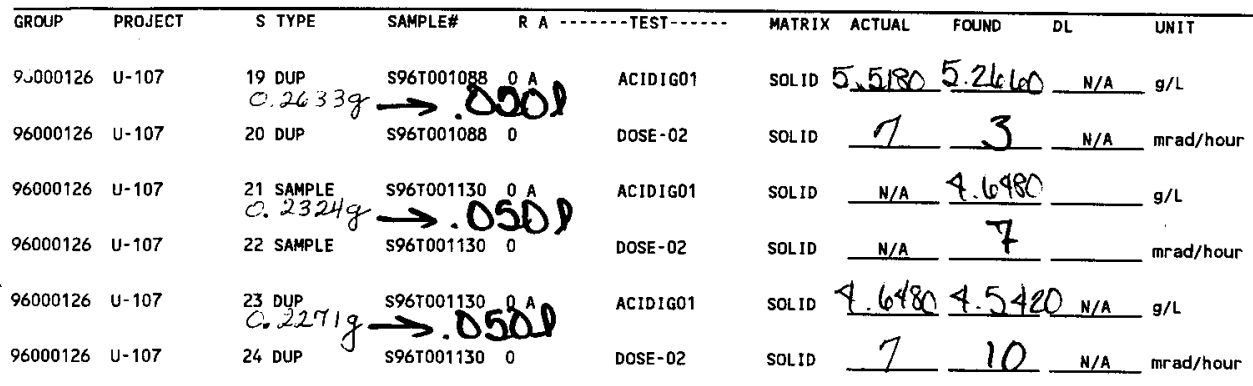

Final page for worklist \#

6421

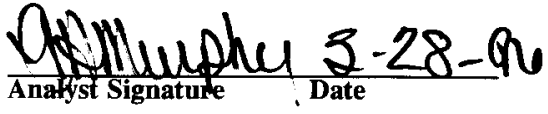

$$
\begin{aligned}
5967001058 & \rightarrow 5967001085 \\
1068 & \rightarrow 1086 \text { (GeAvucm } \\
1059 & \rightarrow 1087 \\
1070 & \rightarrow 1088 \\
1128 & \rightarrow 1130
\end{aligned}
$$

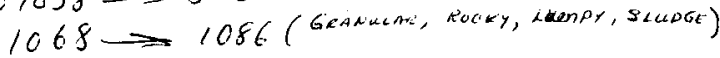

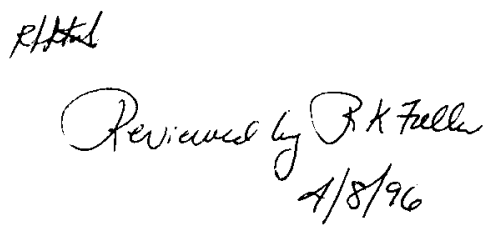

Data Entry Comments:

Units shown for $Q C$ (SPK \& STD) may not reflect the actual units. $D L=$ Detection Limit, $S=$ Worklist Slot Number, $R=$ Replicate Number, $A=$ Aliquot Code. 
worklistrpt Version 2.1 05/15/95

WHC-SD-WM-DP-184, REV. I

$03 / 19 / 9614.54$

LABCORE Data Entry Template for Worklist\#

Page: $\quad I$

6536

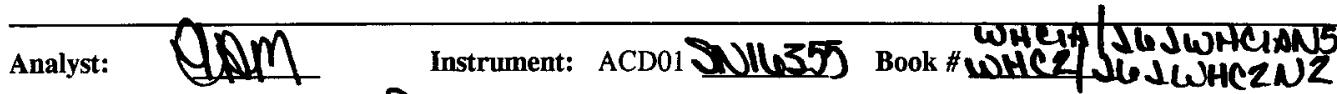

Method: LA-505-159 Rev/Mod $1-0$

Worklist Comment: TK\#U-107,CORE 129,SEG 3R,COMP ACIDIG01 RTS!

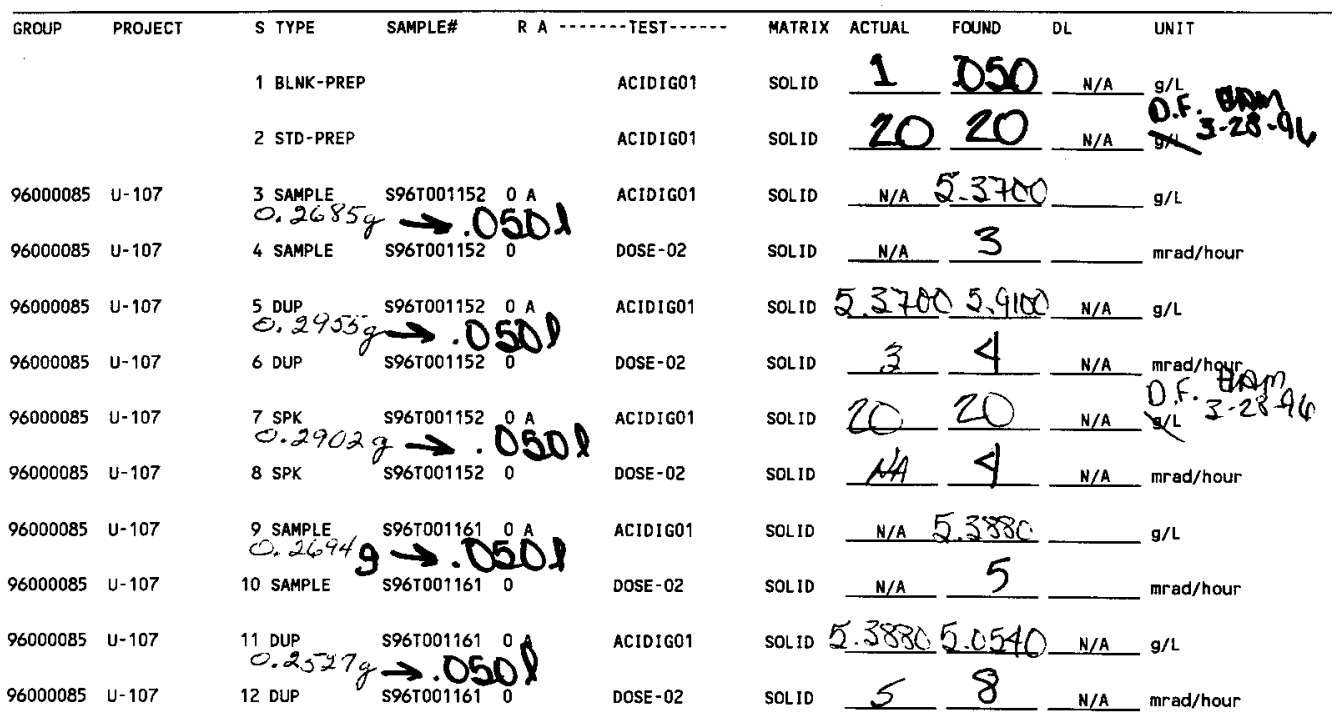

Final page for worklist \# $\quad 6536$

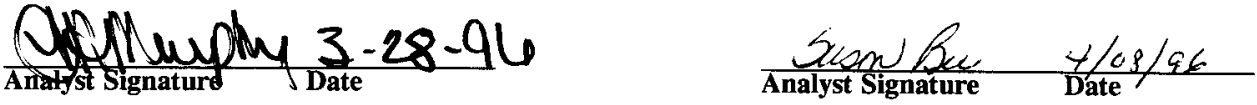

$$
\begin{aligned}
& \text { S46.50.143 } \rightarrow \text { Sc. T61152 } \\
& \text { S46T00 1154 } \rightarrow \text { 544T }
\end{aligned}
$$

Data Entry Comments: $<$ To Dor ids

HPT-Scou Corbally

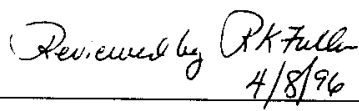

Units shown for $Q C$ (SPK \& STD) may not reflect the actual units. DL = Detection Limit, $S=$ Worklist Slot Number, $R=$ Replicate Number, $A=$ Aliquot Code.

151 


\section{LABCORE Data Entry Template for Worklist\#}

\section{Analyst: YUWM Instrument: ACD01_ Book \# \\ Method: LA-505-159 Rev/Mod

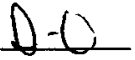

\section{Worklist Comment: U-107 C135 2RA,2RB,2A ACIDIG01 SKB}

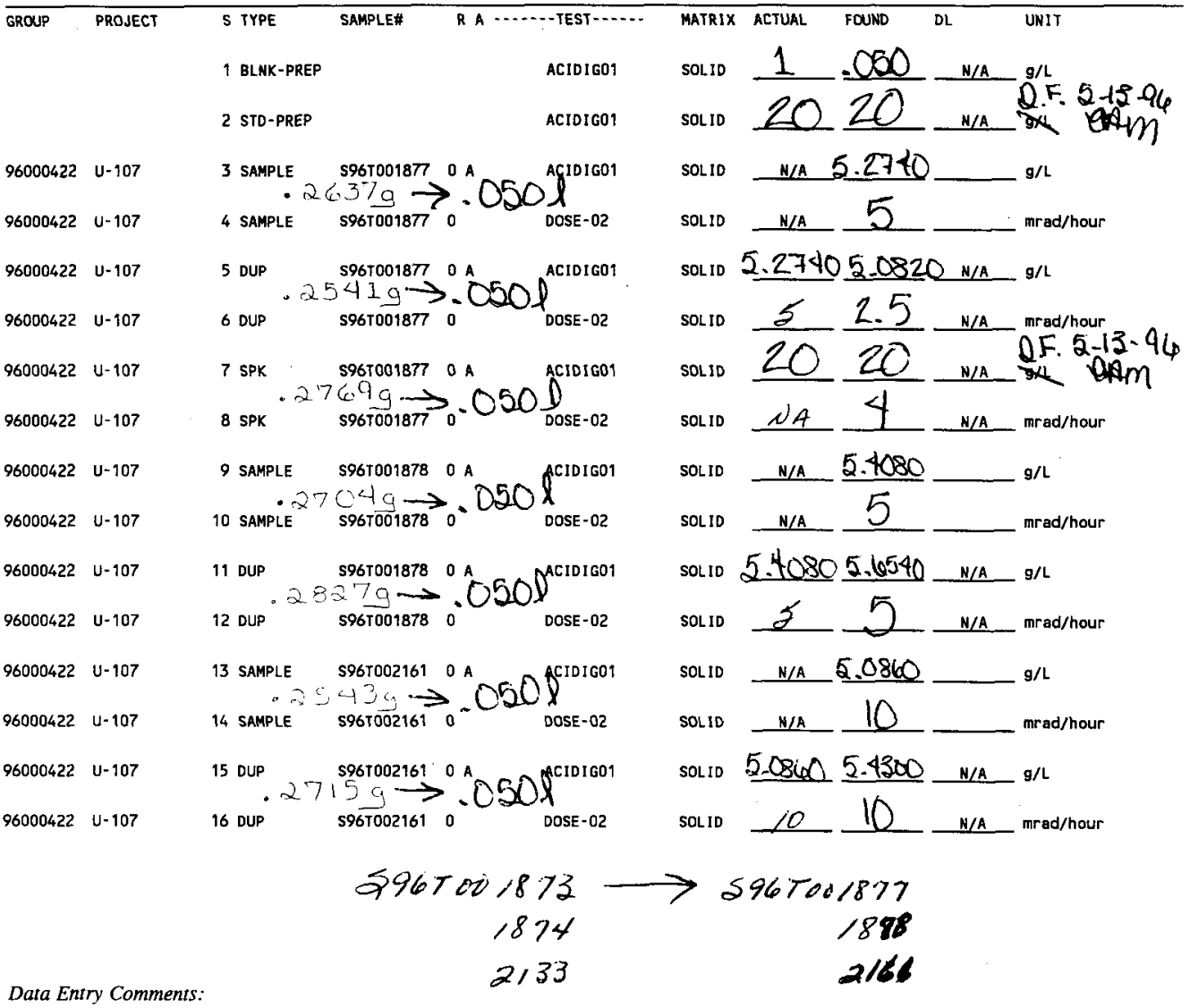

Units shown for $Q C$ (SPK \& STD) may not reflect the actual units. $D L=$ Detection Limit, $S=$ Worklist Slot Number, $R=$ Replicate Number, $A=$ Aliquot Code. 
WHC-SD-WM-DP-184, REV. I

Page: 2

worklistrpt Version 2.1 05/15/95

04/26/96 13:00

LABCORE Data Entry Template for Worklist\#

8103

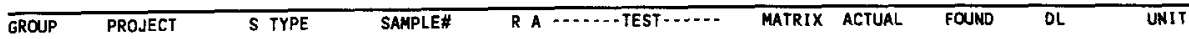

\section{Final page for worklist \# $\quad 8103$}

$\frac{\text { MNDWhath }}{\text { Analyst Signatore }} \frac{\overline{2}+3-96}{\text { Date }}$

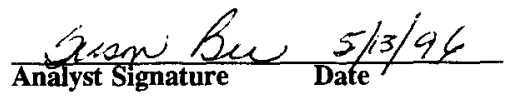




\section{LABCORE Data Entry Template for Worklist\#}

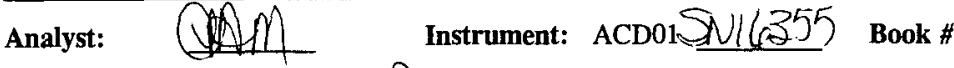 \\ Method: LA-505-159 Rev/Mod D-C}

Worklist Comment: U-107 C134 S2,S3,S4L,S4QA,S4QB ACIDIG01 SKB

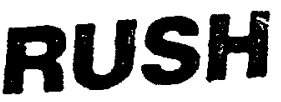

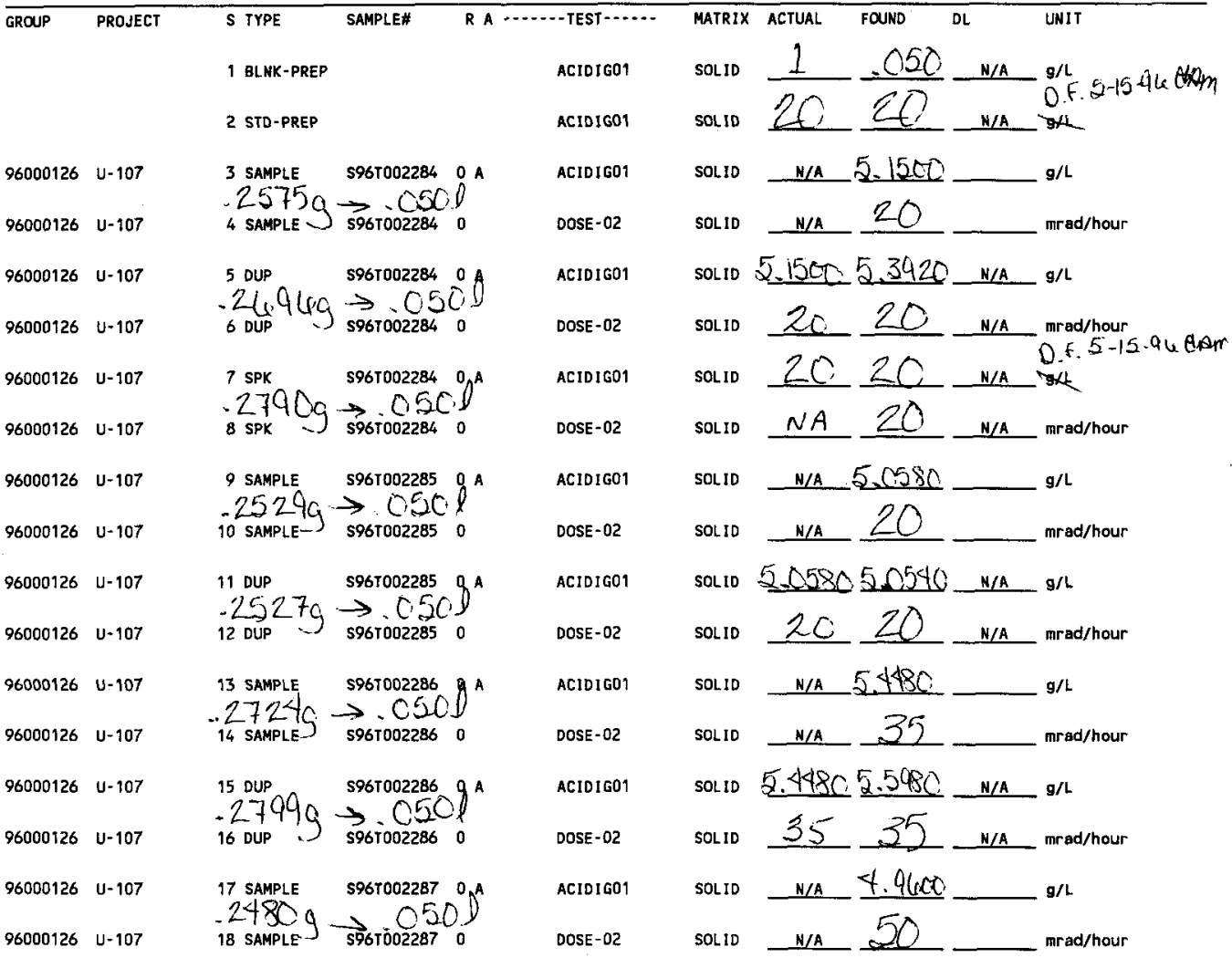

Data Entry Comments:

Units shown for QC (SPK \& STD) may not reflect the actual units. $D L=$ Detection Limit, $S=$ Worklist Slot Number, $R=$ Replicate Number, $A=$ Aliquot Code. 


\section{LABCORE Data Entry Template for Worklist\#}

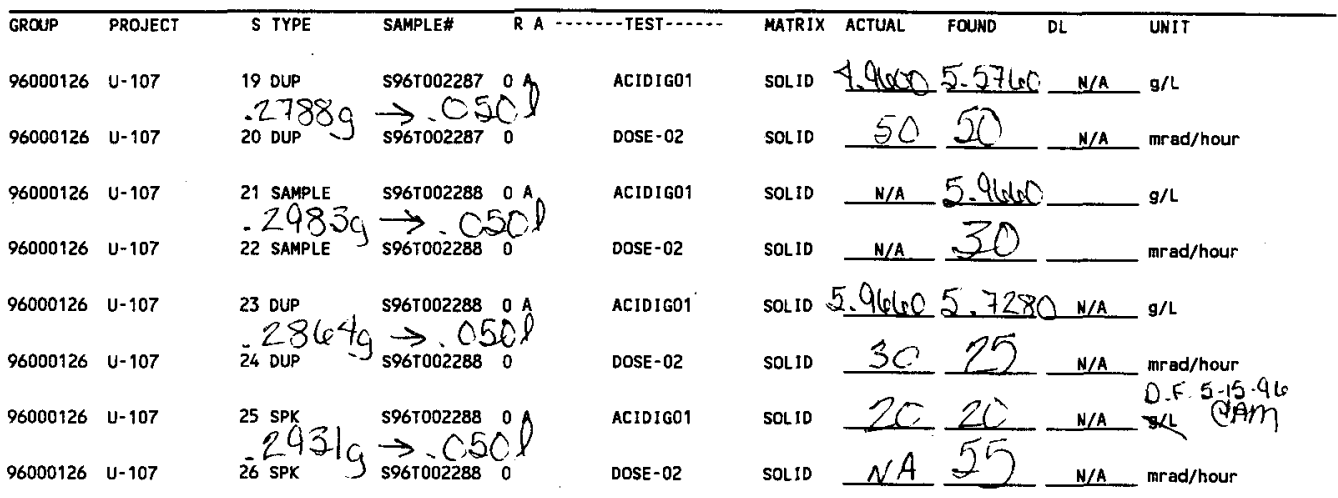

\section{Final page for worklist \# $\quad 8209$}
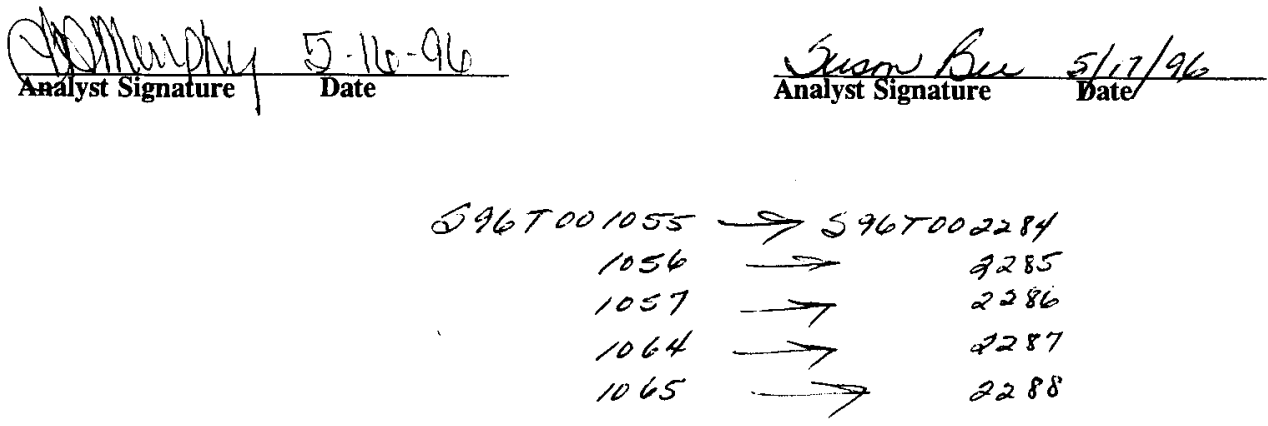

Data Entry Comments.

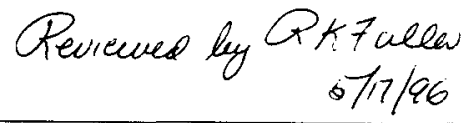

Units shown for $Q C$ (SPK \& STD) may not reflect the actual units. $D L=$ Detection Limit, $S=$ Worklist Slot Number, $R=$ Replicate Number, $A=$ Aliquot Code. 
WHC-SD-WM-DP-184, REV. I

INORGANIC ANALYSES

156 
WHC-SD-WM-DP-184, REV. I

THIS PAGE WAS INTENTIONALLY LEFT BLANK 


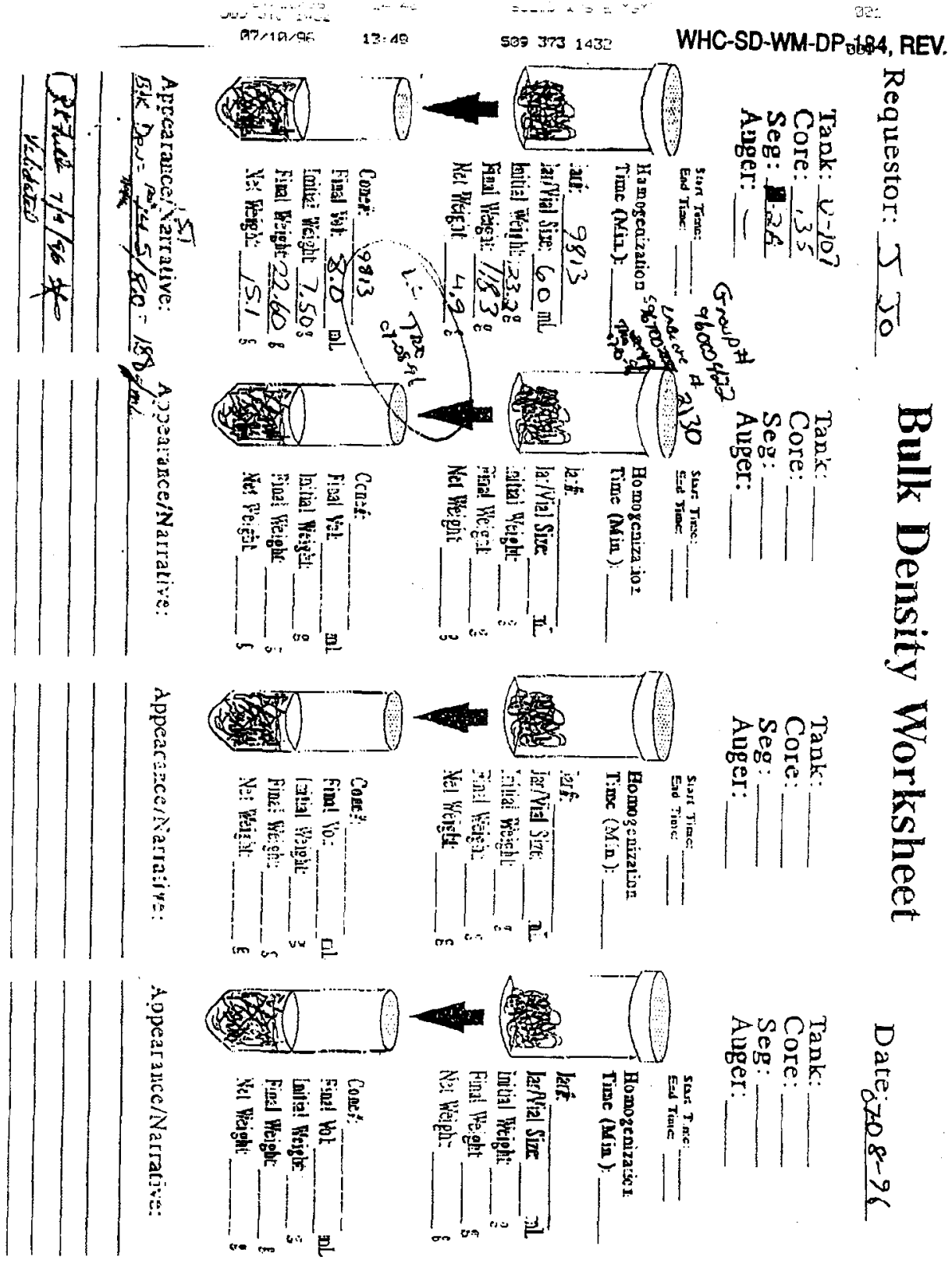




\section{LABCORE Data Entry Template for Worklist\#}

\section{Analyst: $\quad K$ G}

Method: LA-510-112 Rev/Mod C-3

Worklist Comment: U-107 SPG-01 RUN DIRECT. RCJ-

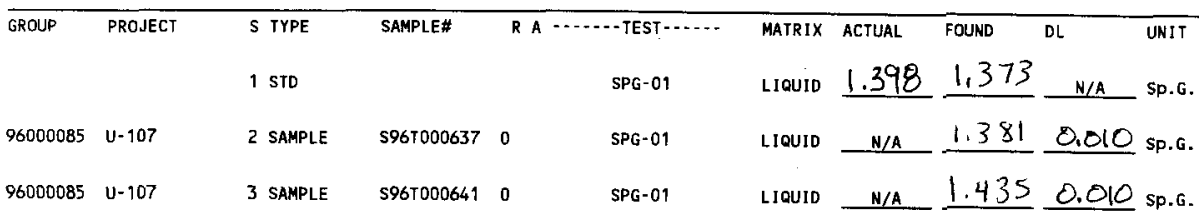

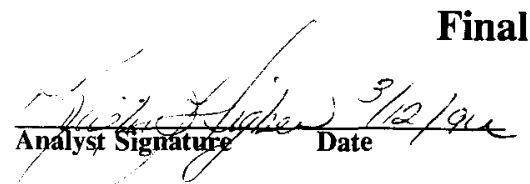

Final page for worklist \# 6053

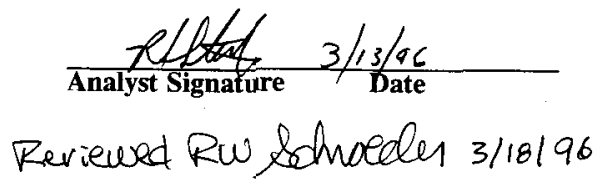

Data Entry Comments:

Units shown for QC (SPK \& STD) may not reflect the actual units. $D L=$ Detection Limit, $S=$ Worklist Slot Number, $R=$ Replicate Number, $A=$ Aliquot Code. 
WHC-SD-WM-DP-184, REV. I

SPECIFIC GRAVITY ANALYSIS

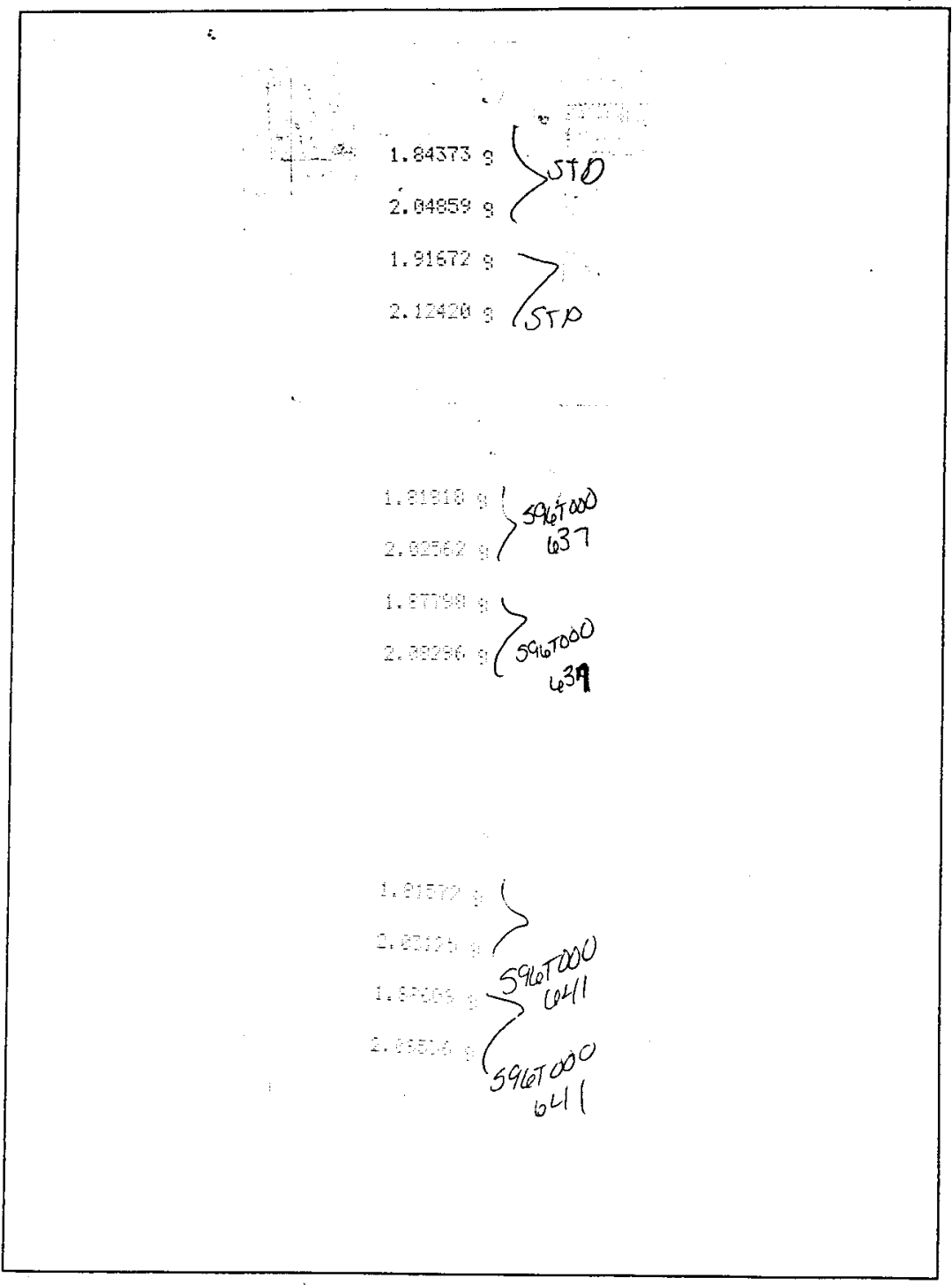

160 
PLACE ANALYTICAL CARD IN BOX BELOW OR ATTACH TRAVELER

\section{SPECIFIC GRAVITY : LA-510-112 (C-3)}

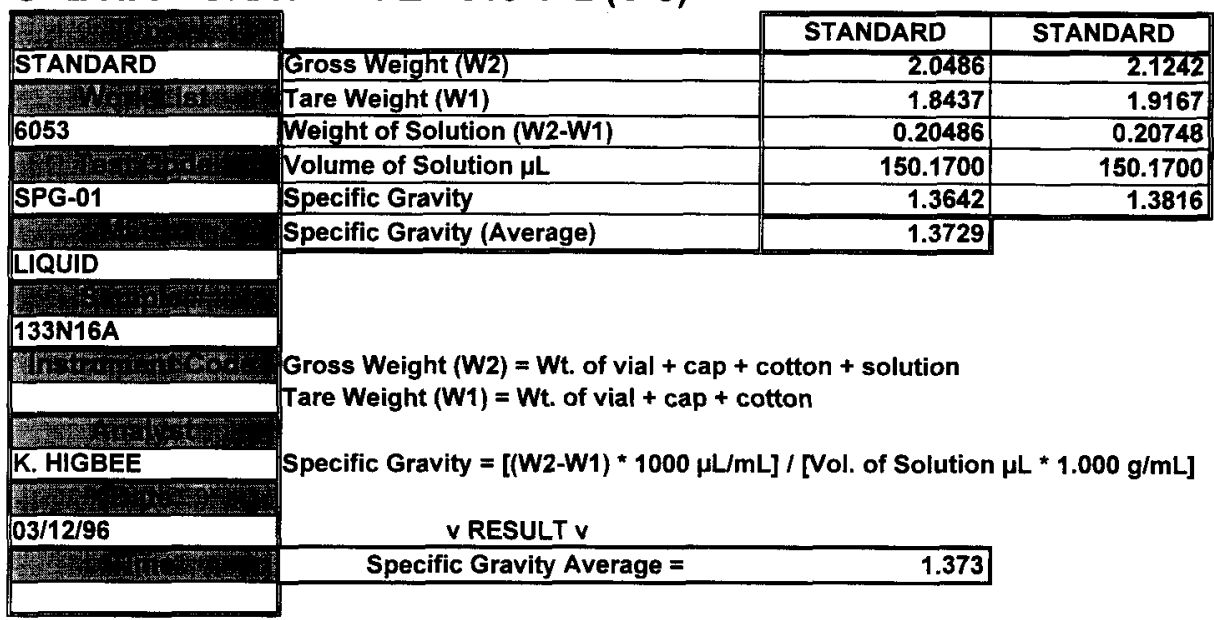

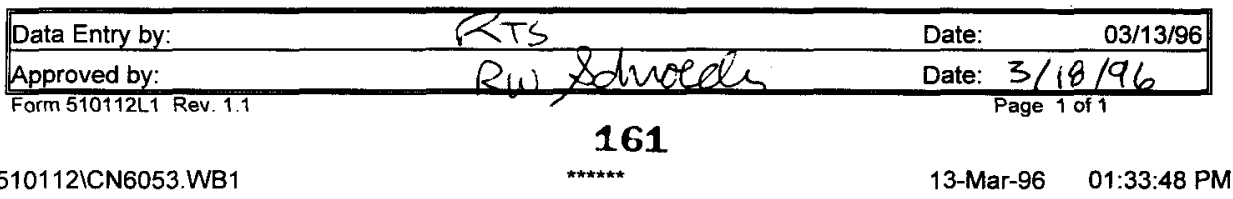


PLACE ANALYTICAL CARD IN BOX BELOW OR ATTACH TRAVELER

WHC-SD-WM-DP-184, REV. I

SPECIFIC GRAVITY : LA-510-112 (C-3)

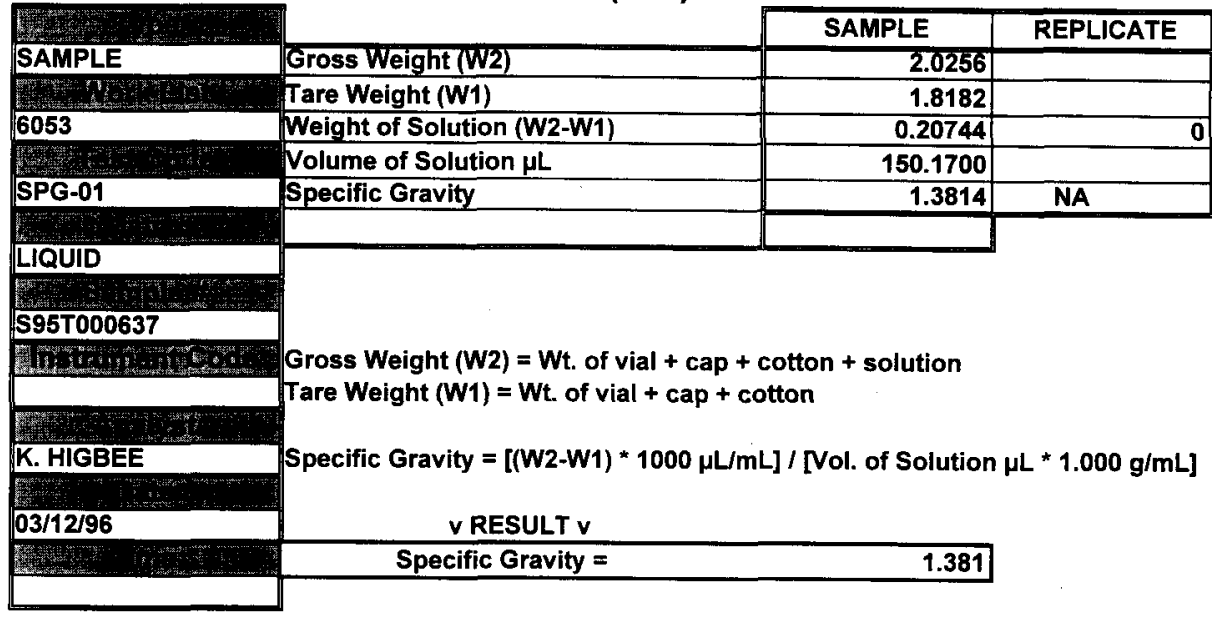

\begin{tabular}{|lccc|}
\hline Data Entry by: & $R T S$ & Date: & $03 / 13 / 96$ \\
\hline Approved by: & $R W$ sduroed & Date: $3 / 18 / 96$ \\
\hline Form 510112L1 Rev.1.1 & PG2 & & Page 1 of 1 \\
\hline
\end{tabular}


PLACE ANALYTICAL CARD IN BOX BELOW OR ATTACH TRAVELER

WHC-SD-WM-DP-184, REV. $\mid$

\section{SPECIFIC GRAVITY : LA-510-112 (C-3)}

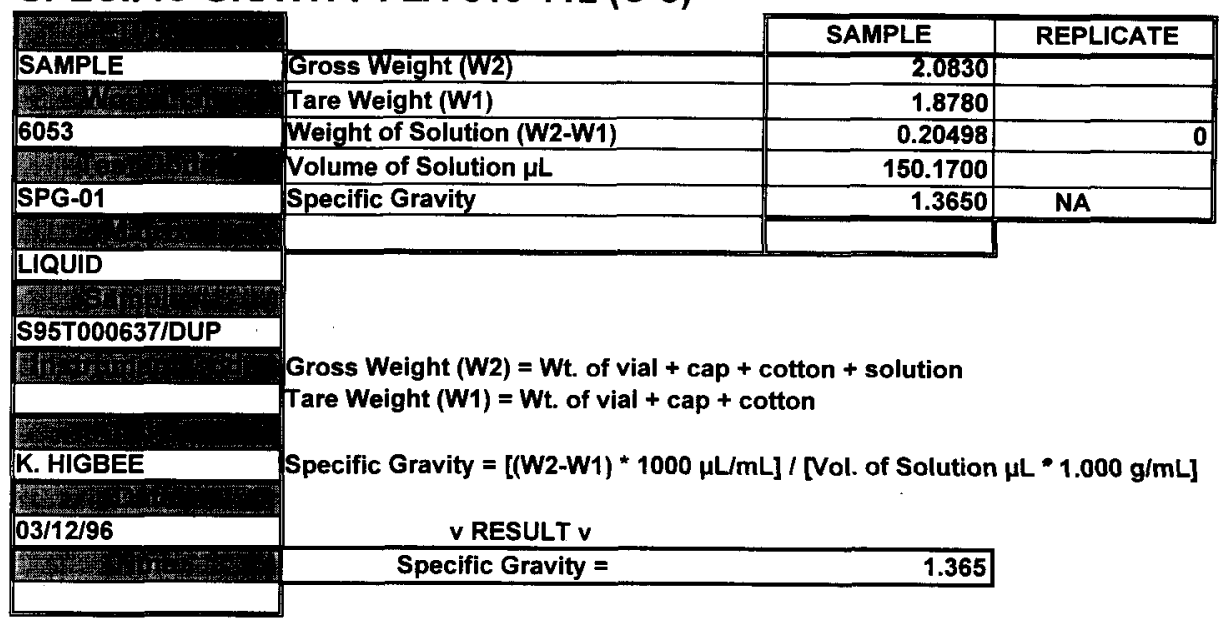

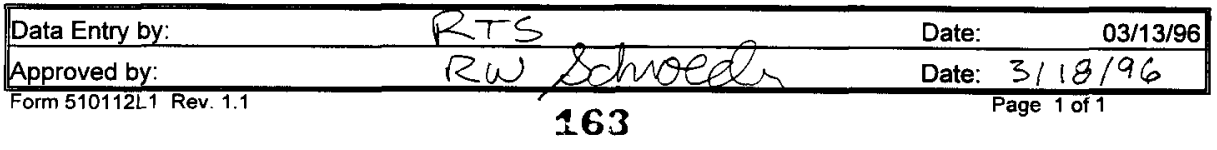


SPECIFIC GRAVITY : LA-510-112 (C-3)

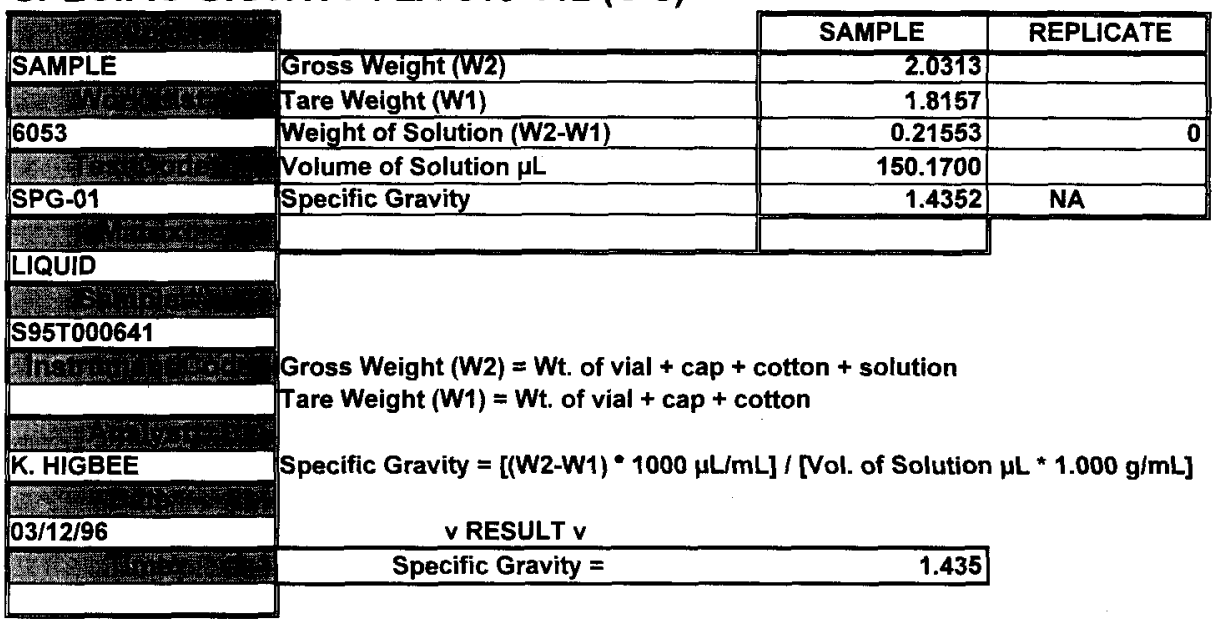

\begin{tabular}{|lll|}
\hline Data Entry by: & Date: & 03/13/96 \\
\hline Approved by: & Dote: $3 / 18 / 96$ \\
\hline Form 510112L1 Rev. 1.1 & 164 & Page 1 of 1
\end{tabular}


WHC-SD-WM-DP-184, REV. I

\section{SPECIFIC GRAVITY : LA-510-112 (C-3)}

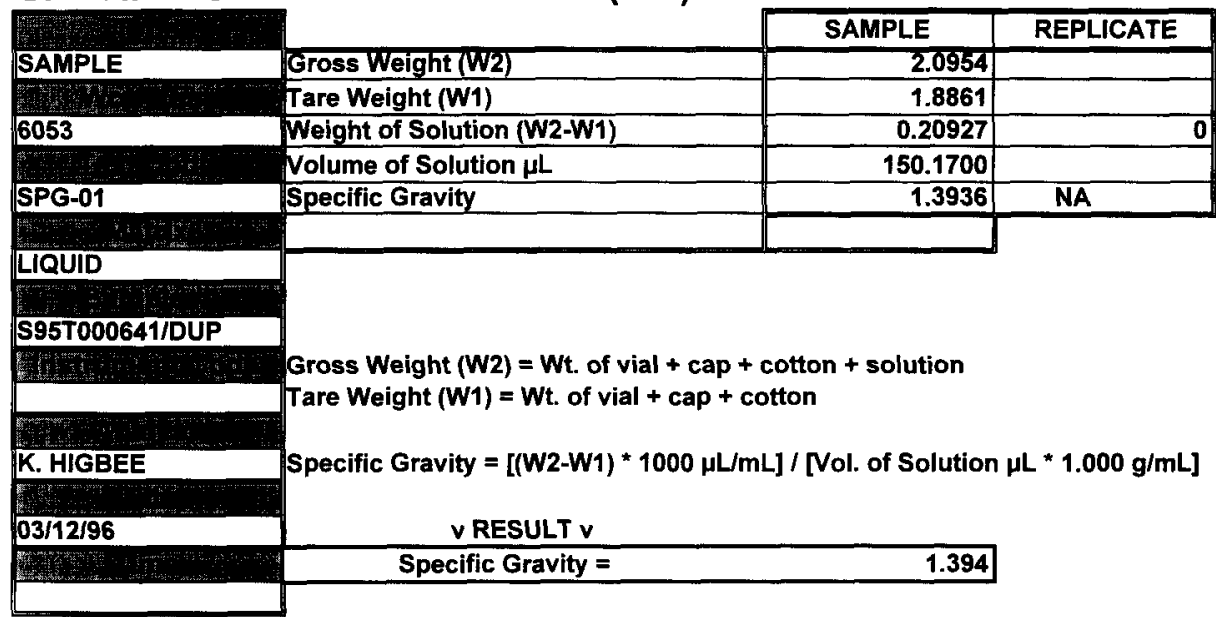

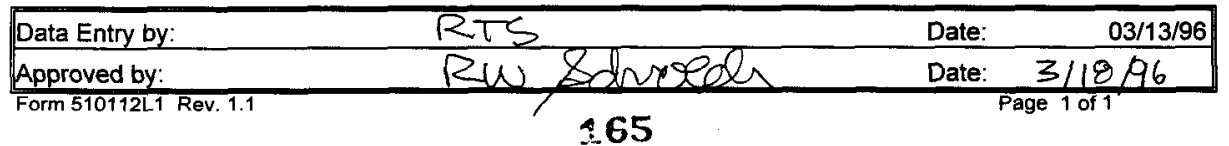


Analyst: $\quad$ SMF $\quad$ Instrument: BA001

Book $\# 133 N_{16}-T$

Method: LA-510-112 Rev/Mod C -3

Worklist Comment: U-107 AND S-102 SPG. RCJ

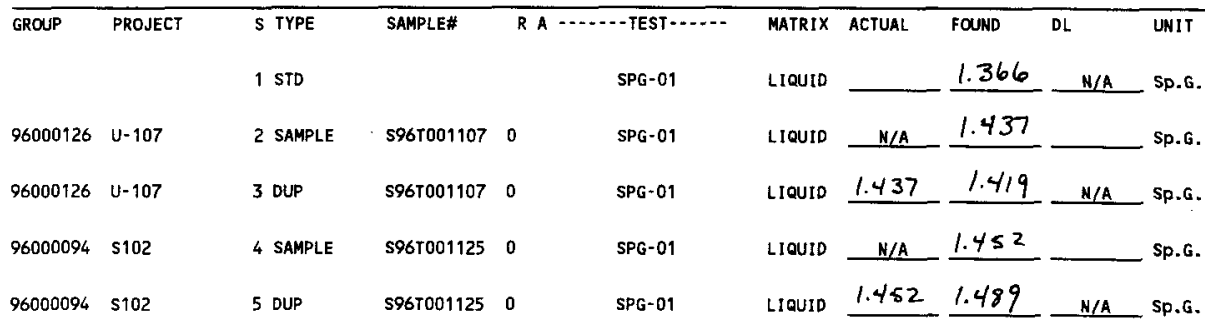

\section{Final page for worklist \#}
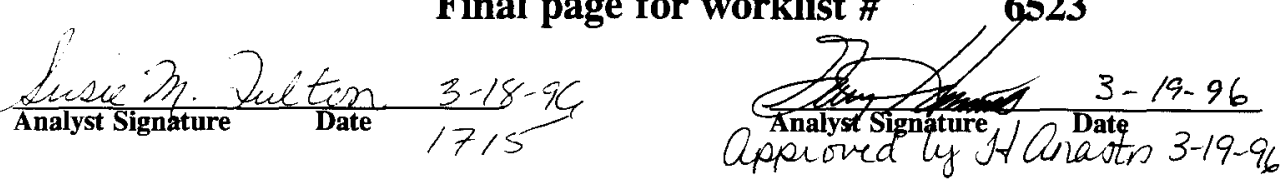
$3 T 0,33016.9$
sTD DuP
S94TCO11OF * Dup
$5967001125+$ DuP
Thit 1.33232 .5
1. $3533 / 8$
Guss, i. 53787
$1.55857 \hat{\gamma}$
i. 306058
$1.36727 \mathrm{~g}$
$1.52190 \%$
$.15017 \mathrm{mi}$
$.15017 \mathrm{ind}$
l. $31619 z$
$1.58036 \mathrm{~g}$
$1.53428 \mathrm{~g}$
$1,35348 \mathrm{~g}$
$1.57715 \mathrm{~g}$
.15017 in

Data Entry Comments:

$\sin$ \& SAMPLE SIEE : . 15017 me

Units shown for $Q C$ (SPK \& STD) may not reflect the actual units. $D L=$ Detection Limit, $S=$ Worklist Slot Number,

$R=$ Replicate Number, $A=$ Aliquot Code. 


\section{SPECIFIC GRAVITY : LA-510-112 (C-3)}

\begin{tabular}{|c|c|c|c|}
\hline 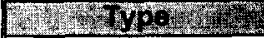 & & STANDARD & STANDARD \\
\hline STANDARD & Gross Weight (W2) & 4.5379 & 1.5586 \\
\hline 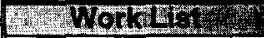 & Tare Weight (W1) & 1.3328 & 1.3533 \\
\hline 6523 & Weight of Solution (W2-W1) & 0.20505 & 0.20526 \\
\hline DExy yot & Volume of Solution $\mu \mathrm{L}$ & 150.1700 & 150.1700 \\
\hline SPG-01 & Specific Gravity & 1.3655 & 1.3669 \\
\hline W & Specific Gravity (Average) & 1.3662 & \\
\hline LIQUID & \\
\hline Samplow & & & \\
\hline STD & & & \\
\hline BA001 & \multirow{2}{*}{\multicolumn{3}{|c|}{$\begin{array}{l}\text { Gross Weight }(W 2)=W t \text {. of vial + cap + cotton + solution } \\
\text { Tare Weight }(W 1)=W t . \text { of vial + cap + cotton }\end{array}$}} \\
\hline 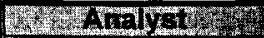 & & & \\
\hline SMF & \multicolumn{3}{|c|}{ Specific Gravity $=[(\mathrm{W} 2-\mathrm{W} 1) * 1000 \mu \mathrm{L} / \mathrm{mL}] /[\mathrm{Vol}$. of Solution $\mu \mathrm{L} * 1.000 \mathrm{~g} / \mathrm{mL}]$} \\
\hline W. & & & \\
\hline $03 / 18 / 96$ & \multicolumn{3}{|l|}{ v RESULT $v$} \\
\hline Hentrims & Specific Gravity Average $=$ & 1.366 & \\
\hline 05:15 PM & & & \\
\hline
\end{tabular}

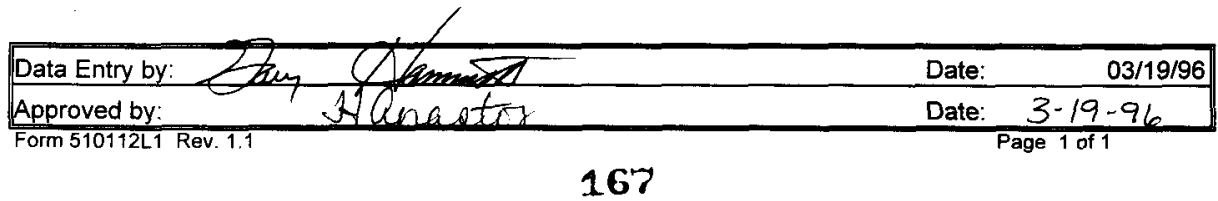


SPECIFIC GRAVITY : LA-510-112 (C-3)

\begin{tabular}{|c|c|c|c|}
\hline H & & SAMPLE & REPLICATE \\
\hline SAMPLE & Gross Weight (W/2) & 1.5219 & \\
\hline 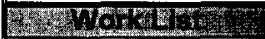 & Tare Weight (W1) & 1.3061 & \\
\hline 6523 & Weight of Solution (W2-W1) & 0.21585 & 0 \\
\hline 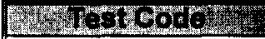 & Volume of Solution $\mu \mathrm{L}$ & 150.1700 & \\
\hline SPG-01 & Specific Gravity & 1.4374 & NA \\
\hline Prow & & & \\
\hline 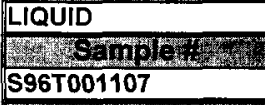 & \multirow{5}{*}{\multicolumn{3}{|c|}{$\begin{array}{l}\text { Gross Weight }(W 2)=W t . \text { of vial }+ \text { cap }+ \text { cotton }+ \text { solution } \\
\text { Tare Weight }(W 1)=W t \text {. of vial }+ \text { cap }+ \text { cotton } \\
\text { Specific Gravity }=[(W 2-W 1) * 1000 \mu L / m L] /[\text { Vol. of Solution } \mu \mathrm{L} \text { * } 1.000 \mathrm{~g} / \mathrm{mL}]\end{array}$}} \\
\hline 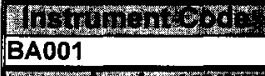 & & & \\
\hline $\begin{array}{l}\text { W } \\
\text { SMF }\end{array}$ & & & \\
\hline 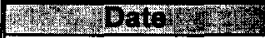 & & & \\
\hline $03 / 18 / 96$ & & & \\
\hline Sher & \multirow{2}{*}{\multicolumn{2}{|c|}{ Specific Gravity = }} & \\
\hline $05: 15$ PM & & & \\
\hline
\end{tabular}

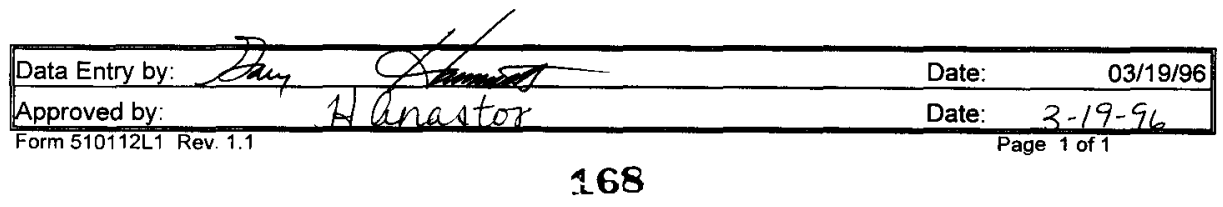




\section{WHC-SD-WM-DP-184, REV. /}

PLACE ANALYTICAL CARD IN BOX BELOW OR ATTACH TRAVELER

\section{SPECIFIC GRAVITY : LA-510-112 (C-3)}

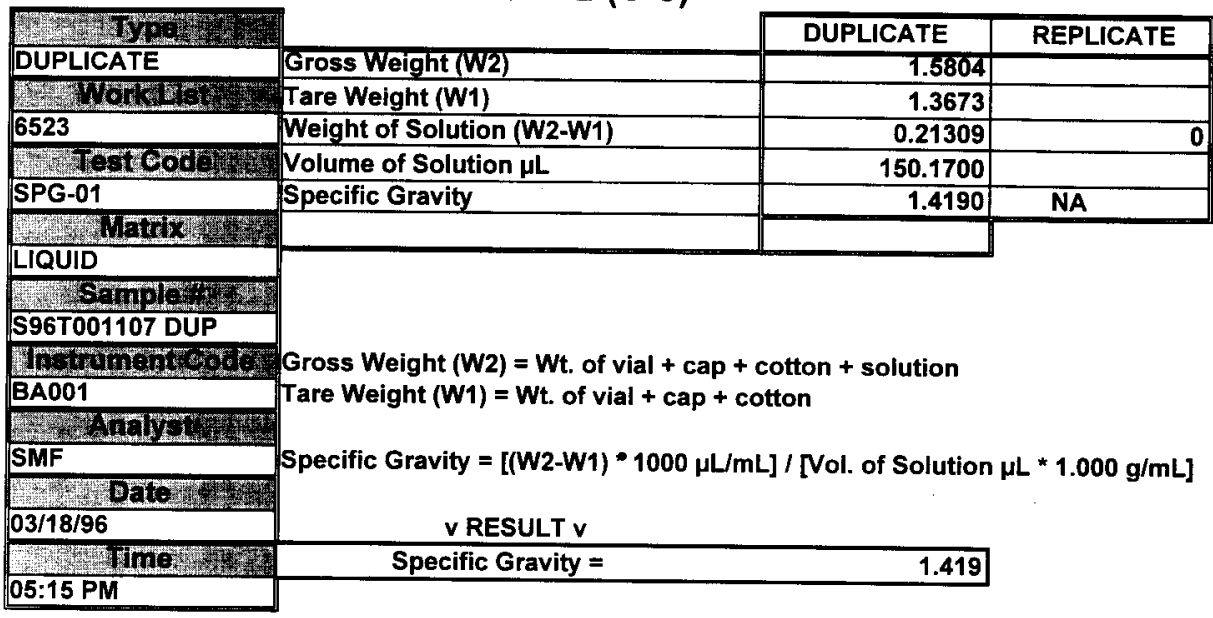

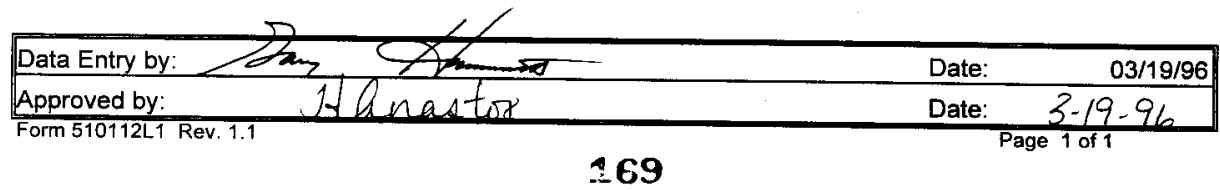




\section{.. BCORE Data Entry Template for Worklist\#}

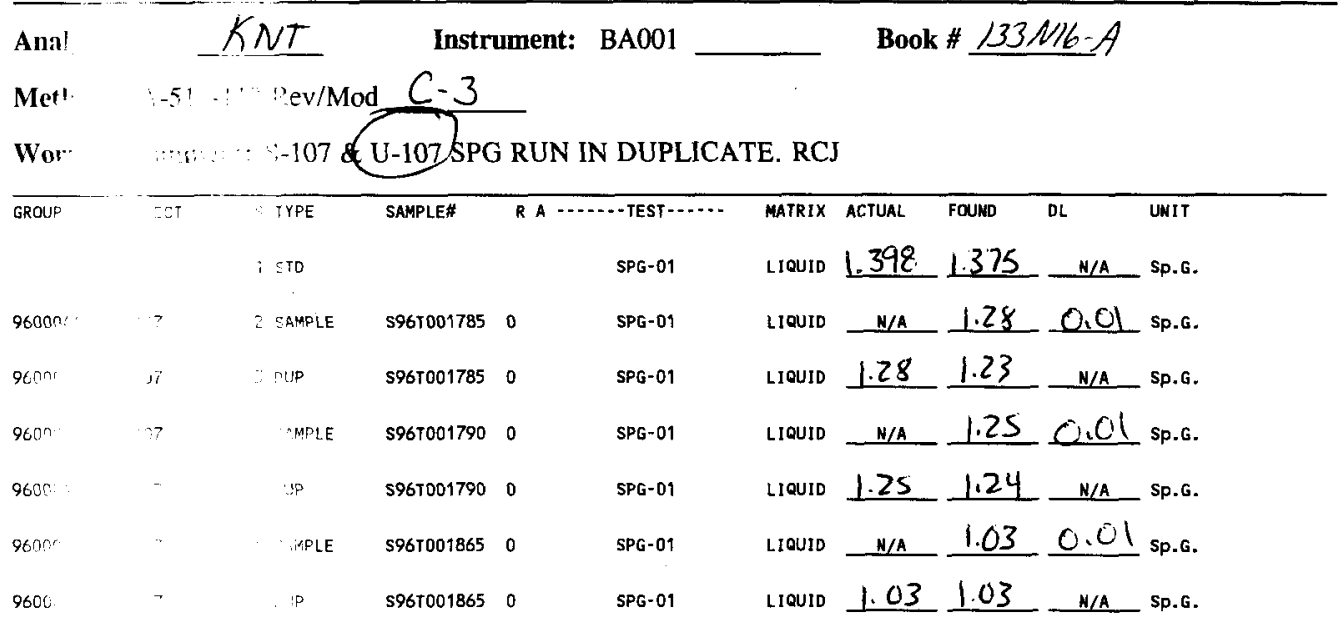

\section{Final page for worklist \# 7984}

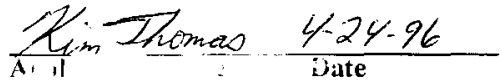

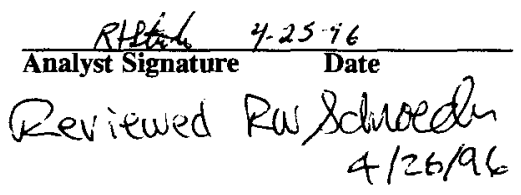

\section{DEST AVAILABLE COPY}




\section{WHG-SD-WM-DP-184, REV.।}

\section{SPECIFIC GRAVITY : LA-510-112 (C-3)}

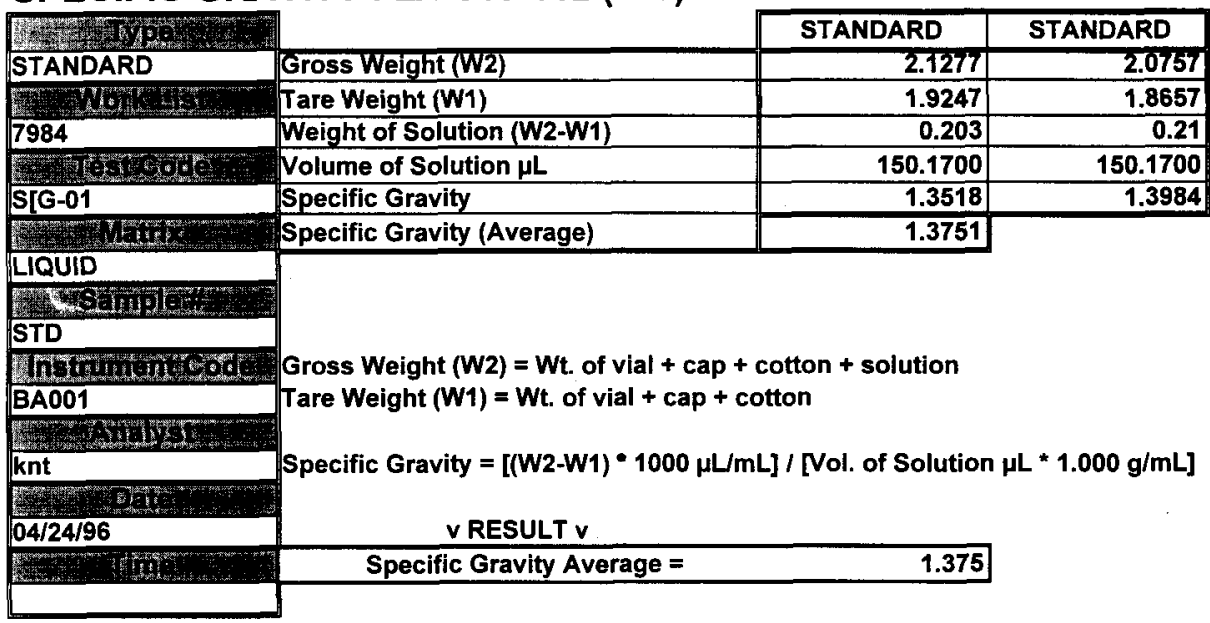

\begin{tabular}{|lllr|}
\hline Data Entry by: & RTS & Date: & $04 / 25 / 96$ \\
\hline Approved by: & Rus Schuceel & Date: $4 / 26 / 96$ \\
\hline Form 510112L1 Rev. 1.1 & \multicolumn{1}{c}{ Page 1 of 1}
\end{tabular}




\section{WHC-SD-WM-DP-184, REV. I}

\section{SPECIFIC GRAVITY : LA-510-112 (C-3)}

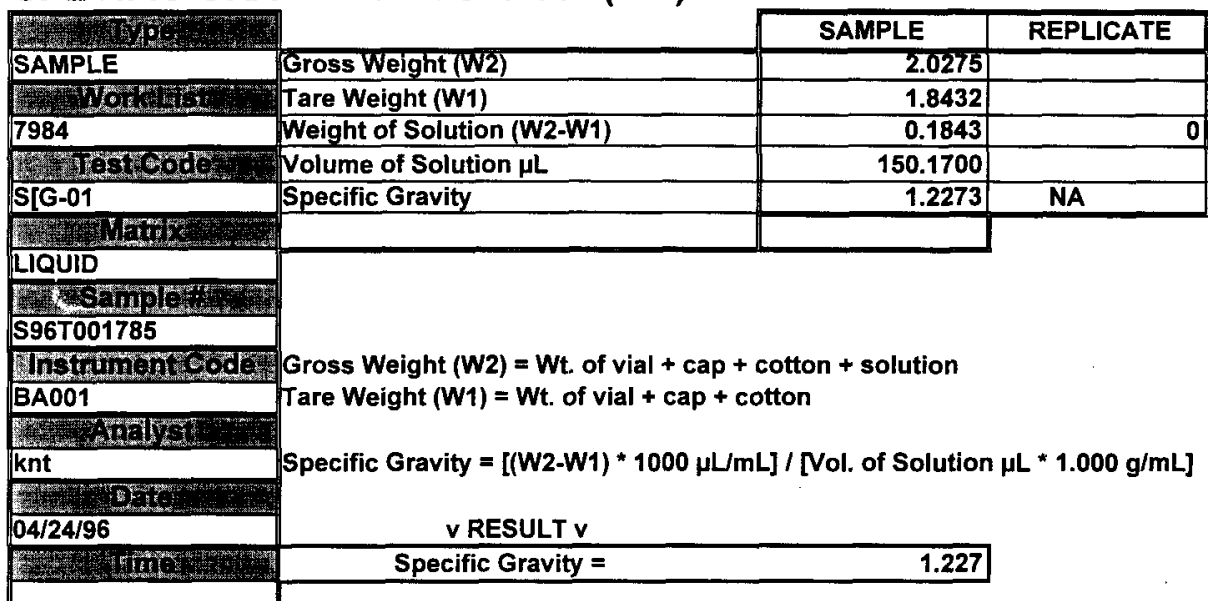

\begin{tabular}{|c|c|c|c|}
\hline Data Entry by: & RTS & Date: & $04 / 25 / 96$ \\
\hline Approved by: & Ru sdunetu & Date: & $4 / 26 / 96$ \\
\hline
\end{tabular}




\section{WHC-SD-WM-DP-184, REV.}

\section{SPECIFIC GRAVITY : LA-510-112 (C-3)}

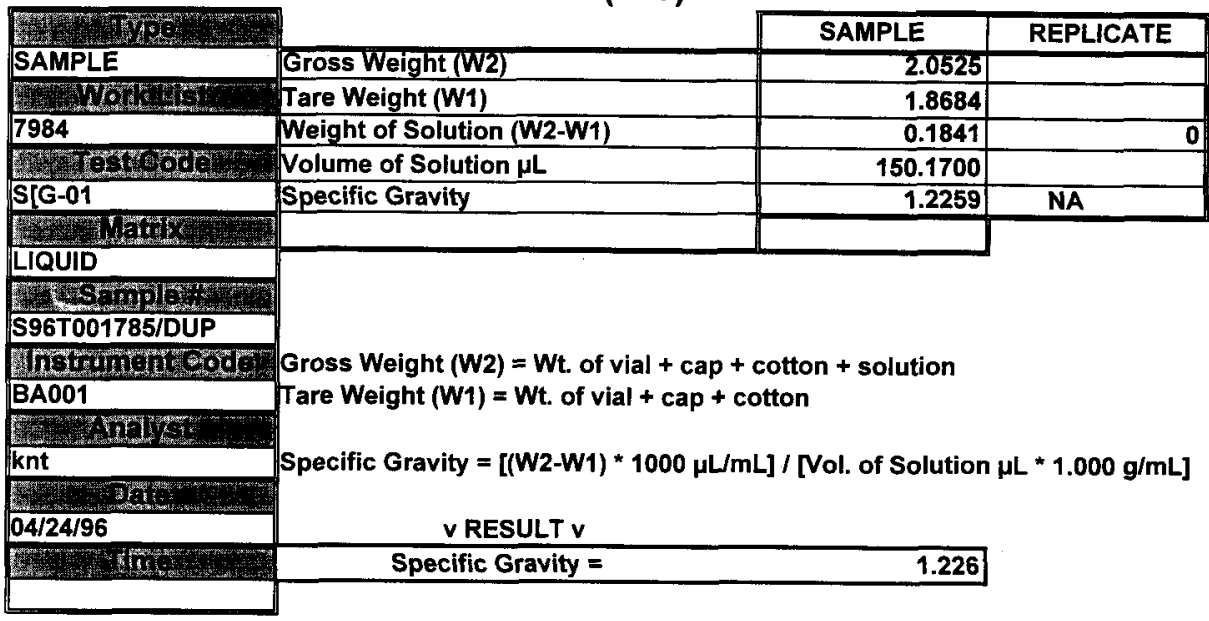

\begin{tabular}{|c|c|c|c|}
\hline Data Entry by: & RTS & Date: & $04 / 25 / 96$ \\
\hline Approved by: & Rus schuoden & Date: & $4 / 26 / 96$ \\
\hline
\end{tabular}


PLACE ANALYTICAL CARD IN BOX BELOW OR ATTACH TRAVELER

\section{WHC-SD-WM-DP-184, REV. I}

\section{SPECIFIC GRAVITY : LA-510-112 (C-3)}

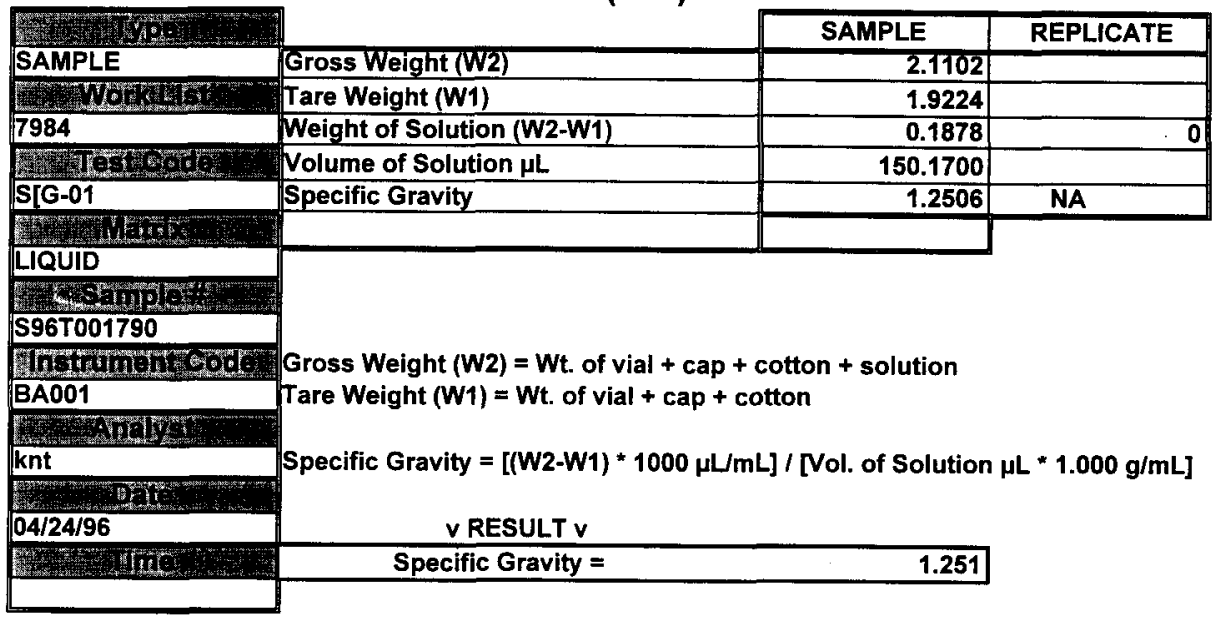

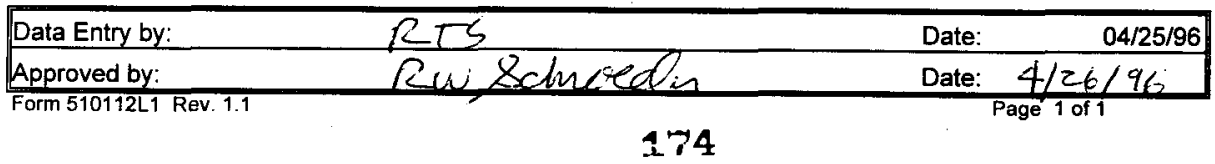




\section{WHC-SD-WM-DP-184, REV. I}

\section{SPECIFIC GRAVITY : LA-510-112 (C-3)}

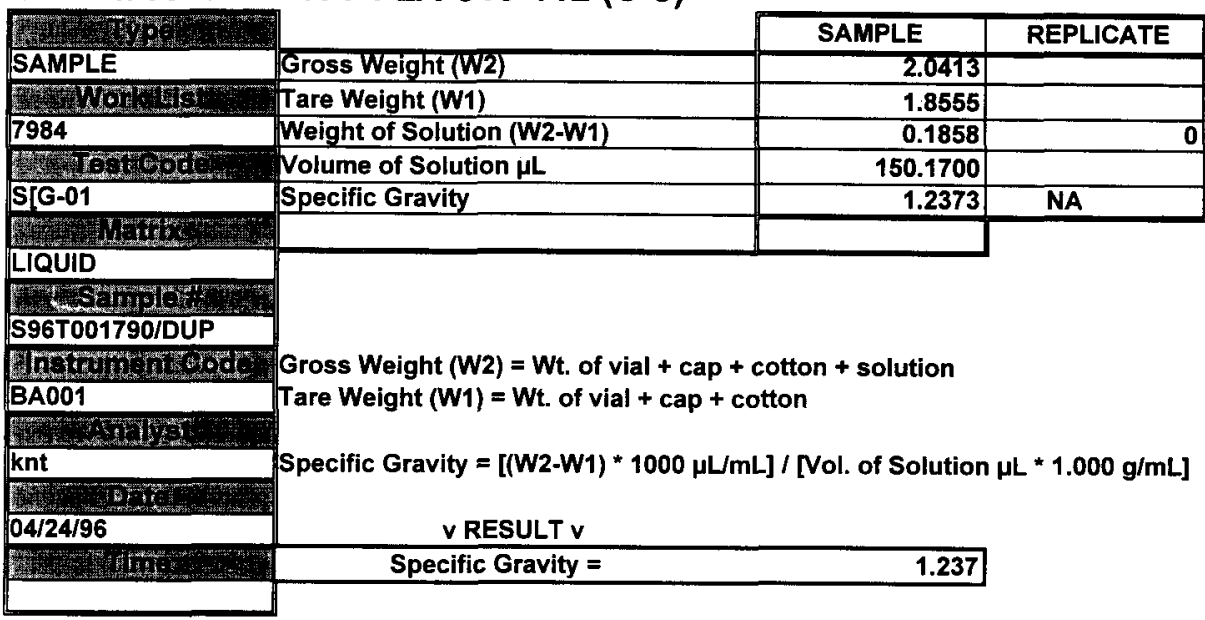

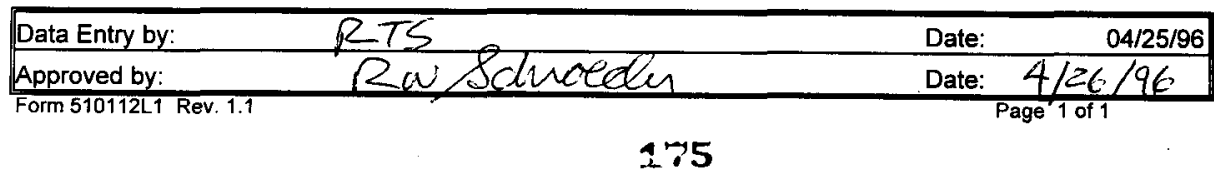




\section{SPECIFIC GRAVITY : LA-510-112 (C-3)}

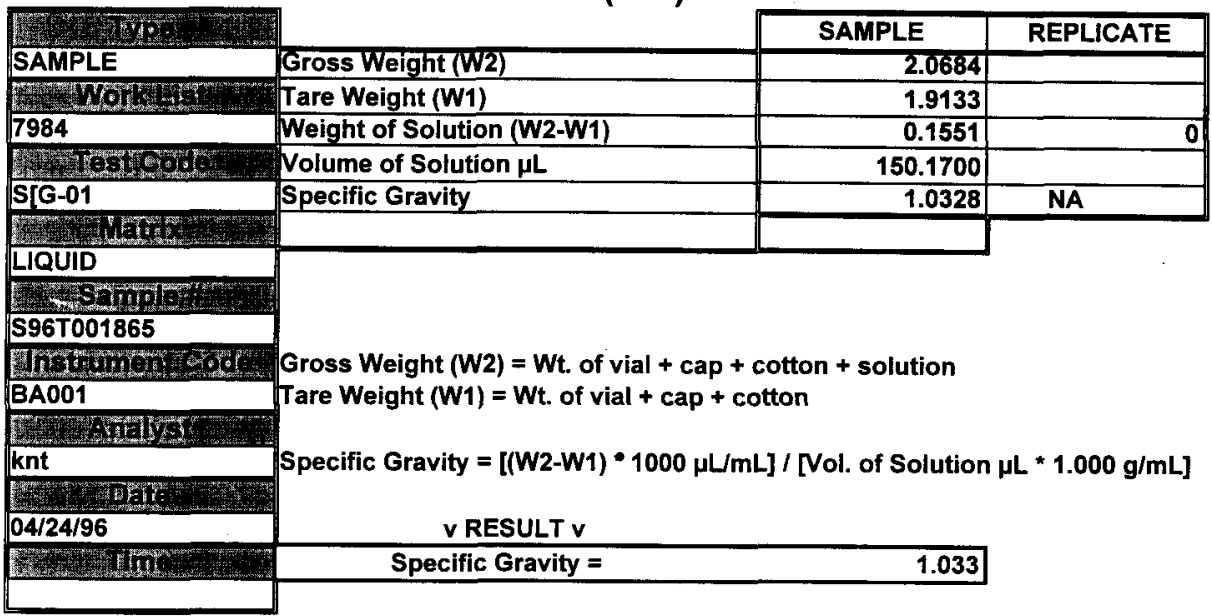

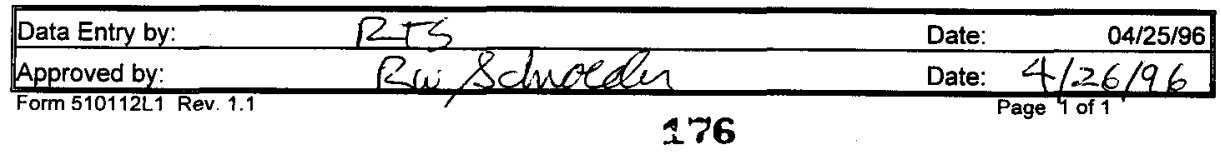




\section{SPECIFIC GRAVITY : LA-510-112 (C-3)}

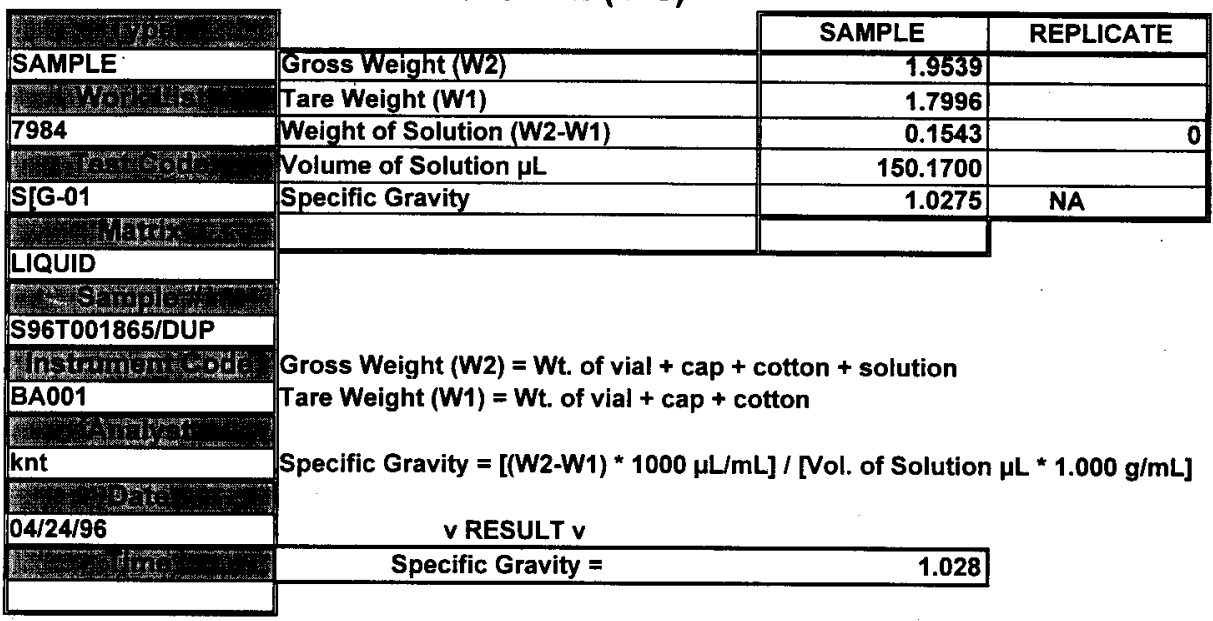

\begin{tabular}{|c|c|c|c|}
\hline Data Entry by: & $2+5$ & Date: & $04 / 25 / 96$ \\
\hline \begin{tabular}{|l} 
Approved by: \\
Form 51011211
\end{tabular} & Rui \&donce & Date: & $6 / 96$ \\
\hline
\end{tabular}




Analyst: $\quad$ ADP $\quad$ Instrument: BA001 Book \#133016A

Method: LA-510-112 Rev/Mod $\mathrm{C}-3$

Worklist Comment: U-107 SPG. RCJ

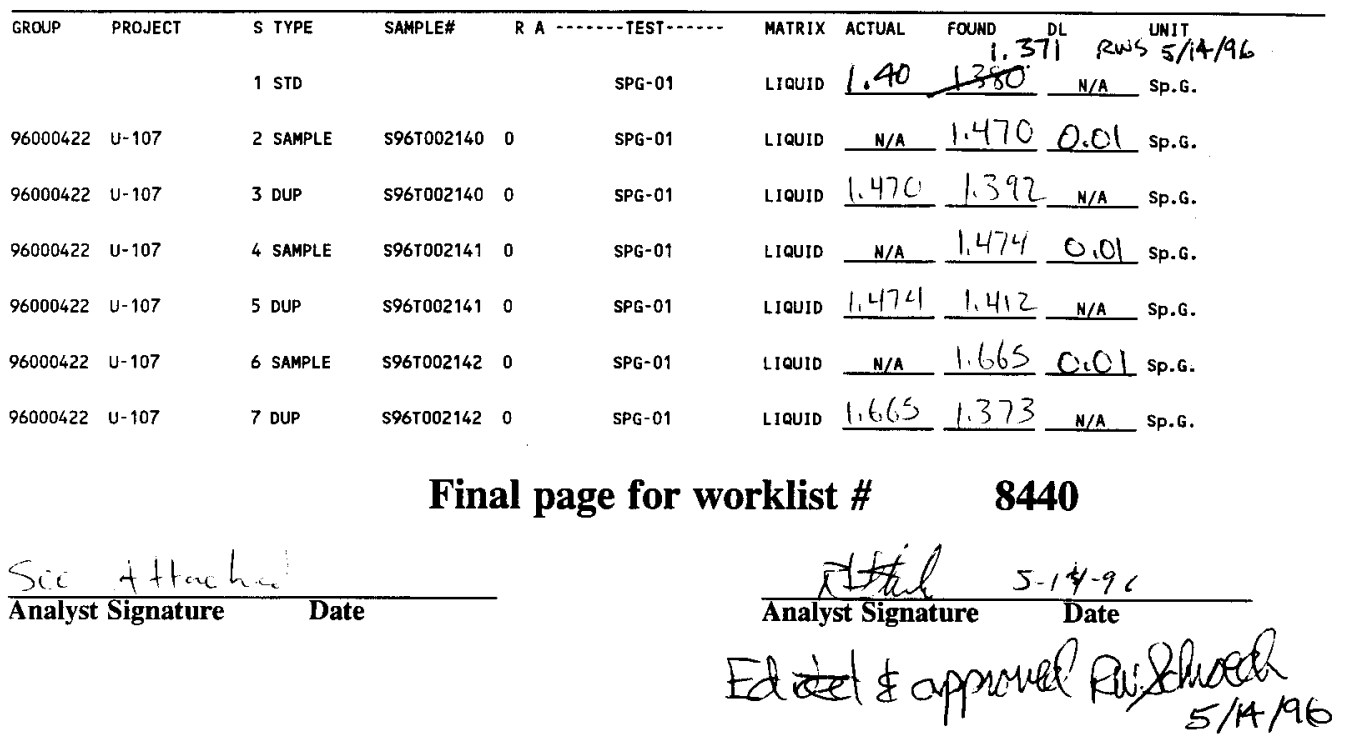

Data Entry Comments:

Units shown for $Q C$ (SPK \& STD) may not reflect the actual units. $D L=$ Detection Limit, $S=$ Worklist Slot Number, $R=$ Replicate Number, $A=$ Aliquot Code . 
work: :F: Vrsion 2.1 05/15/95 WHC-SD-WM-DP-184, REV.

Page: 1

05/07/96 11:19

\section{LABCORE Data Entry Template for Worklist\#}

8440

Analyst: ADP Instrument: BA001_ Book \#133N164

Method: LA-510-112 Rev/Mod $\mathrm{C}_{-} 3$

Worklist Comment: U-107 SPG. RCJ

\begin{tabular}{|c|c|c|c|c|c|c|c|c|c|c|}
\hline GROUP & PROJECT & S TYPE & SAMPLE\# & R A & - & MATRIX & ACTUAL & FOUND & $\mathbf{D L}$ & UNIT \\
\hline 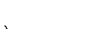 & & 1 SID & & & SPG-01 & LIQUID & & 1.380 & $\mathrm{~N} / \mathrm{A}$ & Sp.G. \\
\hline 96000422 & U- 107 & 2 SAMPLE & S961002140 & 0 & SPG-01 & LIQUID & $\mathbf{N} / \mathbf{A}$ & 1.470 & & Sp.G. \\
\hline 96000422 & $U-107$ & 3 SAMPLE & S96T002141 & 0 & SPG-01 & LIQUID & N/A & & & Sp.G. \\
\hline 96000422 & $U-107$ & 4 SAMPLE & s96T002142 & 0 & SPG-01 & LIQUID & $\mathbf{N} / \mathbf{A}$ & & & Sp.G. \\
\hline
\end{tabular}

\section{Final page for worklist \# $\mathbf{8 4 4 0}$}

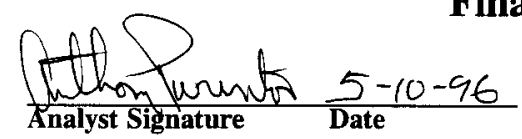

Analyst Signature Date

Data Entry Comments:

Units shown for QC (SPK \& STD) mary not reflect the actual units. $D L=$ Detection Limit, $S=$ Worklist Slot Number, $R=$ Replicate Number, $A=$ Aliquot Code. 


\section{WHC-SD-WM-DP-184, REV. I}

\section{SPECIFIC GRAVITY : LA-510-112 (C-3)}

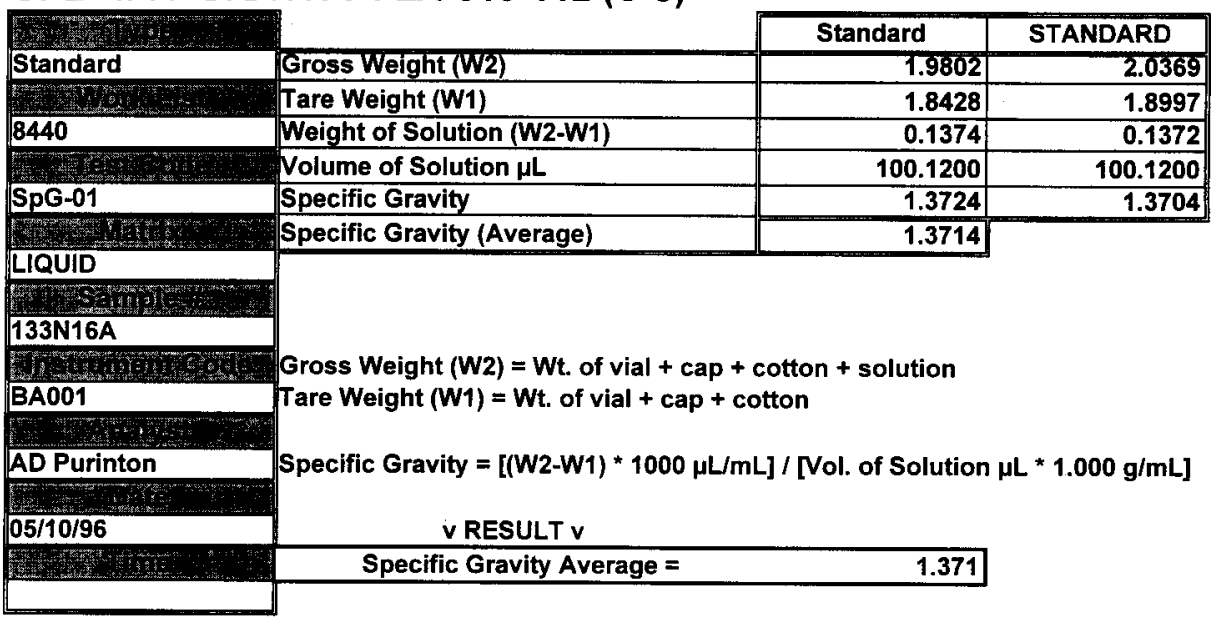

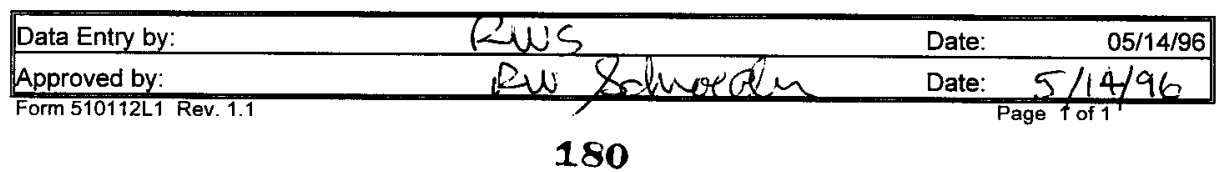


WHC-SD-WM-DP-184, REV. I

\section{SPECIFIC GRAVITY : LA-510-112 (C-3)}

\begin{tabular}{|c|c|c|c|}
\hline 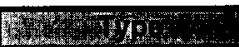 & & SAMPLE & REPLICATE \\
\hline SAMPLE & Gross Weight (W/2) & 2.0416 & \\
\hline 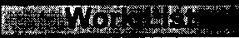 & Tare Weight (W1) & 1.8944 & \\
\hline 8440 & Weight of Solution (W2-W1) & 0.1472 & $\mathbf{0}$ \\
\hline Whe & Volume of Solution $\mu \mathrm{L}$ & 100.1200 & \\
\hline SPG-01 & Specific Gravity & 1.4702 & NA \\
\hline 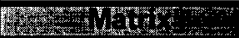 & & & \\
\hline $\begin{array}{l}\text { LIQUID } \\
\text { S96002140 } \\
\text { S960 }\end{array}$ & \multirow{4}{*}{\multicolumn{3}{|c|}{$\begin{array}{l}\text { Gross Weight }(\mathrm{W} 2)=\mathrm{Wt} \text {. of vial + cap + cotton + solution } \\
\text { Tare Weight }(\mathrm{W} 1)=\text { Wt. of vial + cap + cotton } \\
\text { Specific Gravity }=[(\mathrm{W} 2-\mathrm{W} 1) * 1000 \mu \mathrm{L} / \mathrm{mL}] /[\text { Vol. of Solution } \mu \mathrm{L} * 1.000 \mathrm{~g} / \mathrm{mL}]\end{array}$}} \\
\hline 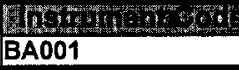 & & & \\
\hline $\begin{array}{l}\text { TID } \\
\text { ADP }\end{array}$ & & & \\
\hline $\begin{array}{l}\text { D. } \\
05 / 10 / 96\end{array}$ & & & \\
\hline W & Specific Gravity = & 1.470 & \\
\hline
\end{tabular}

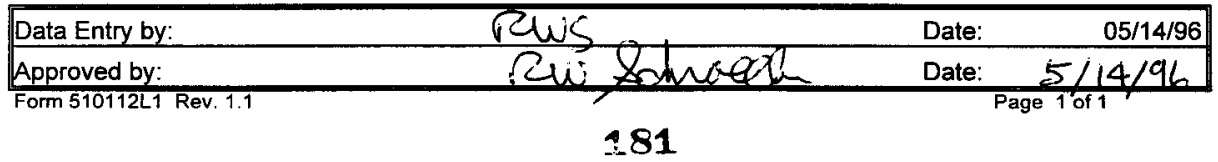




\section{WHC-SD-WM-DP-184, REV. $\mid$}

\section{SPECIFIC GRAVITY : LA-510-112 (C-3)}

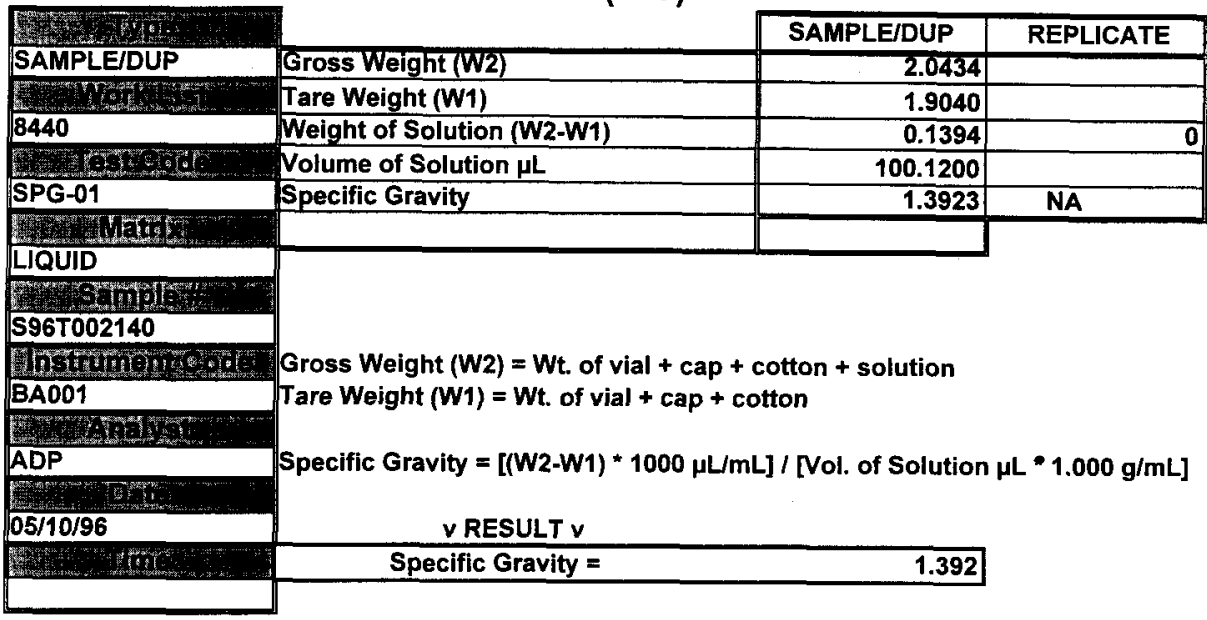

\begin{tabular}{|c|c|c|c|}
\hline Data Entry by & ievs & Date: & $05 / 14 / 96$ \\
\hline Approved by: & Cur solumedr & Date: & $5 / 14 / 96$ \\
\hline
\end{tabular}


SPECIFIC GRAVITY : LA-510-112 (C-3)

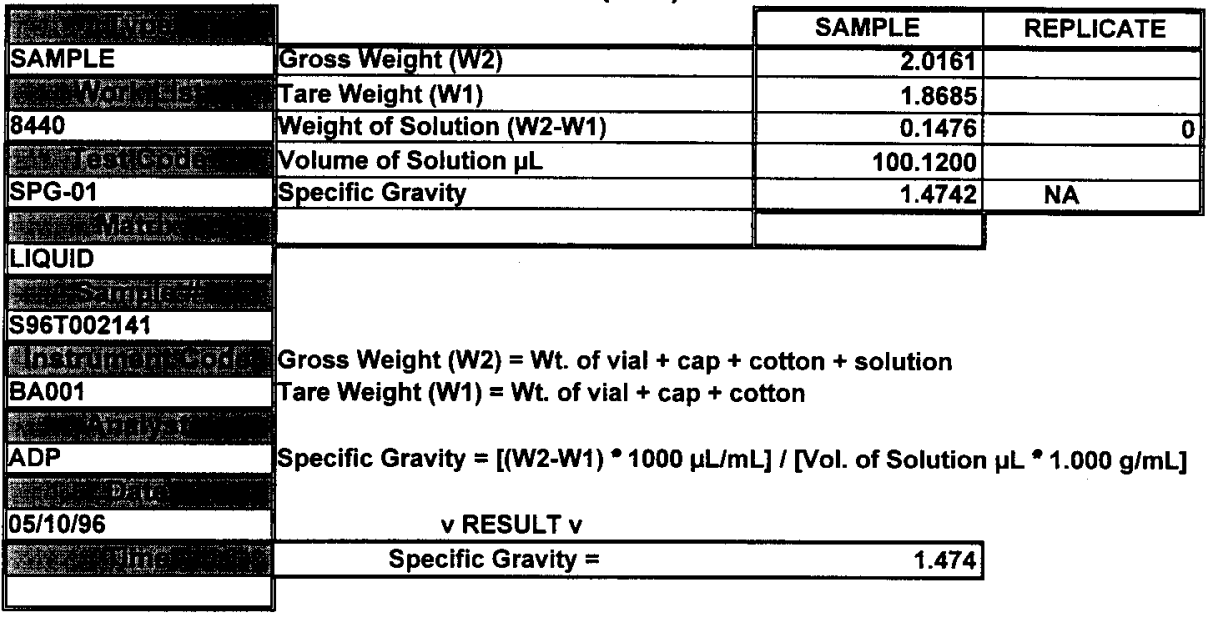

\begin{tabular}{|c|c|c|c|}
\hline Data Entry by: & zuns & Date: & $05 / 14 / 96$ \\
\hline Approved by: & Gu & Date: & $5 / 14 / 96$ \\
\hline
\end{tabular}




\section{SPECIFIC GRAVITY : LA-510-112 (C-3)}

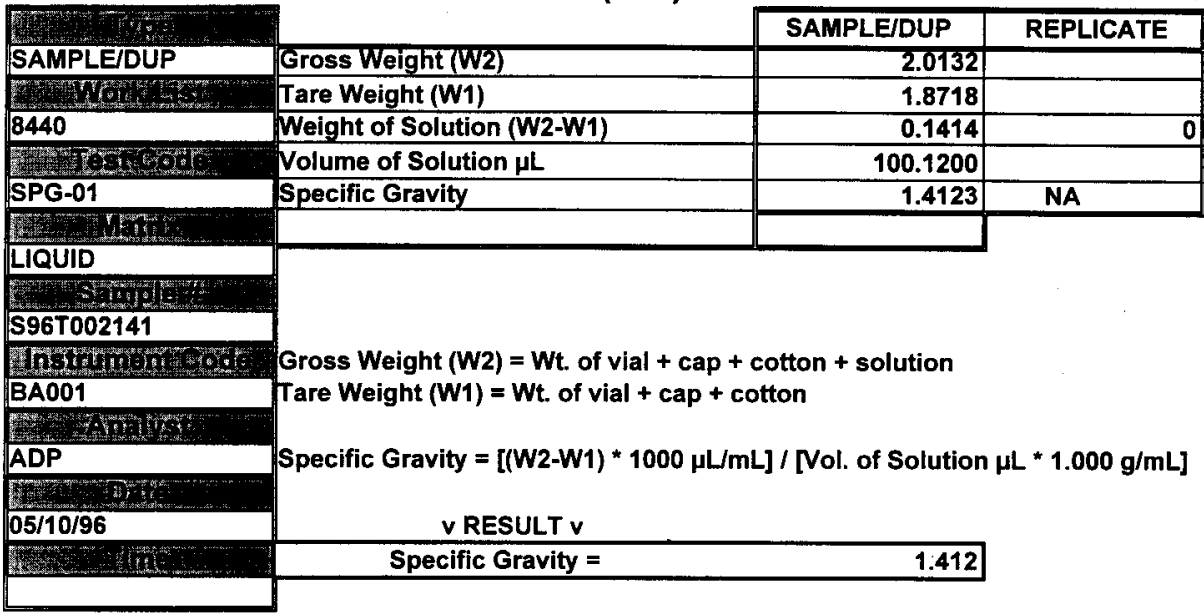

\begin{tabular}{|llll|}
\hline Data Entry by: & Dis & Date: & $05 / 14 / 96$ \\
\hline Approved by: & Dut & Date: & $5 / 14 / 96$ \\
\hline Form 510112L1 Rev. 1.1 & & & \\
\hline
\end{tabular}


SPECIFIC GRAVITY : LA-510-112 (C-3)

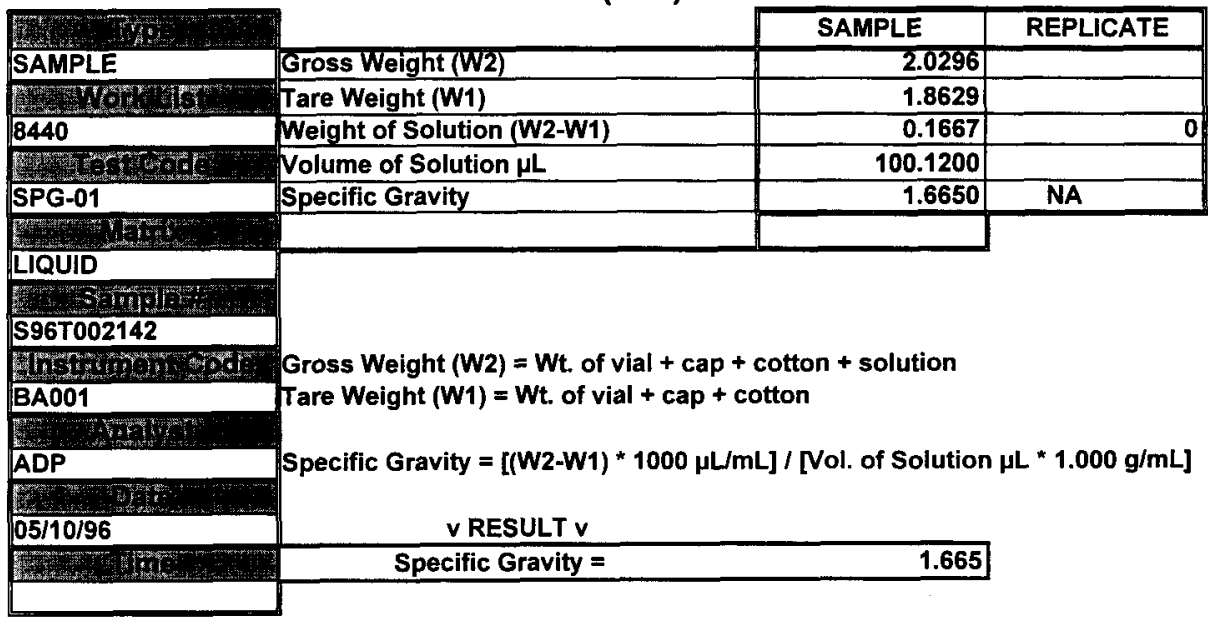

\begin{tabular}{|c|c|c|}
\hline Data Entry by: & Date: & $05 / 14 / 96$ \\
\hline Approved by: & Date: & $5 / 14 / 96$ \\
\hline
\end{tabular}




\section{SPECIFIC GRAVITY : LA-510-112 (C-3)}

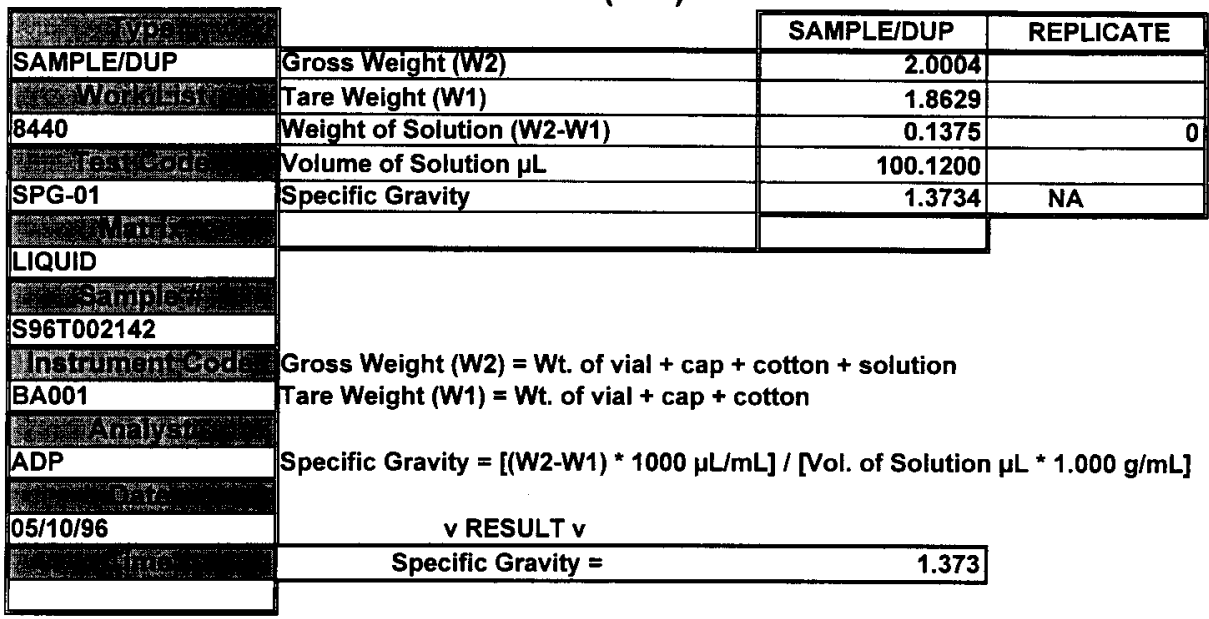

\begin{tabular}{|c|c|c|c|}
\hline Data Entry by: & rus & Date: & $05 / 14 / 96$ \\
\hline Approved by: & Rui sdivoenger & Date: & $5 / 14 / 96$ \\
\hline
\end{tabular}




\section{WHC-SD-WM-LP-184, REV.I}

worklistrpt Version 2.1 05/15/95

05/15/96/3:55

\section{LABCORE Data Entry Template for Worklist\#}

Page: $\quad I$

8729

Analyst: $\quad \not h R$ Instrument: BA001 Book \# $133416 \mathrm{~A}$

Method: LA-510-112 Rev/Mod $<-3$

Worklist Comment: U-107 \& HERTING FOR SPG-01 RTS!

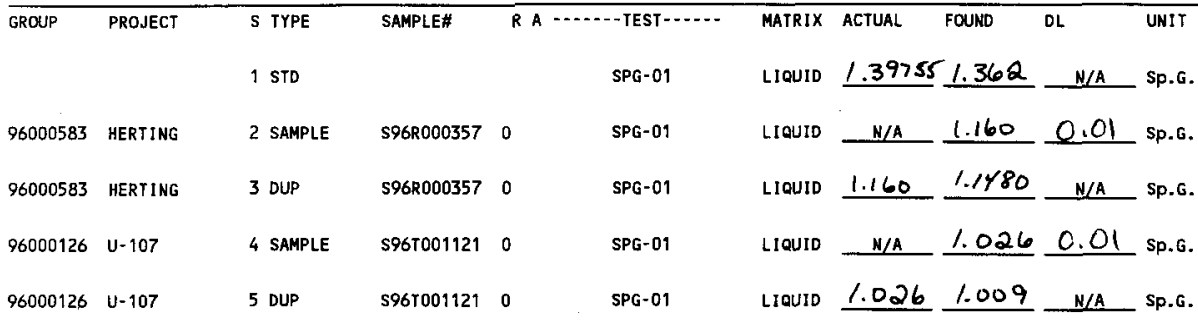

Final page for worklist \#
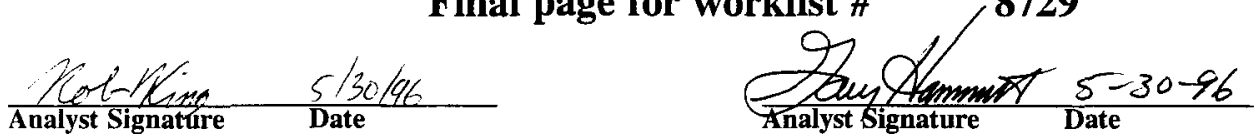

Approvel Ro Somveech

$6 / 03 / 96$

Data Entry Comments:

Units shown for $Q C$ (SPK \& STD) may not reflect the actual units. $D L=$ Detection Limit, $S=$ Worklist Slot Number, $R=$ Replicate Number, $A=$ Aliquot Code. 


\section{SPECIFIC GRAVITY : LA-510-112 (C-3)}

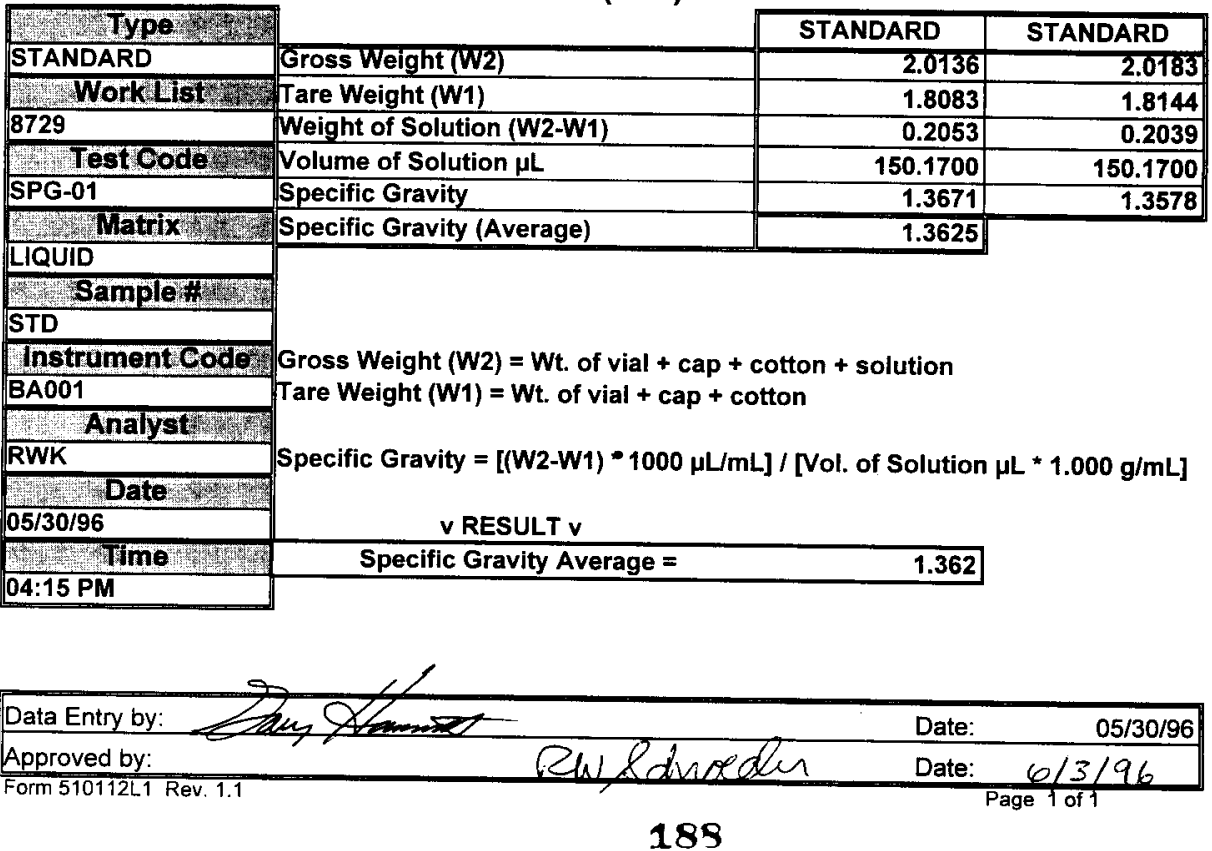




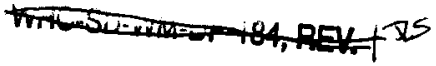

PLACE ANALYTICAL CARD IN BOX BELOW OR ATTACH TRAVELER

WHC-SD-WM-DP-184, REV. ।

\section{SPECIFIC GRAVITY : LA-510-112 (C-3)}

\begin{tabular}{|c|c|c|c|}
\hline 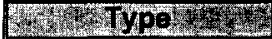 & & SAMPLE & REPLICATE \\
\hline SAMPLE & Gross Weight (W2) & 2.0448 & \\
\hline 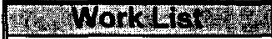 & Tare Weight (W1) & 1.8907 & \\
\hline 8729 & Weight of Solution (W2-W1) & 0.1541 & 0 \\
\hline 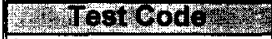 & Volume of Solution $\mu \mathrm{L}$ & 150.1700 & \\
\hline SPG-01 & Specific Gravity & 1.0262 & NA \\
\hline W & & & \\
\hline $\begin{array}{l}\text { LIQUID } \\
\text { S96R001121 }\end{array}$ & \multirow{6}{*}{\multicolumn{3}{|c|}{$\begin{array}{l}\text { Gross Weight }(W 2)=W t \text {. of vial }+ \text { cap }+ \text { cotton }+ \text { solution } \\
\text { Tare Weight }(W 1)=W t \text {. of vial + cap }+ \text { cotton } \\
\text { Specific Gravity }=[(W 2-W 1) * 1000 \mu \mathrm{L} / \mathrm{mL}] /[\text { Vol. of Solution } \mu \mathrm{L} * 1.000 \mathrm{~g} / \mathrm{mL}]\end{array}$}} \\
\hline$\frac{7 \text { notrument } \mathrm{Fog}}{\mathrm{BA001}}$ & & & \\
\hline RWK & & & \\
\hline 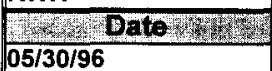 & & & \\
\hline Heforime & & & \\
\hline $04: 15$ PM & & & \\
\hline
\end{tabular}

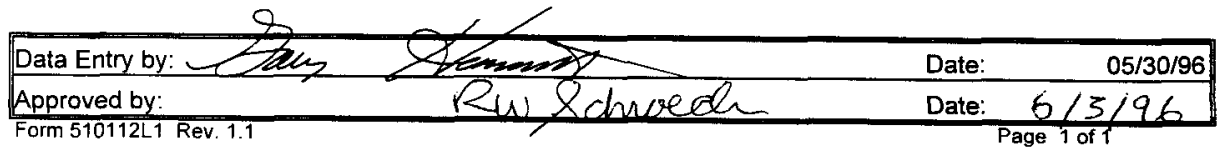


SPECIFIC GRAVITY : LA-510-112 (C-3)

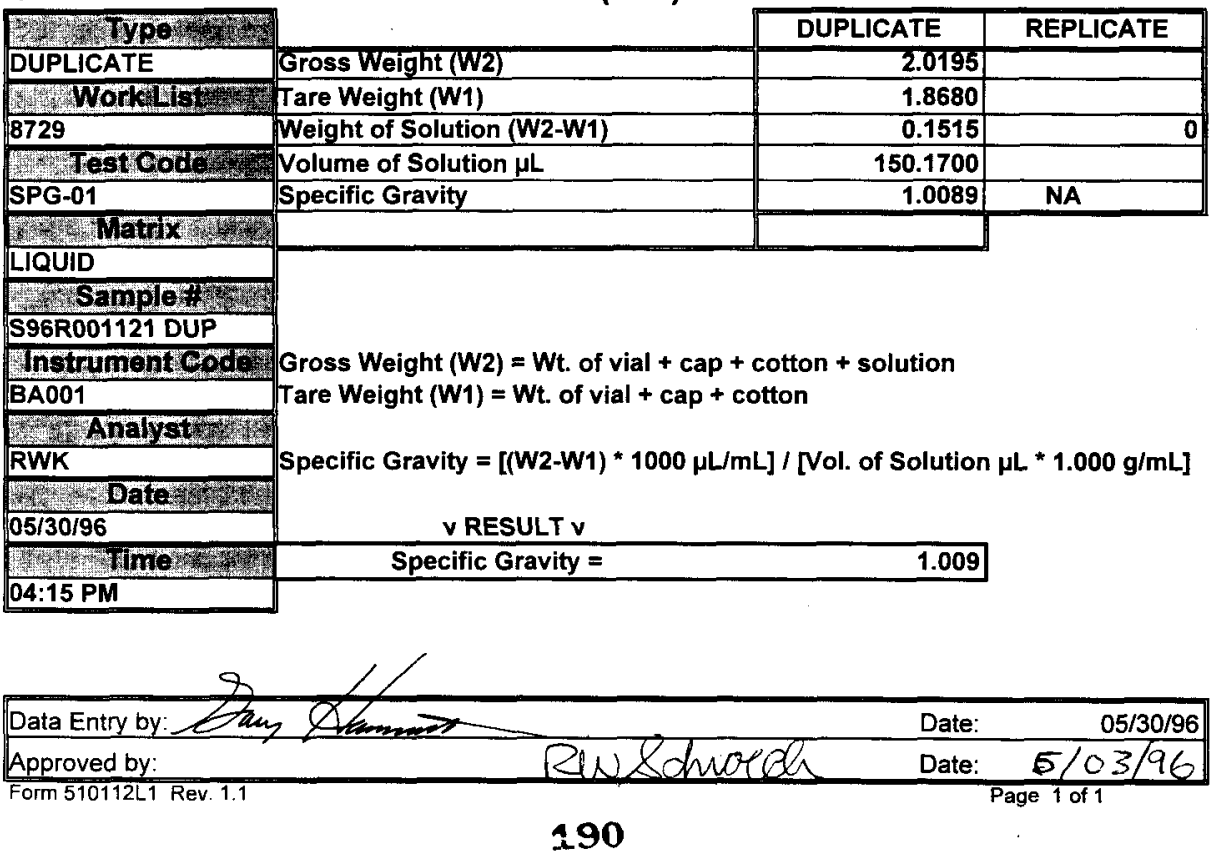


WHC-SD-WM-DP-184, REV. I

THE FOLLOWING ANALYSES WERE RERUN AND INCLUDED IN THE DATA PACKAGE, BUT THE RESULTS HAVE NOT BEEN REPORTED IN THE FINAL SUMMARY REPORTS. 
worklistrpt Version 2.1 05/15/95 WHC-SD-WM-DP-184, REV. I. .

$03 / 18 / 9612: 51$

LABCORE Data Entry Template for Worklist\#

Page: I

6606

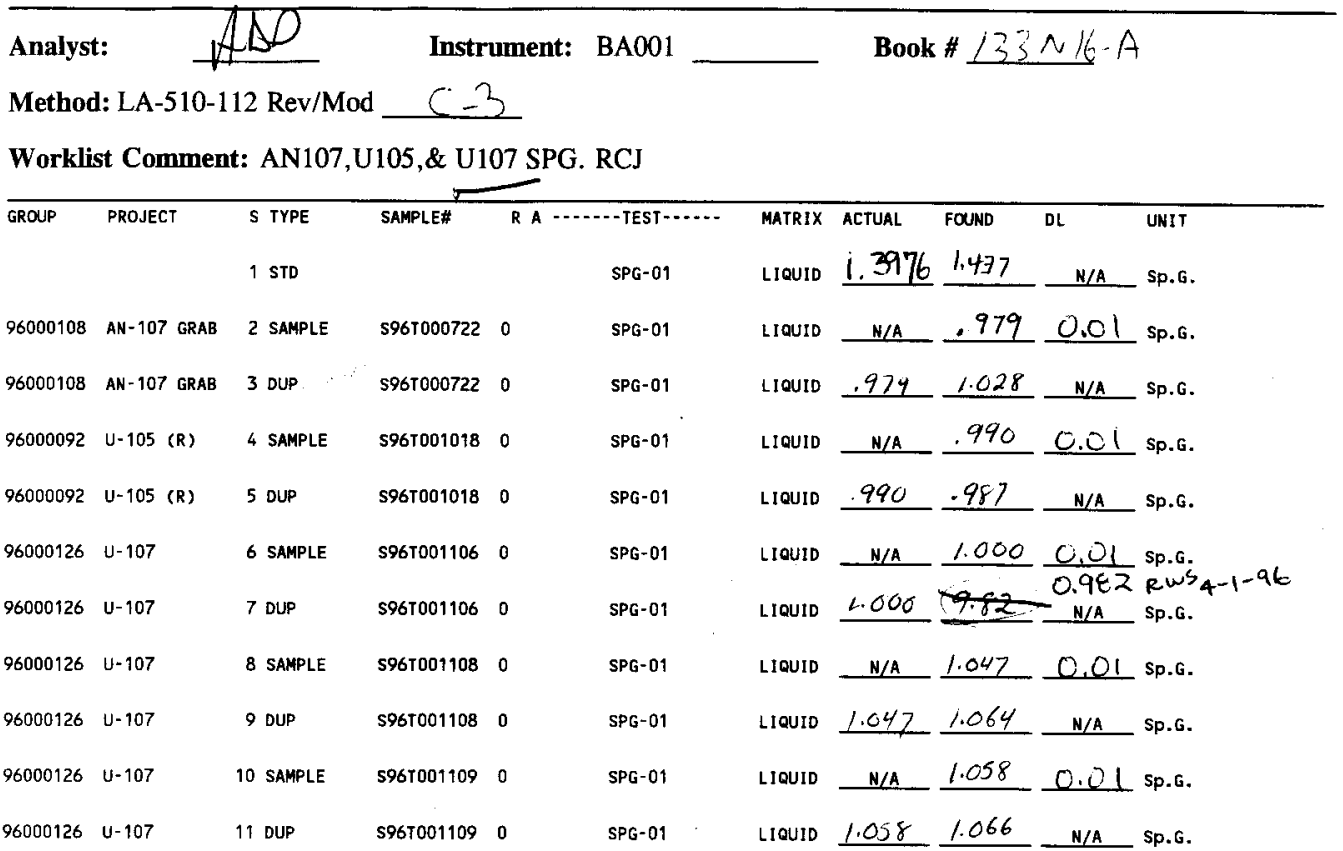

Final page for worklist \# $\quad 6606$

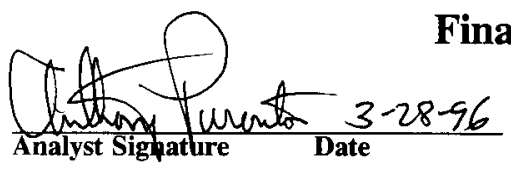

$$
\frac{R \text { t }(3 / 2, / 45}{\text { Analyst Signature Date }}
$$

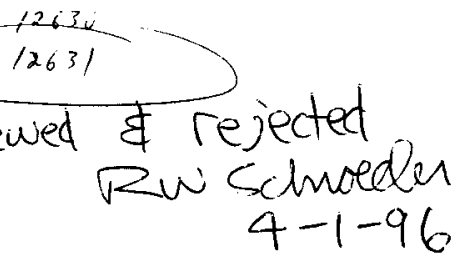

Data Entry Comments:

Results rejected due to batch LCCS failure.

Revs 4-1-96

Units shown for $Q C$ (SPK \& STD) may not reflect the actual units. $D L=$ Detection Limit, $S=$ Worklist Slot Number, $R=$ Replicate Number, $A=$ Aliquot Code.

192 


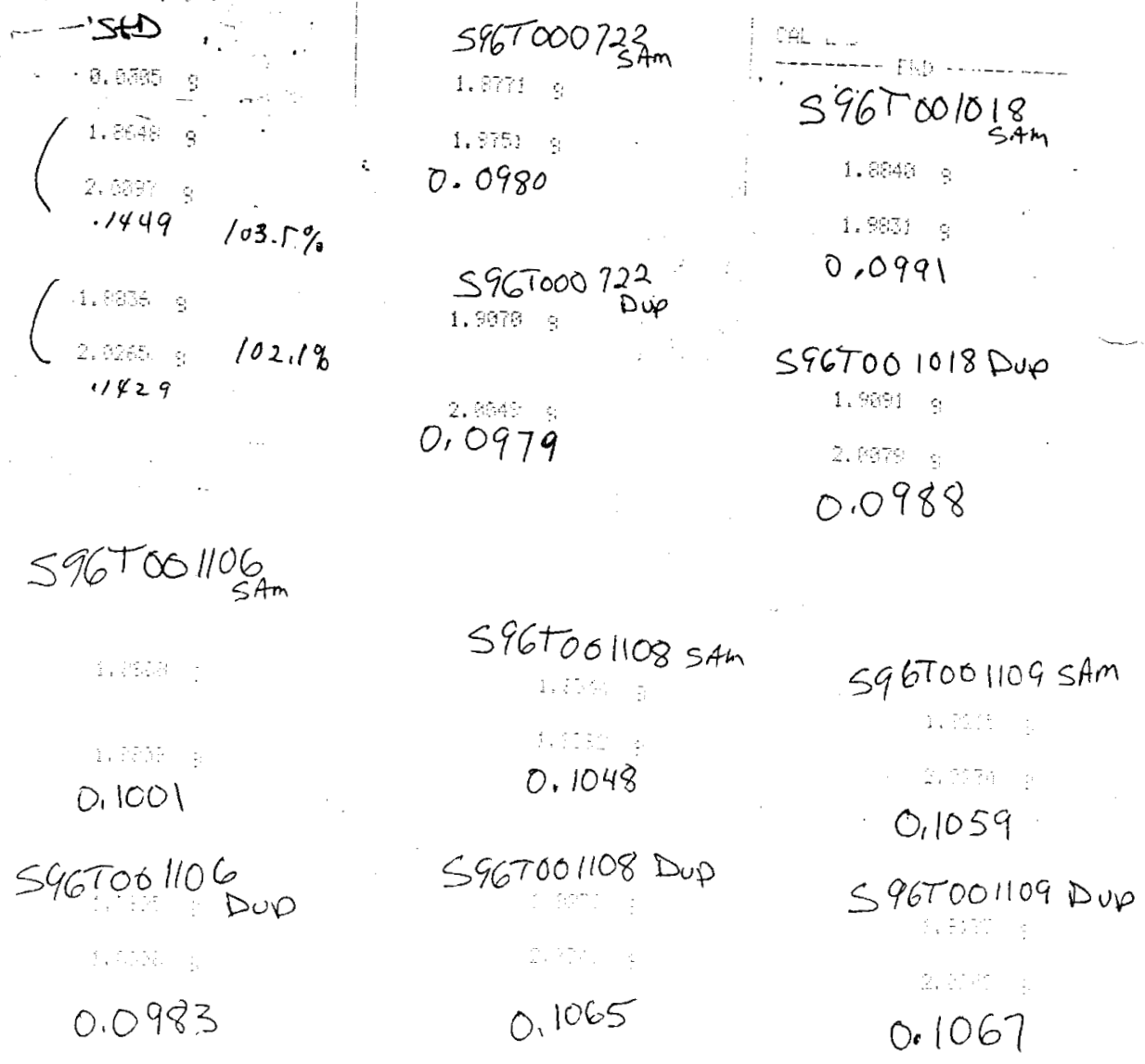


PLACE ANALYTICAL CARD IN BOX BELOW OR ATTACH TRAVELER

\section{WHC-SD-WM-DP-184, REV. I}

\section{SPECIFIC GRAVITY : LA-510-112 (C-3)}

\begin{tabular}{|c|c|c|c|}
\hline & & STANDARD & STANDARD \\
\hline STANDARD & Gross Weight (W2) & 2.0097 & 2.0265 \\
\hline man? & Tare Weight (W1) & 1.8648 & 1.8836 \\
\hline 6606 & Weight of Solution (W2-W1) & 0.1449 & 0.1429 \\
\hline 2.8 & Volume of Solution $\mu \mathrm{L}$ & 100.1200 & 100.1200 \\
\hline SPG-01 & Specific Gravity & 1.4473 & 1.4273 \\
\hline$r=0$ & Specific Gravity (Average) & 1.4373 & \\
\hline
\end{tabular}

LIQUID

STD/133N16A

(16)

Tare Weight $(W 1)=W t$. of vial + cap + cotton

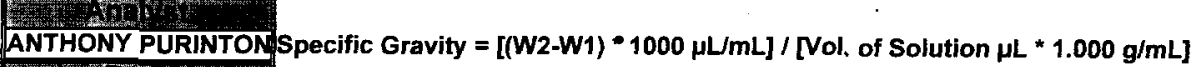
.

$03 / 28 / 96$

v RESULT $v$

\begin{tabular}{|l|l}
\hline Specific Gravity Average $=$ &
\end{tabular}

1.437

DATA

NOT USED

IN PACKAGE

Data Entry by:

Approved by:

Form 510112L_1 Rev. 1.1
Date:

Date:

194 


\section{WHC-SD-WM-DP-184, REV. I}

\section{SPECIFIC GRAVITY : LA-510-112 (C-3)}

\begin{tabular}{|c|c|c|c|}
\hline$\quad \quad \quad$ & & SAMPLE & REPLICATE \\
\hline SAMPLE & Gross Weight (W2) & 9.9751 & \\
\hline 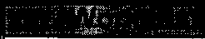 & Tare Weight (W1) & 1.8771 & \\
\hline 6606 & Weight of Solution (W2-W1) & 0.098 & 0 \\
\hline WI & Volume of Solution $\mu \mathrm{L}$ & 100.1200 & \\
\hline SPG-01 & Specific Gravity & 0.9788 & NA \\
\hline
\end{tabular}

\section{LIQUID}

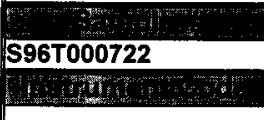

A.

ANTHONY PURINTON Specific Gravity $=[(\mathrm{W} 2-\mathrm{W} 1) * 1000 \mu \mathrm{L} / \mathrm{mL}] /[$ Vol. of Solution $\mu \mathrm{L} * 1.000 \mathrm{~g} / \mathrm{mL}]$

\begin{tabular}{l} 
03/28/96 \\
\hline
\end{tabular}

Gross Weight $(\mathrm{W} 2)=W \mathrm{t}$. of vial + cap + cotton + solution

Tare Weight $(W 1)=W t$. of vial + cap + cotton

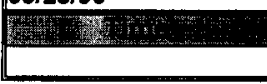

\section{v RESULT $v$}

Specific Gravity $=$

0.979

Data Entry by:

Date:

03/29/96

Date: 
PLACE ANALYTICAL CARD IN BOX BELOW OR ATTACH TRAVELER

WHC-SD-WM-DP-184, REV. I

\section{SPECIFIC GRAVITY : LA-510-112 (C-3)}

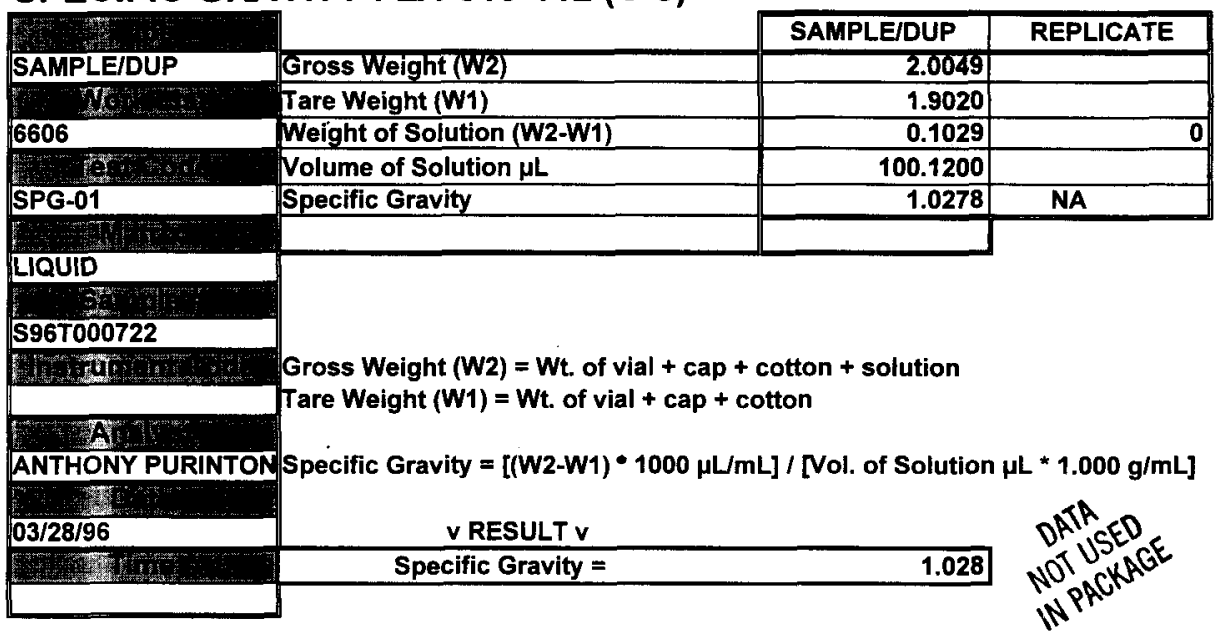

\begin{tabular}{|lll|}
\hline Data Entry by: & Date: & $03 / 29 / 96$ \\
\hline Approved by: & Date: & \\
\hline
\end{tabular}


PLACE ANALYTICAL CARD IN BOX BELOW OR ATTACH TRAVELER

WHC-SD-WM-DP-184, REV. I

- SPECIFIC GRAVITY : LA-510-112 (C-3)

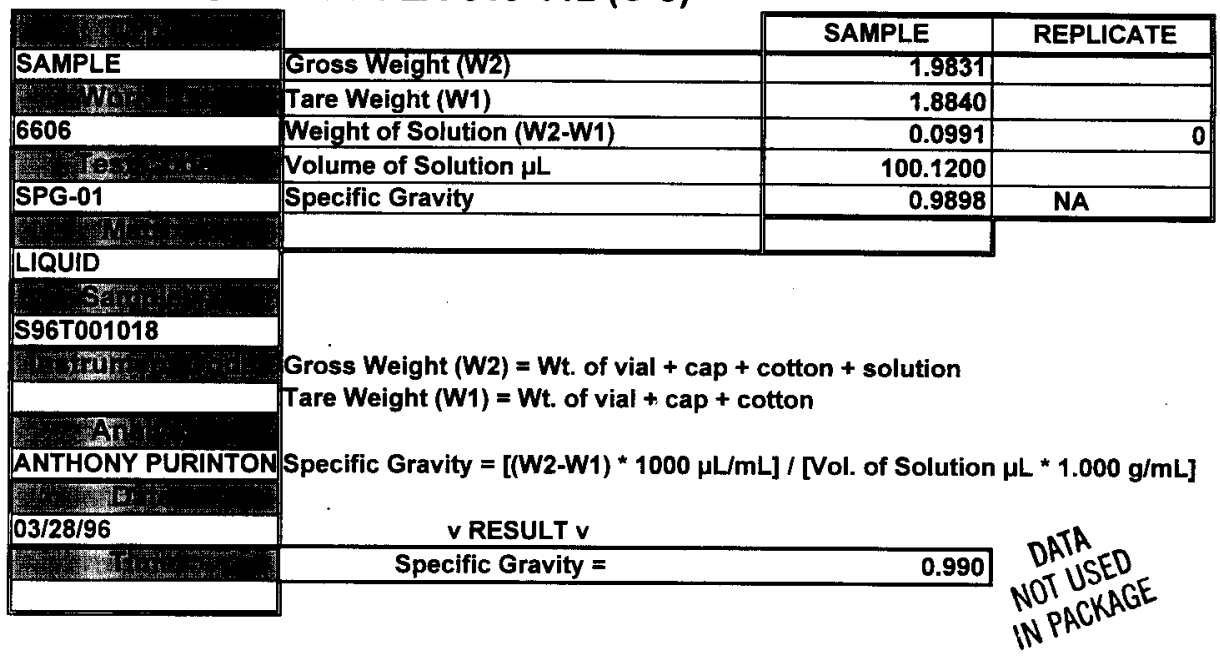

Data Entry by:

Date:

$03 / 29 / 96$

Approved by:

Date:

Form 510112L1 Rev. 1.1

Page 1 of 1 
PLACE ANALYTICAL CARD IN BOX BELOW OR ATTACH TRAVELER

WHC-SD-WM-DP-184, REV.I

SPECIFIC GRAVITY : LA-510-112 (C-3)

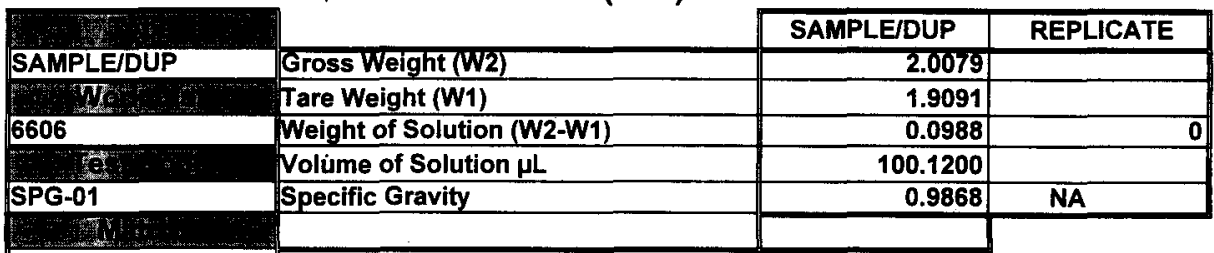

LIQUID

S. $34:-$

S96T001018

Whary, Gross Weight $\left(W_{2}\right)=W t$. of vial + cap + cotton + solution

Tare Weight $(W 1)=W t$. of vial + cap + cotton

ANTHONY PURINTONSpecific Gravity $=[(W 2-W 1) * 1000 \mu \mathrm{L} / \mathrm{mL}] /[$ Vol. of Solution $\mu \mathrm{L} * 1.000 \mathrm{~g} / \mathrm{mL}]$

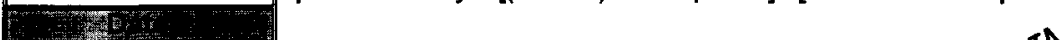

$03 / 28 / 96$

v RESULT v

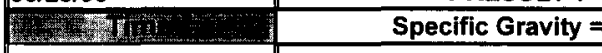

0.987

\begin{tabular}{|lll|}
\hline Data Entry by: & Date: & 03/29/96 \\
\hline Approved by: & Date: \\
\hline
\end{tabular}


PLACE ANALYTICAL CARD IN BOX BELOW OR ATTACH TRAVELER

\section{WHC-SD-WM-DP-184, REV. I}

\section{SPECIFIC GRAVITY : LA-510-112 (C-3)}

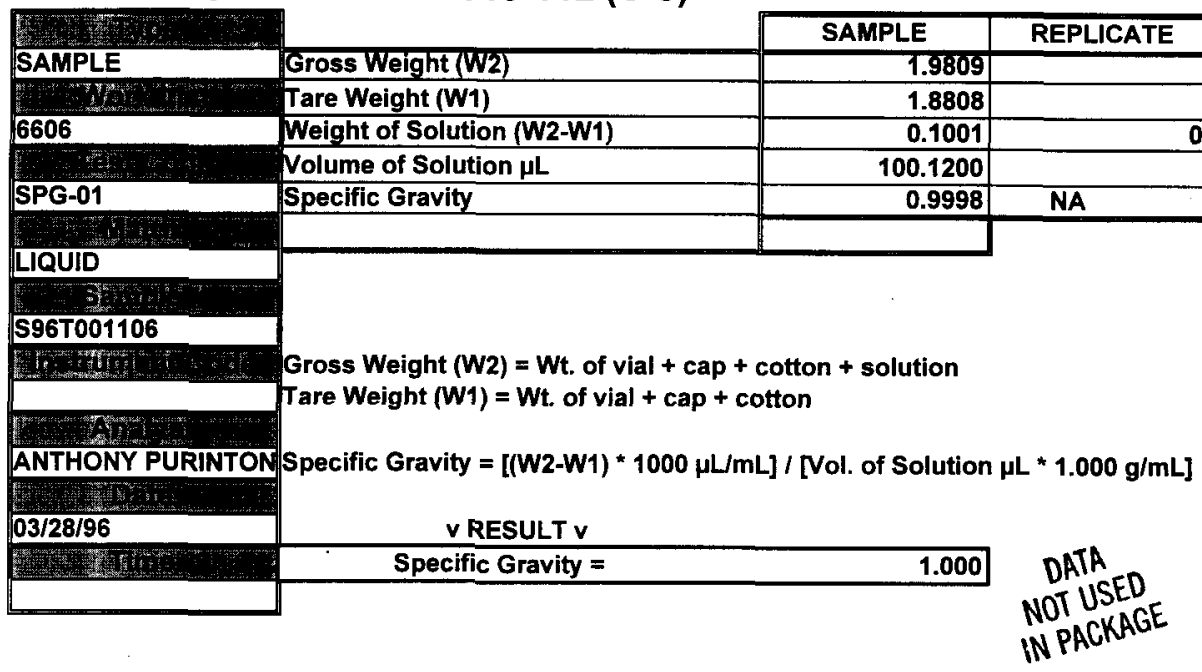

\begin{tabular}{|c|c|c|c|}
\hline Data Entry by: & & Date: & $03 / 29 / 96$ \\
\hline Approved by: & & Date: & \\
\hline Form 510112L1 Rev. 1.1 & 199 & Page & of 1 \\
\hline 10112ISP6606.WB1 & 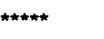 & 29-Mar-96 & $08: 36: 30 \mathrm{~A}$ \\
\hline
\end{tabular}


PLACE ANALYTICAL CARD IN BOX BELOW OR ATTACH TRAVELER

\section{WHC-SD-WM-DP-184, REV. 1}

\section{SPECIFIC GRAVITY : LA-510-112 (C-3)}

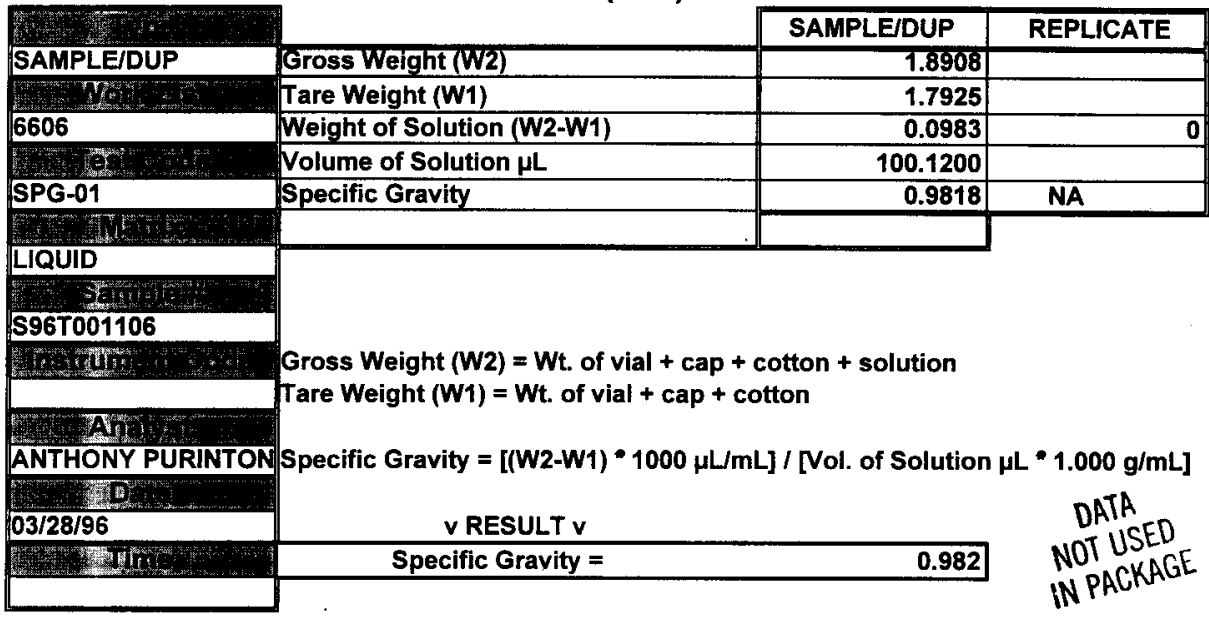

Data Entry by:

Approved by:

Form 510112L1 Rev. 1.1
Date:

Date:

$$
2 \mathrm{CO}
$$

$03 / 29 / 96$

Page 1 of 1


PLACE ANALYTICAL CARD IN BOX BELOW OR ATTACH TRAVELER

WHC-SD-WM-DP-184, REV. I

SPECIFIC GRAVITY : LA-510-112 (C-3)

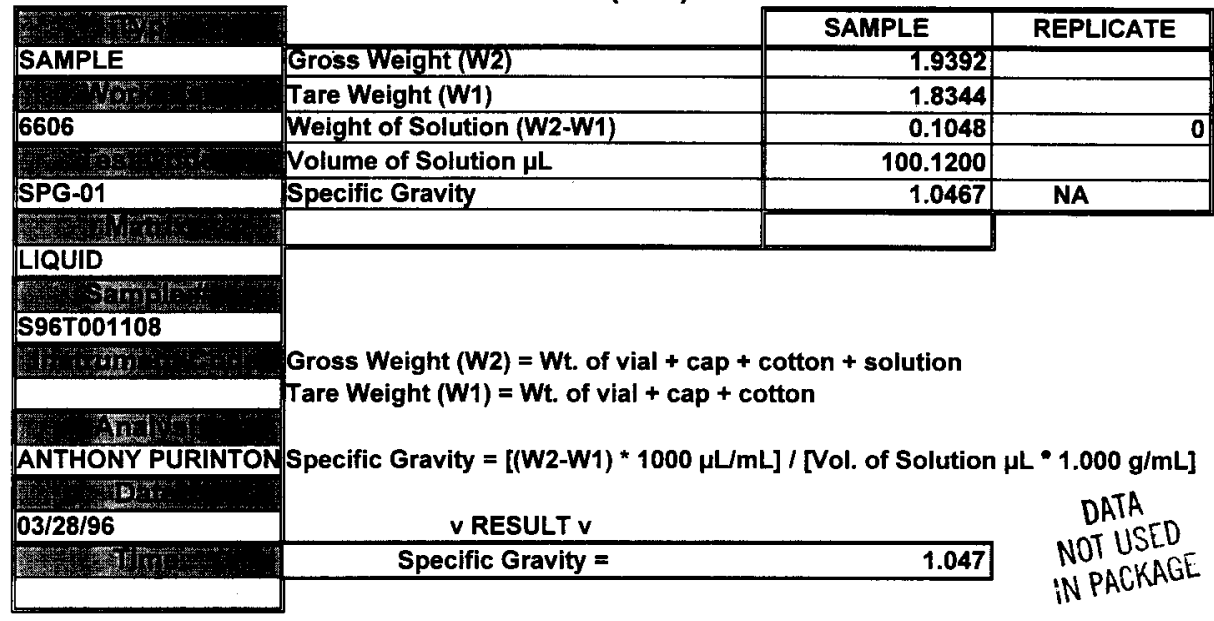

Data Entry by:

Approved by:

Form 510112L1 Rev. 1.1
Date:

Date:

$$
2 \mathrm{C} 1
$$

03/29/96 
PLACE ANALYTICAL CARD IN BOX BELOW OR ATTACH TRAVELER

WHC-SD-WM-DP-184, REV.

SPECIFIC GRAVITY : LA-510-112 (C-3)

\begin{tabular}{|c|c|c|c|}
\hline Sis & & SAMPLE/DUP & REPLICATE \\
\hline SAMPLE/DUP & Gross Weight (W2) & 2.0341 & \\
\hline natin? & Tare Weight (W1) & 1.9276 & \\
\hline 6606 & Weight of Solution (W2-W1) & 0.1065 & $\mathrm{O}$ \\
\hline 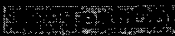 & Volume of Solution $\mu \mathrm{L}$ & 100.1200 & \\
\hline SPG-01 & Specific Gravity & 1.0637 & NA \\
\hline
\end{tabular}

LIQUID

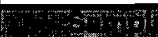

S96T001108

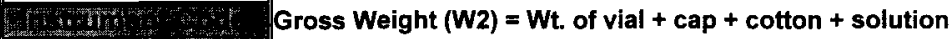

Tare Weight $(\mathrm{W} 1)=\mathrm{Wt}$. of vial + cap + cotton

ANTHONY PURINTON Specific Gravity $=\left[\left(W_{2}-W 1\right) * 1000 \mu \mathrm{L} / \mathrm{mL}\right] /[$ Vol. of Solution $\mu \mathrm{L} * 1.000 \mathrm{~g} / \mathrm{mL}]$ TII

$03 / 28 / 96$

v RESULT $v$

Specific Gravity =

1.064

DATA

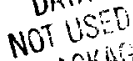

IN PACGATEL

\begin{tabular}{|lll|}
\hline Data Entry by: & Date: & $03 / 29 / 96$ \\
\hline Approved by: & & Date: \\
\hline Form 510112L1 Rev. 1.1 & CC2 & Page 1 of 1 \\
\hline
\end{tabular}


PLACE ANALYTICAL CARD IN BOX BELOW OR ATTACH TRAVELER

WHC-SD-WM-DP-184, REV. 1

\section{SPECIFIC GRAVITY : LA-510-112 (C-3)}

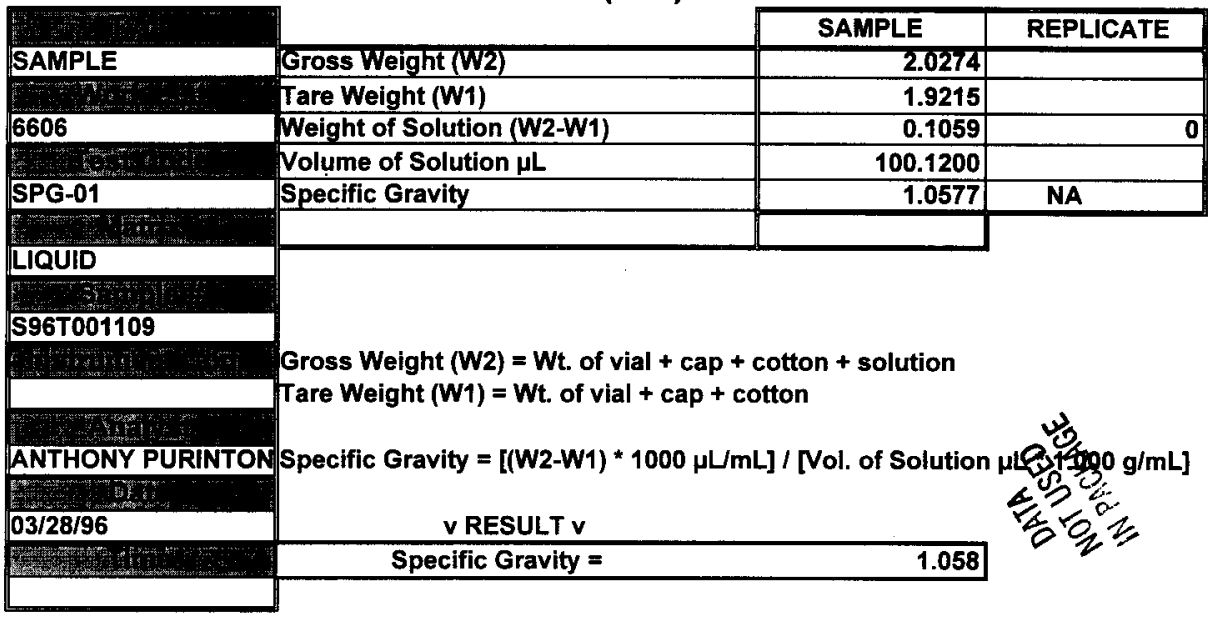


PLACE ANALYTICAL CARD IN BOX BELOW OR ATTACH TRAVELER

WHC-SD-WM-DP-184, REV.|

SPECIFIC GRAVITY : LA-510-112 (C-3)

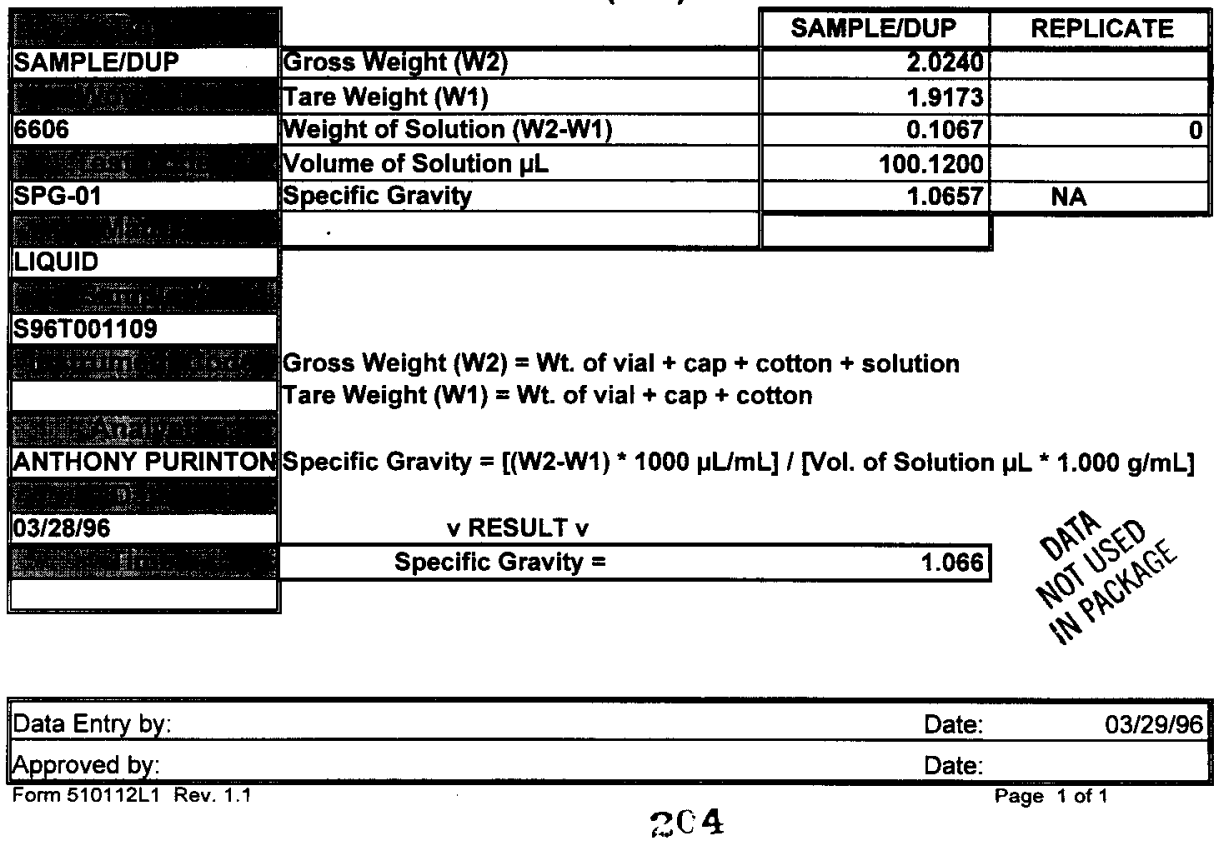


WHC-SD-WM-DP-184, REV.|

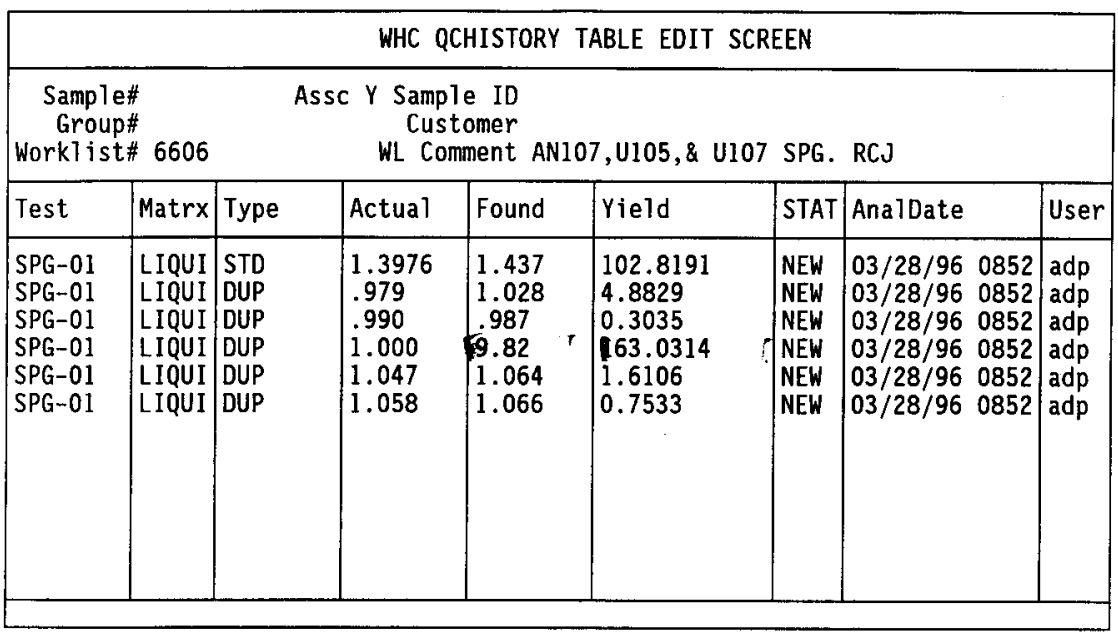

Save(F12) End(F3)

Incorrect

Cuspchueder 4-1-96

205 
WHC-SD-WM-DP-184, REV. I

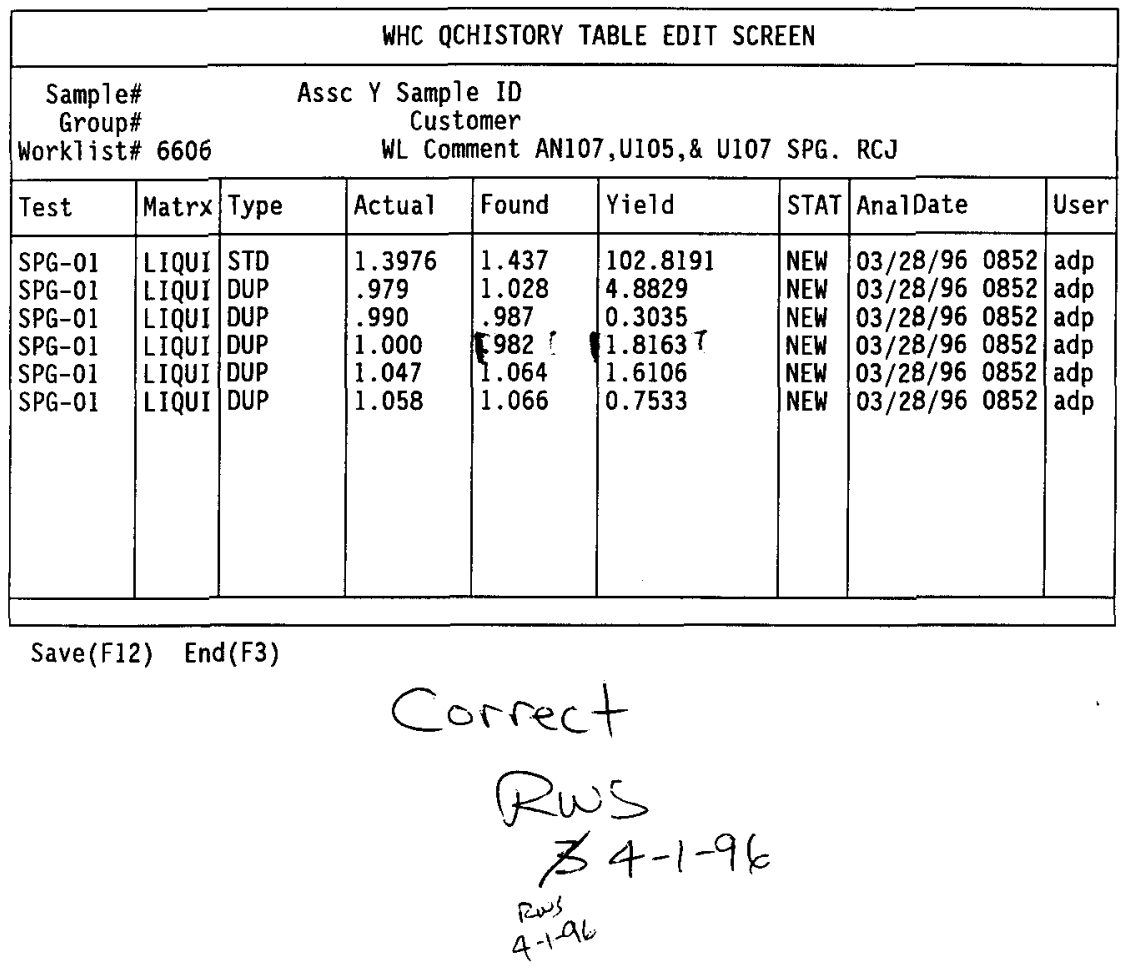

206 
worklistrpt Version 2.1 05/15/95

WHC-SD-WM-DP-184, REV. I

$03 / 19 / 96 / 1: 02$

LABCORE Data Entry Template for Worklist\#

Page: $\quad 1$

6687

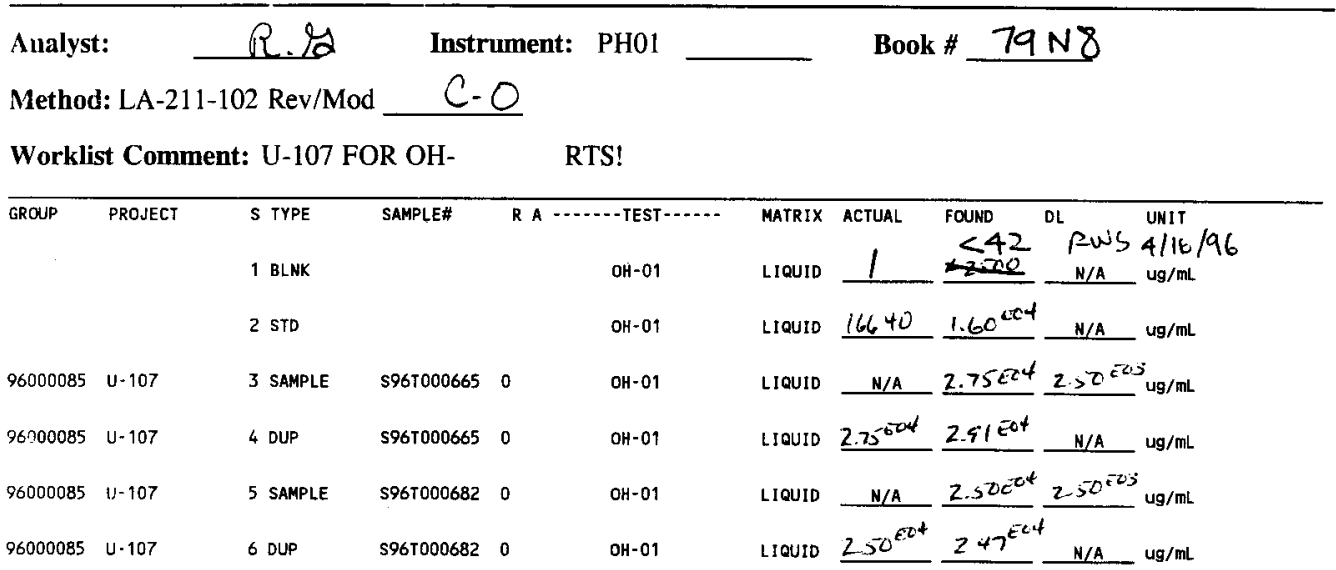

Final page for worklist \# $\quad \mathbf{6 6 8 7}$

Rae ln Treen 4-15-96 Analyst Signature Date $4-16-96$

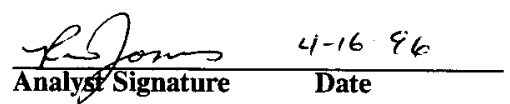

Reviewed \& edited Fu'sodvoed 4/16/96

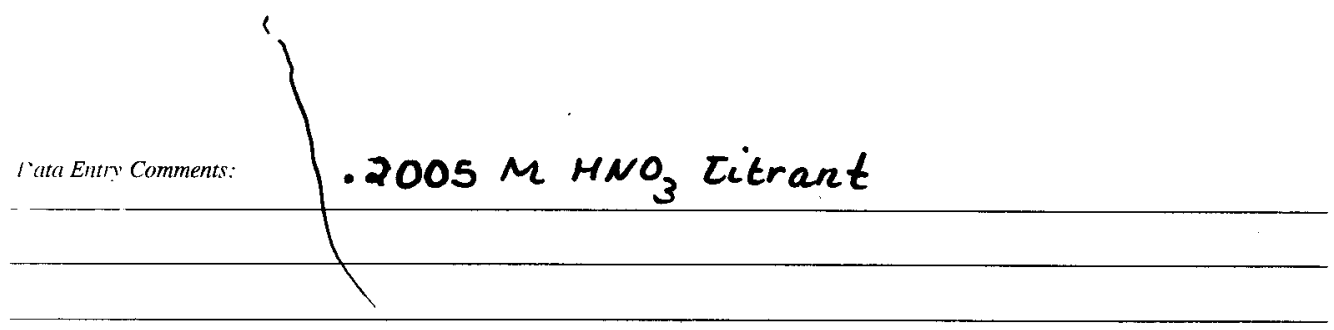

Units shown for $Q C$ (SPK \& STD) may not reflect the actual units. $D L=$ Detection Limit, $S=$ Worklist Slot Number, $R=$ Replicate Number, $A=$ Aliquot Code.

207 


\section{WHC-SD-WM-DP-184, REV. 1}

PLACE ANALYTICAL CARD IN BOX BELOW OR ATTACH TRAVELER
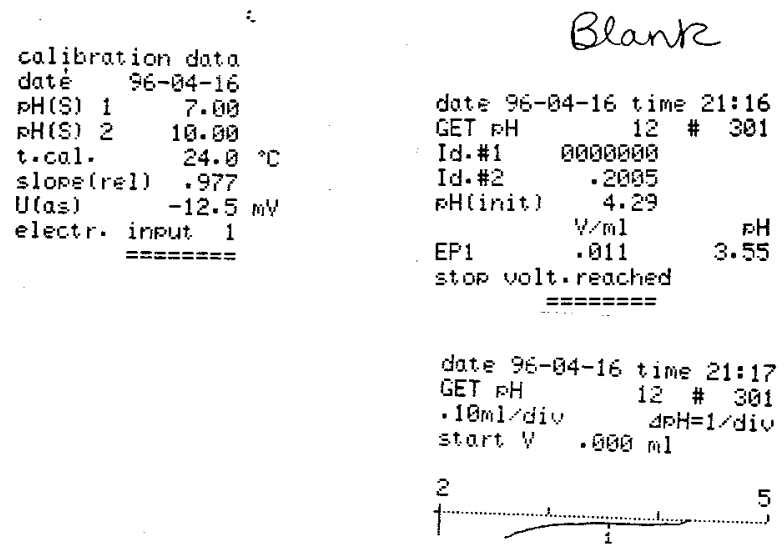

OH (AUTO) : LA-211-102 (C-0)

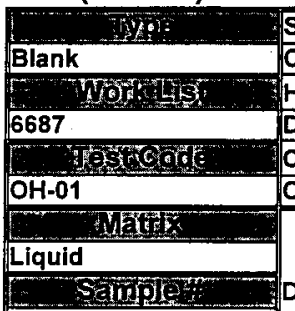

Sample Size (mL) SS

Concentration of $\mathrm{HNO} 3$ (Molarity)

HNO3 Titrant at OH end-point in $\mathrm{mL}$

Dilution Factor DF

Concentration of $\mathrm{OH}$ in Sample (Molarity)

$\mathrm{OH}$ in Sample in $\mu \mathrm{g} / \mathrm{mL}$ (PPM) 5

BLNK

\begin{tabular}{|c|c|c|}
\hline Dhstronento ofe & Detection Limit $(\mu \mathrm{g} / \mathrm{mL})$ & $4.17 E+01$ \\
\hline
\end{tabular}

\begin{tabular}{|c|c|c|}
\hline \multirow[t]{2}{*}{$\mathrm{OH}$ Molarity $=\left((\mathrm{mL} \text { HNO3 })^{*}(\mathrm{M} H \mathrm{HO} 3)\right) /$ Sample Size in $\mathrm{mL}$ "Dilution Factor } & \multirow{2}{*}{\multicolumn{2}{|c|}{ OH Molarity $=\left((\mathrm{mL} \text { HNO3 })^{*}(\mathrm{M} \mathrm{HNO} 3)\right) /$ Sample Size in $\mathrm{mL}$ "Dilution Factor }} \\
\hline & & \\
\hline Gint: & $\mathrm{OH}$ in $\mu \mathrm{g} / \mathrm{mL}=(\mathrm{OH} \text { MOLARITY })^{*}(17.0 \mathrm{~g} / \mathrm{mole}$ & /(1000mL/L)) \\
\hline $04 / 16 / 96$ & & \\
\hline $\mathrm{Tin}$ & & Blank \\
\hline & Concentration of OH in Sample (Molarity) & $0.00 \mathrm{E}+00$ \\
\hline & $\mathrm{OH}$ in Sample in $\mu \mathrm{g} / \mathrm{mL}$ (PPM) & $<42$ \\
\hline
\end{tabular}

The Result is < Detection Limit

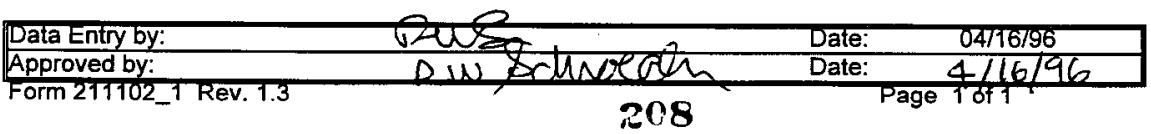




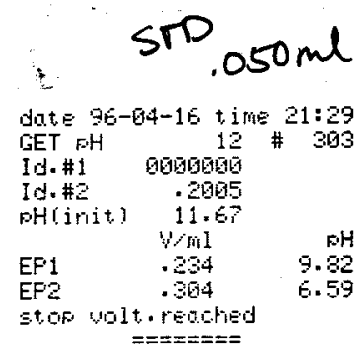

date 96-94-16 time $21: 35$

GET FH 12 \# 393

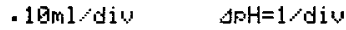

start $y=690 \mathrm{ml}$

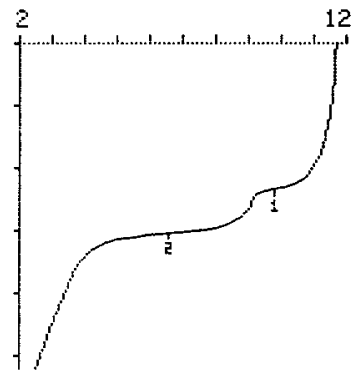

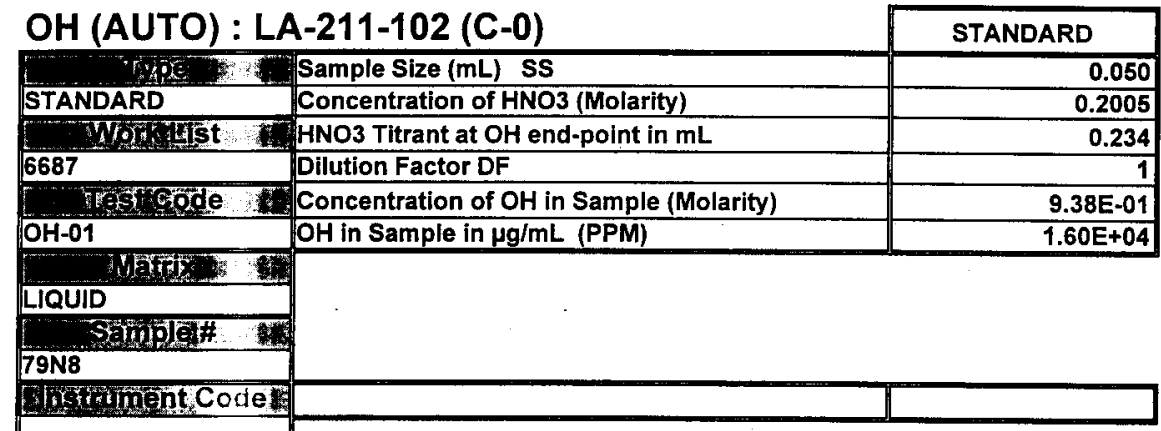

MAIIyst
RAG

Difer in $\mu \mathrm{g} / \mathrm{mL}=(\mathrm{OH} \text { MOLARITY })^{*}(17.0 \mathrm{~g} / \mathrm{mole})^{*}((1000000 \mu \mathrm{g} / \mathrm{g}) /(1000 \mathrm{~mL} / \mathrm{L}))$

$04 / 15 / 96$

\begin{tabular}{|c|c|c|}
\hline ITES & & STANDARD \\
\hline 03:15 AM & Concentration of $\mathrm{OH}$ in Sample (Molarity) & $9.38 E-01$ \\
\hline & $\mathrm{OH}$ in Sample in $\mu \mathrm{g} / \mathrm{mL}$ (PPM) & $1.60 E+04$ \\
\hline
\end{tabular}

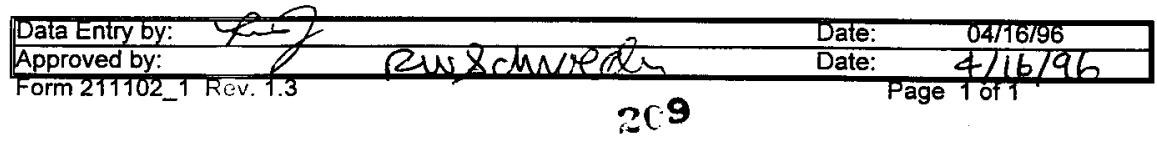




\section{WHC-SD-WM-DP-184, REV. I}

PLACE ANALYTICAL CARD IN BOX BELOW OR ÁTTACH TRAVELER

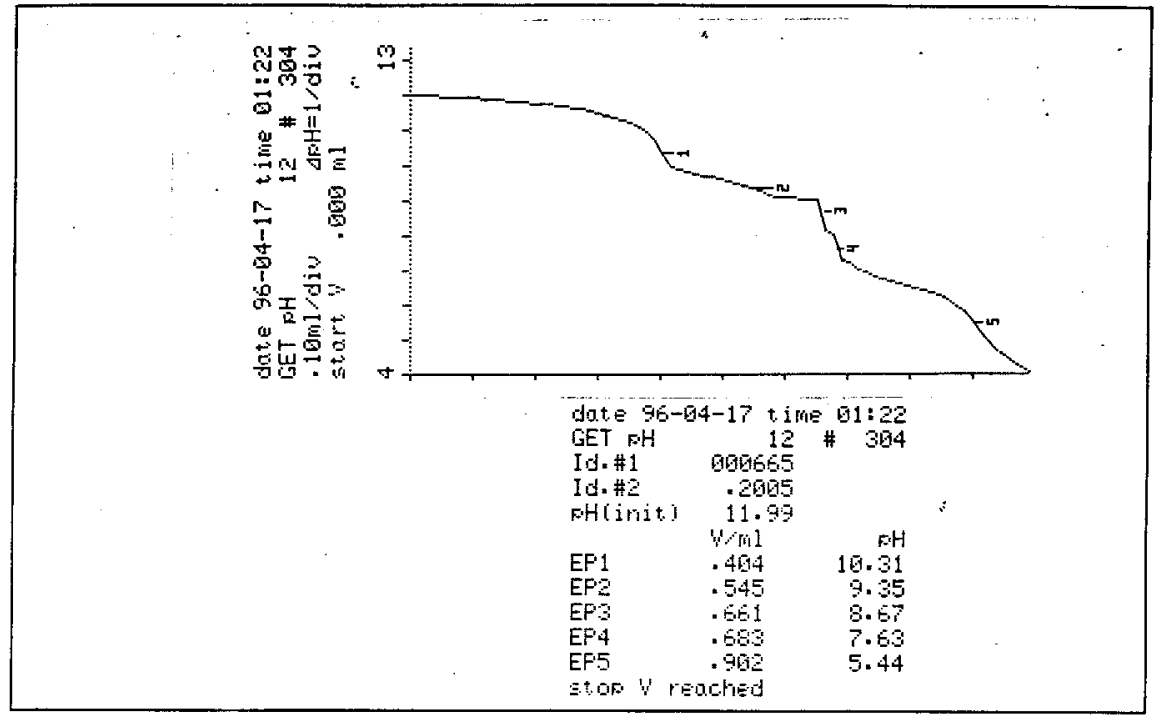

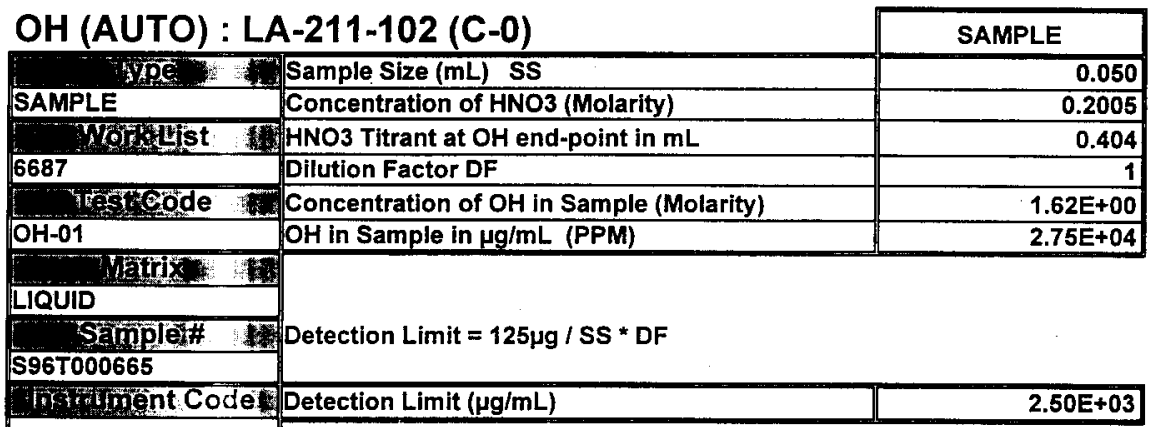

\begin{tabular}{|c|c|c|c|}
\hline \multirow{2}{*}{$\begin{array}{l}\text { AJulyst } \\
\text { RAG }\end{array}$} & 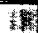 & \multicolumn{2}{|c|}{$\mathrm{OH}$ Molarity $=\left((\mathrm{mL} \text { HNO3 })^{\star}(\mathrm{M} \mathrm{HNO})\right) /$ Sample Size in $\mathrm{mL}^{*}$ Dilution Factor } \\
\hline & & & \\
\hline Dfte: & 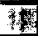 & $\mathrm{OH}$ in $\mu \mathrm{g} / \mathrm{mL}=(\mathrm{OH} \text { MOLARITY })^{\star}(17.0 \mathrm{~g} / \mathrm{mole})^{\star}((1000$ & $000 \mu \mathrm{g} / \mathrm{g}) /(1000 \mathrm{~mL} / \mathrm{L}))$ \\
\hline $15 / 96$ & & & \\
\hline They & 1. & & SAMPLE \\
\hline 03:15 AM & & Concentration of OH in Sample (Molarity) & $1.62 E+00$ \\
\hline & & $\mathrm{OH}$ in Sample in $\mu \mathrm{g} / \mathrm{mL}$ (PPM) & $2.75 E+04$ \\
\hline
\end{tabular}

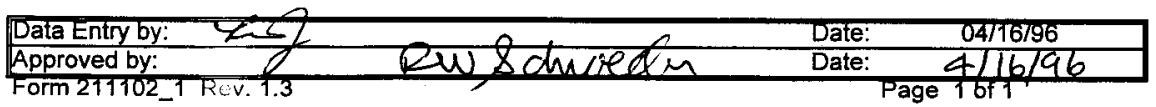


PLACE ANALYTICAL CARD IN BOX BELOW OR ATTACH TRAVELER

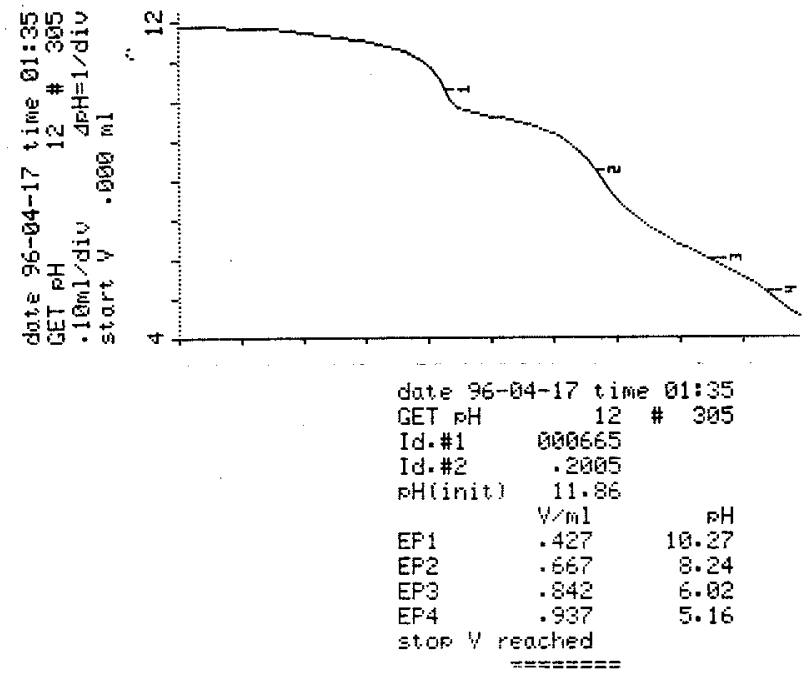

\begin{tabular}{|c|c|c|}
\hline \multicolumn{2}{|c|}{ OH (AUTO) : LA-211-102 (C-O) } & DUPLICATE \\
\hline me +4 & Sample Size (mL) SS & 0.050 \\
\hline DUPLICATE & Concentration of HNO3 (Molarity) & 0.2005 \\
\hline 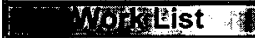 & HNO3 Titrant at OH end-point in $\mathrm{mL}$ & 0.427 \\
\hline 6687 & Dilution Factor DF & 1 \\
\hline ingtreode & Concentration of OH in Sample (Molarity) & $1.71 \mathrm{E}+00$ \\
\hline $\mathrm{OH}-01$ & $\mathrm{OH}$ in Sample in $\mu \mathrm{g} / \mathrm{mL}$ (PPM) & 2.91E+04 \\
\hline
\end{tabular}

LIQUID

\begin{tabular}{|c|c|c|}
\hline Sypple\# & Detection Limit $=125 \mu \mathrm{g} / \mathrm{SS}$ * DF & \\
\hline S96T000665DUP & & \\
\hline Therofont Code & Detection Limit ( $\mu \mathrm{g} / \mathrm{mL})$ & $2.50 E+03$ \\
\hline
\end{tabular}
RAG

$04 / 15 / 96$

Date $\mathrm{OH}$ in $\mu \mathrm{g} / \mathrm{mL}=(\mathrm{OH} \text { MOLARITY })^{\star}(17.0 \mathrm{~g} / \mathrm{mole})^{\star}((1000000 \mu \mathrm{g} / \mathrm{g}) /(1000 \mathrm{~mL} / \mathrm{L}))$

\begin{tabular}{|l|l|r|}
\cline { 2 - 3 } 03:15 AM & \multicolumn{1}{c|}{ DUPLICATE } \\
\hline & Concentration of OH in Sample (Molarity) & $1.71 E+00$ \\
\hline OH in Sample in $\mu \mathrm{g} / \mathrm{mL}$ (PPM) & $2.91 E+04$ \\
\hline
\end{tabular}

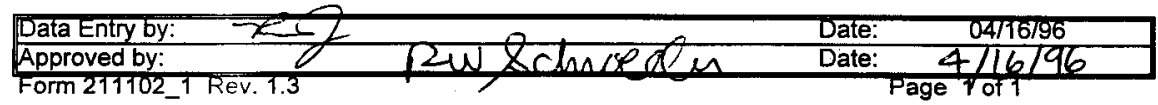




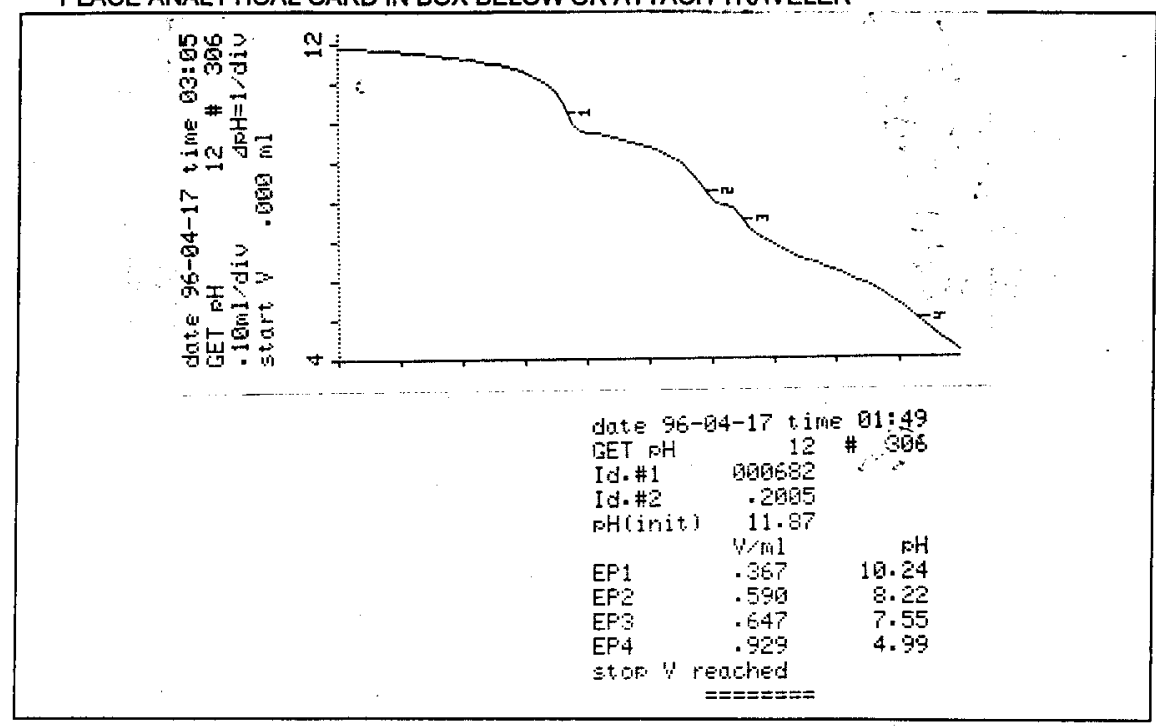

\begin{tabular}{|c|c|c|}
\hline OH (AUTO) : L & $-211-102(\mathrm{C}-0)$ & SAMPLE \\
\hline pe $x$ & Sample Size (mL) SS & 0.050 \\
\hline SAMPLE & Concentration of $\mathrm{HNO} 3$ (Molarity) & 0.2005 \\
\hline Thorgutist & HNO3 Titrant at $\mathrm{OH}$ end-point in $\mathrm{mL}$ & 0.367 \\
\hline 6687 & Dilution Factor DF & 1 \\
\hline WHode & Concentration of $\mathrm{OH}$ in Sample (Molarity) & $1.47 E+00$ \\
\hline $\mathrm{OH}-01$ & OH in Sample in $\mu \mathrm{g} / \mathrm{mL}$ (PPM) & $2.50 \mathrm{E}+04$ \\
\hline
\end{tabular}

\begin{tabular}{l|l|} 
LIQUID & Detection Limit $=125 \mu \mathrm{g} / \mathrm{SS}$ * DF \\
S96T000682 & Detection Limit $(\mu \mathrm{g} / \mathrm{mL})$
\end{tabular}

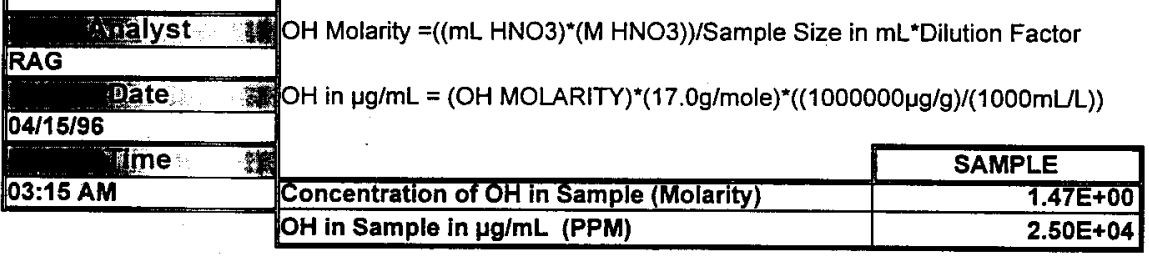

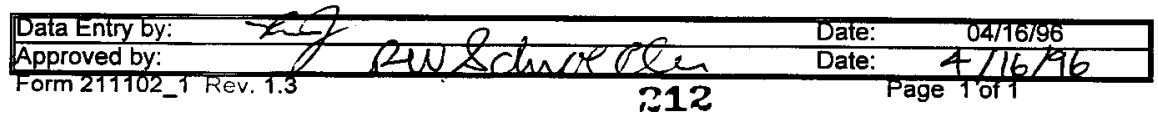


PLACE ANALYTICAL CARD IN BOX BELOW OR ATTACH TRAVELER
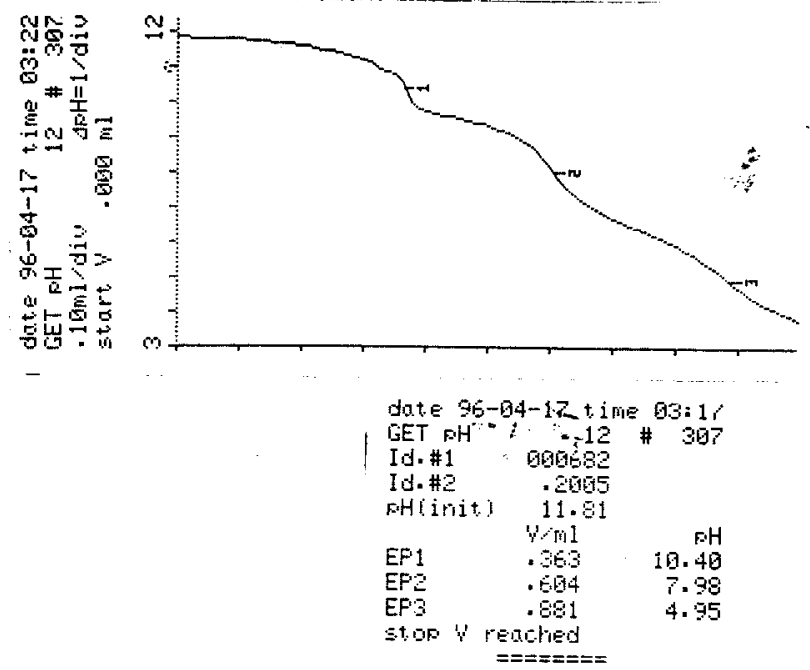

OH (AUTO) : LA-211-102 (C-0)

DUPLICATE

\begin{tabular}{|c|c|c|}
\hline NPE $6 x$ & Sample Size (mL) SS & 0.050 \\
\hline DUPLICATE & Concentration of $\mathrm{HNO}$ (Molarity) & 0.2005 \\
\hline Mrowbst & HNO3 Titrant at OH end-point in $\mathrm{mL}$ & 0.363 \\
\hline 6687 & Dilution Factor DF & 1 \\
\hline ingegde & Concentration of OH in Sample (Molarity) & $1.46 \mathrm{E}+00$ \\
\hline $\mathrm{OH}-01$ & OH in Sample in $\mu \mathrm{g} / \mathrm{mL}$ (PPM) & 2.47E+04 \\
\hline
\end{tabular}

LIQUID

Tatrix

SIMplê\#

S96T000682DUP

6) ipent Coded Detection Limit $(\mu \mathrm{g} / \mathrm{mL})$

$2.50 E+03$

Thalyst $\mathrm{OH}$ Molarity $=\left((\mathrm{mL} H N O 3)^{\star}(\mathrm{M}\right.$ HNO3) $) /$ Sample Size in $\mathrm{mL}$ *Dilution Factor RAG

$04 / 15 / 96$

Date

\begin{tabular}{|c|c|c|}
\hline Iime & & DUPLICATE \\
\hline 03:15 AM & Concentration of OH in Sample (Molarity) & $1.46 E+00$ \\
\hline & $\mathrm{OH}$ in Sample in $\mu \mathrm{g} / \mathrm{mL}$ (PPM) & 2.47E+04 \\
\hline
\end{tabular}

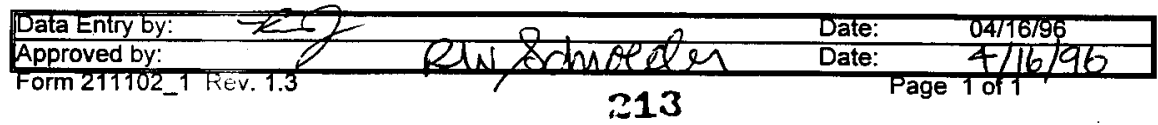




\section{LABCORE Data Entry Template for Worklist\#}

Analyst: Ry Instrument: PH01 _ Book \# 79N8

Method: LA-211-102 Rev/Mod C. O

Worklist Comment: U-107 OH'S RUN IN DUPLICATE. RCJ

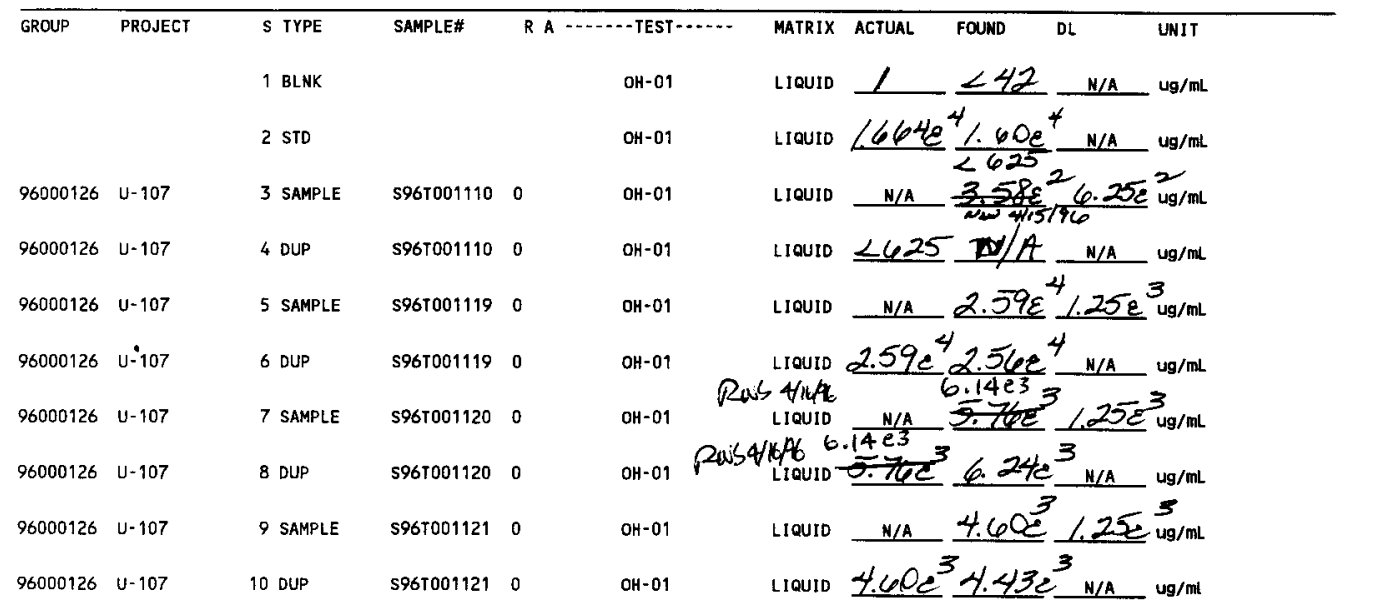

\section{Final page for worklist \# 7581}

Rave Qurze thear 4-14-96

Analyst Signature Date

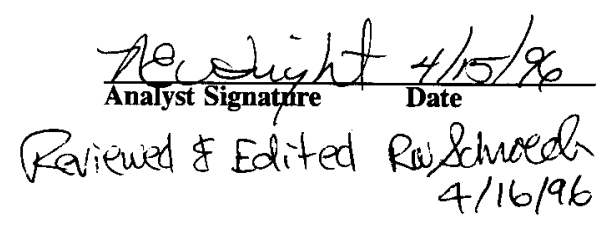

$.2005 \mathrm{~m} \mathrm{HNO}_{3}$ Titrant

Data Entry Comments:

Sample \# S96500110 used all sample in plocess wed max sample sis of $200 \mathrm{ml}$ - unaile to nundiuplecedes

Units shown for $Q C$ (SPK \& STD) may not reflect the actual units. $D L=$ Detection Limit, $S=$ Worklist Slot Number, $R=$ Replicate Number, $A=$ Aliquot Code. 


\section{WHC-SD-WM-DP-184, REV. I}

PLACE ANALYTICAL CARD IN BOX BELOW OR ATTACH TRAVELER
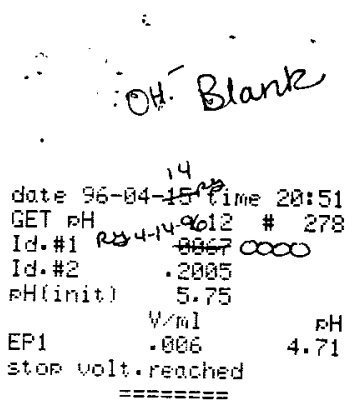

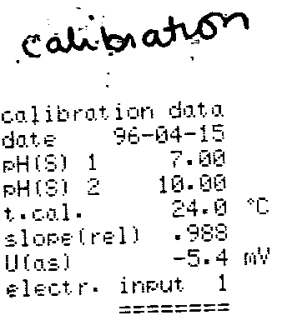

\section{BEST AVAILABLE COPY}

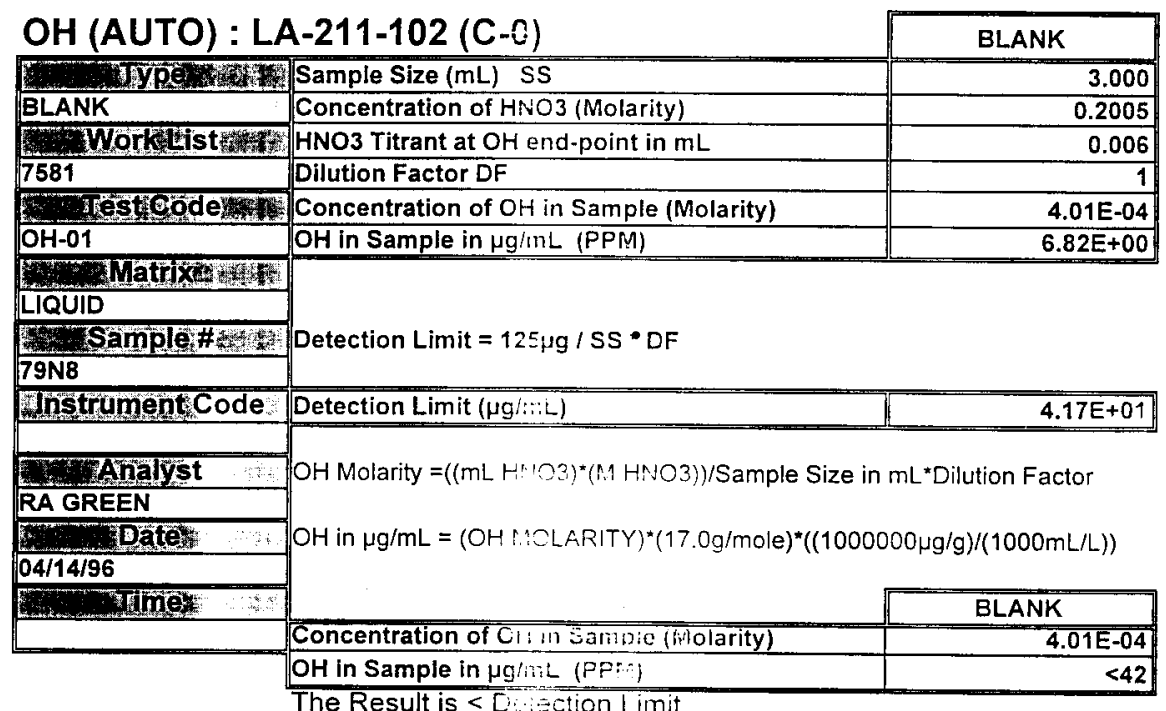

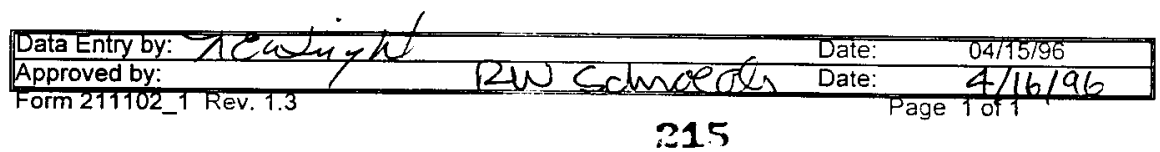




\section{WHC-SD-WM-DP-184, REV. |}

PLACE ANALYTICAL CARD IN BOX BELOW OR ATTACH TRAVELER

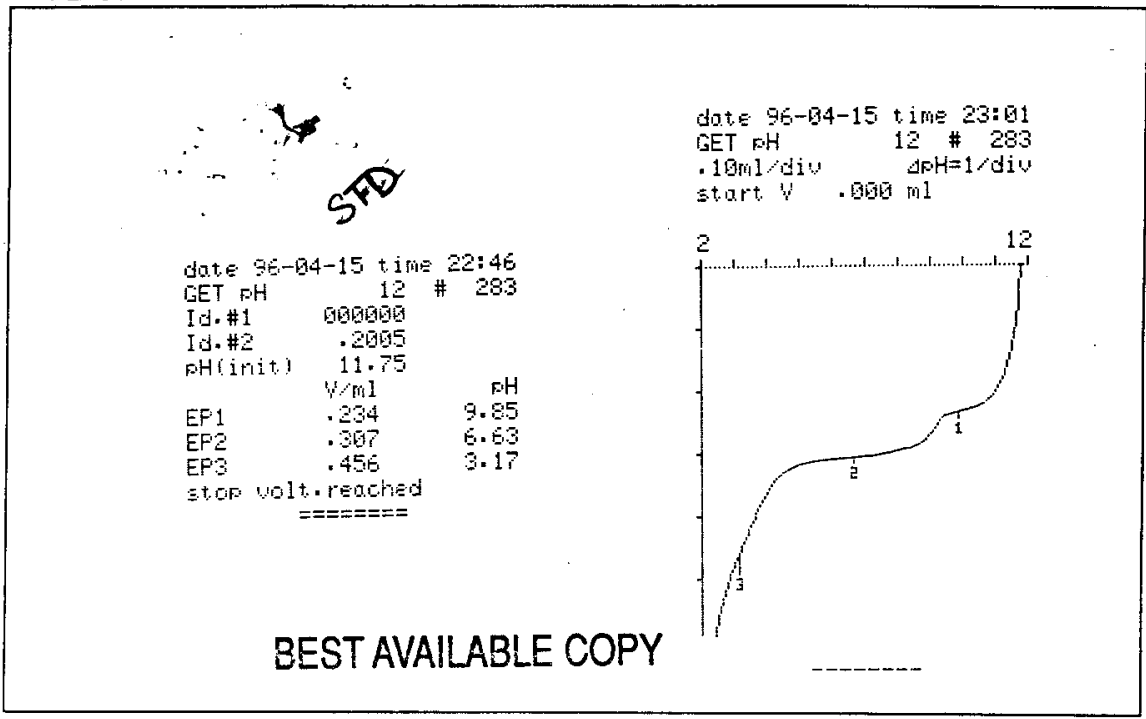

\begin{tabular}{|c|c|c|}
\hline OH (AUTO) : L & A-2 & STANDARD \\
\hline 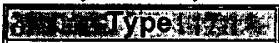 & Sample Size $(\mathrm{mL}) \subseteq s$ & 0.050 \\
\hline STANDARD & Concentration of $\mathrm{Hr}^{+} \mathrm{O} 3$ (Molarity) & 0.2005 \\
\hline WWormList & HNO3 Titrant at $\mathrm{OH}$ end-point in $\mathrm{mL}$ & 0.234 \\
\hline 7581 & Dilution Factor DF & 1 \\
\hline Erinestogde, & Concentration of $\mathrm{OH}$ in Sample (Molarity) & $9.38 \mathrm{E}-01$ \\
\hline $\mathrm{OH}-01$ & $\mathrm{OH}$ in Sample in $\mu \mathrm{g} / \mathrm{mL}$ (PPM) & $1.60 E+04$ \\
\hline
\end{tabular}

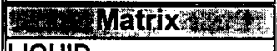

LIQUID

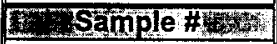

$79 \mathrm{N8}$

Instriment Code.

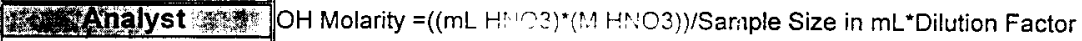

RA GREEN

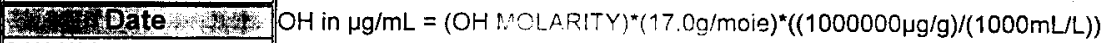

$04 / 14 / 96$

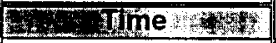

\begin{tabular}{|l|r|}
\hline Concentration of $\mathrm{OH}$ in Sample (Molarity) & $9.38 \mathrm{E}-01$ \\
\hline $\mathrm{OH}$ in Sample in $\mu \mathrm{g}^{\prime} \mathrm{mL}$ (PPM) & $1.60 \mathrm{E}+04$ \\
\hline
\end{tabular}

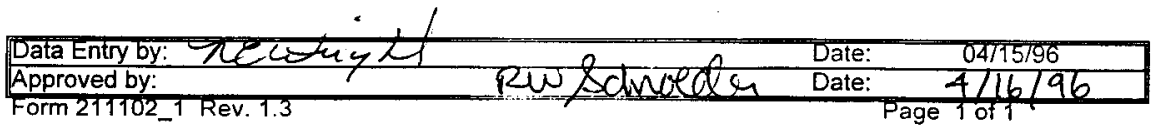




\section{WHC-SD-WM-DP-184, REV.|}

PLACE ANALYTICAL CARD IN BOX BELOW OR ATTACH TRAVELER

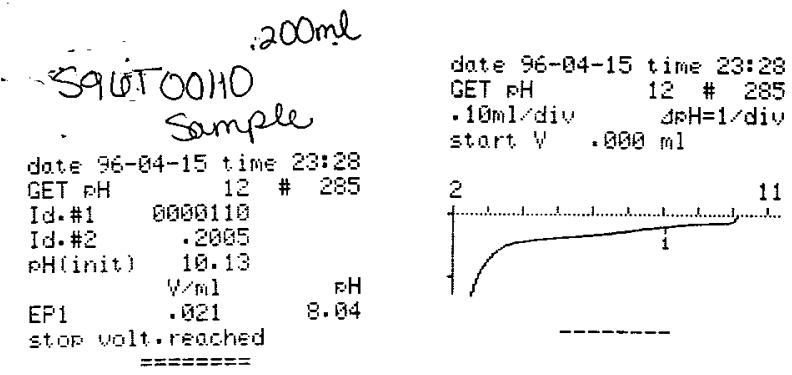

\section{BEST AVAILABLE COPY}

\begin{tabular}{|c|c|c|}
\hline \multicolumn{2}{|c|}{ OH (AUTO) : LA-211-102 (C-G) } & SAMPLE \\
\hline 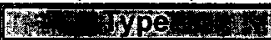 & Sample Size (mL) SS & 0.200 \\
\hline SAMPLE & Concentration of Hivo3 (Molarity) & 0.2005 \\
\hline 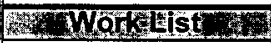 & HNO3 Titrant at $\mathrm{OH}$ end-point in $\mathrm{mL}$ & 0.021 \\
\hline 7581 & Dilution Factor DF & \\
\hline 4esteode, & Concentration of $\mathrm{OH}$ in Sample (Molarity) & 2.11E-02 \\
\hline $\mathrm{OH}-01$ & $\mathrm{OH}$ in Sample in $\mu \mathrm{g} i \mathrm{~nL}$ (PPMi) & $3.58 \mathrm{E}+02$ \\
\hline
\end{tabular}

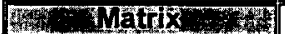

LIQUID

Wample Detection Limit $=125 \mu \mathrm{g} / \mathrm{SS} \cdot \mathrm{DF}$

S96T001110

Instimentropde Detection Limit ( $\mathrm{gg}(\cdots \mathrm{L})$

$6.25 E+52$

WADAlyst,

RA GREEN

Date $\mathrm{OH}$ in $\mu \mathrm{g} / \mathrm{mL}=(\mathrm{OH}: \text {. } 2 \text { LARITY })^{*}\left(17.0 \mathrm{~g} / \mathrm{mole}^{*}((1000000 \mu \mathrm{g} / \mathrm{g}) /(1000 \mathrm{~mL} / \mathrm{L}))\right.$

$04 / 14 / 96$

\begin{tabular}{|c|c|c|}
\hline 4IIme & & SANIFLE \\
\hline & Concentration of $\mathrm{C}:$ in Samp s infolarity) & $2.11 \mathrm{E}-02$ \\
\hline & $\mathrm{OH}$ in Sample in $\mu \mathrm{g} / \mathrm{ir} \mathrm{L}$ (PF & $<625$ \\
\hline
\end{tabular}

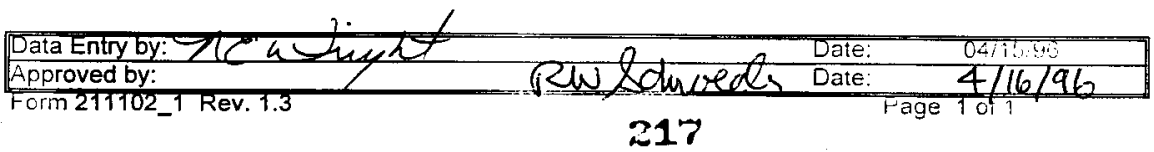




\section{WHC-SD-WM-DP-184, REV. $\mid$}

PLACE ANALYTICAL CARD IN BOX BELOW OR ATTACH TRAVELER

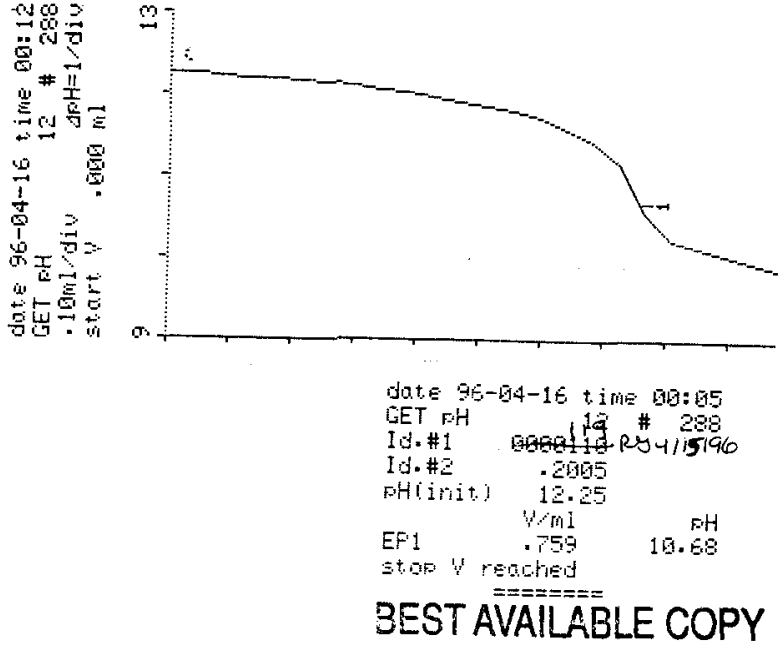

OH (AUTO) : LA-211-102 (C-O)

SAMPLE

STPE $\quad$ Sample Size (mL) S5

SAMPLE

Concentration of $\mathrm{HNO} 3$ (Wolarity)

0.100

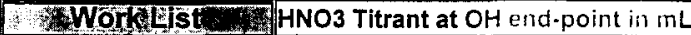

7581

Dilution Factor DF

0.759

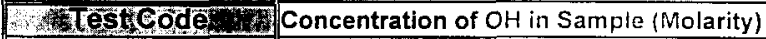

$\mathrm{OH}-01$

$\mathrm{OH}$ in Sample in $\mu \mathrm{g} / \mathrm{mL}$ (PPM)

$2.59 \mathrm{E}+04$

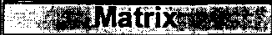

LIQUID

SSample

S96T001119

InstrimentCoder Detection Limit ( $\mu \mathrm{g}$

$+20+2$

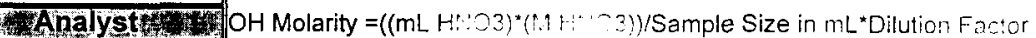

RA GREEN

Date $\mathrm{OH}$ in $\mu \mathrm{g} / \mathrm{mL}=(\mathrm{OH}$ MOLARITY $)\left(17.0 \mathrm{~g} / \mathrm{mole}^{*}((1000000 \mu \mathrm{g} / \mathrm{g}) /(1000 \mathrm{~m} / \mathrm{L} / \mathrm{L}))\right.$

$04 / 14 / 96$

Ime

Concentration of 0 : i: Sampio tholarity)

SAAB.TLE

$\mathrm{OH}$ in Sample in $\mu \mathrm{gti}-\mathrm{L}$ (PPM

$1.52 E+00$

$2.59 E+04$

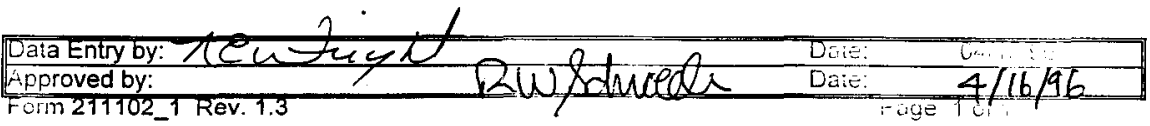




\section{WHC-SD-WM-DP-184, REV. I}

PLACE ANALYTICAL CARD IN BOX BELOW OR ATTACH TRAVELER
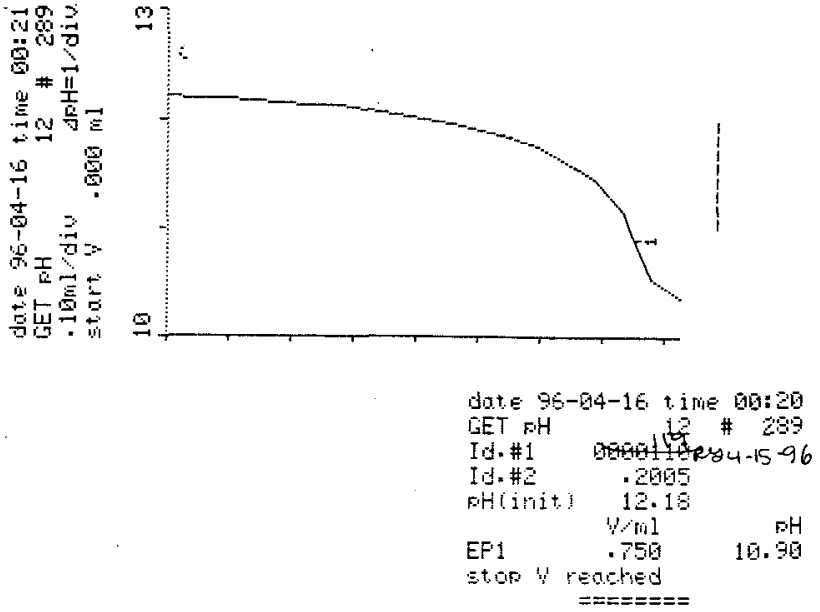

BEST AVAILABLE COPY

\begin{tabular}{|c|c|c|}
\hline \multicolumn{2}{|c|}{ OH (AUTO) : LA-211-102 (C-0) } & DUPLISATE \\
\hline WYPO & Sample Size $(\mathrm{mL})$ SS & 0.100 \\
\hline DUPLICATE & Concentration of $\mathrm{Hi} \cdot 03$ (Molariig) & 0.2205 \\
\hline 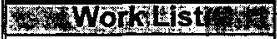 & HNO3 Titrant at $\mathrm{OH}$ end-point in $\mathrm{mL}$ & 0.750 \\
\hline 7581 & Dilution Factor DF & 1 \\
\hline 10stegore & Concentration of $\mathrm{OH}$ in Sample (Molarity) & $1.50 E+00$ \\
\hline $\mathrm{OH}-01$ & $\mathrm{OH}$ in Sample in $\mu \mathrm{g} i \mathrm{~mL}$ (PPM) & $2.565+04$ \\
\hline W. Natrix & \multirow[b]{3}{*}{ Detection Limit $=125 \mu \mathrm{g} / \mathrm{SS} * \mathrm{FF}$} & \\
\hline LIQUID & & \\
\hline $\begin{array}{l}\text { SappleA } \\
\text { S96T001119 }\end{array}$ & & \\
\hline Tnstrument Code & Detection Limit ( $\mu \mathrm{g},:$ ) & $\therefore \cdots$ \\
\hline $\begin{array}{l}\text { Analyst } \\
\text { RA GREEN }\end{array}$ & \multicolumn{2}{|c|}{$\mathrm{OH}$ Molarity $=\left(\left(\mathrm{mL} H^{\prime \prime}: 33\right)^{*}(n+1,-3)\right) /$ Sample Size in $\mathrm{mL}^{*}$ Dilutic: $-\cdots$} \\
\hline $\begin{array}{l}\text { Ala Date } \\
04 / 14 / 96\end{array}$ & \multicolumn{2}{|c|}{ 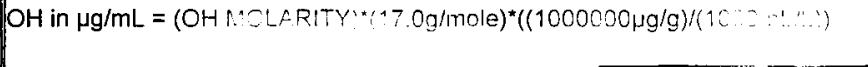 } \\
\hline 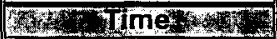 & & $\mathrm{DU}, \mathrm{ZE}$ \\
\hline & Concentration of Oli in Sampis volarity) & $5 u=60$ \\
\hline & $\mathrm{OH}$ in Sample in $\mu \mathrm{g}$ & $2.5=+04$ \\
\hline
\end{tabular}

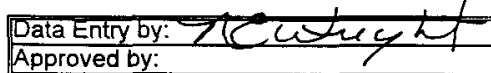

Rusdwoeds 219 


\section{$\sim$ WHC-SD-WM-DP-184, REV.}

PLACE ANALYTICAL CARD IN BOX BELOW OR ATTACH TRAVELER

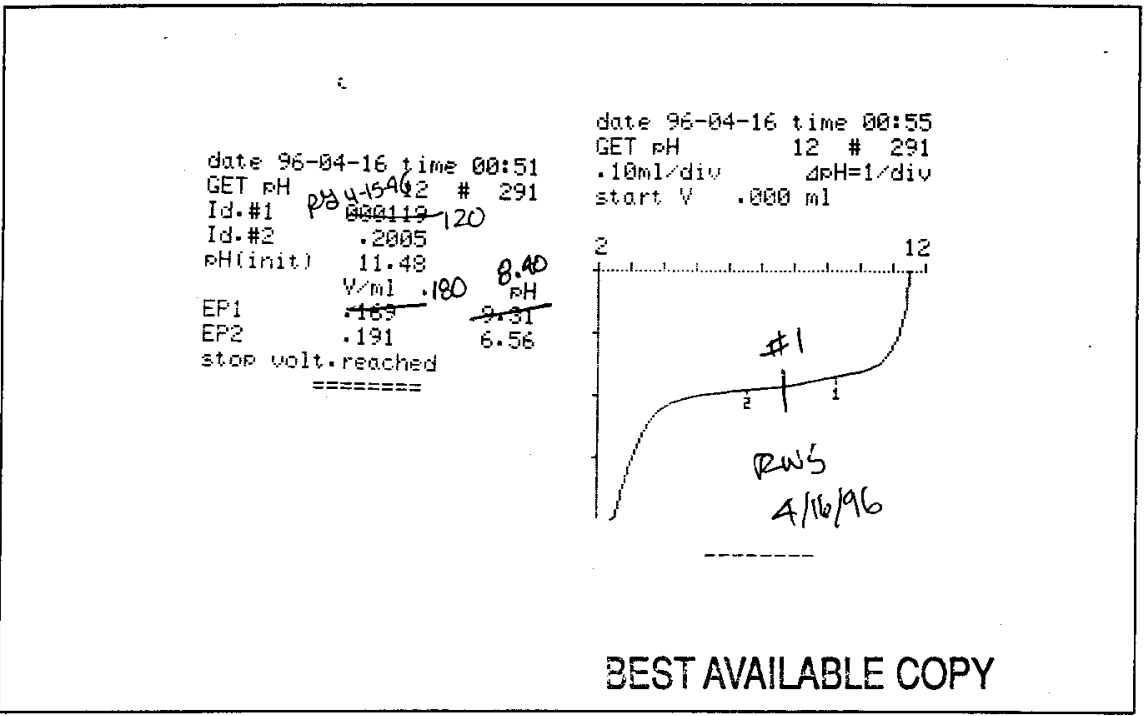

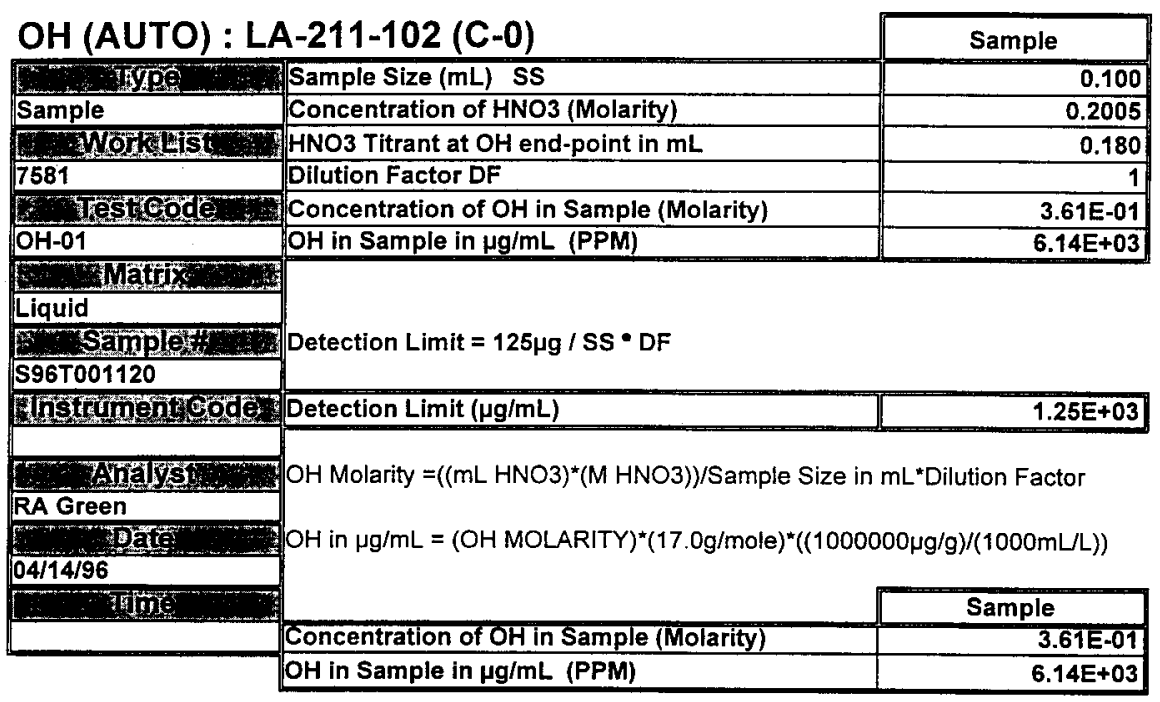

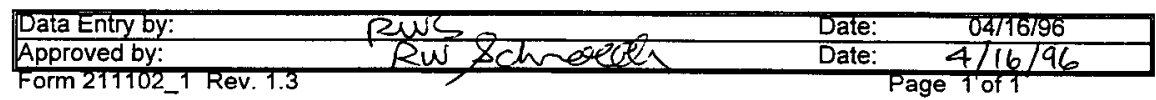




\section{WHC-SD-WM-DP-184, REV. I}

PLACE ANALYTICAL CARD IN BOX BELOW OR ATTACH TRAVELER

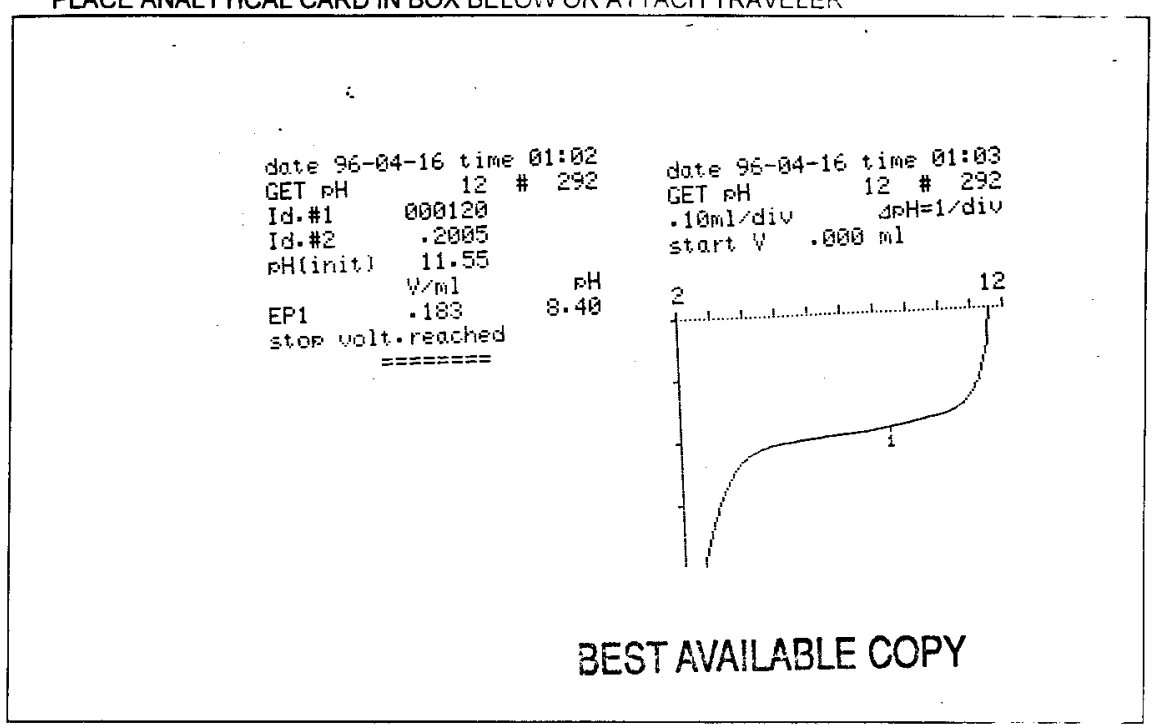

OH (AUTO) : LA-211-102 (C-0)

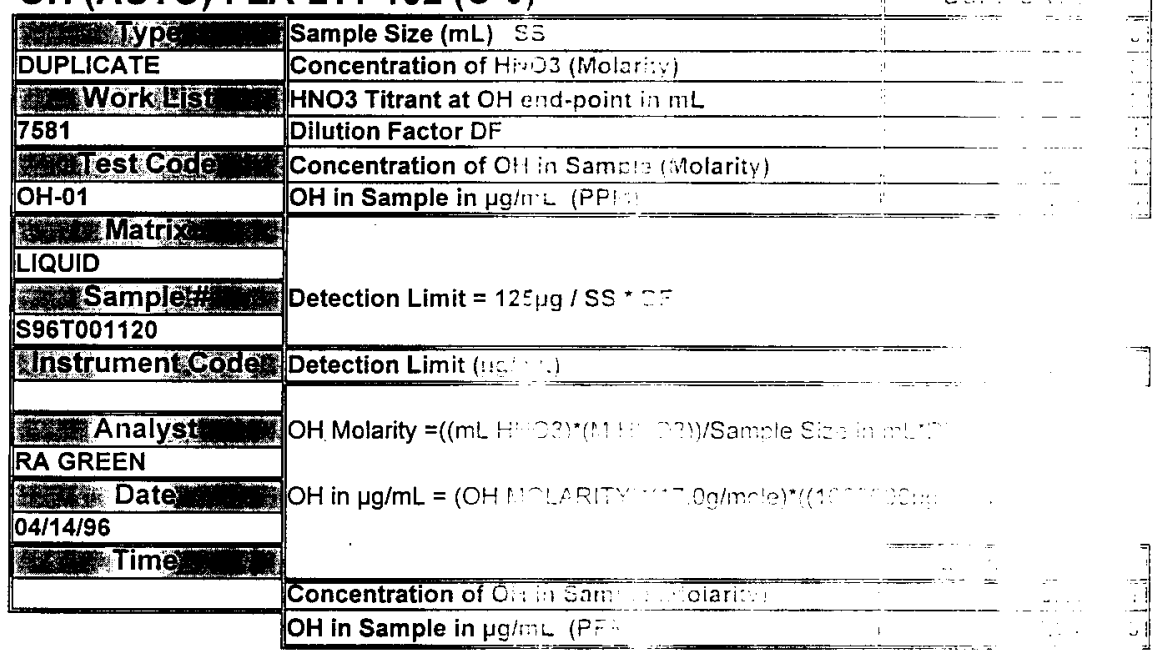

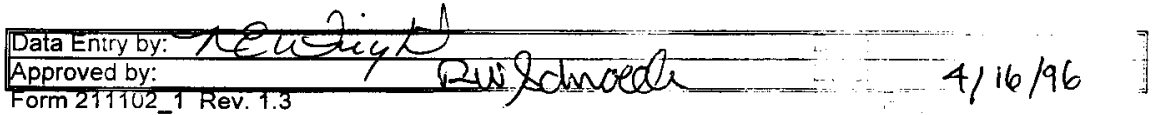


PLACE ANALYTICAL CARD IN BOX BELOW OR ATTACH TRAVELER

PLACEANALYTICAL CARDINBOXBELOW ORATTACH TRAVELER

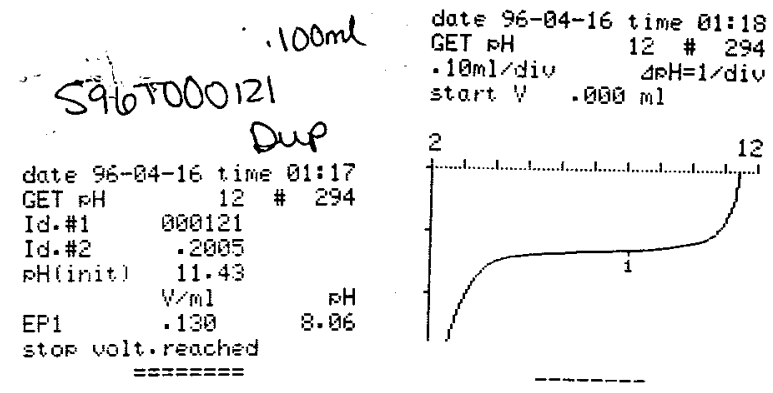

\section{BEST AVAILABLE COPY}

\section{OH (AUTO) : LA-211-102 (C-0)}

\begin{tabular}{|c|c|}
\hline WTE & Sample Size $(\mathrm{mL})$ SS \\
\hline DUPLICATE & Concentration of $\mathrm{HldO} 3$ (Mola \\
\hline Norast & HNO3 Titrant at $\mathrm{OH}$ end-poin \\
\hline 7581 & Dilution Factor DF \\
\hline sestode & Concentration of $\mathrm{OH}$ in Sami : arity) \\
\hline $\mathrm{OH}-01$ & OH in Sample in $\mu g / m L \quad(P P i)$ \\
\hline Matik & \\
\hline LIQUID & \\
\hline Sampla & Detection Limit $=125 \mu \mathrm{g} / \mathrm{SS} *$ \\
\hline S96T001121 & \\
\hline IIstromenteode & Detection Limit (1) -) \\
\hline Analyst & $\mathrm{OH}$ Molarity $=\left((\mathrm{mL}+\mathrm{H}:=3) * \mathrm{C}^{\prime}\right.$ \\
\hline RA GREEN & \\
\hline Date 3 pros & $\mathrm{OH}$ in $\mu \mathrm{g} / \mathrm{mL}=\left(\mathrm{OH} M \mathrm{OL}^{\prime}, \mathrm{R}^{\prime} !^{\cdots}\right.$ \\
\hline $04 / 14 / 96$ & \\
\hline $\operatorname{Limp}, x_{2}$ & \\
\hline & Concentration of $\mathrm{OH}$ in Sait. ......ariayl \\
\hline & $\mathrm{OH}$ in Sample in $\mu \mathrm{g} / \mathrm{m} \mathrm{L} \quad(\mathrm{P})$ \\
\hline
\end{tabular}

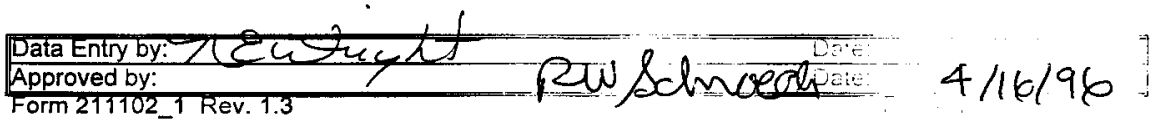




\section{LABCORE Data Entry Template for Worklist\#}

\section{Analyst: $\quad R_{\Omega}$ Instrument: PH01 Book \# $79 \mathrm{NQ}$}

Method: LA-211-102 Rev/Mod $C-C$

Worklist Comment: U-107 OH. RCJ USE $0.050 \mathrm{ml}$ Sample size

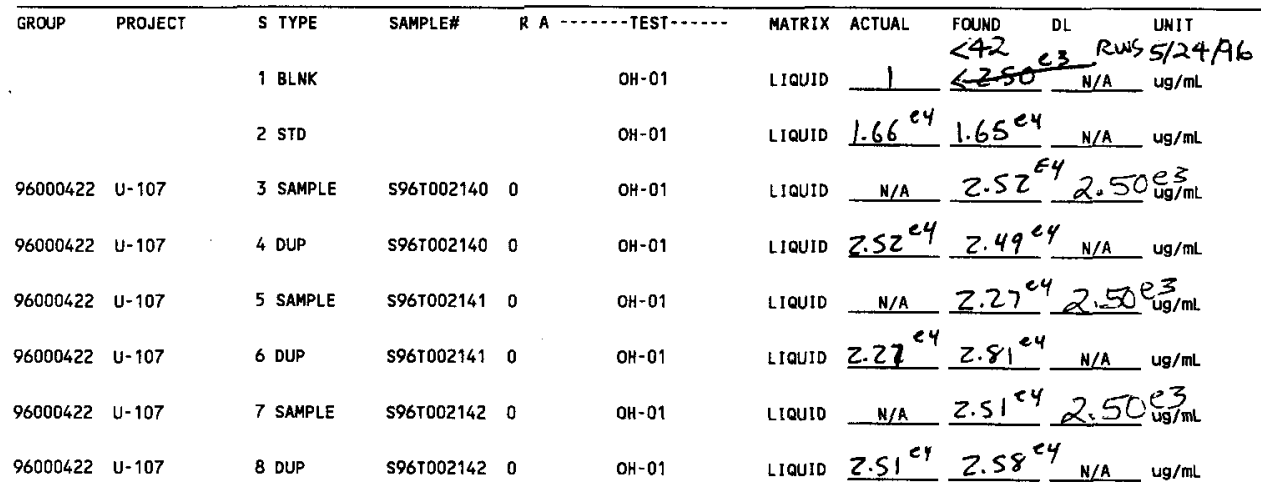

\section{Final page for worklist \# $\mathbf{8 4 2 6}$}
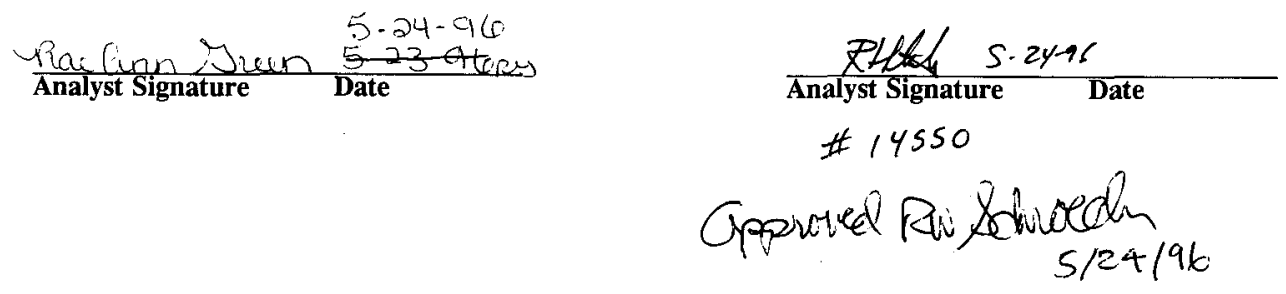

Data Entry Comments:

Units shown for $Q C$ (SPK \& STD) may not reflect the actual units. $D L=$ Detection Limit, $S=$ Worklist Slot Number, $R=$ Replicate Number, $A=$ Aliquot Code. 


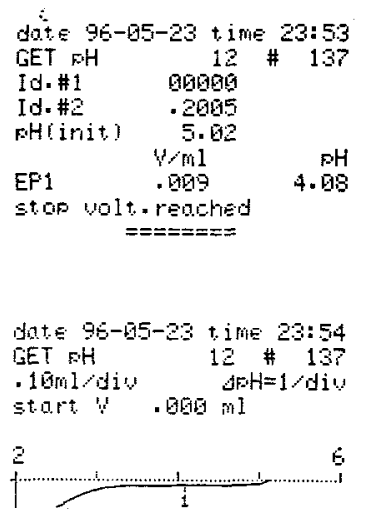

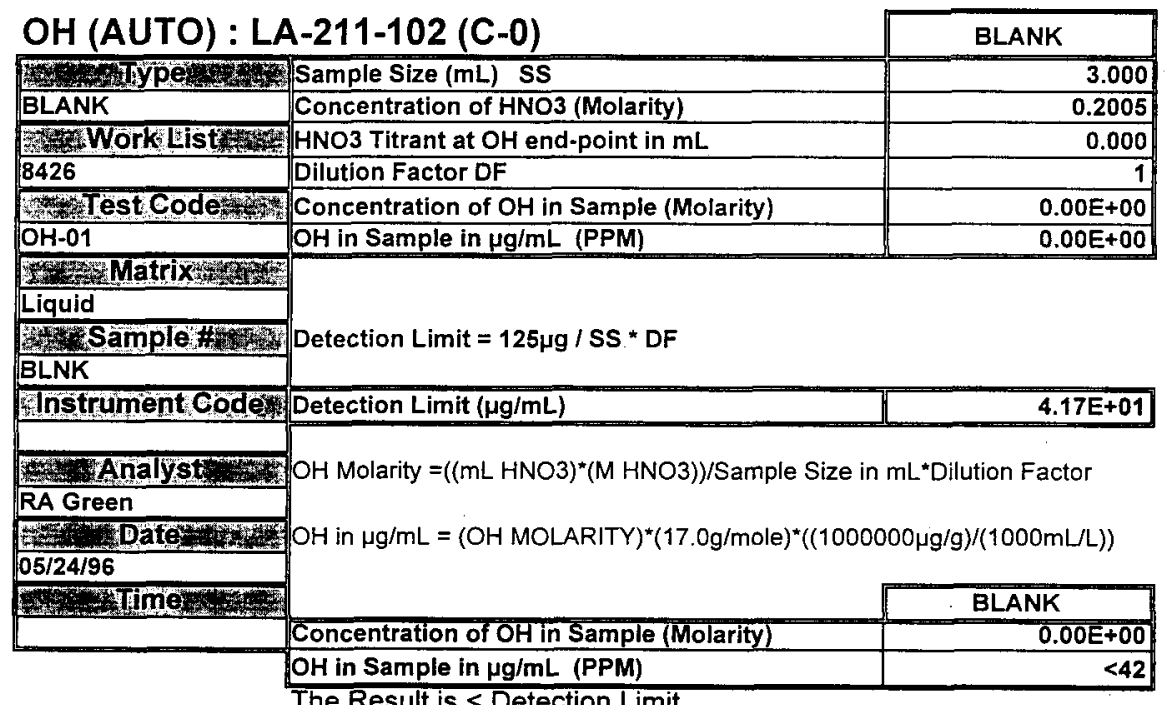

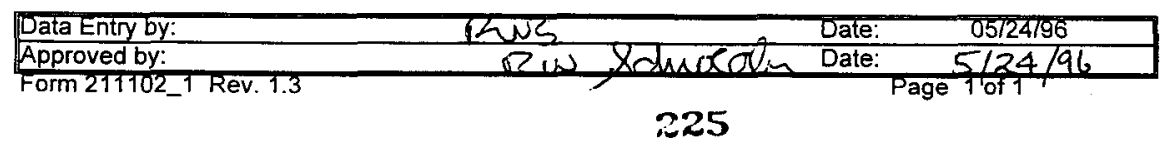




\section{WHC-SD-WM-DP-184, REV. I}

PLACE ANALYTICAL CARD IN BOX BELOW OR ATTACH TRAVELER

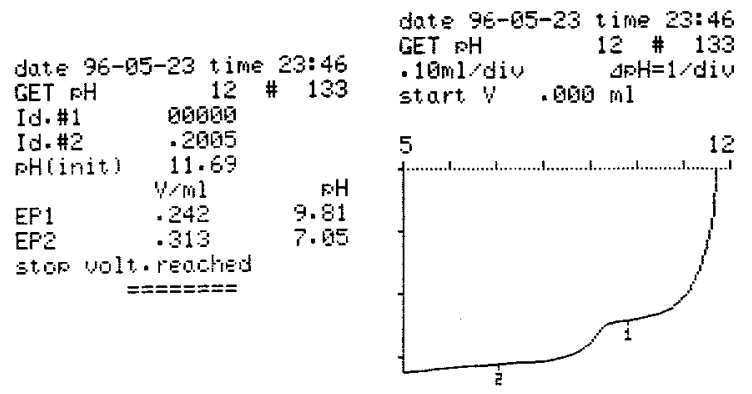

\begin{tabular}{|c|c|c|}
\hline \multirow{2}{*}{\multicolumn{2}{|c|}{ OH (AUTO) : LA-211-102 (C-0) }} & \multirow{3}{*}{ STANDARD } \\
\hline & & \\
\hline 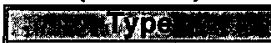 & Sample Size (mL) SS & \\
\hline STANDARD & Concentration of HNO3 (Molarity) & 0.2005 \\
\hline WW Whist & HNO3 Titrant at $\mathrm{OH}$ end-point in $\mathrm{mL}$ & 0.242 \\
\hline 8426 & Dilution Factor DF & 1 \\
\hline GTestoode & Concentration of OH in Sample (Molarity) & 9.70E-01 \\
\hline $\mathrm{OH}-01$ & $\mathrm{OH}$ in Sample in $\mu \mathrm{g} / \mathrm{mL}$ (PPM) & $1.65 \mathrm{E}+04$ \\
\hline \multirow{2}{*}{\multicolumn{3}{|c|}{ LIQUID }} \\
\hline & & \\
\hline \multicolumn{3}{|l|}{ STamplem } \\
\hline \multicolumn{3}{|l|}{ STD } \\
\hline \multicolumn{3}{|l|}{ Unstrumphode } \\
\hline \multicolumn{3}{|l|}{$\mathrm{PH}-01$} \\
\hline Thas & \multicolumn{2}{|c|}{ OH Molarity $=\left((\mathrm{mL} H N O 3)^{\star}(\mathrm{M} \mathrm{HNO})\right) /$ Sample Size in $\mathrm{mL}^{*}$ Dilution Factor } \\
\hline \multicolumn{3}{|c|}{ L } \\
\hline \multicolumn{3}{|c|}{$\mathrm{OH}$ in $\mu \mathrm{g} / \mathrm{mL}=(\mathrm{OH} \text { MOLARITY })^{*}(17.0 \mathrm{~g} / \mathrm{mole})^{\star}((1000000 \mu \mathrm{g} / \mathrm{g}) /(1000 \mathrm{~mL} / \mathrm{L}))$} \\
\hline \multicolumn{3}{|c|}{ 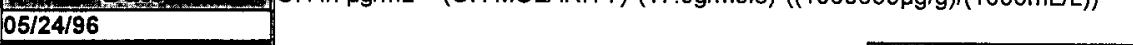 } \\
\hline \multirow[t]{3}{*}{ 2.n.me } & & STANDARD \\
\hline & Concentration of OH in Sample (Molarity) & 9.70E-01 \\
\hline & $\mathrm{OH}$ in Sample in $\mu \mathrm{g} / \mathrm{mL}$ (PPM) & $1.65 E+04$ \\
\hline
\end{tabular}

Data Entry by:

Approved by:

Form 211102_1 Rev. 1.3

Puis ant, $x$ dinerden 226
$05724 / 96^{\circ}$

Date: $5 / 24 / 96$ Page 4 of 1 

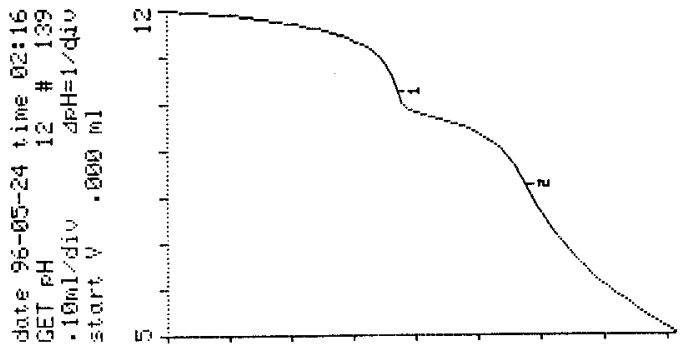

dutE 96-65-24 t. ims - $12: 14$

GET EH

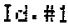

12 \# 139

Id. $\#$

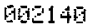

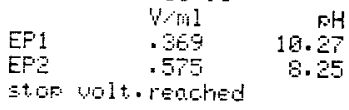

シニニニニニ

\begin{tabular}{|c|c|c|}
\hline \multicolumn{2}{|c|}{ OH (AUTO) : LA-211-102 (C-0) } & \multirow{2}{*}{$\frac{\text { SAMPLE }}{0.050}$} \\
\hline 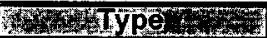 & Sample Size $(\mathrm{mL})$ SS & \\
\hline SAMPLE & Concentration of $\mathrm{HNO}$ (Molarity) & 0.2005 \\
\hline Whork wist & HNO3 Titrant at $\mathrm{OH}$ end-point in $\mathrm{mL}$ & 0.369 \\
\hline 8426 & Dilution Factor DF & \\
\hline Westeode & Concentration of $\mathrm{OH}$ in Sample (Molarity) & $1.48 \mathrm{E}+00$ \\
\hline $\mathrm{OH}-01$ & $\mathrm{OH}$ in Sample in $\mu \mathrm{g} / \mathrm{mL}$ (PPM) & $2.52 E+04$ \\
\hline \multirow{2}{*}{\multicolumn{3}{|c|}{ LIQUID Matrix }} \\
\hline & \multirow{3}{*}{ Detection Limit $=125 \mu \mathrm{g} / \mathrm{SS} * \mathrm{DF}$} & \\
\hline Sample Sam & & \\
\hline \multicolumn{2}{|c|}{ S96T002140 } & \\
\hline Mnstrumenteodo & Detection Limit $(\mu \mathrm{g} / \mathrm{mL})$ & $2.50 \mathrm{E}+03$ \\
\hline \multicolumn{3}{|c|}{$\mathrm{PH}-01$} \\
\hline Analyst & \multicolumn{2}{|c|}{$\mathrm{OH}$ Molarity $=\left((\mathrm{mL} H N O 3)^{*}(\mathrm{M} \mathrm{HNO})\right) /$ Sample Size in $\mathrm{mL}$."Dilution Factor } \\
\hline rag & & \\
\hline T5/24/96 & \multicolumn{2}{|c|}{$\mathrm{OH}$ in $\mu \mathrm{g} / \mathrm{mL}=(\mathrm{OH} \text { MOLARITY })^{*}(17.0 \mathrm{~g} / \mathrm{mole})^{*}((1000000 \mu \mathrm{g} / \mathrm{g}) /(1000 \mathrm{~mL} / \mathrm{L}))$} \\
\hline \multirow[t]{3}{*}{ 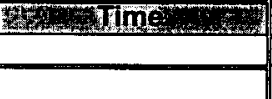 } & & SAMPLE \\
\hline & Concentration of OH in Sample (Molarity) & $1.48 \mathrm{E}+00$ \\
\hline & $\mathrm{OH}$ in Sample in $\mu \mathrm{g} / \mathrm{mL}$ (PPM) & $2.52 E+04$ \\
\hline
\end{tabular}

QNS Nw Schaed

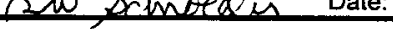

Date: Date: Page $5 / 24 / 9$
05724796 
PLACE ANALYTICAL CARD IN BOX BELOW OR ATTACH TRAVELER
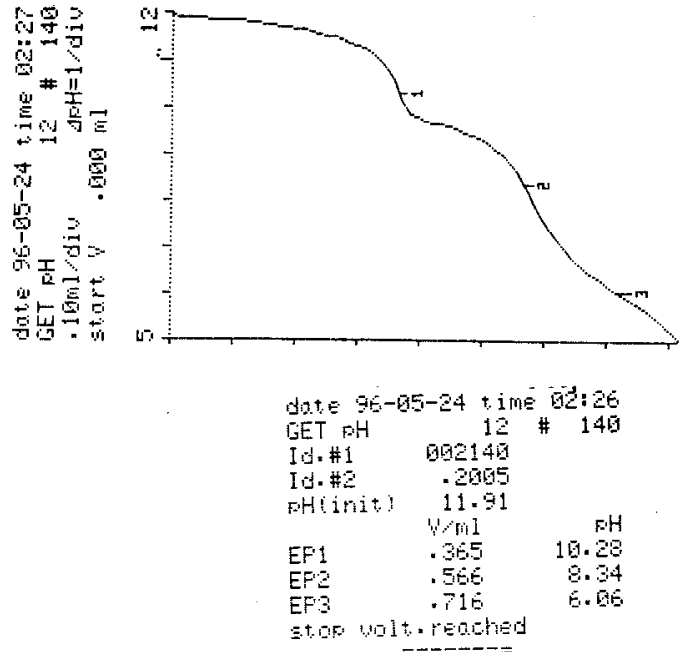

\begin{tabular}{|c|c|c|}
\hline \multicolumn{2}{|c|}{ OH (AUTO) : LA-211-102 (C-0) } & SAMPLE/DUP \\
\hline 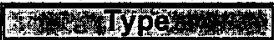 & Sample Size (mL) SS & 0.050 \\
\hline SAMPLE/DUP & Concentration of HNO3 (Molarity) & 0.2005 \\
\hline 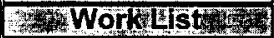 & HNO3 Titrant at $\mathrm{OH}$ end-point in $\mathrm{mL}$ & 0.365 \\
\hline 8426 & Dilution Factor DF & 1 \\
\hline 2y-Testcode & Concentration of OH in Sample (Molarity) & $1.46 \mathrm{E}+00$ \\
\hline $\mathrm{OH}-01$ & $\mathrm{OH}$ in Sample in $\mu \mathrm{g} / \mathrm{mL}$ (PPM) & $2.49 E+04$ \\
\hline
\end{tabular}

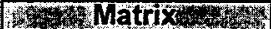

LIQUID

3. Samplo \# Detection Limit $=125 \mu \mathrm{g} / \mathrm{SS}$ * DF

S96T002140

Instrumentgode Detection Limit $(\mu \mathrm{g} / \mathrm{mL})$

PH-01

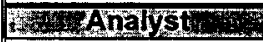

rag

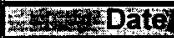

$05 / 24 / 96$

ItTC

$\mathrm{OH}$ Molarity $=\left((\mathrm{mL} H N O 3)^{\star}(\mathrm{M} H N O 3)\right) /$ Sample Size in $\mathrm{mL}$ "Dilution Factor

$\mathrm{OH}$ in $\mu \mathrm{g} / \mathrm{mL}=(\mathrm{OH} \text { MOLARITY })^{\star}(17.0 \mathrm{~g} / \mathrm{mole})^{\star}((1000000 \mu \mathrm{g} / \mathrm{g}) /(1000 \mathrm{~mL} / \mathrm{L}))$

\begin{tabular}{|l|r|}
\cline { 2 - 2 } \multicolumn{1}{c|}{} & \multicolumn{1}{c|}{ SAMPLE/DUP } \\
\hline Concentration of $\mathrm{OH}$ in Sample (Molarity) & $1.46 \mathrm{E}+00$ \\
\hline
\end{tabular}

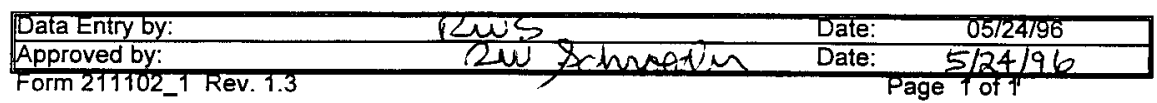




\section{WHC-SD-WM-DP-184, REV. |}

PLACE ANALYTICAL CARD IN BOX BELOW OR ATTACH TRAVELER
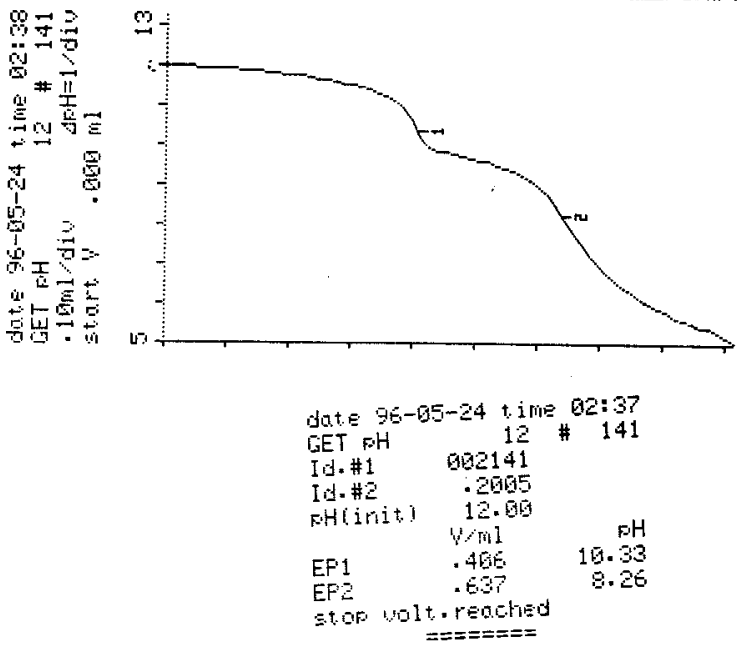

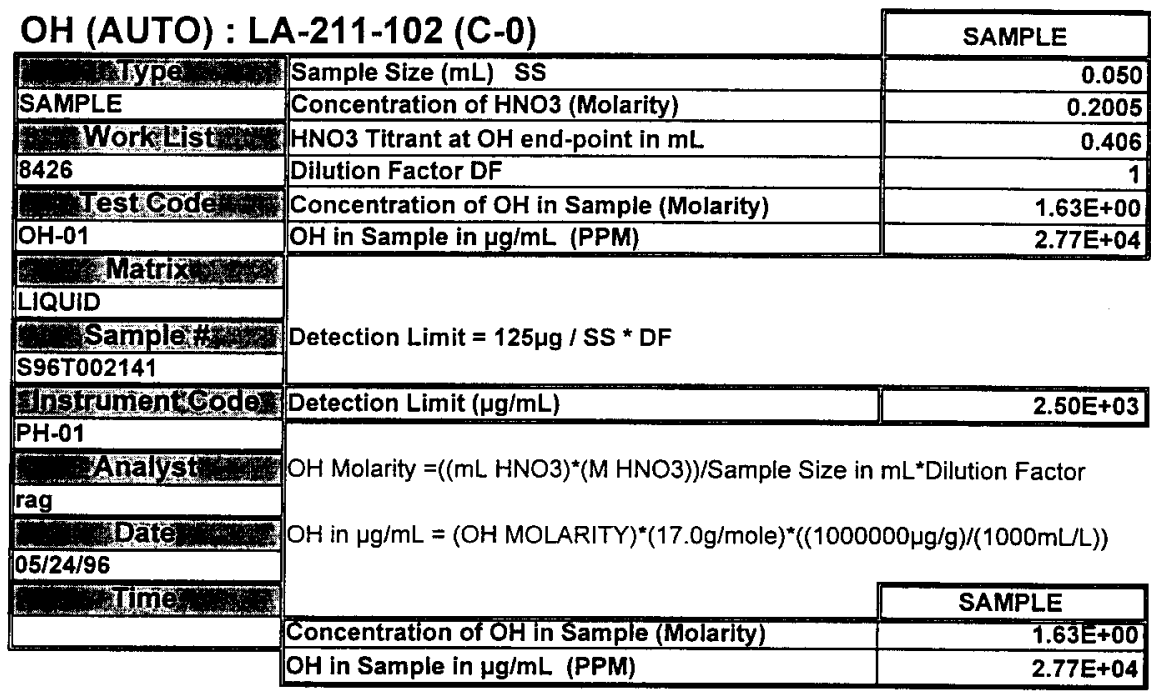

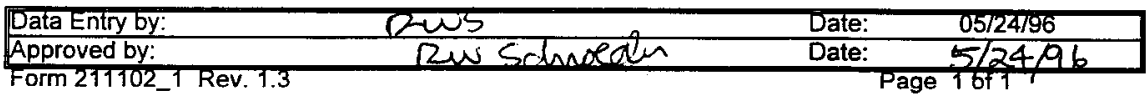




\section{WHC-SD-WM-DP-184, REV.|}

PLACE ANALYTICAL CARD IN BOX BELOW OR ATTACH TRAVELER
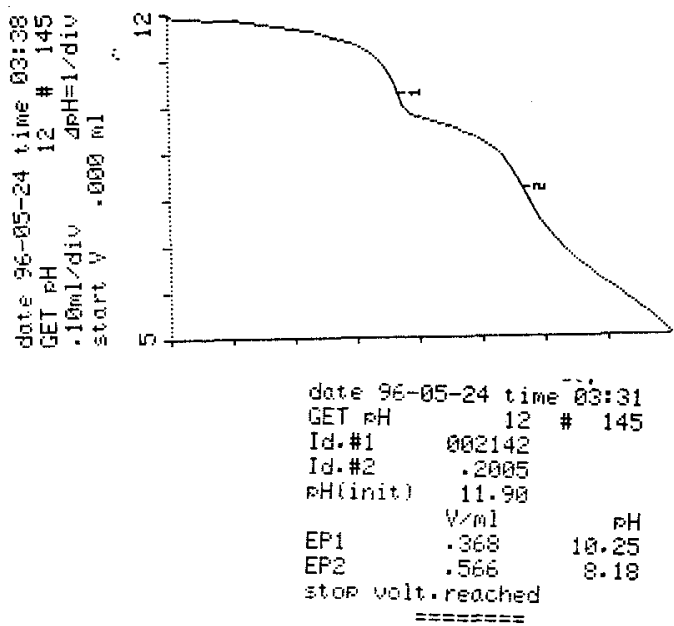

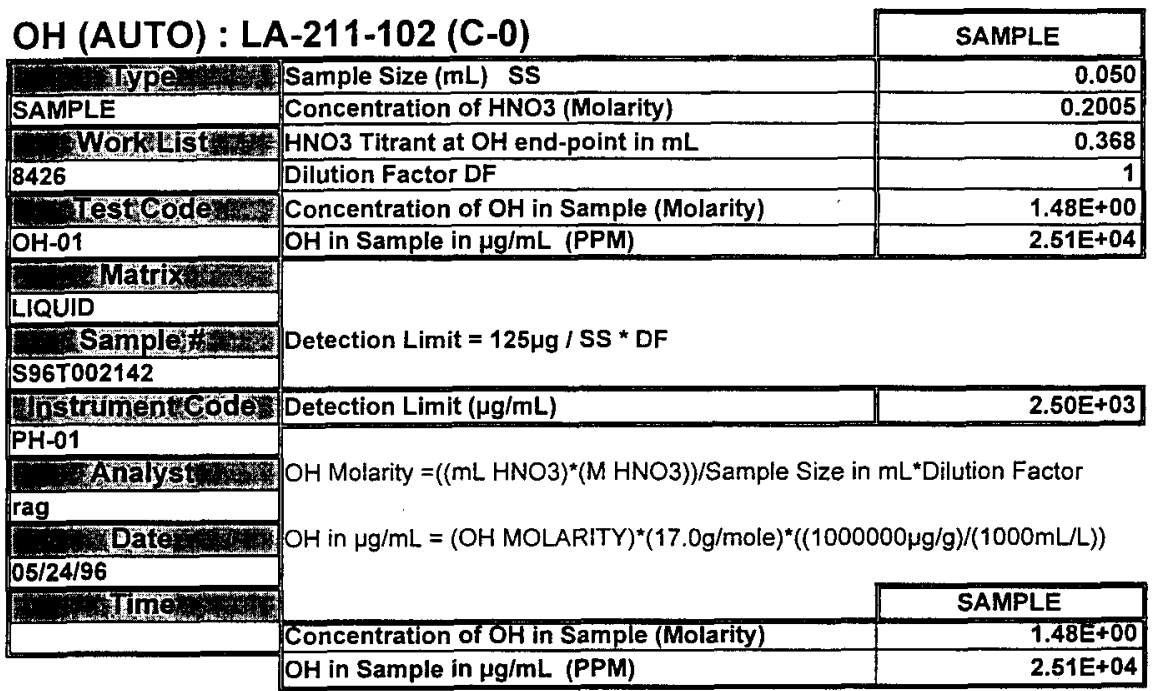

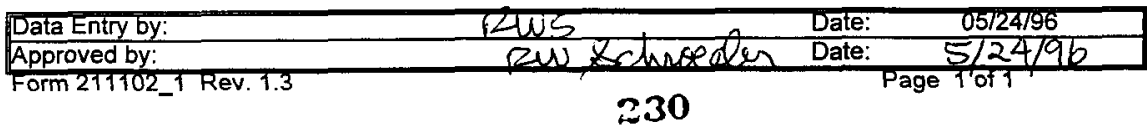




\section{WHC-SD-WM-DP-184, REV. |}

PLACE ANALYTICAL CARD IN BOX BELOW OR ATTACH TRAVELER

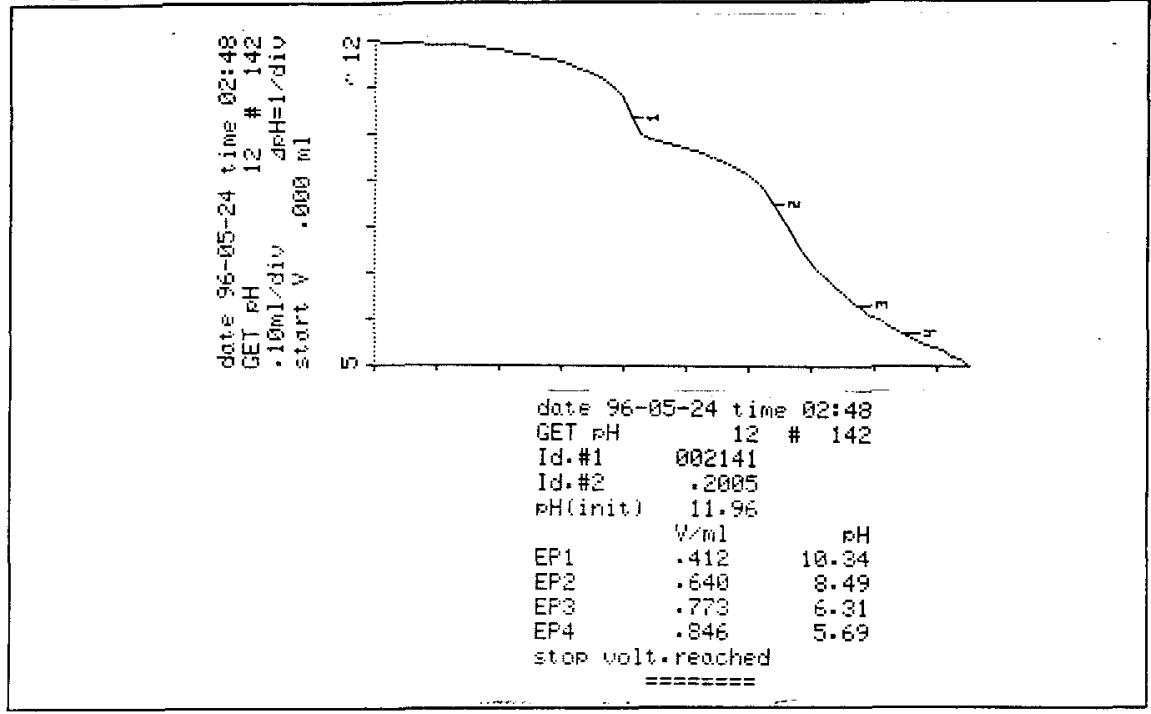

\begin{tabular}{|c|c|c|}
\hline \multicolumn{2}{|c|}{ OH (AUTO) : LA-211-102 (C-0) } & SAMPLE/DUP \\
\hline ThPo & Sample Size $(\mathrm{mL})$ SS & 0.050 \\
\hline SAMPLE/DUP & Concentration of HNO3 (Molarity) & 0.2005 \\
\hline WorkList. & HNO3 Titrant at $\mathrm{OH}$ end-point in $\mathrm{mL}$ & 0.412 \\
\hline 8426 & Dilution Factor DF & 1 \\
\hline Testaodg & Concentration of $\mathrm{OH}$ in Sample (Molarity) & $1.65 \mathrm{E}+00$ \\
\hline $\mathrm{OH}-01$ & $\mathrm{OH}$ in Sample in $\mu \mathrm{g} / \mathrm{mL}$ (PPM) & $2.81 E+04$ \\
\hline \\
\hline \multicolumn{3}{|c|}{ LIQUID } \\
\hline Samplet & \multirow{2}{*}{\multicolumn{2}{|c|}{ Detection Limit $=125 \mu \mathrm{g} / \mathrm{SS} * \mathrm{DF}$}} \\
\hline S96T002141 & & \\
\hline 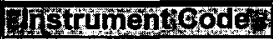 & Detection Limit $(\mu \mathrm{g} / \mathrm{mL})$ & $2.50 \mathrm{E}+03$ \\
\hline \multicolumn{3}{|c|}{ Do } \\
\hline 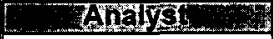 & \multirow{4}{*}{\multicolumn{2}{|c|}{$\begin{array}{l}\mathrm{OH} \text { Molarity }=\left((\mathrm{mL} \text { HNO3 })^{*}(\mathrm{M} \text { HNO3) }) / \text { Sample Size in } \mathrm{mL} * \text { Dilution Factor }\right. \\
\mathrm{OH} \text { in } \mu \mathrm{g} / \mathrm{mL}=(\mathrm{OH} \text { MOLARITY })^{*}(17.0 \mathrm{~g} / \mathrm{mole})^{*}((1000000 \mu \mathrm{g} / \mathrm{g}) /(1000 \mathrm{~mL} / \mathrm{L}))\end{array}$}} \\
\hline & & \\
\hline $\mathrm{DaY}$ & & \\
\hline $05 / 24 / 96$ & & \\
\hline Minte & & SAMPLE/DUP \\
\hline & Concentration of ÖH in Sample (Molarify) & $1.65 E+00$ \\
\hline & $\mathrm{OH}$ in Sample in $\mu g / \mathrm{mL}$ (PPM) & $2.81 E+04$ \\
\hline
\end{tabular}

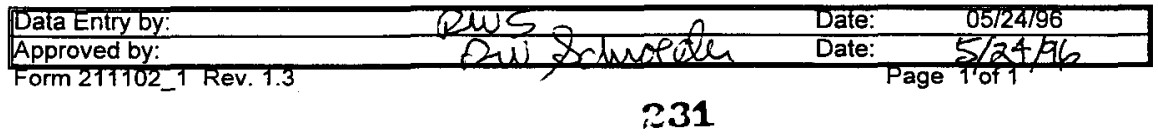




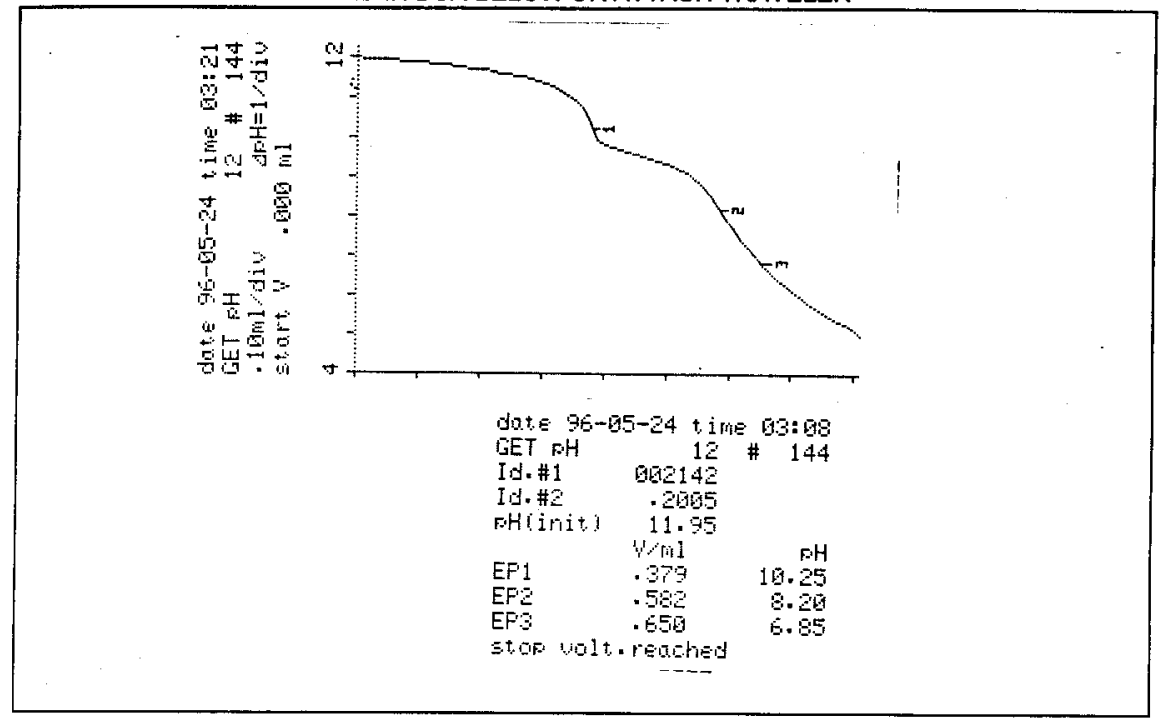

\begin{tabular}{|c|c|c|}
\hline \multicolumn{2}{|c|}{ OH (AUTO) : LA-211-102 (C-0) } & \multirow{2}{*}{$\frac{\text { SAMPLE/DUP }}{0.050}$} \\
\hline D. $T \mathrm{PPE}$ & Sample Size (mL) SS & \\
\hline SAMPLE/DUP & Concentration of $\mathrm{HNO}_{3}$ (Molarity) & 0.2005 \\
\hline Work [ISE & $\mathrm{HNO} 3$ Titrant at $\mathrm{OH}$ end-point in $\mathrm{mL}$ & 0.379 \\
\hline 8426 & Dilution Factor DF & 1 \\
\hline Lesticoded & Concentration of $\mathrm{OH}$ in Sample (Molarity) & $1.52 \mathrm{E}+00$ \\
\hline $\mathrm{OH}-01$ & $\mathrm{OH}$ in Sample in $\mu \mathrm{g} / \mathrm{mL}$ (PPM) & $2.58 E+04$ \\
\hline \begin{tabular}{|l} 
LIQUID \\
LITatrix \\
S96T002142
\end{tabular} & \multicolumn{2}{|l|}{ Detection Limit $=125 \mu \mathrm{g} / \mathrm{SS}$ * DF } \\
\hline Mostruenteode & Detection Limit $(\mu \mathrm{g} / \mathrm{mL})$ & $2.50 E+03$ \\
\hline PH-01 & \multirow{3}{*}{\multicolumn{2}{|c|}{$\begin{array}{l}\text { OH Molarity }=\left((\mathrm{mL} \text { HNO3 })^{*}(\mathrm{M} \mathrm{HNO})\right) / \text { Sample Size in } \mathrm{mL} \mathrm{L}^{*} \text { Dilution Factor } \\
\mathrm{OH} \text { in } \mu \mathrm{g} / \mathrm{mL}=(\mathrm{OH} \text { MOLARITY })^{*}(17.0 \mathrm{~g} / \mathrm{mole})^{*}((1000000 \mathrm{~g} / \mathrm{g}) /(1000 \mathrm{~mL} / \mathrm{L}))\end{array}$}} \\
\hline rag & & \\
\hline$\frac{D \text { Date }}{05 / 24 / 96}$ & & \\
\hline$=\operatorname{sins}$ & & SAMPLE/DUP \\
\hline & Concentration of OH in Sample (Molarity) & $1.52 E+00$ \\
\hline & $\mathrm{OH}$ in Sample in $\mu \mathrm{g} / \mathrm{mL}$ (PPM) & $2.58 E+04$ \\
\hline
\end{tabular}

Data Entry by: Approved by: Form 211102_1 Rev. 1.3

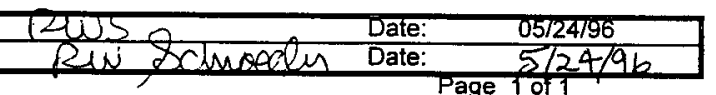




\section{LABCORE Data Entry Template for Worklist\#}

Analyst:

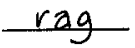

Instrument: PH01

Book \# 79 N 8

Method: LA-211-102 Rev/Mod C-O

Worklist Comment:U-107)AND AY-102 OH. RCJ

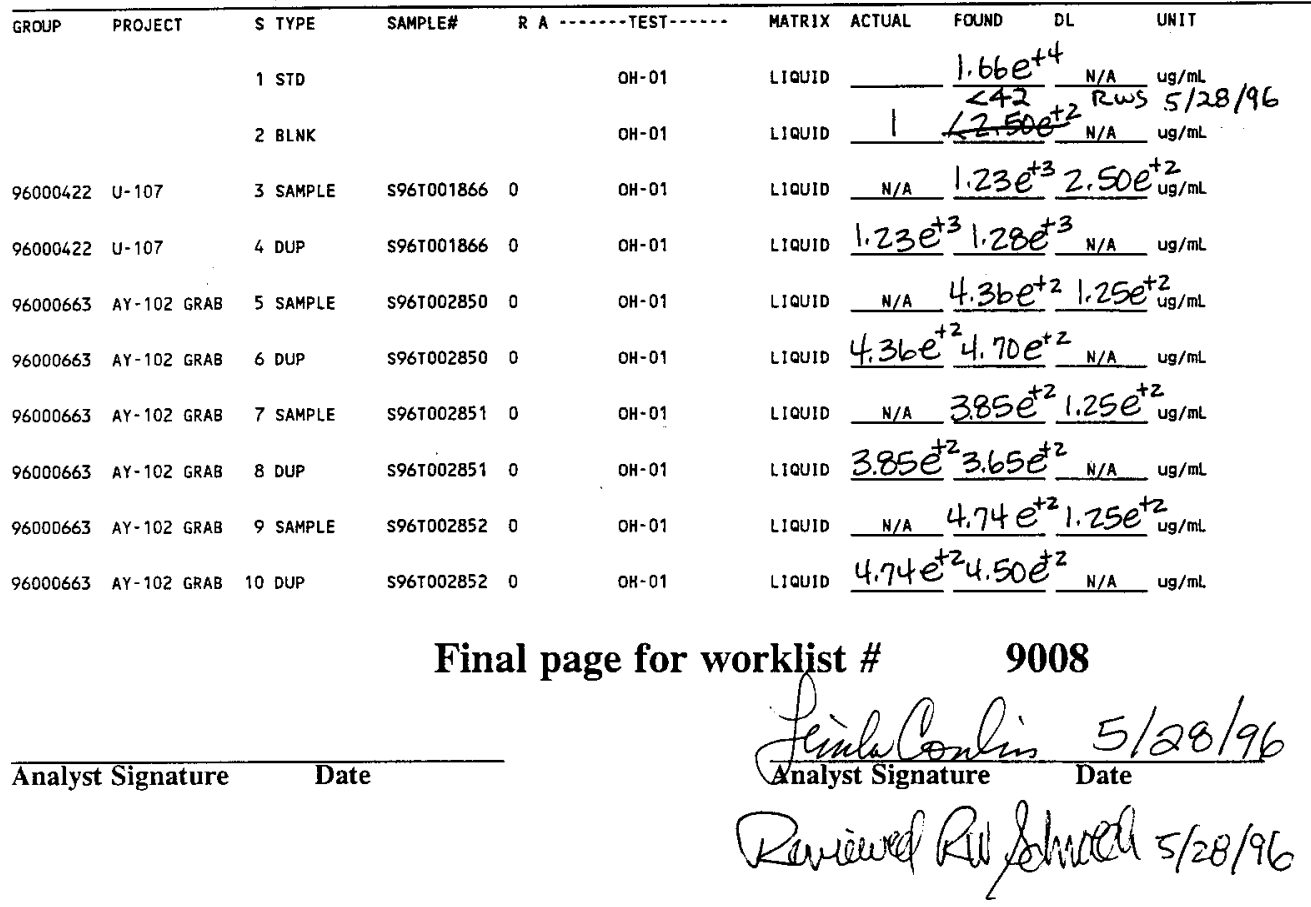

Data Entry Comments:

Units shown for $Q C$ (SPK \& STD) may not reflect the actual units. $D L=$ Detection Limit, $S=$ Worklist Slot Number, $R=$ Replicate Number, $A=$ Aliquot Code. 
Analyst: RY Instrument: PH01 Book \# 79 N8

Method: LA-211-102 Rev/Mod C.O

Worklist Comment: U-107 AND AY-102 OH. RCJ

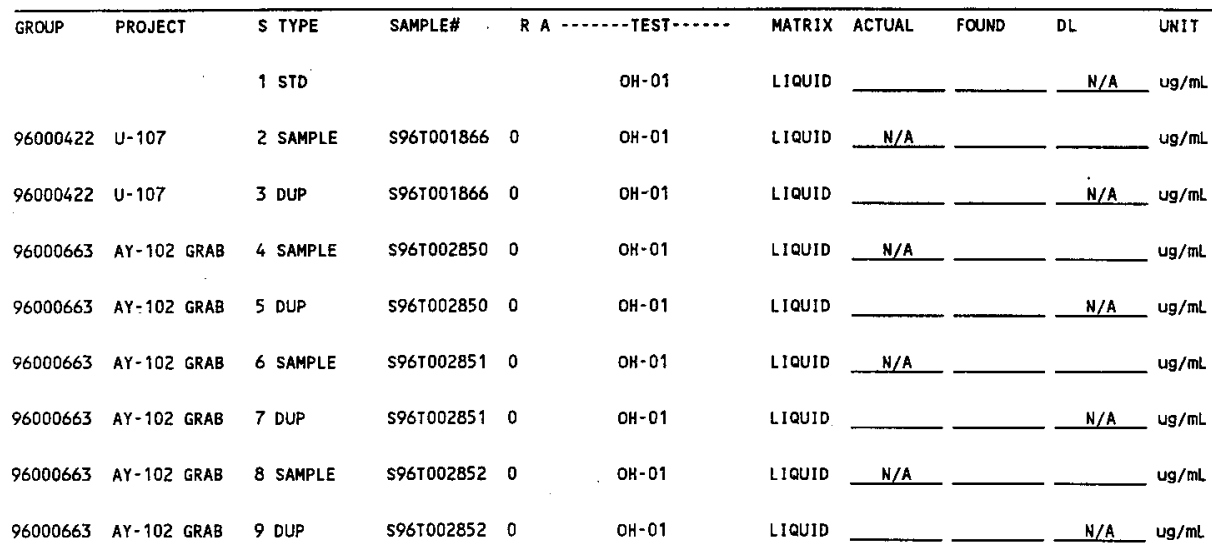

\section{Final page for worklist \# 9008}

Rac Aun Jreen $5.27-96$

Analyst Signature Date
Analyst Signature

Date

Data Entry Comments: $\quad .2006 \mathrm{MHNO}_{3}$ titrant

Units shown for $Q C$ (SPK \& STD) may not reflect the actual units. $D L=$ Detection Limit, $S=$ Worklist Slot Number, $R=$ Replicate Number, $A=$ Aliquot Code. 


\begin{tabular}{|c|c|c|}
\hline \multirow{2}{*}{\multicolumn{2}{|c|}{ OH (AUTO) : LA-211-102 (C-0) }} & \multirow[b]{2}{*}{ STANDARD } \\
\hline & & \\
\hline 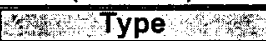 & Sample Size $(\mathrm{mL})$ sS & 0.050 \\
\hline STANDARD & Concentration of $\mathrm{HNO}_{3}$ (Molarity) & 0.2005 \\
\hline Whatk List & HNO3 Titrant at $\mathrm{OH}$ end-point in $\mathrm{mL}$ & 0.243 \\
\hline 9008 & Dilution Factor DF & 1 \\
\hline Test Code & Concentration of $\mathrm{OH}$ in Sample (Molarity) & $9.74 \mathrm{E}-01$ \\
\hline OH-OI & $\mathrm{OH}$ in Sample in $\mu \mathrm{g} / \mathrm{mL}$ (PPM) & $1.66 \mathrm{E}+04$ \\
\hline \multicolumn{3}{|c|}{ Matrix } \\
\hline \multicolumn{3}{|l|}{ LIQUID } \\
\hline \multicolumn{3}{|l|}{ 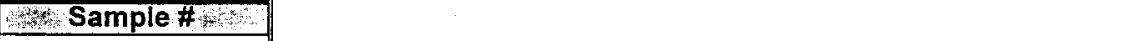 } \\
\hline \multicolumn{3}{|l|}{ Instrument Code? } \\
\hline $\begin{array}{l}\text { Analyst } \\
\text { R A GREEN }\end{array}$ & \multirow{3}{*}{\multicolumn{2}{|c|}{$\begin{array}{l}\text { OH Molarity }=\left((\mathrm{mL} \text { HNO3 })^{*}(\mathrm{M} \mathrm{HNO} 3)\right) / \text { Sample Size in } \mathrm{mL}{ }^{*} \text { Dilution Factor } \\
\mathrm{OH} \text { in } \mu \mathrm{g} / \mathrm{mL}=(\mathrm{OH} \text { MOLARITY })^{*}(17.0 \mathrm{~g} / \mathrm{mole})^{*}((1000000 \mu \mathrm{g} / \mathrm{g}) /(1000 \mathrm{~mL} / \mathrm{L}))\end{array}$}} \\
\hline Pefrote Date & & \\
\hline \multirow{2}{*}{\multicolumn{2}{|c|}{$05 / 27 / 96$}} & \\
\hline & & STANDARD \\
\hline \multirow[t]{2}{*}{ 11:55 AM } & Concentration of $\mathrm{OH}$ in Sample (Molarity) & $9.74 \mathrm{E}-01$ \\
\hline & $\mathrm{OH}$ in Sample in $\mu \mathrm{g} / \mathrm{mL}$ (PPM) & $1.66 E+04$ \\
\hline
\end{tabular}

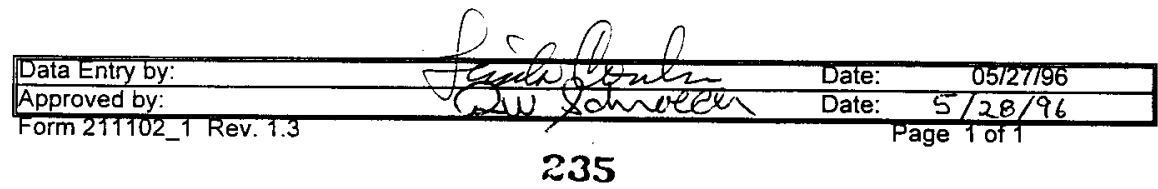




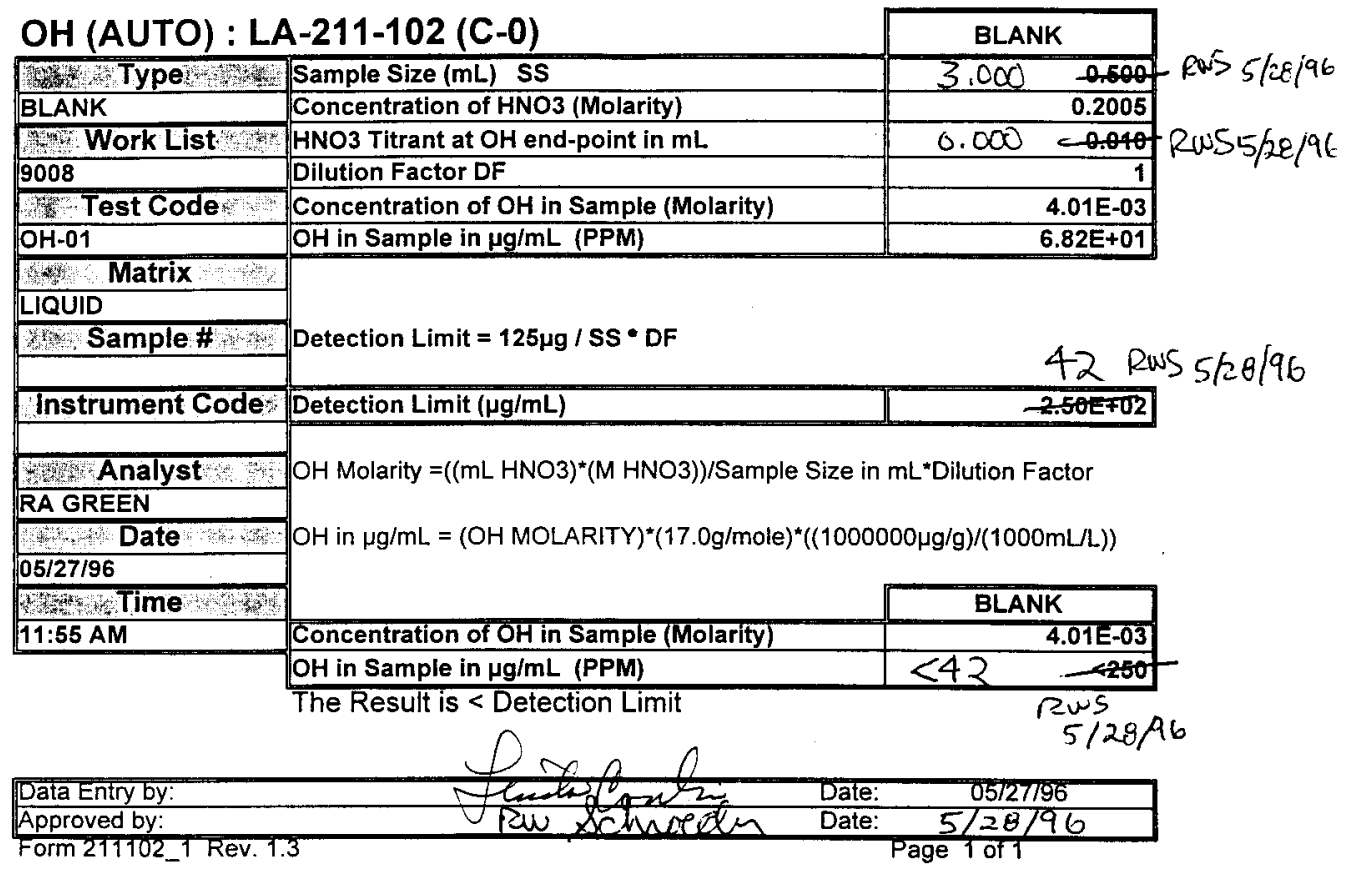




\section{WHC-SD-WM-DP-184, REV.}

\begin{tabular}{|c|c|c|}
\hline OH (AUTO) : LA & $-211-102(C-0)$ & SAMPLE \\
\hline SPIType & Sample Size (mL) SS & 0.500 \\
\hline SAMPLE & Concentration of HNO3 (Molarity) & 0.2005 \\
\hline WorkList & HNO3 Titrant at OH end-point in $\mathrm{mL}$ & 0.181 \\
\hline 9008 & Dilution Factor DF & 1 \\
\hline thestcode & Concentration of $\mathrm{OH}$ in Sample (Molarity) & $7.26 E-02$ \\
\hline $\mathrm{OH}-01$ & $\mathrm{OH}$ in Sample in $\mu \mathrm{g} / \mathrm{mL}$ (PPM) & $1.23 E+03$ \\
\hline Holnatrix & & \\
\hline LIQUID & & \\
\hline Sample\# & Detection Limit $=125 \mu \mathrm{g} / \mathrm{SS}$ * DF & \\
\hline S96T001866 & & \\
\hline InstrumentCode & Detection Limit $(\mu \mathrm{g} / \mathrm{mL})$ & $2.50 E+02$ \\
\hline
\end{tabular}

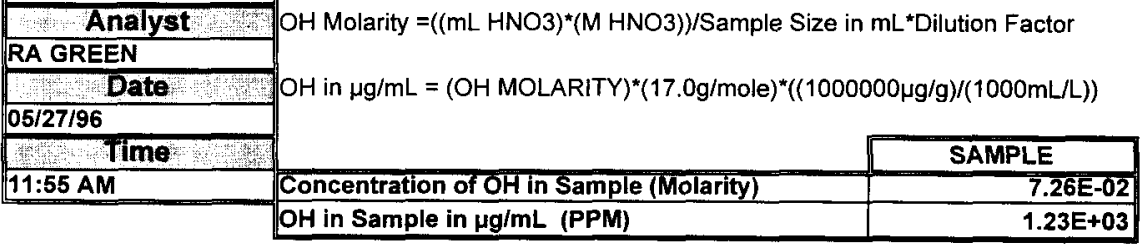

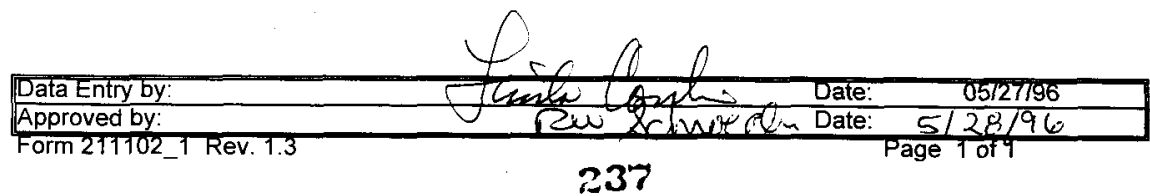


WHC-SD-WM-DP-184, REV.

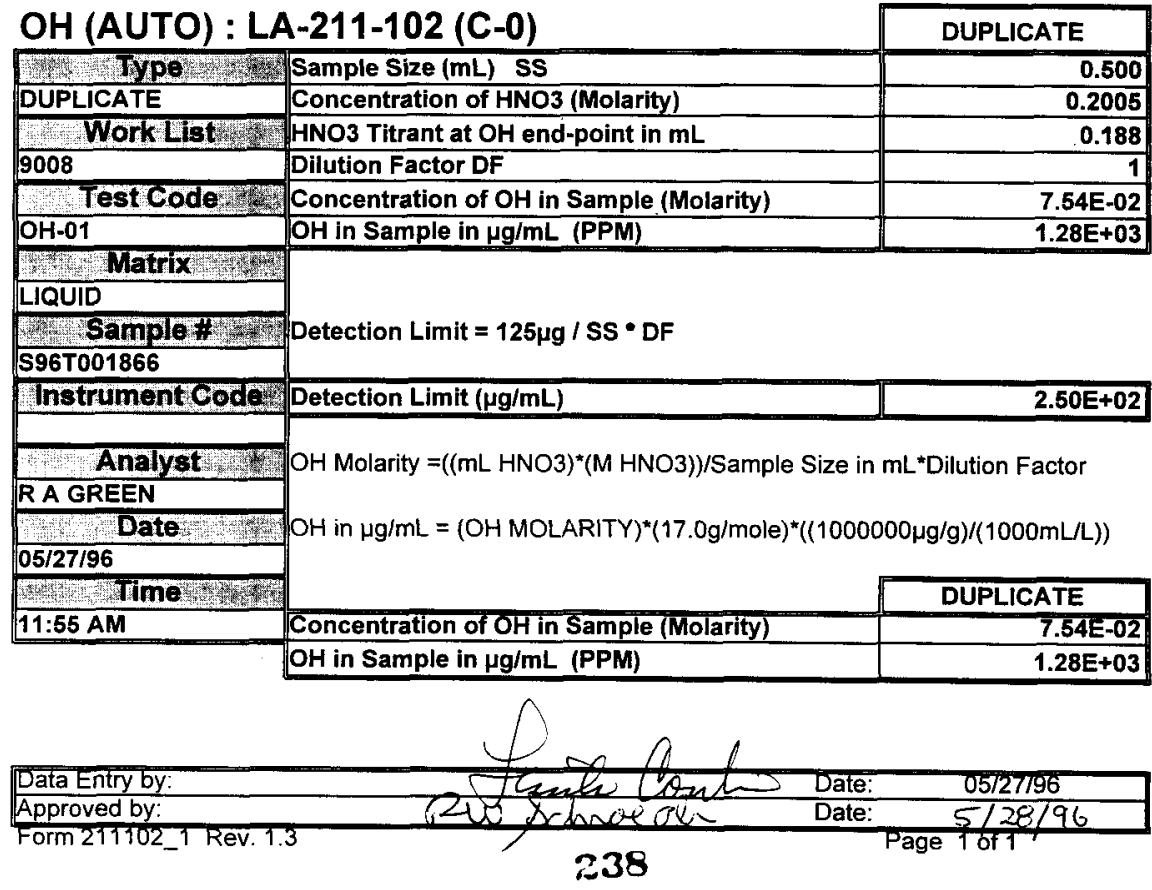


colibration dota dote $96-65-27$ FH[S] 1 7.00

애이 $\varepsilon \quad 10.60$

t.col. $24.0 \mathrm{t}$

slope [rel) .967

U(as) $-7.3 \mathrm{mv}$

electr. infut 1

$S T D$

\section{WHC-SD-WM-DP-184, REV. I BLK}

$$
\text { ..... }
$$

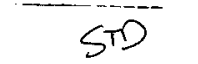

date 96-95-27 time 69:22

GET FH II \# 151

I. \#1 1 G0100

ld. \#2 . 2015

oHinit) 11.79

Whl EH

$\begin{array}{lll}\text { EF1 } & .243 & 9.99 \\ \text { EF2 } & .313 & 7.68\end{array}$

st.

date 95-65-27 time 69:24

GET FHH 12 \# 152

Id.\#1 10000

Id. $\# 2$. 2065

PH(init) 5.27

Wrill

EF1 $1010 \quad 4.35$

stop volt. reached

dote $96-65-27$ tine $09: 24$

LET $\mathrm{PH} 12$ \# 152

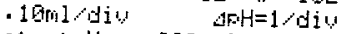

Etart $y$. g60 fil

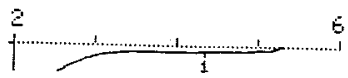

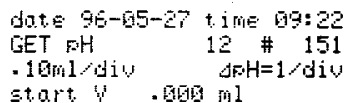

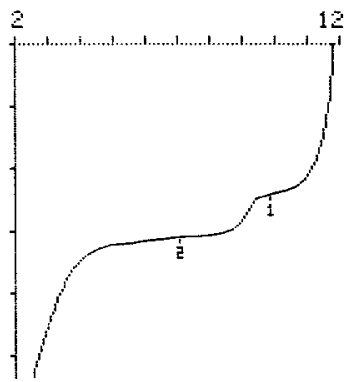

BEST AVAILABLE COPY 


\section{OH ANALYSIS}

WHE-SOHAM-OP 184, REY

$$
59050018106
$$

$$
\begin{aligned}
& \text { date } 96-05-27 \text { time 69:39 }
\end{aligned}
$$

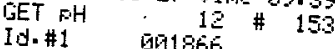

$$
\begin{aligned}
& \text { Id. \#2 .2005 } \\
& \text { FH(init) } 11.66 \\
& \begin{array}{llr}
\text { EF1 } & \text { Wmi } & \text { EH } \\
\text { EF2 } & .181 & 19.44 \\
\text { EF3 } & .305 & 9.15 \\
\text { EF4 } & .489 & 6.79 \\
\text { Etion y reached } & 4.42
\end{array}
\end{aligned}
$$$$
\text { dote } 96-05-27 \text { time 09:43 }
$$$$
\text { GET FH } 12 \text { \# } 153
$$

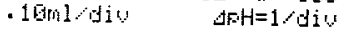$$
\text { start } y \text {. Q060 ml }
$$

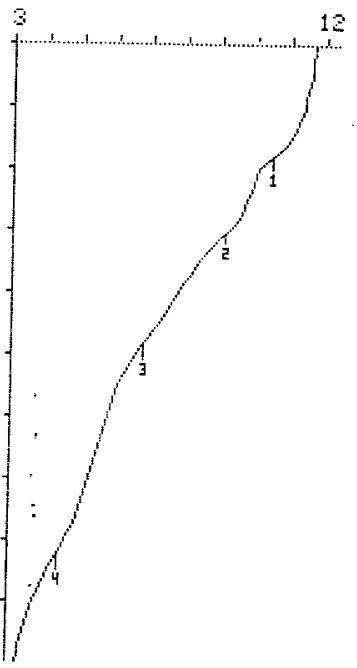

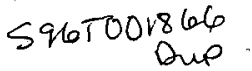

date $96-65-27$ t ime $09: 55$

CET $\mathrm{FH}$ 12 \# 154

Id. \#1 001605

Id. \#2 .2005

PH[init? 11.54

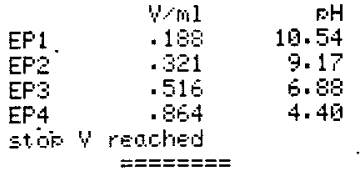

dote 96-65-27 time 0905

LET FH 12 \# 154

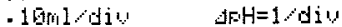

stort $\quad .060 \mathrm{ml}$

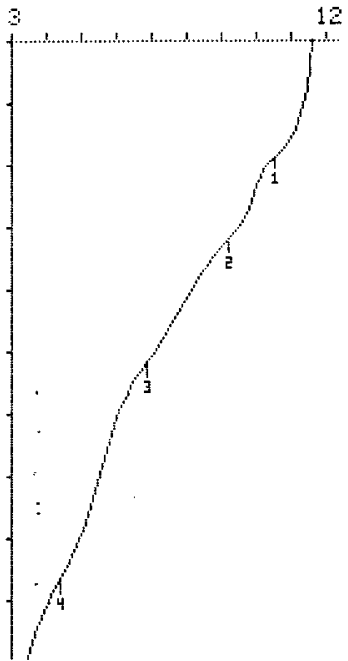

\section{BEST AVAILABLE COPY}




\section{LABCORE Data Entry Template for Worklist\#}

\section{Analyst: उLF Instrument: PH01 _ Book \#144N16E}

Method: LA-212-106 Rev/Mod $A \circlearrowright$

Worklist Comment: U-107 PH'S. RCJ

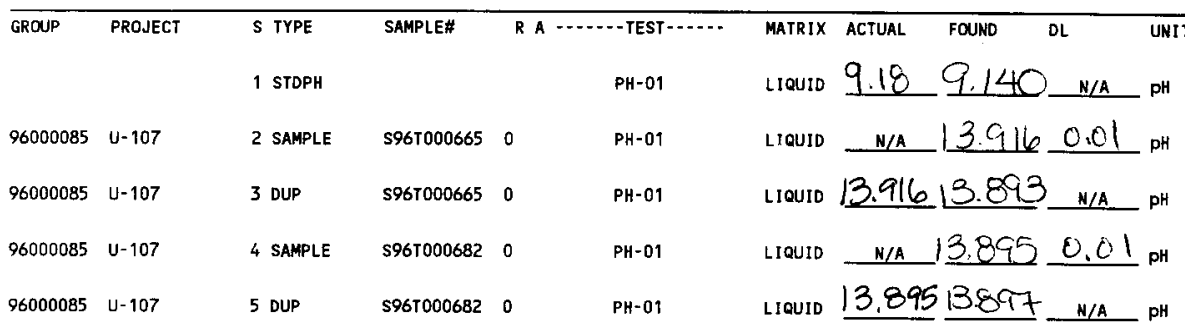

\section{Final page for worklist \# 6770}
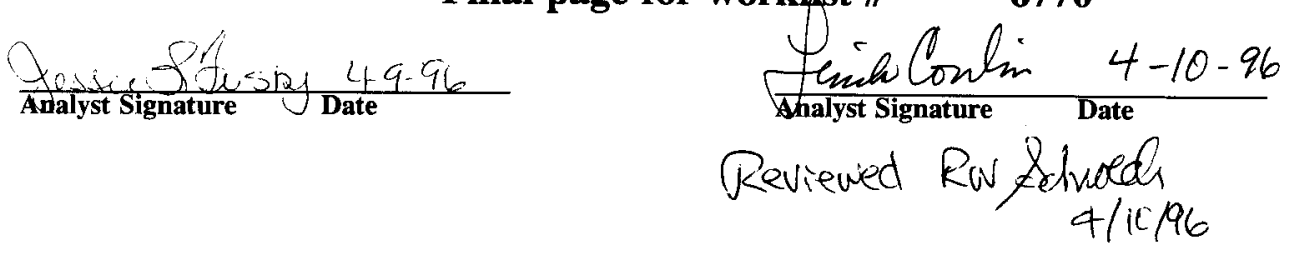

Data Entry Comments:

Units shown for $Q C$ (SPK \& STD) may not reflect the actual units. $D L=$ Detection Limit, $S=$ Worklist Slot Number, $R=$ Replicate Number, $A=$ Aliquot Code. 


Analyst: BS Instrument: PH01 27 Book\# 144 N16-E

Method: LA-212-106 Rev/Mod A-O

Worklist Comment: U-107 PH RUN IN DUPLICATE. RCJ

\begin{tabular}{|c|c|c|c|c|c|c|c|c|c|c|}
\hline \multirow[t]{2}{*}{ GROUP } & \multirow[t]{2}{*}{ PROJECT } & S TYPE & \multirow[t]{2}{*}{ SAMPLE\# } & \multicolumn{2}{|c|}{ R A } & \multirow{2}{*}{$\begin{array}{l}\text { MATRIX } \\
\text { LIQUID }\end{array}$} & \multirow{2}{*}{\multicolumn{2}{|c|}{$\begin{array}{ll}\text { ACTUAL } & \text { FOUND } \\
9.18 \quad 9.19 \\
\end{array}$}} & \multirow[b]{2}{*}{ N/A } & UNI \\
\hline & & $1 \mathrm{STDPH}$ & & & PH-09 & & & & & \\
\hline 96000126 & U-107 & 2 SAMPLE & S967001110 & 0 & $\mathrm{PH}-01$ & LIQUID & N/A & 12.42 & 0.01 & $\mathrm{pH}$ \\
\hline 96000126 & $U-107$ & 3 DUP & S96T001110 & 0 & PH-01 & LIQUID & 12.42 & 12.43 & N/A & pH \\
\hline 96000126 & U-107 & 4 SAMPLE & s96T001119 & 0 & PH-01 & Liouid & N/A & 12.82 & 0.01 & $\mathrm{pH}$ \\
\hline 96000126 & U-107 & 5 DUP & S96T001119 & 0 & PH-01 & LIQUID & 12.82 & 12.87 & N/A & pH \\
\hline 96000126 & U-107 & 6 SAMPLE & S96T001120 & o & PH-01 & LIQUID & W/A & 12604 & 0.01 & $\mathrm{pH}$ \\
\hline 96000126 & $u-107$ & 7 DUP & S96T001120 & 0 & PH-01 & LIQUID & 12.64 & 12.58 & N/A & $\mathrm{pH}$ \\
\hline 96000126 & $U-107$ & 8 SAMPLE & S96T001921 & 0 & $\mathrm{PH}-01$ & LIQUID & N/A & 12.84 & 0.01 & $\mathrm{pH}$ \\
\hline 96000126 & $U-107$ & 9 DUP & S96T001121 & 0 & PH-01 & LIQUID & 12.84 & 12.78 & H/A & pH \\
\hline
\end{tabular}

\section{Final page for worklist \# $\mathbf{7 5 8 2}$}
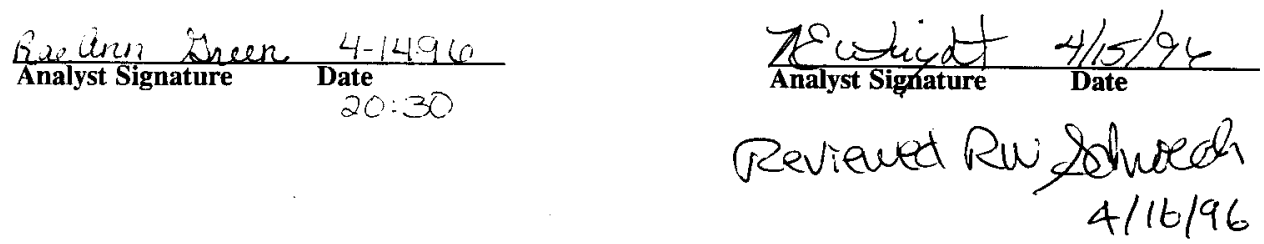

Data Entry Comments:

Units shown for QC (SPK \& STD) may not reflect the actual units. $D L=$ Detection Limit, $S=$ Worklist Slot Number, $R=$ Replicate Number, $A=$ Aliquot Code. 


\section{LABCORE Data Entry Template for Worklist\#}

\section{Analyst: Ry Instrument: PH01 Book \# $14416-D$}

Method: LA-212-106 Rev/Mod A. $Q$

Worklist Comment: U-107 PH. RCJ

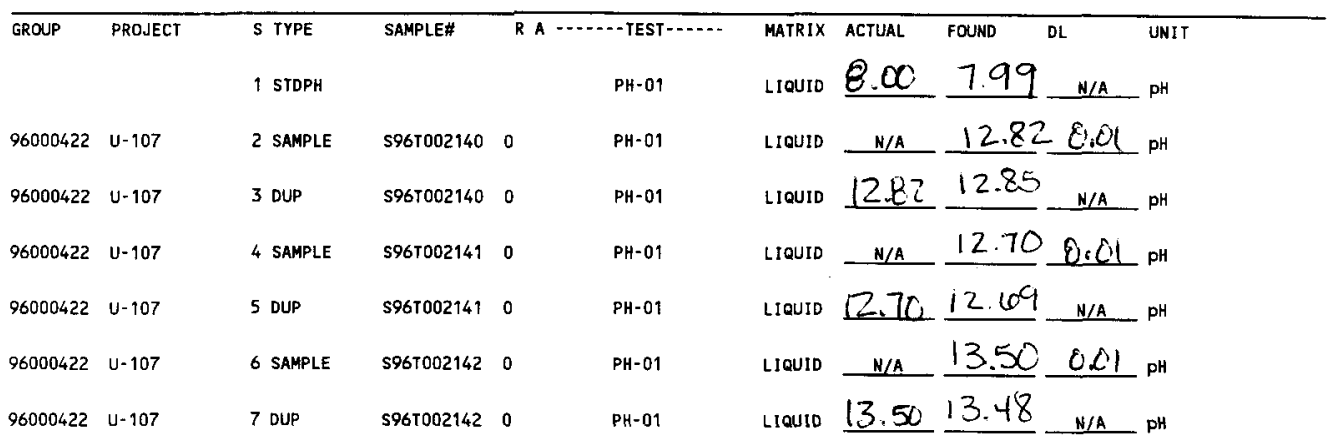

\section{Final page for worklist \# $\quad \mathbf{8 4 2 8}$}

- Par Cine Misen S-1296
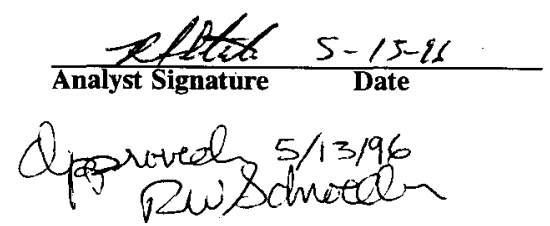

Data Entry Comments:

Units shown for QC (SPK \& STD) may not reflect the actual units. $D L=$ Detection Limit, $S=$ Worklist Slot Number, $R=$ Replicate Number, $A=$ Aliquot Code. 


\section{Analyst: \\ Instrument: PH01 \\ Book \# $2 \mu / 1 /$ LO}

Method: LA-212-106 Rev/Mod

Worklist Comment: C-106ه 102 PH. RCJ 4107

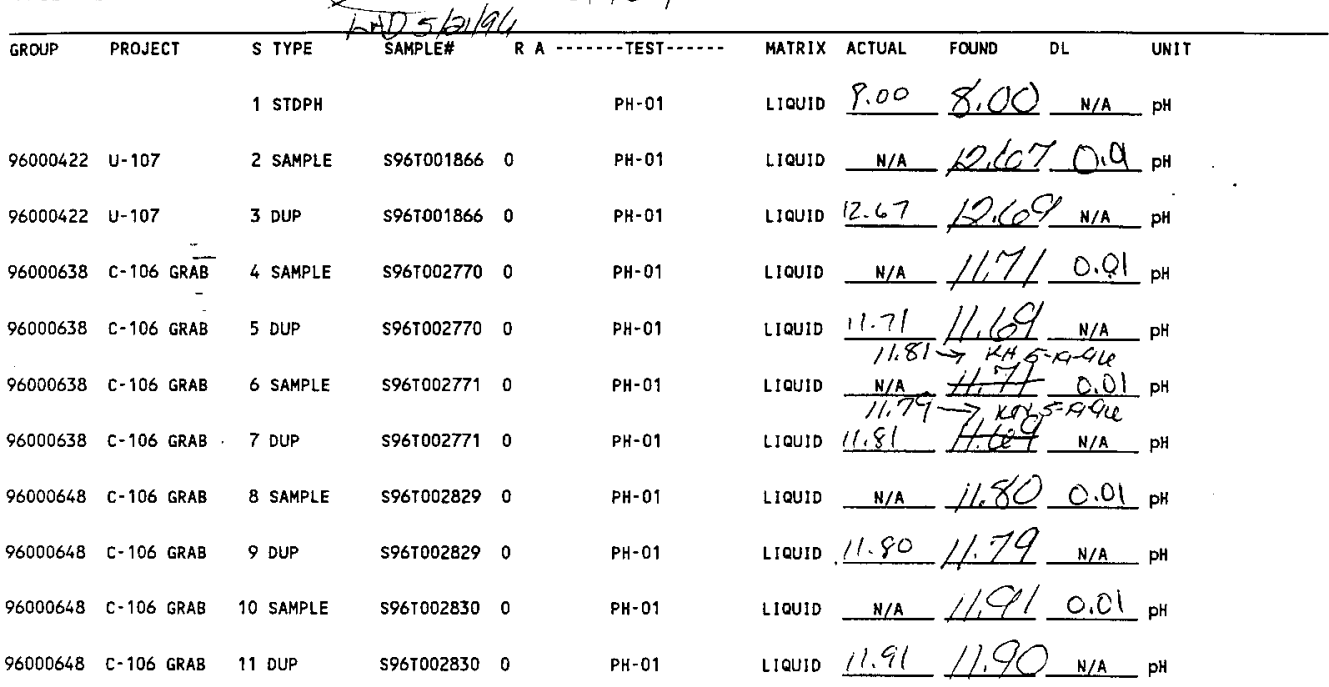

\section{Final page for worklist \# 8913}
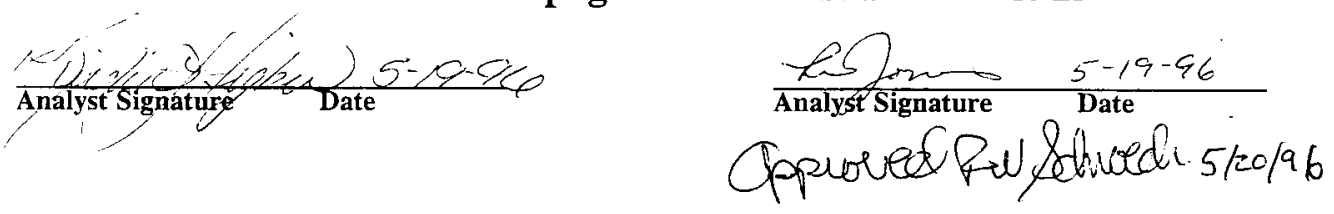

Data Entry Comments:

Units shown for $Q C$ (SPK \& STD) may not reflect the actual units. $D L=$ Detection Limit, $S=$ Worklist Slot Number, $R=$ Replicate Number, $A=$ Aliquot Code. 


\section{LABCORE Data Entry Template for Worklist\# 6322}

Analyst: $\therefore 6$ Instrument: ICO0 $I 01$

Book\# 1334:7

Method: LA-533-105 Rev/Mod L : : -

Worklist Comment: U-107 @IC-01. RCJ

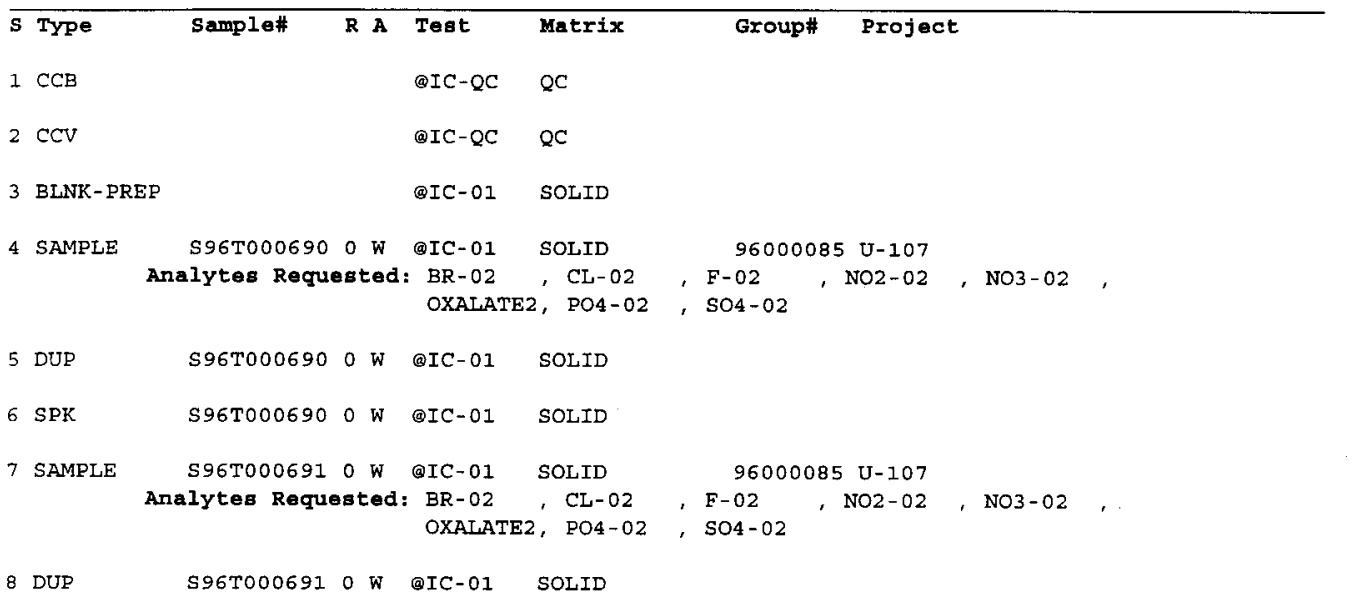

\section{Final page for worklist \# 6322}

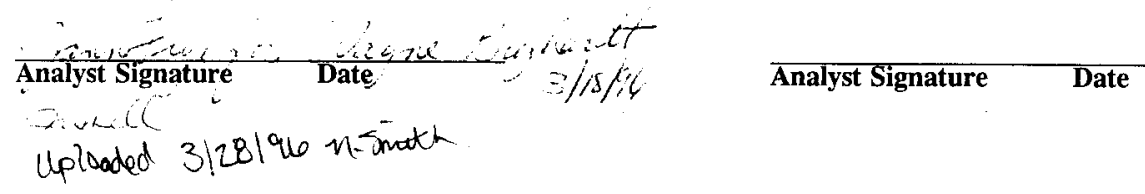

Data Entry Comments:

$S=$ Worklist Slot Number, $R=$ Replicate Number, $A=$ Aliquot Code. 


\section{LABCORE Completed Worklist Report for Worklist\# 6322}

Analyst: wgb

Instrument: ICO1

Book\# $133 N 9_{A} A$

Method: LAS33105 Rev/Mod D-1

Worklist Comment: U-107 @IC-01. RCJ

\begin{tabular}{|c|c|c|c|c|c|c|c|c|c|}
\hline Seq Туре & Sample\# R & & & est & Matrix & Actual & Found & DL or Yield & Unit \\
\hline $1 \operatorname{ces}$ & & 0 & eIc-Rc & $\mathbf{F}$ & $\mathrm{Q}^{\circ}$ & 1 & $<1 ; 300-2$ & & ug/mx \\
\hline $1 \mathrm{ccB}$ & & 0 & DIC-QC & CL & $Q^{C}$ & 1 & $<1.700-2$ & & $\mathbf{u g} / \mathbf{m L}$ \\
\hline $1 \mathrm{CCB}$ & & o & arc-oc & NO2 & oc & 1 & $<1.07 \cdot-1$ & & $\mathrm{ug} / \mathrm{mL}$ \\
\hline $1 \mathrm{ccB}$ & & 0 & -IC-QC & $\mathbf{B R}$ & $Q^{c}$ & 1 & $<1.260-1$ & & ug/mi \\
\hline $1 \operatorname{ccs}$ & & 0 & aIc-8c & no3 & $Q$ & 1 & $<1.400-1$ & & $\mathrm{ug} / \mathrm{mr}:$ \\
\hline $1 \mathrm{ccB}$ & & 0 & DIC-OC & PO4 & oc & 1 & $<1.190-1$ & & ug/mt \\
\hline $1 \mathrm{CCB}$ & & 0 & -IC-QC & so4 & QC & 1 & $<1.360-1$ & & $\mathbf{u g} / \mathbf{m l t}$ \\
\hline $1 \mathrm{CcB}$ & & 0 & DC-QC & OXALATE2 & $O c$ & 1 & $<1.050-1$ & & $\mathrm{ug} / \mathrm{mL}$ \\
\hline $2 \mathrm{ccv}$ & & 0 & eIC-Qc & $\mathbf{z}$ & $Q C$ & 39 & $5.82=+01$ & 98.64 & It R Recovery \\
\hline $2 \mathrm{cev}$ & & 0 & arc-Qc & CL & $Q c$ & 79 & $7.440+01$ & 94.17 & 77 \& Recovery \\
\hline $2 \mathrm{ccv}$ & & 0 & -IC-QC & $\mathrm{NO} 2$ & Qc & 537 & $5.25 \bullet+02$ & .97 .76 & 55 Recovery \\
\hline $2 \operatorname{cov}$ & & 0 & arc- $2 \mathrm{C}$ & BR & $Q c$ & 535 & $5.550+02$ & 96.52 & 22 * xecovery \\
\hline $2 \mathrm{ccv}$ & & 0 & QIC-QC & No3 & $Q c$ & 614 & $6.050+02$ & 98.53 & 34 \& Recovery \\
\hline $2 \mathrm{cct}$ & & 0 & arc-ec & PO4 & Qc & 546 & $5.28 \bullet+02$ & 96.70 & 33 \& Recovery \\
\hline $2 \cdot \mathrm{ccv}$ & & 0 & erc-oc & $\mathrm{SO} 4$ & $8 c$ & 631 & $6.14 \div+02$ & 97.30 & 36 Recovery \\
\hline $2 \mathrm{cCV}$ & & 0 & IC-QC & OXALATE2 & $2 c$ & 509 & $5.120+02$ & $100.5 \mathrm{~B}$ & 39 Recovery \\
\hline 3 BLNT - PREF & & 0 & $\operatorname{arc-01}$ & E-02 & SOLID & $\mathbf{I}$ & $<1,300-2$ & & ug/g \\
\hline 3 BLNK-PRBP & & 0 & -1C-01 & CL-02 & SOLID & 1 & $3.30 \cdot-02$ & 0.03 & ug/g \\
\hline 3 BLWX-PRFP & & 0 & arc-01 & $\mathrm{NO} 2-02$ & SOLXD. & 1 & $<1.07 \cdot-1$ & & $49 / 9$ \\
\hline 3 BLWK-ERIP & & 0 & IC-01 & $B R-02$ & SOLID & 1 & $<1.26 \bullet-1$ & & $\mathrm{ug} / \mathrm{g}$ \\
\hline 3 BLNK-PREP & & 0 & anc-01 & NO3-O2 & SOLID & 1 & $<1.40<-1$ & & $\mathrm{ug} / \mathrm{g}$ \\
\hline 3 BLNK - PRBP & & 0 & -1C-01 & P04-02 & SOLID & 1 & $<1.190-1$ & & $\mathbf{u g} / \mathrm{g}$ \\
\hline 3 BLNK-PREP & & 0 & - $10-01$ & so4-02 & SOLID & 1 & $<1.360-1$ & & $\mathrm{ug} / \mathrm{g}$ \\
\hline 3 BINKK- PREP & & 0 & - 2 IC-01 & OXALATE2 & soLID & 1 & $<1.05 \bullet-1$ & & $\mathrm{ug} / \mathrm{g}$ \\
\hline 4 SAMPLE & S967000690 & ow & $\operatorname{aIC-01}$ & $F-02$ & SOLID & $N / A$ & $4.670 \cdot 402$ & 103.900 & $\mathrm{ug} / \mathrm{g}$ \\
\hline 4 RIJICT & $596 \mathrm{~T} 000690$ & ow & -IC-01 & $F-02$ & SOLID & S/A & $4.670 \bullet+02$ & & $\mathrm{ug} / \mathrm{g}$ \\
\hline 4 SAMPLE & S96T000690 & ow & arc-01 & CL- 02 & SOLID & $\mathbf{N} / \mathrm{A}$ & $4.603 \cdot+03$ & 135.800 & $u g / g$ \\
\hline 4 REJECT & S96T000690 & ow & aIC-01 & $C L-02$ & SOLID & N/A & $4.603 e+03$ & & $\mathrm{ug} / \mathrm{g}$ \\
\hline 4 SAMPLF & $596 \mathrm{T000690}$ & a $w$ & arc-01 & $\mathrm{NO2-02}$ & SOLID & $N / x$ & $5.148 \cdot+04$ & 854.900 & ug/g \\
\hline 4 RENECT & 5967000690 & o w & aIC-01 & $\mathrm{NO2}-02$ & SOLID & $N / A$ & $5.148 \bullet+04$ & & $\mathrm{ug} / \mathrm{g}$ \\
\hline 4 SNAPLE & 5967000690 & O w & -IC-01 & $B R-02$ & SOLID & N/A $<$ & 1007.000 & 1007.000 & $u g / g$ \\
\hline 4 REJECT & S96T000690 & o w & aIC-01 & $B R-02$ & SOLID & $\mathrm{N} / \mathrm{A}$ & $1.006 * 003$ & & $\mathrm{ug} / \mathrm{g}$ \\
\hline 4 SAMPLE & $596 \mathrm{T000690}$ & o $w$ & arc-01 & $N 03.02$ & SOIID & $N / A$ & $1.354 \bullet+05$ & 1118.000 & $\mathrm{ug} / \mathrm{g}$ \\
\hline 4 REJECT & $596 \mathrm{T000690}$ & o w & Orc-01 & No3-02 & SOLID & $\underline{N / A}$ & $1.3540+05$ & & $\mathrm{ug} / \mathrm{g}$ \\
\hline 4 SAMPIIX & 5967000690 & o w & $\triangle I C-01$ & PO4-02 & SOLID & $N / A$ & $3.213 \bullet+04$ & 949.600 & ug $/ g$ \\
\hline 4 REJECT & $S 967000690$ & o w & (1)-01 & PO4-02 & SOLID & $N / A$ & $3.213 e+04$ & & $\mathrm{ug} / \mathrm{g}$ \\
\hline 4 SAMPLE & 5967000690 & ow & aIC-01 & $504-02$ & SOLID & $\mathbf{N} / \mathrm{A}$ & $4.954 e+03$ & 1086.000 & $\mathrm{ug} / \mathrm{g}$ \\
\hline 4 REJECT & 5967000690 & O $\mathrm{w}$ & $\operatorname{arc}-a 1$ & so4-02 & SOLID & $\mathbb{N} / \mathrm{A}$ & $4.954 e+03$ & & $u g / g$ \\
\hline 4 SAMPLE & 5967000690 & o w & $\operatorname{axc}-01$ & OXALATE2 & SOIID & N/A & $9.8740+03$ & 838.900 & ug $/ g$ \\
\hline 4 REJECT & 5967000690 & O w & aIC-01 & OXATATE2 & sOLID & $\mathbf{N} / \mathbf{A}$ & $9.874+403$ & & $\lg / 9$ \\
\hline 5 DTP & 5967000690 & a w & AIC-01 & $7-02$ & SOLID & $4.67 *+02$ & $4.16 \bullet+02$ & 11.55 & 52 RPD \\
\hline
\end{tabular}




\section{LABCORE Completed Worklist Report for Worklist\# 6322}

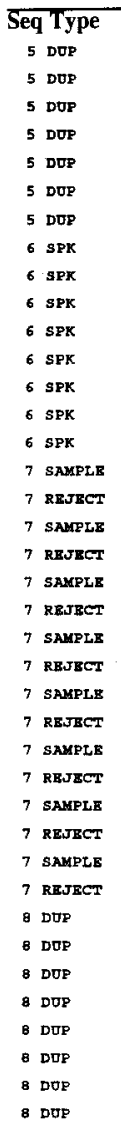

\begin{tabular}{|c|c|c|c|c|}
\hline \multicolumn{2}{|c|}{ Sample\# R A } & \multicolumn{2}{|c|}{ Test } & \multirow{2}{*}{$\begin{array}{r}\text { Matrix } \\
\text { solid }\end{array}$} \\
\hline$s 96 T 000690$ & $0 \mathrm{w}$ & erc-01 & CL-02 & \\
\hline 5967000690 & ow & eIC-01 & พо2 -02 & SOLID \\
\hline $596 \mathrm{TO00690}$ & ow & -IC-01 & R -02 & SOLID \\
\hline $896 \mathrm{T000690}$ & $0 \mathrm{w}$ & erc-01 & No3-02 & SOLID \\
\hline 5967000690 & $0 \mathrm{w}$ & $\operatorname{arc-01}$ & $\mathrm{PO4}_{-02}$ & SOLID \\
\hline $596 \mathrm{~T} 000690$ & o w & exc-01 & $s 04-02$ & sOLID \\
\hline $596 \mathrm{T000690}$ & $0 \mathrm{w}$ & arc-02 & OXALATE2 & 2 SOI.ID \\
\hline s96T000690 & $0 \mathrm{w}$ & -IC-01 & F-02 & SOLID \\
\hline $596 \mathrm{TO000690}$ & $0 w$ & arc-01 & CL-02 & SOLID \\
\hline $396 \mathrm{~T} 000690$ & O W & -IC-01 & NO2-02 & SOLID \\
\hline $896 \mathrm{~T} 000590$ & $0 W$ & arc-01 & BR-02 & SOLID \\
\hline $596 T 000590$ & $0 \mathrm{~W}$ & OIC-01 & $N 03-02$ & SOLID \\
\hline S96T000690 & $0 \mathrm{w}$ & arc-01 & $204-02$ & SOLID \\
\hline $396 \mathrm{~T} 000690$ & ow & aIc-01 & $504-02$ & SOLID \\
\hline$s 96 \mathrm{T000690}$ & ow & - Ic-01 & OXALATE 2 & SOEID \\
\hline s96T000692 & $0 \mathrm{w}$ & exc-01 & $F-02$ & SOLID \\
\hline $896 \mathrm{~T} 000691$ & $0 \mathrm{~W}$ & eIc-01 & F- 02 & SOLID \\
\hline 5967000691 & $0 \%$ & Ic-01 & $C L-02$ & SOLID \\
\hline 5965000691 & $0 \mathrm{w}$ & alc-01 & $C L-02$ & SOLID \\
\hline 5967000691 & O w & DIC-0I & NO2-02 & SOLID \\
\hline \$96T000691 & $0 \mathrm{w}$ & OIC-0i & No2-02 & soLID \\
\hline S96T000691 & o w & eIc-01 & $8 R-02$ & SOLID \\
\hline s96ro000691 & o $w$ & $\operatorname{arc}-01$ & $B R-02$ & SOLID \\
\hline S96T000691 & $0 \mathrm{w}$ & arc-01 & NO3 -02 & SOLID \\
\hline s967000691 & $0 \mathrm{~W}$ & QIC-0I & & SOLID \\
\hline $\mathrm{s} 96 \mathrm{~T} 000691$ & o w & 1C- 01 & PO4-02 & SOLID \\
\hline $596 \mathrm{T000691}$ & $0 \mathrm{w}$ & arc-01 & PO4-02 & SOLID \\
\hline S96T000691 & $0 w$ & OIC-01 & SO4-02 & SOLID \\
\hline $596 \mathrm{~T} 000691$ & ow & aIC-01 & SO4-02 & SOLID \\
\hline 5962000691 & ow & QIC-0I & OXAIATTE2 & SOLID \\
\hline s96T000691 & $0 \mathrm{w}$ & $O I C-0 I$ & OXALATE2 & solID \\
\hline S96T000691 & $0 \mathrm{w}$ & eIC-01 & $\mathbf{F}-02$ & SOLID \\
\hline$\$ 967000691$ & ow & QIC-01 & CL-02 & SOLID \\
\hline $596 T 000691$ & $0 \mathrm{w}$ & MIC- 01 & so2 -02 & SOLID \\
\hline S96T000691 & $0 \mathrm{w}$ & $\operatorname{arc}-01$ & BR-02 & SOLID \\
\hline$\$ 967000691$ & $0 \mathrm{~W}$ & (2IC-0] & No3-02 & SOLID \\
\hline S96T000691 & $0 \mathrm{~W}$ & aIC-01 & PO4-02 & SOLID \\
\hline$S 96 \mathrm{T000061}$ & $0 \mathrm{H}$ & QIC-01 & $504-02$ & SOLID \\
\hline $596 T 000691$ & $0 \mathrm{~W}$ & AIC-01 & OXALATE2 & SOLID \\
\hline
\end{tabular}

\begin{tabular}{|c|c|}
\hline $\begin{array}{l}\text { Actual } \\
4.600+03\end{array}$ & $\begin{array}{l}\text { Found } \\
4.54 \oplus+03\end{array}$ \\
\hline $5.15 \bullet+04$ & $4.920+04$ \\
\hline$<1.0103$ & $<1,080$ \\
\hline $1.35 e+05$ & $1.27=+0$ \\
\hline $3.220+04$ & $2.63=+0$ \\
\hline $4.95 \bullet+03$ & $5.03 €+03$ \\
\hline $9.87 \bullet+03$ & B. $920+0=$ \\
\hline 59 & $4.240+02$ \\
\hline 79 & $7.310+01$ \\
\hline 537 & $5.37 \bullet+02$ \\
\hline 575 & $5.42=+02$ \\
\hline 614 & $6.510+02$ \\
\hline 546 & $5.090+02$ \\
\hline 631 & $5.94 \neq+02$ \\
\hline 509 & $5.03 e+02$ \\
\hline$N / \mathbf{A}$ & $3.0010+0$ \\
\hline N/A & $3.0020+02$ \\
\hline$N / A$ & $2.06900+03$ \\
\hline $\boldsymbol{H} / \mathrm{A}$ & $2.069 \bullet+03$ \\
\hline$y / x$ & $2.280 \bullet+04$ \\
\hline Es/a & $2.2800+04$ \\
\hline$N / A<$ & $1.788 \bullet+03$ \\
\hline $\mathbf{S} / \mathrm{A}$ & $1.7880+03$ \\
\hline$N / A$ & $3.177 \bullet+05$ \\
\hline$y / 2$ & $3.177=+05$ \\
\hline $\mathbb{N} / \mathrm{A}$ & $7.1240+03$ \\
\hline$N / A$ & $7.1240+03$ \\
\hline$N / A$ & $4.650+03$ \\
\hline N/A & $4.650 e+03$ \\
\hline $\mathrm{N} / \mathrm{A}$ & $2.925 \bullet+03$ \\
\hline $\mathbf{N} / \mathbf{A}$ & $2.925 e+03$ \\
\hline $3.000+02$ & $2.910+02$ \\
\hline $2.070+03$ & $1.700+03$ \\
\hline $2.28 \bullet+04$ & $2.040+04$ \\
\hline$<1.79 .43$ & $<1.81 .3$ \\
\hline $3.18 \bullet+05$ & $5.71 \bullet+05$ \\
\hline $7.120+03$ & $6.960+03$ \\
\hline $4.65 \cdot+03$ & $4.88 \backsim+03$ \\
\hline $2.92 \bullet+03$ & $2.780+0$ \\
\hline
\end{tabular}

\section{DL or Yield Unit}

1.313 RFD

$4.56 \mathrm{~B}$ RPD

6.107 RPD

$19.863 . \mathrm{RPD}$

$1.603 \mathrm{RPD}$

10. $112 \mathrm{RPD}$

71.864 \& Recovery

92.532 * Recovery

100.000 \% Recovery

94.263 : Recovery

106.026 \& Recovery

93.223 Recovery

94.136 \% Recovery

98.021 o Recorery

$184.500 \mathrm{ug} / \mathrm{g}$

$u g / g$

$241.200 \mathrm{ug} / \mathrm{g}$

$u g / g$

$1519.000 \mathrm{ug} / \mathrm{g}$

ug/g

$1788.000 \mathrm{ug} / \mathrm{g}$

$\mathrm{ug} / \mathrm{g}$

$1985.000 \mathrm{ug} / \mathrm{g}$

$49 / 9$

$1687.000 \mathrm{ug} / \mathrm{g}$

$\mathrm{ug} / \mathrm{g}$

$1929.000 \mathrm{ug} / \mathrm{g}$

ug $/ g$

$1490.000 \mathrm{ug} / \mathrm{g}$

$\mathrm{ug} / \mathrm{g}$

3.046 RPD

15.065 RFD

11.111 RPD

RPD

56.91日 RPD

2.273 RPD

4.827 RPD

4.912 RPD

\section{Final page for worklist\# 6322}

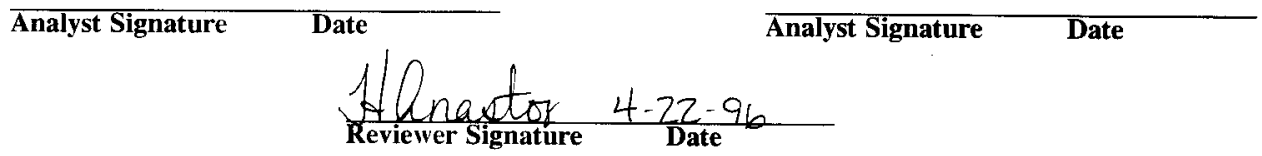

Units shown for $\overline{Q C}(B L K / B K G)$ may not reflect the actual units. 
WHC-SD-WM-DP-184, REV. I

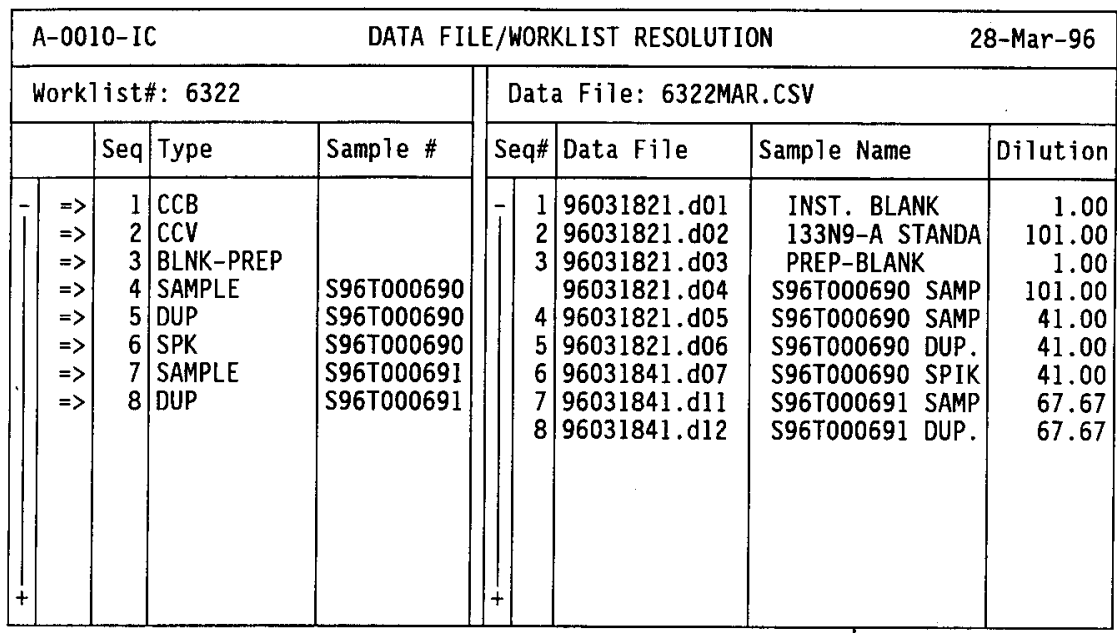

Save(F4) Abort(Shift-F3) ListFiles(Shift-F1) Uploadfile(F8) 
Data Reprocessed On 03/19/1996 09:26:41

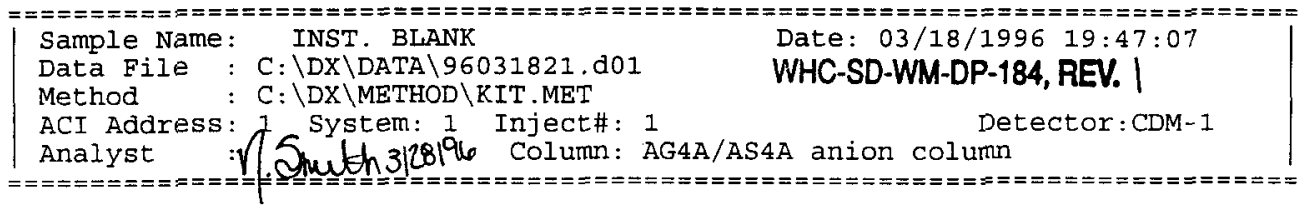

Calibration Volume Dilution Points Rate start Stop Area Reject

- - - - - - - - - - - - -

$\begin{array}{llllllll}\text { External } & 1 & 1 & 3150 & 5 \mathrm{~Hz} & 0.00 & 10.50 & 100\end{array}$

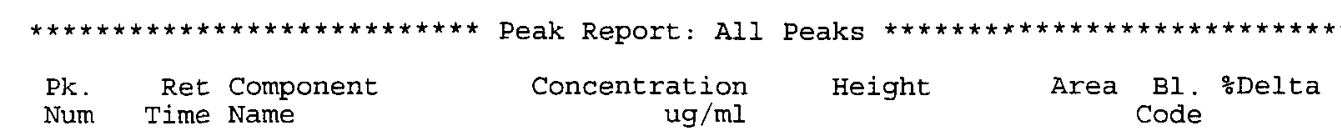

Totals

0.000

0

0

File: 96031821.d01 Sample: INST. BLANK

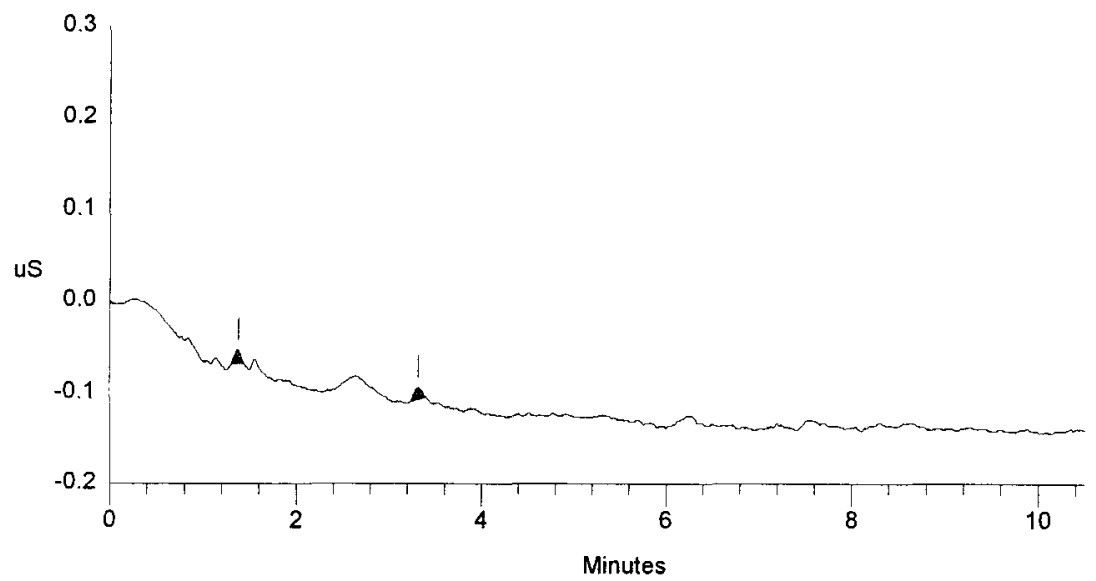

SIGNATURE ABOVE REPRESENTS CHEMICAL TECHNOLOGIST/CHEMIST THAT COMPLETED/VERIFIED THE CALIBRATION/ANALYSIS ON PAGES 244 T0.25 8 
Data Reprocessed on 03/19/1996 09:26:44

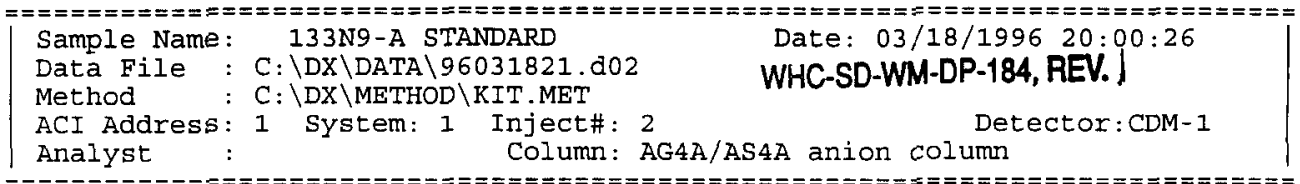

Calibration Volume Dilution Points Rate Start Stop Area Reject

\begin{tabular}{|c|}
\hline External \\
\hline
\end{tabular}

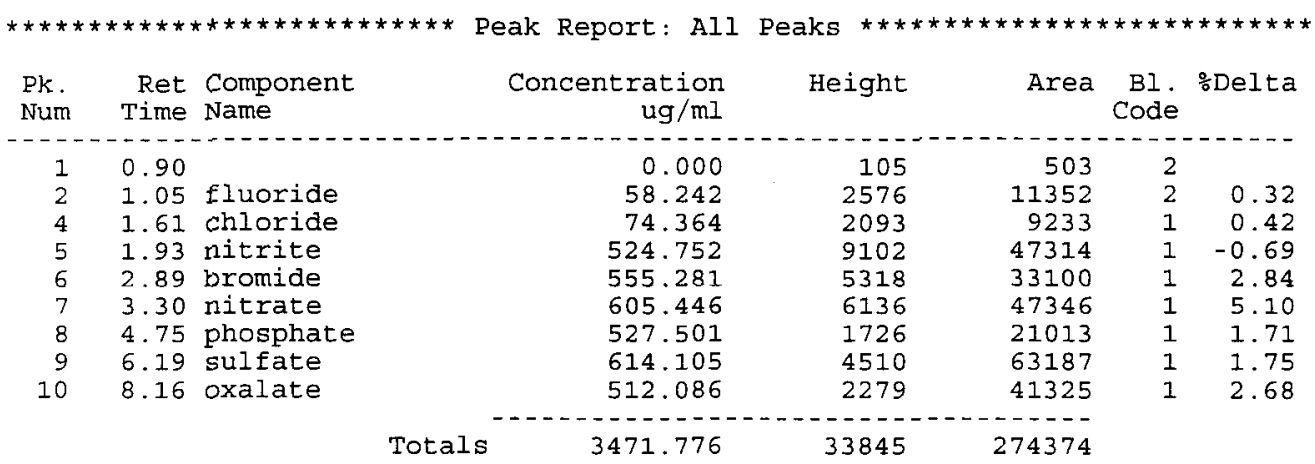

File: 96031821.d02 Sample: 133N9-A STANDARD

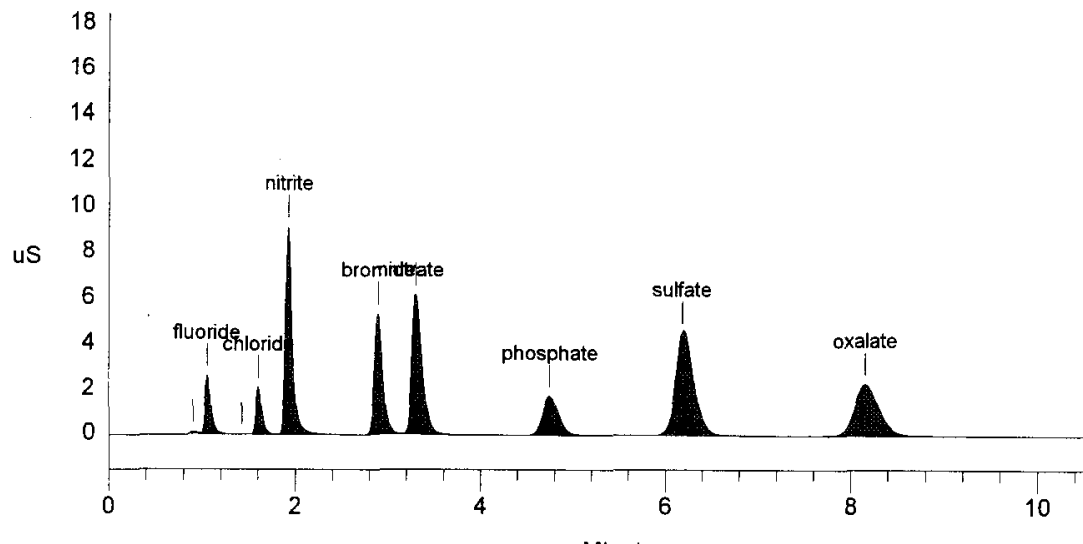

Minutes 
Data Reprocessed On 03/19/1996 09:26:46

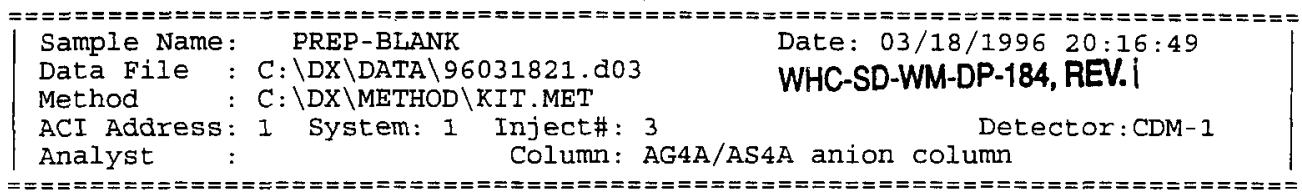

Calibration Volume Dilution Points Rate start stop Area Reject

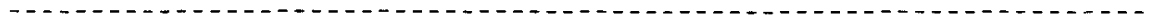

$\begin{array}{llllllll}\text { External } & 1 & 1 & 3150 & 5 \mathrm{~Hz} & 0.00 & 10.50 & 100\end{array}$

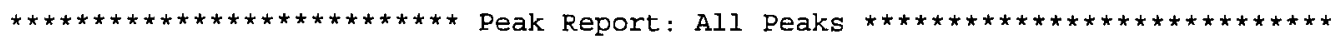

$\begin{array}{lrrr}\text { Pk. Ret Component } & \text { Concentration } & \text { Height } & \text { Area Bl. } \\ \text { Num Delta } & \text { Time Name }\end{array}$

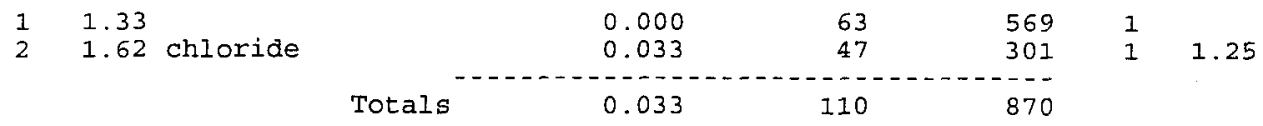

File: 96031821.d03 Sample: PREP-BLANK

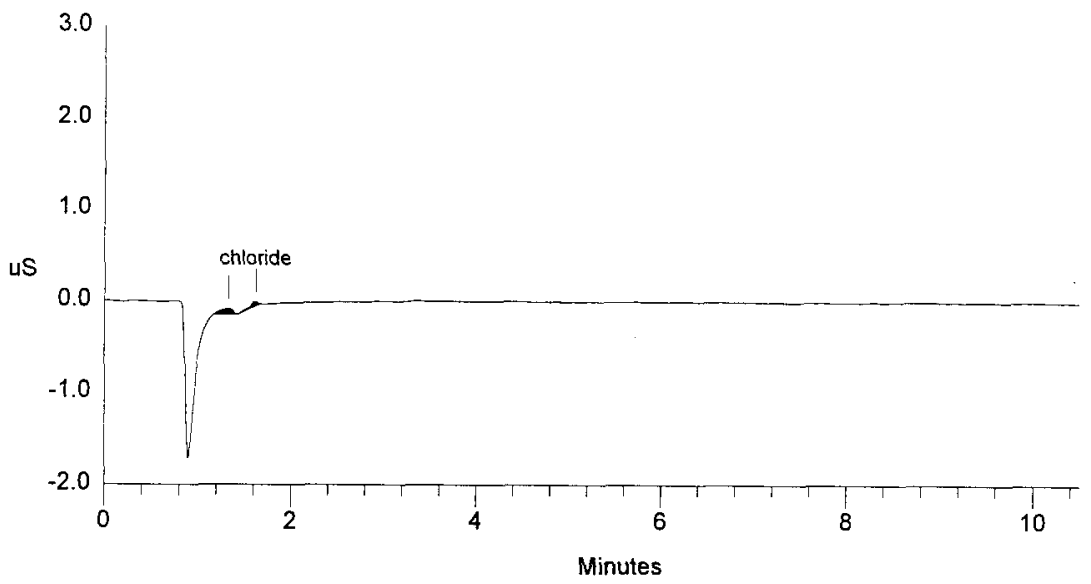


Data Reprocessed On 03/19/1996 09:26:49

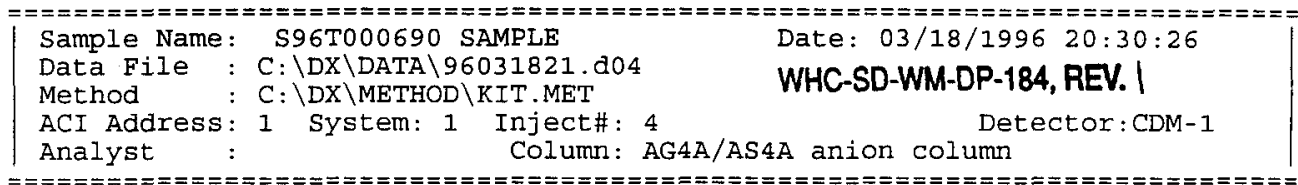

Calibration Volume Dilution Points Rate start Stop Area Reject

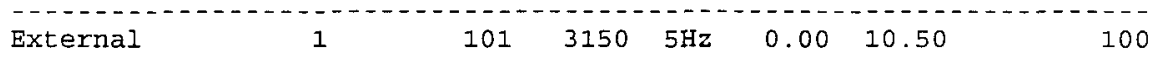

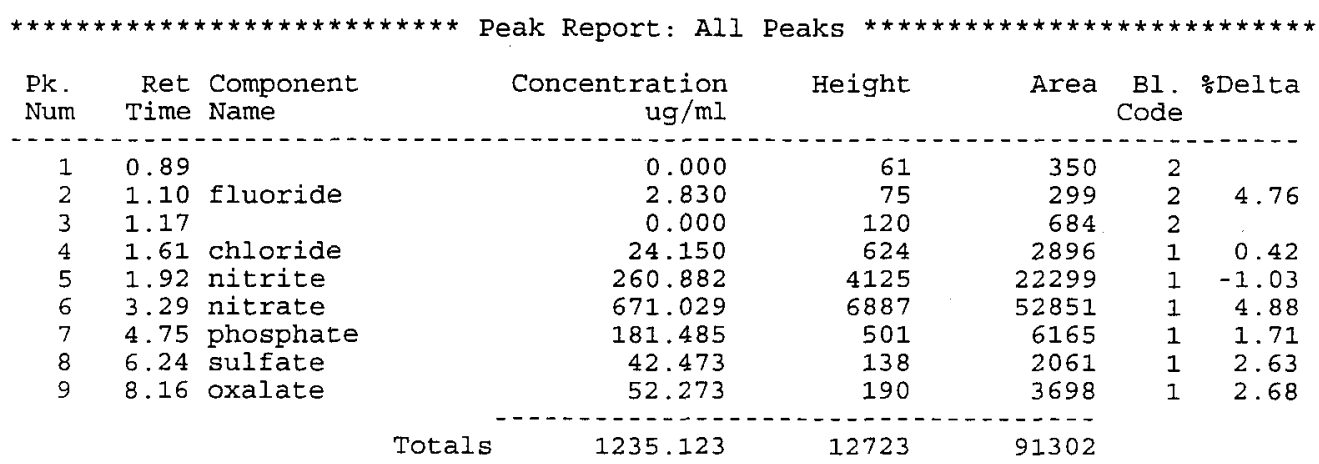

File: 96031821.d04 Sample: S96T000690 SAMPLE

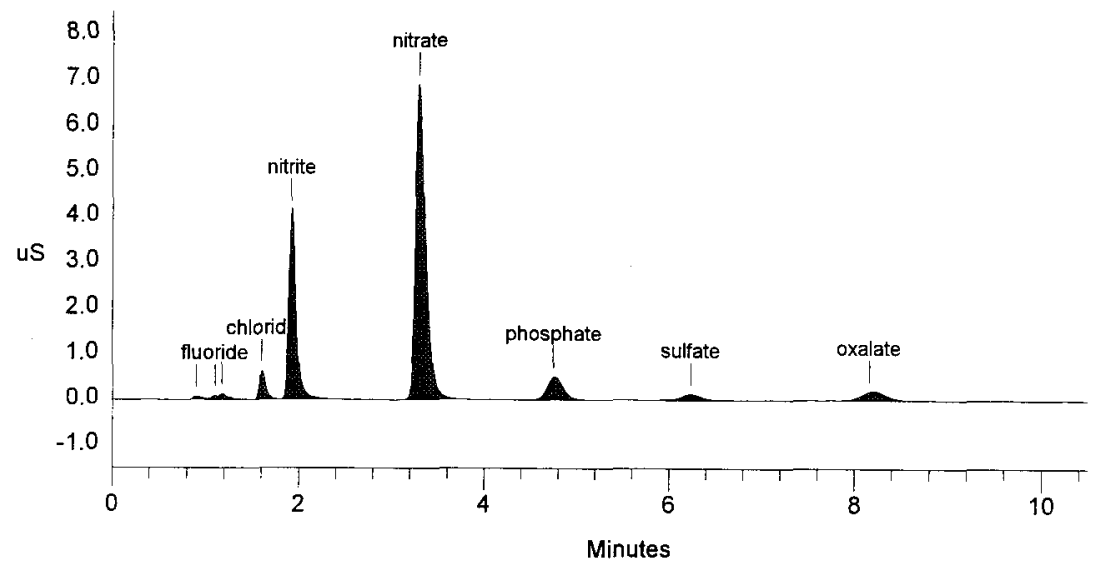


Data Reprocessed On 03/19/1996 09:26:51

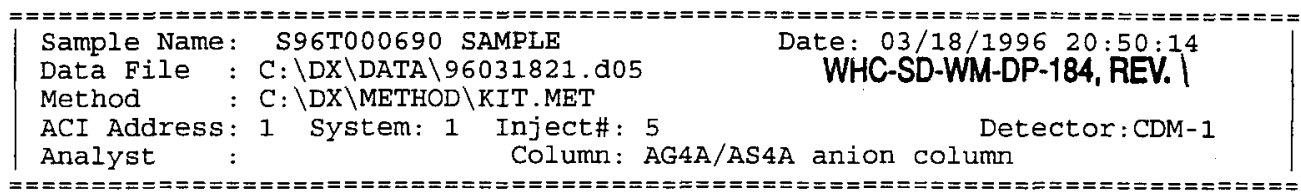

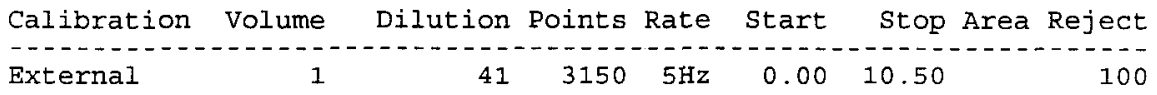

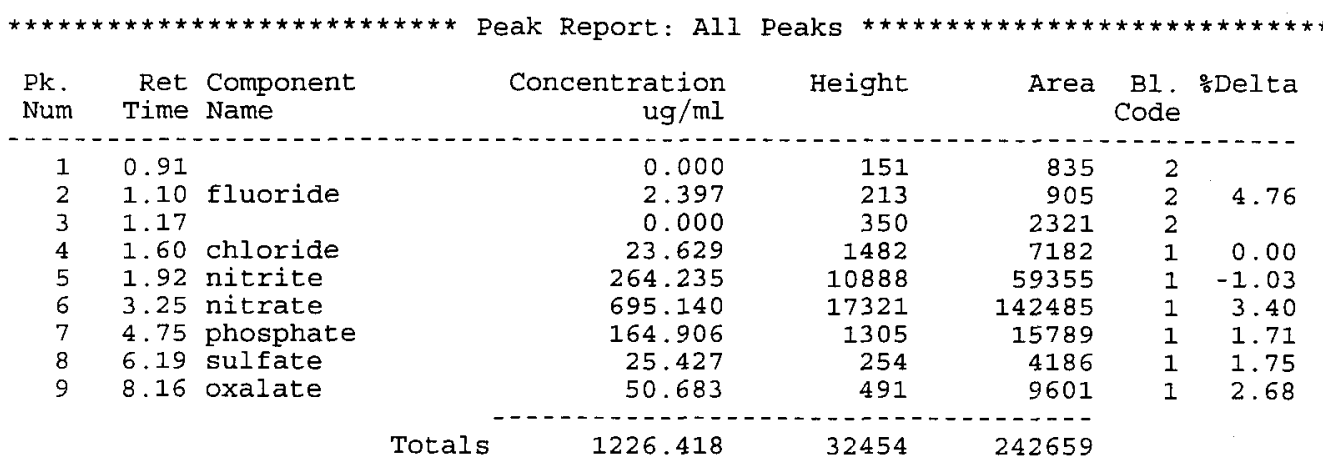

File: 96031821.d05 Sample: S96T000690 SAMPLE

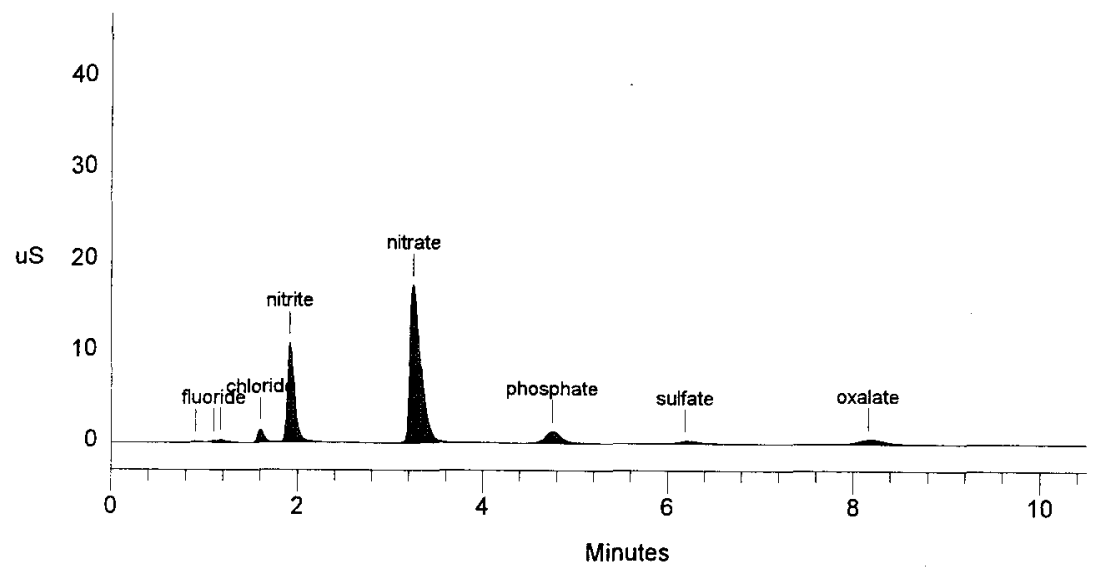


Data Reprocessed On 03/19/1996 09:26:54

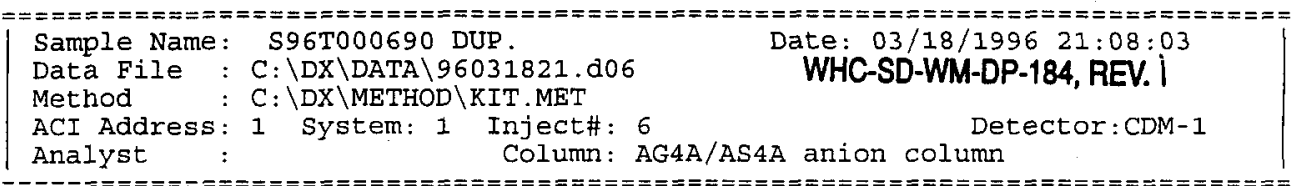

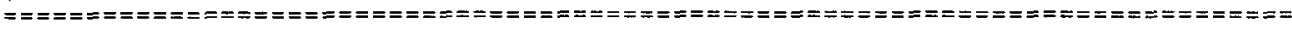

Calibration Volume Dilution Points Rate start stop Area Reject

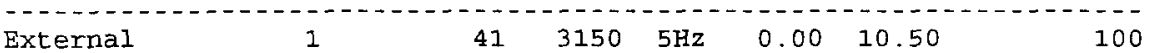

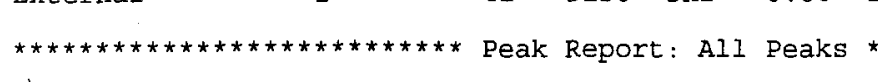

$\begin{array}{lrrr}\text { Pk. Ret Component } & \text { Concentration } & \text { Height } & \text { Area Bl. } \\ \text { Num } & \text { Time Name } & \text { Code }\end{array}$

Num Time Name

$\begin{array}{lll}1 & 0.91 \\ 2 & 1.10 & \text { fluoride } \\ 3 & 1.18 & \\ 4 & 1.60 & \text { chloride } \\ 5 & 1.92 & \text { nitrite } \\ 6 & 3.25 & \text { nitrate } \\ 7 & 4.75 & \text { phosphate } \\ 8 & 6.19 & \text { sulfate } \\ 9 & 8.16 \text { oxalate }\end{array}$

0.000
1.982
0.000
21.610
234.462
604.418
125.114
23.951
42.507

132

181

325

1392

9762

15178

930

226

415

Totals

1054.044

28542

7462

$703 \quad 2$

4.76

2114

6554

52349

122882

11582

3803

7962

2

2

0.00

$\begin{array}{rr}1 & -1.03 \\ 1 & 3.61\end{array}$

11.71

11.71

12.68

File: 96031821.d06 Sample: S96T000690 DUP.

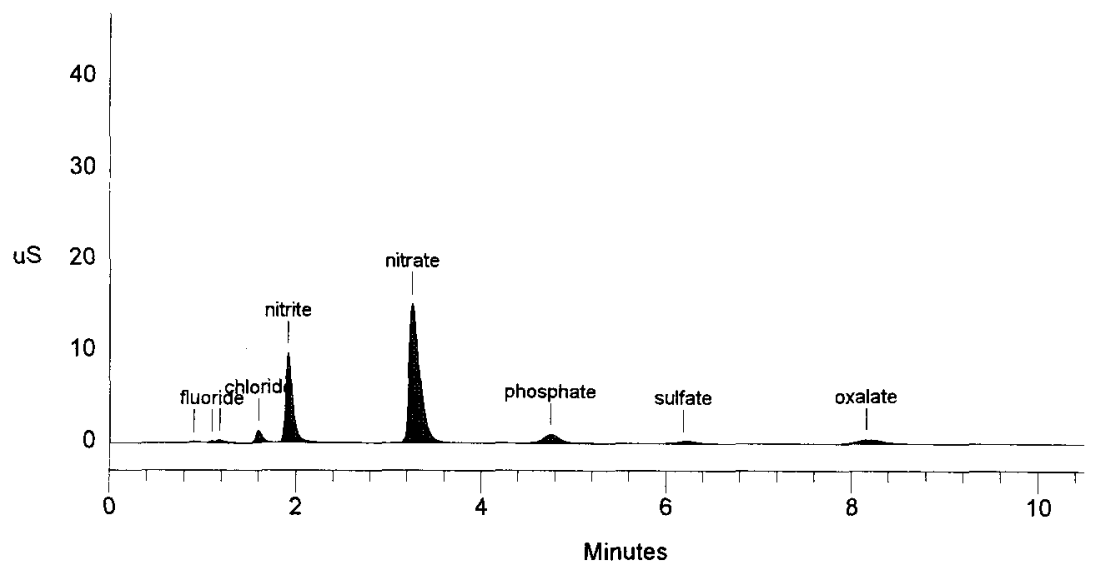


Data Reprocessed On 03/19/1996 09:26:56

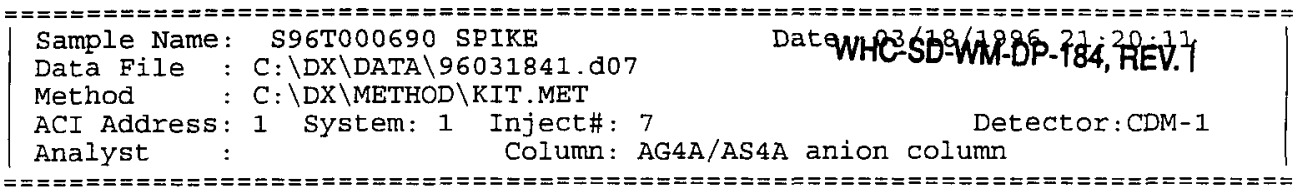

Calibration Volume Dilution Points Rate start Stop Area Reject

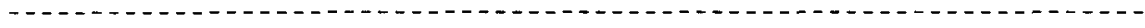

$\begin{array}{llllllll}\text { External } & 1 & 41 & 3150 & 5 \mathrm{~Hz} & 0.00 & 10.50 & 100\end{array}$

Peak Report: All Peaks

Pk. Ret Component Concentration Height Area B1. $\frac{\circ}{D}$ Delta Num Time Name ug/ml

$\begin{array}{lll}1 & 0.90 \\ 2 & 1.05 & \text { fluoride } \\ 3 & 1.60 & \text { chloride } \\ 4 & 1.93 & \text { nitrite } \\ 5 & 2.88 & \text { bromide } \\ 6 & 3.22 & \text { nitrate } \\ 7 & 4.72 & \text { phosphate } \\ 8 & 6.19 & \text { sulfate } \\ 9 & 8.11 \text { oxalate }\end{array}$

\begin{tabular}{|c|c|c|c|c|c|}
\hline & $\begin{array}{r}0.000 \\
19.350 \\
52.864 \\
478.956 \\
216.612 \\
955.358 \\
368.324 \\
263.214 \\
251.875\end{array}$ & $\begin{array}{r}196 \\
2351 \\
3449 \\
20723 \\
5262 \\
23191 \\
3026 \\
4796 \\
2573\end{array}$ & $\begin{array}{r}843 \\
9220 \\
16412 \\
110420 \\
31728 \\
199919 \\
37517 \\
66915 \\
50275\end{array}$ & $\begin{array}{l}1 \\
1 \\
1 \\
1 \\
1 \\
1 \\
1 \\
1 \\
1\end{array}$ & $\begin{array}{r}0.32 \\
0.00 \\
-0.69 \\
2.37 \\
2.55 \\
1.14 \\
1.75 \\
2.01\end{array}$ \\
\hline Totals & 2606.554 & 65567 & 523248 & & \\
\hline
\end{tabular}

File: 96031841.d07 Sample: S96T000690 SPIKE

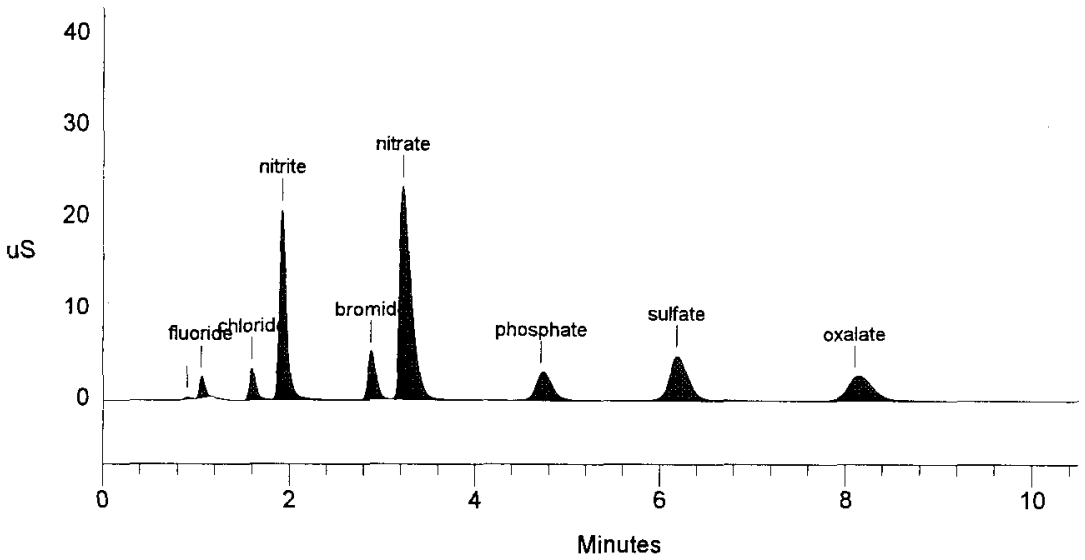


Data Reprocessed on 03/19/1996 09:26:59

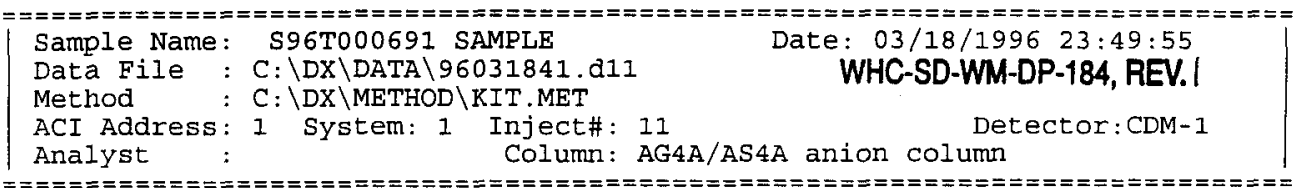

Calibration Volume Dilution Points Rate start Stop Area Reject

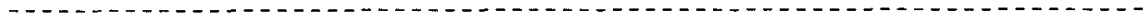

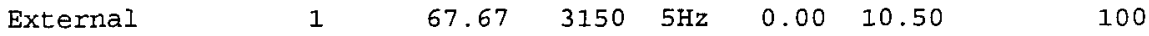

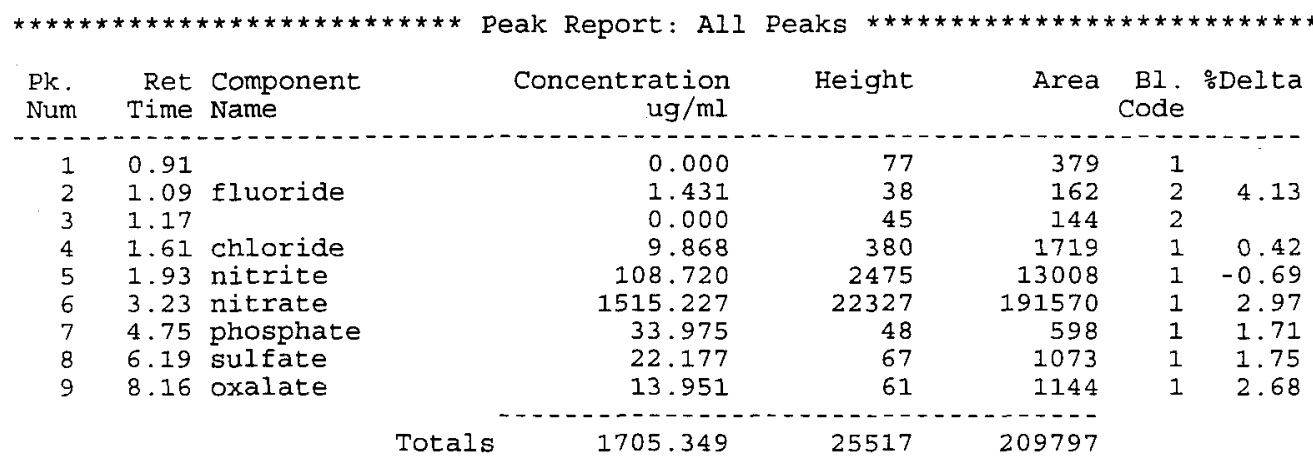

File: 96031841.d11 Sample: S96T000691 SAMPLE

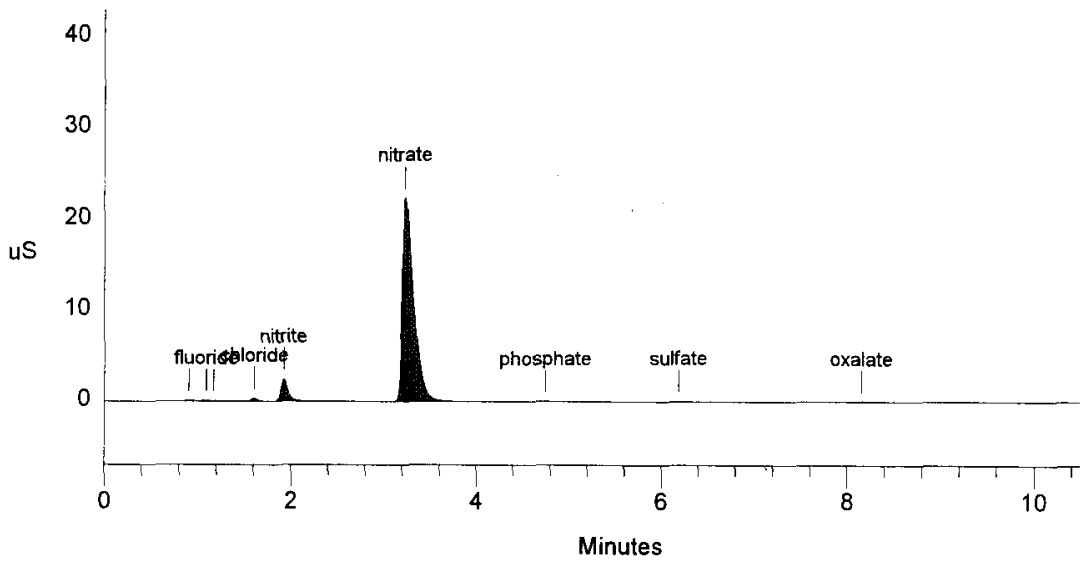


Data Reprocessed On 03/19/1996 09:27:02

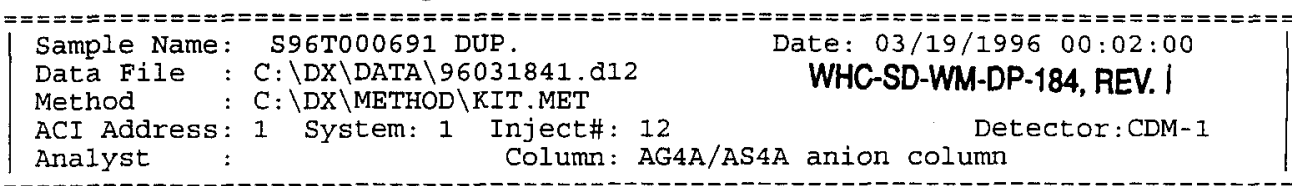

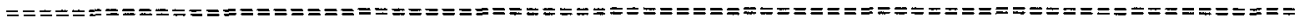

Calibration Volume Dilution Points Rate start Stop Area Reject

Cal ibration

$\begin{array}{llllllll}\text { External } & 1 & 67.67 & 3150 & 5 \mathrm{~Hz} & 0.00 & 10.50 & 100\end{array}$

\begin{tabular}{|c|c|c|c|c|c|c|c|}
\hline $\begin{array}{l}\text { Pk. } \\
\text { Num }\end{array}$ & $\begin{array}{l}\text { Ret } \\
\text { Time }\end{array}$ & $\begin{array}{l}\text { Component } \\
\text { Name }\end{array}$ & $\begin{array}{r}\text { Concentration } \\
\mathrm{ug} / \mathrm{ml}\end{array}$ & Height & Area & $\begin{array}{l}\text { Bl. } \\
\text { Code }\end{array}$ & $\div$ Delta \\
\hline & ---- & & & & & & \\
\hline 1 & 0.90 & & 0.000 & 125 & 666 & 2 & \\
\hline 2 & 1.09 & fluoride & 1.372 & 32 & 145 & 2 & 3.49 \\
\hline 3 & 1.17 & & 0.000 & 37 & 119 & 2 & \\
\hline 4 & 1.61 & chloride & 8.415 & 325 & 1449 & 1 & 0.42 \\
\hline 5 & 1.92 & nitrite & 96.301 & 2104 & 11264 & 1 & -1.03 \\
\hline 6 & 3.19 & nitrate & 2697.032 & 37947 & 359356 & 1 & 1.70 \\
\hline 7 & 4.75 & phosphate & 32.847 & 44 & 526 & 1 & 1.71 \\
\hline 8 & 6.19 & sulfate & 23.036 & 68 & 1209 & 1 & 1.75 \\
\hline 9 & 8.16 & oxalate & 13.137 & 57 & 1045 & 1 & 2.68 \\
\hline
\end{tabular}

Totals $\quad 2872.139 \quad 40739 \quad 375778$

File: 96031841.d12 Sample: S96T000691 DUP.

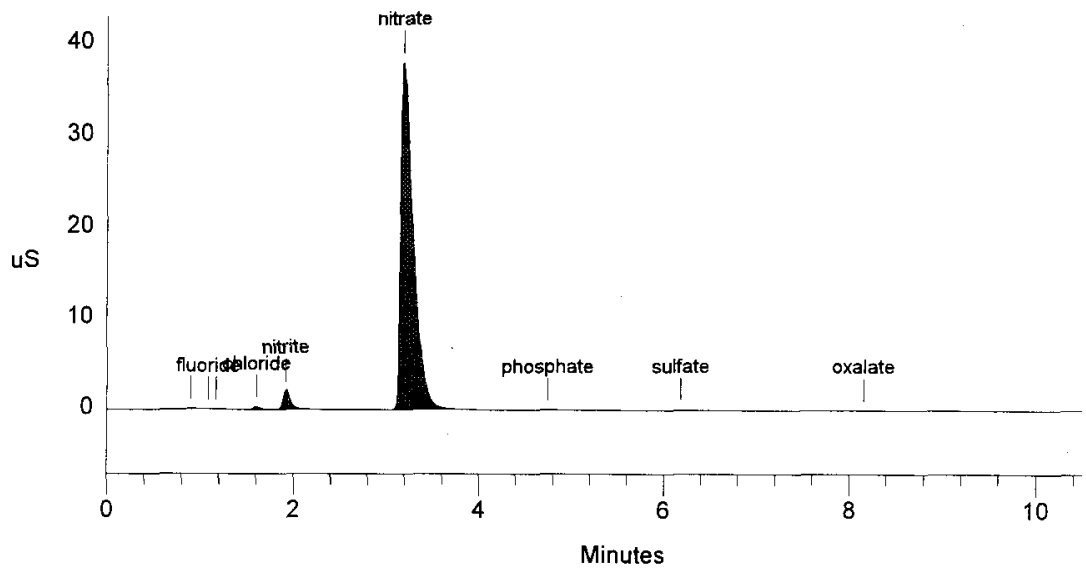




\section{LABCORE Data Entry Template for Worklist\# 6327}

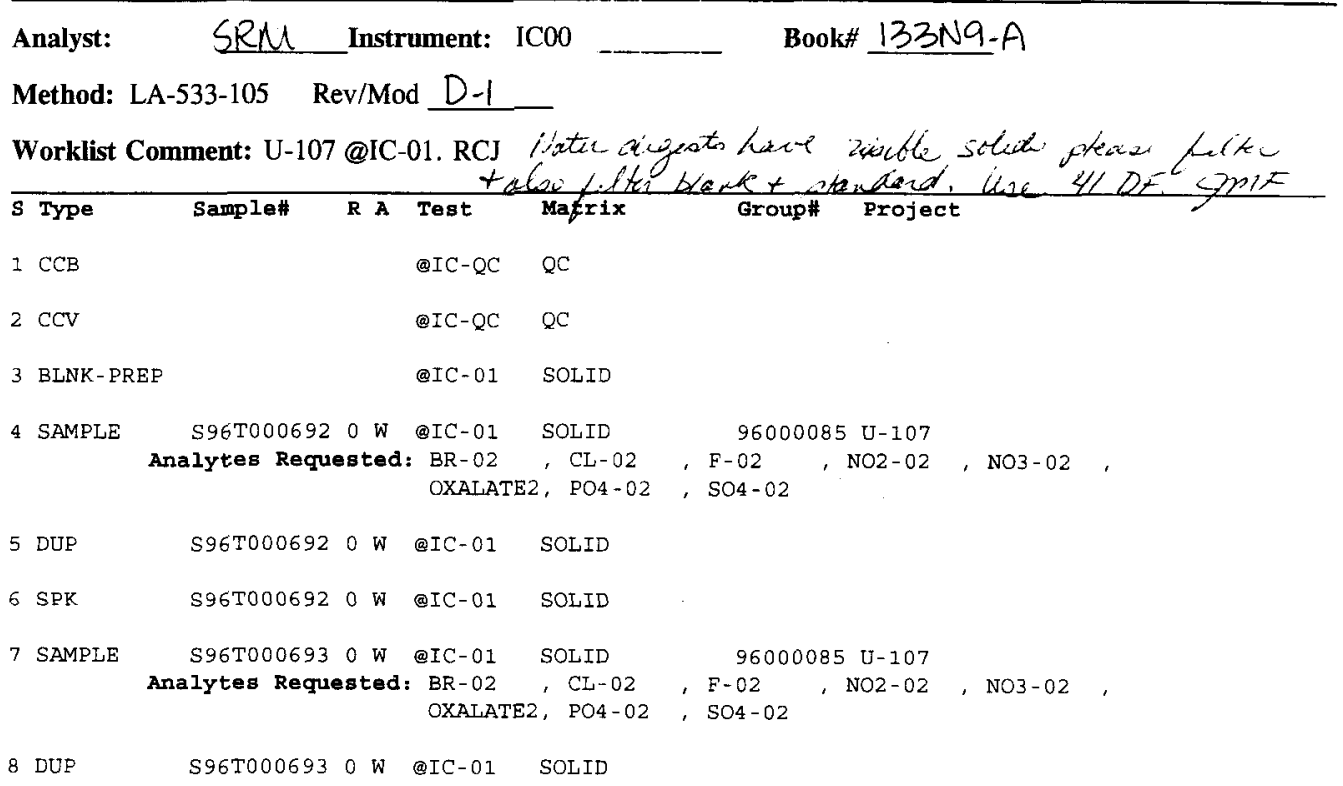

\section{Final page for worklist \# 6327}

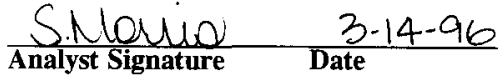

Exponted

Inshell

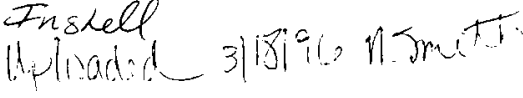

\section{Analyst Signature Date}

Data Entry Comments:

$S=$ Worklist Slot Number, $R=$ Replicate Number, $A=$ Aliquot Code. 


\section{LABCORE Completed Worklist Report for Worklist\# 6327}

Analyst: srm

Instrument: $\mathrm{IC} 01$

Method: $1 A-5,33-105 \operatorname{Rev} / \operatorname{Mod} D-1$

Book\# B3N9,A

Worklist Comment: U-107 @IC-01. RCJ

\begin{tabular}{|c|c|c|c|c|c|c|c|c|c|}
\hline Seq Type & Sample\# $\mathbf{F}$ & $A$ & & & Matrix & Actual & Found & DL or Yie & Unit \\
\hline $1 \mathrm{CCB}$ & & 0 & eIc-oc & 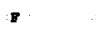 & $O C$ & 1 & $<1.300-2$ & & ug/m \\
\hline $1 \mathrm{CCB}$ & & 0 & -IC-QC & CL & oc & 1 & $<1.700-2$ & & ug/mf. \\
\hline $1 \mathrm{ces}$ & & 0 & $\operatorname{arc-oc}$ & s02 & ec & 1 & $<1.07 \bullet-1$ & & $\mathbf{u g} / \mathbf{m L}$ \\
\hline$I C C B$ & & 0 & IC-QC & $\mathbf{B R}$ & ec & 1 & $<1.260-1$ & & $\mathbf{u g} / \mathbf{m L}$ \\
\hline $1 \mathrm{CCB}$ & & 0 & -TC-QC & No3 & oc & $I$ & $<3.400-1$ & & ug $/ \mathrm{mz}$ \\
\hline $1 \mathrm{CCB}$ & & 0 & AIC-QC & PO4 & $Q c$ & 1 & $<1.190-1$ & & ug/mL \\
\hline $1 \mathrm{ccB}$ & & a & -IC-oc & sod & oe & 1 & $<1,36-1$ & & $4 \mathrm{~g} / \mathrm{mr}$ \\
\hline $1 \mathrm{CCB}$ & & 0 & aIc-Qc & OXALATE2 & 20 & 2 & $<1.050-1$ & & ug/mL \\
\hline $2 \mathrm{ecv}$ & & 0 & IC-gC & $\div$ & Qc & 59 & $6.010+01$ & 101.060 & o \& Ancovexy \\
\hline $2 \mathrm{ccV}$ & & 0 & arc-oc & cL & ec & 79 & $7.460+01$ & 94.430 & o \& Recovery \\
\hline $2 \operatorname{cov}$ & & 0 & -IC-gc & no2: & Qce & 537 & $5.390+02$ & 100.370 & 0 : Recovery \\
\hline $2 \mathrm{ecv}$ & & 0 & arc-oc & BR & ec & 575 & $5.760+02$ & 100.170 & o \& Recovery \\
\hline $2 \mathrm{ccv}$ & & 0 & aIC-OC & no3 & $\mathrm{gc}$ & 614 & $6.260+02$ & 101.630 & o t Rndovery \\
\hline $2 \mathrm{ccv}$ & & 0 & arc-gc & Po4 & $o$ & 546 & $5.460+02$ & 100.000 & o \& Recovery \\
\hline $2 \mathrm{ccv}$ & & 0 & orc-Qc & so4 & $\mathrm{ec}$ & 631 & $6: 390+02$ & 101.270 & o : Recovary \\
\hline $2 \mathrm{ccv}$ & & 0 & IC-QC & OXALATE2 & $2 \mathrm{Qc}$ & 509 & $5.450+02$ & 107.070 & o \& Recovery \\
\hline 3 BLNTK - PREF & & 0 & arc-03 & $=-02$ & soLID & 1 & $<1.30<-2$ & & $\mathrm{ug} / \mathrm{g}$ \\
\hline 3 BLRK-PREP & & 0 & -IC-01 & CL- 02 & SOLID & 1 & $6.100-02$ & $5.0000-002$ & $2 \mathrm{ug} / \mathrm{g}$ \\
\hline 3 BLNX-PREP & & 0 & eIC-01 & $402-02$ & SOLID & $\mathbf{1}$ & $<1.070-1$ & & ug/g \\
\hline 3 BLNR-PREP & & 0 & erc-01 & BR-02 & SOLID & 1 & $<1.260-1$ & & $\mathrm{ug} / \mathrm{g}$ \\
\hline 3 BLNK-PREP & & 0 & tec-01 & vos -02 & SOLID & 2 & $5.180-02$ & 0.520 & $4 g / g$ \\
\hline 3 BLSK - PREF & & 0 & erc-01 & $004-02$ & SOLID & $\mathbf{1}$ & $<1.200-1$ & & $\operatorname{ug} / \mathrm{g}$ \\
\hline 3 BLNK-PRBP & & 0 & exc-01 & so4-02 & SOZID & 1 & $<2.360-1$ & & ug $/ \mathrm{g}$ \\
\hline 3 BLANK-PREF & & 0 & -IC-01 & OXALATE2 & 2 SOLID & 1 & $<1.050-1$ & & $\mathrm{ug} / \mathrm{g}$ \\
\hline 4 SANPLI & S965000892 & $0 \%$ & eIC-01 & $F-02$ & SOLID & N/A/A & $6.5200+02$ & 161.300 & $4 g / g$ \\
\hline 4 SAMPLE & 3962000692 & ow & AIC-01 & CL- 02 & SOLID & $\mathbb{N} / \mathbf{A}$ & $2.2380+03$ & 210.900 & $\mathrm{ug} / \mathrm{g}$ \\
\hline + SAMPLI & $996 \mathrm{~T} 000692$ & $0 . W$ & $\operatorname{erc-01}$ & No2 -.02 & SOLID & N/A & $2.7800 * 04$ & $1.3280+003$ & ug/g \\
\hline 4 SAMPLE & 5967000692 & $0 \%$ & -re-01 & $8 R-02$ & SOLID & E/A & $1.563 \bullet+03$ & $1.5630+003$ & $\mathbf{u g} / g$ \\
\hline 4 SAKOLIX & 3967000692 & $0 w$ & $\operatorname{arc-01}$ & $1003-02$ & SOEID. & N/n & $5.6620+05$ & $1.736 \bullet+003$ & $\mathrm{ug} / \mathrm{g}$ \\
\hline 4 SAMPLE & S96T000692 & $0 \mathrm{~W}$ & arc-01 & PO4-02 & SOLID & E $/ A$ & $7.8610+03$ & $1.4880+003$ & $\mathrm{ug} / \mathrm{g}$ \\
\hline 4 SAMPLiR & 3967000692 & $0 \%$ & erc-01. & $504-02$ & SOIID & $\mathbb{N} / \mathrm{A}$ & $6.217 \bullet+03$ & $1.686 e+003$ & $\pm g / g$ \\
\hline 4 SAMPLE & S967000692 & OW & erc-01 & OXALATrs? & SOLID & $\mathbb{N} / \mathrm{A}$ & $1.959 \bullet+03$ & $1.303 *+003$ & $\mathrm{ug} / \mathrm{g}$ \\
\hline 5 DUP & 5967000692 & $0 \%$ & Ic-01 & $8-02$ & sosid & $6.520+02$ & $5.83 \bullet+02$ & 4.640 & $\mathbf{R P D}$ \\
\hline 5 DUP & 5967000692 & ow & erc-01 & CL-02 & SOLTD & 2.240403 & $2.66 \bullet \bullet 03$ & 17.140 & $R P D$ \\
\hline 5 DUP & $\$ 967000692$ & $0 \%$ & eIc-01 & N02-02 & SOIID & $2.780+04$ & $3.18 \bullet+04$ & 13.420 & RPD \\
\hline $5 \mathrm{DUP}$ & S96T000692 & ow & IC-01 & $B R-02$ & SOLTD & $<1.5603$ & $<1.59 .3$ & & RPD \\
\hline $5 \mathrm{DUP}$ & $s 967000692$ & ow & erc-01 & $x 03-02$ & SOLID & $5.660+05$ & $5.270+05$ & 7.340 & npo \\
\hline 5 DOP & S96T000692 & OW & arc-01 & PO4-02 & SOLID & $7.860+03$ & $8.48 \bullet+03$ & 7.590 & $R R D$ \\
\hline 5 DOP & s967000692 & O w & erc-01 & $304-02$ & SOLID & $6.22 \theta+03$ & $6.410+03$ & 3.010 & RPD \\
\hline 5 DUP & $s 967000692$ & o w & erc-01 & OXALATB 2 & SOLID & $1.960+03$ & $<1.33 .3$ & & RPD \\
\hline $6 \operatorname{SPK}$ & 5967000692 & 0 พ & IC-01 & $8-02$ & soIIt & 59 & 60.212 & 102.050 & Recovery \\
\hline
\end{tabular}

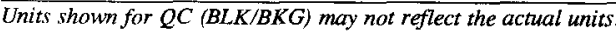




\section{LABCORE Completed Worklist Report for Worklist\# 6327}

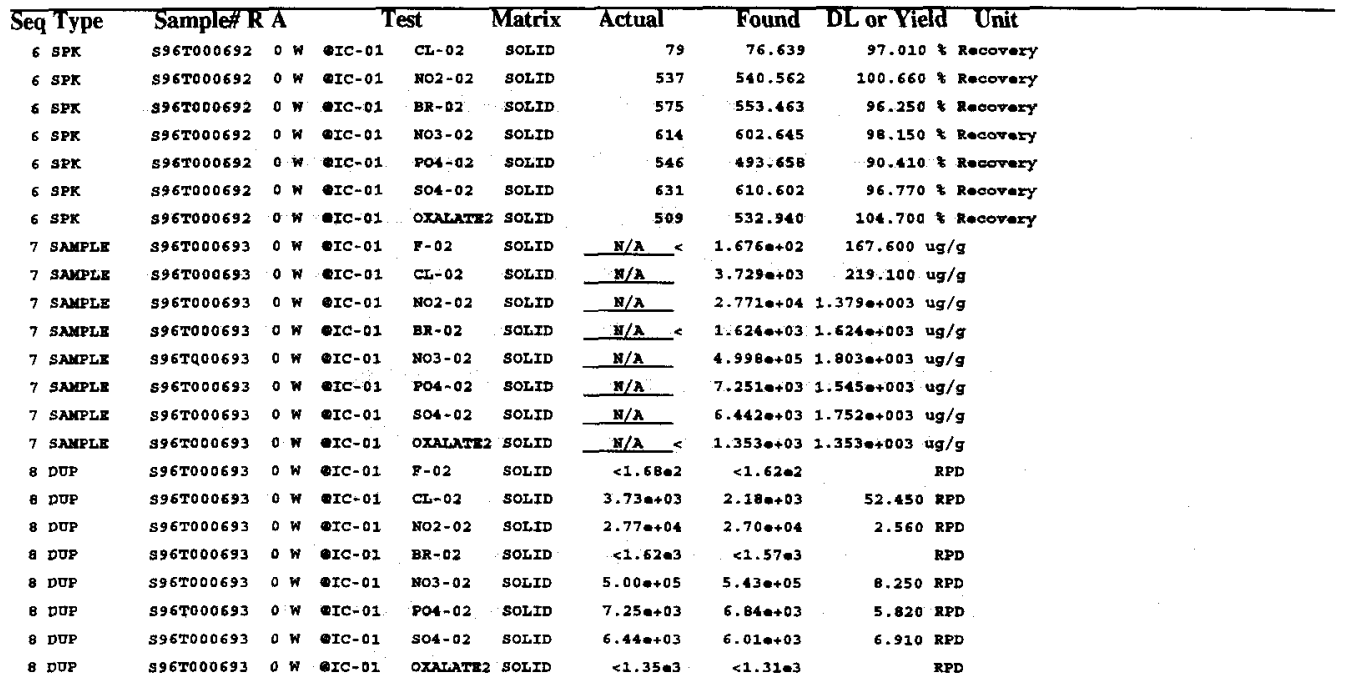

\section{Final page for worklist\# 6327}

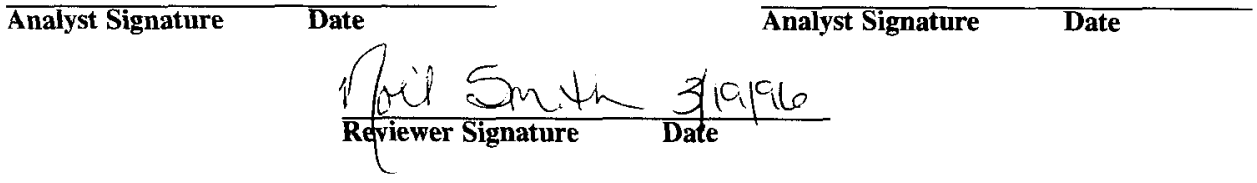


WHC-SD-WM-DP-184, REV. |

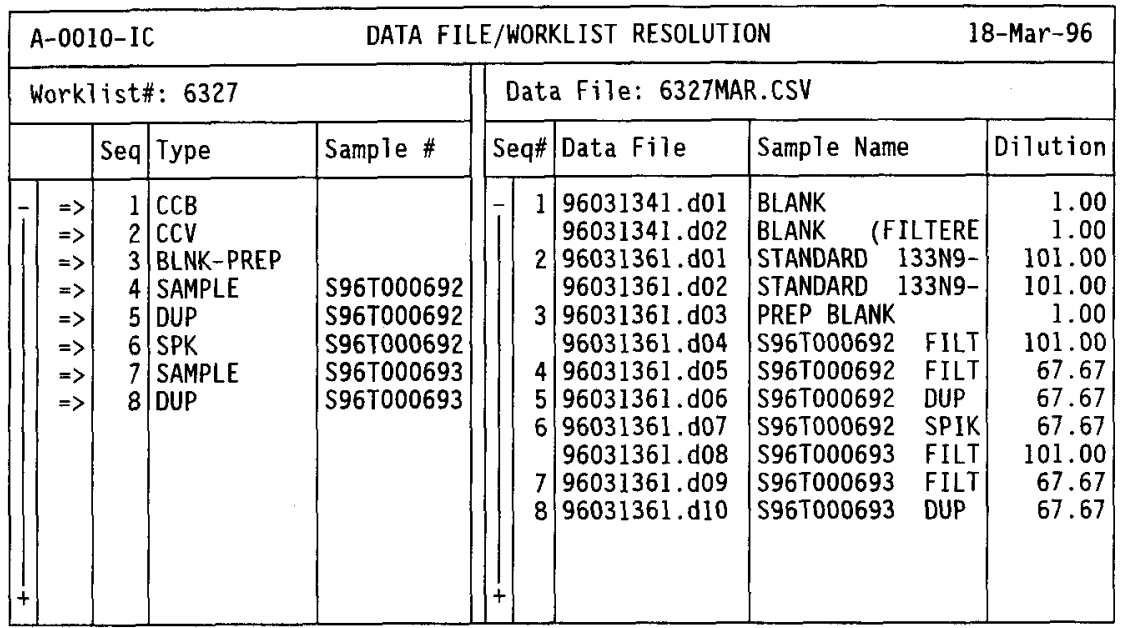

Save(F4) Abort(Shift-F3) ListFiles(Shift-F1) UploadFile(F8) 
Data Reprocessed On 03/15/1996 09:41:45

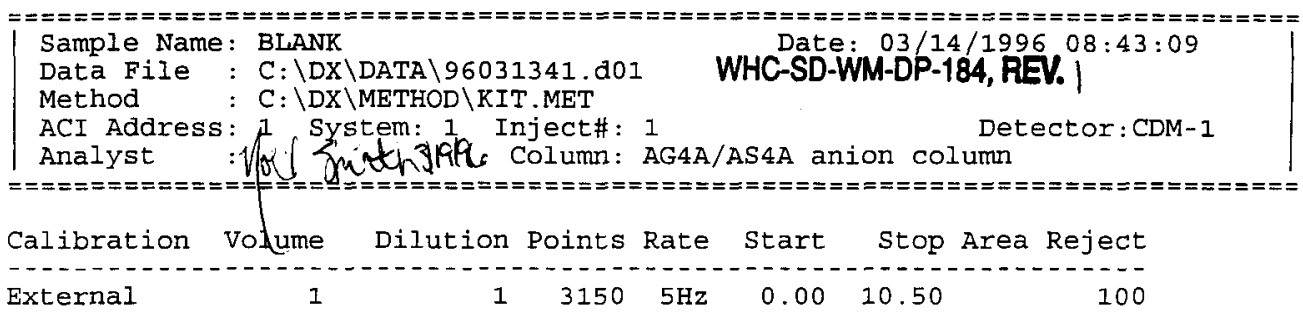

$\begin{array}{lrrr}\text { Pk. Ret Component } & \text { Concentration } & \text { Height } & \text { Area } / \mathrm{ml} \\ \text { Num } & \text { Time Name } & \text { Code } & \end{array}$

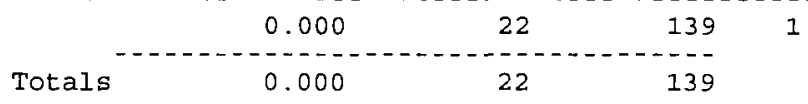

File: 96031341.d01 Sample: BLANK

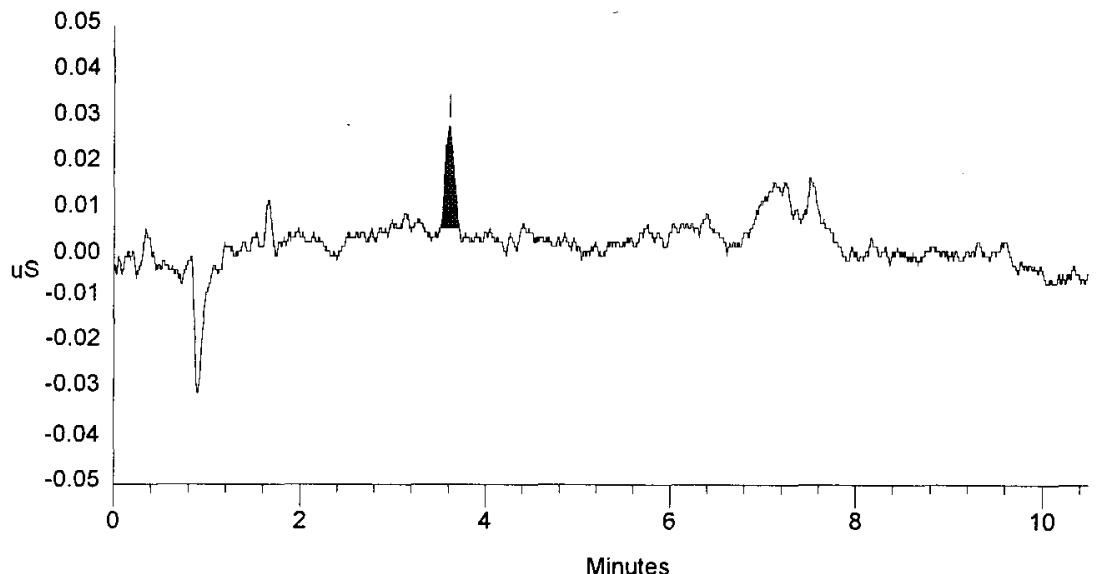

SIGNATURE ABOVE REPRESENTS CHEMICAL TECHNOLOGIST/CHEMIST THAT COMPLETED/VERIFIED THE CALIBRATION/ANALYSIS ON PAGES 262 T0 273. 
Data Reprocessed on 03/15/1996 09:41:48

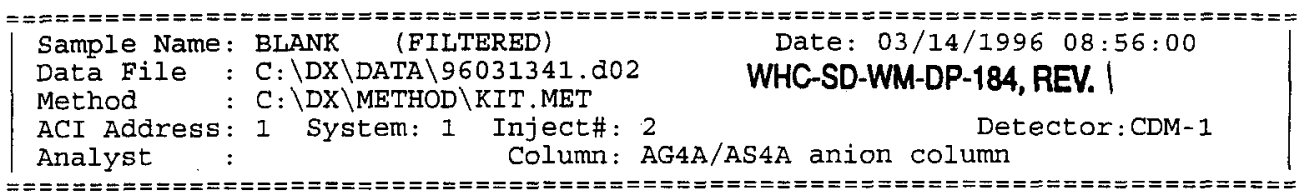

Calibration Volume Dilution Points Rate start stop Area Reject

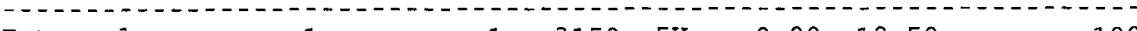

Externa1

1

$13150 \quad 5 \mathrm{~Hz} \quad 0.00 \quad 10.50$

100

Peak Report: All Peaks

Pk. Ret Component

Concentration

Height

Area Bl. \%Delta

Num Time Name $\mathrm{ug} / \mathrm{ml}$ Code

11.19

21.65 chloride

0.000

23

0.022

28

145

112

1

13.33

Totals

0.022

51

257

File: 96031341.d02 Sample: BLANK (FILTERED)

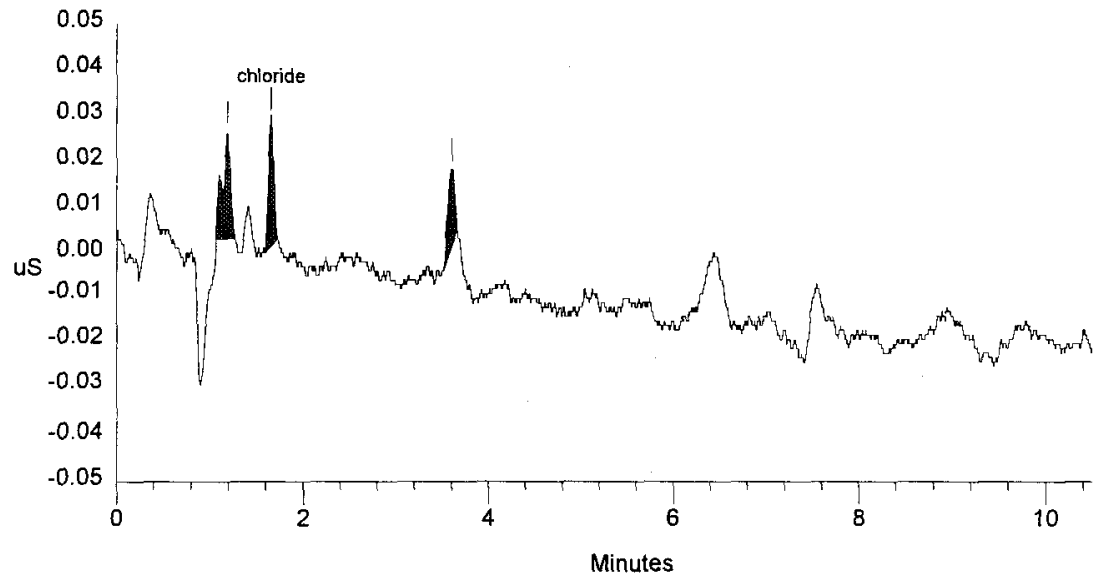


Data Reprocessed on 03/15/1996 09:41:50

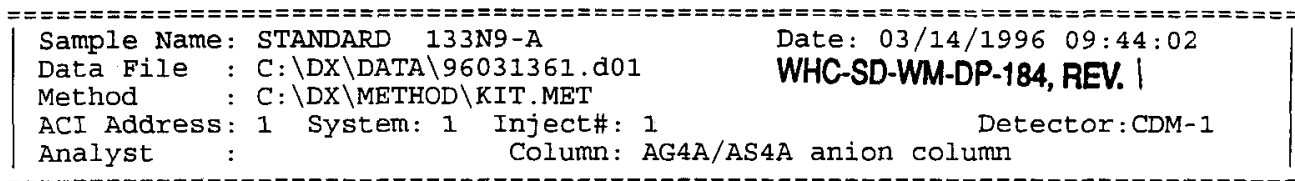

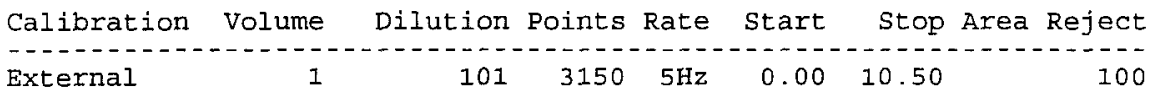

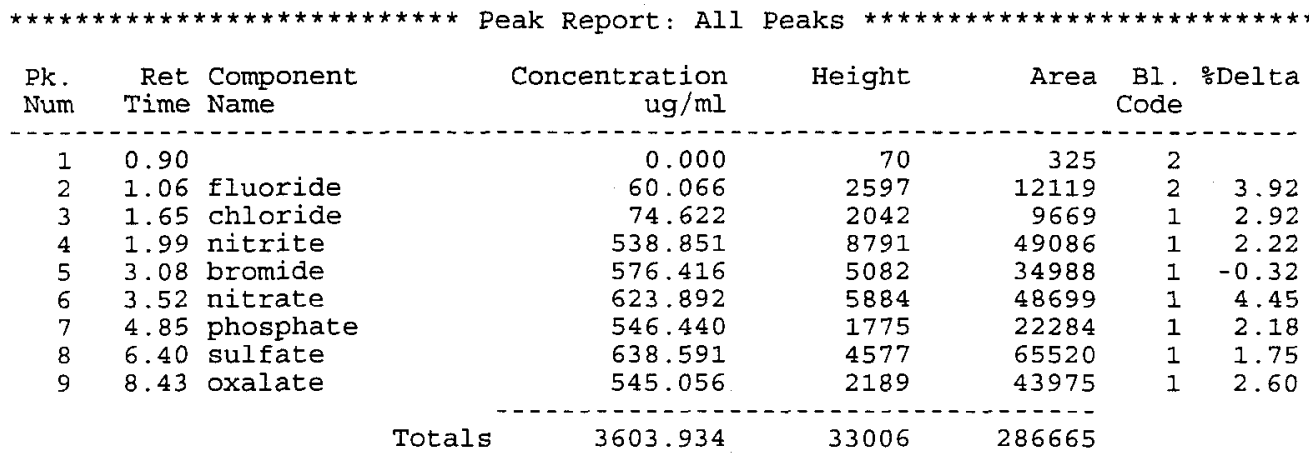

File: 96031361.d01 Sample: STANDARD 133N9-A

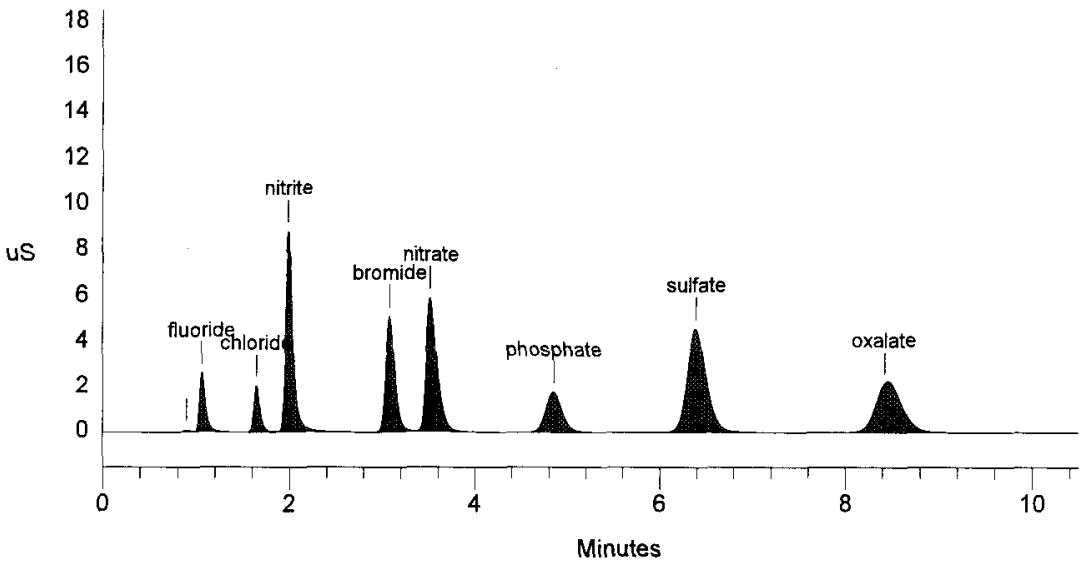


Data Reprocessed On 03/15/1996 09:41:53

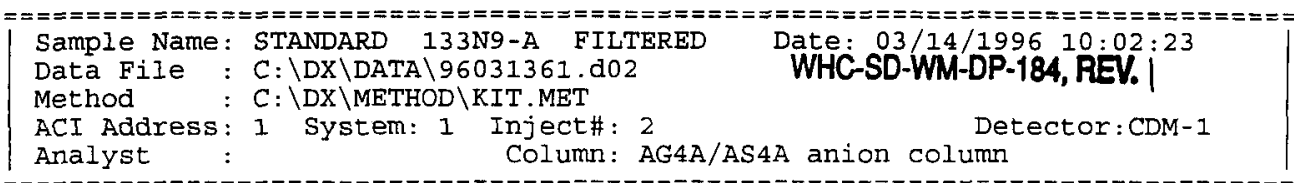

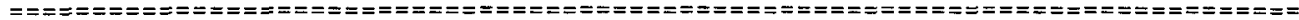

Calibration Volume Dilution Points Rate start stop Area Reject

Calibration

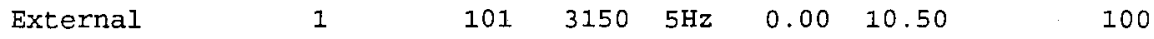

Peak Report: All Peaks

Pk. Ret Component Concentration Height Area Bl. \%Delta

Num Time Name ug/ml

$\begin{array}{rrlrrrr}1 & 0.90 & 0.000 & 77 & 354 & 2 \\ 2 & 1.06 \text { fluoride } & 60.081 & 2597 & 12123 & 2 & 3.92 \\ 3 & 1.42 & 0.000 & 39 & 252 & 2 & \\ 4 & 1.65 \text { chloride } & 74.641 & 2096 & 9671 & 1 & 2.92 \\ 5 & 1.99 \text { nitrite } & 534.752 & 8866 & 48683 & 1 & 2.22 \\ 6 & 3.07 \text { bromide } & 566.576 & 5064 & 34345 & 1 & -0.54 \\ 7 & 3.51 \text { nitrate } & 642.662 & 6070 & 50287 & 1 & 4.25 \\ 8 & 4.85 \text { phosphate } & 541.101 & 1763 & 22044 & 1 & 2.18 \\ 9 & 6.35 \text { sulfate } & 636.194 & 3834 & 65256 & 1 & 0.90 \\ 10 & 8.43 \text { oxalate } & 543.141 & 2176 & 43816 & 1 & 2.60 \\ & & \text { Totals } & 3599.149 & 32582 & 286830 & \end{array}$

File: 96031361.d02 Sample: STANDARD 133N9-A FILTERED

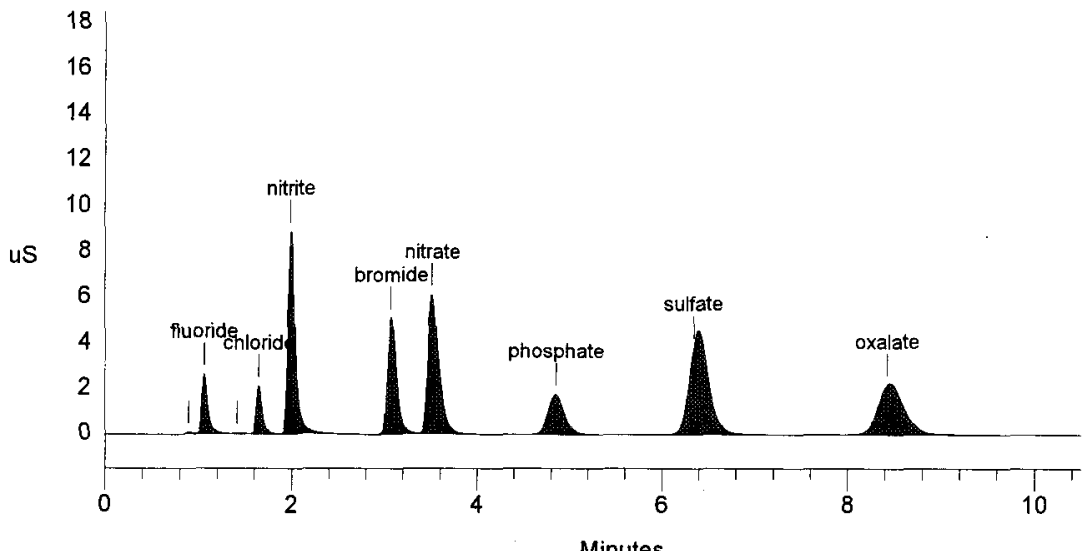

Minutes 
Data Reprocessed On 03/15/1996 09:41:56

\begin{tabular}{|c|c|c|}
\hline Sample Name & PREP BLANK & Date: 03/14/1996 10:17:37 \\
\hline $\begin{array}{l}\text { Data File } \\
\text { Method }\end{array}$ & $\begin{array}{l}C: \backslash D X \backslash D A T A \backslash 96031361 . d 03 \\
C: \backslash D X \backslash M E T H O D \backslash R I T . M E T\end{array}$ & WHC-SD-WM-DP-184, REV. I \\
\hline $\begin{array}{l}\text { ACI Address } \\
\text { Analyst }\end{array}$ & 1 System: $1 \begin{array}{r}\text { Inject\#: } 3 \\
\text { Column: } A\end{array}$ & $\begin{array}{l}\text { Detector: CDM-1 } \\
1 \mathrm{~A} \text { anion column }\end{array}$ \\
\hline
\end{tabular}

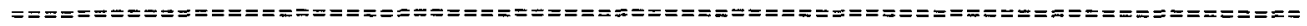

Calibration Volume Dilution Points Rate start Stop Area Reject

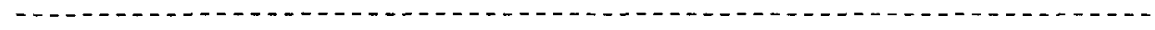

$\begin{array}{llllllll}\text { External } & 1 & 1 & 3150 & 5 \mathrm{~Hz} & 0.00 & 10.50 & 100\end{array}$

\begin{tabular}{|c|c|c|c|c|c|c|c|}
\hline $\begin{array}{l}\text { Pk. } \\
\text { Num }\end{array}$ & $\begin{array}{r}\text { Ret } \\
\text { Time }\end{array}$ & $\begin{array}{l}\text { Component } \\
\text { Name }\end{array}$ & $\begin{array}{r}\text { Concentration } \\
\mathrm{ug} / \mathrm{ml}\end{array}$ & Height & Area & Bl. & :Delta \\
\hline----- & ---- & & & & --- & $\ldots--$ & \\
\hline 1 & 1.34 & & 0.000 & 58 & 552 & 1 & \\
\hline 2 & 1.66 & chloride & 0.061 & 97 & 619 & 1 & 3.75 \\
\hline \multirow[t]{2}{*}{3} & 3.57 & nitrate & 0.518 & 144 & 1070 & 1 & 6.03 \\
\hline & & & 0.578 & 299 & 2241 & & \\
\hline
\end{tabular}

File: 96031361.d03 Sample: PREP BLANK

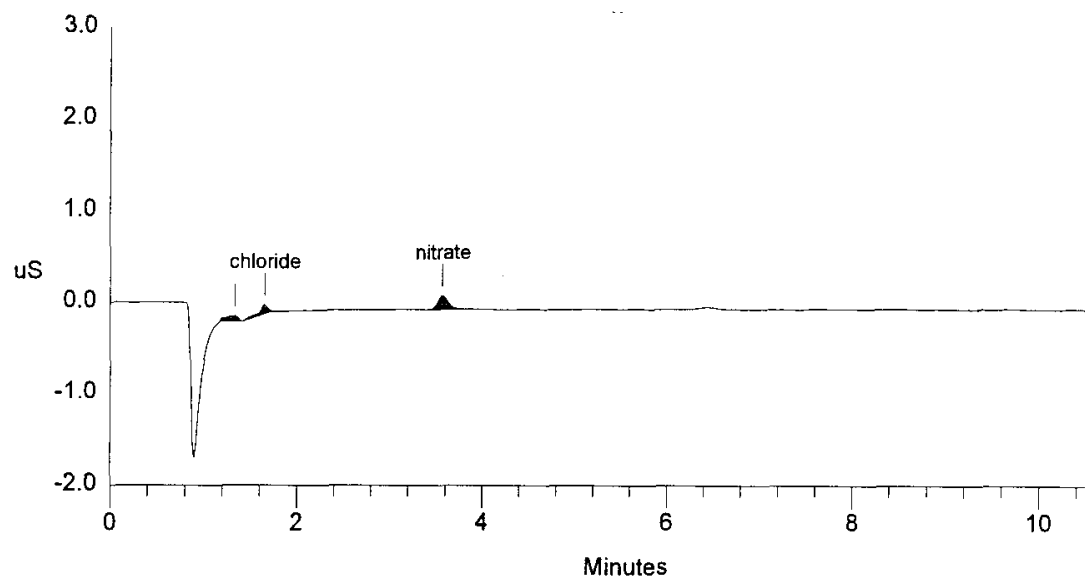


Data Reprocessed on 03/15/1996 09:41:58

\begin{tabular}{|c|c|c|}
\hline $\begin{array}{l}\text { Sample Name } \\
\text { Data File } \\
\text { Method }\end{array}$ & $\begin{array}{l}\text { S96T000692 } \text { FILTERED } \\
C: \backslash \mathrm{DX} \backslash \mathrm{DATA} \backslash 96031361 . \mathrm{d04} \\
\mathrm{C}: \backslash \mathrm{DX} \backslash \mathrm{METHOD} \backslash \mathrm{KIT} . \mathrm{MET}\end{array}$ & $\begin{array}{l}\text { Date: 03/14/1996 13:28:05 } \\
\text { WHC-SD-WM-DP-184, REV. I }\end{array}$ \\
\hline $\begin{array}{l}\text { ACI Address } \\
\text { Analyst }\end{array}$ & $\begin{array}{c}\text { System: } 1 \text { Inject\# : } \\
\text { Column: } ?\end{array}$ & $\begin{array}{l}\text { Detector: CDM-1 } \\
1 \text { A anion column }\end{array}$ \\
\hline
\end{tabular}

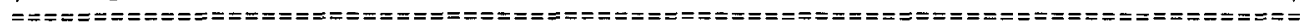

Calibration Volume Dilution Points Rate start stop Area Reject

Calibration

$\begin{array}{llllllll}\text { External } & 1 & 101 & 3150 & 5 \mathrm{~Hz} & 0.00 & 10.50 & 100\end{array}$

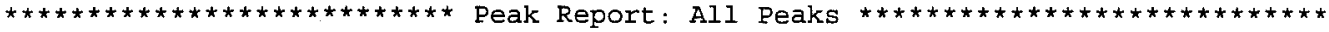

Pk. Ret Component Concentration Height Area Bl. \%Delta

Num Time Name $\mathrm{ug} / \mathrm{ml}$ Code

$\begin{array}{lll}1 & 0.89 & \\ 2 & 1.09 & \text { fluoride } \\ 3 & 1.18 & \\ 4 & 1.63 & \text { chloride } \\ 5 & 1.97 & \text { nitrite } \\ 6 & 3.34 & \text { nitrate } \\ 7 & 4.85 & \text { phosphate } \\ 8 & 6.40 & \text { sulfate }\end{array}$

$\begin{array}{rrrr}0.000 & 72 & 343 \\ 4.936 & 44 & 205 \\ 0.000 & 41 & 135 \\ 12.800 & 332 & 1488 \\ 163.198 & 2262 & 12260 \\ & 3226.608 & 29855 & 285700 \\ & 57.776 & 44 & 513 \\ \text { Totals } & 46.632 & 79 & 1296 \\ & 3511.949 & 32729 & 301939\end{array}$

File: 96031361.d04 Sample: S96T000692 FILTERED

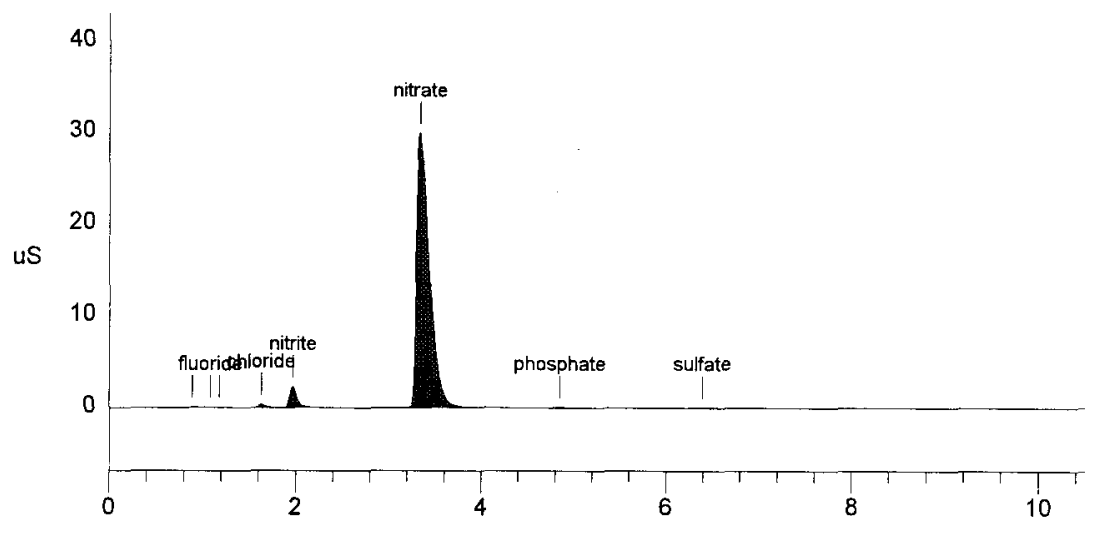

Minutes 
Data Reprocessed on 03/15/1996 09:42:01

\begin{tabular}{|c|c|c|c|}
\hline $\begin{array}{l}\text { Sample Name: } \\
\text { Data File: } \\
\text { Method : }\end{array}$ & $\begin{array}{l}S 96 T 000692 \\
C: \backslash D X \backslash D A T A \backslash S \\
C: \backslash D X \backslash M E T H O I\end{array}$ & $\begin{array}{l}\text { FILTERED } \\
96031361 . \mathrm{d} 05 \\
\mathrm{D} \backslash \mathrm{KIT} . \mathrm{MET}\end{array}$ & $\begin{array}{c}\text { Date: } 03 / 14 / 1996 \quad 13: 43: 21 \\
\text { WHC-SD-WM-DP-184, REV. I }\end{array}$ \\
\hline $\begin{array}{l}\text { ACI Address: } \\
\text { Analyst : }\end{array}$ & 1 System: 1 & 1 Inject\# : 5 & $\begin{array}{l}\text { Detector: CDM-1 } \\
4 \mathrm{~A} \text { anion column }\end{array}$ \\
\hline
\end{tabular}

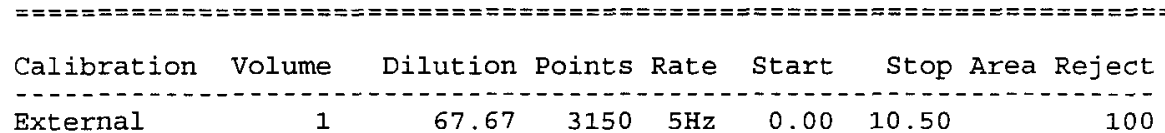

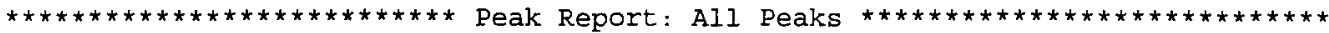

Pk. Ret Component Concentration Height Area Bl. $\div$ Delta

Num Time Name $\mathrm{ug} / \mathrm{ml}$

$\begin{array}{lll}1 & 0.90 & \\ 2 & 1.09 & \text { fluoride } \\ 3 & 1.19 & \\ 4 & 1.63 & \text { chloride } \\ 5 & 1.97 & \text { nitrite } \\ 6 & 3.29 & \text { nitrate } \\ 7 & 4.85 & \text { phosphate } \\ 8 & 6.40 & \text { sulfate } \\ 9 & 8.48 & \text { oxalate }\end{array}$

$\begin{array}{rr}0.000 & 108 \\ 3.556 & 58 \\ 0.000 & 60 \\ 12.204 & 467 \\ 151.601 & 3305 \\ 3088.209 & 42083 \\ 42.873 & 67 \\ 33.905 & 109 \\ 10.683 & 20\end{array}$

108

58

60

467

3305

2083

67

109

20 Code

$517 \quad 2$

2842

2042

$2197 \quad 1$

18426

$429457 \quad 1$

787

1721

350
6.54

2.08

0.85

$-2.27$

2.18

1. 75

3. 25

Totals

3343.030

46277

453942

File: 96031361.d05 Sample: S96T000692 FILTERED

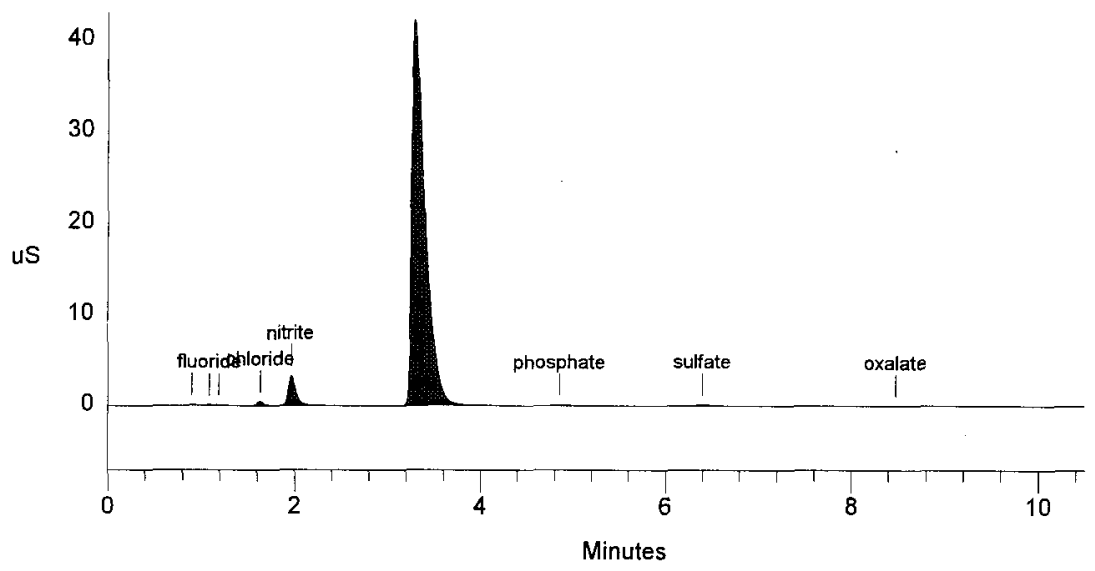


Data Reprocessed On 03/15/1996 09:42:03

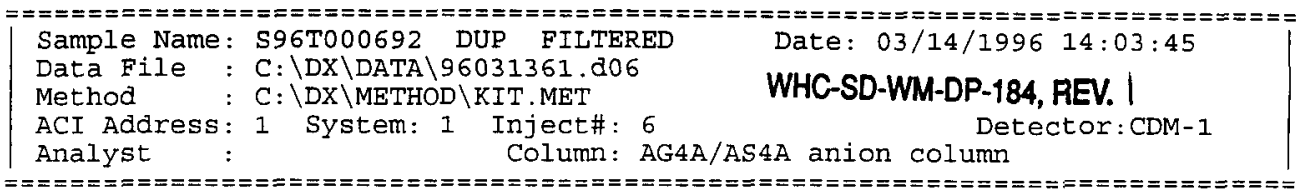

Calibration Volume Dilution Points Rate Start Stop Area Reject

Con

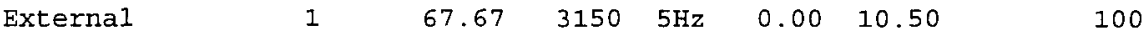

\begin{tabular}{|c|c|c|c|c|c|c|c|}
\hline $\begin{array}{l}\text { Pk. } \\
\text { Num }\end{array}$ & $\begin{array}{l}\text { Ret } \\
\text { Time }\end{array}$ & $\begin{array}{l}\text { Component } \\
\text { Name }\end{array}$ & $\begin{array}{r}\text { Concentration } \\
\mathrm{ug} / \mathrm{ml}\end{array}$ & Height & Area & $\begin{array}{l}\text { Bl. } \\
\text { Code }\end{array}$ & $\because$ Delta \\
\hline & ---- & & & & & & \\
\hline 1 & 0.90 & & 0.000 & 103 & 495 & 1 & \\
\hline 2 & 1.09 & fluoride & 3.660 & 65 & 317 & 2 & 7.19 \\
\hline 3 & 1.18 & & 0.000 & 71 & 239 & 2 & \\
\hline 4 & 1.63 & chloride & 14.257 & 551 & 2599 & 1 & 1.67 \\
\hline 5 & 1.97 & nitrite & 170.569 & 3851 & 21195 & 1 & 0.85 \\
\hline 6 & 3.29 & nitrate & 2822.239 & 38500 & 386572 & 1 & -2.27 \\
\hline 7 & 4.85 & phosphate & 45.429 & 79 & 956 & 1 & 2.18 \\
\hline 8 & 6.40 & sulfate & 34.348 & 125 & 1791 & 1 & 1.75 \\
\hline
\end{tabular}

Totals $\quad 3090.503 \quad 43344 \quad 414164$

File: 96031361.d06 Sample: S96T000692 DUP FILTERED

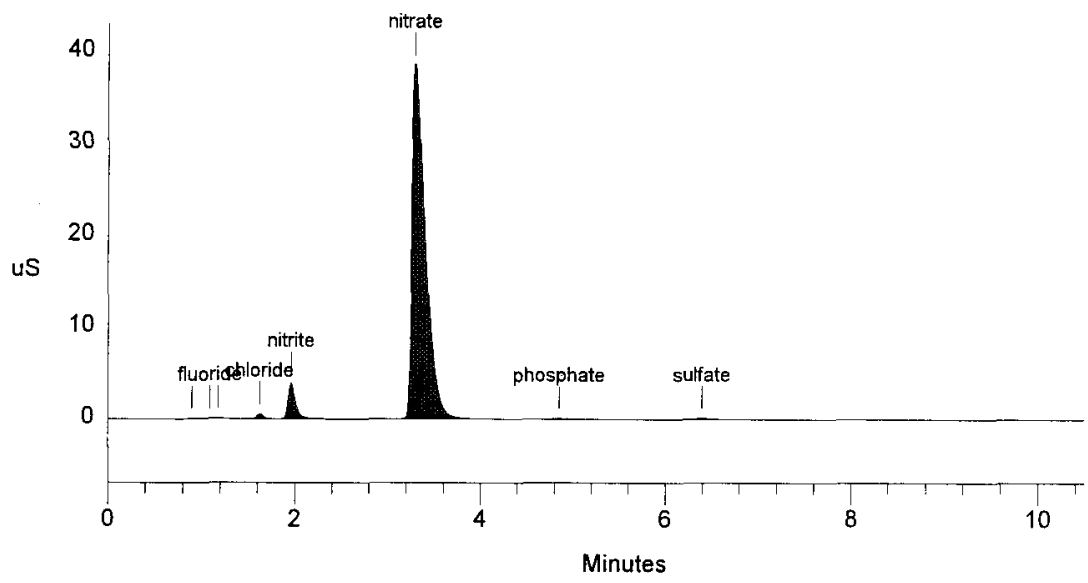


Data Reprocessed On 03/15/1996 09:42:06

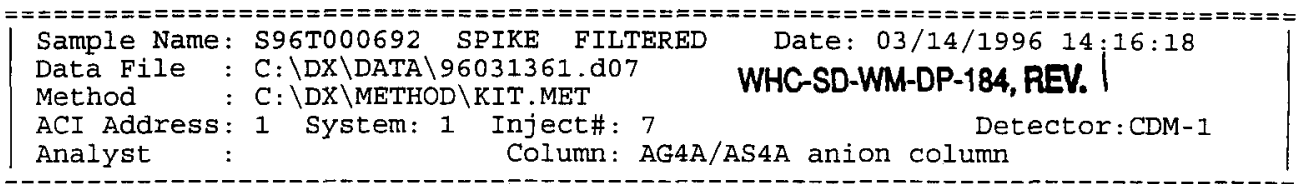

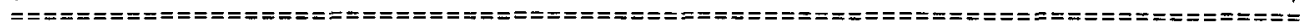

Calibration Volume Dilution Points Rate start stop Area Reject

Cal-

$\begin{array}{llllllll}\text { External } & 1 & 67.67 & 3150 & 5 \mathrm{~Hz} & 0.00 & 10.50 & 100\end{array}$

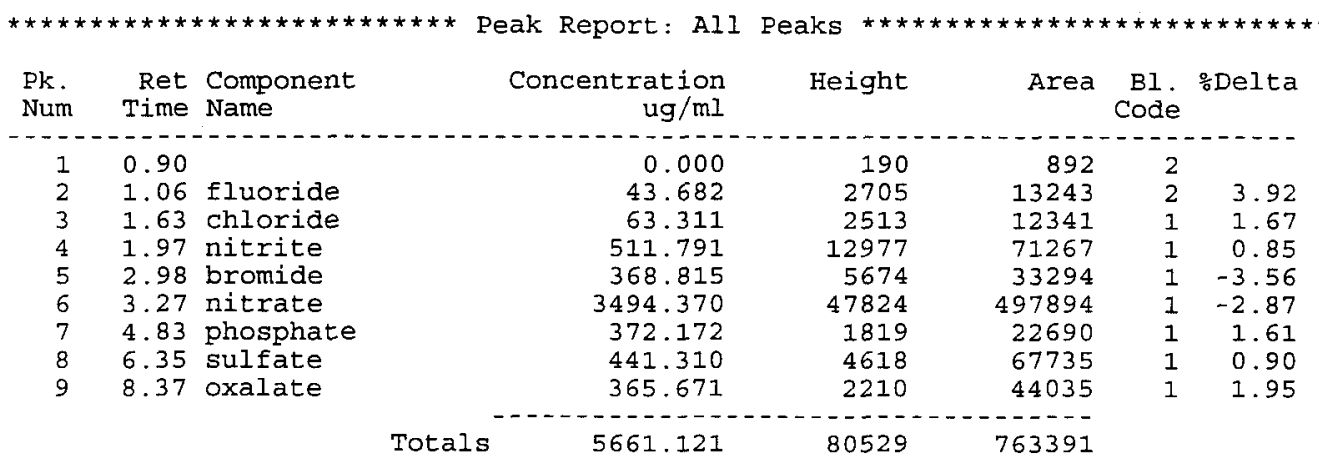

File: 96031361.d07 Sample: S96T000692 SPIKE FILTERED

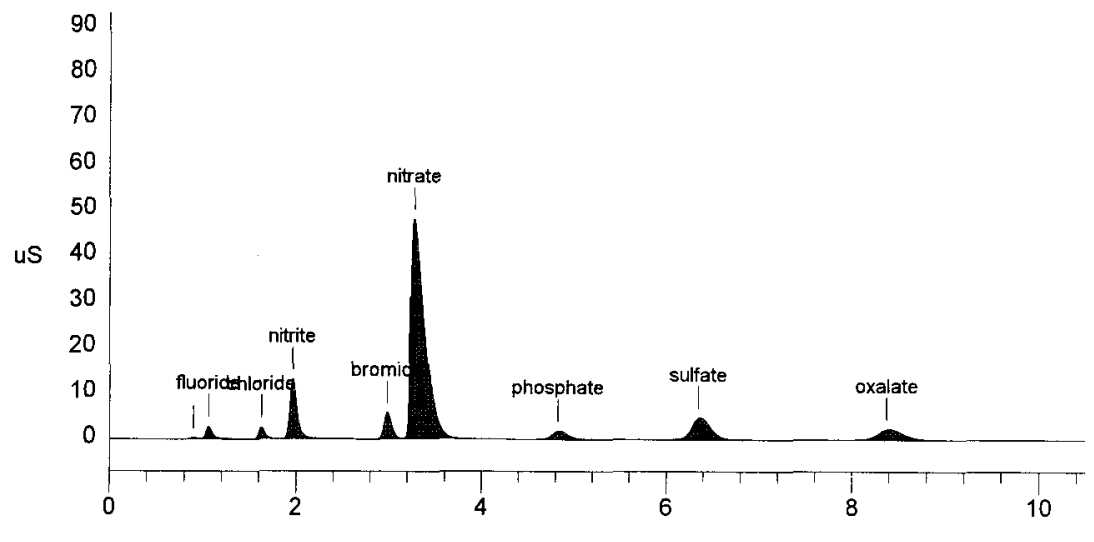

Minutes 
Data Reprocessed on 03/15/1996 09:42:09

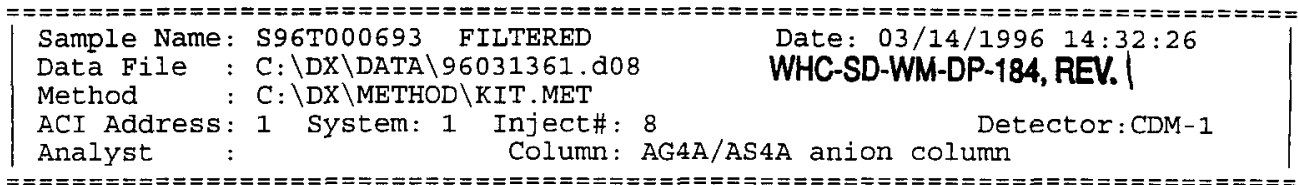

Calibration Volume Dilution Points Rate start stop Area Reject

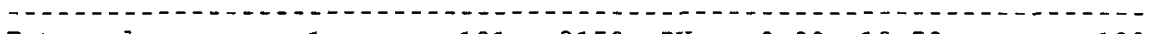

$\begin{array}{llllllll}\text { External } & 1 & 101 & 3150 & 5 \mathrm{~Hz} & 0.00 & 10.50 & 100\end{array}$

\begin{tabular}{|c|c|c|c|c|c|c|c|}
\hline $\begin{array}{l}\text { Pk. } \\
\text { Num. }\end{array}$ & $\begin{array}{l}\text { Ret } \\
\text { Time }\end{array}$ & $\begin{array}{l}\text { Component } \\
\text { Name }\end{array}$ & $\begin{array}{r}\text { Concentration } \\
\mathrm{ug} / \mathrm{ml}\end{array}$ & Height & Area & $\begin{array}{l}\text { Bl. } \\
\text { Code }\end{array}$ & $\div$ Delta \\
\hline 1 & 0.91 & & 0.000 & 50 & 292 & 1 & \\
\hline 2 & 1.18 & & 0.000 & 33 & 181 & 1 & \\
\hline 3 & 1.63 & chloride & 11.472 & 291 & 1315 & 1 & 2.08 \\
\hline 4 & 1.97 & nitrite & 143.973 & 1921 & 10382 & 1 & 0.85 \\
\hline 5 & 3.34 & nitrate & 2356.289 & 22483 & 202270 & 1 & -0.89 \\
\hline 6 & 6.40 & sulfate & 43.787 & 69 & 992 & 1 & 1.75 \\
\hline
\end{tabular}

Totals $2555.521 \quad 24847 \quad 215433$

File: 96031361.d08 Sample: S96T000693 FILTERED

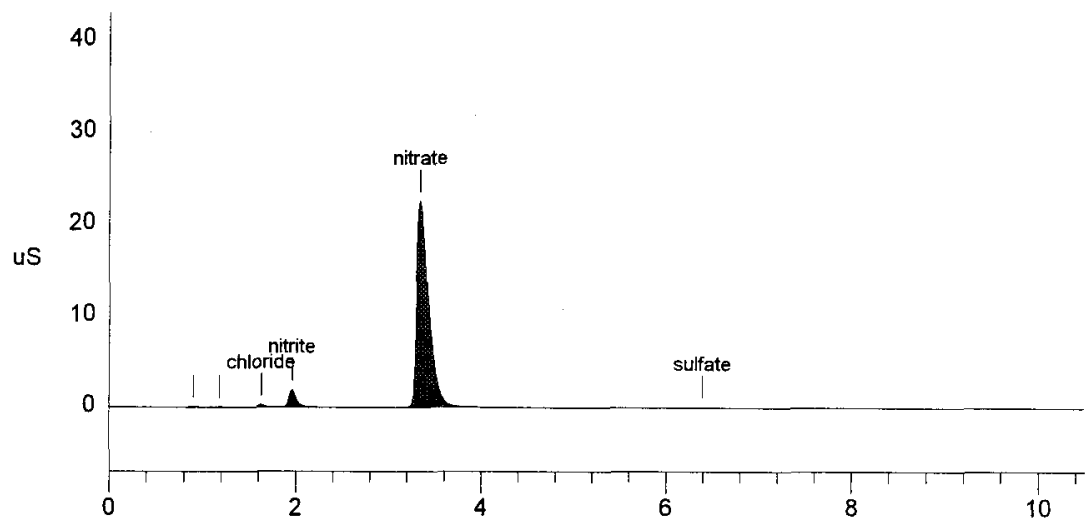

Minutes 
Data Reprocessed on 03/15/1996 09:42:11

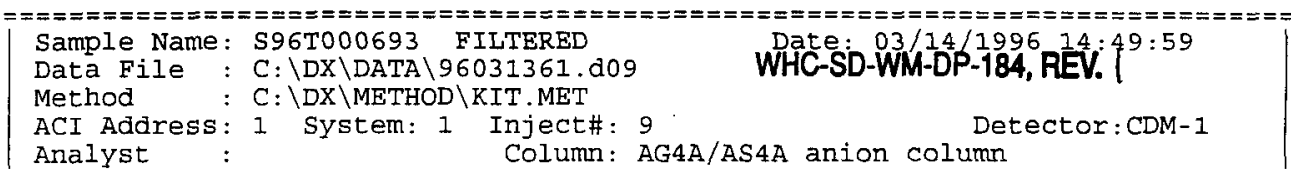

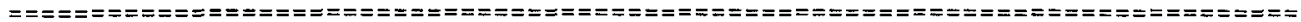

Calibration Volume Dilution Points Rate Start stop Area Reject

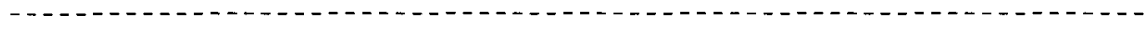

$\begin{array}{llllllll}\text { External } & 1 & 67.67 & 3150 & 5 \mathrm{~Hz} & 0.00 & 10.50 & 100\end{array}$

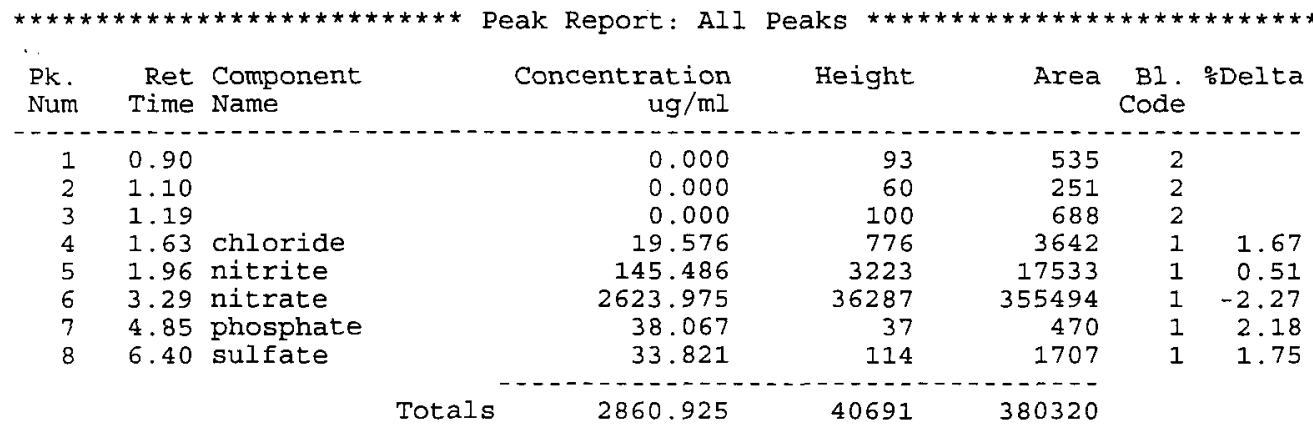

File: 96031361.d09 Sample: S96T000693 FILTERED

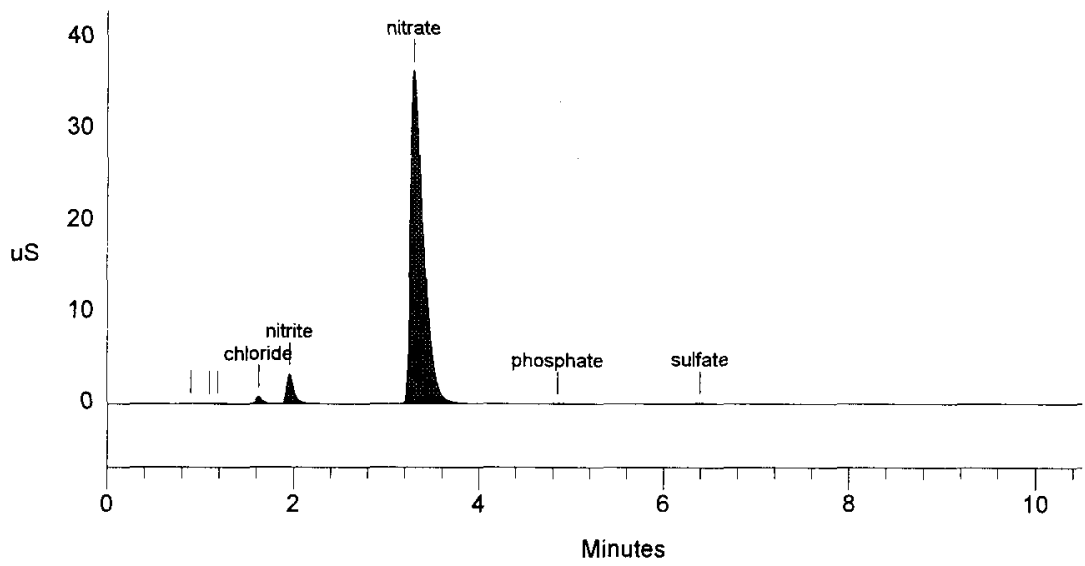


Data Reprocessed on 03/15/1996 09:42:14

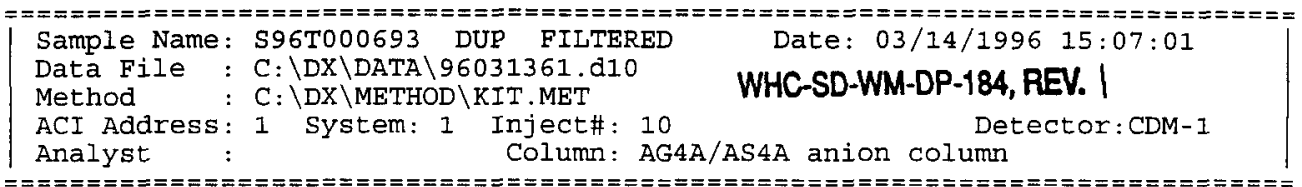

Calibration Volume Dilution Points Rate start stop Area Reject

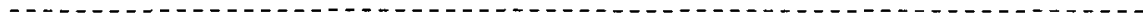

External

1

$67.67 \quad 3150 \quad 5 \mathrm{~Hz}$

$0.00 \quad 10.50$

100

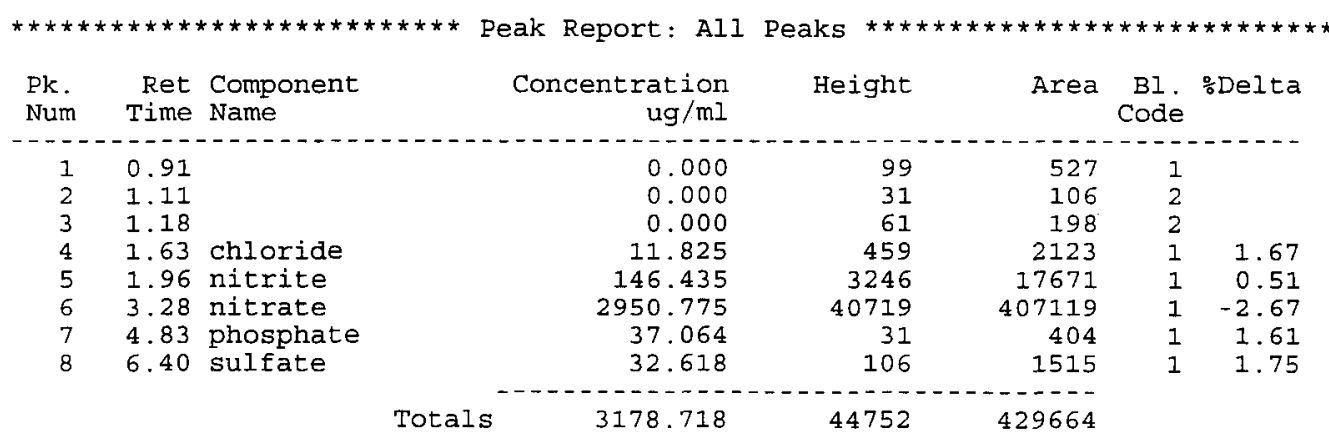

File: 96031361.d10 Sample: S96T000693 DUP FILTERED

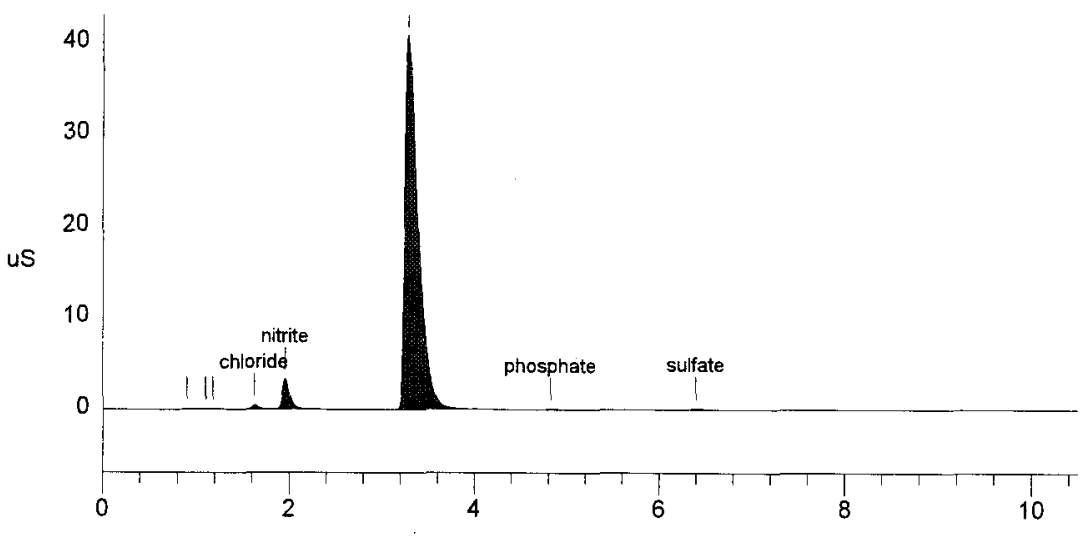

Minutes 


\section{LABCORE Data Entry Template for Worklist\# 6622}

Analyst: $\quad$ KRQQ Instrument: IC00 Book\# $133 N 9-C$

Method: LA-533-105 Rev/Mod D-/

Worklist Comment: U-107 IC. nds

S Type Sample\# R A Test Matrix Group\# Project

$1 \mathrm{CCB} \quad$ QIC-QC QC

$2 \mathrm{CCV} \quad$ @IC-QC QC

3 SAMPLE S96T000682 $0 \quad$ @IC-01 LIQUID 96000085 U-107

Analytes Requested: BR-02, CL-02, F-02, NO2-02, NO3-02,

4 DUP $\quad \$ 96 T 0006820 \quad @ I C-01 \quad$ LIQUID

5 SPK S96T000682 0 @IC-01 LIQUID

6 SAMPLE S96T000665 O OIC-01 LIQUID 96000085 U-107 Analytes Requested: BR-02, CL-02, F-02, $\mathrm{NO2}-02, \mathrm{NO}-02$, OXALATE2, PO4-02, SO4-02

7 DUP S96T000665 $0 \quad @ I C-01 \quad$ LIQUID

\section{Final page for worklist \# 6622}

$\rightarrow$

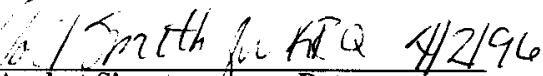
Analyst Signature $($ Date Thice

\section{Analyst Signature Date}

Data Entry Comments: 


\section{LABCORE Completed Worklist Report for Worklist\# 6622}

Analyst: krq

Instrument: ICO1

Book\#, 3 3NAC

Method: $E A-533 / 05 \operatorname{Rev} / \operatorname{Mod} D 1$

Worklist Comment: U-107 IC. nds

\begin{tabular}{|c|c|c|c|c|c|c|c|c|c|}
\hline$\overline{\text { Seq Type }}$ & Sample\# $\mathbf{F}$ & & & & Matrix & Actual & Found & DL or Yield & Unit \\
\hline $1 \operatorname{ccs}$ & & 0 & aIC-ge & $\mathbf{F}$ & $Q c$ & 1 & $<1.300-2$ & & ug/mi \\
\hline $1 \operatorname{ccB}$ & & 0 & -re-pe & cr. & oc & 1 & $<1.70<-2$ & & ug $/ \mathrm{mI}$ \\
\hline $1 \mathrm{CCB}$ & & 0 & DIC-RC & พоO2 & ec & 1 & $<1.07 \bullet-1$ & & ug/mI \\
\hline $1 \mathrm{CCB}$ & & 0 & are-qe & BR & oc & 1 & $<1.260-1$ & & ug/mu \\
\hline $1 \mathrm{CCB}$ & & 0 & ote-oc & nos & $\infty$ & 1 & $<1.400-2$ & & ug $/=\mathbf{m I}$ \\
\hline $1 \mathrm{CCB}$ & & 0 & arc-gc & PO4 & Qc & 1 & $<1.19 \bullet-1$ & & $\mathrm{ug} / \mathrm{mt}$ \\
\hline $1 \mathrm{ccs}$ & & 0 & are-ac & so 4 & oc & 1 & $<1.360-1$ & & ug/mL \\
\hline $1 \mathrm{CCB}$ & & 0 & IC-RC & OXALATE2 & $2 \mathrm{Qc}$ & 1 & $<1.05 \bullet-1$ & & $u g / m$ \\
\hline $2 \mathrm{ccv}$ & & 0 & ere-ge & $\mathbf{F}$ & $Q C$ & 59 & $6.07 a+01$ & 102.881 & 91. \% Recovery \\
\hline $2 \mathrm{ccv}$ & & 0 & DC-QC & CL & gc & 79 & $7.77 \bullet+01$ & 98.354 & 54 \& Recovary \\
\hline $2 \mathrm{ccv}$ & & 0 & erc-oc & Ho2 & oc & 534 & $5.45 \bullet+02$ & 102.060 & 50 \& Recorvery \\
\hline $2 \mathrm{ccv}$ & & 0 & aIC-QC & BR & $\mathrm{Qc}$ & 575 & $5.730+02$ & 99.652 & 52 \& Recovery \\
\hline $2 \mathrm{ccv}$ & & 0 & erc-oc & MO3 & Qc & 614 & $6.29++02$ & $102 \times 443$ & I3 \& Reoovary \\
\hline $2 \mathrm{ccv}$ & & 0 & are-ec & Po4 & oc & 546 & $5.400+02$ & 98.901 & I \& Recovery \\
\hline $2 \mathrm{ccv}$ & & 0 & -IC-QC & so4 & gc & 631 & $6.38 m+02$ & 101.109 & If \& Recovery \\
\hline $2 \mathrm{ccv}$ & & 0 & erc-oc & OTALATE: & $2 \mathrm{oc}$ & 504 & $5.210+02$ & 103.373 & 3 \& Recovery \\
\hline 3 SAKPLI & 5967000682 & 0 & eIC-0.1 & F-02 & LIQUID & $w / \pi$ & $4.9900+02$ & $53.830 \mathrm{u}$ & $\mathrm{ug} / \mathrm{mL}$ \\
\hline 3 REJRCT & $596 T 000682$ & 0 & arc-01 & F-02 & CrovID & $N / \pi$ & $4.9900+02$ & & ug/mL \\
\hline 3 SAHPLI & $S 965000582$ & 0 & erc-02 & CL-O2 & LIQUID & N/A & $7.7420+03$ & 70.400 & ug/mi \\
\hline 3 REJBCT & $\$ 967000682$ & 0 & -rc-01 & $c t-02$ & LIQUID & $N / A$ & $7.7420+03$ & & $\mathrm{ug} / \mathrm{mL}$ \\
\hline 3 SAMTLE & 5957000682 & 0 & exc-01 & $\mathrm{NO2}-02$ & EIQUID & $\mathbf{w / 2}$ & $9.395=404$ & 443.100 & $\mathbf{u g} / \mathbf{m L}$ \\
\hline 3 REJECT & $S 96 \mathrm{~T} 0006 \mathrm{B2}$ & 0 & DIC-01 & NO2-02 & LIQDID & $\mathrm{N} / \mathrm{A}$ & $9.395 \bullet+04$ & & $\mathrm{ug} / \mathrm{mL}$ \\
\hline 3 SAMPLE & S96T000682 & 0 & arc-01 & $B R-02$ & LIRUID & N/A & $5.2180+02$ & 521.800 & ug/mI \\
\hline 3 REJECT & S96T000682 & 0 & OIC-0I & $B R-02$ & LIQUID & $\mathrm{N} / \mathrm{A}$ & $5.218 \bullet+02$ & & $\mathrm{ug} / \mathrm{mL}$ \\
\hline 3 SAMPLI & 5967000682 & 0 & arc-01 & $103-02$ & LIOOID & $\mathbb{N} / \mathbf{A}$ & $2.2070+05$ & 579.700 & ug/unL \\
\hline 3 REJECT & S96T000682 & 0 & erc-01 & No3-02 & LIOUID & $N / \mathbf{A}$ & $2.207 * 0.05$ & & ug/m \\
\hline 3 SAMPLII & s967000682 & 0 & erc-01 & PO4-02 & LIQUID & $N / A$ & $3.6990+03$ & 492.400 & $\mathrm{ug} / \mathrm{mr}$ \\
\hline 3 REJRCT & 3967000682 & 0 & arc-01 & PO4-02 & LIQUID & $N / A$ & $3.6990+03$ & & $\mathbf{u g} / \mathrm{mL}$ \\
\hline 3 SAMPLE & $\$ 967000682$ & 0 & exc-01 & $504-02$ & LIOUID & $\mathrm{M} / \mathrm{A}$ & $6.3740+03$ & 562.800 & $\mathrm{ug} / \mathrm{mr}$ \\
\hline 3 REJECT & 5967000682 & 0 & eIc-01 & $504-02$ & IIQUID & $\mathbf{N} / \mathbf{A}$ & $6.3740+03$ & & $\mathrm{ug} / \mathrm{mL}$ \\
\hline 3 SAMPLE & 5969000682 & 0 & erc-02 & OXATATT22 & 2 LIQUID & B/A & $5.3050+02$ & 434.800 & $\mathrm{ug} / \mathrm{mc}$ \\
\hline 3 REJECT & $596 \mathrm{~T} 000682$ & 0 & IC-01 & OXALATR2 & 2 LIQUID & $N / A$ & $5.305 \bullet+02$ & & $\mathbf{u g} / \mathbf{m L}$ \\
\hline 4 DOP & 5967000682 & 0 & aIc-0I & $z-02$ & IIQUTO & $4.990+02$ & $4.960+02$ & 0.603 & 3 APD \\
\hline $4 \mathrm{DDP}$ & 5967000682 & 0 & arc-01 & CL-02 & IIQUID & $7.740+03$ & $7.670+03$ & 0.909 & 9 RPD \\
\hline 4 DOP & 3967000682 & 0 & Ire-01 & $\mathrm{NO2}-02$ & LIQUID & $9.400+04$ & $9.380+04$ & 0.213 & 13 RPD \\
\hline 4 DOP & S967000682 & 0 & OIC-01 & ER-02 & IIQUID & $<5.22 \bullet 2$ & $<5.2202$ & & RPD \\
\hline $4 \mathrm{DDP}$ & S96T000682 & 0 & बIC-01 & No3 -02 & LIOJID & $2.21 *+0.5$ & 2.210405 & 0.000 & DO RPD \\
\hline 4 DOP & $s 967000682$ & 0 & erc-01 & PO4-02 & IIQUID & $3.700+03$ & $3.48 *+03$ & 6.128 & $8 \mathrm{RPD}$ \\
\hline 4 DOP & S96T000682 & 0 & $\operatorname{arc}+01$ & $504-02$ & LIQUTD & $6.37 *+03$ & $6.28 \bullet+0.3$ & 1.423 & $23 \cdot$ RPD \\
\hline $4 \mathrm{DUP}$ & 5967000682 & 0 & aIC-01 & OXATATE2 & 2 LIgTtD & $5.30 \cdot+02$ & $5.180+02$ & 2.290 & 0 RPD \\
\hline 5 SPK & 5967000682 & 0 & QIC-01 & P-02 & IIQUID & 59 & $5.03 \bullet+01$ & 85.254 & is \& Recol \\
\hline
\end{tabular}

Units shown for $Q C(B L K / B K G)$ may not reflect the actual units. 


\section{LABCORE Completed Worklist Report for Worklist\# 6622}

\begin{tabular}{|c|c|c|c|c|c|c|c|c|c|c|}
\hline Seq & Type & Sample\# R & $A$ & & st & Matrix & Actual & Found & DL or Yied & Onit \\
\hline 5 & SPK & 5967000682 & 0 & $\operatorname{arc}-01$ & $\mathrm{CL}-02$ & LIQTID & 79 & B. $25 \bullet+01$ & 104.430 & 0 F Recovery \\
\hline 5 & SPK & S96T0006B2 & 0 & Ac- 01 & $\mathrm{NO2}-02$ & LIQJID & 534 & $5.290+02$ & 99.064 & \& is Recovery \\
\hline 5 & sPx & 5967000682 & 0 & arc-01 & BR-02 & ETQUTD & 575 & $5.55 \bullet+02$ & 96.522 & 2 * R-covery \\
\hline 5 & SEK & $596 T 000682$ & 0 & -IC-01 & $2003-02$ & LIQUID & 614 & $5.700+02$ & 92.834 & 4 is Recovery \\
\hline 5 & $\mathbf{S P K}$ & 5962000602 & 0 & eIc-01 & PO4-02 & LIQUID & 546 & $5.31=402$ & 97.253 & 3 i Recovery \\
\hline 5 & SPK & 5967000682 & 0 & arc-01 & $504-02$ & LIQUID & 631 & $6.17 \bullet+02$ & 97.781 & 1 Recovery \\
\hline 5 & $\operatorname{spK}$ & 3967000682 & 0 & erc-02 & OXATATE2 & 2 EIQUID & 504 & $5.10 \bullet+0.2$ & 101.190 & o Redovery \\
\hline 6 & SAMULII & S96T000665 & 0 & $\operatorname{erc-01}$ & F-02 & LIQUID & N/A & $4.575 \bullet+02$ & 53.830 & $\mathrm{gg} / \mathrm{mr}$ \\
\hline 6 & REJBCT & $398 \mathrm{~T} 000665$ & 0 & exc-01 & $7 \div 02$ & xIOUID & $w / A$ & $4.575 \bullet+02$ & & $u g / m$ \\
\hline 6 & SAMPLI & S96T000665 & 0 & eIC-01 & $C t-02$ & LIOUID & $N / A$ & $7.1990+03$ & 70.400 & ug/mI \\
\hline 6 & REJECT & 3967000665 & 0 & -xe-01 & $\mathrm{CE}-02$ & IIQUID & $\mathbf{N} / \mathrm{A}$ & $7.299 \bullet+03$ & . & ug/ax \\
\hline 6 & SAMPLI & S96T000665 & 0 & arc-01 & $102-02$ & LIOUID & $N / A$ & $8.827 \bullet+04$ & 413.100 & $\mathrm{~g} / \mathrm{me}$ \\
\hline 6 & RETECT & 3967000665 & 0 & $\operatorname{arc-01}$ & NO2-02 & IXgerd & IN/A & $8.827 \bullet+04$ & & $\mathrm{ug} / \mathrm{mr}$ \\
\hline 6 & SAMPLI & s96T000665 & 0 & erc-01 & $B R-02$ & IIQIID & $N / A$ & $5.218 \bullet+02$ & 521.800 & $\mu g / m L$ \\
\hline 6 & REJTCT & 5967000665 & a & erc-01 & $B R-02$ & ETOUID & $\mathbf{N} / \mathbf{A}$ & $5.218_{0+02}$ & . & ug/mL \\
\hline 6 & SAMPLI & S96T000665 & 0 & ax-01 & No3-02 & LIQUID & $N / A$ & $2.036 e+05$ & 579.700 & $\mathrm{dg} / \mathrm{m}$ \\
\hline 6 & REJECT & $\$ 967000665$ & 0 & aIc-01 & $1503-02$ & IIQJID & $x / \pi$ & $2.036 \bullet+05$ & & $\mathrm{~g} / \mathrm{g}$ \\
\hline 6 & SAMPLE & $\$ 96 \mathrm{T000665}$ & 0 & $\operatorname{arc}-a 1$ & FO4-02 & IIOUID & $\mathrm{N} / \mathrm{A}$ & $3.534-+03$ & 492.400 & Ig/mI \\
\hline 6 & REJECT & 5967000665 & 0 & erc-01 & $504-02$ & IIOUID & $N / A$ & $3.5340+03$ & & $\mathrm{~g} / \mathrm{g} / \mathrm{mI}$ \\
\hline 6 & SAMPLI & S\$ETO00665 & 0 & $\operatorname{arc}=01$ & $504-02$ & LIO0ID & $y / 2$ & $5.340 *+03$ & 562.800 & $\mathrm{~g} / \mathrm{mt}$ \\
\hline 6 & RRJECT & 5967000665 & 0 & aIC-01 & $504-02$ & IIQUID & $x / A$ & $5.9400+03$ & & $\mathrm{~g} / \mathrm{g} / \mathrm{mL}$ \\
\hline 6 & SAMPLE & $\$ 96 \mathrm{T000665}$ & 0 & arc-0I & OxAIATEz & IIQOID & $N / A$ & $5.6580+02$ & 434,800 & g/gI \\
\hline 6 & RETECT & 5967000665 & 0 & $\operatorname{erc-01}$ & OXAIATE2 & IIOUID & $N / 2$ & $5.6580+02$ & & \\
\hline 7 & DUP & 5965000665 & 0 & aIC-01 & P-02 & LIOOID & $4.58++02$ & $4.51 \bullet+02$ & 1.540 & O RPD \\
\hline 7 & DOP & $596 \mathrm{~T} 000665$ & 0 & aIC-0I & $C L-02$ & LIOETD & $7.200+03$ & $7.33-+03$ & 1.7 .89 & RPD \\
\hline 7 & DOP & S96T000665 & 0 & DIC-01 & No2-02 & IIOUID & $0.03 \bullet+04$ & $0.94 m+04$ & $1.23 \theta$ & G RPD \\
\hline 7 & DUP & s96T000665 & 0 & erc-01 & BR- -02 & IIOUID & $<5.2202$ & $<5,22 \theta z$ & & RPD \\
\hline 7 & DUP & $S 967000665$ & 0 & DIC-01 & N03-02 & LIQUID & $2.040+05$ & $2.09 e+05$ & 2.421 & 1 RPD \\
\hline 7 & DEP & 5967000665 & 0 & aIc+01 & $504-02$ & LIQOID & $3.53 e+03$ & $3.74 *+03$ & 5.777 & RPD \\
\hline 7 & DUP & s96T000665 & 0 & IC-01 & SO4-02 & LIOUID & $5.94 \bullet+03$ & $6.180+03$ & 3.960 & 0 RPD \\
\hline 7 & DOP & 8965000665 & 0 & MIC-01 & OXALATZZ2 & LIQOID & $5.66 \bullet+02$ & $6.87-+02$ & 29.314 & 4 RPD \\
\hline
\end{tabular}

\section{Final page for worklist\# 6622}

Analyst Signature Date

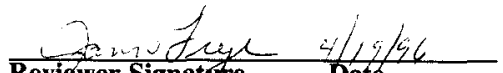

Reviewer Signatifre

Daté

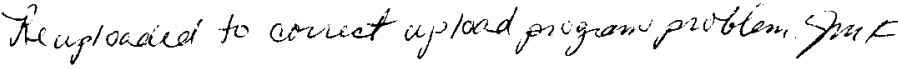




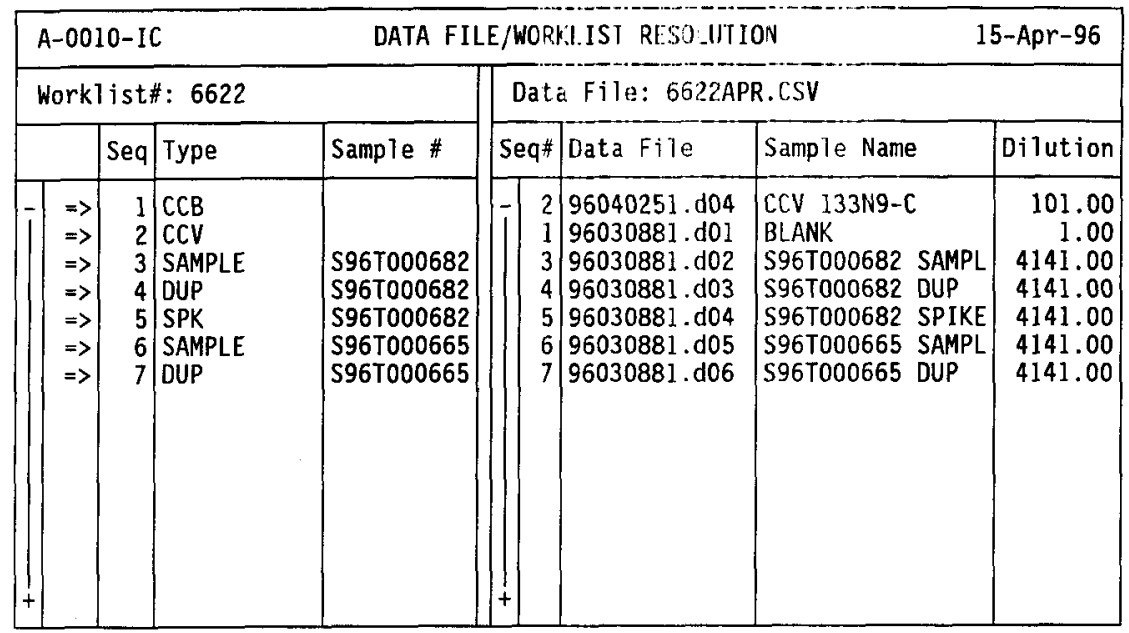

Save(F4) Abort(Shift-F3) ListFiles(Shift-F1) UploadFile(F8) 
Data Reprocessed on 04/09/1996 10:52:05

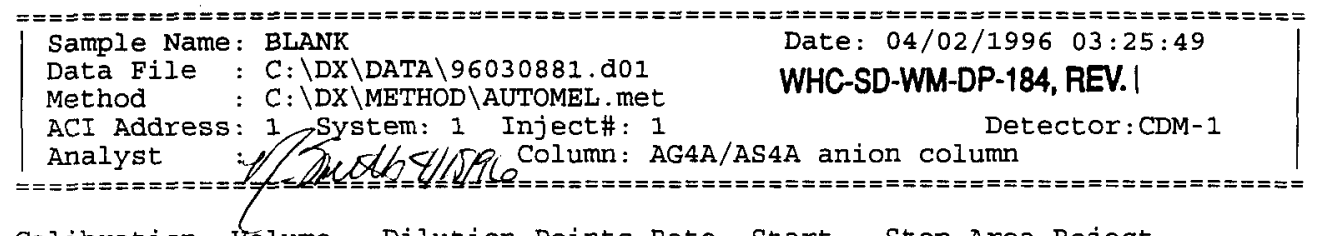

Calibration Volume Dilution points Rate start stop Area Reject

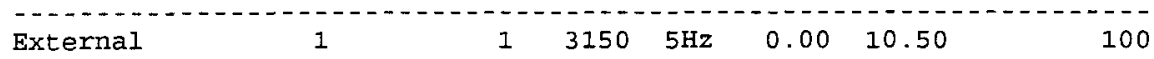

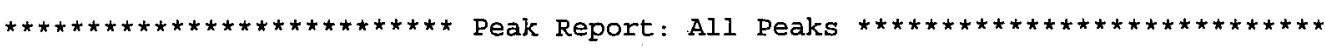

Pk. Ret Component Concentration Height Area Bl. $\%$ Delta Num Time Name $\mathrm{ug} / \mathrm{ml}$ Code

Totals

0.000

0

0

File: 96030881.d01 Sample: BLANK

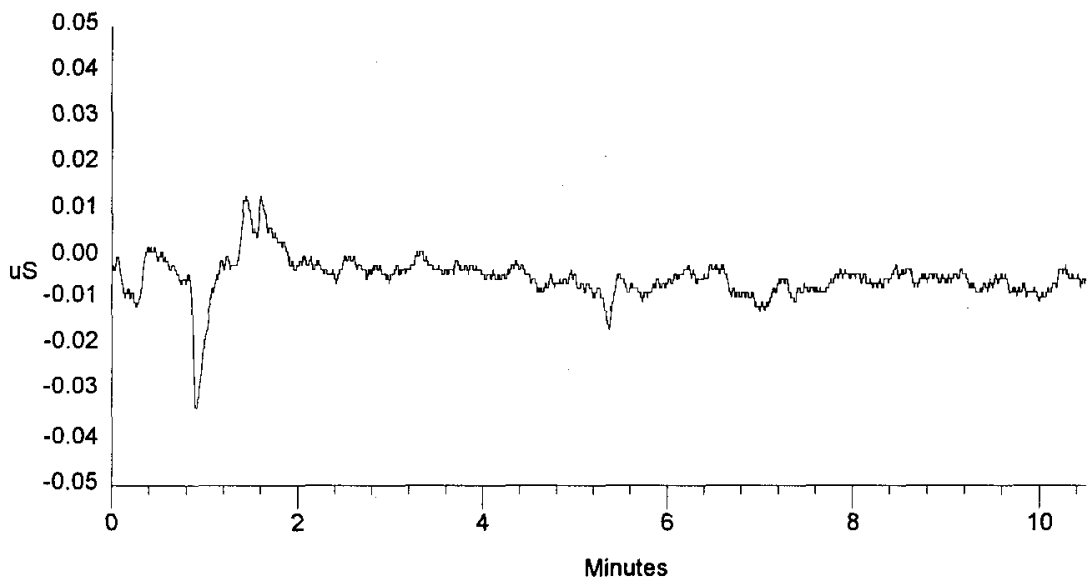

SIGNATURE ABOVE REPRESENTS CHEMICAL TECHMOLOGIST/CHEMIST THAT COMPLETED/VERIFIED THE CALIBRATION/ANALYSIS ON PAGES 279 TO 25 
Data Reprocessed On 04/09/1996 10:52:03

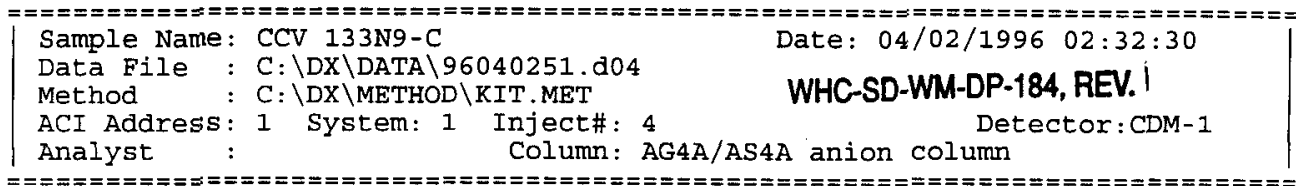

Calibration Volume Dilution Points Rate start stop Area Reject

Calibation

External

1

$101 \quad 3150 \quad 5 \mathrm{~Hz}$

$0.00 \quad 10.50$

100

Peak Report: All Peaks

Pk. Ret Component

Num Time Name
Concentration $\mathrm{ug} / \mathrm{ml}$
Area Bl. \%Delta

Code
0.90
1.06
fluoride
1.60 chloride
1.92 nitrite
2.88 bromide
3.28 nitrate
4. 67 phosphate
6.19 sulfate
8.11
oxalate

0.000

60.688

77.665

545.368

573.108

629.118

540.112

638.094

521.246

Height

$\begin{array}{rrrr}61 & 272 & 2 & \\ 2662 & 11847 & 2 & 0.95 \\ 2096 & 9653 & 1 & 0.00 \\ 9292 & 49278 & 1 & -1.03 \\ 5488 & 34231 & 1 & -0.69 \\ 6424 & 49332 & 1 & -2.67 \\ 1654 & 21558 & 1 & 0.00 \\ 4745 & 65794 & 1 & 1.75 \\ 2113 & 42081 & 1 & 2.01\end{array}$

Totals

3585.398

34536

284044

File: 96040251.d04 Sample: CCV 133N9-C

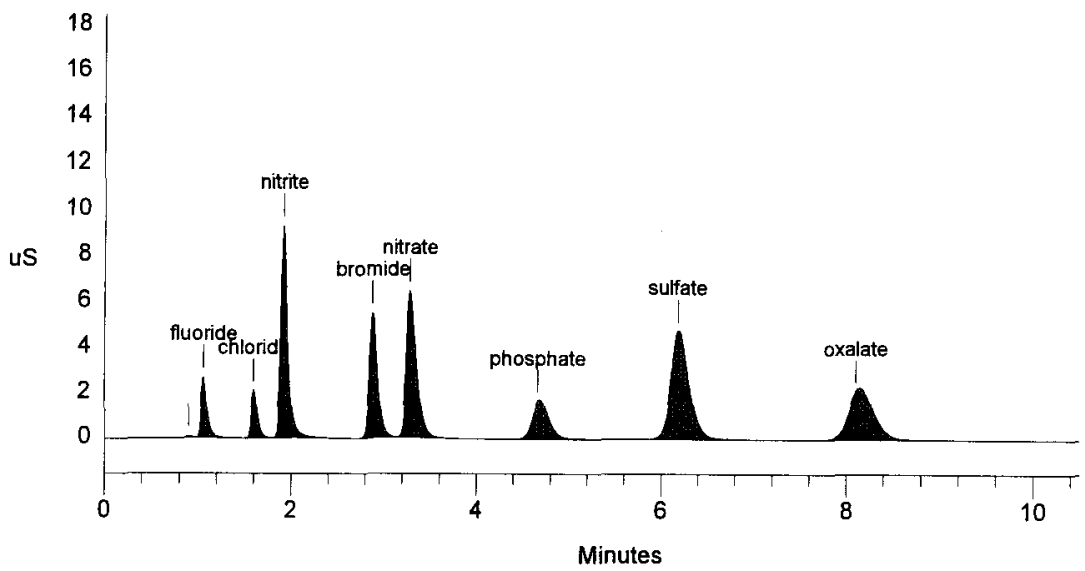


Data Reprocessed on 04/09/1996 10:52:07

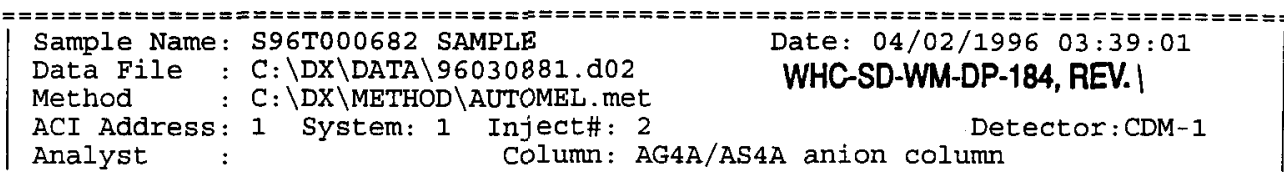

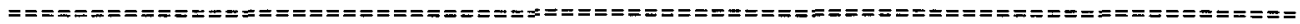

Calibration Volume Dilution points Rate start stop Area Reject

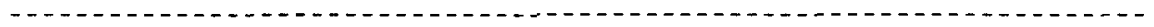

$\begin{array}{llllllll}\text { External } & 1 & 4141 & 3150 & 5 \mathrm{~Hz} & 0.00 & 10.50 & 100\end{array}$

\begin{tabular}{|c|c|c|c|c|c|c|c|}
\hline $\begin{array}{l}\mathrm{Pk} \\
\text { Num }\end{array}$ & $\begin{array}{l}\text { Ret } \\
\text { Time }\end{array}$ & $\begin{array}{l}\text { Component } \\
\text { Name }\end{array}$ & $\begin{array}{r}\text { Concentration } \\
\mathrm{ug} / \mathrm{mI}\end{array}$ & Height & Area & $\begin{array}{l}\text { Bl. } \\
\text { Code }\end{array}$ & \%Delta \\
\hline---- & ---- & & & & & & \\
\hline 1 & 0.91 & & 0.000 & 416 & 2434 & 2 & \\
\hline 2 & 1.11 & fluoride & 499.044 & 620 & 2142 & 2 & 5.40 \\
\hline 3 & 1.17 & & 0.000 & 1168 & 7987 & 2 & \\
\hline 4 & 1.59 & chloride & 7742.103 & 4731 & 24100 & 1 & -0.83 \\
\hline 5 & 1.92 & nitrite & 93952.114 & 38015 & 221012 & 1 & -1.03 \\
\hline 6 & 3.15 & nitrate & 220674.294 & 50346 & 501449 & 1 & 0.21 \\
\hline 7 & 4.69 & phosphate & 3699.219 & 187 & 2277 & 1 & 0.57 \\
\hline 8 & 6.19 & sulfate & 6373.956 & 892 & 13998 & $\bar{I}$ & 1.75 \\
\hline 9 & 8.16 & oxalate & 530.497 & 25 & 504 & 1 & 2.68 \\
\hline
\end{tabular}

Totals $333471.227 \quad 96400 \quad 775903$

File: 96030881.d02 Sample: S96T000682 SAMPLE

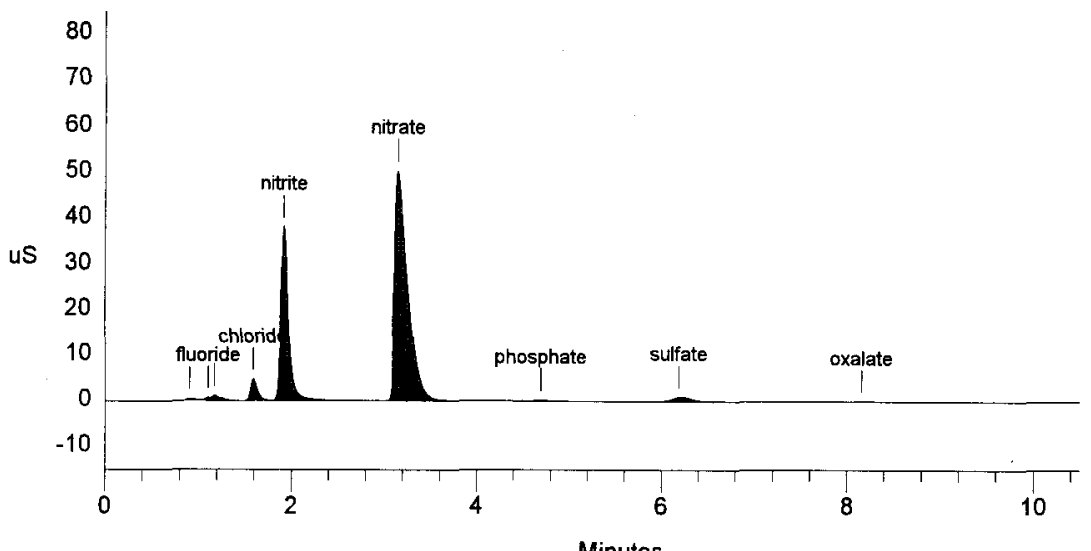

Minutes 
Data Reprocessed On 04/09/1996 10:52:09

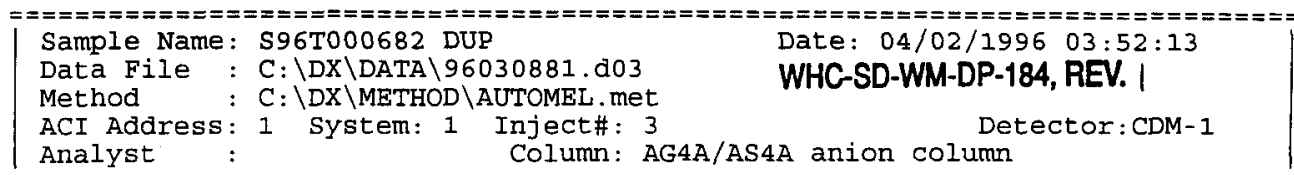

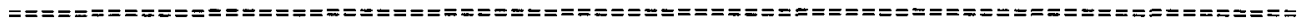

Calibration Volume Dilution Points Rate start stop Area Reject

\begin{tabular}{|c|}
\hline External \\
\hline
\end{tabular}

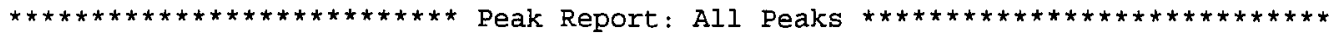

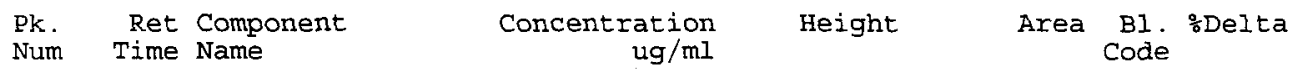

Num Time Name ug/ml

$\begin{array}{lll}1 & 0.91 & \\ 2 & 1.11 & \text { fluoride } \\ 3 & 1.17 & \\ 4 & 1.59 & \text { chloride } \\ 5 & 1.93 & \text { nitrite } \\ 6 & 3.15 & \text { nitrate } \\ 7 & 4.69 & \text { phosphate } \\ 8 & 6.19 & \text { sulfate } \\ 9 & 8.21 \text { oxalate }\end{array}$

$\begin{array}{rrrrr}0.000 & 406 & 2366 & 2 & \\ 495.952 & 613 & 2127 & 2 & 5.40 \\ 0.000 & 1149 & 7961 & 2 & \\ 7672.506 & 4784 & 23875 & 1 & -0.42 \\ 93849.775 & 38140 & 220758 & 1 & -0.69 \\ 220664.772 & 50169 & 501424 & 1 & 0.21 \\ 3481.919 & 172 & 2052 & 1 & 0.57 \\ 6282.827 & 886 & 13763 & 1 & 1.75 \\ 517.714 & 24 & 479 & 1 & 3.36\end{array}$

Totals $332965.465 \quad 96344 \quad 774803$

File: 96030881.d03 Sample: S96T000682 DUP

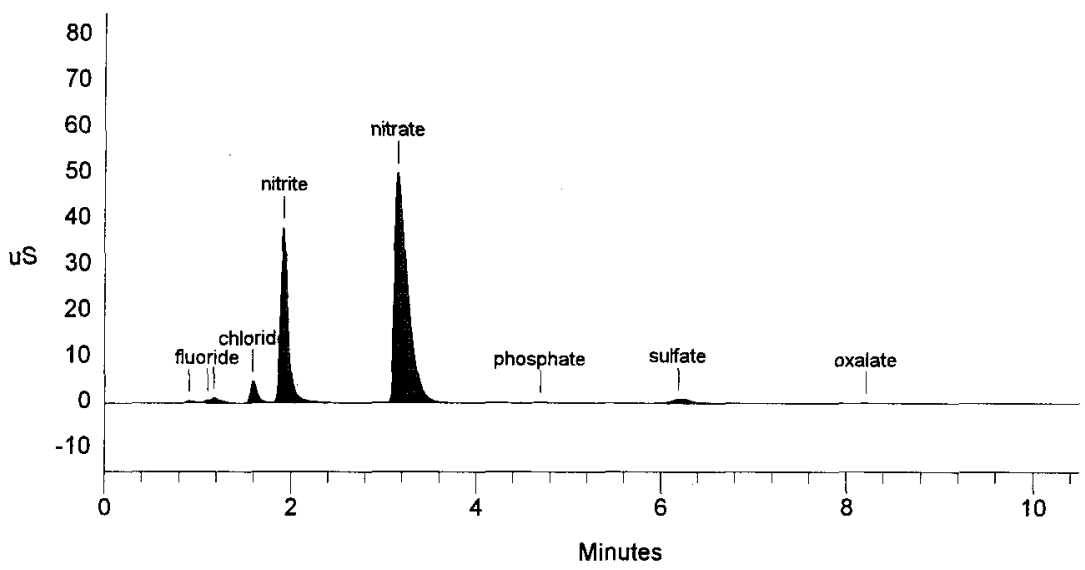


Data Reprocessed On 04/09/1996 10:52:11

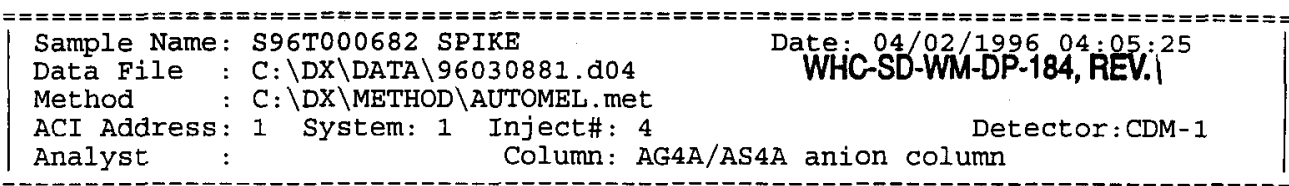

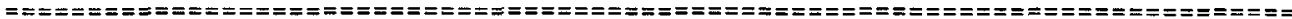

Calibration Volume Dilution Points Rate Start Stop Area Reject

Calibation

$\begin{array}{llllllll}\text { External } & 1 & 4141 & 3150 & 5 \mathrm{~Hz} & 0.00 & 10.50 & 100\end{array}$

\begin{tabular}{|c|c|c|c|c|c|c|c|}
\hline $\begin{array}{l}\text { Pk. } \\
\text { Num }\end{array}$ & $\begin{array}{r}\text { Ret } \\
\text { Time }\end{array}$ & $\begin{array}{l}\text { Component } \\
\text { Name }\end{array}$ & $\begin{array}{r}\text { Concentration } \\
\mathrm{ug} / \mathrm{ml}\end{array}$ & Height & Area & Bl. & $\div$ Delta \\
\hline$I$ & 0.91 & & 0.000 & 437 & 2197 & 2 & \\
\hline 2 & 1.07 & fluoride & 2526.926 & 2476 & 12038 & 2 & 1.59 \\
\hline 3 & 1.17 & & 0.000 & 1279 & 8480 & 2 & \\
\hline 4 & 1.59 & chloride & 11068.550 & 6795 & 35025 & 1 & -0.83 \\
\hline 5 & 1.93 & nitrite & 115290.868 & 47310 & 274443 & 1 & -0.69 \\
\hline 6 & 2.87 & bromide & 22370.415 & 5689 & 32488 & 1 & 2.02 \\
\hline 7 & 3.13 & nitrate & 243667.246 & 55474 & 564377 & 1 & -0.2 \\
\hline 8 & 4.67 & phosphate & 25100.069 & 1874 & 24674 & 1 & 0.00 \\
\hline 9 & 6.19 & sulfate & 31262.104 & 5738 & 79363 & 1 & 1.75 \\
\hline 10 & 8.11 & oxalate & 21088.734 & 2158 & 41513 & 1 & 2.01 \\
\hline
\end{tabular}

Totals $\quad 472374.913 \quad 129231 \quad 1074598$

File: $96030881 . d 04$ Sample: S96T000682 SPIKE

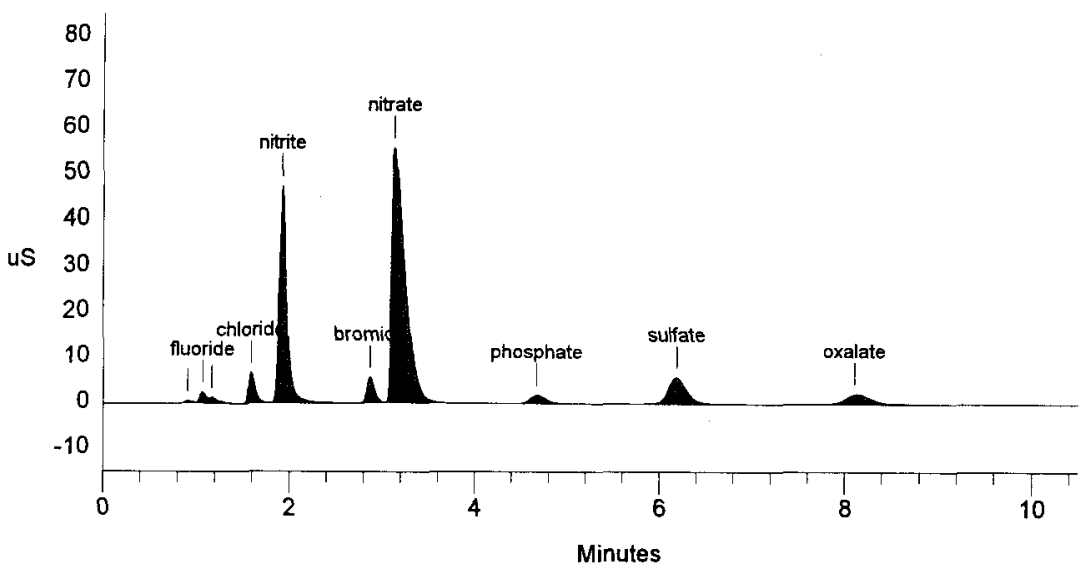


Data Reprocessed on 04/09/1996 10:52:14

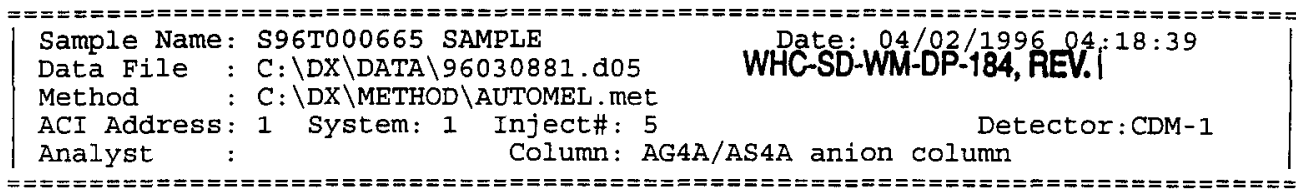

Calibration Volume Dilution Points Rate Start stop Area Reject

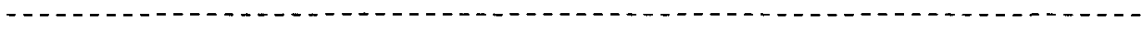

$\begin{array}{llllllll}\text { External } & 1 & 4141 & 3150 & 5 \mathrm{~Hz} & 0.00 & 10.50 & 100\end{array}$

\begin{tabular}{|c|c|c|c|c|c|c|c|}
\hline $\begin{array}{l}\mathrm{Pk} . \\
\text { Num }\end{array}$ & $\begin{array}{l}\text { Ret } \\
\text { Time }\end{array}$ & $\begin{array}{l}\text { Component } \\
\text { Name }\end{array}$ & $\begin{array}{r}\text { Concentration } \\
\mathrm{ug} / \mathrm{ml}\end{array}$ & Height & Area & $\begin{array}{l}\text { B1. } \\
\text { Code }\end{array}$ & oDelta \\
\hline & ---- & --------- & -----------1 & & ---- & --- & \\
\hline 1 & 0.91 & & 0.000 & 389 & 2249 & 2 & \\
\hline 2 & 1.11 & fluoride & 457.548 & 565 & 1942 & 2 & 5.40 \\
\hline 3 & 1.18 & & 0.000 & 1095 & 7479 & 2 & \\
\hline 4 & 1.59 & chloride & 7198.510 & 4486 & 22346 & 1 & -0.42 \\
\hline 5 & 1.92 & nitrite & 88267.132 & 35559 & 206961 & 1 & -1.0 \\
\hline 6 & 3.16 & nitrate & 203641.323 & 46590 & 456523 & 1 & 0.6 \\
\hline 7 & 4.69 & phosphate & 3533.886 & 191 & 2106 & 1 & 0.5 \\
\hline 8 & 6.19 & sulfate & 5940.353 & 773 & 12878 & 1 & 1.7 \\
\hline 9 & 8.16 & oxalate & 565.813 & 27 & 574 & 1 & 2.68 \\
\hline
\end{tabular}

Totals $\quad 309604.564 \quad 89675 \quad 713058$

File: 96030881.d05 Sample: S96T000665 SAMPLE

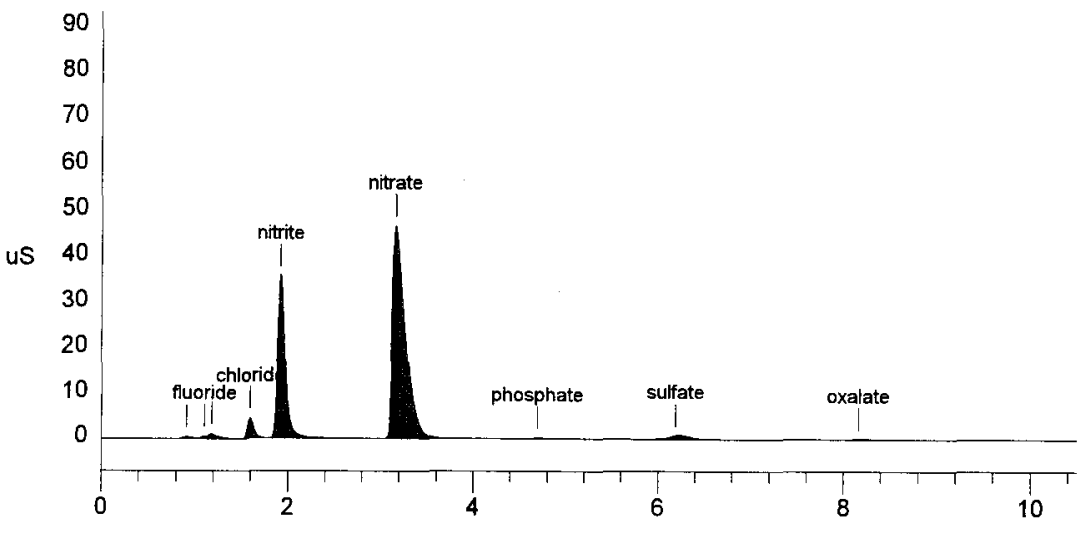

Minutes 
Data Reprocessed On 04/09/1996 10:52:16

\begin{tabular}{|c|c|c|}
\hline $\begin{array}{l}\text { Sample Name: } \\
\text { Data File: } \\
\text { Method : }\end{array}$ & $\begin{array}{l}S 96 T 000665 \text { DUP } \\
C: \backslash D X \backslash D A T A \backslash 96030881 . \text { do6 } \\
C: \backslash D X \backslash M E T H O D \backslash A U T O M E L \text {. met }\end{array}$ & $\begin{array}{l}\text { Date: } 04 / 02 / 199604: 31: 51 \\
\text { WHC-SD-WM-DP-184, REV.| }\end{array}$ \\
\hline $\begin{array}{l}\text { ACI Adaress: } \\
\text { Analyst : }\end{array}$ & $\begin{array}{r}1 \text { System: } 1 \text { Inject\# : } 6 \\
\text { Column: } A\end{array}$ & $\begin{array}{l}\text { Detector: CDM-1 } \\
\text { column }\end{array}$ \\
\hline
\end{tabular}

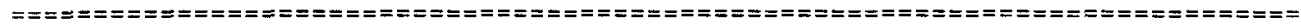

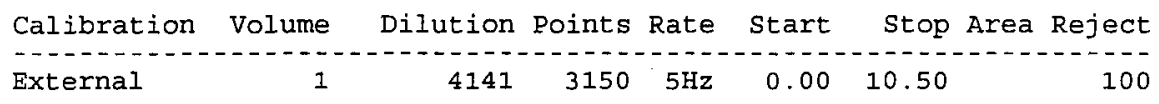

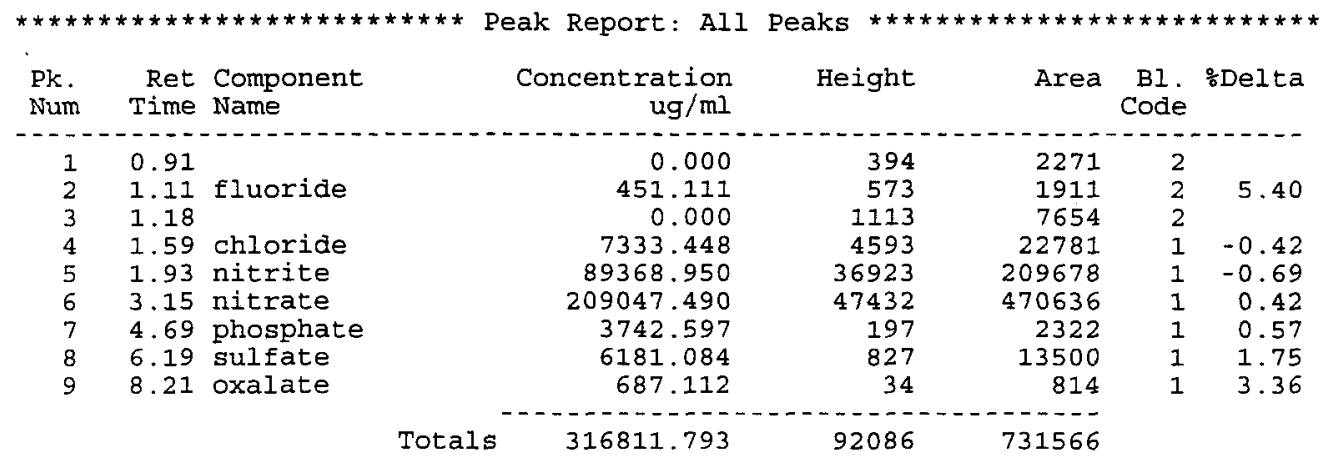

File: 96030881.d06 Sample: S96T000665 DUP

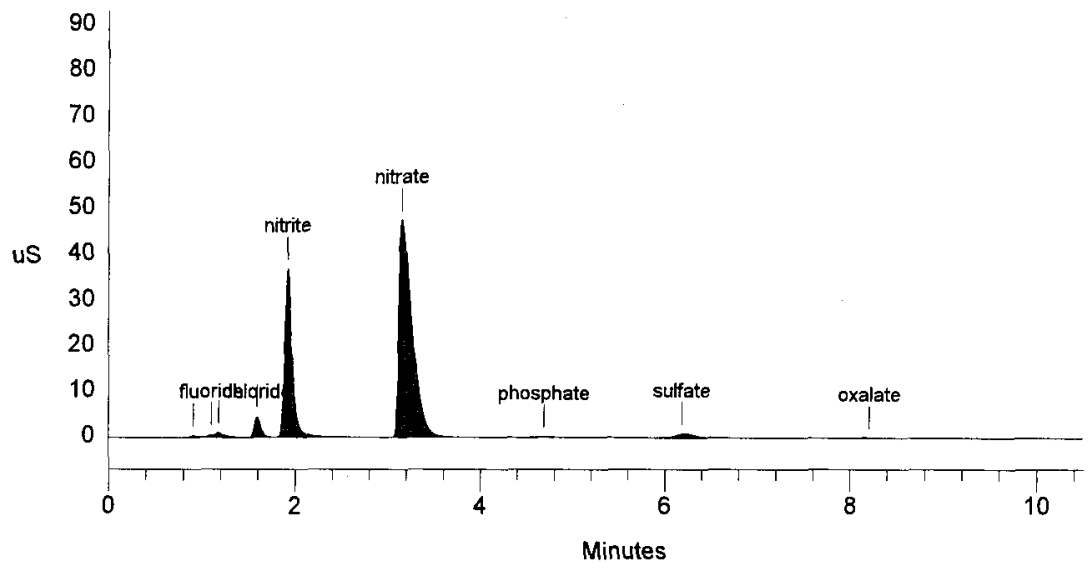




\section{LABCORE Data Entry Template for Worklist\# 7493} $9604 / 801.009 \rightarrow$ Dol

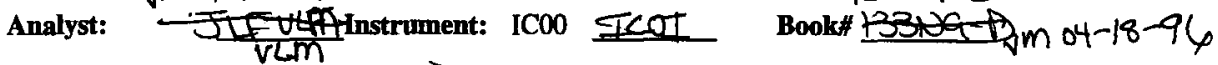
Method: LA-533-105 Rev/Mod D-1

Worklist Comment: U-107 FOR @IC-01 RTS!

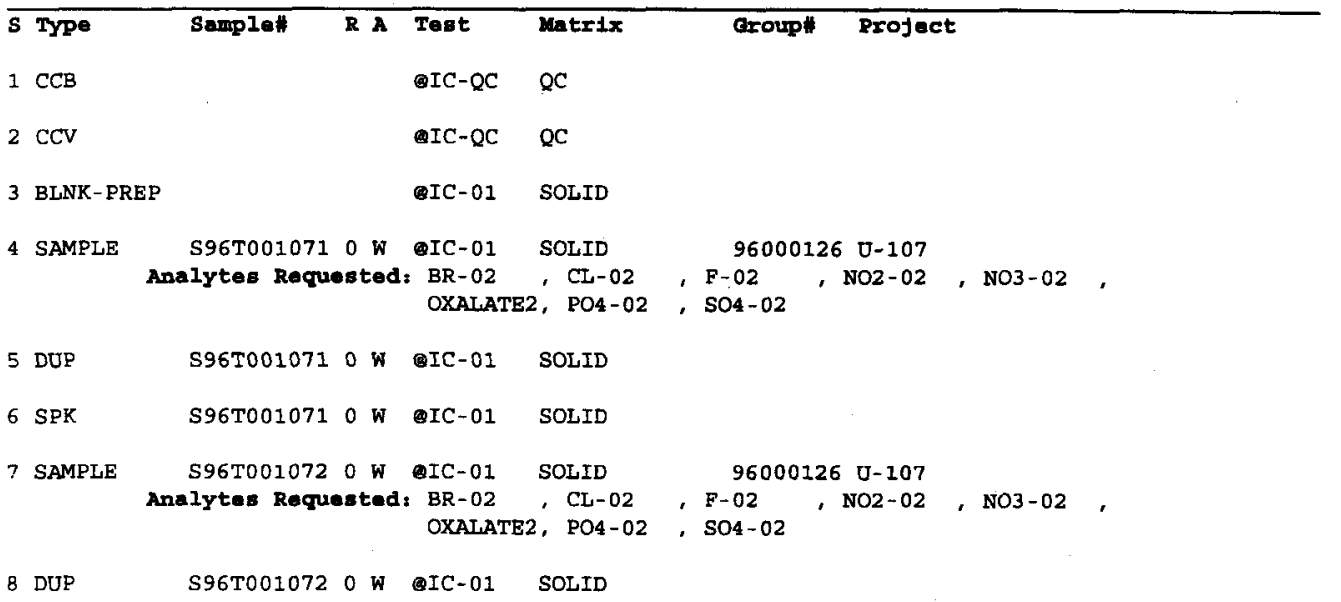

\section{Final page for worklist \# 7493}

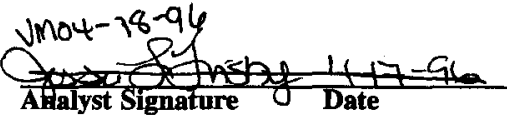

Exponted 7493 ap. Cor

Inshell

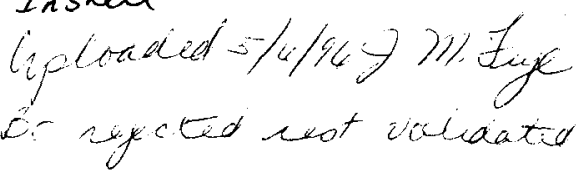

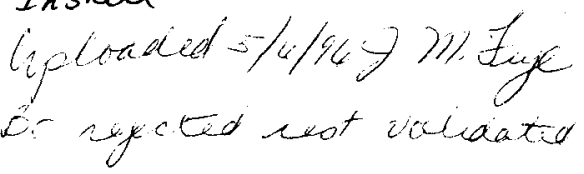

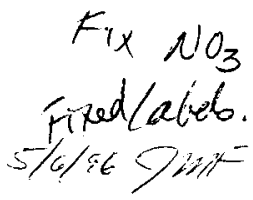

Data Entry Comments:

$S=$ Worklist Slot Number, $R=$ Replicate Number, $A=$ Alquot Code.

285 


\section{LABCORE Completed Worklist Report for Worklist\# 7493}

Analyst: $V L m$

Instrument: IC01

Book\# $133 N q=$

Method: $b A-533-105 \mathrm{Rev} / \mathrm{Mod} D-1$

Worklist Comment: U-107 FOR @IC-01 RTS!

\begin{tabular}{|c|c|c|c|c|c|c|c|c|c|}
\hline \multirow{2}{*}{$\begin{array}{l}\text { Seq Type } \\
1 \text { ccs }\end{array}$} & \multicolumn{2}{|c|}{ Sample\# R A } & \multicolumn{2}{|c|}{ Test } & \multirow{2}{*}{$\begin{array}{l}\text { Matrix } \\
\text { oc }\end{array}$} & \multirow{2}{*}{$\begin{array}{l}\text { Actual } \\
1\end{array}$} & \multirow{2}{*}{$\begin{array}{l}\text { Found } \\
<1.30<-2\end{array}$} & \multirow{2}{*}{ DL or Yield } & \multirow{2}{*}{$\begin{array}{l}\text { Unit } \\
\text { ug/mx }\end{array}$} \\
\hline & & 0 & IC-QC & 8 & & & & & \\
\hline $1 \mathrm{cCB}$ & & 0 & DIC-QC & $\mathrm{CI}$ & $\mathrm{gc}$ & 1 & $<1.700+2$ & & ug/me \\
\hline $1 \cos$ & &. & eIC-QC & No2 2 & ge & 1 & $<1.07=-1$ & & $\mathrm{ug} / \mathrm{mL}$ \\
\hline $1 \operatorname{ccs}$ & & 0 & eIc-oc & $B R$ & $\mathbf{Q C}$ & 1 & $<1.260-1$ & & ug/mL \\
\hline $1 \operatorname{ccs}$ & & 0 & aIC-QC & No3 & oc & 1 & $3.65=-01$ & 0.365 & $. \mathrm{tg} / \mathrm{mI}$ \\
\hline $1 \mathrm{CCB}$ & & 0 & aIC-QC & PO4 & oc & 1 & $<1.190-1$ & & $\mathrm{ug} / \mathrm{mL}$ \\
\hline $1 \mathrm{ccs}$ & & 0 & arc-gc & so4 & oc & 1 & $<1: 36--1$ & & ug/mL \\
\hline $1 \mathrm{CCB}$ & & 0 & IC-OC & OXALATE2 & oc & 1 & $<1.05 \bullet-1$ & & $v g / m \mathbf{m}$ \\
\hline $2 \mathrm{ccv}$ & & 0 & IC-QC & I & $\mathrm{gc}$ & 5.90001 & 5.690401 & 96.441 & I Recovary \\
\hline $2 \mathrm{ccs}$ & & 0 & aIc-oc & CL & oc & 7.90001 & $7.63 \bullet+01$ & 96.582 & R Recovery \\
\hline $2 \mathrm{ccv}$ & & 0 & eIc-Qc & No2 & Qc & 5.76002 & $5.690+02$ & 98.785 & \& Recovexy \\
\hline $2 \mathrm{ccV}$ & & 0 & are-Qc & BR & $Q C$ & 5.75 .02 & $5.65 \bullet+02$ & 98.261 & t Recovery \\
\hline $2 \mathrm{ccv}$ & & 0 & atc-Qc & No3 & QC & 6.14002 & $5.960+02$ & 97.068 & Recovery \\
\hline $2 \mathrm{ccv}$ & & 0 & QIC-QC & po4 & $\alpha c$ & 5.46 .02 & $5.11=+02$ & 93.590 & \& Recovery \\
\hline $2 \mathrm{ccv}$ & & 0 & eIc-QC & so4 & oc & 6.31 .02 & $6.200+02$ & 98.257 & \& Recovery \\
\hline $2 \mathrm{ccv}$ & & 0 & are-ge & OXALATE2 & $\mathrm{oc}$ & 5.37002 & $5.37 \bullet+02$ & 100.000 & \& Recovery \\
\hline 3 BLNK-PEKP & & 0 & atc-0I & F-02 & SOLID & $\because \quad 1$ & $<1.300-2$ & & $\mathrm{ug} / \mathrm{g}$ \\
\hline 3 BLNK-PREP & & 0 & QIC-0I & CL- 02 & SOLID & 1 & $<1.70 \bullet-2$ & & $\mathrm{ug} / \mathrm{g}$ \\
\hline 3 BLAK-PRIP & & 0 & aIC-01 & No2-02 & SOLID & 1 & $<1.07-1$ & & $u g / g$ \\
\hline 3 BLMK-PREF & & 0 & DIc-01 & BR-02 & SOLID & 1 & $<1.26 \bullet-1$ & ' & $\mathrm{ug} / \mathrm{g}$ \\
\hline 3 BLNK-PRBP & & 0 & DC-01 & No3 -02 & SOLID & 1 & $3.86 e-01$ & 0.386 & $\mathbf{u g} / \mathbf{g}$ \\
\hline 3 ELNKK-PRIP & & 0 & DIC-0I & P04-02 & SOLID & 1 & $<1.190-1$ & & $\mathrm{ug} / \mathrm{g}$ \\
\hline 3 BLKK-PREP & & 0 & aIc-02 & $504-02$ & SOLID & 1 & $<2.360-1$ & & ug $/ \mathrm{g}$ \\
\hline 3 BLNTK-PREF & & 0 & $\operatorname{atc}-01$ & OKALATE2 & SOLID & 1 & $<1.05 \bullet-1$ & & ug $/ g$ \\
\hline 4 SAMPLE & S96T001071 & $0 \mathrm{~W}$ & arc-01 & $F-02$ & SOLID & $\mathrm{y} / \mathrm{A}<$ & $2.392 *+02$ & 239.300 & $g / g$ \\
\hline 4 SAMPLI & 5957001071 & o w & -IC-01 & CL- 02 & SOLID & $N / A$ & $1.412 a+03$ & 312.900 & $g / g$ \\
\hline 4 SAUPLB & $396 \mathrm{T001071}$ & $0 \mathrm{w}$ & $\cos -02$ & $\mathrm{NO} 2-02$ & SOLID & $\mathbf{N} / \mathbf{A}$ & $1.6220+04$ & 1970.000 & $g / g$ \\
\hline 4 REJRCT & 5962001071 & $0 \mathrm{w}$ & $\operatorname{arc}-01$ & BR-02 & SOLID & $g / A<$ & $2.319 \bullet+03$ & & $g / g$ \\
\hline 4 SAMPLE & $\$ 96 T 001071$ & O W & arc-01 & No3 -02 & SOLID & $\underline{N / A}$ & $6.503 e+05$ & 2574.000 & $g / g$ \\
\hline 4 SAMPLE & S96T001071 & $0 \mathrm{~W}$ & $\operatorname{arc-01}$ & PO4-02 & SOLID & $\mathbf{N} / \mathbf{A}$ & $1.165 *+04$ & 2189.000 & $g / g$ \\
\hline 4 SNAPLE & 5967001071 & $0 . w$ & arc-02 & $504-02$ & SOLID & $\mathbf{N} / \mathrm{A}$ & $6.307 e+03$ & 2501.000 & $g / g$ \\
\hline 4 SAMPLB & S96T001071 & 0 พ & aIC-01 & OXALATE2 & SOLID & $w / A<$ & $1.932 \bullet+03$ & 1932.000 & $g / g$ \\
\hline 5 DUP & $s 96 \mathrm{~T} 001071$ & $0 \mathrm{~W}$ & arc-01 & F-02 & SOLID & $<2.3902$ & $<2.3902$ & & RPD \\
\hline 5 DDP & 5967001071 & o w & MIC-01 & CL-02 & SOLID & $1.41 \bullet+03$ & $1.30=+03$ & 8.118 & RPD \\
\hline 5 DOP & 5967001071 & $0 \mathrm{w}$ & Dre-01 & No2 -02 & SOL.ID & $1.620+04$ & $1.620+04$ & 0.000 & RPD \\
\hline 5 DUP & $596 \mathrm{~T} 001071$ & $0 \mathrm{w}$ & aIC-01 & BR-02 & SOLID & $<2.3203$ & $<2.31 .3$ & & RPD \\
\hline 5 DOP & 5965001071 & o w & $\operatorname{exc} 01$ & NO3-02 & SOLID & $6.500+05$ & $6.444+05$ & 0.927 & RPD \\
\hline $5 \mathrm{DDP}$ & $S 96 T 002071$ & $0 \mathrm{w}$ & arc-01 & P04-02 & SOLID & $1.160+04$ & $1.200+04$ & 3.390 & RPD \\
\hline 5 DOP & S96T001071 & $0 W$ & axc-01 & so4-02 & SOLID & $6.310+03$ & $6.350+03$ & 0.632 & RPD \\
\hline 5 DUP & $596 \mathrm{~T} 001071$ & ow & exc-01 & OXALATE2 & SOLID & $<1.93 \times 3$ & $2.580+03$ & & RPD \\
\hline $6 \mathrm{SPK}$ & S96T001071 & $0 w$ & erc-01 & $F-02$ & SOLID & 5.90001 & $5.87 \cdot+02$ & 99.492 & s Recovery \\
\hline
\end{tabular}




\section{LABCORE Completed Worklist Report for Worklist\# 7493}

\begin{tabular}{|c|c|c|c|c|c|c|c|c|c|c|c|}
\hline Seq & Type & Sample\# & $\mathbf{A}$ & & & & & Actual & Found & DL or Yied & Unit \\
\hline 6 & SPK & $\$ 96 \mathrm{T001071}$ & 0 & $w$ & aIc-01 & CL-02 & SOLID & 7.90001 & $7.200+02$ & 91.139 & \& Recovery \\
\hline 6 & SPK & 5967001071 & 0 & $\mathbf{w}$ & OIC-01 & $\mathrm{NO}_{2}-\mathrm{O}_{2}$ & SOIID & $5.76 \bullet 02$ & $5 \cdot 47 \cdot+02$ & 94.965 & \& Recovery \\
\hline 6 & SPK & $\$ 967001071$ & 0 & $w$ & $\operatorname{arc}-01$ & ER-02 & SOLID & $5.75=02$ & $5.35+02$ & 93.043 & \% Recovery \\
\hline 6 & SPK & 5967001071 & 0 & $w$ & $\triangle I C-01$ & $\mathrm{NO3}-02$ & SOLID & $6.14 \bullet 02$ & $6.16 e+02$ & 100.326 & i Recovery \\
\hline 6 & SPK & 5967001071 & 0 & $\omega$ & AIC-01 & $P 04-02$ & SOLID & $5.46 \oplus 02$ & $4.780+02$ & $97-546$ & \& Recorery \\
\hline 6 & SPK & $\$ 967001071$ & 0 & $w$ & eIc-01 & $504-02$ & SOLID & $6.31 \bullet 02$ & $5.87=+02$ & 93.027 & \% necorery \\
\hline 6 & SPX & 9967002071 & 0 & $\omega$ & IC-01 & OXALAII2 & SOIIID & 5.37002 & $5.43=+02$ & 101.117 & if Recerery \\
\hline 7 & SAMPLE & $596 T 001072$ & 0 & $w$ & $\operatorname{arc}-01$ & $F-02$ & SOLID & $\mathbf{n} / \mathbf{A}<$ & $2.677 \bullet+02$ & $267.600 \mathrm{ug}$ & $/ g$ \\
\hline 7 & SAMPLE & 5967001072 & 0 & $\boldsymbol{w}$ & eIc-01 & CL-02 & sotid & $N / \mathbf{A}$ & $2.2560+03$ & 350.100 & $/ 9$ \\
\hline 7 & SAMPLE & S96T001072 & 0 & $w$ & arc-01 & $102-02$ & SOLID & $N / \mathbf{A}$ & $2.373 \bullet+04$ & 2203.000 & $/ 9$ \\
\hline 7 & REJECT & 9967001072 & 0 & $\omega$ & AIC-01 & BR-02 & SOIID & $B / A<$ & $2.594+03$ & ug & $9 / 9$ \\
\hline 7 & SAXPLE & 5967001072 & 0 & $w$ & $\operatorname{arc}-01$ & $\operatorname{No3}-02$ & SOLID & $\mathbf{n} / \mathbf{A}$ & $5.5330+05$ & 2081.000 & $/ 9$ \\
\hline 7 & SAMPEE & s96T001072 & 0 & $w$ & QIC-0I & $\mathrm{PO4-02}$ & SOLID & $\mathbf{n} / \mathbf{x}$ & $1.3360+04$ & 2448.000 & $9 / 9$ \\
\hline 7 & SAMPLE & $\mathrm{S} 96 \mathrm{T001072}$ & 0 & $\mathbf{w}$ & QIC-0I & $504-02$ & SOLID & $\mathbf{N} / \mathbf{A}$ & $0.0580+03$ & 2799.000 & $9 / 9$ \\
\hline 7 & SAXPLE & $596 T 001072$ & 0 & $w$ & arc-01 & OXALATE2 & sorID & $\mathbf{N} / \mathbf{A}$ & $3.213 \bullet+03$ & 2162.000 & $3 / 9$ \\
\hline 8 & DUP & 5967001072 & 0 & $w$ & eIc-01 & $F-02$ & SOLID & $<2.68 \notin 2$ & $<2.7602$ & & RPD \\
\hline 8 & DUP & $595 T 001072$ & 0 & $w$ & arc-01 & Cl- 02 & sourd & $2.26=003$ & $2.40 .+03$ & 6.009 & RPD \\
\hline$B$ & DUP & $S 96 T 001072$ & 0 & $w$ & Orc-02 & $\mathrm{NO2}-02$ & EOLID & $2.37 \cdot+04$ & $2.56 \bullet+04$ & 7.708 & RPD \\
\hline $\mathbf{a}$ & DOP & $\$ 96 T 001072$ & 0 & $w$ & $\operatorname{PIC}-01$ & $\mathrm{BR}-02$ & softs & $<2.59 .3$ & $<2.67 .3$ & & RPD \\
\hline e & ndp & S96T001072 & 0 & $w$ & $\operatorname{erc-01}$ & No3-O2 & sorID & $5.53=0.05$ & $5.35=+05$ & $3.309=$ & $\mathrm{RPD}$ \\
\hline 8 & DUP & S95T001072 & 0 & $W$ & IC-OI & PO4-02 & soLID & $1.34 \cdot+04$ & $1.43 \bullet+04$ & 6.498 & RPD \\
\hline $\mathbf{8}$ & DOP & S96T001072 & 0 & $w$ & $\operatorname{AIC-01}$ & $504-02$ & SOLTD & B. $06+0+03$ & $1.000+04$ & 12.009 & RPD \\
\hline $\mathbf{8}$ & DUP & s96T001072 & 0 & W & Oxc-01 & OXALATE2 & sotID & $3.11 \cdot+03$ & $3.48 \cdot+0.3$ & 11.229 & RPD \\
\hline
\end{tabular}

\section{Final page for worklist\# 7493}

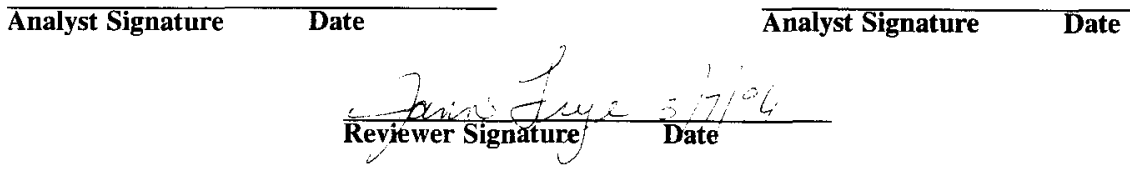

Units shown for $Q C(\overline{B L K} / B K G)$ may not reflect the actual units. 
WHC-SD-WM-DP-184, REV. I

\begin{tabular}{|c|c|c|c|c|c|c|c|}
\hline \multicolumn{3}{|c|}{$A-0010-I C$} & \multicolumn{4}{|c|}{ DATA FILE/WORKLIST RESOLUTION } & J6-May-96 \\
\hline \multicolumn{4}{|c|}{ Workl ist\#: 7493} & \multicolumn{4}{|c|}{ Data File: 7493AP.CSV } \\
\hline & Seq & Type & Sample \# & Seq\# & Data File & Sample Name & Dilution \\
\hline $\begin{array}{l}\Rightarrow \\
\Rightarrow \\
\Rightarrow \\
\Rightarrow \\
\Rightarrow \\
\Rightarrow \\
\Rightarrow \\
\Rightarrow\end{array}$ & $\begin{array}{l}1 \\
2 \\
3 \\
4 \\
5 \\
6 \\
7 \\
8\end{array}$ & $\begin{array}{l}\text { CCB } \\
\text { CCV } \\
\text { BLNK-PREP } \\
\text { SAMPLE } \\
\text { DUP } \\
\text { SPK } \\
\text { SAMPLE } \\
\text { DUP }\end{array}$ & $\begin{array}{l}\text { S96T001071 } \\
\text { S96T001071 } \\
\text { S96T001071 } \\
\text { S96T001072 } \\
\text { S96T001072 }\end{array}$ & & $\begin{array}{l}96041801 . d 01 \\
96041801 . d 02 \\
96041801 . d 03 \\
96041801 . d 04 \\
96041801 . d 05 \\
96041801 . d 06 \\
96041801 . d 07 \\
96041801 . d 08 \\
96041801 . d 09\end{array}$ & 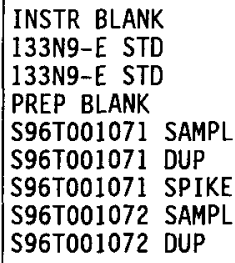 & $\begin{array}{r}1.00 \\
101.00 \\
101.00 \\
1.00 \\
101.00 \\
101.00 \\
101.00 \\
101.00 \\
101.00\end{array}$ \\
\hline
\end{tabular}

Save(F4) Abort(Shift-F3) ListFiles(Shift-F1) UploadFile(F8) 


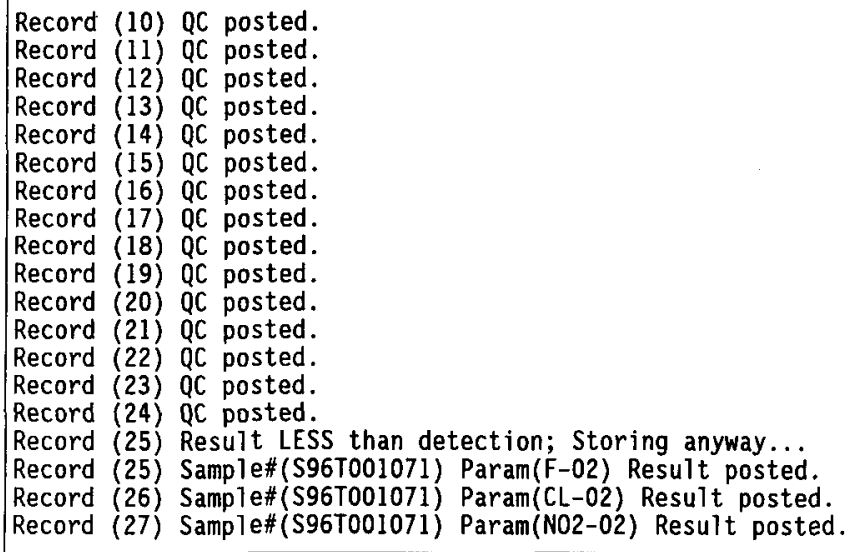

Keys(F5) NextPage(Page Dn) PrevPage(Page Up) Help(F2) > 
HELP -- LogFile Viewer

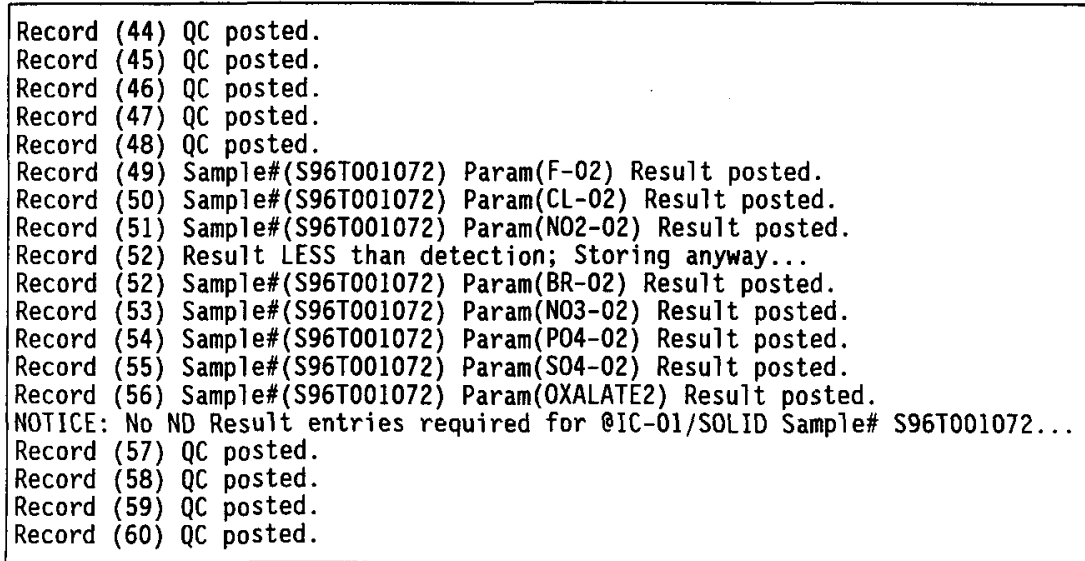

Keys(F5) NextPage(Page Dn) PrevPage(Page Up) Help(F2) > 
Data Reprocessed On 04/29/1996 11:58:28

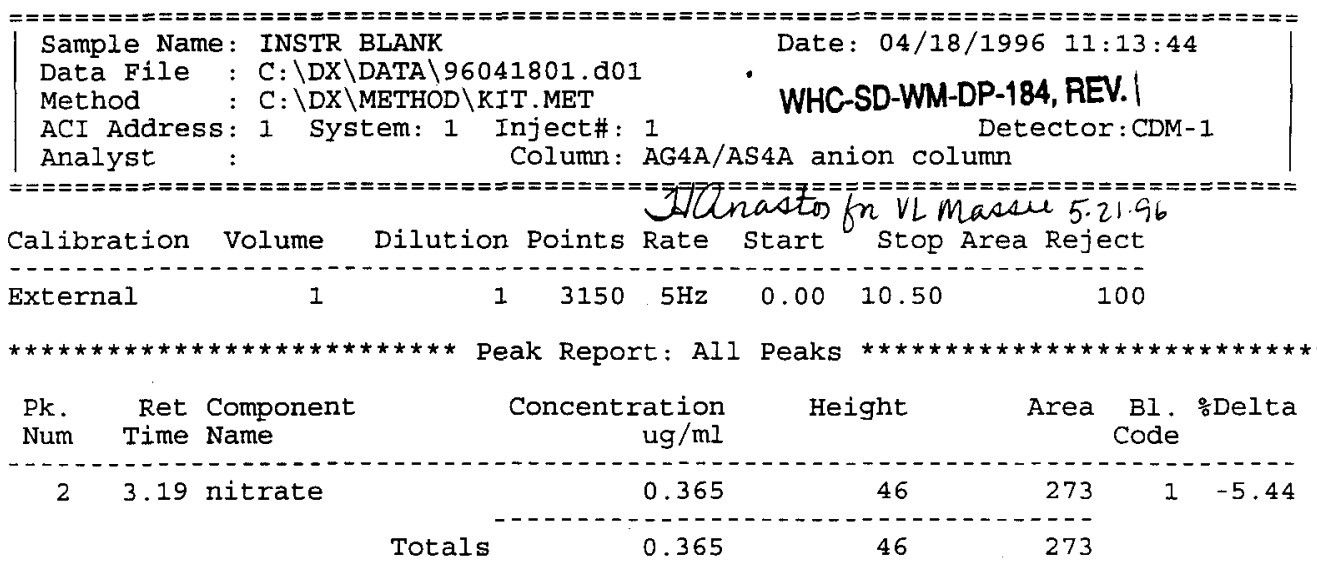

File: 96041801.d01 Sample: INSTR BLANK

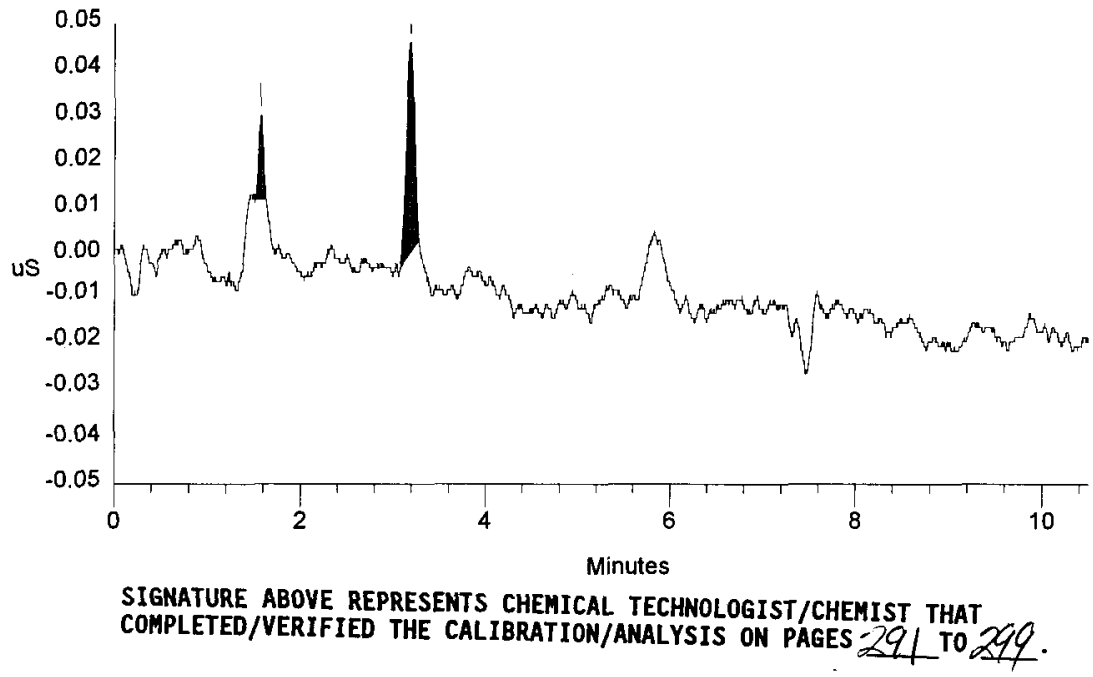


Data Reprocessed On 04/29/1996 11:58:33

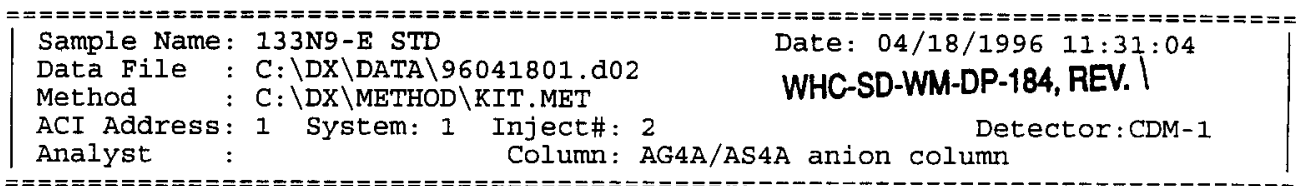

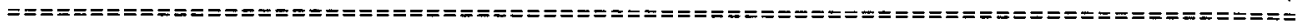

Calibration Volume Dilution Points Rate Start Stop Area Reject

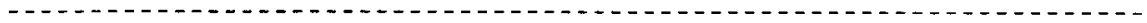

$\begin{array}{llllllll}\text { External } & 1 & 101 & 3150 & 5 \mathrm{~Hz} & 0.00 & 10.50 & 100\end{array}$

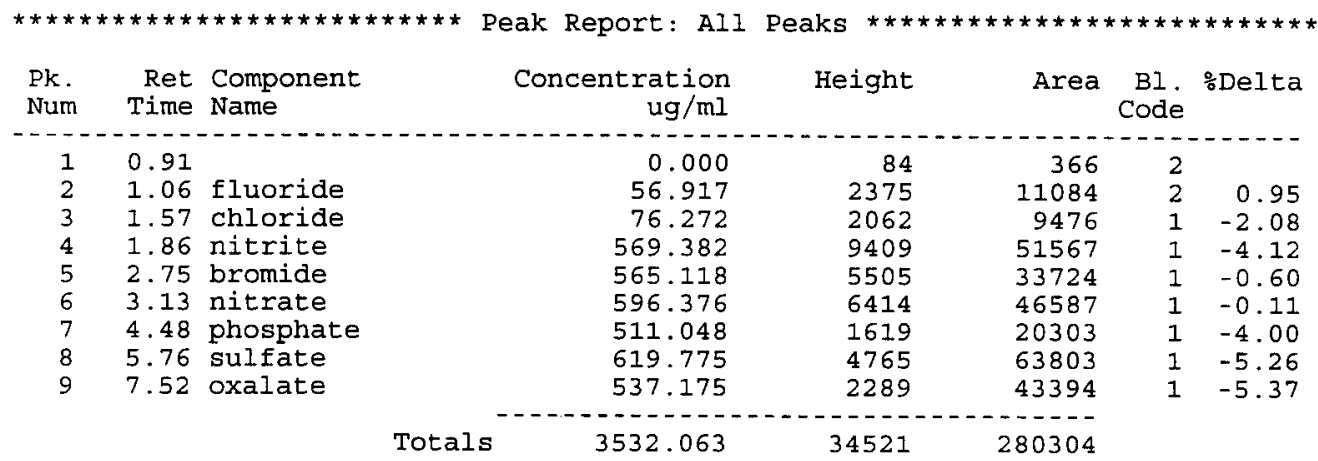

File: 96041801.d02 Sample: 133N9-E STD

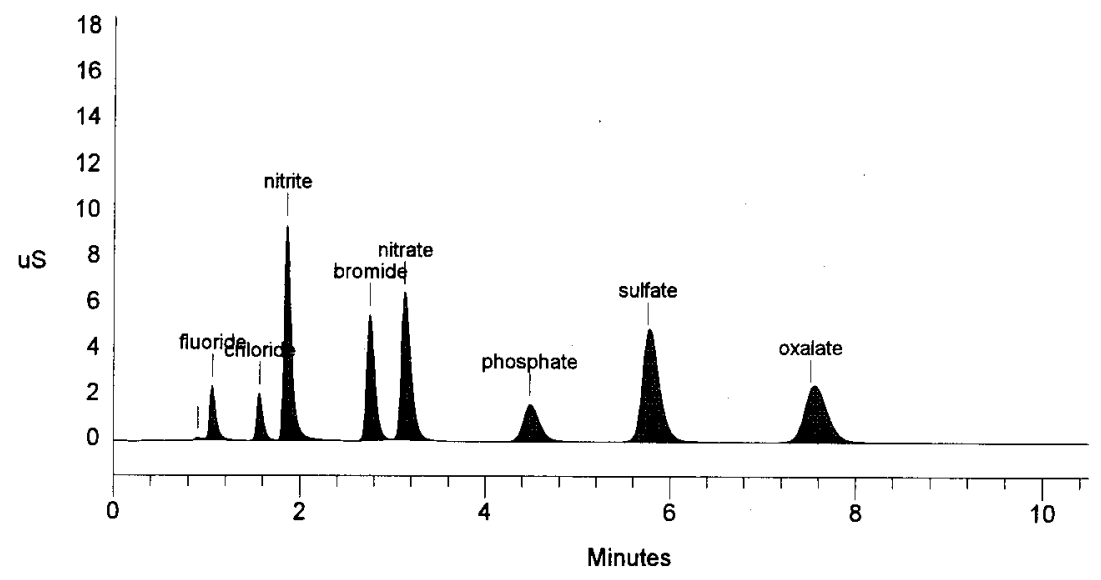

